\title{
Do. 253
}

\section{X-Ray Fluorescence Cross Sections for $K$ and $L X$ Rays of the Elements}

$$
\begin{aligned}
& \text { M O Krause } \\
& C \text { W Nestor, Jr } \\
& \text { C J Sparks, Jr } \\
& \text { E RicCl }
\end{aligned}
$$

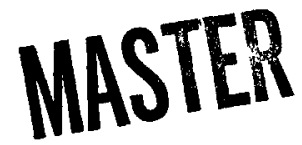

\section{OAK RIDGE NATIONAL LABORATORY} OPERATED BY UNION CARBIDE CORPORATION - FOR THE DEPARTMENT OF ENERGY 


\section{DISCLAIMER}

This report was prepared as an account of work sponsored by an agency of the United States Government. Neither the United States Government nor any agency Thereof, nor any of their employees, makes any warranty, express or implied, or assumes any legal liability or responsibility for the accuracy, completeness, or usefulness of any information, apparatus, product, or process disclosed, or represents that its use would not infringe privately owned rights. Reference herein to any specific commercial product, process, or service by trade name, trademark, manufacturer, or otherwise does not necessarily constitute or imply its endorsement, recommendation, or favoring by the United States Government or any agency thereof. The views and opinions of authors expressed herein do not necessarily state or reflect those of the United States Government or any agency thereof. 


\section{DISCLAIMER}

Portions of this document may be illegible in electronic image products. Images are produced from the best available original document. 


\section{Printed in the United States of America. Available from}

National Technical Information Service

U.S. Department of Commerce 5285 Port Royal Road, Springfield, Virginia 22161

Price: Printed Copy $\$ 8.00$; Microfiche $\$ 3.00$

This report was prepared as an account of work sponsored by an agency of the United States Government Neither the United States Government nor any agency thereof, nor any of their employees, contractors, subcontractors, or their employees, makes any warranty, express or implied, nor assumes any legal liability or responsibility for any third party's use or the results of such use of any information, apparatus, product or process disclosed in this report, nor represents that its use by such third party would not infringe privately owned rights 

X-RAY FLUORESCENCE CROSS SECTIONS FOR $K$ AND $\stackrel{L}{=}$ X RAYS OF THE ELEMENTS
M. O. Krause ${ }^{*}$
C. W. Nestor, $\mathrm{Jr} .^{\dagger}$
C. J. Sparks, Jr. $\ddagger$
E. Ricci ${ }^{\S}$

\footnotetext{
*hemistry Division

${ }^{\dagger}$ Computer Sciences Division

$\ddagger$

Metals and Ceramics Division

§Analytical Chemistry Division
}

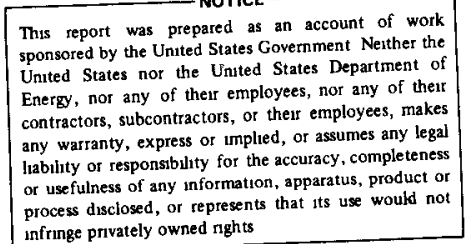

contractors, subcontractors, or

eroduct or

infringe privately owned rights

Date Published: June 1978

OAK RIDGE NATIONAL LABORATORY

Oak Ridge, Tennessee 37830

operated by

UNION CARBIDE CORPORATION

for the

DEPARTMENT OF ENERGY 
0

○ 


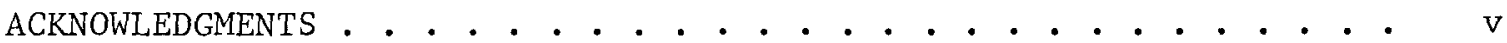
ABSTRACT • . . . . . . . . . . . . . . . . . . . . . . . . . . 1

1. INTRODUCTION . . . . . . . . . . . . . . . . . . . . . . . . . . 1

1.1 Formulae . . . . . . . . . . . . . . . . . . . . . . . 2

1.2 Source Material . . . . . . . . . . . . . . . . . . . 4

1.3 Estimate of Uncertainties . . . . . . . . . . . . . . . 4

1.4 Remarks . . . . . . . . . . . . . . . . . . 11

2. TABLES AND GRAPHS OF X-RAY FLUORESCENCE CROSS SECTIONS

FOR INCIDENT PHOTONS IN THE ENERGY RANGE 1 TO $200 \mathrm{keV} \mathrm{•} \mathrm{•} \mathrm{•} \mathrm{•} 13$

3. TABLES AND GRAPHS OF X-RAY FLUORESCENCE CROSS SECTIONS

FOR SPECIFIC PHOTON SOURCES . • . . . . . . . . . . . . . . . 99

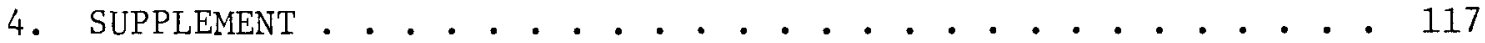

REFERENCES • • • • • • • • • • • • • • • • • • • • • • • • 
0

O 


\section{ACKNOWLEDGMENTS}

We express our sincere appreciation to J. H. Scofield, Lawrence Livermore Laboratory, who put at our disposal a computer tape containing his calculation of the partial photoionization cross sections. We acknowledge helpful discussions with L. D. Hulett, ORNL, on the applications of $\mathrm{x}$-ray fluorescence analysis. 
X-RAY FLUORESCENCE CROSS SECTIONS FOR $K$ AND $L X$ RAYS OF THE ELEMENTS*

M. O. Krause, C. W. Nestor, Jr., C. J. Sparks, Jr., and E. Ricci

\section{ABSTRACT}

$X$-ray fluorescence cross sections are calculated for the major $x$ rays of the $K$ series $5 \leqslant z \leqslant 101$, and the three $L$ series $12 \leqslant Z \leqslant 101$ in the energy range 1 to $200 \mathrm{keV}$. This calculation uses Scofield's theoretical partial photoionization cross sections, Krause's evaluation of fluorescence and Coster-Kronig yields, and Scofield's theoretical radiative rates. Values are presented in table and graph format, and an estimate of their accuracy is made. The following $x$ rays are considered: $K \alpha_{1}, K \alpha_{1}, 2, K \beta_{1}, K \beta_{1,3}, L \alpha_{1}, L \alpha_{1,2}, L \beta_{1}$, $L \beta_{2,15}, L \beta_{3}, L \ell, L \gamma_{1}, L \gamma_{4}$, and $L_{1} \rightarrow L_{2,3}$. For use in $x$-ray fluorescence analysis, $K \alpha$ and $L \alpha$ fluorescence cross sections are presented at specific energies: TiK $\equiv 4.55 \mathrm{keV}$, $\mathrm{Cr} K \equiv 5.46 \mathrm{keV}, \operatorname{CoK} \equiv 7.00 \mathrm{keV}, \mathrm{Cu} K \equiv 8.13 \mathrm{keV}, \operatorname{Mo} K \alpha \equiv 17.44 \mathrm{keV}$, $\mathrm{Ag} K \equiv 22.5 \mathrm{keV}$, DyK $\equiv 47.0 \mathrm{keV}$, and $241_{\mathrm{Am}} \equiv 59.54 \mathrm{keV}$. Supplementary material includes fluorescence and CosterKronig yields, fractional radiative rates, fractional fluorescence yields, total $L$-shell fluorescence cross sections, fluorescence and Coster-Kronig yields in condensed matter, effective fluorescence yields, average $L$-shell fluorescence yield, L-subshell photoionization cross section ratios, and conversion factors from barns per atom to square centimeters per gram.

\section{INTRODUCTION}

$\mathrm{X}$-ray fluorescence cross sections are important in various applied fields, such as radiation transport in matter, dosimetry, and especially $x$-ray fluorescence analysis using either traditional photon sources or synchrotron radiation. In all cases, an accurate knowledge of fluorescence cross sections is desirable. This requires reliable values of

* Research sponsored by the Division of Nuclear Sciences, Office of Basic Energy Sciences, of the U.S. Department of Energy under contract with Union Carbide Corporation. 
the basic parameters that enter into the calculation of the fluorescence cross section - namely, partial photoionization cross sections, radiative rates, fluorescence yields, and Coster-Kronig yields. Until recently, accurate values were known only for the $K$-shell parameters, but now reliable values have become available also for the parameters pertaining to the $L$ subshells. Thus it has becone feasible for the first time to calculate, for both the $K$ and the $L$ shells, fluorescence cross sections of sufficient accuracy to be useful in the various applications of innershell ionization by photons.

In this report, we present tables and graphs of fluorescence cross sections calculated for the major $\mathrm{x}$ ravs of the $K$ and $L$ series in the photon energy range 1 to $200 \mathrm{keV}$. To facilitate use, results are given in two formats: (1) for each element, cross sections are tabulated and plotted for the various characteristic $K$ and $L \mathrm{x}$ rays as a function of photon energy, and (2) for each of the important $K$ and $L \mathrm{x}$ rays, cross sections are tabulated and plotted for regularly spaced photon energies as a function of atomic number 2 . For the special needs of the $x$-ray fluorescence spectroscopist, $K \alpha$ and $L \alpha$ fluorescence cross sections are tabulated for several frequently used excitation sources and are plotted with the cross sections of the less intense, but still strong $x$-ray lines. A detailed estimate of the uncertainties of the calculated values is made on the basis of the known or inferred uncertainties of the input parameters. Supplementary tables contain background and auxiliary material for the benefit of the user of the principal tables.

\subsection{Formulae}

The fluorescence cross section $\sigma_{i j}$ for an $x$ ray $i j$ is the product of the partial or subshell photoionization cross section $\sigma_{i}$, the fractional radiative rate $F_{i j}$, the fluorescence yield $\omega_{i}$, and the hole transfer factor $T_{i, k}\left(T_{i, k} \geqslant 1\right)$ :

$$
\sigma_{i j}=\sigma_{i} \omega_{i} F_{i j}{ }^{T}, k,
$$


where $i$ refers to the subshell photoionized, $j$ to the final state of an $\mathrm{x}$-ray transition, and $k$ to the subshell to which a vacancy has been shifted by a Coster-Kronig transition. Alternatively, $i j$ could be considered the designation of a characteristic $\mathrm{x}$ ray, for example, $K \alpha_{1}$, $L \beta_{2}$, etc. The partial rate $F_{i j}$ is given by the radiative rate $S_{i j}$ for the $\mathrm{x}$ ray $i j$ relative to the total radiative rate $S_{i, R}$ for a vacancy in the subshell $i$ :

$$
F_{i j}=S_{i j} / S_{i, R}
$$

Obviously, for the $K$ shell, and for any $s$ subshell, the hole transfer factor $T_{i, k}$ is unity, and we have for an $x$ ray of the $K$ series

$$
\sigma_{K j}=\sigma_{K} \omega_{K} F_{K j}
$$

or for $K \alpha_{1}$, using an example,

$$
\sigma_{K \alpha_{1}}=\sigma_{K}^{\omega} F_{K \alpha_{1}}
$$

Similarly for an $x$ ray originating in the $L_{1}$ subshell, we have

$$
\sigma_{L_{1} j}=\sigma_{L_{1}}{ }^{\omega_{1}} F_{L_{1} j}
$$

where $\omega_{1}$ is an abbreviation of $\omega_{L_{1}}$.

For an $x$ ray of the $L_{2}$ emission series, we have

$$
\sigma_{L_{2} j}=\sigma_{L_{2}} \omega_{2} F_{L_{2} j}\left(1+\frac{\sigma_{L_{1}}}{\sigma_{L_{2}}} f_{1,2}\right) \text {, }
$$

and for an $\mathrm{x}$ ray of the $L_{3}$ emission series,

$$
\sigma_{L_{3 j} j}=\sigma_{L_{3} \omega_{3} F_{L_{3} j}}\left[1+\frac{\sigma_{L_{2}}}{\sigma_{L_{3}}} f_{2,3}+\frac{\sigma_{L_{1}}}{\sigma_{L_{3}}}\left(f_{1,3}+f_{1,3}^{\prime}+f_{1,2} f_{1,3}\right)\right] \text {, }
$$

where the bracketed terms define the hole transfer factor $T_{i, k^{*}}$ 
In Eqs. 5 and 6 , the $f_{i, k}$ factors are the Coster-Kronig yields that shift a vacancy from subshell $i$ to subshell $k$ before the emission of an $x$ ray. Yields $f_{1,2}, f_{1,3}$, and $f_{2,3}$ refer to radiationless transitions and $f^{\prime} 1,3$ to the radiative transition $L_{1} \rightarrow L_{3}$.

Equations 4 to 6 apply to photon energies in the range $E_{L_{i}} \lesssim h \nu<E_{K^{*}}$, where $i=1,2,3$, and $E_{K^{*}}$ is the minimum energy required to excite a $K$ electron. The effect of hole transfer for $L_{2}$ and especially $L_{3}$ series $\mathrm{x}$ rays may be large as discussed earlier. ${ }^{1}$

Equation 1 and its detailed versions, Eqs. 3 to 6 , were used to calculate $x$-ray fluorescence cross sections tabulated and plotted in Sects. 2 and 3 .

\subsection{Source Materia1}

Partial photoionization cross sections were obtained from Scofield's relativistic Hartree-Slater calculation, ${ }^{2}$ fluorescence and Coster-Kronig yields from Krause's recent evaluation, ${ }^{3}$ and fractional radiative rates and intrashell radiative yield $f^{\prime} 1,3$ from Scofield's relativistic Hartree-Fock calculations. ${ }^{4-8}$

\subsection{Estimate of Uncertainties}

Uncertainties of the calculated values are estimated on the basis of the known or inferred uncertainties of the input parameters.

\section{Partial photoionization cross sections}

In the absence of systematic experimental tests of theoretical partial cross sections over an extended $Z$ range, an idea of the uncertainty of the cross section values may be obtained by comparing theoretical total photoionization cross sections with experimental determinations in regions where either the $K$-shell component $\sigma_{K}$ or the $L$-shell component $\sigma_{L}$ predominates. In Tables 1 to 4 , the semiempirical values by Plechaty et al. 9,10 and the experimental (evaluated) values by Veigele ${ }^{11}$ are compared with Scofield's predictions. ${ }^{2}, 12$ Agreement is good, generally within $3 \%$, except for regions close to absorption edges. Because the 
Table 1. Comparison of evaluated ${ }^{a}$ and theoretical ${ }^{b}$ photoionization cross sections above $K$ edge: $100\left(\sigma_{1}-\sigma_{2}\right) / \sigma_{2}$

(The relative $K$-shell cross section is also given.)

\begin{tabular}{lccccccc}
\hline & & & \multicolumn{5}{c}{ Range of photon energies (keV) } \\
\cline { 5 - 8 } Element & Atomic & & & Near & & & \\
number & $\sigma_{K} / \sigma_{\text {total }}$ & $K$ edge & $5-10$ & $10-60$ & $60-100$ & $100-400$ \\
\hline Sulfur & 16 & $>0.90$ & +6 & +2 & -3 & \pm 1 & \\
Selenium & 34 & $>0.88$ & +7 & & \pm 2 & -3 & -3 \\
Barium & 56 & $>0.84$ & +10 & & +5 & \pm 2 & -3 \\
Tantalum & 73 & $>0.81$ & +7 & & & +3 & -3 \\
Uranium & 92 & $>0.77$ & +5 & & & & -2 \\
\hline
\end{tabular}

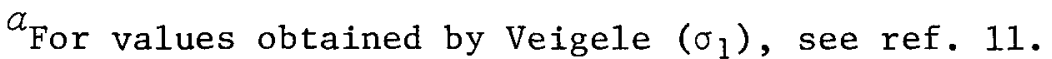

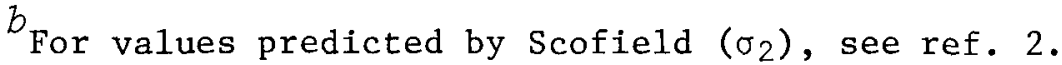

Table 2. Comparison of semiempirical ${ }^{a}$ and theoretical ${ }^{b}$ photoionization cross sections above $K$ edge: $100\left(\sigma_{1}-\sigma_{2}\right) / \sigma_{2}$

\begin{tabular}{|c|c|c|c|c|c|c|}
\hline \multirow[b]{2}{*}{ Element } & \multirow[b]{2}{*}{$\begin{array}{l}\text { Atomic } \\
\text { number }\end{array}$} & \multicolumn{5}{|c|}{ Range of photon energies (keV) } \\
\hline & & $\begin{array}{c}\text { Near } \\
K \text { edge }\end{array}$ & $5-10$ & $10-60$ & $60-100$ & $100-400$ \\
\hline Sulfur & 16 & +2 & \pm 2 & -4 & -2 & \\
\hline Selenium & 34 & -2 & & +2 & +1 & +2 \\
\hline Barium & 56 & -2 & & +2 & +1 & \pm 1 \\
\hline Tantalum & 73 & 0 & & & -2 & -2 \\
\hline Uranium & 92 & +6 & & & & -1 \\
\hline Californium & 98 & +4 & & & & -1 \\
\hline
\end{tabular}

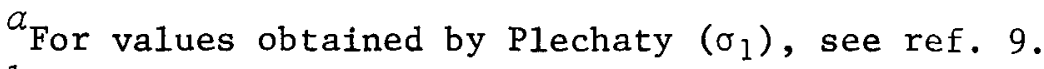

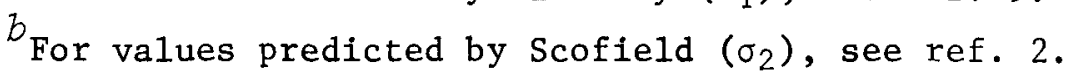


Table 3. Comparison of evaluated ${ }^{a}$ and theoretical ${ }^{b}$ photoionization cross sections between $L$ and $K$ edges: $100\left(\sigma_{1}-\sigma_{2}\right) / \sigma_{2}$

(The relative $L$-shel1 cross section for $E_{L_{1}}<E<E_{K}$ is also given.)

\begin{tabular}{lccccc}
\hline & & & \multicolumn{2}{l}{ Range of photon energies between edges } \\
\cline { 5 - 6 } Element & $\begin{array}{c}\text { Atomic } \\
\text { number }\end{array}$ & $\sigma_{L} / \sigma_{\text {total }}$ & $\begin{array}{c}\text { Near } \\
\text { edges }\end{array}$ & $\begin{array}{c}\text { First } \\
\text { half }\end{array}$ & $\begin{array}{c}\text { Second } \\
\text { half }\end{array}$ \\
\hline Sulfur & 16 & $>0.87$ & & +1 & +1 \\
Selenium & 34 & $>0.85$ & +10 to 20 & +2 & +3 \\
Barium & 56 & $>0.78$ & +3 to 8 & +3 & +0 \\
Tantalum & 73 & $>0.76$ & +2 to 5 & -2 & +1 \\
Uranium & 92 & $>0.74$ & +1 to 5 & -3 & -2 \\
\hline \multicolumn{2}{c}{$\begin{array}{l}a_{F} \\
b_{F}\end{array}$} & &
\end{tabular}

Table 4. Comparison of semiempirical ${ }^{a}$ and theoretical ${ }^{b}$ photoionization cross sections between $L$ and $K$ edges: $100\left(\sigma_{1}-\sigma_{2}\right) / \sigma_{2}$

\begin{tabular}{lcccc}
\hline & \multicolumn{2}{c}{ Range of photon energies between edges } \\
\cline { 4 - 5 } Element & $\begin{array}{c}\text { Atomic } \\
\text { number }\end{array}$ & $\begin{array}{c}\text { Near } \\
\text { Ledges }\end{array}$ & $\begin{array}{c}\text { First } \\
\text { half }\end{array}$ & $\begin{array}{c}\text { Second } \\
\text { half }\end{array}$ \\
\hline Sulfur & 16 & & +4 & +2 \\
Selenium & 34 & +10 to 15 & +6 & +3 \\
Barium & 56 & +3 to 8 & +3 & +0 \\
Tantalum & 73 & \pm 1 to 3 & -1 & -0 \\
Uranium & 92 & -2 to 4 & -2 & -1 \\
Californium & 98 & \pm 2 to 4 & -3 & -3 \\
\hline
\end{tabular}

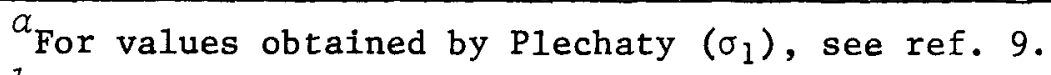

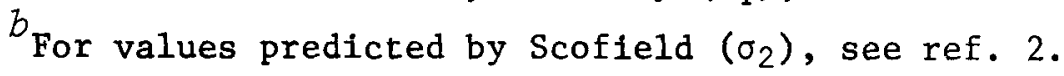


partial cross sections $\sigma_{K}$ and $\sigma_{L}$ account for more than $75 \%$ and often $90 \%$ of the total cross sections, the errors in $\sigma_{K}$ and $\sigma_{L}$ will not be much greater than those for $\sigma_{\text {total }}$. Partition of $\sigma_{L}$ into its components increases the uncertainty for $\sigma_{L_{1}}, \sigma_{L_{2}}$, and $\sigma_{L_{3}}$. However, the effect on the $x$-ray fluorescence cross section from this source should be less than $10 \%$ for $E_{L_{i}}<h \nu<E_{K}$ because of considerable compensation by hole transfer (Eqs. 5 and 6). Further discussions of data on subshell cross sections for low $Z$ elements and at low photon energies can be found elsewhere.13,14 The estimated uncertainties of the theoretical partial photoionization cross section must finally be combined with the uncertainties reported for the tabulated total photoionization cross section values (for example, those of refs. 9 to 11). For the latter, we relied on the assessment by Pratt et al. 15

\section{Fractional radiative rates}

Theoretical values have been checked against experimental data throughout the periodic table. 4-7,16 From these comparisons, uncertainty of the fractional $K \alpha$ rates can be surmised to be 1 ess than $2 \%$ and those of the fractional $L \alpha$ rates less than $4 \%$. For $L \beta_{1}$ and the weaker lines of the $K$ and $L_{3}$ series, these uncertainties should be doubled, and for the remaining $L$ lines uncertainties may exceed $10 \%$.

\section{Fluorescence and Coster-Kronig yields}

Estimates of uncertainties of these parameters are given in Table 5 . Uncertainty of the intrashel1 radiative yield $f^{\prime} 1,3$, even if large, would have a negligible effect on $\sigma_{L \alpha}$ since $f^{\prime} 1,3$ remains very small at any $Z$.

\section{Combined uncertainty}

Errors in the various contributions have been added quadratically to arrive at the estimated uncertainty in the $\sigma_{K \alpha}$ and $\sigma_{L \alpha}$ values. Results are summarized in Table 6 and displayed in Fig. 1. Note the regions of about $5 \%$ accuracy in $\sigma_{K \alpha}$ and of 10 to $15 \%$ in $\sigma_{L \alpha}$; these regions should be chosen for high-accuracy analytical work. Note also regions of 
Table 5. Estimated uncertainties of adopted values for fluorescence and Coster-Kronig yields (In percent)

\begin{tabular}{|c|c|c|c|c|c|c|c|c|c|c|c|}
\hline \multirow[b]{2}{*}{ Yield } & \multicolumn{11}{|c|}{ Range of atomic numbers } \\
\hline & $5-10$ & $10-20$ & $20-30$ & $30-40$ & $40-50$ & $50-60$ & $60-70$ & $70-80$ & $80-90$ & $90-100$ & $100-110$ \\
\hline$\omega_{K}$ & $40-10^{a}$ & $10-5$ & $5-3$ & 3 & 2 & $2-1$ & 1 & 1 & $i 1$ & $\imath^{<1}$ & 1 \\
\hline$\omega_{1}$ & & $>30^{a}$ & $30^{a}$ & $30^{b}$ & $30-20^{b}$ & $20-15$ & 15 & $15^{b}$ & 15 & $15-20$ & 20 \\
\hline$\omega_{2}$ & & $i^{25^{a}}$ & $25^{a, b}$ & 25 & $25-10$ & 10 & $10-5$ & 5 & 5 & $10^{b}$ & 10 \\
\hline$\omega_{3}$ & & $\imath^{25^{\alpha}}$ & $25^{a}$ & 20 & $20-10$ & $10-5$ & 5 & $5-3$ & 3 & $3-5$ & 5 \\
\hline$f_{1}$ & & $3^{a}$ & $5^{a}$ & 5 & $5-10^{b}$ & 15 & 10 & $10-5^{b}$ & 5 & $5-10$ & 15 \\
\hline$f_{1,2}$ & & $10^{a}$ & $15^{\alpha}$ & 15 & $20^{b}$ & 20 & 15 & $20^{b}$ & 10 & $10-50$ & $50-100$ \\
\hline$f_{1,3}$ & & $5^{a}$ & $10^{a}$ & 10 & $10^{b}$ & 15 & 10 & $10-5^{b}$ & 5 & $5-10$ & 15 \\
\hline$f_{2,3}$ & & & $i^{240^{a}}$ & $30-20$ & 20 & 20 & $20-15$ & 15 & 15 & $15^{b}$ & 20 \\
\hline
\end{tabular}

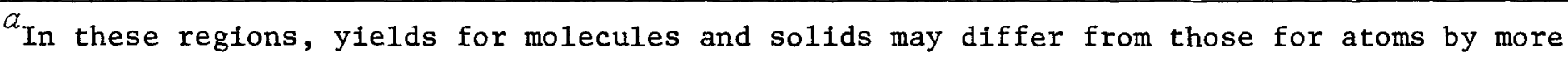
than the values quoted.

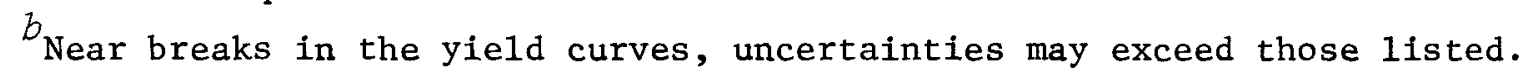


Table 6. Estimated uncertainties of calculated fluorescence cross sections for $K \alpha$ and $L \alpha \mathrm{x}$ rays

(In percent)

\begin{tabular}{|c|c|c|c|c|c|c|c|c|c|}
\hline \multirow{2}{*}{$\begin{array}{r}\text { Photon } \\
\text { energy } \\
(\mathrm{keV})\end{array}$} & \multirow[b]{2}{*}{ ray } & \multicolumn{8}{|c|}{ Range of atomic numbers } \\
\hline & & $5-10$ & $10-20$ & $20-30$ & $30-40$ & $40-60$ & $60-80$ & $80-90$ & $90-100$ \\
\hline \multirow[t]{2}{*}{$1-5$} & $K \alpha$ & $40-10$ & 10 & & & & & & \\
\hline & $L \alpha$ & & $>25$ & 25 & 25 & 25 & & & \\
\hline \multirow[t]{2}{*}{$5-10$} & $K \alpha$ & $40-10$ & 8 & 5 & & & & & \\
\hline & $L \alpha$ & & $>25$ & 25 & 25 & 20 & 20 & & \\
\hline \multirow[t]{2}{*}{$10-30$} & $K \alpha$ & $40-15$ & 10 & 5 & 5 & 5 & & & \\
\hline & $L \alpha$ & & $>30$ & 30 & 25 & $20-15$ & $10-15$ & 15 & $15-20$ \\
\hline \multirow[t]{2}{*}{$30-80$} & $K \alpha$ & $45-15$ & 15 & 8 & 5 & 5 & 5 & & \\
\hline & $L \alpha$ & & $>40$ & 35 & 25 & 20 & 10 & 10 & 15 \\
\hline \multirow[t]{2}{*}{$80-200$} & $K \alpha$ & $45-20$ & 20 & 15 & 10 & 8 & 8 & 10 & 15 \\
\hline & $L \alpha$ & & $\imath^{50}$ & 40 & 35 & 25 & 20 & 20 & 20 \\
\hline
\end{tabular}




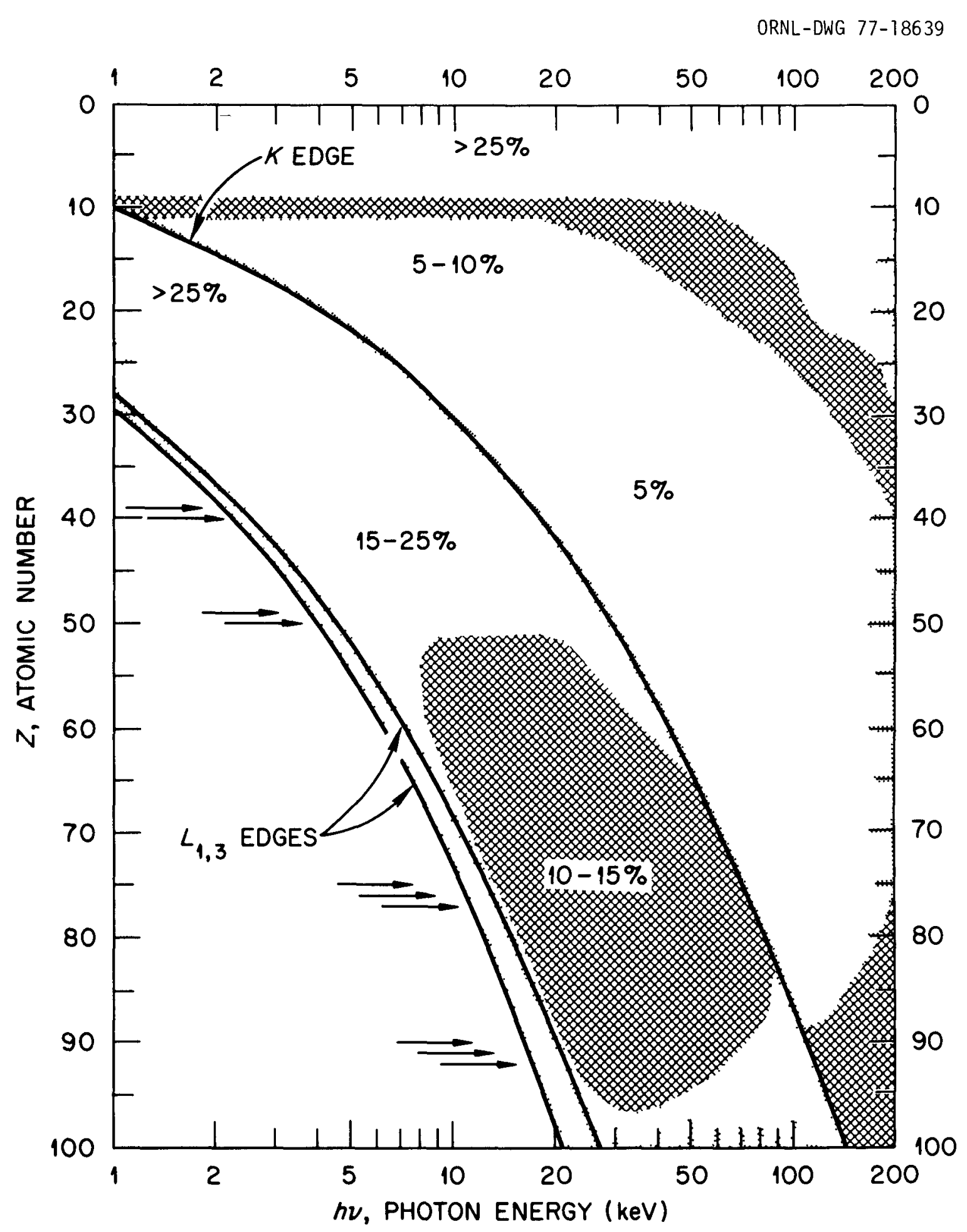

Fig. 1. Estimated uncertainties in calculated fluorescence cross sections for $K \alpha$ and $L \alpha \times$ rays. Arrows indicate regions where CosterKronig discontinuities occur. See also Table 6 . 
decreased accuracy near absorption edges and near the Coster-Kronig discontinuities indicated by arrows in Fig. 1. Innershell ionization by Compton scattering has not been taken into account, but it will make contributions at photon energies far above an absorption edge.

\subsection{Remarks}

Although the calculated $x$-ray fluorescence cross sections pertain to free atoms because of the use of atomic parameters in Eq. 1, they also apply to bound atoms, that is, molecular and solid state systems, in all but a few cases. If $x$-ray transitions involve inner or core electron levels, as in the case of the intense $K$ and $L \mathrm{x}$ rays of most elements of the periodic table, $x$-ray fluorescence cross sections are essentially invariant regardless of chemical environment, because neither transition rates nor innershell photoionization cross sections will be affected to any degree. Thus, the calculated $K \alpha$ cross sections apply for all elements with $Z \gtrsim 15$, and the $L \alpha$ cross sections apply for $Z \gtrsim 30$. However, dependence on the chemical environment may become strong for lighter elements. For $3 d$ transition elements, differences between fluorescence and Coster-Kronig yields for free atoms and those for bound atoms are substantial; corresponding values are given in Tables 15 and 16 .

When relating measured $\mathrm{x}$-ray intensities to calculated $\mathrm{x}$-ray fluorescence cross sections, it is important to note that the cross section for a given $x$-ray line, $L \alpha_{1,2}$ for example, pertains to the diagram line and to the accompanying satellite lines. There are two reasons for this: first, theoretical photoionization cross sections in the frozen-structure approximation contain multiple ionization events that lead to $x$-ray satellites $13,14,17,18$ and second, satellite events arising from Coster-Kronig transitions ${ }^{18}$ are included in the cross sections as implied by Eqs. 5 and 6 . Thus in high-resolution $x$-ray spectroscopy, in which satellite lines are separated from the main line, integration must encompass the entire group.

Uncertainties given in Table 6 are subject to experimental verification. So far few attempts have been made in this direction and only on a relative basis with non-monochromatic photon sources.19-21 Ideally, a 
test should be made on an absolute basis with a monochromatic photon source. However, the bandwidth of such a source should be sufficiently broad to average oscillations in the photoionization cross section caused by extended $x$-ray absorption fine structure (EXAFS) in molecular and solid-state systems. EXAFS modulations may amount to up to $10 \%$ of the average cross section.

At the present level of accuracy, angular correlation effects between the incoming photons and the outgoing $\mathrm{x}$ rays of the $L_{3}$ emission series are of no importance, 22,23 even for measurements in which the photon beams are restricted to very small solid angles.

In $x$-ray fluorescence analysis, measurements are often made relative to a standard, for example the CuKa line. Under these circumstances, uncertainties of the relative $\mathrm{x}$-ray fluorescence cross sections are about one-half of those listed in Table 6 . If in addition elements are not too far apart, uncertainties may be further reduced - to about $1 \%$ for $K \alpha \times$ rays and to less than $5 \%$ for $L \alpha \times$ rays in favorable cases. 
2. TABLES AND GRAPHS OF X-RAY FLUORESCENCE CROSS SECTIONS IN THE PHOTON ENERGY RANGE 1 to $200 \mathrm{keV}$ 
0

O 
In this section, tables and graphs of $x$-ray fluorescence cross sections are given in two forms:

1. In sequence by element (Tables 7 and 8 ) $-K \alpha_{1}, K \alpha_{1,2}, K \beta_{1}$, and $K_{1,3} \times$ rays for $E_{K}<h \nu \leqslant 200 \mathrm{keV} ; L \alpha_{1}, L \beta_{1}, L \beta_{2,15}, L \beta_{3}, L l$, $L_{\gamma_{4}}$, and $L_{1} \rightarrow L_{2,3} \mathrm{x}$ rays for $E_{L_{i}}<h \nu \lesssim E_{K}$, where $E_{L_{i}}$ is the binding energy of $L_{1}, L_{2}$, or $L_{3}$ subshell.

2. In sequence by fluorescent $K$ or $L \times$ ray (Tables 9 and 10) - for all elements at regular photon energy intervals.

X-ray fluorescence cross sections were calculated from Eqs. 3 to 6:

- Example for a $K$ series $\mathrm{x}$ ray:

$$
\sigma_{K \alpha_{1}}=\sigma_{K} \omega_{K} F_{K \alpha_{1}}
$$

- Example for an $L_{1}$ series $\mathrm{x}$ ray:

$$
\sigma_{L \beta_{3}}={ }^{\sigma} L_{1}{ }^{\omega} F_{L \beta_{3}}
$$

- Example for an $L_{2}$ series $\mathrm{x}$ ray:

$$
\sigma_{L B_{1}}=\sigma_{L_{2}} \omega_{2} F_{L B_{1}}\left(1+\frac{\sigma_{L_{1}}}{\sigma_{L_{2}}} f_{1,2}\right) \text {. }
$$

- Example for an $L_{3}$ series $\mathbf{x}$ ray:

$$
\sigma_{L \alpha_{1,2}}=\sigma_{L_{3}}{ }^{\omega_{3} F} F_{L \alpha_{1,2}}\left[1+\frac{\sigma_{L_{2}}}{\sigma_{L_{3}}} f_{2,3}+\frac{\sigma_{L_{1}}}{\sigma_{L_{3}}}\left(f_{1,3}+f_{1,3}^{\prime}+f_{1,2} f_{2,3}\right)\right] \text {. }
$$

Al1 values are given in barns/atom; to convert to $\mathrm{cm}^{2} / \mathrm{g}$, use the factors listed in Table 22. Uncertainties of calculated values are summarized in Table 6 and in Fig. 1.

Note the following details:

1. The first entry above an absorption edge, or binding energy $E_{i}$ of level $i$, occurs at $h \nu=1.007 E_{i}$, and the last entry below an absorption edge occurs at $h v=E_{i} / 1.001 .24$ A11 $E_{i}$ values are theoretical; these theoretical energies are always greater than the experimental energies. 
2. Breaks in the cross section curves for $L \mathrm{x}$ rays are due to discontinuities in the Coster-Kronig yields ${ }^{3} f_{1,2}, f_{1,3}$, and $f_{2,3}$ except for breaks near the high $Z$ ends caused by shell structure. Note that in regions of breaks, uncertainties in the cross sections may exceed those given in Table 6 .

3. In the cases of $K$ series $x$ rays if $Z \leqslant 15$, and $L$ series $x$ rays if $Z \leqslant 30$, cross sections should be modified when investigating molecular or solid-state systems in accordance with the values in Table 16.

4. The relation $\sigma_{L \alpha_{1}, 2}=1.113 \sigma_{L \alpha_{1}}$ holds independent of $Z$.

5. $\operatorname{Read} 4.28 \mathrm{E}-02$ as $4.28 \times 10^{-2}$. 
Table $7 K$ shell $x$-ray fluorescence cross sections arranged in sequence by element

Values are in units of barns/atom See Figs 2 to 5 for graphic representation

\begin{tabular}{rcc}
$\begin{array}{c}\text { Photon } \\
\text { energy } \\
(\mathrm{keV})\end{array}$ & \multicolumn{1}{c}{$K L_{3}$} & $\begin{array}{c}K \alpha_{23} \\
K \alpha_{12}\end{array}$ \\
\hline & & $\mathrm{Z}=5$ \\
1.00 & $2.39 \mathrm{E} \quad 01$ & $3.59 \mathrm{E} 01$ \\
1.50 & $7.31 \mathrm{E} 00$ & $1.10 \mathrm{E}-01$ \\
2.00 & $3.09 \mathrm{E} 00$ & $4.64 \mathrm{~F} 00$ \\
3.00 & $8.98 \mathrm{E}-01$ & $1.35 \mathrm{~F} 00$ \\
4.00 & $3.67 \mathrm{E}-01$ & $5.50 \mathrm{E}-01$ \\
5.00 & $1.81 \mathrm{E}-01$ & $2.72 \mathrm{~F}-01$ \\
6.00 & $1.01 \mathrm{~F}-01$ & $1.52 \mathrm{E}-01$ \\
8.00 & $4.02 \mathrm{E}-02$ & $6.03 \mathrm{~F}-02$ \\
10.00 & $1.95 \mathrm{E}-02$ & $2.92 \mathrm{E}-02$ \\
15.00 & $5.15 \mathrm{E}-03$ & $7.73 \mathrm{~F}-03$ \\
20.00 & $1.99 \mathrm{E}-03$ & $2.98 \mathrm{~F}-03$ \\
30.00 & $5.15 \mathrm{E}-04$ & $7.73 \mathrm{E}-04$ \\
40.00 & $1.97 \mathrm{E}-04$ & $2.95 \mathrm{E}-04$ \\
$50.0 \mathrm{C}$ & $9.30 \mathrm{E}-05$ & $1.40 \mathrm{E}-04$ \\
60.00 & $5.04 \mathrm{E}-05$ & $7.56 \mathrm{E}-05$ \\
80.00 & $1.92 \mathrm{E}-05$ & $2.88 \mathrm{E}-05$ \\
100.00 & $9.11 \mathrm{E}-06$ & $1.37 \mathrm{E}-05$ \\
150.00 & $2.37 \mathrm{E}-06$ & $3.56 \mathrm{E}-06$ \\
200.00 & $9.30 \mathrm{E}-07$ & $1.39 \mathrm{E}-06$ \\
& &
\end{tabular}

Photon
energy
(keV)

$K L_{3}$
$K \alpha_{1}$

$\begin{array}{ccc}K L_{2} 3 & K M_{3} & K M_{23} \\ K \alpha_{1} & K \beta_{1} & K \beta_{13}\end{array}$

C $\quad z=6$

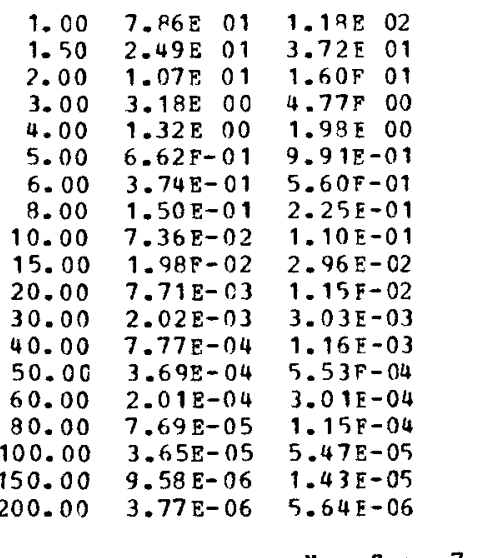

N $\quad 2=7$

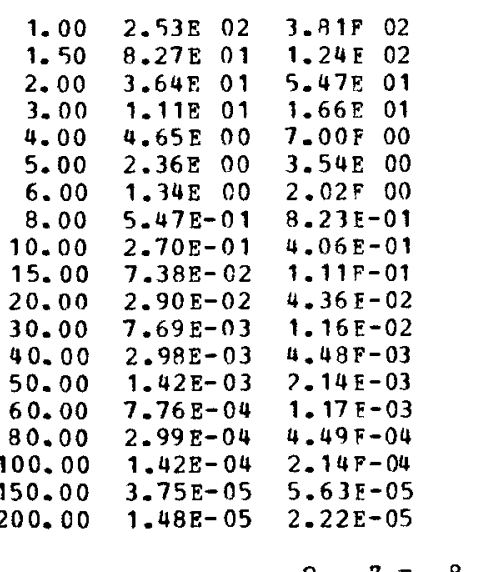

$\begin{array}{lllll}1.00 & 6.39 \mathrm{~F} & 02 & 9.59 \mathrm{E} & 02\end{array}$

$1.50 \quad 2.15 \mathrm{E} \quad 02 \quad 3.24 \mathrm{E} \quad 02$

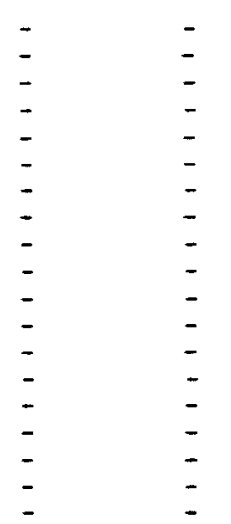

150

\subsection{0}

$66 \mathrm{~F} 01 \quad 1.45 \mathrm{~F} 0 ?$

$3.01 \mathrm{E} 01 \quad 4.52 \mathrm{~L} 01$

4.00 1.2 UE 01 1.93. 01

$\begin{array}{lllll}5.00 & 6.57 \mathrm{E} & 00 & 9.365 & 00\end{array}$

$6.00 \quad 3.77 \mathrm{E}$ OC $5.66 \mathrm{C} 00$

$9.00 \quad 1.55 \mathrm{~F}$ CO $2.33 \mathrm{~F} 00$

$10.00 \quad 7.74 \mathrm{E}-01 \quad 1.16100$

$15.00 \quad 2.14 \mathrm{~F}-01 \quad 3.220-01$

$20.00 \quad 9.51 \mathrm{~F}-\mathrm{C} 2 \quad 1.28^{\circ}-01$

$30.00 \quad 2.23 \mathrm{~F}-02 \quad 3.425-02$

$40.00 \quad 3.87 \mathrm{~F}-03 \quad 1.33 \Gamma-02$

$50.00 \quad 4.26 \mathrm{~F}-03 \quad \mathrm{~s} .40 \mathrm{H}-03$

$60.0 n \quad 2.34 \mathrm{E}-03 \quad 3.51=-03$

$90.00 \quad 9.03 \mathrm{E}-04 \quad 1.36-03$

$100.00 \quad 4.32 \mathrm{~F}-\mathrm{C4} \quad 5.49 \mathrm{~F}-04$

$150.00 \quad 1.14 F-04 \quad 1.72 F-04$

$200.00 \quad 4.52 \mathrm{E}-05 \quad 6.78 \mathrm{P}-05$

$$
P>=9
$$

$\begin{array}{lllll}1.0 n \quad 1.45 \mathrm{~F} & 03 \quad 2.18 \Rightarrow 03\end{array}$

$1.50 \quad 5.10 \mathrm{~F} 02 \quad 7.66572$

$2.00 \quad 2.33 \mathrm{~F} \quad 02 \quad 3.50 \mathrm{~F} \quad 02$

$3.00 \quad 7.43 \mathrm{E} 01$ 1.12 0 ?

$4.00 \quad 3.22 \mathrm{E} 014.84 .01$

$5.00 \quad 1.66 \mathrm{~L} 01 \quad 2.50 \mathrm{~F} 01$

$6.00 \quad 9.61 \mathrm{~F}$ CO $1.44 \mathrm{z} 01$

$8.00 \quad 4.00 \mathrm{E}$ OO $6.01 \mathrm{~F}$ 3n

$\begin{array}{llll}10.00 & 2.01 \mathrm{E} & 00 & 3.02=00\end{array}$

$15.00 \quad 5.64 \mathrm{E}-\mathrm{C} 1 \quad 8.47 \mathrm{~g}-01$

$20.00 \quad 2.26 \mathrm{E}-01 \quad 3.39-01$

$30.00 \quad 6.12 \mathrm{~F}-02 \quad 9.19=-02$

$40.00 \quad 2.40 \mathrm{E}-02 \quad 3.60=-0$ ?

$50.0 C \quad 1.16 E-02 \quad 1.74 E-9 ?$

$00.00 \quad 6.36 \mathrm{E}-\mathrm{C}^{3} \quad 9.555-03$

$80.00 \quad 2.47 \mathrm{E}-\mathrm{C} 3 \quad 3.71 \mathrm{C}-03$

$100.00 \quad 1.19 \mathrm{E}-03 \quad 1.78 \mathrm{~F}-03$

$150.00 \quad 3.1, F-04 \quad 4.74 \mathrm{E}-04$

$200.00 \quad 1.25 E-04 \quad 1.88 \Gamma-04$

$$
\text { NF } 7=10
$$

\begin{tabular}{|c|c|c|}
\hline 1.00 & $2.63 E 03$ & $3.05=03$ \\
\hline 1.50 & $9.50 F \quad 02$ & $1.43 E 03$ \\
\hline 2.00 & $4.43 E$ & $0.66 \mathrm{~F} 02$ \\
\hline 3.00 & $1.44 \mathrm{~F}$ & $2.17 \mathrm{v} \cap ?$ \\
\hline 4.00 & $6.3 \supset F$ & $9.54=01$ \\
\hline 5.00 & $3.31 \mathrm{~F}$ & $4.97 \Gamma$ \\
\hline 6.00 & $1.93 \mathrm{E}$ & $2.90 \mathrm{E}$ \\
\hline 8.00 & $8.11 \mathrm{E}$ & $1.22^{5}$ \\
\hline 10.00 & $4.10 F$ & $6.16=$ \\
\hline 15.00 & $1.17 \mathrm{E}$ & $1.75 \mathrm{p}$ \\
\hline 20.00 & $4.7 \mathrm{CF}-01$ & $7.07 F-01$ \\
\hline 30.00 & 1. $29 F-01$ & $1.937-01$ \\
\hline 00 & 5. $08 \mathrm{E}-0.2$ & $7.64=-02$ \\
\hline 00 & 2. $40 F-02$ & $3.70 \mathrm{~s}-122$ \\
\hline 00 & 1. $36 F-0 ?$ & $2.04 \mathrm{z}-02$ \\
\hline & 5. $29 \mathrm{~F}-03$ & $7.955-03$ \\
\hline 00 & $2.55 F-0 ?$ & $3.83 \mathrm{E}-03$ \\
\hline & & $1.02[-03$ \\
\hline & & $4.07 \pi$ \\
\hline
\end{tabular}

-
-
-
-
-
-
-
-
-
-
-

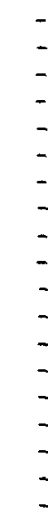

$$
\begin{aligned}
& - \\
& - \\
& - \\
& - \\
& - \\
& - \\
& - \\
& - \\
& - \\
& - \\
& - \\
& - \\
& - \\
& - \\
& - \\
& - \\
& - \\
& - \\
& - \\
& - \\
& - \\
& - \\
& - \\
& - \\
& - \\
& - \\
& - \\
& - \\
& - \\
& -
\end{aligned}
$$


Table 7 (continued)

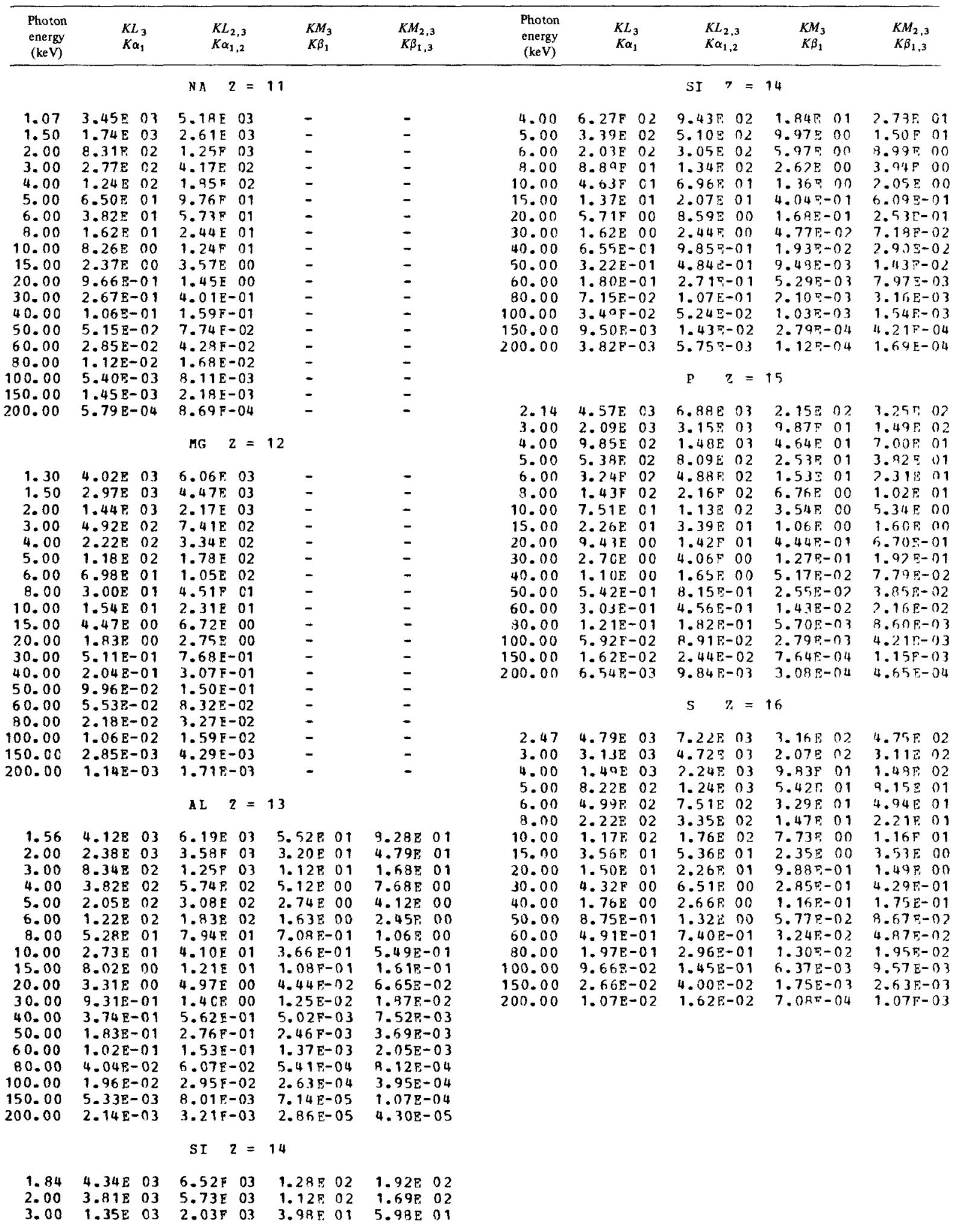


Table 7 (continued)

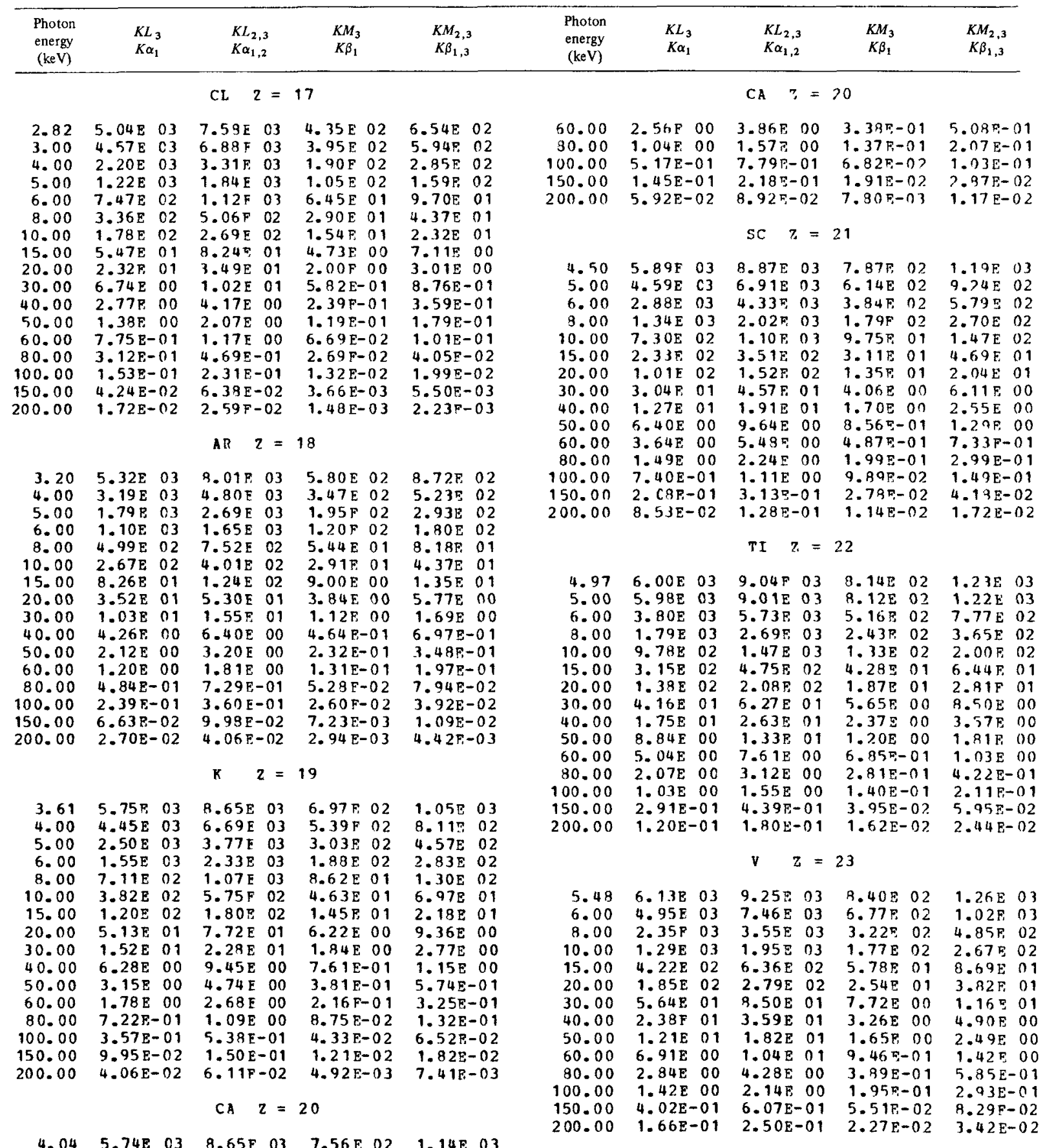


Table 7 (continued)

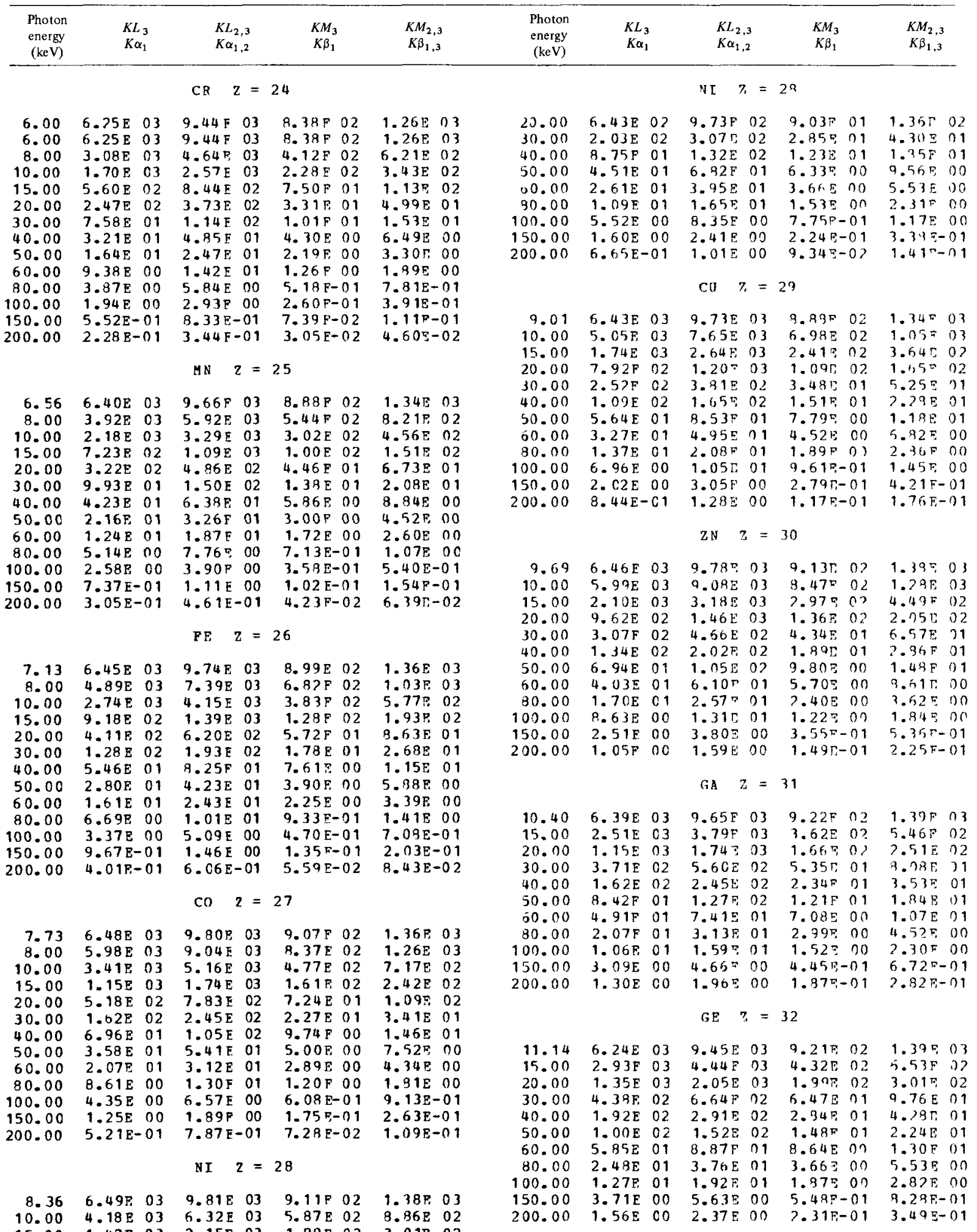


Table 7 (continued)

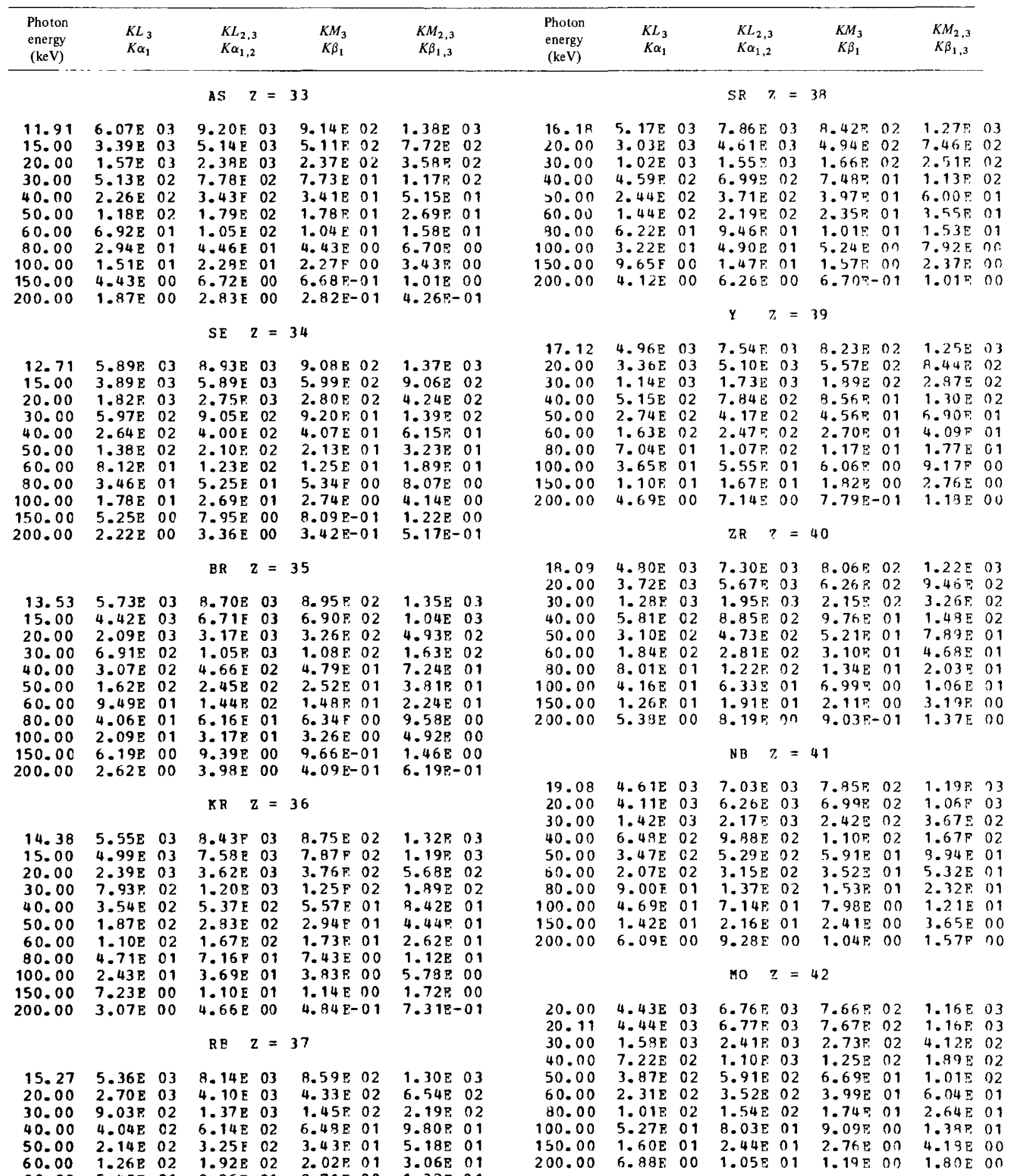


Table 7 (continued)

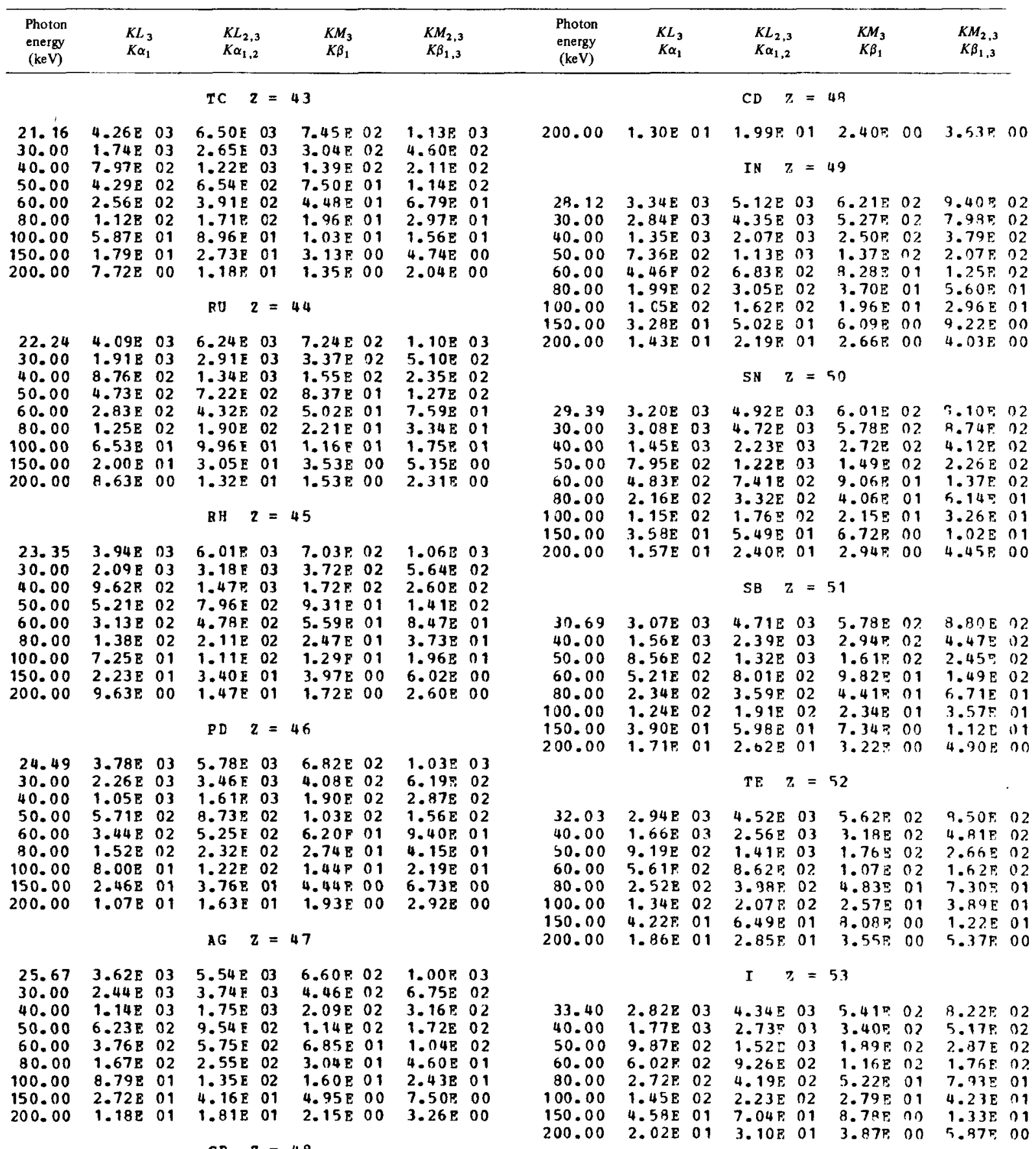

$\begin{array}{rllllllll}26.88 & 3.48 \mathrm{E} & 03 & 5.32 \mathrm{E} & 03 & 6.40 \mathrm{E} & 02 & 9.70 \mathrm{E} & 02 \\ 30.00 & 2.63 \mathrm{E} & 03 & 4.03 \mathrm{E} & 03 & 4.85 \mathrm{E} & 02 & 7.34 \mathrm{E} & 02 \\ 40.00 & 1.24 \mathrm{E} & 03 & 1.90 \mathrm{E} & 03 & 2.29 \mathrm{E} & 02 & 3.47 \mathrm{E} & 02 \\ 50.00 & 6.79 \mathrm{E} & 02 & 1.04 \mathrm{E} & 03 & 1.25 \mathrm{E} & 02 & 1.89 \mathrm{E} & 02 \\ 60.00 & 4.10 \mathrm{E} & 02 & 6.28 \mathrm{E} & 02 & 7.55 \mathrm{E} & 01 & 1.14 \mathrm{E} & 02 \\ 80.00 & 1.82 \mathrm{E} & 02 & 2.79 \mathrm{E} & 02 & 3.36 \mathrm{E} & 01 & 5.09 \mathrm{E} & 01 \\ 100.00 & 9.64 \mathrm{E} & 01 & 1.4 \mathrm{EE} & 02 & 1.78 \mathrm{E} & 01 & 2.69 \mathrm{E} & 01 \\ 150.00 & 2.99 \mathrm{E} & 01 & 4.58 \mathrm{E} & 01 & 5.50 \mathrm{E} & 00 & 8.33 \mathrm{E} & 00\end{array}$


Table 7 (continued)

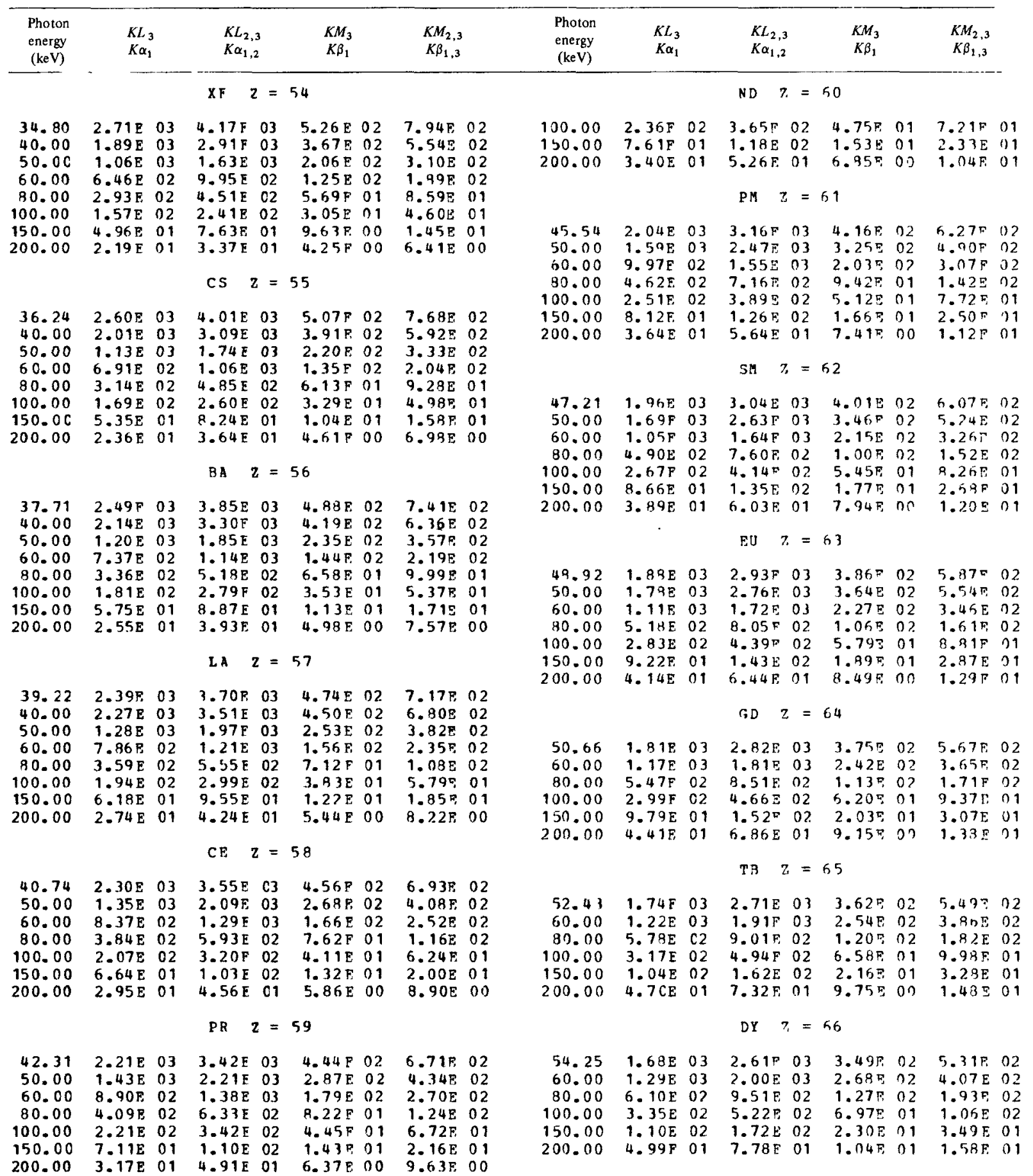

ND $Z=60$

$\begin{array}{lllllllllll}43.91 & 2.12 \mathrm{E} & 03 & 3.29 \mathrm{E} & 03 & 4.27 \mathrm{E} & 02 & 6.49 \mathrm{E} & 02\end{array}$

$\begin{array}{llllllllll}50.00 & 1.51 \mathrm{E} & 03 & 2.34 \mathrm{~F} & 03 & 3.04 \mathrm{E} & 02 & 4.61 \mathrm{E} & 02\end{array}$ $\begin{array}{lllllllll}60.00 & 9.43 \mathrm{E} & 02 & 1.46 \mathrm{~F} & 03 & 1.90 \mathrm{E} & 02 & 2.38 \mathrm{E} & 02\end{array}$

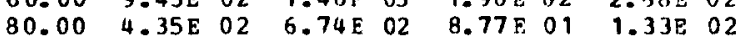


Table 7 (continued)

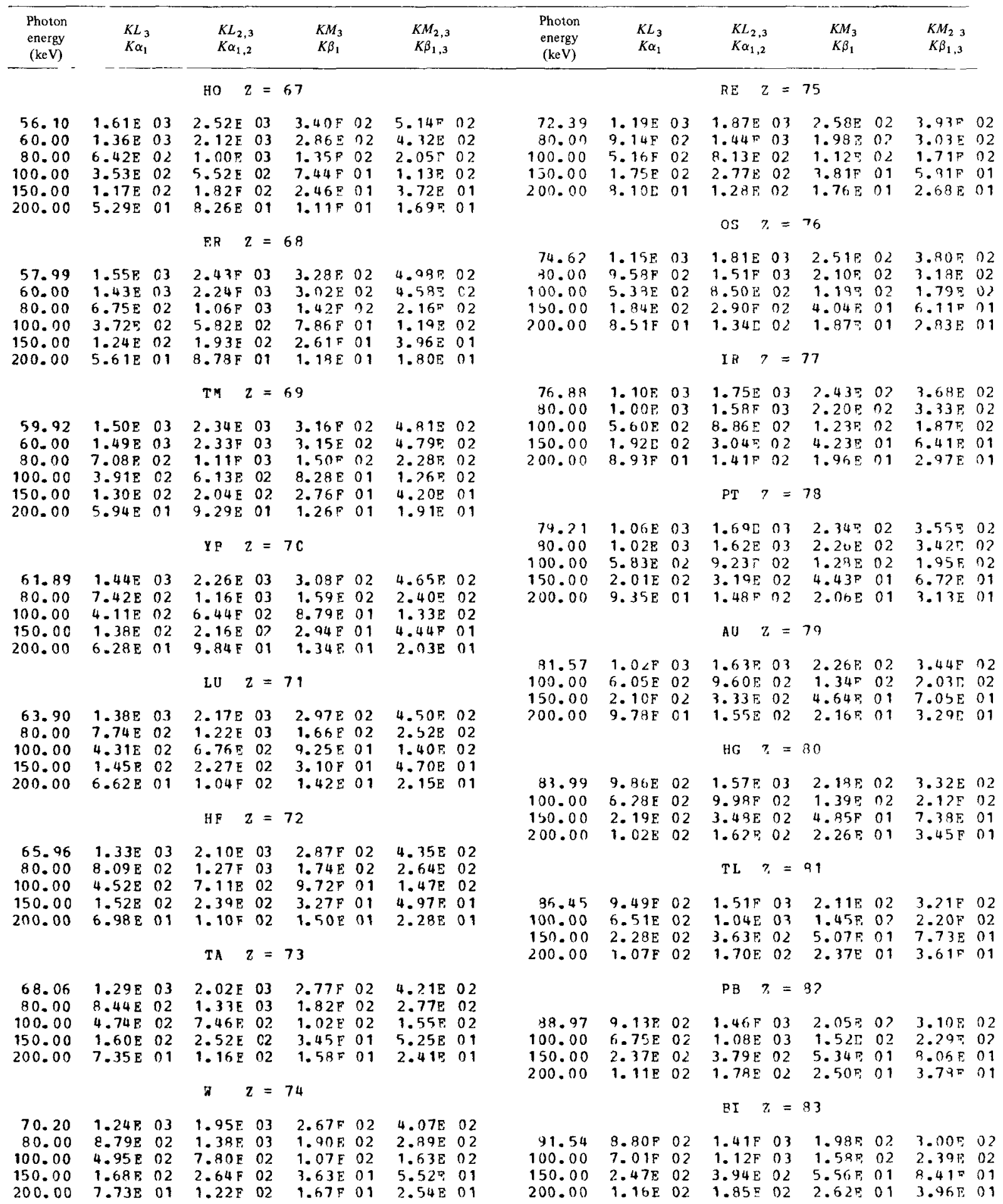


Table 7 (contınued)

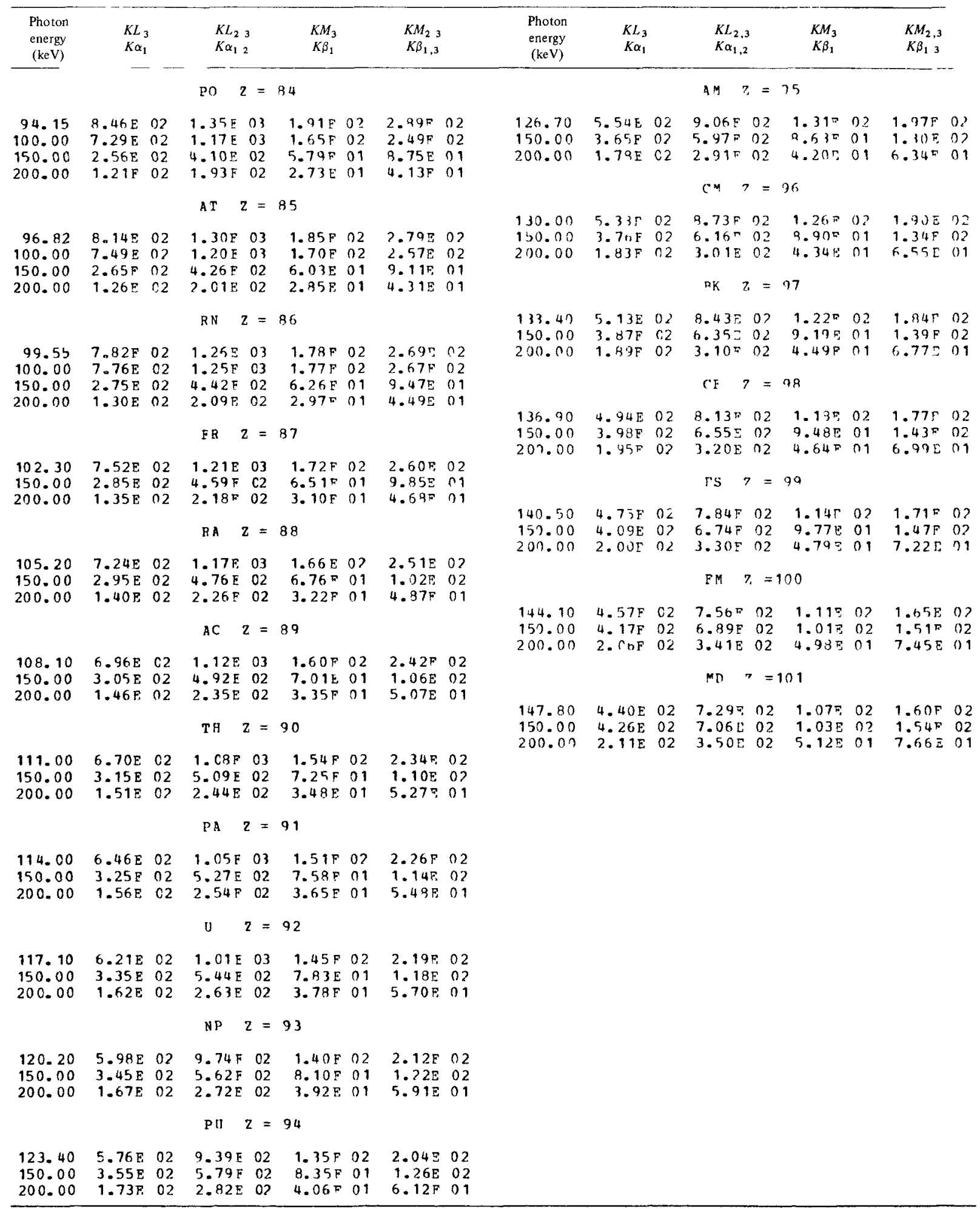


ORNL-DWG 77-2118b.

$X$-ray Production Cross Sections

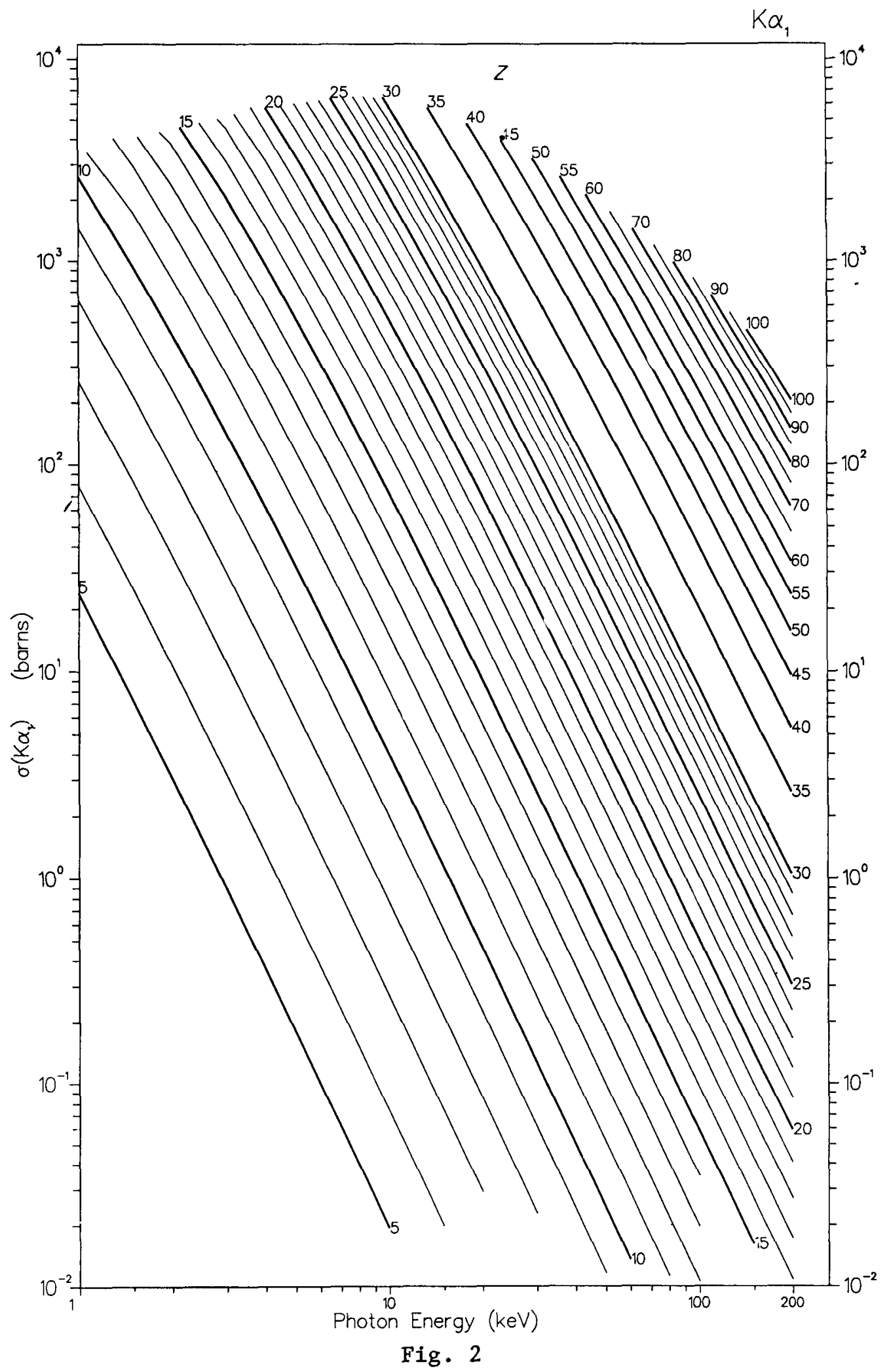


ORNL-DWG 77-18987R

X-ray Production Cross Sections

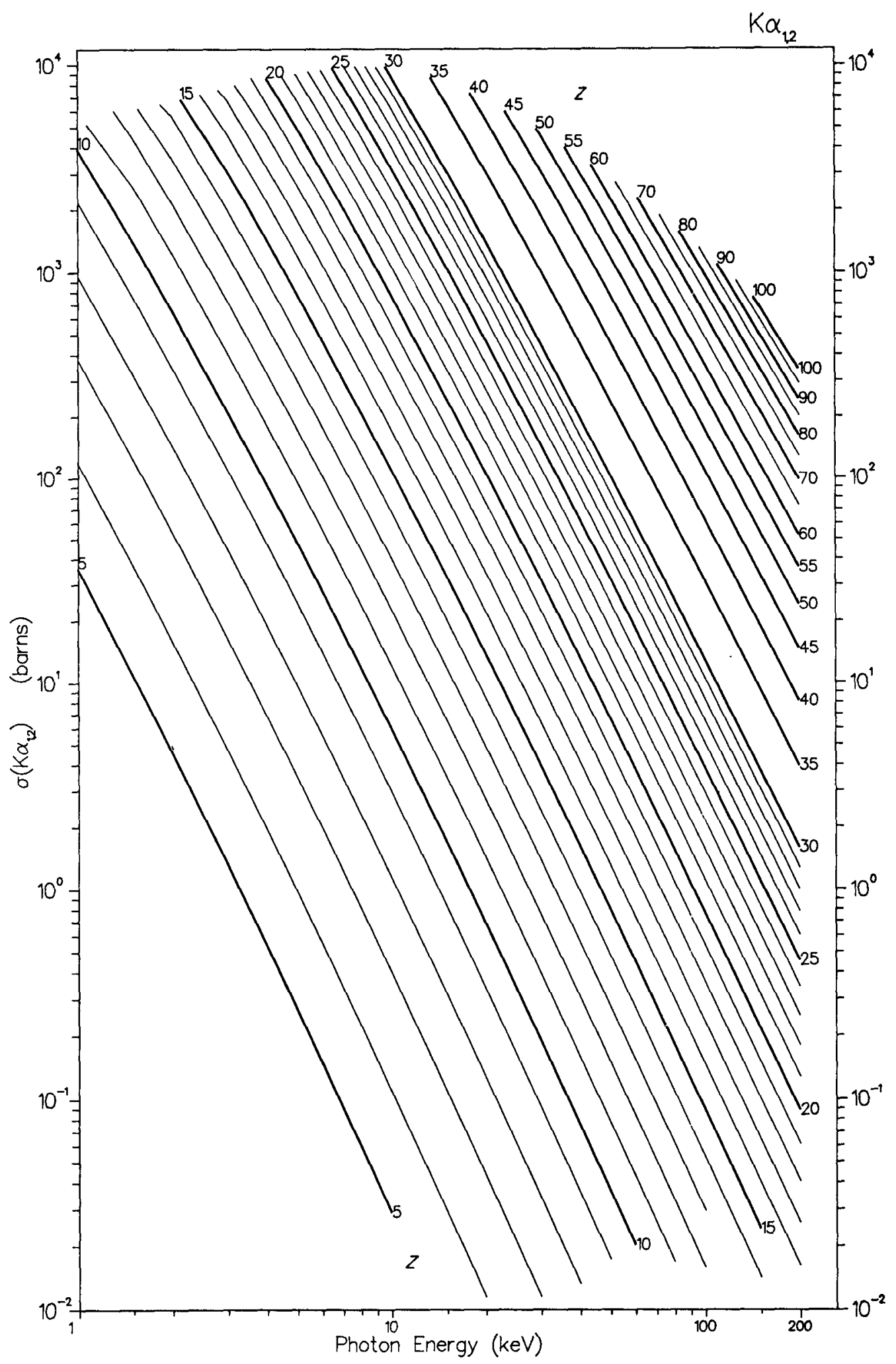

Fig. 3 
ORNL-DWG 77-18988R

X-ray Production Cross Sections

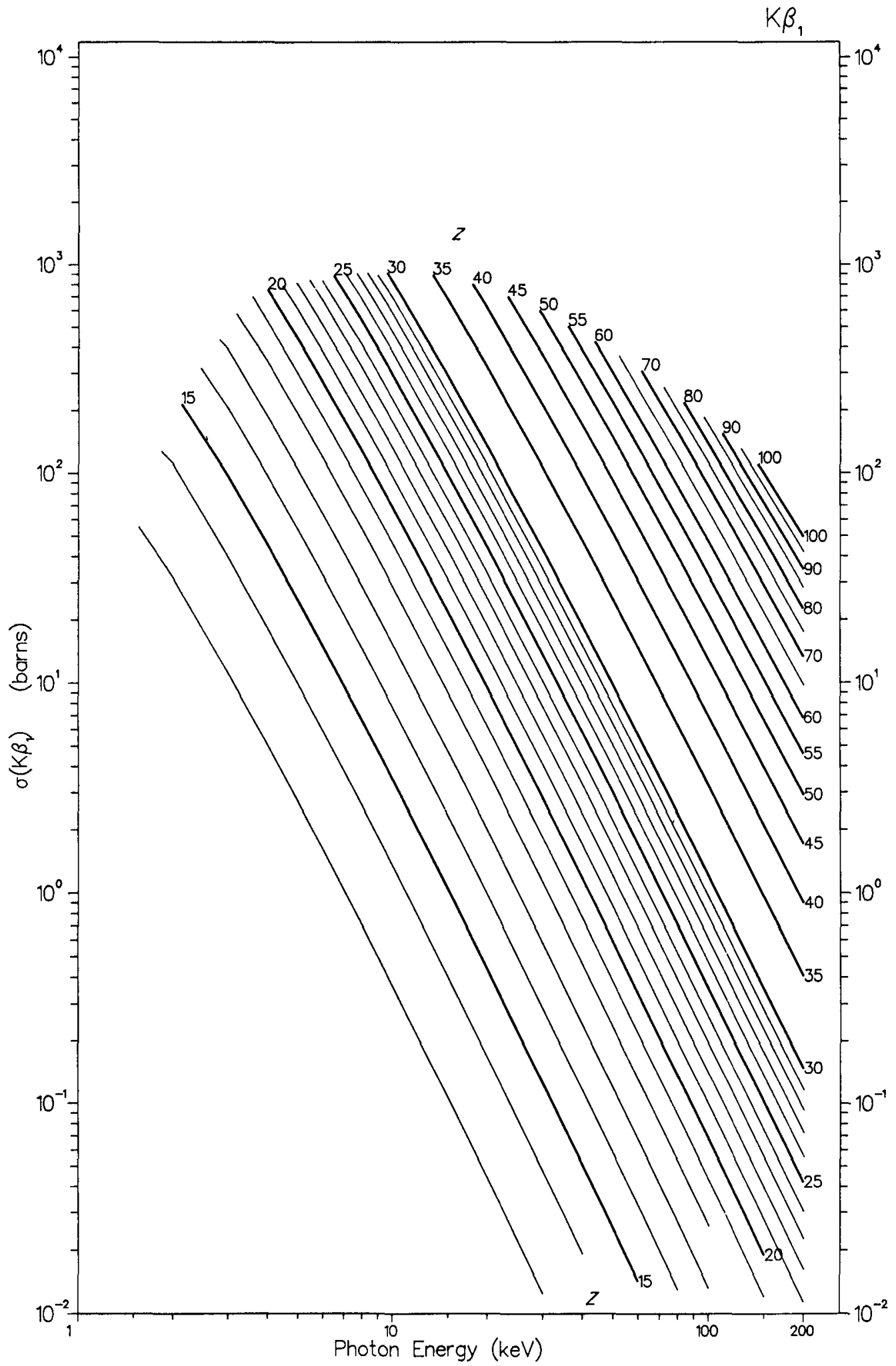

Fig. 4 
$X$-ray Production Cross Sections

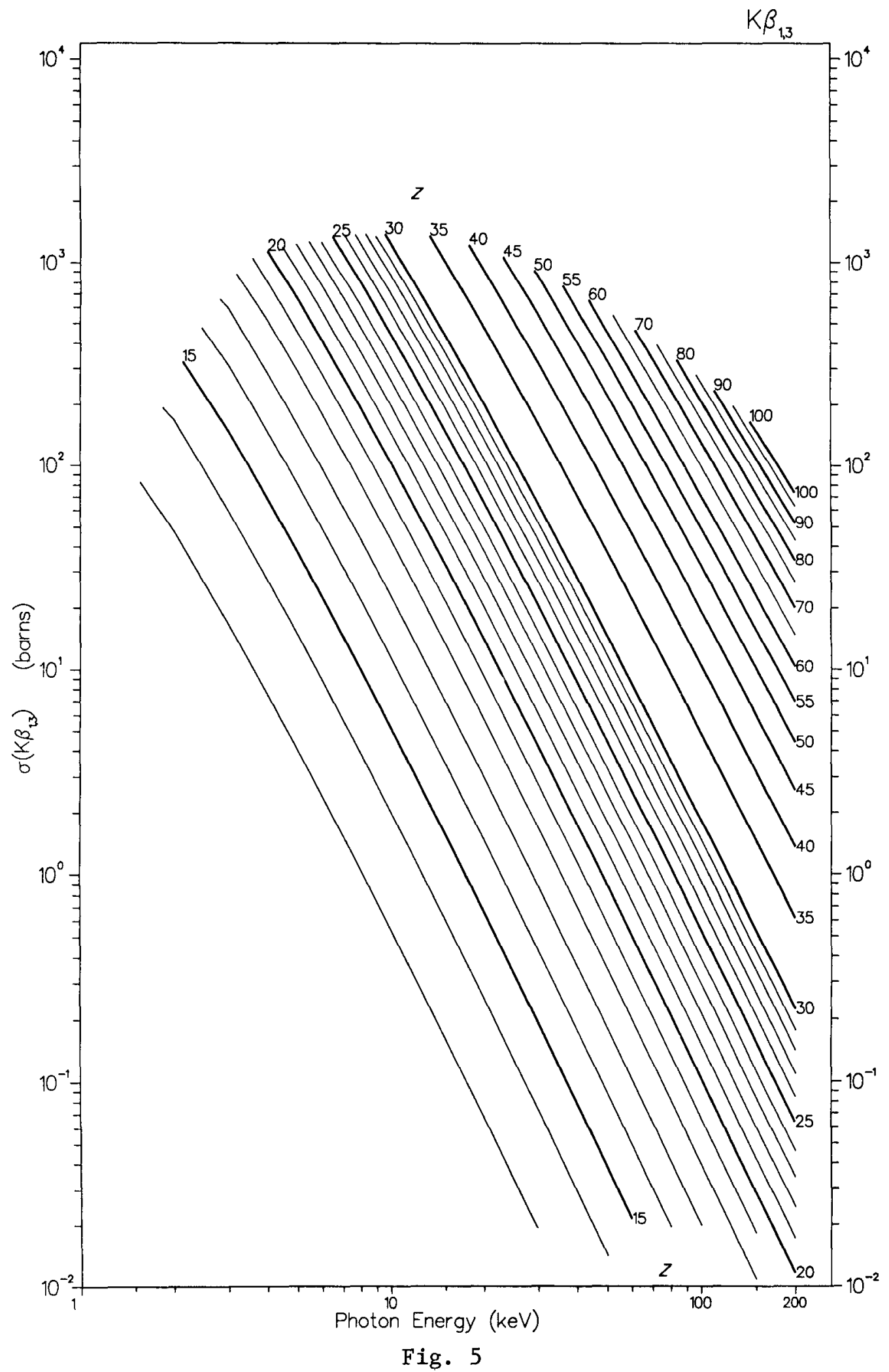


Table 8. $L$-shell $x$-ray fluorescence cross sections arranged in sequence by element

Values are in units of barns/atom. See Figs. 6 to 13 for graphic representation.

Photon energies cover the ranges from just above the absorption edges of the respective $L$ subshells to just below the $K$ absorption edge.

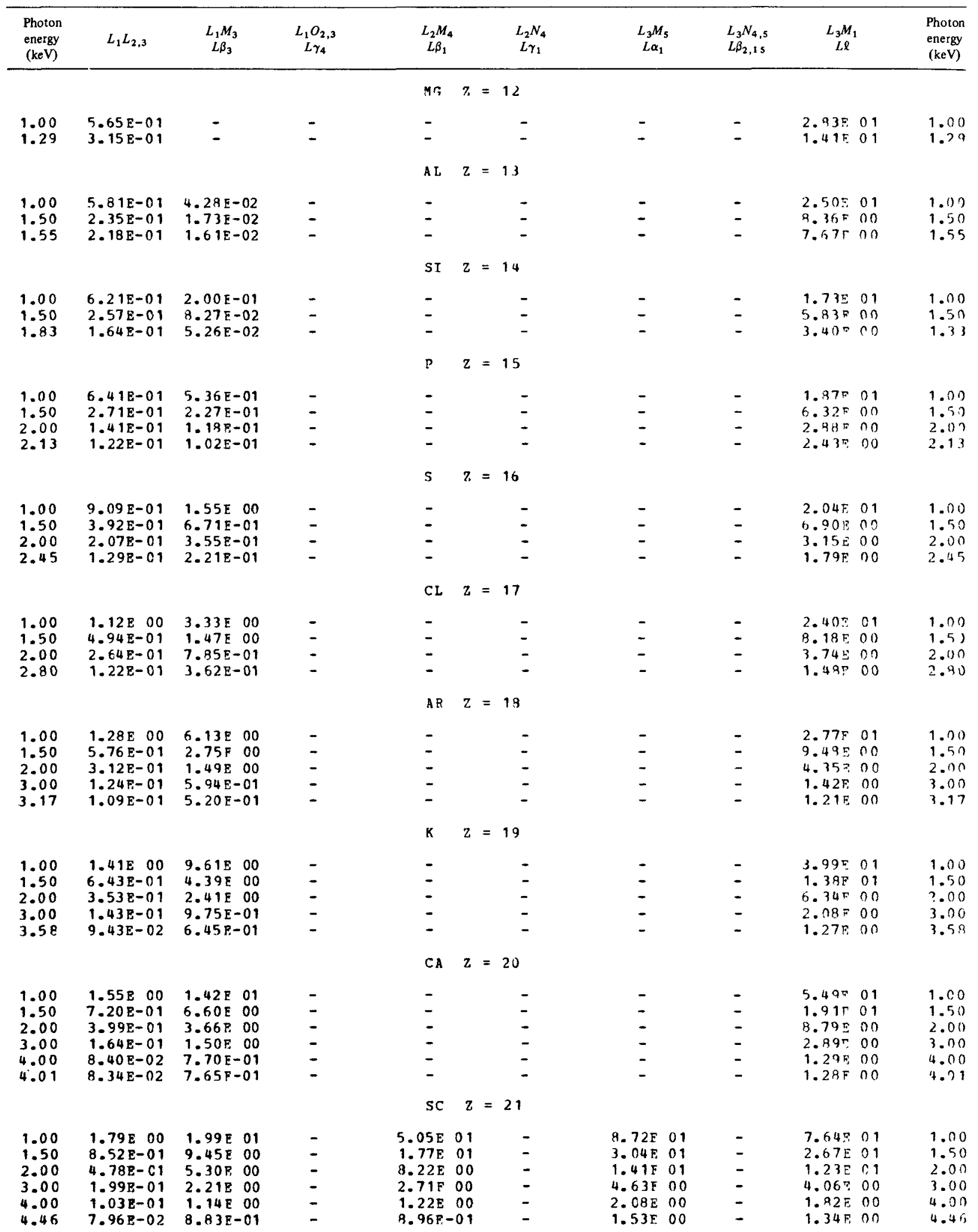


Table 8 (continued)

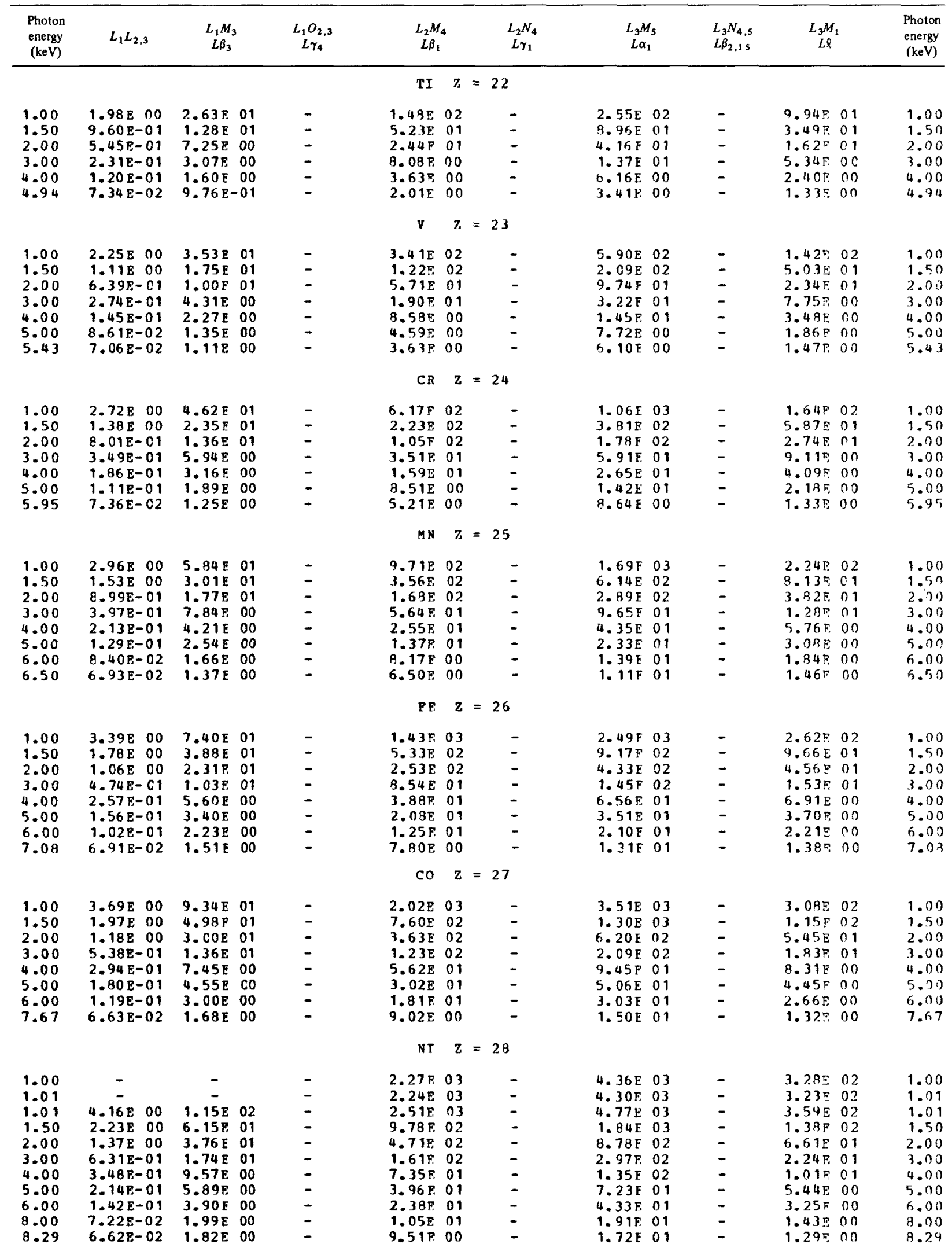


Table 8 (continued)

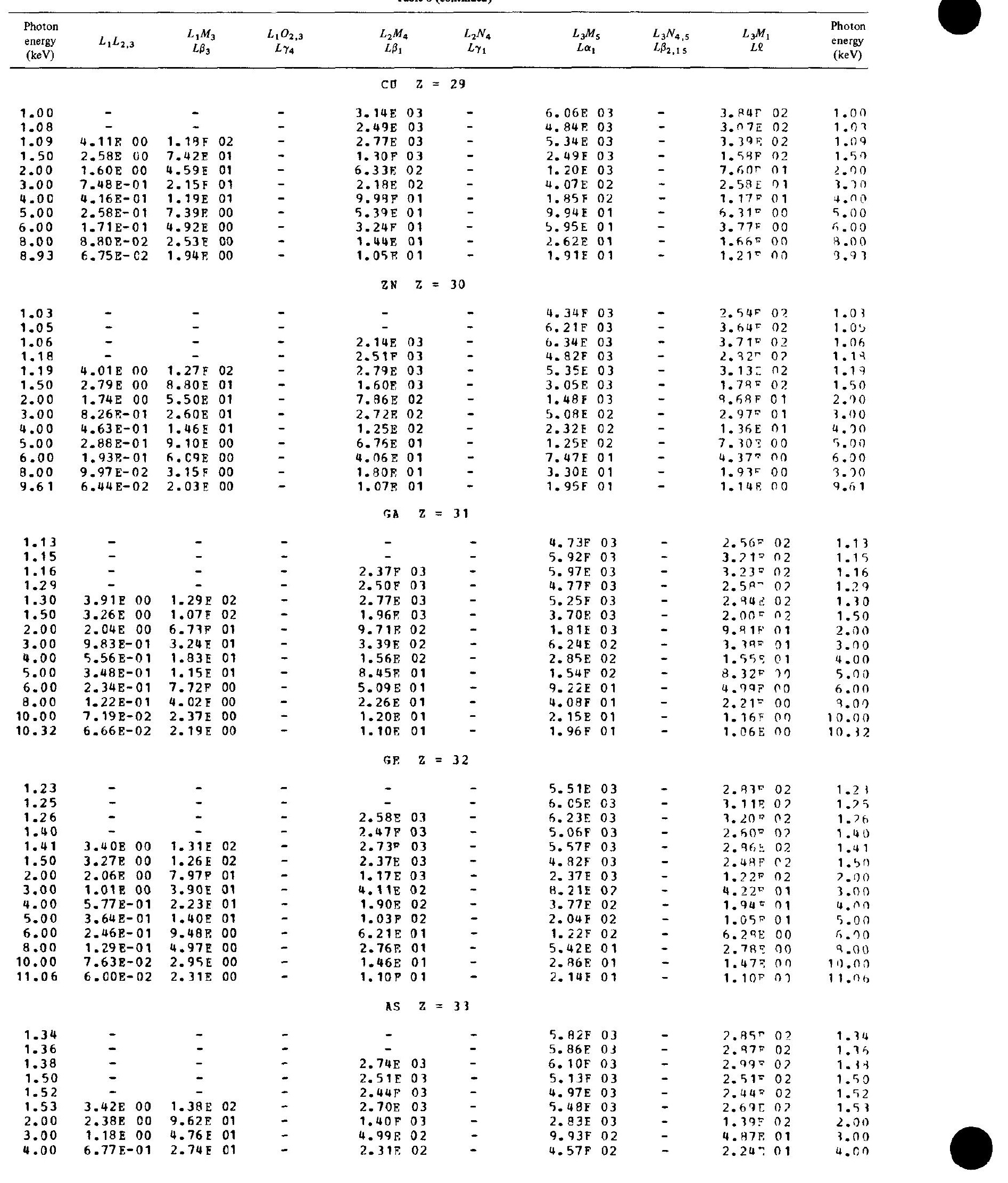


Table 8 (continued)

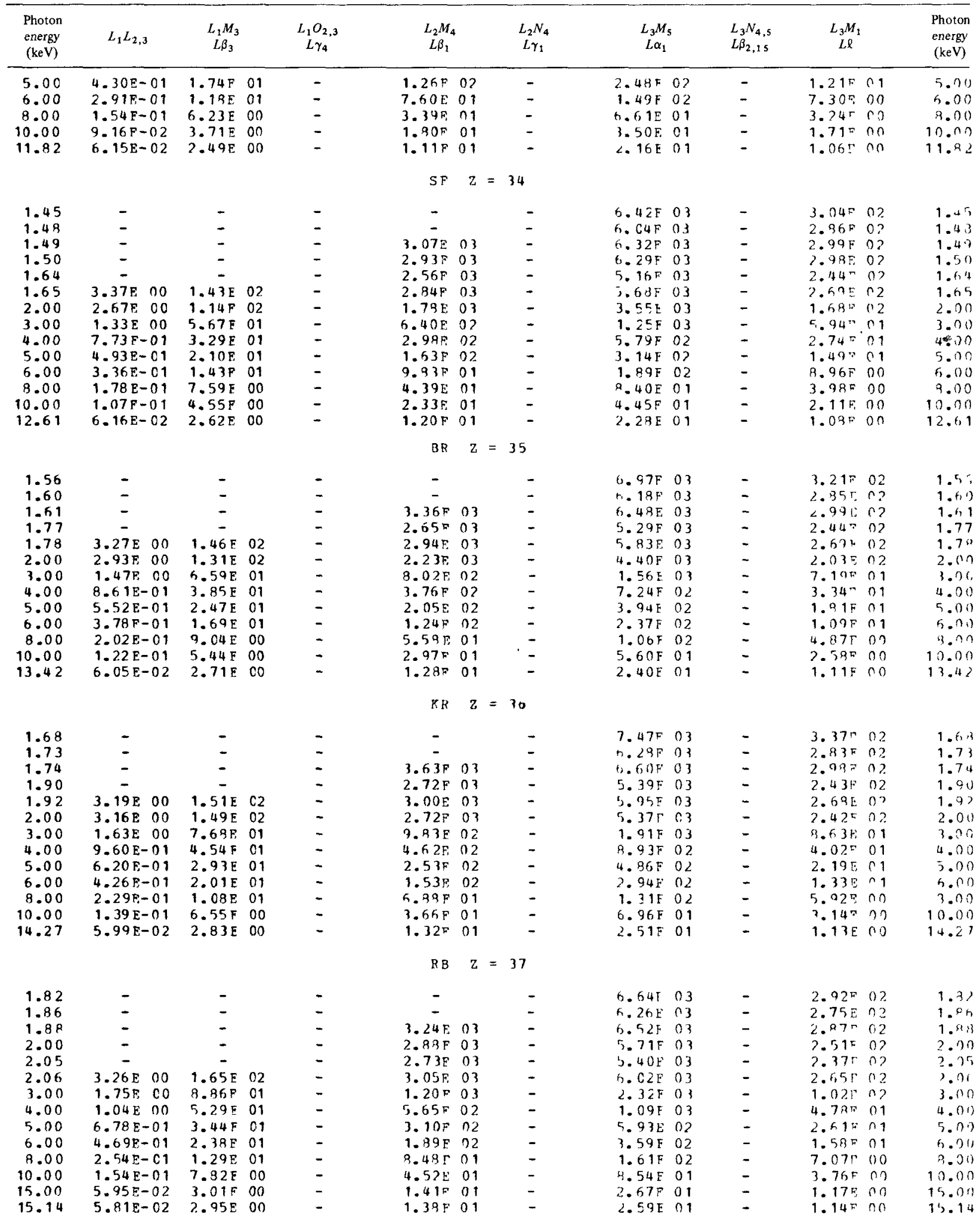


Table 8 (continued)

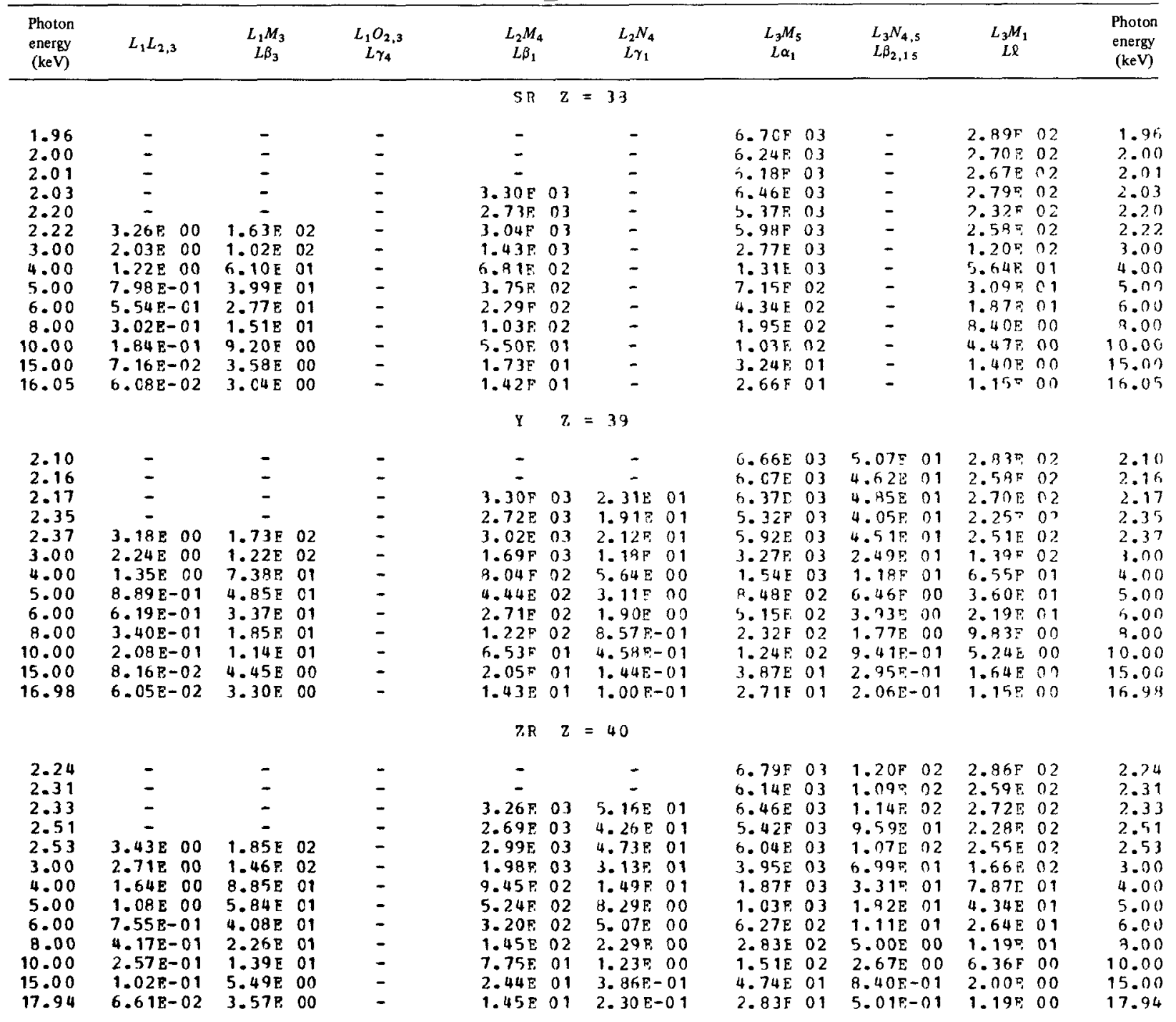

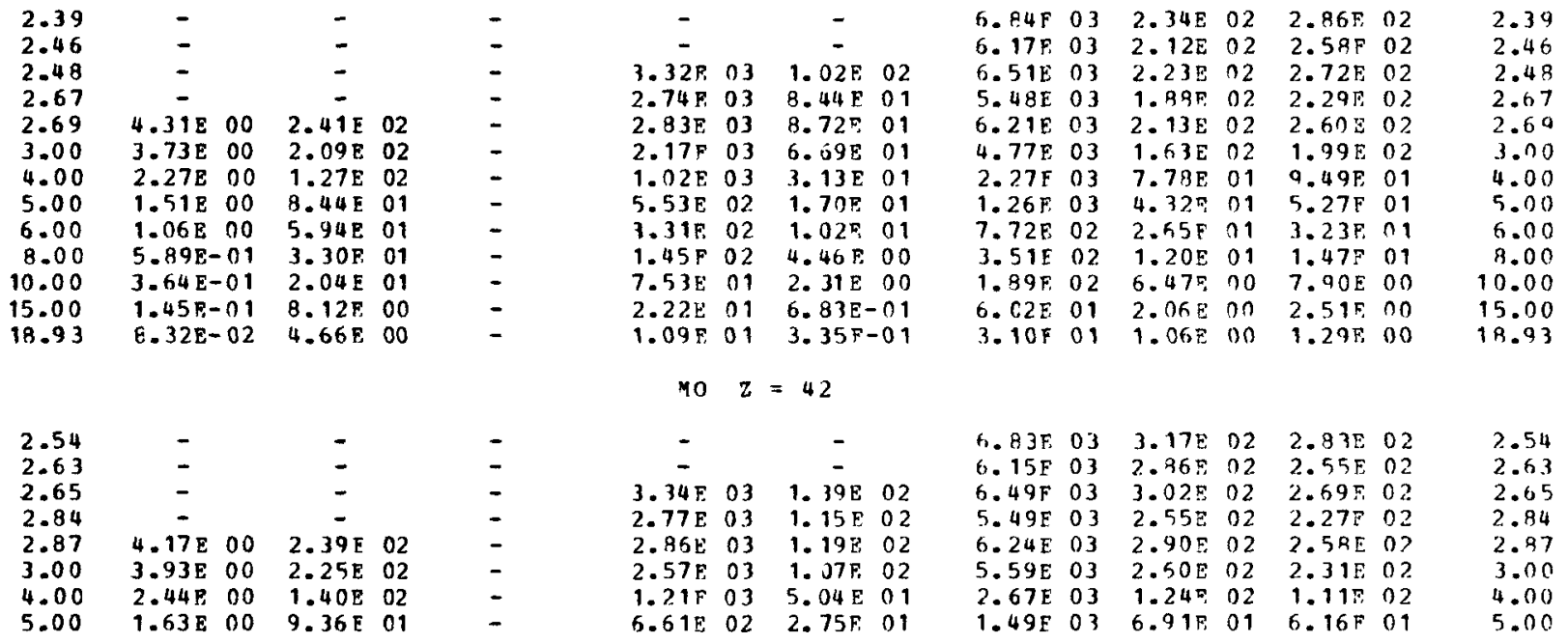


Table 8 (continued)

\begin{tabular}{|c|c|c|c|c|c|c|c|c|c|c|c|}
\hline $\begin{array}{l}\text { Photon } \\
\text { energy } \\
\text { (keV) }\end{array}$ & $L_{1} L_{2,3}$ & $\begin{array}{c}L_{1} M_{3} \\
L \beta_{3}\end{array}$ & & $\begin{array}{c}L_{1} O_{2,3} \\
L \gamma_{4}\end{array}$ & $\begin{array}{c}L_{2} M_{4} \\
L \beta_{1}\end{array}$ & $\begin{array}{c}L_{2} N_{4} \\
L \gamma_{1}\end{array}$ & $\begin{array}{c}L_{3} M_{5} \\
L \alpha_{1}\end{array}$ & $\begin{array}{l}L_{3} N_{4,5} \\
L \beta_{2,15}\end{array}$ & $\begin{array}{c}L_{3} M_{1} \\
L Q\end{array}$ & & $\begin{array}{l}\text { Photon } \\
\text { energy } \\
\text { (keV) }\end{array}$ \\
\hline $\begin{array}{r}6.00 \\
8.00 \\
10.00 \\
15.00 \\
19.95\end{array}$ & $\begin{array}{l}1.15 \mathrm{E} 00 \\
6.44 \mathrm{E}-01 \\
4.00 \mathrm{E}-01 \\
1.60 \mathrm{E}-01 \\
8.14 \mathrm{E}-02\end{array}$ & $\begin{array}{l}6.61 \mathrm{E} \\
3.70 \mathrm{~F} \\
2.30 \mathrm{~F} \\
9.21 \mathrm{E} \\
4.67 \mathrm{~F}\end{array}$ & $\begin{array}{l}01 \\
01 \\
01 \\
00 \\
00\end{array}$ & $\begin{array}{l}- \\
- \\
-\end{array}$ & 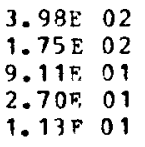 & $\begin{array}{rr}1.66 \mathrm{E} & 01 \\
7.29 \mathrm{E} & 00 \\
3.30 \mathrm{~F} & 00 \\
1.13 \mathrm{~F} & 00 \\
4.71 \mathrm{E}-01\end{array}$ & 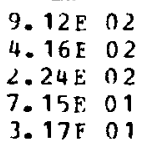 & $\begin{array}{ll}4.24 E & 01 \\
1.93 E & 01 \\
1.04 E & 01 \\
3.32 F & 00 \\
1.47 E & 00\end{array}$ & $\begin{array}{l}3.78 \mathrm{E} \\
1.72 \mathrm{E} \\
9.28 \mathrm{~F} \\
2.96 \mathrm{~F} \\
1.31 \mathrm{~F}\end{array}$ & $\begin{array}{ll}01 \\
01 \\
0 & 0 \\
0 & 0 \\
0 & 0\end{array}$ & $\begin{array}{r}6.00 \\
8.00 \\
10.00 \\
15.00 \\
19.95\end{array}$ \\
\hline
\end{tabular}

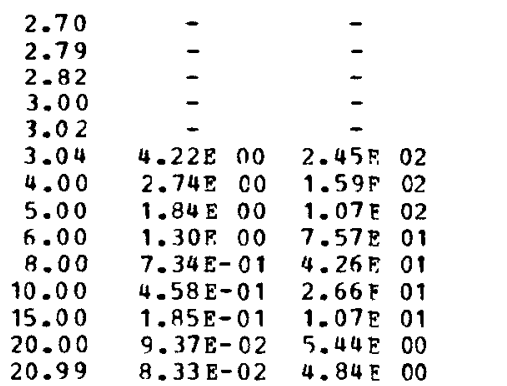

-
-
-
-
-
-
-
-
-

\begin{tabular}{cccc}
- & \multicolumn{2}{c}{-} \\
$3.34 \mathrm{~F}$ & 03 & $1.73 \mathrm{~F}$ & 02 \\
$2.83 \mathrm{~F}$ & 03 & $1.51 \mathrm{~F}$ & 02 \\
$2.79 \mathrm{E}$ & 03 & $1.49 \mathrm{~F}$ & 02 \\
$2.89 \mathrm{E}$ & 03 & $1.54 \mathrm{~F}$ & 02 \\
$1.43 \mathrm{~F}$ & 03 & $7.62 \mathrm{~F}$ & 01 \\
$7.84 \mathrm{E}$ & 02 & $4.19 \mathrm{~F}$ & 01 \\
$4.74 \mathrm{E}$ & 02 & $2.53 \mathrm{E}$ & 01 \\
$2.09 \mathrm{~F}$ & 02 & $1.12 \mathrm{~F}$ & 01 \\
$1.09 \mathrm{~F}$ & 02 & $5.83 \mathrm{E}$ & 00 \\
$3.26 \mathrm{~F}$ & 01 & $1.74 \mathrm{~F}$ & 00 \\
$1.35 \mathrm{E}$ & 01 & $7.23 \mathrm{E}-01$ \\
$1.17 \mathrm{E}$ & 01 & $0.23 \mathrm{E}-01$
\end{tabular}

$$
\text { RU } z=44
$$

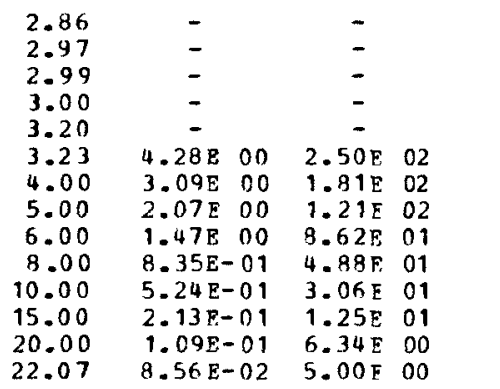

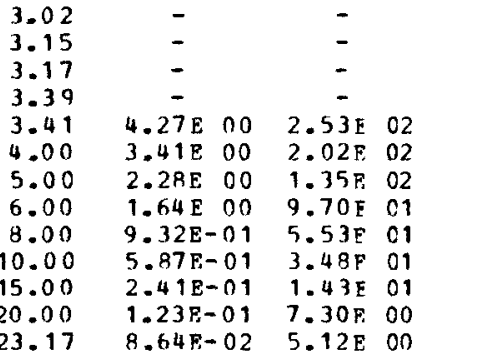

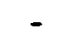

$\begin{array}{lllll}3.31 E & 03 & 2.19 E & 02\end{array}$ $3.34 \mathrm{~F} 03 \quad 2.20 \mathrm{E} 02$ 2.79E $03 \quad 1.83 E \quad 02$ $\begin{array}{lllll}2.88 \mathrm{~F} & 03 & 1.90 \mathrm{E} & 02\end{array}$ $\begin{array}{llll}1.67 \mathrm{~F} & 03 & 1.10 \mathrm{E} & 02\end{array}$ $9.18 E \quad 02 \quad 6.05 F \quad 01$ $\begin{array}{llll}5.57 \mathrm{E} & 02 & 3.67 \mathrm{E} & 01\end{array}$ $2.47 \mathrm{E} \quad 02 \quad 1.63 \mathrm{E} \quad 01$ $\begin{array}{lllll}1.29 \mathrm{~F} & 02 & 8.51 \mathrm{E} & 00\end{array}$ $\begin{array}{llll}3.87 \mathrm{E} & 01 & 2.55 \mathrm{E} & 00\end{array}$ 1.625011 .06500 $1.19 \mathrm{~F} 017.87 \mathrm{E}-01$ $\mathrm{RH} \quad 7=45$

$\begin{array}{cccc}- & & \bar{c} \\ \overline{3} & & \overline{ } & \\ 3.27 \mathrm{~F} & 03 & 2.58 \mathrm{~F} & 02 \\ 2.77 \mathrm{E} & 03 & 2.19 \mathrm{~F} & 02 \\ 2.87 \mathrm{E} & 03 & 2.26 \mathrm{~F} & 02 \\ 1.93 \mathrm{~F} & 03 & 1.53 \mathrm{~F} & 02 \\ 1.07 \mathrm{~F} & 03 & 9.42 \mathrm{~F} & 01 \\ 6.48 \mathrm{~F} & 02 & 5.11 \mathrm{~F} & 01 \\ 2.89 \mathrm{~F} & 02 & 2.28 \mathrm{E} & 01 \\ 1.52 \mathrm{~F} & 02 & 1.20 \mathrm{~F} & 01 \\ 4.57 \mathrm{E} & 01 & 3.01 \mathrm{E} & 00 \\ 1.91 \mathrm{~F} & 01 & 1.51 \mathrm{~F} & 00\end{array}$

$1.22 \mathrm{~F} 019.61 \mathrm{E}-01$

$$
\text { PD } Z=46
$$

\begin{tabular}{|c|c|c|c|c|c|c|}
\hline $6.78 \mathrm{~F}$ & 03 & 4.078 & 07 & $2.90 \mathrm{~F}$ & 02. & 2.70 \\
\hline $6 . \mathrm{CgF}$ & 03 & $3.52 \mathrm{E}$ & 02 & $2.51 \mathrm{E}$ & $n ?$ & 2.73 \\
\hline $6.44 \mathrm{P}$ & 03 & 25 & 02 & & 02 & 2.82 \\
\hline & 03 & $9 \mathrm{E}$ & 02 & $29 F$ & $0 ?$ & \\
\hline & 03 & $4 \mathrm{E}$ & 02 & $26 \bar{F}$ & 02 & \\
\hline h. $22 \mathrm{E}$ & 03 & $3.69 \mathrm{~F}$ & $\cap 2$ & $57 \mathrm{E}$ & $n_{2}$ & \\
\hline 3. $10 \mathrm{E}$ & 03 & $1.84 \mathrm{I}$ & 02 & 2R5 & 02 & \\
\hline $1.73 \mathrm{E}$ & 03 & 1.135 & 02 & $7.15 \mathrm{E}$ & 01 & 00 \\
\hline $1.07 \mathrm{E}$ & 03 & 6.325 & 21 & $4.40 \mathrm{E}$ & 01 & 00 \\
\hline $88 \mathrm{~F}$ & 02 & $2.99 \mathrm{E}$ & 01 & $2.01 \mathrm{E}$ & 01 & 00 \\
\hline $3 \mathrm{~F}$ & 02 & $1.56 \mathrm{E}$ & 01 & $1.09 \mathrm{~F}$ & 01 & 00 \\
\hline $2 E$ & 01 & $5.00 \mathrm{~F}$ & 00 & $3.47 \mathrm{~F}$ & 00 & 00 \\
\hline & 01 & 2.205 & 00 & $53 E$ & no & 00 \\
\hline & 01 & $92 \vec{E}$ & 00 & $33 \mathrm{E}$ & 00 & 20 \\
\hline
\end{tabular}

$\begin{array}{lllllll}6.70 \mathrm{~F} & 03 & 4.86 \mathrm{~F} & 02 & 2.75 \mathrm{~F} & 02\end{array}$ 6. $\mathrm{C} 2 \mathrm{P} \quad 03 \quad 4.37 \mathrm{~F} 02 \quad 2.47 \mathrm{E} 0$ ? $6.38 \mathrm{E} 03 \quad 4.63 \mathrm{E} 02$ 2.62E 02 $\begin{array}{llllll}6.3 F E & 03 & 4.61 F & 02 & 2.617 & 02\end{array}$ $\begin{array}{lllllll}5.43 E & 03 & 3.94 E & 02 & 2.23 E & 02\end{array}$ 6. $19 \mathrm{~F} 03 \quad 4.49 \mathrm{~F} \quad 02 \quad 2.54 \mathrm{E} 02$ $\begin{array}{llllll}3.59 F & 03 & 2.61 \mathrm{~F} & 02 & 1.48 \mathrm{~F} & 02\end{array}$ $\begin{array}{llllll}2 . C 1 E & 03 & 1.46 E & 02 & 8.25 E & 01\end{array}$ $1.24 \mathrm{E} 03 \quad 8.99 \mathrm{E} 01$ 5.09E 01 $5.67 \mathrm{~F} 02 \quad 4.12 \mathrm{~F} 01 \quad 2.33 \mathrm{~F} 01$

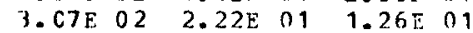
9. $83 \mathrm{E}$ O1 7.14E nO $4.04 \mathrm{E} 00$ $4.34 E \quad 01 \quad 3.15 E$ DO 1.79200 $3.27 \mathrm{E} 01$ 2.38F $00 \quad 1.35 \mathrm{E} 00$

2.86 2.97 2.99 3.00 3.20 3.23 4.00 4.00
5.00 6. 00 4. 00 10.00 15.00 20.00 22.07

$\begin{array}{lllllll}6.59 \mathrm{E} & 03 & 5.75 \mathrm{~F} & 02 & 2.71 \mathrm{~F} & 02 & 3.02 \\ 5.92 \mathrm{~F} & 03 & 5.17 \mathrm{~F} & 02 & 2.44 \mathrm{E} & 02 & 3.15 \\ 6.28 \mathrm{E} & 03 & 5.48 \mathrm{~F} & 02 & 2.58 \mathrm{E} & 02 & 3.17 \\ 5.37 \mathrm{E} & 03 & 4.68 \mathrm{~F} & 02 & 2.21 \mathrm{~F} & 0 ? & 3.39 \\ 6.11 \mathrm{E} & 03 & 5.33 \mathrm{~F} & 02 & 2.51 \mathrm{~F} & 02 & 3.41 \\ 4.11 \mathrm{~F} & 03 & 3.59 \mathrm{~F} & 02 & 1.69 \mathrm{~F} & 02 & 4.00 \\ 2.30 \mathrm{~F} & 03 & 2.00 \mathrm{E} & 02 & 9.44 \mathrm{~F} & 01 & 5.00 \\ 1.42 \mathrm{~F} & 03 & 1.24 \mathrm{~F} & 02 & 5.83 \mathrm{~F} & 01 & 6.00 \\ 6.51 \mathrm{~F} & 02 & 5.68 \mathrm{E} & 01 & 2.68 \mathrm{~F} & 01 & 8.00 \\ 3.52 \mathrm{~F} & 02 & 3.07 \mathrm{~F} & 01 & 1.45 \mathrm{~F} & 01 & 10.00 \\ 1.13 \mathrm{~F} & 02 & 9.96 \mathrm{~F} & 00 & 4.65 \mathrm{E} & 0 \mathrm{C} & 15.00 \\ 4.99 \mathrm{~F} & 01 & 4.35 \mathrm{~F} & 00 & 2.05 \mathrm{~F} & 00 & 20.00 \\ 3.27 \mathrm{E} & 01 & 2.86 \mathrm{~F} & 00 & 1.35 \mathrm{~F} & 00 & 23.17\end{array}$

$\begin{array}{llllllll}6.37 \mathrm{~F} & 03 & 6.69 \mathrm{~F} & 02 & 2.64 \mathrm{~F} & 02\end{array}$ 5. $82 \mathrm{~F} 03 \quad 6.11 \mathrm{~F}, 02$ 2.41F 02 6. 16E $03 \quad 6.47 \mathrm{~F} 02 \quad 2.55 \mathrm{~F} 02$ $\begin{array}{llllll}5.29 \mathrm{E} & 03 & 5.56 \mathrm{~F} & 02 & 2.19 \mathrm{~F} & 02\end{array}$ 6. C4F 03 b.35E 02 2.50F O2 $4.67 \mathrm{~F} 03 \quad 4.90 \mathrm{~F} \quad 02 \quad 1.93 \mathrm{~F} 02$ $2.61 E 03 \quad 2.74 \mathrm{E} 02$ 1.03E 02 $\begin{array}{llllll}1.61 \mathrm{E} & 03 & 1.69 \mathrm{E} & 02 & 6.67 \mathrm{E} & 01\end{array}$

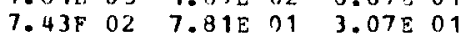
$\begin{array}{llllll}4.02 \mathrm{E} & 02 & 4.23 \mathrm{E} & 01 & 1.67 \mathrm{~F} & 01\end{array}$ $\begin{array}{lllllll}1.30 \mathrm{~F} & 02 & 1.36 \mathrm{~F} & 01 & 5.36 \mathrm{E} & 00\end{array}$ 5. $73 \mathrm{~F} 01 \quad 6.02 \mathrm{P}, 00 \quad 2.37 \mathrm{~F} 00$ $\begin{array}{lllll}3.28 \mathrm{~F} & 01 & 3.45 \mathrm{~F} & 00 & 1.36 \mathrm{~F}\end{array}$

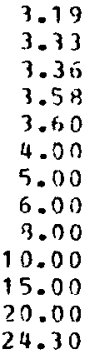


Table 8 (contmued)

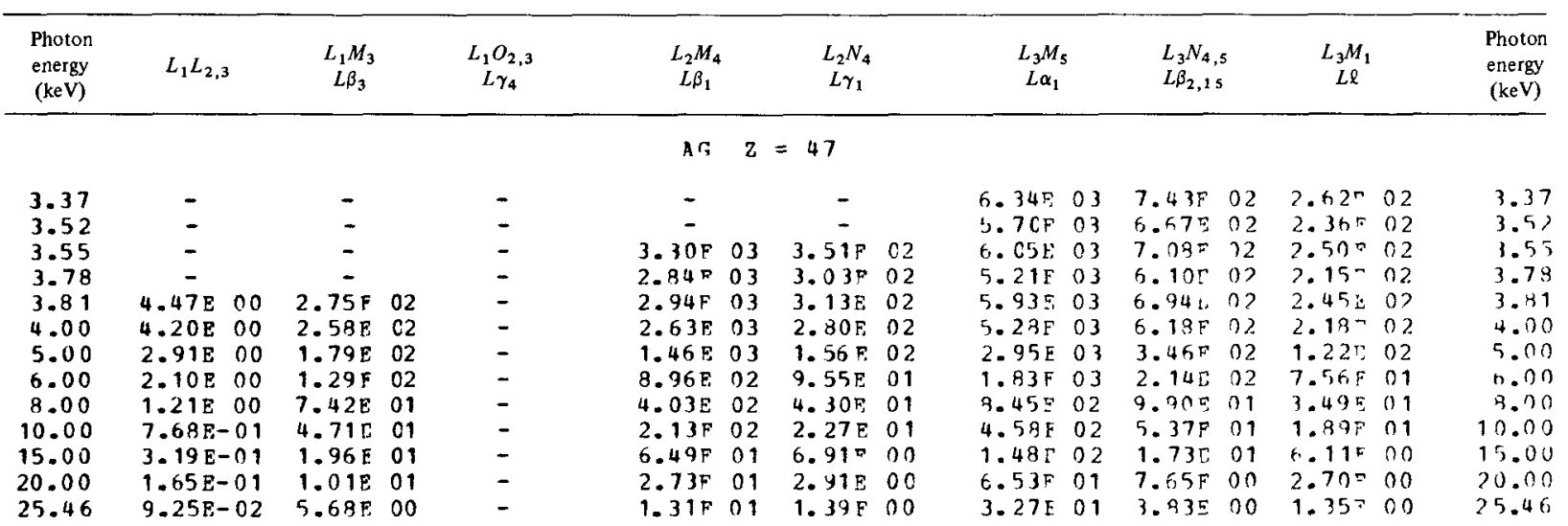

\begin{tabular}{|c|c|c|c|c|c|c|}
\hline $0.33 \mathrm{~F}$ & 03 & 8. $09=$ & 02 & $2.63 \%$ & $0 ?$ & 3.50 \\
\hline 5. $6,0 \mathrm{E}$ & 03 & $7.24 \mathrm{Fi}$ & 02 & 2.35 & $0 ?$ & 3.73 \\
\hline 6. C1F: & 03 & $7.69 \mathrm{r}$ & 02 & $2.49 \%$ & 1) 7 & 3.70 \\
\hline $5.20^{\circ}$ & 03 & 6.657 & 02 & 2.165 & $0 ?$ & 3.17 \\
\hline 6. CGF & 03 & $7.74 \mathrm{~F}$ & 02 & $2.51 \mathrm{E}$ & 02 & 4.00 \\
\hline & 03 & $7.56 \mathrm{~F}$ & $n 2$ & $2.45 \mathrm{r}$ & 02 & 4.02 \\
\hline $3.41 \mathrm{E}$ & 03 & 4.365 & 02 & $1.41 \mathrm{~F}$ & 02 & 5.00 \\
\hline $2.11 \mathrm{~F}$ & 03 & $2.70 \mathrm{~F}$ & 02 & $8.75 \mathrm{E}$ & 01 & 6.00 \\
\hline $4.76 \mathrm{E}$ & 02 & $1.25 \mathrm{r}$ & 02 & $4.05 \mathrm{~F}$ & 01 & 3.20 \\
\hline $5.31 \mathrm{~F}$ & 02 & 6.785 & 01 & $2.20^{\circ}$ & 01 & 10.00 \\
\hline $1.71 \mathrm{~F}$ & 02 & $2.19 \mathrm{~F}$ & 01 & $7.11 \mathrm{~F}$ & $0 n$ & 15.00 \\
\hline $7.60 \mathrm{E}$ & 01 & $9.71 \mathrm{~F}$ & 00 & $3.15 \%$ & 1) & 20.00 \\
\hline $3.34 \mathrm{~F}$ & 01 & $4.26 \pi$ & 00 & 1.397 & 00 & 26.66 \\
\hline
\end{tabular}

$$
C D \quad Z=43
$$

\begin{tabular}{|c|c|c|c|}
\hline $\begin{array}{l}3.56 \\
3.73 \\
3.76 \\
3.99\end{array}$ & $\begin{array}{l}- \\
- \\
- \\
-\end{array}$ & $\begin{array}{l}- \\
- \\
-\end{array}$ & \\
\hline 4.00 & $5.18 \mathrm{E} \cap 0$ & $3.11 \mathrm{E}$ & 02 \\
\hline 4.02 & $4.77 \mathrm{E} \quad 00$ & $2.87 \mathrm{E}$ & 02 \\
\hline 5.00 & $3.45 \mathrm{E} \quad 00$ & 2. C8E & 02 \\
\hline 6.00 & $2.47 \mathrm{E} \quad 00$ & $1.49 \mathrm{~F}$ & 02 \\
\hline 8.00 & $1.44 \mathrm{E} \quad 00$ & $8.65 E$ & 01 \\
\hline 10.00 & $9.18 E-01$ & $5.52 \mathrm{E}$ & 01 \\
\hline 15.00 & $3.85 \mathrm{E}-01$ & $2.31 E$ & 01 \\
\hline 20.00 & $2.00 E-01$ & 1. $20 \mathrm{E}$ & 01 \\
\hline 26.66 & $1.01 F-01$ & $6 . C 5 F$ & $\mathrm{CO}$ \\
\hline
\end{tabular}

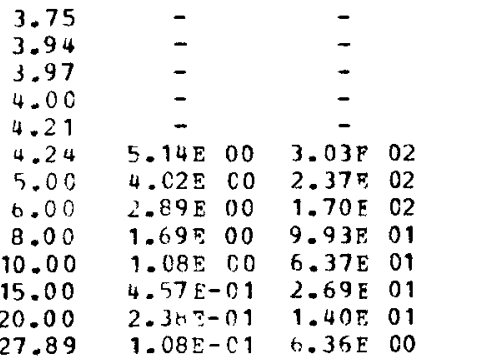

$27.89 \quad 1.08 \mathrm{E}-\mathrm{C} 1$ 6. $36 \mathrm{E} 00$

$=$
$=$
$=$
$=$
$=$
$=$
$=$
$=$

IN $Z=49$

\begin{tabular}{|c|c|c|c|c|}
\hline 3.95 & - & - & & - \\
\hline 4.00 & - & - & & - \\
\hline 4.16 & - & - & & - \\
\hline 4.19 & - & - & & - \\
\hline 4.44 & - & - & & \\
\hline 4.47 & $9.24 \mathrm{E} 00$ & $5.32 \mathrm{~F}$ & 02 & $5.52 \mathrm{~F} 00$ \\
\hline 5.00 & $7.72 \mathrm{E} \quad 00$ & $4.45 \mathrm{E}$ & 02 & $4.61 \mathrm{E} 00$ \\
\hline 6.00 & $5.63 \mathrm{E} 00$ & $3.24 \mathrm{E}$ & 02 & $3.36 \mathrm{E} \quad 00$ \\
\hline 8.00 & $3.30 E 00$ & $1.90 \mathrm{E}$ & 02 & $1.97 \mathrm{P} 00$ \\
\hline 10.00 & $2.12 E \quad 00$ & 1.228 & 02 & $1.27 \mathrm{~F} 00$ \\
\hline 15.00 & $9.03 \mathrm{E}-01$ & $5.20 \mathrm{E}$ & 01 & $5.40 \mathrm{E}-01$ \\
\hline & $4.73 \mathrm{E}-01$ & $2.72 \mathrm{E}$ & 01 & $2.83 F-01$ \\
\hline
\end{tabular}

\section{$-$}

29.16

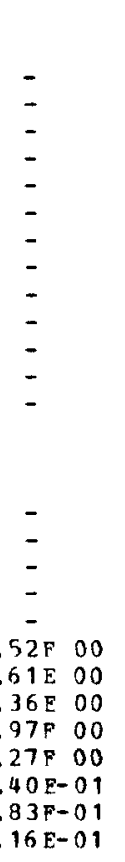

$\begin{array}{llll}3.34 \mathrm{E} & 03 & 3.89 & 02\end{array}$ $2.90 \mathrm{E} O 3 \quad 3.39 \mathrm{E} 02$

$3.05 \mathrm{E} \quad \mathrm{n} \quad 3.35 \mathrm{~F} \quad 2$

$3.00 \mathrm{E} \quad 03 \quad 3.49 \mathrm{~F} \quad 02$

$\begin{array}{llll}1.73 \mathrm{~F} & 03 & 2.02 \mathrm{E} & 02\end{array}$

$1.06 \mathrm{~F} 03 \quad 1.24 \mathrm{~F} 02$

4.30E 02 ל. ל9. 01

$2.55 \mathrm{E} 02 \quad 2.96 \mathrm{E} 01$

$7.79 \mathrm{E} \quad 01 \quad 9.06 \mathrm{E} \quad 00$

$3.29 \mathrm{E} 01 \quad 3.32 \mathrm{E}$ OO

$1.37 \mathrm{E} 01 \quad 1.59 \mathrm{E}$ ก

$3.30 F \quad 03 \quad 4.15=02$ $3.36 \mathrm{E} 03 \quad 4.22 \mathrm{E} \quad 02$

$2.94 \mathrm{E} \quad 03 \quad 3.70 \mathrm{E} \quad 02$

$3.04 \mathrm{E} 03 \quad 3.83 \mathrm{~F}, 02$

$2.03 \mathrm{~F} 03 \quad 2.55 \mathrm{E} 02$

$1.24 \% 03 \quad 1.56 \mathrm{E} \quad 02$

$5.66 \mathrm{E} \quad 02 \quad 7.11 \mathrm{R} 01$

$3.01 \mathrm{E} 02 \quad 3.79 \mathrm{E} 01$

$9.25 \mathrm{~F}, 01 \quad 1.16 \mathrm{E} 01$

$3.92 E 014.92 \mathrm{E} 00$

$1.43 \times 01 \quad 1.79 \mathrm{E} \mathrm{O}^{2}$

$$
\text { SN } z=50
$$

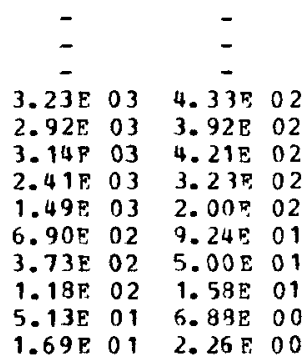

$$
5 B \quad z=51
$$

\begin{tabular}{|c|c|c|c|c|c|c|}
\hline $6.18 \mathrm{~F}$ & 03 & $8.53 \mathrm{E}$ & 02 & $2.57 \%$ & 02 & 3.75 \\
\hline $5.61 \mathrm{E}$ & 03 & $7.74 \Gamma$ & 72 & $2.33 \mathrm{E}$ & 02 & 3.94 \\
\hline 5.968 & 03 & $9.22 \mathrm{E}$ & 02 & 2.478 & 02 & 3.97 \\
\hline $5.86 \mathrm{E}$ & 03 & $8.09 \mathrm{E}$ & 02 & $2.43 F$ & 02 & 4.00 \\
\hline $5.18 \mathrm{~F}$ & 03 & $7.14 \%$ & 02 & $2.15 \mathrm{E}$ & 02 & 4.21 \\
\hline $5.91 \mathrm{E}$ & 03 & ค. $15 E$ & 02 & $2.45 \mathrm{E}$ & 02 & 4.24 \\
\hline $3.91 \mathrm{~F}$ & 03 & $5.40 \mathrm{~F}$ & 02 & $1.63 \mathrm{E}$ & 02 & 5.00 \\
\hline $2.42 \mathrm{E}$ & 03 & $3.34 \mathrm{E}$ & 02 & $1.01 \mathrm{E}$ & 02 & 6.00 \\
\hline $1.12 \mathrm{E}$ & 03 & $1.55 \%$ & 32 & $4.67 \mathrm{E}$ & 01 & 8.00 \\
\hline $6.92 \mathrm{~F}$ & 02 & $8.44 E$ & 01 & $2.54 \pi$ & 01 & 10.00 \\
\hline $1.98 \mathrm{~F}$ & 02 & $2.73 \mathrm{E}$ & 01 & $8.23 \mathrm{E}$ & 00 & 15.00 \\
\hline & 01 & $1.21 \mathrm{E}$ & 01 & $3.65 \mathrm{~F}$ & $n \mathrm{C}$ & 20.00 \\
\hline & 1 & $.69 \mathrm{E}$ & 00 & $1.41 \mathrm{~F}$ & 00 & \\
\hline
\end{tabular}

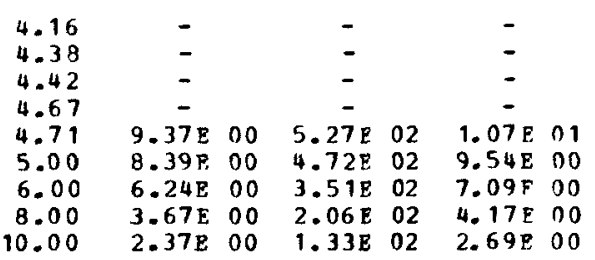

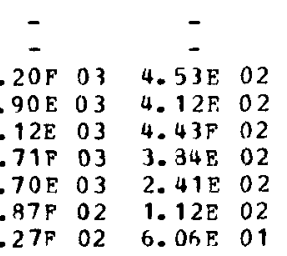

$\begin{array}{lllllll}6.04 F & 03 & 8.93 E & 02 & 2.52 \Gamma & 02 & 3.95 \\ 6.17 \mathrm{E} & 03 & 9.07 \mathrm{E} & 02 & 2.57 \mathrm{E} & 02 & 4.00 \\ 5.54 \mathrm{~F} & 03 & 8.15 \mathrm{~F} & 02 & 2.31 \mathrm{E} & 02 & 4.16 \\ 5.88 \mathrm{E} & 03 & 8.64 \mathrm{E} & 02 & 2.45 \mathrm{~F} & 02 & 4.19 \\ 5.12 \mathrm{E} & 03 & 7.53 \mathrm{E} & 02 & 2.14 \mathrm{E} & 02 & 4.44 \\ 5.44 \mathrm{E} & 03 & 7.99 \mathrm{E} & 02 & 2.27 \mathrm{E} & 02 & 4.47 \\ 4.09 \mathrm{E} & 03 & 6.01 \mathrm{E} & 02 & 1.71 \mathrm{~F} & 02 & 5.00 \\ 2.49 \mathrm{E} & 03 & 3.66 \mathrm{E} & 02 & 1.04 \mathrm{E} & 02 & 5.00 \\ 1.13 \mathrm{~F} & 03 & 1.66 \mathrm{~F} & 02 & 4.71 \mathrm{~F} & 01 & 9.00 \\ 6.00 \mathrm{~F} & 02 & 8.92 \mathrm{E} & 01 & 2.50 \mathrm{~F} & 01 & 10.00 \\ 1.85 \mathrm{E} & 02 & 2.72 \mathrm{E} & 01 & 7.72 \mathrm{~F} & 00 & 15.00 \\ 7.88 \mathrm{E} & 01 & 1.16 \mathrm{~F} & 01 & 3.29 \mathrm{E} & 00 & 20.00 \\ 2.54 \mathrm{~F} & 01 & 3.73 \mathrm{E} & 00 & 1.06 \mathrm{~F} & 00 & 29.16\end{array}$

$\begin{array}{lllllll}6.07 \mathrm{~F} & 03 & 9.49 \mathrm{E} & 02 & 2.54 \mathrm{~T} & 02\end{array}$ $5.56 \mathrm{E}$ 03 $8.68 \mathrm{E} 02$ 2.32E 02 $\begin{array}{lllllll}5.89 \mathrm{~F} & 03 & 9.20 \mathrm{E} & 02 & 2.46 \mathrm{E} & 02\end{array}$ $\begin{array}{lllllll}5.15 \mathrm{~F} & 03 & 8.04 \mathrm{~F} & 02 & 2.15 \mathrm{~F} & 02\end{array}$ $\begin{array}{llllll}4.48 \mathrm{~F} & 03 & 8.57 \mathrm{E} & 02 & 2.29 \mathrm{E} & 02\end{array}$ $\begin{array}{llllll}4.71 \mathrm{~F} & 03 & 7.36 \mathrm{5} & 02 & 1.97 \mathrm{E} & 02\end{array}$ $2.89 \mathrm{~F} 03 \quad 4.52 \mathrm{~F} \quad 02 \quad 1.21 \% 02$ $\begin{array}{llllll}1.31 E & 03 & 2.05 E & 02 & 5.49 \vec{F} & 01\end{array}$

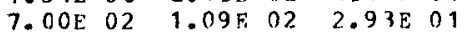


Table 8 (continued)

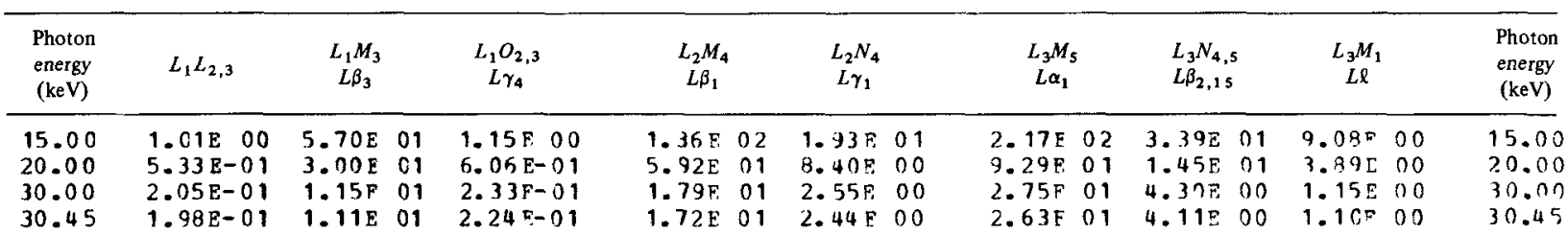

TE $\quad Z=52$

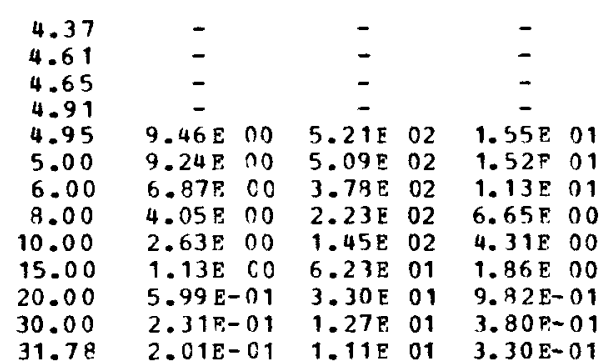

4.59

4.85

4.89
5.00

5.16

5.20

6.00

8.00

10.00

15.00

20.00

30.00

33.13
$2.66 \mathrm{E}-011.43 \mathrm{E} 01$

2. C9E-C1 1.13E OI

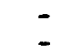

$-$

$-$

$2.06 \mathrm{E} \quad 01$

$1.62 \mathrm{E} 01$

$9.63 \mathrm{E} 00$ $6.27 \mathrm{E} 00$ $2.72 E 00$

1. $45 \mathrm{E} 00$

5. $62 \mathrm{E}-01$

4. 42 2 -01
4.8

5.00

5.10
5.14

5.41

5.45

6.00

8.00

10.00

15.00

20.00

30.00

34.52
$-$

$3.21 \mathrm{E} \quad 03 \quad 4.30 \mathrm{E} \quad 02$ $2.92 \mathrm{~F} 03 \quad 4.36 \mathrm{E} 02$ $\begin{array}{lllll}3.15 \mathrm{~F} & 03 & 4.71 \mathrm{~F} & 02\end{array}$ $\begin{array}{lllll}3.08 \mathrm{~F} & 03 & 4.59 \mathrm{P}_{1} & 02\end{array}$ $1.97 \mathrm{~F} 03 \quad 2.93 \mathrm{E} \quad 02$ $9.13 \mathrm{~F} \quad 02 \quad 1.36 \mathrm{E} \quad 02$ 4.98F $02 \quad 7.43 \mathrm{E} 01$ $\begin{array}{lllll}1.60 \mathrm{~F} & 02 & 2.39 \mathrm{E} 01\end{array}$ $6.99 \mathrm{~F} 01 \quad 1.04 \mathrm{E} \quad 01$ $2.13 \mathrm{E} 01 \quad 3.19 \mathrm{E}$ on $\begin{array}{llll}1.80 \mathrm{~F} 01 & 2.69 \mathrm{E} & 00\end{array}$

$$
\text { I } \quad z=53
$$

$-$

$3.23 \mathrm{E} \quad 03 \quad 5.03 \mathrm{E} \quad 02$ $3.19803 \quad 4.98 \mathrm{~F} 02$ $\begin{array}{lllll}2.93 \mathrm{E} & 03 & 4.57 \mathrm{~F} & 02\end{array}$ $3.16 \mathrm{E} \quad 03 \quad 4.93 \mathrm{~F} 02$ $2.24 \mathrm{E} \quad 0.3 \quad 3.49 \mathrm{~F} 02$ $1.05 \mathrm{~F} 03 \quad 1.63 \mathrm{E} \quad 02$ $5.72 \mathrm{E} \quad 02 \quad 8.92 \mathrm{E} \quad 01$ $\begin{array}{lllll}1.84 \mathrm{~F} & 02 & 2.88 \mathrm{E} & 01\end{array}$ $8.09 \mathrm{~F}, 01 \quad 1.26 \mathrm{E} \quad 01$ $2.48 \mathrm{E} 01$ 3. $06 \mathrm{E} 00$

$$
X \mathrm{~F} \quad Z=54
$$

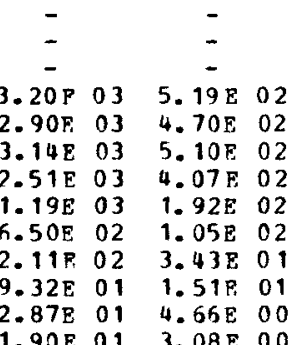

$1.90 \mathrm{E} 01 \quad 3.08 \mathrm{E} 00$

$$
\operatorname{cs} z=55
$$

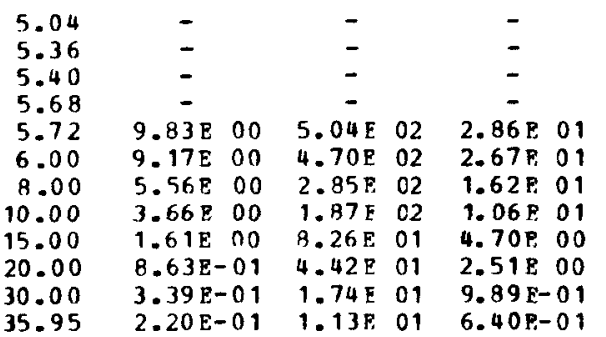

$\begin{array}{lllllll}6.11 \mathrm{E} & 03 & 1.00 \mathrm{~F} & 03 & 2.56 \mathrm{E} & 02 & 4.37 \\ 5.54 \mathrm{E} & 03 & 9.11 \mathrm{E} & 02 & 2.33 \mathrm{~F} & 02 & 4.61 \\ 5.87 \mathrm{E} & 03 & 9.66 \mathrm{E} & 02 & 2.46 \mathrm{~F} & 02 & 4.65 \\ 5.15 \mathrm{E} & 03 & 8.46 \mathrm{E} & 02 & 2.16 \mathrm{~F} & 02 & 4.91 \\ 5.49 \mathrm{E} & 03 & 9.03 \mathrm{E} & 02 & 2.30 \mathrm{~F} & 02 & 4.95 \\ 5.35 \mathrm{~F} & 03 & 8.90 \mathrm{~F} & 02 & 2.25 \mathrm{E} & 02 & 5.00 \\ 3.32 \mathrm{~F} & 03 & 5.46 \mathrm{E} & 02 & 1.39 \mathrm{~F} & 02 & 6.00 \\ 1.51 \mathrm{E} & 03 & 2.48 \mathrm{E} & 02 & 6.32 \mathrm{E} & 01 & 9.00 \\ 8 . \mathrm{C} \mathrm{E} & 02 & 1.33 \mathrm{E} & 02 & 3.39 \mathrm{~F} & 01 & 10.00 \\ 2.51 \mathrm{~F} & 02 & 4.13 \mathrm{E} & 01 & 1.05 \mathrm{~F} & 01 & 15.00 \\ 1 . C 8 \mathrm{E} & 02 & 1.77 \mathrm{E} & 01 & 4.52 \mathrm{~F} & 00 & 20.00 \\ 3.20 \mathrm{E} & 01 & 5.27 \mathrm{E} & 00 & 1.34 \mathrm{E} & 10 & 30.00 \\ 2.69 \mathrm{E} & 01 & 4.43 \mathrm{E} & 00 & 1.13 \mathrm{~F} & 00 & 31.7 \mathrm{~B}\end{array}$

6. 13F 03 1.05E $03 \quad 2.59 F \quad 02$ $\begin{array}{llllll}5.51 \mathrm{E} & 03 & 9.45 \mathrm{~F} & 02 & 2.33 \mathrm{~F} & 0\end{array}$ $\begin{array}{llllll}5.84 \mathrm{~F} & 03 & 1.00 \mathrm{E} & 03 & 2.47 \mathrm{E} & 02\end{array}$ $\begin{array}{llllll}5.54 \mathrm{E} & 03 & 9.50 \mathrm{~S} & \mathrm{~T} 2 & 2.34 \mathrm{~F} & 02\end{array}$ $\begin{array}{llllll}5.13 \mathrm{E} & 03 & 8.78 \mathrm{E} & 02 & 2.17 \mathrm{E} & 02\end{array}$ $\begin{array}{lllllll}5.48 E & 03 & 9.39 \mathrm{E} & 02 & 2.31 \mathrm{E} & 02\end{array}$ $\begin{array}{llllll}3.78 \mathrm{~F} & 03 & 6.48 \mathrm{~F} & 02 & 1.60 \mathrm{~F} & 02\end{array}$ $\begin{array}{llllll}1.72 E & 03 & 2.74 F & 02 & 7.265 & 01\end{array}$ $9.23 \mathrm{E} 02 \quad 1.58 \mathrm{~F} 023.90 \mathrm{~F} 01$ $\begin{array}{llllllll}2.89 F & 02 & 4.945 & 01 & 1.22 \mathrm{~F} & 01\end{array}$ $\begin{array}{lllllllll}1.24 \mathrm{E} & 02 & 2.12 \mathrm{E} & 01 & 5.24 \mathrm{E} & 00\end{array}$ $\begin{array}{lllll}3.7 \mathrm{CE} O 1 & 6.34 \mathrm{E} & \mathrm{OO} & 1.56 \mathrm{E} & 00\end{array}$ $2.74 E 01 \quad 4.70800 \quad 1.16 F 00$

4.59 14.85 4.89 5.00 5.16

5.20

6. 00

3.00 10.00 15.00 20.00 30.00 33.13

$\begin{array}{lllllll}6.23 \mathrm{E} & 03 & 1.10 \mathrm{E} & 03 & 2.65 \mathrm{~F} & 02 & 4.81 \\ 5.85 \mathrm{E} & 03 & 1.04 \mathrm{~F} & 03 & 2.49 \mathrm{E} & 02 & 5.00 \\ 5.53 \mathrm{E} & 03 & 9.90 \mathrm{~F} & 02 & 2.35 \mathrm{E} & 02 & 5.10 \\ 5.87 \mathrm{E} & 03 & 1.04 \mathrm{E} & 03 & 2.49 \mathrm{~F} & 02 & 5.14 \\ 5.16 \mathrm{E} & 03 & 9.15 \mathrm{~F} & 02 & 2.19 \mathrm{~F} & 02 & 5.41 \\ 5.52 \mathrm{E} & 03 & 9.78 \mathrm{E} & 02 & 2.34 \mathrm{E} & 02 & 5.45 \\ 4.33 \mathrm{E} & 03 & 7.67 \mathrm{~F} & 02 & 1.94 \mathrm{~F} & 02 & 6.00 \\ 1.98 \mathrm{E} & 03 & 3.50 \mathrm{~F} & 02 & 8.40 \mathrm{~F} & 01 & 8.00 \\ 1.07 \mathrm{E} & 03 & 1.99 \mathrm{~F} & 02 & 4.52 \mathrm{~F} & 01 & 10.00 \\ 3.34 \mathrm{E} & 02 & 5.92 \mathrm{~F} & 01 & 1.42 \mathrm{~F} & 01 & 15.00 \\ 1.44 \mathrm{~F} & 02 & 2.55 \mathrm{E} & 01 & 6.11 \mathrm{~F} & 00 & 2.000 \\ 4.30 \mathrm{E} & 01 & 7.62 \mathrm{~F} & 0 \mathrm{O} & 1.83 \mathrm{E} & 00 & 30.00 \\ 2.82 \mathrm{~F} & 01 & 5.00 \mathrm{~F} & 00 & 1.20 \mathrm{~F} & 00 & 34.52\end{array}$

$\begin{array}{cccc}- & & - & \\ 3.33 \mathrm{E} & 03 & 5.59 \mathrm{~F} & 02\end{array}$ $2.96 \mathrm{E} 03 \quad 4.97 \mathrm{E} 02$ $\begin{array}{llll}3.21 \mathrm{E} & 03 & 5.39 \mathrm{E} & 02\end{array}$ $2.87 \mathrm{~F} .03 \quad 4.82502$ $\begin{array}{llll}1.37 \mathrm{~F} 03 & 2.31 \mathrm{~F} & 02\end{array}$ $\begin{array}{llll}7.56 \% & 02 & 1.27 E & 02\end{array}$ $2.47 \mathrm{~F} \quad 02 \quad 4.15 \mathrm{~F} \quad 01$ $1.09 \mathrm{E} 02 \quad 1.33 \mathrm{E} 01$ $3.38 \mathrm{E} 01 \quad 5.67 \mathrm{E} 00$ 1.99F 01 3.34F, 00

$$
B A \quad Z=56
$$

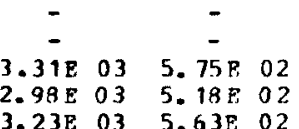

$\begin{array}{lllllll}6.44 \mathrm{E} & 03 & 1.18 \mathrm{E} & 03 & 2.75 \mathrm{~F} & 02 & 5.04 \\ 5.53 \mathrm{~F} & 03 & 1.02 \mathrm{E} & 03 & 2.36 \mathrm{~F} & 02 & 5.36 \\ 5.87 \mathrm{E} & 03 & 1.08 \mathrm{E} & 03 & 2.51 \mathrm{E} & 02 & 5.40 \\ 5.17 \mathrm{~F} & 03 & 9.50 \mathrm{E} & 02 & 2.21 \mathrm{~F} & 02 & 5.68 \\ 5.53 \mathrm{~F} & 03 & 1.01 \mathrm{E} & 03 & 2.36 \mathrm{E} & 02 & 5.72 \\ 4.92 \mathrm{E} & 03 & 9.03 \mathrm{E} & 02 & 2.10 \mathrm{E} & 02 & 6.00 \\ 2.27 \mathrm{~F} & 03 & 4.16 \mathrm{E} & 02 & 9.67 \mathrm{~F} & 01 & 8.00 \\ 1.22 \mathrm{~F} & 03 & 2.24 \mathrm{~F} & 02 & 5.21 \mathrm{~F} & 01 & 10.00 \\ 3.84 \mathrm{E} & 02 & 7.06 \mathrm{~F} & 01 & 1.64 \mathrm{E} & 01 & 15.00 \\ 1.66 \mathrm{E} & 02 & 3.05 \mathrm{E} & 01 & 7.09 \mathrm{~F} & 00 & 20.00 \\ 4.97 \mathrm{E} & 01 & 9.13 \mathrm{~F} & 00 & 2.12 \mathrm{~F} & 00 & 30.00 \\ 2.89 \mathrm{E} & 01 & 5.30 \mathrm{~F} & 10 & 1.23 \mathrm{~F} & 00 & 35.95\end{array}$

$\begin{array}{lllllll}6.38 E & 03 & 1.21 E & 03 & 2.755 & 02 & 5.74\end{array}$

\begin{tabular}{|c|c|c|c|c|c|c|c|c|c|c|c|}
\hline $\begin{array}{l}5.29 \\
5.63 \\
5.67 \\
5.95 \\
6.00\end{array}$ & $\begin{array}{c}- \\
- \\
- \\
\overline{-} \\
1.02 \mathrm{E} \\
01\end{array}$ & $\begin{array}{c}- \\
- \\
- \\
- \\
5.06 \mathrm{E} \quad 02\end{array}$ & $\begin{array}{c}- \\
- \\
- \\
- \\
3.27 \mathrm{E}\end{array}$ & $\begin{array}{cc}\overline{-} & \\
3.31 \mathrm{E} & 03 \\
2.98 \mathrm{E} & 03 \\
3.23 \mathrm{E} & 03\end{array}$ & $\begin{array}{cc}- & \\
-\overline{5} & \\
5.75 \mathrm{P} & 02 \\
5.18 \mathrm{P} & 02 \\
5.63 \mathrm{E} & 02\end{array}$ & $\begin{array}{l}6.38 \mathrm{E} \\
5.50 \mathrm{~F} \\
5.84 \mathrm{E} \\
5.15 \mathrm{E} \\
5.52 \mathrm{E}\end{array}$ & $\begin{array}{l}03 \\
03 \\
03 \\
03 \\
03\end{array}$ & $\begin{array}{l}1.21 \mathrm{E} \\
1.05 \mathrm{~F} \\
1.11 \mathrm{E} \\
9.798 \\
1.05 \mathrm{E}\end{array}$ & $\begin{array}{l}03 \\
03 \\
03 \\
02 \\
03\end{array}$ & $\begin{array}{l}2.75 \mathrm{~F} \\
2.37 \mathrm{E} \\
2.51 \mathrm{E} \\
2.22 \mathrm{~F} \\
2.37 \mathrm{E}\end{array}$ & $\begin{array}{l}02 \\
02 \\
02 \\
02 \\
02\end{array}$ \\
\hline
\end{tabular}
$\begin{array}{lllllll}5.50 \mathrm{~F} & 03 & 1.05 \mathrm{~F}, 03 & 2.37 \mathrm{~F} & 02 & 5.63\end{array}$ $\begin{array}{lllllll}5.84 \mathrm{E} & 03 & 1.112 & 03 & 2.51 \mathrm{E} & 02 & 5.67\end{array}$ $\begin{array}{lllllll}5.15 \mathrm{~F} & 03 & 9.79 \mathrm{E} & 02 & 2.22 \mathrm{~F} & 02 & 5.75\end{array}$ 
Table 8 (continued)

\begin{tabular}{|c|c|c|c|c|c|c|c|c|c|c|c|c|c|c|c|c|}
\hline \multirow{2}{*}{$\begin{array}{r}\begin{array}{r}\text { Photon } \\
\text { energy } \\
\text { (keV) }\end{array} \\
6.00 \\
8.00 \\
10.00 \\
15.00 \\
20.00 \\
30.00 \\
37.41\end{array}$} & $L_{1} L_{2,3}$ & \multicolumn{2}{|l|}{$\begin{array}{c}L M_{1} M_{3} \\
L \beta_{3}\end{array}$} & \multicolumn{2}{|l|}{$\begin{array}{c}L_{1} O_{2,3} \\
L \gamma_{4}\end{array}$} & \multicolumn{2}{|c|}{$\begin{array}{c}L_{2} M_{4} \\
L \beta_{1}\end{array}$} & \multicolumn{2}{|c|}{$\begin{array}{c}L_{2} N_{4} \\
L \gamma_{1}\end{array}$} & \multicolumn{2}{|c|}{$\begin{array}{c}L_{3} M_{5} \\
L \alpha_{1}\end{array}$} & \multicolumn{2}{|c|}{$\begin{array}{l}L_{3} N_{4,5} \\
L \beta_{2,15}\end{array}$} & \multicolumn{2}{|c|}{$\begin{array}{c}L_{3} M_{1} \\
L \ell\end{array}$} & \multirow{2}{*}{$\begin{array}{r}\begin{array}{r}\text { Photon } \\
\text { energy } \\
\text { (keV) }\end{array} \\
6.00 \\
9.00 \\
10.00 \\
15.00 \\
20.00 \\
30.00 \\
37.41\end{array}$} \\
\hline & 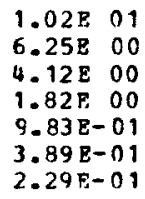 & $\begin{array}{l}5.05 \mathrm{E} \\
3.10 \mathrm{E} \\
2.04 \mathrm{~F} \\
9.04 \mathrm{E} \\
4.87 \mathrm{E} \\
1.93 \mathrm{E} \\
1.13 \mathrm{E}\end{array}$ & $\begin{array}{l}02 \\
02 \\
02 \\
01 \\
01 \\
01 \\
01\end{array}$ & $\begin{array}{l}3.26 \mathrm{E} \\
2.00 \mathrm{E} \\
1.32 \mathrm{~F} \\
5.84 \mathrm{E} \\
3.15 \mathrm{E} \\
1.24 \mathrm{E} \\
7.33 \mathrm{E}-\end{array}$ & $\begin{array}{ll}0 & 1 \\
0 & 1 \\
0 & 1 \\
0 & 0 \\
0 & 0 \\
0 & 0 \\
0 & 1\end{array}$ & $\begin{array}{l}3.23 \mathrm{~F} \\
1.57 \mathrm{~F} \\
8.61 \mathrm{E} \\
2.93 \mathrm{E} \\
1.26 \mathrm{~F} \\
3.90 \mathrm{E} \\
2.04 \mathrm{~F}\end{array}$ & $\begin{array}{l}03 \\
03 \\
02 \\
02 \\
02 \\
01 \\
01\end{array}$ & $\begin{array}{l}5.62 \mathrm{~F} \\
2.73 \mathrm{E} \\
1.50 \mathrm{E} \\
4.93 \mathrm{E} \\
2.19 \mathrm{~F} \\
6.78 \mathrm{E} \\
3.55 \mathrm{~F}\end{array}$ & $\begin{array}{l}02 \\
02 \\
02 \\
01 \\
01 \\
00 \\
00\end{array}$ & $\begin{array}{l}5.51 \mathrm{~F} \\
2.57 \mathrm{~F} \\
1.39 \mathrm{E} \\
4.39 \mathrm{~F} \\
1.90 \mathrm{~F} \\
5.71 \mathrm{E} \\
2.94 \mathrm{~F}\end{array}$ & $\begin{array}{ll}0 & 3 \\
03 \\
03 \\
02 \\
02 \\
01 \\
01\end{array}$ & $\begin{array}{l}1.05= \\
4.99 F \\
2.54= \\
8.342 \\
3.615 \\
1.090 \\
5.59 \mathrm{E}\end{array}$ & $\begin{array}{l}03 \\
02 \\
07 \\
01 \\
01 \\
01 \\
00\end{array}$ & $\begin{array}{l}2.37 \mathrm{E} \\
1.11 \mathrm{E} \\
5.97 \pi \\
1.395 \\
8.175 \\
2.46 \pi \\
1.27 \mathrm{r}\end{array}$ & $\begin{array}{l}02 \\
02 \\
01 \\
01 \\
00 \\
00 \\
0\end{array}$ & \\
\hline & & & & & & LA & $z$ & $=57$ & & & & & & & & \\
\hline $\begin{array}{r}5.52 \\
5.90 \\
5.94 \\
6.00 \\
6.24 \\
6.29 \\
8.00 \\
10.00 \\
15.00 \\
20.00 \\
30.00 \\
38.91\end{array}$ & $\begin{array}{cc}- & \\
- & \\
- & \\
- & \\
1.05 \mathrm{E} & 01 \\
6.99 \mathrm{E} & 00 \\
4.61 \mathrm{E} & 00 \\
2.06 \mathrm{~F} & 00 \\
1.11 \mathrm{~F} & 00 \\
4.43 \mathrm{E}-01 \\
2.38 \mathrm{E}-01\end{array}$ & $\begin{array}{c}- \\
\bar{z} \\
\overline{-} \\
\overline{-} \\
5.03 \mathrm{~F} \\
3.36 \mathrm{E} \\
2.22 \mathrm{E} \\
9.87 \mathrm{E} \\
5.34 \mathrm{E} \\
2.12 \mathrm{E} \\
1.14 \mathrm{E}\end{array}$ & $\begin{array}{l}02 \\
02 \\
02 \\
01 \\
01 \\
01 \\
01\end{array}$ & $\begin{array}{c}- \\
- \\
- \\
- \\
- \\
3.23 \mathrm{~F} \\
2.16 \mathrm{~F} \\
1.42 \mathrm{E} \\
6.34 \mathrm{~F} \\
3.43 \mathrm{~F} \\
1.36 \mathrm{~F} \\
7.33 \mathrm{E}-\end{array}$ & $\begin{array}{ll}0 & 1 \\
0 & 1 \\
0 & 1 \\
0 & 0 \\
0 & 0 \\
0 & 0 \\
0 & 1\end{array}$ & $\begin{array}{c}- \\
3.34 \mathrm{E} \\
3.33 \mathrm{E} \\
3.02 \mathrm{E} \\
3.27 \mathrm{E} \\
1.79 \mathrm{E} \\
9.87 \mathrm{E} \\
3.26 \mathrm{E} \\
1.45 \mathrm{E} \\
4.52 \mathrm{E} \\
2.11 \mathrm{E}\end{array}$ & $\begin{array}{l}03 \\
03 \\
03 \\
03 \\
03 \\
02 \\
02 \\
02 \\
01 \\
01\end{array}$ & $\begin{array}{c}- \\
5.99 \mathrm{E} \\
5.97 \mathrm{E} \\
5.41 \mathrm{E} \\
5.86 \mathrm{~F} \\
3.21 \mathrm{E} \\
1.77 \mathrm{E} \\
5.85 \mathrm{E} \\
2.60 \mathrm{E} \\
8.09 \mathrm{E} \\
3.79 \mathrm{E}\end{array}$ & $\begin{array}{l}02 \\
02 \\
02 \\
02 \\
02 \\
02 \\
01 \\
01 \\
00 \\
00\end{array}$ & $\begin{array}{l}6.45 \mathrm{E} \\
5.52 \mathrm{~F} \\
5.86 \mathrm{E} \\
5.72 \mathrm{~F} \\
5.18 \mathrm{~F} \\
5.56 \mathrm{E} \\
2.95 \mathrm{~F} \\
1.59 \mathrm{~F} \\
5.06 \mathrm{~F} \\
2.20 \mathrm{~F} \\
6.64 \mathrm{E} \\
3.05 \mathrm{~F}\end{array}$ & $\begin{array}{l}03 \\
03 \\
03 \\
03 \\
03 \\
03 \\
03 \\
03 \\
02 \\
02 \\
01 \\
01\end{array}$ & $\begin{array}{l}1.27 \mathrm{E} \\
1.08 \mathrm{E} \\
1.15 \mathrm{E} \\
1.125 \\
1.025 \\
1.09 \mathrm{E} \\
5.79 \mathrm{E} \\
3.125 \\
9.94 \mathrm{E} \\
4.32 \mathrm{E} \\
1.30 \mathrm{E} \\
5.99 \mathrm{E}\end{array}$ & $\begin{array}{l}03 \\
03 \\
03 \\
03 \\
03 \\
03 \\
02 \\
02 \\
01 \\
01 \\
01 \\
00\end{array}$ & $\begin{array}{l}2.80 \mathrm{~F} \\
2.40 \mathrm{~F} \\
2.54 \mathrm{~F} \\
2.495 \\
2.25 \mathrm{~F} \\
2.41 \mathrm{~F} \\
1.28 \mathrm{~F} \\
6.91 \mathrm{~F} \\
2.20 \mathrm{~F} \\
9.54 \mathrm{5} \\
2.93 \mathrm{~F} \\
1.33 \mathrm{~F}\end{array}$ & $\begin{array}{l}02 \\
0 ? \\
02 \\
02 \\
02 \\
02 \\
02 \\
01 \\
01 \\
00 \\
00 \\
00\end{array}$ & $\begin{array}{l}5.57 \\
3.90 \\
5.94 \\
6.00 \\
6.24 \\
6.29 \\
9.00 \\
10.00 \\
15.00 \\
20.07 \\
30.00 \\
37.01\end{array}$ \\
\hline
\end{tabular}

$C F \quad Z=58$

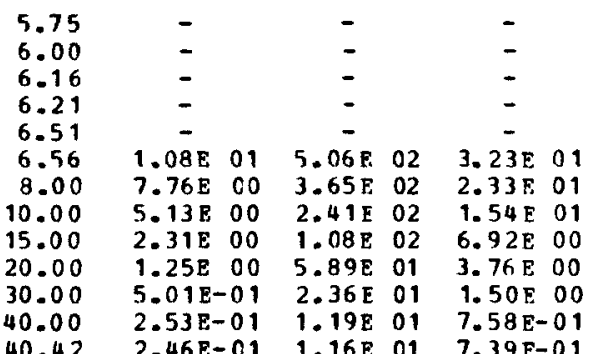

$2.46 \mathrm{~F}-01 \quad 1.16 \mathrm{E} 01 \quad 7.39 \mathrm{E}-01$

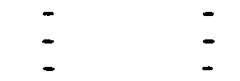

$3.41 \mathrm{E} 03 \quad 6.06 \mathrm{E} 0 ?$ $3.09 \mathrm{E} \quad 03 \quad 5.48 \mathrm{E} \quad 02$ $3.34 \mathrm{E} 03 \quad 5.94 \mathrm{E} 02$ $2.05 \mathrm{E} \quad 03 \quad 3.64 \mathrm{E} 02$ $\begin{array}{llll}1.13 \mathrm{~F} & 03 & 2.01 \mathrm{~B} & 02\end{array}$ $\begin{array}{llll}3.76 \mathrm{E} & 02 & 6.68 \mathrm{E} & 01\end{array}$ $1.69 \mathrm{E} 02 \quad 2.98 \mathrm{E} 01$ $5.24 \mathrm{P} 019.30 \mathrm{E} 00$ $2.26 \mathrm{E} 01 \quad 4.02 \mathrm{E} 00$ 2. $20 \mathrm{~F} 01 \quad 3.90 \mathrm{~F} 00$

$$
\text { PR } z=59
$$

\begin{tabular}{|c|c|c|c|c|}
\hline $\begin{array}{r}6.00 \\
6.00 \\
6.44 \\
6.49 \\
6.79 \\
6.85 \\
8.00 \\
10.00 \\
15.00 \\
20.00 \\
30.00\end{array}$ & 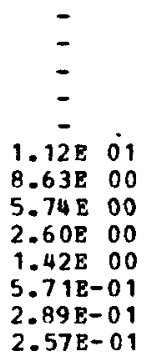 & $\begin{array}{c}- \\
- \\
- \\
- \\
- \\
5.06 \mathrm{~F} \\
3.91 \mathrm{E} \\
2.60 \mathrm{E} \\
1.18 \mathrm{E} \\
6.44 \mathrm{E} \\
2.59 \mathrm{E} \\
1.31 \mathrm{E}\end{array}$ & $\begin{array}{l}02 \\
02 \\
02 \\
02 \\
01 \\
01 \\
01 \\
01\end{array}$ & $\begin{array}{c}- \\
- \\
- \\
- \\
- \\
3.20 \mathrm{~F} \\
2.48 \mathrm{~F} \\
1.65 \mathrm{~F} \\
7.47 \mathrm{~F} \\
4.07 \mathrm{~F} \\
1.64 \mathrm{E} \\
8.30 \mathrm{~F}\end{array}$ \\
\hline
\end{tabular}

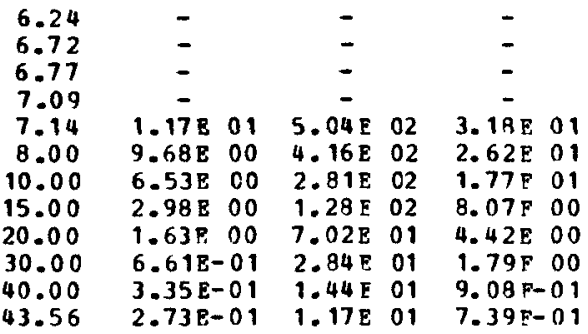

\begin{tabular}{|c|c|c|c|}
\hline & & & \\
\hline - & & - & \\
\hline - & & - & \\
\hline $.44 \mathrm{E}$ & 03 & $6.18 \mathrm{E}$ & 02 \\
\hline $12 \mathrm{E}$ & 03 & $5.59 \mathrm{~F}$ & 02 \\
\hline $.38 \mathrm{~F}$ & 03 & $6.06 \Gamma$ & 02 \\
\hline $31 \mathrm{~F}$ & 03 & $4.15 \mathrm{E}$ & 02 \\
\hline $.29 E$ & 03 & $2.31 \mathrm{E}$ & 02 \\
\hline $.2 \mathrm{BF}$ & 02 & $7.68^{\circ}$ & 01 \\
\hline .925 & 02 & $3.44 \mathrm{E}$ & 01 \\
\hline $.01 \mathrm{E}$ & 01 & $1.08 \mathrm{E}$ & 01 \\
\hline $60 \mathrm{~F}$ & 01 & $4.66 \mathrm{~F}$ & 00 \\
\hline 260 & & $4.05 \mathrm{E}$ & 00 \\
\hline
\end{tabular}

ND $Z=60$

\begin{tabular}{cccc}
- & \multicolumn{1}{c}{-} \\
$3.48 \mathrm{~F}$ & 03 & $6.28 \mathrm{P}$ & 02 \\
$3.15 \mathrm{E}$ & 03 & $5.70 \mathrm{~F}$ & 02 \\
$3.42 \mathrm{~F}$ & 03 & $6.17 \mathrm{E}$ & 02 \\
$2.60 \mathrm{E}$ & 03 & $4.69 \mathrm{E}$ & 02 \\
$1.45 \mathrm{~F}$ & 03 & $2.63 \mathrm{E}$ & 02 \\
$4.97 \mathrm{E}$ & 02 & $8.79 \mathrm{E}$ & 01 \\
$2.19 \mathrm{E}$ & 02 & $3.95 \mathrm{E}$ & 01 \\
$6.88 \mathrm{E}$ & 01 & $1.24 \mathrm{E}$ & 01 \\
$2.98 \mathrm{~F}$ & 01 & $5.39 \mathrm{E}$ & 00 \\
$2.33 \mathrm{E}$ & 01 & $4.20 \mathrm{E}$ & 00
\end{tabular}

P1 $2=61$

\begin{tabular}{|c|c|c|c|c|c|c|}
\hline $6.6 \mathrm{CF}$ & 03 & $1.27 \%$ & 03 & 2. 3 म & $0 ?$ & 5.75 \\
\hline $6 . \mathrm{C3F}$ & 03 & $1.16 \mathrm{E}$ & 03 & $2.63 \mathrm{E}$ & 12 & 6.00 \\
\hline $5.59 E$ & 03 & 1. & 03 & $2.44 \mathrm{E}$ & 07 & $6.1 \mathrm{~h}$ \\
\hline $94 \mathrm{E}$ & 03 & 1. 15E & 03 & $2.59 \mathrm{~F}$ & 02 & 6.21 \\
\hline $5.27 \mathrm{~F}$ & 03 & $1.02 \mathrm{E}$ & 03 & $2.30 \mathrm{~F}$ & 02 & 6.51 \\
\hline $5.66 \mathrm{~F}$ & 03 & 1.095 & 03 & $2.47 \mathrm{r}$ & 02 & $6.5 \mathrm{n}$ \\
\hline $37 \mathrm{E}$ & 03 & 6.508 & 02 & 1.475 & 02 & 0.00 \\
\hline $1.82 \mathrm{E}$ & 03 & 3.518 & 02 & $93 \mathrm{~F}$ & 01 & 10.00 \\
\hline $5.81 \mathrm{~F}$ & 02 & $1.12 \mathrm{E}$ & 02 & 7.545 & 01 & \\
\hline $3 \mathrm{E}$ & 02 & 4. १९F & 01 & $1.10=$ & 01 & 20.01 \\
\hline $6 E$ & 01 & $.48 \mathrm{~F}$ & 01 & $3.34 \mathrm{~F}$ & 00 & 30.00 \\
\hline $25 \mathrm{E}$ & 01 & $6.26 \mathrm{~F}$ & 00 & 1.425 & $0 n$ & 40 \\
\hline $5 F$ & 01 & $6.06 \mathrm{E}$ & 00 & $1.37 \pi$ & 00 & 40. \\
\hline
\end{tabular}

$6.67 \mathrm{E} 03 \quad 1.30 \mathrm{E} 03 \quad 2.94 \Gamma \quad 0 ?$ $\begin{array}{lllllll}6.67 \mathrm{~F} & 03 & 1.30 \mathrm{E} & 03 & 2.94 \mathrm{E} & \mathrm{O} 2\end{array}$

$\begin{array}{llllll}5.617 & 03 & 1.092 & 03 & 2.47 \mathrm{E} & 02\end{array}$

$\begin{array}{llllll}5.96 E & 03 & 1.16 F & 03 & 2.63 \mathrm{~F} & 02\end{array}$

$5.30 \mathrm{~F} 03 \quad 1.03 \mathrm{~L}$ 03 $2.33 \mathrm{~F} 02$

$5 . \mathrm{G} 9 \mathrm{E} 03 \quad 1.11 \mathrm{~F} 03 \quad 2.51 \mathrm{~F} 02$

$\begin{array}{llllll}3.81 \mathrm{~F} & 03 & 7.43 \mathrm{~F} & 02 & 1.69 \mathrm{P} & 02\end{array}$

$2.06 \mathrm{~F} 03 \quad 4.02 \mathrm{E} 02 \quad 9.07 \mathrm{~F} 01$

$6.61 \mathrm{~F} 02 \quad 1.29502 \quad 2.91501$

$\begin{array}{llllll}2.88 \mathrm{~F} & 02 & 5.62 \mathrm{~F} & 01 & 1.270 & 01\end{array}$

$8.75 \mathrm{~F} 01 \quad 1.71$, त1 3.95F 00

$3.71 \mathrm{E} 01 \quad 7.24 \mathrm{E}$ on $1.64 \mathrm{5} 00$

$3.22 \mathrm{~F} 01 \quad 6.27 \mathrm{E} 00 \quad 1.42 \% 00$

6.00

6.00

0.44

6.49

6.79

6.85

9.00

10.00

15.01

20.00

30.07

40.00

41.77

$0.77 \mathrm{~F} 03 \quad 1.33503 \quad 3.00=02$

$5.64 \mathrm{~F} 03 \quad 1.13 \mathrm{~F} 03 \quad 2.50 \mathrm{~F} 03$

$5.99 \mathrm{E} 03 \quad 1.17 \mathrm{E} 03$ 2.6FF 0 ?

$5.34 \mathrm{E} 03 \quad 1.05 \mathrm{~F} 03 \quad 2.37 \mathrm{E} 0$ ?

$\begin{array}{lllllll}5.75 F & 03 & 1.13 & 03 & 0.55 F & 02\end{array}$

$4.31 E_{03} 8.44802 \quad 1.9110 ?$

$2.34 \mathrm{~F} 03 \quad 4.59 \% 02 \quad 1.04 \mathrm{~F} 0$ ?

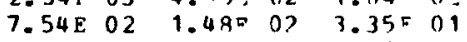

$3.30 \mathrm{~F} 02$ 6.478 $71 \quad 1.47 \mathrm{E} 01$

$1.01 \mathrm{~F} 02 \quad 1.075014 .47 \mathrm{~F} 00$

$4.29 \mathrm{E} 01 \quad 8.40 \mathrm{E}$ Oก $1.90 \mathrm{~T}$ OO

$3.33 \mathrm{E} 01$ 6.51E 09 1.49 0 ก

6.24

5.7 ?

5.77

7.09

4.00

10.00

15.00

30.00

30.00

40.00

$43.5 n$ 
Table 8 (continued)

\begin{tabular}{|c|c|c|c|c|c|c|c|c|c|c|c|c|c|c|c|}
\hline $\begin{array}{c}\begin{array}{l}\text { Photon } \\
\text { energy } \\
(\mathrm{keV})\end{array} \\
7.39 \\
7.45 \\
8.00\end{array}$ & $L_{1} L_{2,3}$ & \multicolumn{2}{|l|}{$\begin{array}{c}L_{1} M_{3} \\
L \beta_{3}\end{array}$} & $\begin{array}{c}L_{1} O_{2,3} \\
L \gamma_{4}\end{array}$ & \multicolumn{2}{|c|}{$\begin{array}{c}L_{2} M_{4} \\
L \beta_{1}\end{array}$} & \multicolumn{2}{|c|}{$\begin{array}{c}L_{2} N_{4} \\
L \gamma_{1}\end{array}$} & \multicolumn{2}{|c|}{$\begin{array}{c}L_{3} M_{5} \\
L \alpha_{1}\end{array}$} & \multicolumn{2}{|c|}{$\begin{array}{l}L_{3} N_{4,5} \\
L \beta_{2,15}\end{array}$} & \multicolumn{2}{|l|}{$\begin{array}{c}L_{3} M_{1} \\
L l\end{array}$} & $\begin{array}{c}\text { Photon } \\
\text { energy } \\
\text { (keV) }\end{array}$ \\
\hline $\begin{array}{r}7.39 \\
7.45 \\
8.00 \\
10.00 \\
15.00 \\
20.00 \\
30.00 \\
40.00 \\
45.18\end{array}$ & $\begin{array}{cc}1.20 \mathrm{E} & 01 \\
1.06 \mathrm{E} & 01 \\
7.23 \mathrm{E} & 00 \\
3.31 \mathrm{E} & 00 \\
1.82 \mathrm{E} & 00 \\
7.42 \mathrm{E}-01 \\
3.78 \mathrm{E}-01 \\
2.82 \mathrm{E}-01\end{array}$ & $\begin{array}{l}- \\
4.94 \mathrm{E} \\
4.36 \mathrm{E} \\
2.98 \mathrm{~F} \\
1.36 \mathrm{~F} \\
7.49 \mathrm{E} \\
3 . \mathrm{C} \mathrm{E} \\
1.35 \mathrm{E} \\
1.16 \mathrm{~F}\end{array}$ & $\begin{array}{l}02 \\
02 \\
02 \\
02 \\
01 \\
01 \\
01 \\
01\end{array}$ & $\begin{array}{cc}- \\
3.08 \mathrm{~F} & 01 \\
2.71 \mathrm{~F} & 01 \\
1.85 \mathrm{~F} & 01 \\
8.49 \mathrm{~F} & 00 \\
4.67 \mathrm{~F} & 00 \\
1.90 \mathrm{~F} & 00 \\
9.69 \mathrm{~F}-01 \\
7.22 \mathrm{~F}-01\end{array}$ & $\begin{array}{l}3.21 \mathrm{~F} \\
3.47 \mathrm{E} \\
2.91 \mathrm{~F} \\
1.65 \mathrm{E} \\
5.53 \mathrm{E} \\
2.57 \mathrm{E} \\
7.89 \mathrm{~F} \\
3.43 \mathrm{~F} \\
2.40 \mathrm{~F}\end{array}$ & $\begin{array}{l}03 \\
03 \\
03 \\
03 \\
02 \\
02 \\
01 \\
01 \\
01\end{array}$ & $\begin{array}{l}5.83 \mathrm{E} \\
6.31 \mathrm{~F} \\
5.29 \mathrm{E} \\
3.01 \mathrm{~F} \\
1.01 \mathrm{~F} \\
4.34 \mathrm{~F} \\
1.43 \mathrm{E} \\
6.24 \mathrm{~F} \\
4.37 \mathrm{~F}\end{array}$ & $\begin{array}{l}02 \\
02 \\
02 \\
02 \\
02 \\
01 \\
01 \\
00 \\
00\end{array}$ & $\begin{array}{l}5.36 \mathrm{~F} \\
5.73 \mathrm{~F} \\
4.82 \mathrm{E} \\
2.64 \mathrm{E} \\
3.51 \mathrm{E} \\
3.74 \mathrm{E} \\
1.14 \mathrm{E} \\
4.87 \mathrm{E} \\
3.39 \mathrm{E}\end{array}$ & $\begin{array}{l}03 \\
03 \\
03 \\
03 \\
02 \\
02 \\
02 \\
01 \\
01\end{array}$ & $\begin{array}{l}1.05 \% \\
1.14 \mathrm{~F} \\
9.47 \mathrm{E} \\
5.195 \\
1.675 \\
7.345 \\
2.25 \mathrm{~F} \\
9.57 \mathrm{5} \\
6.66 \mathrm{E}\end{array}$ & $\begin{array}{l}03 \\
03 \\
32 \\
02 \\
02 \\
01 \\
01 \\
00 \\
00\end{array}$ & $\begin{array}{l}2.40 \mathrm{I} \\
2.59 \mathrm{E} \\
2.16 \mathrm{E} \\
1.18 \mathrm{5} \\
3.81 \mathrm{~F} \\
1.67 \mathrm{E} \\
5.11 \mathrm{~F} \\
2.18 \mathrm{~F} \\
1.52 \mathrm{~F}\end{array}$ & $\begin{array}{l}0 ? \\
0 ? \\
02 \\
02 \\
01 \\
01 \\
00 \\
00 \\
00\end{array}$ & $\begin{array}{r}7.31 \\
7.45 \\
9.00 \\
10.00 \\
15.00 \\
20.00 \\
30.00 \\
40.00 \\
45.18\end{array}$ \\
\hline & & & & & SM & $\mathrm{Z}$ & $=62$ & & & & & & & & \\
\hline $\begin{array}{r}6.75 \\
7.31 \\
7.37 \\
7.70 \\
7.76 \\
8.00 \\
10.00 \\
15.00 \\
20.00 \\
30.00 \\
40.00 \\
46.84\end{array}$ & $\begin{array}{cc}- & \\
- & \\
- & \\
1.28 \mathrm{E} & 01 \\
1.23 \mathrm{E} & 01 \\
8.33 \mathrm{E} & 00 \\
3.82 \mathrm{E} & 00 \\
2.12 \mathrm{E} & 00 \\
8.66 \mathrm{E}-01 \\
4.43 \mathrm{E}-01 \\
3.03 \mathrm{E}-01\end{array}$ & $\begin{array}{c}- \\
- \\
- \\
- \\
5.05 \mathrm{E} \\
4.84 \mathrm{E} \\
3.29 \mathrm{E} \\
1.51 \mathrm{E} \\
8.35 \mathrm{E} \\
3.42 \mathrm{E} \\
1.75 \mathrm{E} \\
1.20 \mathrm{E}\end{array}$ & $\begin{array}{l}02 \\
02 \\
02 \\
02 \\
01 \\
01 \\
01 \\
01\end{array}$ & $\begin{array}{cc}- & \\
- & \\
- & \\
3.12 \mathrm{~F} & 01 \\
2.99 \mathrm{E} & 01 \\
2.03 \mathrm{E} & 01 \\
9.32 \mathrm{~F} & 00 \\
5.16 \mathrm{~F} & 00 \\
2.11 \mathrm{~F} & 00 \\
1.08 \mathrm{~F} & 00 \\
7.39 \mathrm{~F}-01\end{array}$ & $\begin{array}{c}- \\
- \\
3.58 \mathrm{~F} \\
3.26 \mathrm{E} \\
3.53 \mathrm{E} \\
3.26 \mathrm{E} \\
1.87 \mathrm{~F} \\
6.31 \mathrm{E} \\
2.85 \mathrm{E} \\
9.03 \mathrm{~F} \\
3.94 \mathrm{~F} \\
2.49 \mathrm{E}\end{array}$ & $\begin{array}{l}03 \\
03 \\
03 \\
03 \\
03 \\
02 \\
02 \\
01 \\
01 \\
01\end{array}$ & $\begin{array}{c}- \\
- \\
6.55 \% \\
5.97 \% \\
6.46 \mathrm{~F} \\
5.97 \% \\
3.43 \mathrm{~F} \\
1.15 \mathrm{~F} \\
5.21 \mathrm{~F} \\
1.55 \mathrm{~F} \\
7.20 \mathrm{~F} \\
4.55 \%\end{array}$ & $\begin{array}{l}02 \\
02 \\
02 \\
02 \\
02 \\
02 \\
01 \\
01 \\
00 \\
00\end{array}$ & $\begin{array}{l}6.86 \mathrm{E} \\
5.62 \mathrm{E} \\
5.98 \mathrm{~F} \\
5.35 \mathrm{~F} \\
5.77 \mathrm{~F} \\
5.35 \mathrm{E} \\
2.94 \mathrm{E} \\
9.52 \mathrm{E} \\
4.19 \mathrm{~F} \\
1.29 \mathrm{~F} \\
5.49 \mathrm{E} \\
3.43 \mathrm{~F}\end{array}$ & $\begin{array}{l}03 \\
03 \\
03 \\
03 \\
03 \\
03 \\
03 \\
02 \\
02 \\
02 \\
01 \\
01\end{array}$ & $\begin{array}{l}1.365 \\
1.115 \\
1.185 \\
1.065 \\
1.145 \\
1.055 \\
5.915 \\
1.885 \\
8.295 \\
2.545 \\
1.985 \\
6.785\end{array}$ & $\begin{array}{l}03 \\
03 \\
03 \\
03 \\
03 \\
23 \\
02 \\
02 \\
71 \\
01 \\
01 \\
00\end{array}$ & $\begin{array}{l}3.105 \\
2.54 \mathrm{~F} \\
2.70 \mathrm{E} \\
2.425 \\
2.61 \mathrm{~F} \\
2.425 \\
1.335 \\
4.30 \mathrm{~F} \\
1.89 \mathrm{E} \\
5.81 \mathrm{~F} \\
2.48 \mathrm{E} \\
1.55 \mathrm{5}\end{array}$ & $\begin{array}{l}02 \\
07 \\
02 \\
02 \\
02 \\
02 \\
02 \\
01 \\
01 \\
00 \\
00 \\
01\end{array}$ & $\begin{array}{l}5.75 \\
7.31 \\
7.37 \\
7.70 \\
7.76 \\
4.00 \\
10.00 \\
15.00 \\
20.00 \\
30.00 \\
40.00 \\
46.94\end{array}$ \\
\hline
\end{tabular}

$$
\text { EI } z=63
$$

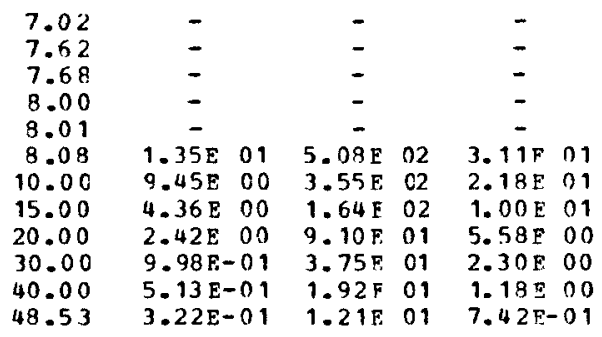

$\begin{array}{cccc}- & & - \\ 3.64 \mathrm{E} & 03 & 0.67 \mathrm{E} & 02 \\ 3.37 \mathrm{~F} & 03 & 6.11 \mathrm{~F} & 02 \\ 3.32 \mathrm{E} & 03 & 6.09 \mathrm{~F} & 02 \\ 3.59 \mathrm{~F} & 03 & 6.59 \mathrm{~F} & 02 \\ 2.12 \mathrm{~F} & 03 & 3.99 \mathrm{~F} & 02 \\ 7.18 \mathrm{E} & 02 & 1.32 \mathrm{~F} & 02 \\ 3.25 \mathrm{~F} & 02 & 5.97 \mathrm{~F} & 01 \\ 1.03 \mathrm{E} & 02 & 1.90 \mathrm{~F} & 01 \\ 4.52 \mathrm{E} & 01 & 9.29 \mathrm{~F} & 00 \\ 2.5 \mathrm{BE} & 01 & 4.73 \mathrm{~F} & 00\end{array}$

GD $z=64$

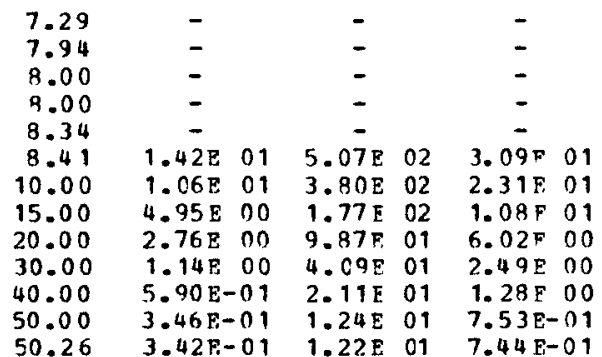

$$
-
$$

$\begin{array}{llll}7.67 E & 0.3 & 6.84 E & 02\end{array}$ $3.67 \mathrm{E} 03 \quad 6.34 \%, 02$ $3.35 \mathrm{E} 03 \quad 5.25 \mathrm{~F} 02$ $\begin{array}{llll}3.63 \mathrm{~F} & 0.3 & 6.77 \mathrm{~F} & 02\end{array}$ $\begin{array}{lllll}2.37 \mathrm{~F} & 03 & 4.43 \mathrm{E} & 02\end{array}$ $8.09 \mathrm{~F} \quad 02 \quad 1.51 \mathrm{E} 02$ 3.69E $02 \quad 6.96 \mathrm{E} 01$ 1. $17 \mathrm{E} 02 \quad 2.19 \mathrm{E} 0$ $5.14 \mathrm{~F} 019.59 \mathrm{~F} 00$ 2.69F $015.02 \% 00$ $2.65 \mathrm{E} 014.95 \mathrm{~F} 00$

$T B \quad Z=65$

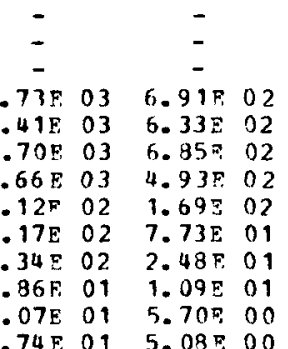

$6.96503 \quad 1.38 \mathrm{E} 03 \quad 3.1750 ?$

5.65E $03 \quad 1.12 \% 03 \quad 2.59702$

$6.01 \mathrm{E} 03 \quad 1.19 \mathrm{P}, 13 \quad 2.74 \mathrm{P} 02$

$\begin{array}{llllll}5.41 \mathrm{~F} & 03 & 1.075 & 03 & 2.47 \mathrm{E} & 02\end{array}$

$\begin{array}{llllll}5.39 \mathrm{~L} & 03 & 1.07 \mathrm{E} & 03 & 2.46 \mathrm{~F} & 02\end{array}$

5.82F 03 1.15\% 03 2.65\% 02

$\begin{array}{lllllll}3.32 \mathrm{~F} & 03 \quad 6.57 \mathrm{E} & 02 & 1.51 \mathrm{~F} & 02\end{array}$

$\begin{array}{llll}1.07 \mathrm{~F} & 03 & 2.13 \mathrm{~F} & 0\end{array}$

$4.74 \mathrm{~F} 02$ 9.385 01 2.15 त1

$1.46 \mathrm{E} 02$ 2.98E 01 6.64\% 00

$6.23 \mathrm{E} 01 \quad 1.23 \mathrm{E} 01 \quad 2.94 \mathrm{E} 00$

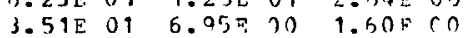

7.02
7.62
7.62
8.00
9.01
7.09
10.00
15.00
20.00
30.00
40.00
48.51

$\begin{array}{llllll}7.005 & 03 & 1.395 & 03 & 3.23 \% & 07\end{array}$

$5.63 \mathrm{E} 03 \quad 1.12503 \quad 2.50 \mathrm{~F} 02$

$5.99 \mathrm{E} \quad 03 \quad 1.19 \pi 03 \quad 2.7 \mathrm{~h}, 02$

$\begin{array}{lllllll}5.98 E & 03 & 1.19 \mathrm{E} & 03 & 2.76=0 ?\end{array}$

5.38E $03 \quad 1.07 \% 03$ 2.49F 0?

$5.80 E 03 \quad 1.16503 \quad 2.68502$

$\begin{array}{llllll}3.7 C E & 03 & 7.36 \mathrm{~F} & 02 & 1.71 \mathrm{~F} & 0 ?\end{array}$

$\begin{array}{llllll}1.20 \mathrm{E} & 03 & 2.39 \mathrm{E} & 0 ? & 5.54 \mathrm{~F} & 01\end{array}$

$5.30 \mathrm{P} 02 \quad 1.76 \mathrm{3} 02$ 2.45 11

$\begin{array}{llll}1.64 \mathrm{E} & 02 & 3.26 \% 01 & 7.55 \mathrm{E} 00\end{array}$

7.01E 01 1.40F 013.23500

$3.61 \mathrm{~F} 01 \quad 7.20 \mathrm{~F} 0 \mathrm{O} 1.67 \mathrm{~F} 0 \mathrm{n}$

$3.56 \mathrm{E} 01$ 7.093 00 1.64: 00

7.27

7.34

9.00

8.09

ค. 34

B. 41

10.00

15.00

20.07

$30.01)$

40.00

52.30

50.25 $\begin{array}{lllllll}7.12 \mathrm{E} & 03 & 1.42 \mathrm{E} & 03 & 3.335 & 02 & 7.56\end{array}$ $\begin{array}{lllllll}0.20 \mathrm{E} & 03 & 1.24 \mathrm{~F} & 03 & 2.89 \mathrm{E} & 0 ? & 9.00\end{array}$ $5.68 F, 0.3 \quad 1.13503 \quad 2.65 \mathrm{E} \cap 2 \quad 9.25$

5. $03 \mathrm{~F} 03$ 1.205 ก3 2.82 02 3.32

$\begin{array}{lllllll}5.43 \mathrm{E} & 03 & 1.03 \mathrm{E} & 0.3 & 7.54 \mathrm{E} & 0 ? & 8.67\end{array}$

$\begin{array}{lllllllll}5.87 \mathrm{~F} & 03 & 1.17 \mathrm{E} & 03 & 2.74 \mathrm{~F} & \mathrm{C} 2 & 3.73\end{array}$

$\begin{array}{llllllll}4.13 F & 03 & 8.24 \% 02 & 1.93 \% & 02 & 10.00\end{array}$

$\begin{array}{lllllll}1.35 \mathrm{E} & 03 & 2.69 \mathrm{E} & 02 & 6.31 \mathrm{~F} & 1 & 15.0 \mathrm{~J}\end{array}$

$\begin{array}{lllllll}5.97 \mathrm{E} & 02 & 1.19 \mathrm{~F} & 02 & 2.73 \mathrm{E} & 01 & 20.00\end{array}$

$\begin{array}{llllll}1.85 \mathrm{~F} & 02 & 3.69 \mathrm{~F} & 01 & 8.63500 & 30.00\end{array}$

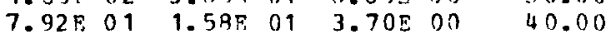

$\begin{array}{llllll}4.09 \mathrm{E} 01 & 8.15 \mathrm{E} \text { OO } & 1.91 \mathrm{E} & 00 & 50.00\end{array}$ 
Table 8 (continued)

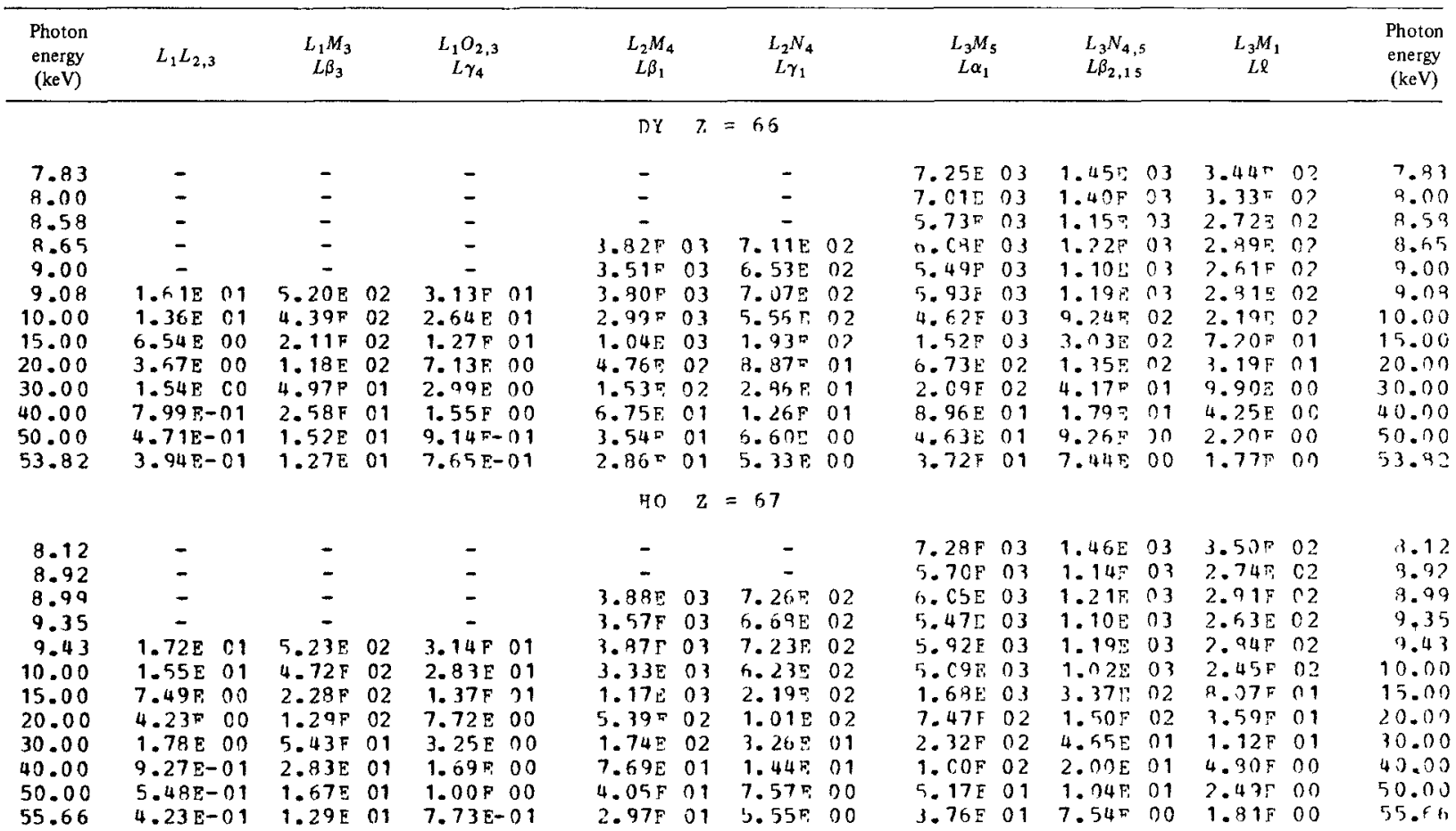

$\begin{array}{rcccccc}8.41 & - & - & - & \\ 9.27 & - & - & - & - & \\ 9.34 & - & - & - & & - & \\ 9.71 & - & & - & & & \\ 9.79 & 1.83 \mathrm{E} & 01 & 5.30 \mathrm{E} & 02 & 3.15 \mathrm{~F} & 01 \\ 10.00 & 1.77 \mathrm{E} & 01 & 5.15 \mathrm{~F} & 02 & 3.06 \mathrm{~F} & 01 \\ 15.00 & 8.54 \mathrm{E} & 00 & 2.49 \mathrm{E} & 02 & 1.47 \mathrm{E} & 01 \\ 20.00 & 4.85 \mathrm{E} & 00 & 1.41 \mathrm{~F} & 02 & 8.36 \mathrm{~F} & 00 \\ 30.00 & 2.05 \mathrm{E} & 00 & 5.96 \mathrm{E} & 01 & 3.54 \mathrm{E} & 00 \\ 40.00 & 1.07 \mathrm{~F} & 00 & 3.12 \mathrm{~F} & 01 & 1.85 \mathrm{~F} & 00 \\ 50.00 & 6.36 \mathrm{E}-01 & 1.85 \mathrm{E} & 01 & 1.10 \mathrm{E} & 00 \\ 57.53 & 4.54 \mathrm{E}-01 & 1.32 \mathrm{E} & 01 & 7.83 \mathrm{~F}-01\end{array}$

57.53

8.70

9.7
9.62
9.7

10.00

10.07

10.15

15.00

20.00

30.00

40.00

50.00

59.44
$4.54 \mathrm{E}-01$

$$
\mathrm{ER} \quad Z=68
$$

$\begin{array}{cccc}- & & - & - \\ 3.34 \mathrm{E} & 0.3 & 7.39 \mathrm{~F} & 02 \\ 3.63 \mathrm{~F} & 03 & 6.31 \mathrm{E} & 02 \\ 3.93 \mathrm{~F} & 03 & 7.37 \mathrm{E} & 02 \\ 3.71 \mathrm{~F} & 03 & 6.96 \mathrm{~F} & 0 ? \\ 1.32 \mathrm{E} & 03 & 2.49 \mathrm{~F} & 02 \\ 6.03 \mathrm{E} & 02 & 1.14 \mathrm{~F} & 02 \\ 1.98 \mathrm{~F} & 02 & 3.70 \mathrm{E} & 01 \\ 3.73 \mathrm{~F} & 01 & 1.64 \mathrm{E} & 01 \\ 4.60 \mathrm{E} & 01 & 8.63 \mathrm{~F} & 00 \\ 3.07 \mathrm{~F} & 01 & 5.75 \mathrm{E} & 00\end{array}$

$$
\mathrm{TM} \quad 7_{1}=69
$$

$\begin{array}{cccc}- & & - \\ - & & \\ .99 F & 03 & 7.50 \mathrm{E} & 02 \\ .76 \mathrm{~F} & 03 & 7.07 \mathrm{E} & 02 \\ .68 \mathrm{~F} & 03 & 6.92 \mathrm{~F} & 02 \\ .98 \mathrm{~F} & 03 & 7.49 \mathrm{E} & 02 \\ .49 \mathrm{~F} & 03 & 2.78 \mathrm{E} & 02 \\ .82 \mathrm{~F} & 02 & 1.29 \mathrm{~F} & 02 \\ .23 \mathrm{E} & 02 & 4.19 \mathrm{~F} & 01 \\ .86 \mathrm{~F} & 01 & 1.96 \mathrm{~F} & 01 \\ .21 \mathrm{~F} & 01 & 9.80 \mathrm{~F} & 00 \\ .16 \mathrm{E} & 01 & 5.95 \mathrm{E} & 00\end{array}$

$$
Y R \quad Z=70
$$

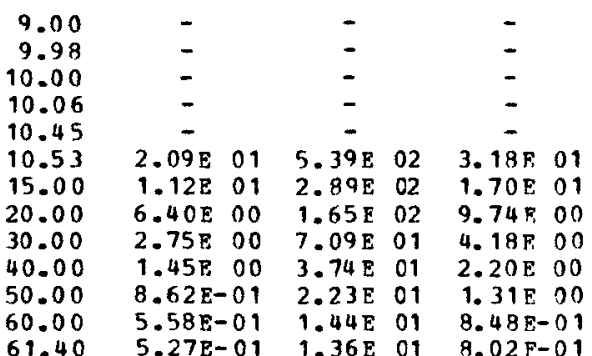

$\begin{array}{lllllll}7.39 \mathrm{E} & 03 & 1.49 \mathrm{~F} & 03 & 3.56 \mathrm{~F} & 02 & 8.41 \\ 5.74 \mathrm{E} & 03 & 1.15 \mathrm{E} & 03 & 2.76 \mathrm{~F} & 02 & 9.27 \\ 6 . \mathrm{C} 9 \mathrm{~F} & 03 & 1.22 \mathrm{E} & 03 & 2.93 \mathrm{~F} & 02 & 9.34 \\ 5.51 \mathrm{~F} & 03 & 1.11 \mathrm{E} & 03 & 2.66 \mathrm{~F} & 02 & 9.71 \\ 5.96 \mathrm{E} & 03 & 1.19 \mathrm{E} & 03 & 2.87 \mathrm{~F} & 02 & 9.79 \\ 5.65 \mathrm{~F} & 03 & 1.13 \mathrm{E} & 03 & 2.72 \mathrm{~F} & 02 & 11.00 \\ 1.87 \mathrm{~F} & 03 & 3.75 \mathrm{~F} & 02 & 0.03 \mathrm{~F} & 01 & 15.00 \\ 3.36 \mathrm{E} & 02 & 1.68 \mathrm{~F} & 02 & 4.03 \mathrm{~F} & 01 & 20.00 \\ 2.61 \mathrm{~F} & 02 & 5.22 \mathrm{E} & 01 & 1.26 \mathrm{~F} & 01 & 30.00 \\ 1.12 \mathrm{~F} & 02 & 2.25 \mathrm{E} & 01 & 5.42 \mathrm{~F} & 00 & 40.00 \\ 5.82 \mathrm{E} & 01 & 1.17 \mathrm{E} & 01 & 2.80 \mathrm{~F} & 00 & 50.00 \\ 3.84 \mathrm{E} & 01 & 7.70 \mathrm{E} & 00 & 1.85 \mathrm{E} & 00 & 57.53\end{array}$

$\begin{array}{llllll}7.43 \mathrm{E} & 03 & 1.49 \mathrm{E} & 03 & 3.63 \mathrm{~F} & 02\end{array}$ $\begin{array}{llllll}5.72 F & 03 & 1.15 E & 03 & 2.79 F & 02\end{array}$

$\begin{array}{lllllll}6.07 F & 03 & 1.228 & 03 & 2.96 \mathrm{~F} & 02\end{array}$

$\begin{array}{llllll}5.61 \mathrm{~F} & 03 & 1.13 \mathrm{~F} & 03 & 2.745 & 03\end{array}$

$\begin{array}{lllllll}5.51 \mathrm{E} & 03 & 1.11 \mathrm{E} & 03 & 3.69 \mathrm{~F} & 02\end{array}$

$5.94 \mathrm{~F} 03$ 1.19F O3 $2.90 \mathrm{~F} 02$

$\begin{array}{llllll}2.06 \mathrm{~F} & 03 & 4.14 \mathrm{E} & 02 & 1.01 \mathrm{~F} & 02\end{array}$

$\begin{array}{llllll}3.22 \mathrm{E} & 02 & 1.95 \mathrm{E} & 02 & 4.50 \mathrm{~F} & 01\end{array}$

$2.87 \mathrm{E} \quad 02 \quad 5.78 \mathrm{~F} \quad 01 \quad 1.40 \mathrm{E} 01$

$\begin{array}{llllll}1.24 \mathrm{~F} & 02 & 2.49 \mathrm{E} & 01 & 6.05 \mathrm{~F} & 00\end{array}$

$6.42 \mathrm{E} 01 \quad 1.29 \mathrm{E} \quad 01 \quad 3.13 \mathrm{~F} 00$

$3.85 \mathrm{E} 01 \quad 7.73 \mathrm{E}$ oO $1.88=00$

8.70

9.62

9.70

10.00

10.07

10.15

15.00
20.00

30.00

40.00

50.00

59.44 $\begin{array}{lllllll}7.48 \mathrm{E} & 03 & 1.51 \mathrm{E} & 03 & 3.70 \mathrm{E} & 02 & 9.00\end{array}$ $\begin{array}{llllll}5.7 C E & 03 & 1.15 E & 03 & 2.92 E & 02\end{array}$ $6.17 \mathrm{E} \quad 03 \quad 1.24 \mathrm{~F}, 03 \quad 3.06 \mathrm{E} \quad 2$ $\begin{array}{llllll}0.04 \mathrm{E} & 03 & 1.22 \mathrm{~F} & 03 & 2.99 \mathrm{E} & 02\end{array}$

$\begin{array}{lllllll}5.49 \mathrm{E} & 03 & 1.11 \mathrm{~F} & 03 & 2.72 \mathrm{E} & 02\end{array}$

$\begin{array}{lllllll}5.93 \mathrm{~F} & 03 & 1.19 \mathrm{E} & 03 & 2.94 \mathrm{E} & 02\end{array}$

$\begin{array}{llllll}2.28 \mathrm{E} & 03 & 4.58 \mathrm{~F} & 02 & 1.13 \mathrm{~F} & 02\end{array}$

$1.02 \mathrm{E} 03 \quad 2.05 \mathrm{~F} 02 \quad 5.05 \mathrm{E} 01$

$\begin{array}{lllllll}3.19 \mathrm{E} & 02 & 6.42 \mathrm{E} & 01 & 1.58 \mathrm{E} & 01\end{array}$

$\begin{array}{llllll}1.38 \mathrm{~F} & 02 & 2.77 \mathrm{E} & 01 & 6.82 \mathrm{~F} & 0 \mathrm{n}\end{array}$

$7.14 \mathrm{E} 01 \quad 1.44 \mathrm{~F} 01 \quad 3.54 \mathrm{~F} 0 \mathrm{O}$

$4.17 \mathrm{~F} 01 \quad 8.39 \mathrm{E} 00$ 2.05E 00

$3.89 \mathrm{E} 01 \mathrm{7.83F}$ 0O $1.93 \mathrm{~F}$,
9.98

10.00

10.06

10.45

10.5 ?

15.00

20.00

40.00

50.00

60.00

61.40 
Table 8 (contınued)

\begin{tabular}{|c|c|c|c|c|c|c|c|c|c|c|c|c|c|c|c|}
\hline $\begin{array}{l}\text { Photon } \\
\text { energy } \\
\text { (keV) }\end{array}$ & $L_{1} L_{23}$ & $\begin{array}{c}L L_{1} M_{3} \\
L \beta_{3}\end{array}$ & & $\begin{array}{c}L_{1} O_{23} \\
L \gamma_{4}\end{array}$ & $\begin{array}{c}L_{2} M_{4} \\
L \beta_{1}\end{array}$ & & $\begin{array}{c}L_{2} N_{4} \\
L \gamma_{1}\end{array}$ & & $\begin{array}{c}L_{3} M_{5} \\
L \alpha_{1} \\
\quad\end{array}$ & & $\begin{array}{l}L_{3} N_{4} \\
L \beta_{2} \\
15\end{array}$ & & $\begin{array}{c}L_{3} M_{1} \\
L \ell\end{array}$ & & $\begin{array}{l}\text { Photon } \\
\text { energy } \\
(\mathrm{keV})\end{array}$ \\
\hline & & & & & LU & $z$ & $=71$ & & & & & & & & \\
\hline 9.31 & - & - & & - & - & & - & & $7.51 \mathrm{~F}$ & 03 & $1.53 \mathrm{E}$ & 03 & $3.79^{\circ}$ & $0 ?$ & 9.71 \\
\hline 10.00 & - & - & & - & - & & - & & $6.25 \mathrm{E}$ & 03 & $1.27 \mathrm{E}$ & $\cap 3$ & 3.155 & n? & 10.07 \\
\hline 10.36 & - & - & & - & - & & - & & $3.67 \mathrm{~F}$ & 03 & $1.15 \mathrm{E}$ & 03 & $2.86 \mathrm{E}$ & 02 & 17.35 \\
\hline 10.45 & - & - & & - & $4.06 \mathrm{~F}$ & 03 & $7.76 \mathrm{~F}$ & $0 ?$ & h. $01 \mathrm{~F}$ & 03 & $1.22 E$ & 03 & $3.04 \pi$ & 02 & 10.45 \\
\hline 10.84 & - & - & & - & $3.76 \mathrm{~F}$ & 03 & 7. $19 \mathrm{E}$ & 02 & $5.47 \mathrm{E}$ & 03 & $1.11 \mathrm{~F}$ & 03 & $2.75 \mathrm{~F}$ & 02 & 10.94 \\
\hline 10.92 & $2.24 \mathrm{E} \quad 01$ & $5.49 \mathrm{~F}$ & 02 & $3.42 F \quad 01$ & $4.07 \mathrm{~F}$ & 03 & 7.780 & 02 & $5.89 \mathrm{E}$ & 03 & $1.20=$ & $n 3$ & $2.99=$ & $n ?$ & 13.92 \\
\hline 15.00 & $1.29 \mathrm{E} \quad 01$ & $3.15 \mathrm{~F}$ & 02 & $1.97 \mathrm{~F} \quad 01$ & $1.84 \mathrm{t}$ & 03 & 3. $د 1 \mathrm{E}$ & 02 & $2.50 \mathrm{E}$ & 03 & $5.09 \mathrm{~F}$ & $0 ?$ & 1. 25 & 02 & 15.00 \\
\hline 20.00 & $7.37 \mathrm{E} \quad 00$ & $1.81 \mathrm{~F}$ & 02 & $1.13 \mathrm{~F} 01$ & $8.53 E$ & 02 & $1.63 \%$ & 02 & $1.12 \mathrm{~F}$ & 03 & $2.28 \mathrm{~F}$ & $0 ?$ & $5.65 \mathrm{~F}$ & 01 & 20.00 \\
\hline 30.00 & $3.18 \mathrm{E} 00$ & $7.80 \mathrm{E}$ & 01 & 4. $86 \mathrm{~F} 02$ & 2.805 & 02 & $5.36 \mathrm{P}$ & 01 & $3.51 \mathrm{~F}$ & 02 & $7.13 F$ & $n 1$ & 1.775 & 01 & 37.70 \\
\hline 40.00 & $1.68 E \quad 00$ & $4.12 \mathrm{E}$ & 01 & $2.57 \mathrm{~F} 00$ & $1.25 \mathrm{E}$ & 02 & $2.39 \mathrm{~F}$ & 01 & $1.52 \mathrm{E}$ & 02 & $3.08 \mathrm{~F}$ & 01 & $7.65 \Gamma$ & or & 43.00 \\
\hline 50.00 & $1.00 \mathrm{E} \quad 00$ & $2.46 \mathrm{E}$ & 01 & $1.54 \mathrm{E} 00$ & $6.62 E$ & 01 & 1. 265 & n & $7.86 \mathrm{~F}$ & 01 & $1.60 \Gamma$ & 01 & 3.975 & 00 & 50.03 \\
\hline 60.00 & $6.51 \mathrm{E}-01$ & $1.60 \mathrm{P}$ & 01 & $9.96 \mathrm{E}-01$ & $3.92 \mathrm{~F}$ & 01 & $7.50 \mathrm{~F}$ & 00 & 4. $5 \mathrm{AL}$ & 01 & $9.31 F$ & 20 & $2.31 \mathrm{~F}$ & no & $6 n .00$ \\
\hline 63.40 & $5.70 E-01$ & $1.40 \mathrm{~F}$ & $\mathrm{Cl}$ & $8.72 \mathrm{~F}-01$ & $3.35 \mathrm{~F}$ & 01 & $6.40 \mathrm{E}$ & 00 & $3.89 F$ & 01 & 7.015 & no & 1.970 & 00 & 63.40 \\
\hline
\end{tabular}

\begin{tabular}{|c|c|c|c|c|}
\hline 9.63 & - & - & & - \\
\hline 10.00 & - & - & & - \\
\hline 10.75 & - & - & & - \\
\hline 10.84 & - & - & & - \\
\hline 11.24 & - & - & & - \\
\hline 11.33 & $2.41 \mathrm{E} \quad 01$ & $5.56 \mathrm{~F}$ & 02 & $3.66 \mathrm{~F}$ \\
\hline 15.00 & $1.48 \mathrm{~F} \quad 01$ & $3.42 F$ & 02 & $2.25 \mathrm{~F}$ \\
\hline 20.00 & $8.52 \mathrm{E} \quad 00$ & $1.96 \mathrm{~F}$ & 02 & 1. $29 \mathrm{~F}$ \\
\hline 30.00 & $3.70 \mathrm{E} \quad 00$ & $8.53 \mathrm{~F}$ & 01 & $62 \mathrm{~F}$ \\
\hline .00 & $1.96 \mathrm{E} \mathrm{CO}$ & $52 F$ & 01 & $2.98 \mathrm{~F}$ \\
\hline & $1.18 \mathrm{E} 00$ & $2.71 \mathrm{E}$ & 01 & $1.79 \mathrm{E}$ \\
\hline & $7.64 \mathrm{E}-01$ & $1.76 \mathrm{E}$ & 01 & $16 \mathrm{~F}$ \\
\hline
\end{tabular}

$$
\text { HE } \quad z=72
$$

\begin{tabular}{|c|c|c|c|}
\hline & & & \\
\hline & & & \\
\hline- & & & \\
\hline $.06 \mathrm{~F}$ & 03 & $7.85 \mathrm{~F}$ & 02 \\
\hline $.77 \mathrm{E}$ & 03 & 7. $28 \mathrm{~F}$ & 02 \\
\hline $.05 \mathrm{~F}$ & 03 & $7.83 \Gamma$ & 02 \\
\hline $.01 \mathrm{~F}$ & 03 & 3. $89 \mathrm{E}$ & 02 \\
\hline $.33 F$ & 02 & 1. $80 \mathrm{~F}$ & 02 \\
\hline $.08 \mathrm{E}$ & 02 & $5.95 \mathrm{E}$ & 01 \\
\hline $.37 \mathrm{~F}$ & 02 & $2.66 \mathrm{~F}$ & 01 \\
\hline $28 \mathrm{~F}$ & 01 & 1.415 & 01 \\
\hline $.31 E$ & 01 & $8.34 \%$ & 00 \\
\hline
\end{tabular}

$3.36 \Gamma 016.47 \mathrm{~F} 00$
9.95

10.00

11.15

11.24

11.65

11.74

15.00

20.00

30.00

40.00

50.00

60.00

67.52
$6.20 \mathrm{E}-01$
$1.43 \mathrm{~F} 01$
$9.42 \mathrm{E}-01$

\begin{tabular}{|c|c|c|c|c|c|c|}
\hline $7.58 \mathrm{E}$ & 03 & $1.55 \mathrm{~F}$ & 03 & $3.85 \mathrm{~F}$ & 07 & 9.63 \\
\hline & 03 & $43 E$ & 03 & $3.53 E$ & $0 ?$ & 10.00 \\
\hline & 03 & 1. 16E & 03 & 2.875 & 02 & .75 \\
\hline :0F & 03 & $.23 \mathrm{~F}$ & 03 & $3.05^{\circ}$ & $0 ?$ & 10.84 \\
\hline $7 \mathrm{E}$ & 03 & $1.12 \mathrm{E}$ & 03 & $2.78 \mathrm{~F}$ & $c^{3}$ & $11.2+$ \\
\hline $8 F$ & 03 & $.21 \mathrm{t}$ & 03 & $2.99 \mathrm{~F}$ & 07 & 11.33 \\
\hline $77 \mathrm{~F}$ & 03 & $5.67 \mathrm{~F}$ & 02 & $1.41 \mathrm{~F}$ & C? & 15.00 \\
\hline $24 \mathrm{E}$ & 0.3 & $2.54 \mathrm{~F}$ & 02 & $0.23 F$ & 01 & 20.00 \\
\hline $89 E$ & 02 & 7.985 & 09 & $1.98 \Gamma$ & 01 & 30.01 \\
\hline $68 \mathrm{E}$ & 02 & $3.45 \Gamma$ & 01 & $0.55 \Gamma$ & $\mathrm{CO}$ & 40.00 \\
\hline $3 \mathrm{E}$ & 01 & $1.79 \mathrm{E}$ & 01 & $4.44 \mathrm{~F}$ & 00 & 50.00 \\
\hline$O E$ & 01 & $1.05 E$ & 01 & $2.59 \mathrm{~F}$ & $n$ & 60.00 \\
\hline $4 \mathrm{~F}$ & 01 & 8.095 & 00 & $2.00 \mathrm{~F}$ & 00 & b 5.44 \\
\hline
\end{tabular}

$\begin{array}{lllllll}7.65 \mathrm{E} & 03 & 1.59 \mathrm{E} & 03 & 3.95 \mathrm{~F} & 02\end{array}$ $\begin{array}{llllll}7.58 \mathrm{E} & 03 & 1.57 \mathrm{E} & 03 & 3.91 \Gamma & 03\end{array}$ $5.65 \mathrm{~F} 03 \quad 1.17 \mathrm{~F}$ 03 $2.91 \mathrm{~F} \mathrm{C} 2$ $\begin{array}{llllll}5.99 \mathrm{~F} & 03 & 1.245 & 03 & 3.09 \mathrm{~F} & 0\end{array}$ $\begin{array}{lllllll}5.46 \mathrm{E} & 03 & 1.131 & 03 & 7.92 \mathrm{~F} & 03\end{array}$ $\begin{array}{llllll}5.88 \mathrm{E} & 03 & 1.22 \mathrm{~F} & 03 & 3.03 \mathrm{E} & 03\end{array}$

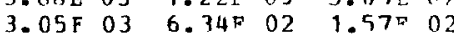
1.37E $03 \quad 2.94 \mathrm{E}$ 0? 7.07E 01 4. $32 \mathrm{E} \quad 02 \quad 8.96 \mathrm{I} \quad 01 \quad 2.23 \mathrm{~F} 01$ $\begin{aligned} & 4.87 \mathrm{~F} 02 \\ & 1.98 \mathrm{~F}\end{aligned} 019.64=00$ $\begin{array}{lllll}1.87 \mathrm{~F} & 0 ? & 3.98 \mathrm{~F} & 01 & 9.64 \mathrm{~F} \\ 9.72 \mathrm{~F} & 01 & 2.02 \mathrm{E} & 01 & 5.01 \mathrm{E}\end{array}$ 5.68E $01 \quad 1.13 \mathrm{E} 01 \quad 2.93 \mathrm{~F} C$ 4. $00 \mathrm{~F} 018.31 \mathrm{\Gamma} 002.06 \mathrm{~F} 00$

a.or 10.70 11.15 11.24 11.1, $11.7+$ 15.00 20.07

30.07 40.00 50.00 8.07 $(7.5)$

$7.71 \mathrm{~F} 03 \quad 1.62 \mathrm{~F} 03 \quad 4.03 \mathrm{~F} 07$ $5.62 \mathrm{E} 03 \quad 1.18 \mathrm{~T} \cap 3 \quad 2.94 \mathrm{~F} 02$ $5.96 \mathrm{~F} 03 \quad 1.25 \mathrm{~F}$ ก $3.11 \mathrm{~F}$ ? $\begin{array}{llllll}5.45 \Gamma & 03 & 1.14 F & 03 & 2.85 \mathrm{~F} & 02\end{array}$ $5.87 \mathrm{~F} 03 \quad 1.23 \mathrm{E} 03 \quad 3.07 \mathrm{~F} 0 ?$ $\begin{array}{llllll}5.87 F & 03 & 1.23 E & 03 & 3.07 F & 0 \\ 3.36 F & 03 & 7.05 \Gamma & 02 & 1.76=\end{array}$ $\begin{array}{llll}1.51=03 & 3.16=02 & 7.90 & 01\end{array}$ $4.76 \mathrm{E} 02 \quad 9.98 \mathrm{~F} 01 \quad 2.49501$ $2.07 \mathrm{~F} 02 \quad 4.33-11 \quad 1.08=01$

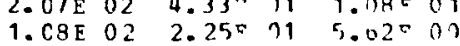
$6.28 \mathrm{E} 01$ 1.32E 01 7.73 $\mathrm{E}$ ก $4.05 \mathrm{~F} 018.49 \mathrm{~F}$ ) $2.11^{-} 01$

10.23 11.56 11.65 12.07 12.17 15.) 22.00 30.00 4). 01 50.00 (0.0) , 9.05

7. POE $03 \quad 1.6503 \quad 4.1303$ $3.62 \Gamma 03 \quad 1.19 \Gamma 03 \quad 39$ ग. 02 $5.95 \mathrm{~F} 03 \quad 1.26 \mathrm{~F} 33 \quad 3.15 \mathrm{~F} 02$ $3.45 \mathrm{E} 31.15 \mathrm{~N} 732.3902$ $3.96^{\circ} 3 \quad 1.36^{\circ} 13 \quad 3.15 E 0$ ? $\begin{array}{lllllll}3.77 & 03 & 7.975 & 02 & 1.995 & 02\end{array}$ $\begin{array}{lllll}1.7 \mathrm{CE} & 03 & 3.60 \mathrm{E} & 02 & 9.01 \mathrm{n}\end{array}$ $\begin{array}{llllll}5.43 \mathrm{E} & 02 & 1.15 \mathrm{~F} & 02 & 2.97 \mathrm{~F} & 01\end{array}$ $2.38 \mathrm{E} 02$ b. $04 \mathrm{E} 01$ 1.26F 01 $1.25 \mathrm{~F} 02 \quad 2.64=016.60 \% 00$

7.33F 01 1.55E 01 3.98F 00

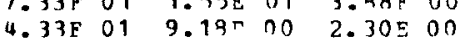

10.51

11.79

12.04

12.50

12.60

15.01

20.00

30.00

40.0 ?

50.00

60.00 
Table 8 (continued)

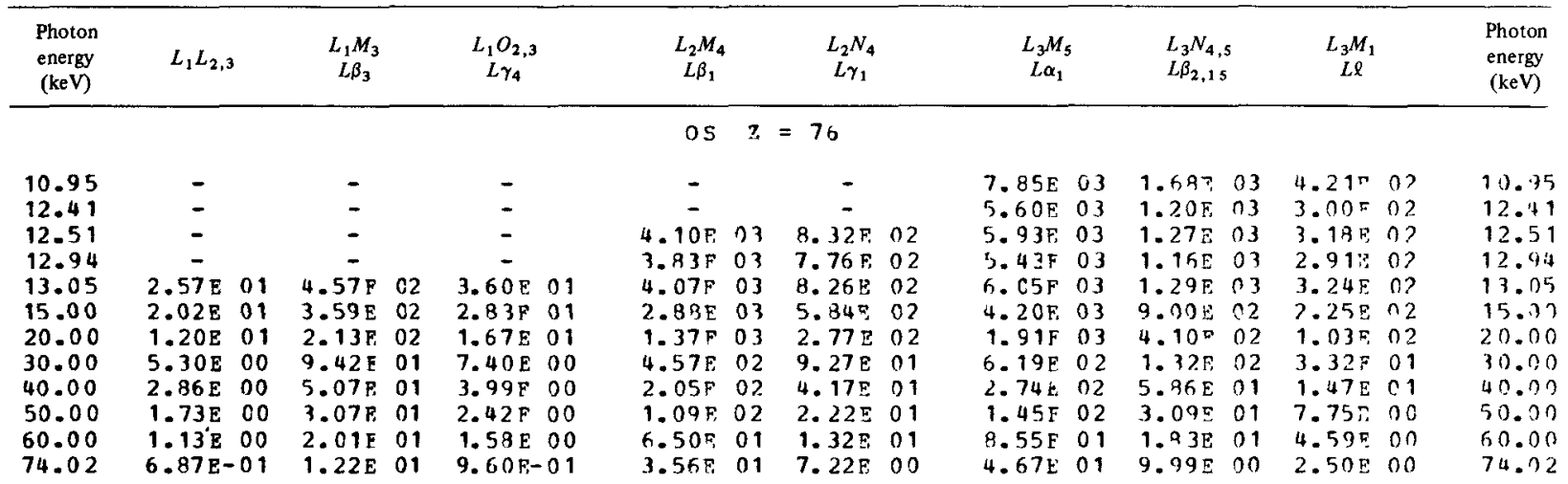

$$
\text { IR } z=77
$$

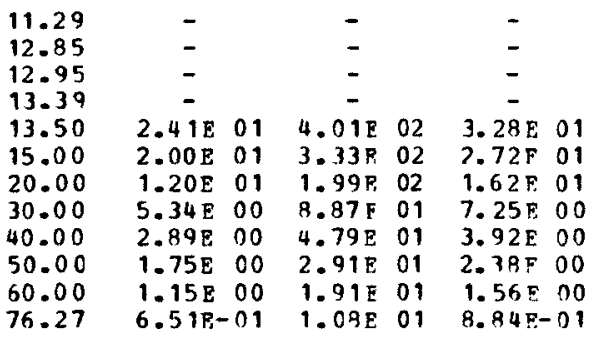

11. 65

13.30

13.41
13.85

13.97

15.00

20.00

30.00

40.00

50.00

60.00

78.58

-

$\begin{array}{lllll}4.11 \mathrm{~F} & 03 & 8.44 \mathrm{E} & 02\end{array}$ $\begin{array}{lllll}3.83 F & 03 & 7.86 \mathrm{E} & 02\end{array}$ $\begin{array}{llll}4.05 \mathrm{~F} & 03 & 8.31 \mathrm{E} & 02\end{array}$ $\begin{array}{lllll}3.105 & 03 & 6.375 & 02\end{array}$ $1.49 \mathrm{~F} 03 \quad 3.05 \mathrm{~F} 02$ $4.99 \mathrm{~F} 02 \quad 1.02 \mathrm{E} 02$ $\begin{array}{llll}2.25 \mathrm{~F} & 02 & 4.61 \mathrm{E} & 01\end{array}$ $1.20 \mathrm{E} 02 \quad 2.45 \mathrm{~F} 01$ $7.12 \mathrm{~F} 01 \quad 1.46 \% 01$ $3.57 \mathrm{E} 01 \quad 7.33 \mathrm{E} 0 \mathrm{O}$

$$
\text { PT } z=78
$$

- $\begin{array}{lllll}4.11 \mathrm{E} & 03 & 8.52 \mathrm{E} & 02\end{array}$ $3.34 \mathrm{~F} 03 \quad 7.95 \mathrm{~F} 02$ $4.05 \mathrm{E} \quad 03 \quad 8.39 \mathrm{~F} 02$ $\begin{array}{lllll}3.35 \mathrm{~F} & 03 & 6.94 \mathrm{~F} & 02\end{array}$ $1.62 \mathrm{E} 03 \quad 3.36 \mathrm{E} \quad 02$ $\begin{array}{llll}5.45 \mathrm{E} & 02 & 1.13 \mathrm{E} & 02\end{array}$ $2.46 \mathrm{~F} \quad 02 \quad 5.09 \mathrm{~F} \quad 01$

$\begin{array}{llll}1.31 \mathrm{E} & 02 & 2.72 \mathrm{E} & 01\end{array}$ 7. $90 \mathrm{E}$ 01 $1.62 \mathrm{P} 01$ $3.60 \mathrm{E} 017.45 \mathrm{E} 00$

$$
\mathrm{AU} \quad \mathrm{Z}=73
$$

12.00 13.76
13.87 14.33 14.44 15.00 20.00 30.00 40.00 50.00 60.00 90.00 80.92
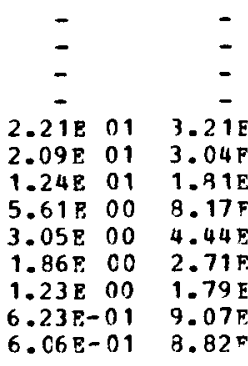

$3.21 \mathrm{E} \quad 02$ 3. $04 \mathrm{~F} 02$ 1. $91 \mathrm{E} 02$ $8.17 \mathrm{~F} 01$ 4. $44 \mathrm{E} 01$ $2.71 \mathrm{~F} .01$ $1.79 \mathrm{E} \quad 01$ $9.07 \mathrm{E} \quad 00$ $8.82=00$

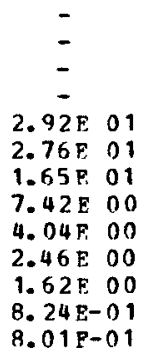

12.37
14.24
14.35
14.82
14.94
15.00
20.00
30.00
49.00
50.00
60.00
80.00
83.32

$\begin{array}{cccc}- & & - & \\ - & & \bar{E} & \\ 4.10 \mathrm{~F} & 03 & 8.56 \mathrm{~F} & 02 \\ 3.84 \mathrm{E} & 03 & 8.02 \mathrm{E} & 02 \\ 4.04 \mathrm{E} & 03 & 8.44 \mathrm{E} & 02 \\ 3.64 \mathrm{~F} & 03 & 7.60 \mathrm{E} & 02 \\ 1.78 \mathrm{~F} & 03 & 3.71 \mathrm{~F} & 02 \\ 5.98 \mathrm{~F} & 02 & 1.25 \mathrm{~F} & 02 \\ 2.71 \mathrm{~F} & 02 & 5.66 \mathrm{~F} & 01 \\ 1.45 \mathrm{E} & 02 & 3.02 \mathrm{E} & 01 \\ 8.63 \mathrm{E} & 01 & 1.80 \mathrm{E} & 01 \\ 3.79 \mathrm{E} & 01 & 7.91 \mathrm{E} & 00 \\ 3.67 \mathrm{~F} & 01 & 7.65 \mathrm{E} & 00\end{array}$

$$
\text { HG } \mathrm{z}=80
$$

$\begin{array}{cccc}- & & - & \\ - & & \overline{1} \\ 4.08 \mathrm{~F} & 03 & 8.59 \mathrm{~F} & 02 \\ 3.83 \mathrm{~F} & 03 & 8.06 \mathrm{~F} & 02 \\ 4.01 \mathrm{E} & 03 & 8.44 \mathrm{E} & 02 \\ 3.96 \mathrm{~F} & 03 & 8.34 \mathrm{E} & 02 \\ 1.92 \mathrm{E} & 03 & 4.05 \mathrm{E} & 02 \\ 6.50 \mathrm{~F} & 02 & 1.37 \mathrm{~F} & 02 \\ 2.95 \mathrm{E} & 02 & 6.20 \mathrm{~F} & 01 \\ 1.59 \mathrm{E} & 02 & 3.32 \mathrm{E} & 01 \\ 9.41 \mathrm{E} & 01 & 1.98 \mathrm{E} & 01 \\ 4.13 \mathrm{E} & 01 & 8.70 \mathrm{E} & 00 \\ 3.68 \mathrm{E} & 01 & 7.74 \mathrm{E} & 00\end{array}$

$\begin{array}{lllllll}7.93 \mathrm{~F} & 03 & 1.72 \mathrm{~F} & 03 & 4.32 \mathrm{~F} & 02 & 11.20 \\ 5.56 \mathrm{E} & 03 & 1.20 \mathrm{E} & 03 & 3.03 \mathrm{~F} & 02 & 12.05 \\ 5.89 \mathrm{E} & 03 & 1.27 \mathrm{~F} & 03 & 3.71 \mathrm{~F} & 02 & 12.95 \\ 5.40 \mathrm{E} & 03 & 1.17 \mathrm{~F} & 03 & 2.94 \mathrm{~F} & 02 & 13.30 \\ 6.12 \mathrm{E} & 03 & 1.32 \mathrm{~F} & 03 & 3.33 \mathrm{~F} & 02 & 13.50 \\ 4.66 \mathrm{~F} & 03 & 1.01 \mathrm{E} & 03 & 2.54 \mathrm{~F} & 02 & 15.00 \\ 2.14 \mathrm{~F} & 03 & 4.63 \mathrm{E} & 02 & 1.17 \mathrm{~F} & 02 & 20.00 \\ 7.01 \mathrm{~F} & 02 & 1.52 \mathrm{E} & 02 & 3.82 \mathrm{E} & 01 & 30.00 \\ 3.13 \mathrm{~F} & 02 & 6.77 \mathrm{E} & 01 & 1.70 \mathrm{E} & 01 & 40.00 \\ 1.66 \mathrm{~F} & 02 & 3.50 \mathrm{~F} & 01 & 9.06 \mathrm{E} & 00 & 50.00 \\ 9.89 \mathrm{~F} & 01 & 2.14 \mathrm{E} & 01 & 5.3 \mathrm{E} & 00 & 60.00 \\ 4.99 \mathrm{E} & 01 & 1.09 \mathrm{E} & 01 & 2.72 \mathrm{E} & 01 & 76.27\end{array}$

$\begin{array}{lllllll}7.93 \mathrm{~F} & 03 & 1.73 \mathrm{E} & 03 & 4.39 \mathrm{~F} & 03\end{array}$

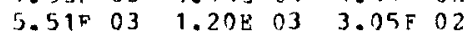
$\begin{array}{lllll}5.83 \mathrm{~F} & 03 \quad 1.27 \mathrm{O} & 03 & 3.23 \mathrm{~F} & 02\end{array}$ $\begin{array}{llllll}5.35 \mathrm{~F} & 03 & 1.17 \mathrm{~F} & 03 & 2.36 \mathrm{~F} & 03\end{array}$ $6.15 \mathrm{~F} 03 \quad 1.34 \mathrm{E}$ ก 3 3.41 02 $\begin{array}{llllll}5.12 \mathrm{E} & 03 & 1.12 \mathrm{E} & 03 & 2.94 \mathrm{E} & 02\end{array}$ $\begin{array}{llllll}2.37 \mathrm{~F} & 03 & 5.17 \mathrm{E} & 02 & 1.31 \mathrm{~F} & 02\end{array}$ $\begin{array}{llll}7.85 E & 02 & 1.71 F \text { O2 } 4.34=01\end{array}$ $3.52 \mathrm{~F} 02 \quad 7.69 \mathrm{E} 01 \quad 1.95 \mathrm{E} 01$ $\begin{array}{llllll}1.28 E & 02 & 4.11 E & 01 & 1.04 F & 01\end{array}$ $\begin{array}{lllll}1.13 F & 02 & 2.46 E & 01 & 6.24500\end{array}$ $5.25 \mathrm{~F}$ OI $1.14 \mathrm{~T} 012.91 \mathrm{~F} 00$

11.65 13.30

13.41 13.85 13.07 15.00 20.00 30.70 10.00

50.00

60.00

$7 \times .59$

$\begin{array}{lllllll}7.97 \mathrm{~F} & 03 & 1.75 \mathrm{~F} & 03 & 4.47 \mathrm{~F} & 02 & 12.07 \\ 5.43 \mathrm{E} & 03 & 1.20 \mathrm{E} & 03 & 3.07 \mathrm{~F} & 02 & 13.75 \\ 5.79 \mathrm{~F} & 03 & 1.27 \mathrm{~F} & 03 & 3.25 \mathrm{E} & 02 & 13.97 \\ 5.33 \mathrm{~F} & 03 & 1.17 \mathrm{~F} & 03 & 2.99 \mathrm{~F} & 02 & 14.33 \\ 5.18 \mathrm{~F} & 03 & 1.36 \mathrm{~F} & 03 & 3.45 \mathrm{~F} & 02 & 14.41 \\ 5.62 \mathrm{~F} & 03 & 1.24 \mathrm{E} & 03 & 3.15 \mathrm{~F} & 02 & 15.00 \\ 2.62 \mathrm{~F} & 03 & 5.75 \mathrm{~F} & 02 & 1.47 \mathrm{~F} & 02 & 20.00 \\ 8.71 \mathrm{E} & 02 & 1.71 \mathrm{E} & 02 & 4.98 \mathrm{E} & 01 & 30.00 \\ 3.93 \mathrm{E} & 02 & 8.63 \mathrm{~F} & 01 & 2.20 \mathrm{E} & 01 & 40.00 \\ 2.11 \mathrm{E} & 02 & 4.63 \mathrm{E} & 01 & 1.19 \mathrm{~F} & 01 & 50.00 \\ 1.26 \mathrm{E} & 02 & 2.78 \mathrm{E} & 01 & 7.09 \mathrm{~F} & 00 & 60.00 \\ 5.62 \mathrm{E} & 01 & 1.24 \mathrm{E} & 01 & 3.15 \mathrm{~F} & 0 \mathrm{C} & 30.00 \\ 5.45 \mathrm{~F} & 01 & 1.20 \mathrm{~F} & 01 & 3.05 \mathrm{E} & 00 & 90.92\end{array}$

$\begin{array}{lllllll}7.97 \mathrm{~F} & 03 & 1.77 \mathrm{E} & 03 & 4.54 \mathrm{~F} & 02\end{array}$ $5.42 \mathrm{~F} 03 \quad 1.20 \mathrm{E} 03 \quad 3.03 \mathrm{E} 0$ ? $\begin{array}{llllll}5.72 \mathrm{E} & 03 & 1.27 \mathrm{~F} & 03 & 3.26 \mathrm{~T} & 0 ?\end{array}$ $\begin{array}{llllll}5.27 E & 03 & 1.17 \mathrm{~F} & 03 & 3.00 \mathrm{Y} & 02\end{array}$ $\begin{array}{llllll}5.17 \mathrm{E} & 03 & 1.37 \mathrm{E} & 03 & 3.52 \mathrm{~F} & 02\end{array}$ $\begin{array}{llllll}6.17 \mathrm{E} & 03 & 1.37 \mathrm{E} & 03 & 3.52 \mathrm{~F} & 02 \\ 6.10 \mathrm{E} & 03 & 1.35 \mathrm{~F} & 03 & 3.4 \mathrm{FF} & 0 ?\end{array}$ 2.87F $03 \quad 6.36 \mathrm{~F} 02$ 1.64F 02 $\begin{array}{llllll}9.60 \mathrm{E} & 02 & 2.13 \mathrm{E} & 02 & 5.47 \mathrm{E} & 01\end{array}$ $\begin{array}{llllll}4.35 \mathrm{~F} & 02 & 9.64 \mathrm{E} & 01 & 2.49 \mathrm{~F} & 01\end{array}$ $\begin{array}{llllll}4.35 F & 02 & 9.64 E & 01 & 2.49 F & 01 \\ 2.34 E & 02 & 5.19 E & 01 & 1.345 & 01\end{array}$ $\begin{array}{llllll}1.41 \mathrm{E} & 02 & 3.12 \mathrm{E} & 01 & 8.03 \mathrm{E} & 0\end{array}$ $6.29 \mathrm{~F} 01 \quad 1.39 \mathrm{~F}, 01 \quad 3.59 \mathrm{~F} 00$ $5.61 \mathrm{~F} 01 \quad 1.24 \% 01 \quad 3.20 \% 00$
12.37

14.24

14.35

14.92

14.94

$15.0 n$

20.00

30.00

40.30

50.00

60.00

33.32 
Table 8 (continued)

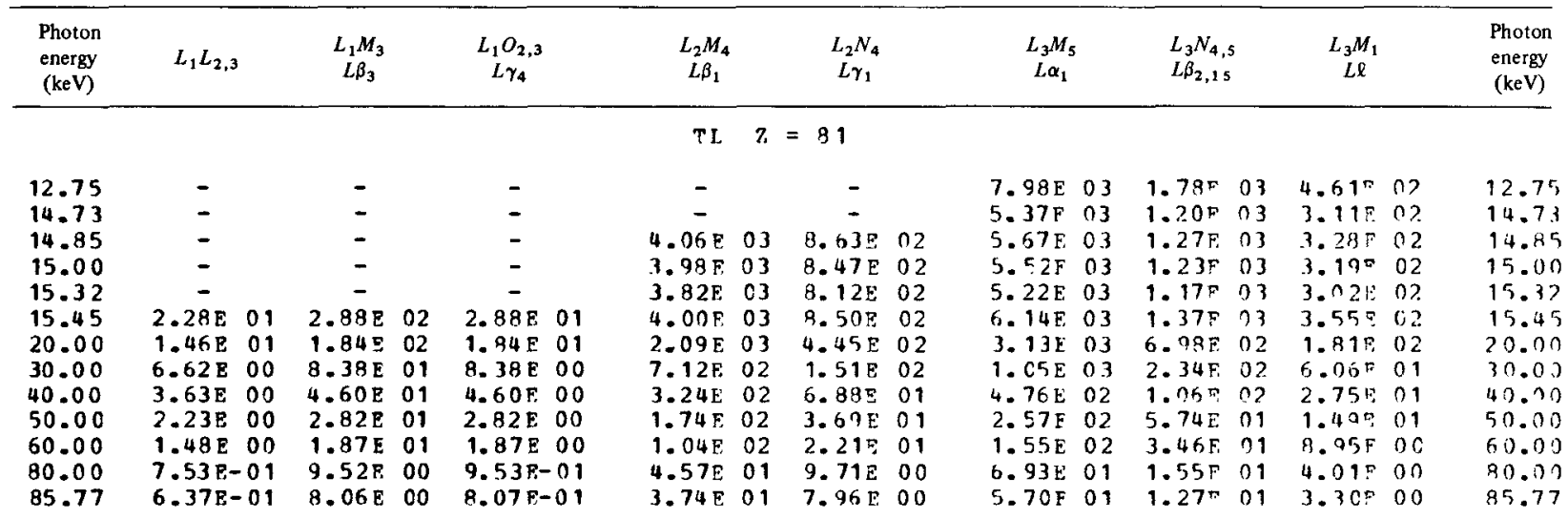

13. 13

15.00

15.24

15.36
15.84

15.97

20.00

30.00

40.00

50.00

60.00

80.00

88.26

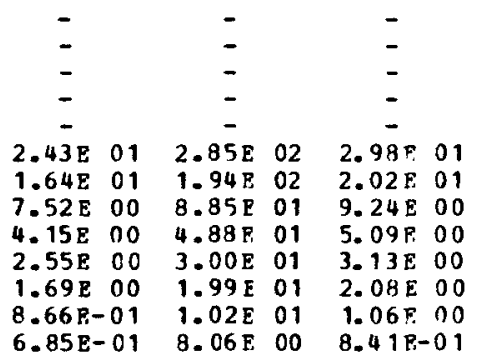

13.52

15.00

15.75

15.88

16.37

16.50

20.00

30.00

40.00

50.00

60.00

80.00

90.81

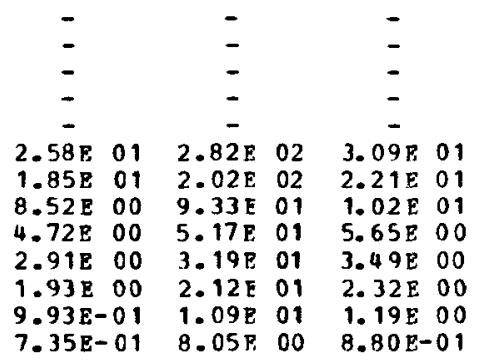

$$
\text { PB } \quad Z=82
$$

$\begin{array}{cccc}- & & - & \\ - & & - & \\ - & & - & \\ 4.03 \mathrm{E} & 03 & 8.66 \mathrm{~F} & 02 \\ 3.80 \mathrm{E} & 03 & 8.15 \mathrm{E} & 02 \\ 3.96 \mathrm{E} & 03 & 8.49 \mathrm{E} & 02 \\ 2.25 \mathrm{E} & 03 & 4.84 \mathrm{E} & 02 \\ 7.70 \mathrm{~F} & 02 & 1.05 \mathrm{E} & 02 \\ 3.51 \mathrm{~F} & 02 & 7.53 \mathrm{~F} & 01 \\ 1.98 \mathrm{~F} & 02 & 4.04 \mathrm{E} & 01 \\ 1.13 \mathrm{E} & 02 & 2.42 \mathrm{E} & 01 \\ 4.96 \mathrm{E} & 01 & 1.07 \mathrm{~F} & 01 \\ 3.74 \mathrm{E} & 01 & 8.04 \mathrm{E} & 00\end{array}$

$$
\text { BI } Z=83
$$
$\begin{array}{llll}1.23 \mathrm{E} & 02 & 2.66 \mathrm{R} & 0\end{array}$ $5.41 \mathrm{P} 01 \quad 1.17 \mathrm{E} 01$ $3.76 \mathrm{E}$ O1 $8.17 \mathrm{E} 00$
$7.97 \mathrm{~F} 03 \quad 1.79 \mathrm{~F} 03 \quad 4.60 \mathrm{P} 02$ $5.52 \mathrm{~F} 03 \quad 1.24 \mathrm{~F} 03$ 3.25F 22 5. 30E $03 \quad 1.19 \mathrm{E} 03 \quad 3.11 \mathrm{E} 02$ $\begin{array}{llllll}5.59 \mathrm{~F} & 03 & 1.268 & 03 & 3.29 \mathrm{~F} & 02\end{array}$ $\begin{array}{lllllll}5.16 E & 03 & 1.16 \mathrm{~F} & 03 & 3.03 \mathrm{E} & 0 ?\end{array}$ 6. C8E 03 1.37E 03 3.57F 02 $\begin{array}{llllll}3.39 \mathrm{E} & 03 & 7.52 \mathrm{E} & 02 & 1.99 \mathrm{E} & 02\end{array}$ $\begin{array}{llllll}1.14 \mathrm{~F} & 03 & 2.565 & 02 & 6.59 \mathrm{~F} & 01\end{array}$ $\begin{array}{lllllll}5.19 \mathrm{E} & 02 & 1.17 \mathrm{~F} & 02 & 3.05 \mathrm{E} & 01\end{array}$ 2.80E 02 6.30F $01 \quad 1.65 \mathrm{E} 01$ $1.69 \mathrm{E} 02 \quad 3.80 \mathrm{E} 01 \quad 9.33500$ 7. $58 \mathrm{~F} 01 \quad 1.71 \mathrm{E} 01 \quad 4.46 \mathrm{E} 0 \mathrm{O}$ $5.77 \mathrm{~F} 01$ 1.30E 01 3.39E 00
13.13 15.00 15.24

15.36 15.94 15.97 20.00 30.07 40.00 50.00 60.00 80.00 38.26
7.95F 03 1. 0 DE $03 \quad 4.74 \Gamma, 02$ $\begin{array}{llllll}5.96 \mathrm{E} & 03 & 1.35 \mathrm{E} & 03 & 3.55 \mathrm{~F} & 02\end{array}$ $\begin{array}{lllllll}5.22 E & 03 & 1.18 E & 03 & 3.11 E & 0 ?\end{array}$ $5.50 E 03$ 1.25E 03 3.28E 02 $\begin{array}{llllll}5 . C 8 F & 03 & 1.15 E & 03 & 3.03 E & 0 ?\end{array}$ $6.00 \mathrm{E} 03 \quad 1.36 \mathrm{E}$ 03 3.57 E 02 $\begin{array}{llllll}3.64 \mathrm{E} & 03 & 8.25 \mathrm{E} & 02 & 2.17 \mathrm{E} & 0 ?\end{array}$ $\begin{array}{llllll}1.23 \mathrm{~F} & 03 & 2.78 \mathrm{E} & 02 & 7.30 \mathrm{E} & 01\end{array}$

$5.6 \mathrm{CE} \quad 02 \quad 1.27 \mathrm{~F}$ 02 $3.34 \mathrm{~F} 01$

$3.03 \mathrm{E} 02 \quad 6.96 \mathrm{~F} 01 \quad 1.80 \% 01$

$\begin{array}{llllll}1.83 \mathrm{E} & 02 & 4.14 \mathrm{~F} & 01 & 1.09 \mathrm{~F} & 01\end{array}$ $8.21 E 01$ 1.86E n1 $4.89 E 0$. $5.77 \mathrm{E} 01 \quad 1.31 \mathrm{~F} 013.44 \mathrm{~F} 00$
13.52 15.00 15.73 15.8 8 16.37 16.50 20.00 30.00 40.00 50.00 60.00

\begin{tabular}{|c|c|c|c|c|c|}
\hline $\begin{array}{l}13.91 \\
15.00 \\
16.28 \\
16.41 \\
16.91 \\
17.05 \\
20.00 \\
30.00 \\
40.00 \\
50.00 \\
50.00 \\
80.00\end{array}$ & $\begin{array}{c}- \\
- \\
- \\
- \\
- \\
2.73 \mathrm{E} \\
2.06 \mathrm{~B} \\
9.60 \mathrm{E} \\
5.34 \mathrm{E} \\
3.30 \mathrm{E} \\
2.20 \mathrm{E} \\
1.13 \mathrm{E} \\
7.85 \mathrm{E}-\end{array}$ & $\begin{array}{r}01 \\
01 \\
00 \\
00 \\
00 \\
00 \\
00 \\
01\end{array}$ & $\begin{array}{c}- \\
= \\
= \\
- \\
2.78 \mathrm{E} \\
2.10 \mathrm{E} \\
9.81 \mathrm{E} \\
5.45 \mathrm{E} \\
3.37 \mathrm{E} \\
2.25 \mathrm{E} \\
1.16 \mathrm{E} \\
8.01 \mathrm{E}\end{array}$ & $\begin{array}{l}02 \\
02 \\
01 \\
01 \\
01 \\
01 \\
01 \\
00\end{array}$ & $\begin{array}{c}- \\
- \\
- \\
- \\
3.17 \mathrm{~F} \\
2.40 \mathrm{P} \\
1.12 \mathrm{E} \\
6.21 \mathrm{~F} \\
3.84 \mathrm{E} \\
2.56 \mathrm{E} \\
1.32 \mathrm{~F} \\
9.13 \mathrm{P}-\end{array}$ \\
\hline
\end{tabular}

$$
\text { Po } z=84
$$

$\begin{array}{lllllll}7.93 E & 03 & 1.81 \mathrm{E} & 03 & 4.80 \mathrm{~F} & 02 & 1.3 .91 \\ 6.47 \mathrm{E} & 03 & 1.48 \mathrm{~F} & 03 & 3.91 \mathrm{E} & 02 & 15.00 \\ 5.14 \mathrm{E} & 03 & 1.17 \mathrm{E} & 03 & 3.11 \mathrm{E} & 02 & 16.29 \\ 5.41 \mathrm{E} & 03 & 1.24 \mathrm{E} & 03 & 3.28 \mathrm{E} & 02 & 16.41 \\ 5.00 \mathrm{E} & 03 & 1.14 \mathrm{~F} & 03 & 3.03 \mathrm{E} & 02 & 16.91 \\ 5.91 \mathrm{E} & 03 & 1.35 \mathrm{E} & 03 & 3.58 \mathrm{E} & 02 & 17.05 \\ 3.92 \mathrm{~F} & 03 & 8.95 \mathrm{E} & 02 & 2.37 \mathrm{~F} & 02 & 20.00 \\ 1.32 \mathrm{E} & 03 & 3.01 \mathrm{E} & 02 & 7.995 & 01 & 30.00 \\ 6 . \mathrm{CHE} & 02 & 1.39 \mathrm{~F} & 02 & 3.65 \mathrm{~F} & 01 & 40.00 \\ 3.27 \mathrm{~F} & 02 & 7.47 \mathrm{~F} & 01 & 1.98 \mathrm{~F} & 01 & 50.00 \\ 1.97 \mathrm{E} & 02 & 4.51 \mathrm{E} & 01 & 1.20 \mathrm{~F} & 01 & 60.00 \\ 8.99 \mathrm{E} & 01 & 2.03 \mathrm{~F} & 01 & 5.38 \mathrm{E} & 00 & 80.00 \\ 5.77 \mathrm{~F} & 01 & 1.32 \mathrm{~F} & 01 & 3.50 \mathrm{E} & 00 & 93.41\end{array}$

$\begin{array}{llllll}7.89 \mathrm{~F} & 03 & 1.82 \mathrm{~F} & 03 & 4.87 \mathrm{~F} & 0 ?\end{array}$ $\begin{array}{llllll}7.04 \mathrm{E} & 03 & 1.62 \mathrm{~F} & 03 & 4.34 \mathrm{E} & 02\end{array}$ 5.06E $03 \quad 1.17 \mathrm{~F} 03 \quad 3.12 \mathrm{~F}$ ก $\begin{array}{llllll}5.33 \mathrm{~F} & 03 & 1.23 \mathrm{~F} & 03 & 3.295 & 02\end{array}$ $\begin{array}{llllll}4.93 F & 03 & 1.147 & 03 & 3.04 \mathrm{~F} & 02\end{array}$ $5.85 \mathrm{~F} 03 \quad 1.35 \mathrm{E} 03 \quad 3.51 \mathrm{~F}$ ? ? 4. $21 \mathrm{~F} 03 \quad 9.71 \mathrm{E} 02$ 2.5OE 02 $\begin{array}{llllll}1.43 \mathrm{E} & 03 & 3.29 \mathrm{~F} & 02 & 8.79 \mathrm{E} & 01\end{array}$ $6.54 \mathrm{E} \quad 02 \quad 1.51 \mathrm{E} \quad 02 \quad 4.03 \mathrm{E} \quad 01$
14.31 15.00 16. 22 16.96 17.47

17.61 20.00 30.00 40.00 
Table 8 (continued)

\begin{tabular}{|c|c|c|c|c|c|c|c|c|c|c|c|c|c|c|c|c|}
\hline \multirow{2}{*}{$\begin{array}{c}\begin{array}{c}\text { Photon } \\
\text { energy } \\
(\mathrm{keV})\end{array} \\
50.00 \\
60.00 \\
80.00 \\
96.05\end{array}$} & $L_{1} L_{23}$ & \multicolumn{2}{|l|}{$\begin{array}{c}L_{1} M_{3} \\
L \beta_{3}\end{array}$} & \multicolumn{2}{|l|}{$\begin{array}{c}L_{1} O_{23} \\
L \gamma_{4}\end{array}$} & \multicolumn{2}{|c|}{$\begin{array}{c}L_{2} M_{4} \\
L \beta_{1}\end{array}$} & \multicolumn{2}{|l|}{$\begin{array}{c}L_{2} N_{4} \\
L \gamma_{1}\end{array}$} & \multicolumn{2}{|c|}{$\begin{array}{c}L_{3} M_{5} \\
L \alpha_{1}\end{array}$} & \multicolumn{2}{|c|}{$\begin{array}{l}L_{3} N_{4} 5 \\
L \beta_{2} 15\end{array}$} & \multicolumn{2}{|l|}{$\begin{array}{c}L_{3} M_{1} \\
L \ell\end{array}$} & \multirow{2}{*}{$\begin{array}{c}\begin{array}{c}\text { Photon } \\
\text { energy } \\
\text { (keV) }\end{array} \\
50.00 \\
60.00 \\
80.00 \\
96.05\end{array}$} \\
\hline & $\begin{array}{ll}3.79 \mathrm{~F} & 00 \\
2.53 \mathrm{E} & 00 \\
1.31 \mathrm{E} & 00 \\
8.48 \mathrm{E}-01\end{array}$ & $\begin{array}{l}3.58 \mathrm{~F} \\
2.39 \mathrm{E} \\
1.24 \mathrm{E} \\
8.02 \mathrm{~F}\end{array}$ & $\begin{array}{l}01 \\
01 \\
01 \\
00\end{array}$ & $\begin{array}{l}4.265 \\
2.85 \mathrm{~F} \\
1.47 \mathrm{~F} \\
9.54 \mathrm{E}-\end{array}$ & $\begin{array}{ll}0 & 0 \\
0 & 0 \\
1 & 0 \\
01\end{array}$ & $\begin{array}{l}2.43 \mathrm{~F} \\
1.46 \mathrm{~F} \\
6.47 \mathrm{~F} \\
3.84 \mathrm{~F}\end{array}$ & $\begin{array}{l}02 \\
02 \\
91 \\
01\end{array}$ & $\begin{array}{l}5.39 E \\
3.23 E \\
1.43 E \\
8.49 F\end{array}$ & $\begin{array}{ll}0 & 1 \\
0 & 1 \\
0 & 1 \\
0 & 0\end{array}$ & $\begin{array}{l}3.55 \mathrm{~F} \\
2.15 \mathrm{~F} \\
9.68 \mathrm{~F} \\
5.83 \mathrm{~F}\end{array}$ & $\begin{array}{l}02 \\
02 \\
01 \\
01\end{array}$ & $\begin{array}{l}8.18 \mathrm{~F} \\
4.95 \mathrm{~F} \\
2.23 \mathrm{~F} \\
1.34 \mathrm{E}\end{array}$ & $\begin{array}{l}01 \\
01 \\
01 \\
01\end{array}$ & $\begin{array}{l}2.19 \mathrm{~F} \\
1.32 \mathrm{E} \\
5.97 \mathrm{E} \\
3.59 \mathrm{~F}\end{array}$ & $\begin{array}{l}01 \\
01 \\
00 \\
01\end{array}$ & \\
\hline & & & & & & $\mathrm{RN}$ & $z$ & $=86$ & & & & & & & & \\
\hline $\begin{array}{l}14.72 \\
15.00 \\
17.38 \\
17.52 \\
18.04 \\
18.18 \\
20.00 \\
30.00 \\
40.00 \\
50.00 \\
60.00 \\
80.00 \\
99.76\end{array}$ & $\begin{array}{cc}- & - \\
- & \\
- & \\
- & \\
3.10 \mathrm{E} & 01 \\
2.60 \mathrm{E} & 01 \\
1.24 \mathrm{E} & 01 \\
6.94 \mathrm{E} & 00 \\
4.32 \mathrm{~F} & 00 \\
2.89 \mathrm{E} & 00 \\
1.50 \mathrm{~F} & 00 \\
9.11 \mathrm{E}-01\end{array}$ & $\begin{array}{c}- \\
- \\
- \\
- \\
- \\
2.72 \mathrm{E} \\
2.28 \mathrm{E} \\
1.09 \mathrm{E} \\
6.09 \mathrm{~F} \\
3.79 \mathrm{E} \\
2.54 \mathrm{~F} \\
1.31 \mathrm{~F} \\
7.99 \mathrm{~F}\end{array}$ & $\begin{array}{l}02 \\
02 \\
02 \\
01 \\
01 \\
01 \\
01 \\
00\end{array}$ & $\begin{array}{c}- \\
- \\
- \\
- \\
3.36 \mathrm{~F} \\
2.92 \mathrm{E} \\
1.35 \mathrm{~F} \\
7.53 \mathrm{~F} \\
4.69 \mathrm{E} \\
3.14 \mathrm{~F} \\
1.62 \mathrm{~F} \\
9.98 \mathrm{~F}-\end{array}$ & $\begin{array}{ll}9 & 1 \\
0 & 1 \\
0 & 1 \\
0 & 0 \\
0 & 0 \\
0 & 0 \\
0 & 0 \\
0 & 1\end{array}$ & $\begin{array}{c}- \\
- \\
- \\
1.95 \mathrm{~F} \\
3.73 \mathrm{E} \\
3.25 \mathrm{~F} \\
3.00 \mathrm{~F} \\
1.06 \mathrm{E} \\
4.91 \mathrm{~F} \\
2.66 \mathrm{~F} \\
1.61) \mathrm{F} \\
7.10 \mathrm{~F} \\
3.90 \mathrm{~F}\end{array}$ & $\begin{array}{l}03 \\
03 \\
03 \\
03 \\
03 \\
02 \\
02 \\
02 \\
01 \\
01\end{array}$ & $\begin{array}{c}- \\
- \\
- \\
8.82 \mathrm{~F} \\
8.33 \mathrm{~F} \\
8.61 \mathrm{~F} \\
6.70 \mathrm{E} \\
2.38 \mathrm{~F} \\
1.10 \mathrm{E} \\
5.95 \mathrm{E} \\
3.58 \mathrm{E} \\
1.59 \mathrm{E} \\
8.73 \mathrm{~F}\end{array}$ & $\begin{array}{l}02 \\
02 \\
02 \\
02 \\
02 \\
02 \\
01 \\
01 \\
01 \\
00\end{array}$ & $\begin{array}{l}7.84 \mathrm{E} \\
7.47 \mathrm{E} \\
4.96 \mathrm{E} \\
5.22 \mathrm{~F} \\
4.84 \mathrm{E} \\
5.73 \mathrm{E} \\
4.48 \mathrm{E} \\
1.52 \mathrm{E} \\
6.97 \mathrm{E} \\
3.79 \mathrm{E} \\
2.29 \mathrm{E} \\
1.03 \mathrm{E} \\
5.76 \mathrm{E}\end{array}$ & $\begin{array}{l}03 \\
03 \\
03 \\
03 \\
03 \\
03 \\
03 \\
03 \\
02 \\
02 \\
02 \\
02 \\
01\end{array}$ & $\begin{array}{l}1.82 \mathrm{E} \\
1.74 \mathrm{~F} \\
1.15 \mathrm{E} \\
1.21 \mathrm{~F} \\
1.12 \mathrm{E} \\
1.335 \\
1.04 \mathrm{~F} \\
3.54 \mathrm{E} \\
1.62 \mathrm{~F} \\
8.81 \mathrm{~F} \\
5.33 \mathrm{~F} \\
2.40 \mathrm{~F} \\
1.34 \mathrm{E}\end{array}$ & $\begin{array}{l}03 \\
03 \\
03 \\
03 \\
03 \\
03 \\
03 \\
02 \\
02 \\
01 \\
01 \\
01 \\
01\end{array}$ & $\begin{array}{l}4.91 \mathrm{~F} \\
4.69 \mathrm{E} \\
3.11 \mathrm{~F} \\
3.27 \mathrm{~F} \\
3.03 \mathrm{~F} \\
3.59 \mathrm{~F} \\
2.80 \mathrm{~F} \\
9.53 \mathrm{~F} \\
4.37 \mathrm{E} \\
2.37 \mathrm{~F} \\
1.43 \mathrm{~F} \\
6.47 \mathrm{~F} \\
3.61 \mathrm{I}\end{array}$ & $\begin{array}{l}02 \\
0 ? \\
0 ? \\
0 ? \\
02 \\
0 ? \\
0 ? \\
01 \\
01 \\
01 \\
01 \\
13 \\
0 \\
0\end{array}$ & $\begin{array}{l}14.72 \\
15.00 \\
17.39 \\
17.52 \\
18.04 \\
18.19 \\
20.00 \\
30.00 \\
49.00 \\
50.00 \\
60.00 \\
80.00 \\
93.76\end{array}$ \\
\hline
\end{tabular}

$R R \quad Z=87$

$\begin{array}{rcccccc}15.14 & - & & - & - & \\ 17.95 & - & - & - & - & \\ 18.10 & - & - & - & - & \\ 18.63 & - & & & - & \\ 18.79 & 3.27 \mathrm{E} & 01 & 2.66 \mathrm{E} & 02 & 3.43 \mathrm{E} & 01 \\ 20.00 & 2.93 \mathrm{E} & 01 & 2.38 \mathrm{E} & 02 & 3.07 \mathrm{~F} & 01 \\ 30.00 & 1.40 \mathrm{E} & 01 & 1.14 \mathrm{~F} & 02 & 1.46 \mathrm{~F} & 01 \\ 40.00 & 7.83 \mathrm{E} & 00 & 6.37 \mathrm{E} & 01 & 8.21 \mathrm{~F} & 00 \\ 50.00 & 4.89 \mathrm{E} & 00 & 3.97 \mathrm{E} & 01 & 5.12 \mathrm{~F} & 00 \\ 60.00 & 3.28 \mathrm{~F} & 00 & 2.66 \mathrm{~F} & 01 & 3.43 \mathrm{~F} & 00 \\ 90.00 & 1.70 \mathrm{E} & 00 & 1.38 \mathrm{~F} & 01 & 1.78 \mathrm{~F} & 00 \\ 100.00 & 1.01 \mathrm{E} & 00 & 8.19 \mathrm{E} & 00 & 1.06 \mathrm{E} & 00 \\ 101.50 & 9.72 \mathrm{~F}-01 & 7.90 \mathrm{~F} & 00 & 1.02 \mathrm{~F} & 00\end{array}$

$9.72 \mathrm{~F}-01 \quad 7.90 \mathrm{~F} \quad 00$ $\begin{array}{cccc}- & & - \\ 3.93 \mathrm{~F} & 03 & 8.86 \mathrm{E} & 02 \\ 3.70 \mathrm{~F} & 03 & 8.34 \mathrm{E} & 02 \\ 3.82 \mathrm{~F} & 03 & 8.62 \mathrm{E} & 02 \\ 3.22 \mathrm{~F} & 03 & 7.27 \mathrm{E} & 02 \\ 1.15 \mathrm{~F} & 03 & 2.60 \mathrm{~F} & 02 \\ 5.34 \mathrm{~F} & 02 & 1.21 \mathrm{~F} & 02 \\ 2.90 \mathrm{~F} & 02 & 6.55 \mathrm{E} & 01 \\ 1.75 \mathrm{~F} & 02 & 3.95 \mathrm{E} & 01 \\ 7.80 \mathrm{E} & 01 & 1.76 \mathrm{E} & 01 \\ 4.14 \mathrm{~F} & 01 & 9.35 \mathrm{~F} & 00 \\ 3.97 \mathrm{~F} & 01 & 8.95 \mathrm{E} & 00\end{array}$

$\begin{array}{lllllll}7.81 \mathrm{~F} & 03 & 1.33 \mathrm{~F} & 03 & 4.97 \mathrm{~F} & 0 ? & 15.14 \\ 4.87 \mathrm{~F} & 03 & 1.14 \mathrm{~F} & 03 & 3.10 \mathrm{~F} & 0 ? & 17.95 \\ 5.13 \mathrm{E} & 03 & 1.20 \mathrm{~F} & 03 & 3.26 \mathrm{~F} & 02 & 18.10 \\ 4.75 \mathrm{~F} & 03 & 1.11 \mathrm{~F} & 03 & 3 . \mathrm{C} F & 02 & 19.63 \\ 5.63 \mathrm{E} & 03 & 1.32 \mathrm{E} & 03 & 3.59 \mathrm{~F} & 02 & 19.79 \\ 4.79 \mathrm{E} & 03 & 1.12 \mathrm{~F} & 03 & 3.05 \mathrm{~F} & 02 & 20.00 \\ 1.63 \mathrm{~F} & 03 & 3.93 \mathrm{E} & 02 & 1.04 \mathrm{~F} & 0 ? & 30.00 \\ 7.49 \mathrm{E} & 02 & 1.76 \mathrm{~F} & 02 & 4.76 \mathrm{~F} & 01 & 40.00 \\ 4.07 \mathrm{E} & 02 & 9.54 \mathrm{E} & 01 & 2.59 \mathrm{~F} & 01 & 50.00 \\ 2.47 \mathrm{~F} & 02 & 5.79 \mathrm{E} & 01 & 1.57 \mathrm{~F} & 01 & 60.00 \\ 1.11 \mathrm{~F} & 02 & 2.51 \mathrm{E} & 01 & 7.0 \mathrm{~F} & 00 & 90.00 \\ 6 . \mathrm{CCF} & 01 & 1.41 \mathrm{E} & 01 & 3.82 \mathrm{E} & 00 & 100.00 \\ 5.76 \mathrm{~F} & 01 & 1.35 \mathrm{E} & 01 & 3.66 \mathrm{~F} & 00 & 101.50\end{array}$

$5.76 \mathrm{~F} 01 \quad 1.35 \mathrm{E} 01 \quad 3.66 \mathrm{r} 00 \quad 101.50$

15.56

18.54

18.69

19.23

19.39

20.00

30.00

40.0 ?

50.00

60.00

80.00

100.00

104.30

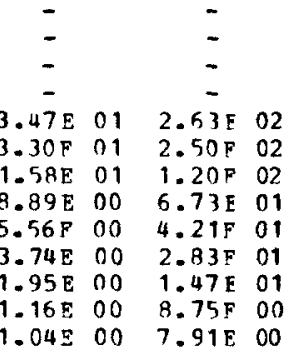

$1.04500 \quad 7.91 \mathrm{E} 00$
RA $Z=88$

$\begin{array}{cccc}- & & - & \\ - & & - & \\ 89 \mathrm{~F} & 03 & 9.86 \mathrm{~F} & 02 \\ 66 \mathrm{E} & 03 & 8.33 \mathrm{E} & 02 \\ 76 \mathrm{~F} & 03 & 8.57 \mathrm{E} & 02 \\ 45 \mathrm{~F} & 03 & 7.87 \mathrm{E} & 02 \\ 24 \mathrm{~F} & 03 & 2.81 \mathrm{~F} & 02 \\ 74 \mathrm{E} & 02 & 1.31 \mathrm{E} & 02 \\ 12 \mathrm{~F} & 02 & 7.12 \mathrm{~F} & 01 \\ 89 \mathrm{~F} & 02 & 4.29 \mathrm{~F} & 01 \\ 41 \mathrm{E} & 01 & 1.92 \mathrm{~F} & 01 \\ 47 \mathrm{~F} & 01 & 1.02 \mathrm{E} & 01 \\ 97 \mathrm{~F} & 01 & 9.04 \mathrm{~F} & 00\end{array}$

$A C \quad z=89$

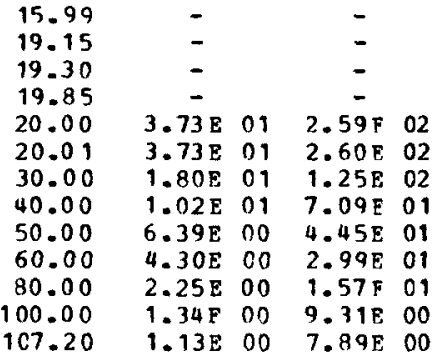

$3.52 \mathrm{~F} 01$

3. $34 \mathrm{~F} 01$

1. $60 \mathrm{E} 01$

$9.01 \mathrm{~F} 00$

$5.64 \mathrm{E} \quad 00$

$3.79 \mathrm{E} 00$

$1.97 \mathrm{E} 00$

1. $17 \mathrm{~F} 00$
$3.89=03 \quad 9.86=02$

$3.66 \mathrm{E} 03 \quad$ R. $33 \mathrm{E} 02$

$\begin{array}{lllll}3.45 \mathrm{~F} & 03 & 7.87 \mathrm{E} & 02\end{array}$

$1.24 \mathrm{~F} 03 \quad 2.81 \mathrm{~F} 02$

1. $31 \mathrm{E} 02$

$1.89 \mathrm{~F} 02 \quad 4.29 \mathrm{~F} 01$

$\begin{array}{llll}8.41 \mathrm{E} & 01 & 1.92 \mathrm{~F} & 01 \\ 4.47 \mathrm{~F} & 01 & 1.02 \mathrm{C} & 01\end{array}$

$3.97 \mathrm{~F} 01 \quad 9.04 \mathrm{~F} 00$

$\begin{array}{lllllll}7.77 \mathrm{~F} & 03 & 1.94 \mathrm{~F} & 03 & 5.03 \mathrm{~F} & 02 & 15.5 \mathrm{~F} \\ 4.78 \mathrm{~F} & 03 & 1.13 \mathrm{~F} & 03 & 3.09 \mathrm{E} & 02 & 19.54 \\ 5 . \mathrm{C} 3 \mathrm{E} & 03 & 1.19 \mathrm{E} & 03 & 3.26 \mathrm{E} & 02 & 18.69 \\ 4.67 \mathrm{E} & 03 & 1.10 \mathrm{E} & 03 & 3 . \mathrm{C} 2 \mathrm{E} & 02 & 19.23 \\ 5.53 \mathrm{E} & 03 & 1.31 \mathrm{~F} & 03 & 3.59 \mathrm{E} & 02 & 19.39 \\ 5.11 \mathrm{E} & 03 & 1.21 \mathrm{E} & 03 & 3.31 \mathrm{~F} & 02 & 20.00 \\ 1.75 \mathrm{~F} & 03 & 4.13 \mathrm{~F} & 02 & 1.13 \mathrm{~F} & 02 & 30.07 \\ 8.03 \mathrm{E} & 02 & 1.90 \mathrm{~F} & 02 & 5.20 \mathrm{E} & 01 & 41.00 \\ 4.37 \mathrm{~F} & 02 & 1.03 \mathrm{E} & 02 & 2.33 \mathrm{~F} & 01 & 50.00 \\ 7.65 \mathrm{~F} & 02 & 6.26 \mathrm{~F} & 01 & 1.71 \mathrm{~F} & 01 & 60.00 \\ 1.20 \mathrm{~F} & 02 & 2.83 \mathrm{~F} & 01 & 7.75 \mathrm{E} & 00 & 80.00 \\ 6.46 \mathrm{E} & 01 & 1.53 \mathrm{~F} & 01 & 4.18 \mathrm{~F} & 00 & 100.00 \\ 5.74 \mathrm{~F} & 01 & 1.36 \mathrm{E} & 01 & 3.72 \mathrm{~F} & 00 & 104.30\end{array}$

$\begin{array}{llllllll}7.72 \mathrm{E} & 03 & 1.84 \mathrm{~F} & 03 & 5.0 \mathrm{RE} & 02\end{array}$ $\begin{array}{llllll}4.68 \mathrm{E} & 03 & 1.11 \mathrm{E} & 03 & 3.08 \mathrm{E} & 02\end{array}$ $\begin{array}{llllll}4.93 F & 03 & 1.17 E & 03 & 3.24 \% & 0 ?\end{array}$ $\begin{array}{lllllll}4.58 \mathrm{~F} & 03 & 1.09 \mathrm{~F} & 03 & 3.01 \mathrm{~F} & 02\end{array}$ $\begin{array}{llllll}5.44 E & 03 & 1.29 \mathrm{~F} & 03 & 3.57 \mathrm{E} & 02\end{array}$ $\begin{array}{llllll}5.43 \mathrm{~F} & 03 & 1.29 \mathrm{~F} & 03 & 3.57 \mathrm{~F} & 02\end{array}$ $\begin{array}{llllll}1.87 \mathrm{E} & 03 & 4.45 \mathrm{E} & 02 & 1.23 \mathrm{~F} & 02\end{array}$ $8.60 \mathrm{~F} 02 \quad 2.05 \mathrm{~F} 02 \quad 5.65 \mathrm{~F} 01$ $4.68 \mathrm{~F} 02 \quad 1.11 \mathrm{E} \cap 2 \quad 3.08 \mathrm{E} 01$ $2.84 \mathrm{~F} 02 \quad 6.76 \mathrm{~F} 01 \quad 1.87 \mathrm{~F} 01$ $\begin{array}{llll}1.29 \mathrm{~F} 02 & 3.06 \mathrm{~F} 09 \quad 8.45 \mathrm{~F} & 0 & 0\end{array}$ $6.94 \mathrm{E} 01 \quad 1.65 \mathrm{E} 11 \quad 4.57 \mathrm{~F} 00$

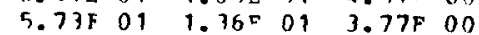

15.99 19.15 19.30 19.85 20.00 20.01 30.00 40.00 50.00 60.00 80.00 100.00 107.20

$$
\mathrm{TH} \quad \mathrm{Z}=90
$$

16.43

19.76

19.92

20.00

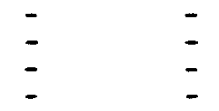

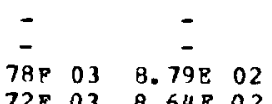

$\begin{array}{llll}3.78 \mathrm{~F} & 03 & 8.79 \mathrm{E} & 02\end{array}$
$7.68 \mathrm{~F} \quad 03$ 4. $58 \mathrm{E} \quad 03$ 4. $93 \mathrm{E} 03$ 4. $78 E 03$
1. $84 \mathrm{~F} \quad 03 \quad 5.14 \mathrm{~F} \quad 02$ 1. $10 \mathrm{E} 03 \quad 3.06 \mathrm{~F} 02$

$\begin{array}{lllll}1.16 \mathrm{~F} & 03 & 3.23 \mathrm{~F} & \mathrm{O} 2\end{array}$ $1.15 \mathrm{~F} 03 \quad 3.20 \mathrm{~F} 0$ ?
16.43 $19.7 \mathrm{~h}$ 20.01 
Table 8 (continued)

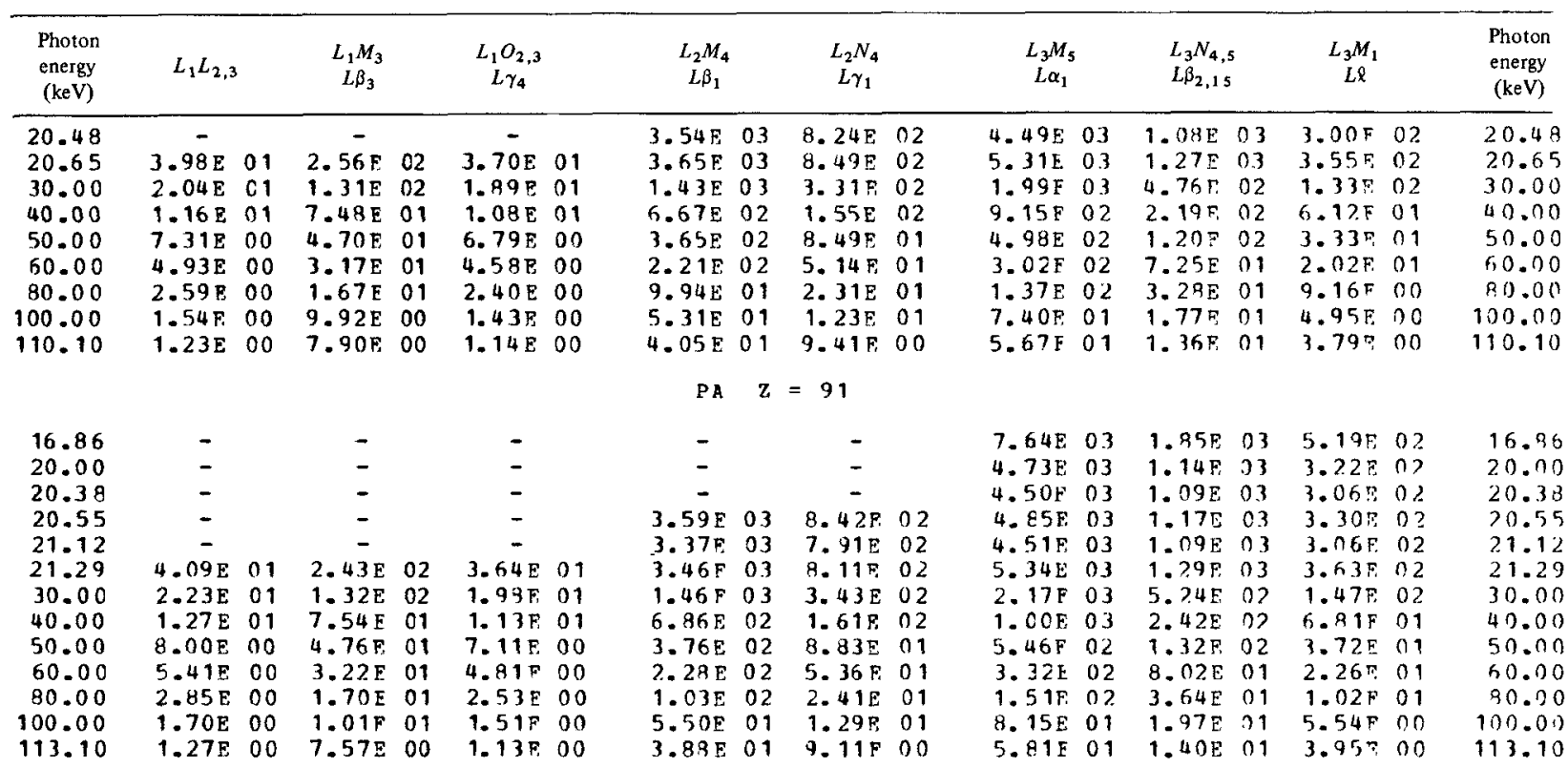

17.30

20.00

21.03

21.19

21.78

21.95

30.00

40.00

50.00

60.00

80.00

100.00

116.20
$1.27 \mathrm{E} \quad 00$
$1.13 F 00$
17.75

20.00

21.68

21.86

22.45

22.63

30.00

40.00

50.00

60.00

80.00

100.00

119.30

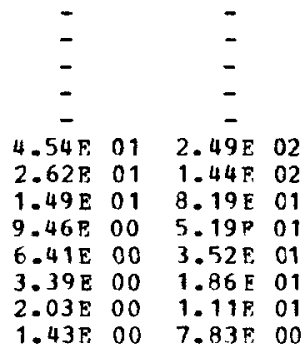

-

3. $85 \mathrm{E} \quad 01$

$2.22 \mathrm{~F} 01$

1.27F 01

$8.02 \mathrm{~F} 00$

$5.44 \mathrm{E} 00$

$2.87 E 00$

1. $72 \mathrm{~F} 00$

1. $21 \mathrm{~F} 00$

$5.81 \mathrm{~F} 01 \quad 1.40 \mathrm{E} 01 \quad 3.95 \% 00$

113.10

$\begin{array}{lllllll}7.59 \mathrm{E} & 03 & 1.85 \mathrm{~F} & 03 & 5.24 \mathrm{E} & 02 & 17.30 \\ 5.04 \mathrm{~F} & 03 & 1.23 \mathrm{E} & 03 & 3.4 \mathrm{FF} & 02 & 20.02 \\ 4.41 \mathrm{E} & 03 & 1.07 \mathrm{~F} & 03 & 3.04 \mathrm{~F} & 02 & 21.03 \\ 4.84 \mathrm{~F} & 03 & 1.18 \mathrm{~F} & 03 & 3.34 \mathrm{~F} & 02 & 21.19 \\ 4.50 \mathrm{~F} & 03 & 1.10 \mathrm{~F} & 03 & 3.11 \mathrm{~F} & 02 & 21.78 \\ 5.31 \mathrm{~F} & 03 & 1.29 \mathrm{~F} & 03 & 3.67 \mathrm{~F} & 02 & 21.95 \\ 2.34 \mathrm{~F} & 03 & 5.70 \mathrm{~F} & 02 & 1.52 \mathrm{~F} & 02 & 10.00 \\ 1 . \mathrm{CFE} & 03 & 2.63 \mathrm{~F} & 02 & 7.48 \mathrm{~F} & 01 & 40.00 \\ 5.91 \mathrm{E} & 02 & 1.44 \mathrm{E} & 02 & 4.08 \mathrm{~F} & 01 & 50.00 \\ 3.59 \mathrm{~F} & 02 & 8.74 \mathrm{~F} & 01 & 2.48 \mathrm{~F} & 01 & 60.00 \\ 1.63 \mathrm{E} & 02 & 3.97 \mathrm{E} & 01 & 1.13 \mathrm{~F} & 01 & 80.00 \\ 8.83 \mathrm{E} & 01 & 2.15 \mathrm{E} & 01 & 6.10 \mathrm{~F} & 00 & 100.00 \\ 5.85 \mathrm{~F} & 01 & 1.42 \mathrm{~F} & 01 & 4.04 \mathrm{~F} & 00 & 116.20\end{array}$

$.42 F .03 \quad 8.09802$ $\begin{array}{lllll}3.21 \mathrm{~F} & 03 & 7.61 \mathrm{E} & 02\end{array}$ $3.30 \mathrm{~F} \quad 03 \quad 7.80 \mathrm{~F} \quad 02$ $\begin{array}{lllll}1.51 \mathrm{~F} & 03 & 3.59 \mathrm{~F} & 02\end{array}$ $\begin{array}{llll}7.13 \mathrm{E} & 02 & 1.69 \mathrm{E} & 02\end{array}$ 3.92F $02 \quad 9.28 \mathrm{~F} 01$ $2.39 \mathrm{~F} 02 \quad 5.65 \mathrm{~F} 01$ 1.08F 02 2.55E 0 $\begin{array}{lllll}5.73 \mathrm{~F}, & 01 & 1.37 \mathrm{E} & 0\end{array}$ $3.80 \mathrm{E} 01 \quad 8.98 \mathrm{E} 00$

NP $\quad 7=93$

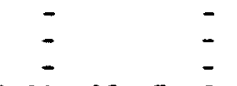

$3.29 \mathrm{~F} 03 \quad 7.85 \mathrm{~F} 02$ $3.09 \mathrm{E} \quad 03 \quad 7.37 \mathrm{~F} 02$ $\begin{array}{llll}3.15 \mathrm{~F} & 03 & 7.53 \mathrm{E} & 02\end{array}$ $1.57 \mathrm{~F} 03 \quad 3.74 \mathrm{E} 02$ $\begin{array}{lllll}7.41 \mathrm{E} & 02 & 1.77 \mathrm{E} & 02\end{array}$ $\begin{array}{llll}4.09 \mathrm{E} \quad 02 \quad 9.76 \mathrm{E} & 0\end{array}$ $2.49 \mathrm{E} \quad 02 \quad 5.94 \mathrm{E} \quad 0$ $1.13 \mathrm{~F}, 02 \quad 2.69 \mathrm{~F}, 01$ $6.05 \mathrm{E} \quad 01 \quad 1.44 \mathrm{E} \quad 01$ $3.69 \mathrm{E} 01 \quad 8.82 \mathrm{E} \quad 00$

$\begin{array}{lllllll}7.54 \mathrm{~F} & 03 & 1.35 \mathrm{E} & 03 & 5.31 \mathrm{E} & 02 & 17.75 \\ 3.38 \mathrm{E} & 03 & 1.32 \mathrm{E} & 03 & 3.79 \mathrm{E} & 02 & 20.0 \mathrm{Z} \\ 4.31 \mathrm{E} & 03 & 1.06 \mathrm{E} & 03 & 3.03 \mathrm{E} & 02 & 21.68 \\ 4.82 \mathrm{~F} & 03 & 1.18 \mathrm{~F} & 03 & 3.39 \mathrm{E} & 02 & 21.96 \\ 4.49 \mathrm{~F} & 03 & 1.10 \mathrm{~F} & 03 & 3.16 \mathrm{E} & 02 & 22.45 \\ 5.29 \mathrm{E} & 03 & 1.70 \mathrm{E} & 03 & 3.72 \mathrm{E} & 02 & 22.63 \\ 2.53 \mathrm{~F} & 03 & 6.20 \mathrm{~F} & 02 & 1.78 \mathrm{~F} & 02 & 30.00 \\ 1.17 \mathrm{E} & 03 & 2.37 \mathrm{E} & 02 & 8.24 \mathrm{E} & 01 & 40.00 \\ 6.40 \mathrm{E} & 02 & 1.57 \mathrm{E} & 02 & 4.51 \mathrm{E} & 01 & 50.00 \\ 3.90 \mathrm{E} & 02 & 9.56 \mathrm{E} & 01 & 2.74 \mathrm{E} & 01 & 60.00 \\ 1.77 \mathrm{E} & 02 & 4.35 \mathrm{E} & 01 & 1.25 \mathrm{E} & 01 & 80.00 \\ 9.6 \mathrm{CE} & 01 & 2.35 \mathrm{E} & 01 & 6.75 \mathrm{E} & 0 \mathrm{O} & 100.00 \\ 5.91 \mathrm{E} & 01 & 1.45 \mathrm{~F} & 01 & 4.16 \mathrm{~F} & 00 & 119.30\end{array}$

$7.48 \mathrm{~F} 03 \quad 1.85 \mathrm{~F} \quad 03 \quad 5.34 \mathrm{~F} 02$ $\begin{array}{llllll}5.74 \mathrm{E} & 03 & 1.42 \mathrm{E} & 03 & 4.10 \mathrm{E} & 02\end{array}$ $4.22 \mathrm{E} \quad 03 \quad 1.04 \mathrm{E} \quad 03 \quad 3.02 \mathrm{E} \quad 22$ $\begin{array}{llllll}4.74 \mathrm{~F} & 03 & 1.17 \mathrm{E} & 03 & 3.39 \mathrm{~F} & 02\end{array}$ $\begin{array}{llllll}4.42 \mathrm{~F} & 03 & 1.09 \mathrm{~F} & 03 & 3.16 \mathrm{E} & 03\end{array}$ $\begin{array}{llllll}5.18 E & 03 & 1.28 \mathrm{E} & 03 & 3.70 \mathrm{~F} & 02\end{array}$ $\begin{array}{llllll}2.68 \mathrm{E} & 03 & 6.61 \mathrm{E} & 02 & 1.91 \mathrm{P} & 02\end{array}$ $1.24 \mathrm{E} 03 \quad 3.07 \mathrm{E} 02 \quad 8.88 \mathrm{~F} 01$ 6. $80 \mathrm{E} \quad 02 \quad 1.69 \mathrm{E} 02 \quad 4.86 \mathrm{~F} \cap 1$ 4. $14 \mathrm{~F} 02 \quad 1.02 \mathrm{~F} \quad 02 \quad 2.96 \mathrm{~F} 01$

$\begin{array}{llllll}1.88 \mathrm{~F} & 02 & 4.64 \mathrm{E} & 01 & 1.34 \mathrm{E} & 01\end{array}$

$\begin{array}{llllll}1.02 F & 02 & 2.52 E & 01 & 7.29 F & 00\end{array}$ $5.95 \mathrm{E} 01$ 1.44E 01 4.18E 00
19.20

20.00

22.35

22.53

23.14

23.32

30.00

40.00

50.00

60.00

80.00

100.00 
Table 8 (continued)

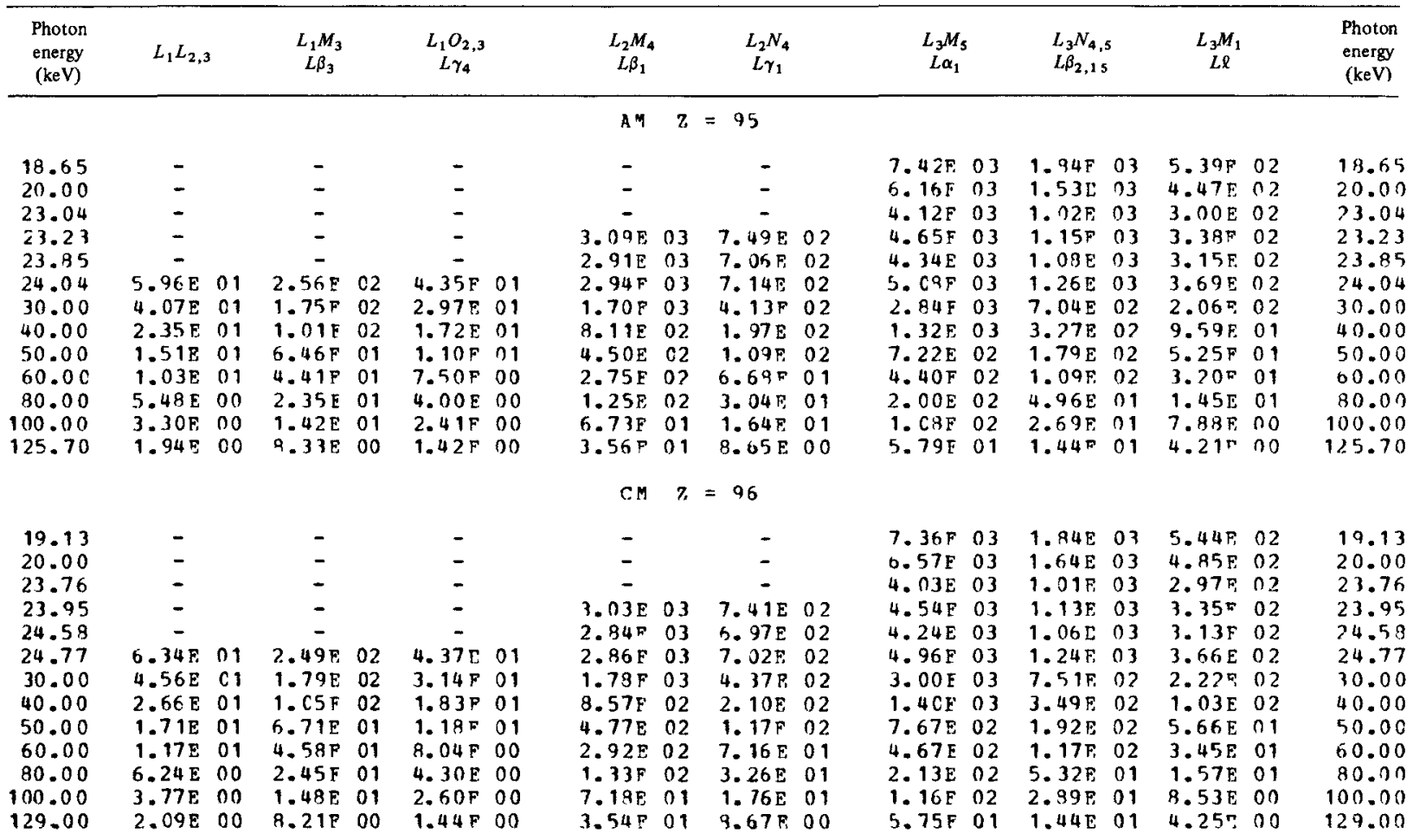

$$
\text { BK } \quad Z=97
$$

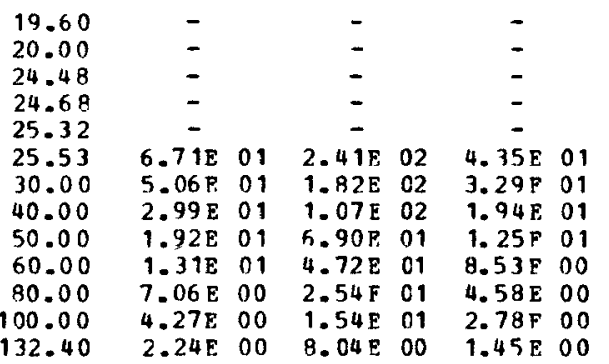

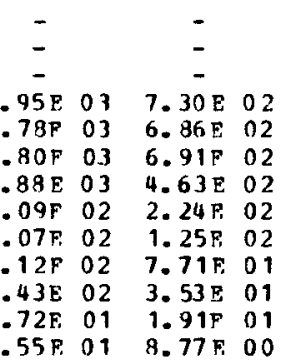

$3.55 \mathrm{~F} 01 \quad 8.77 \mathrm{~F} 00$

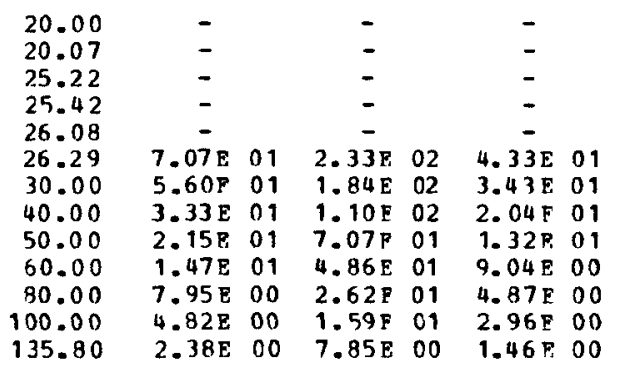$$
C F \quad Z=98
$$

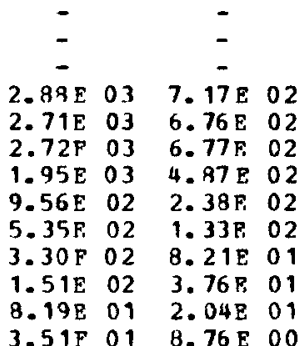

$3.51 \mathrm{~F} 01$ B.76 E 00

$\begin{array}{lllllll}7.28 \mathrm{~F} & 03 & 1.83 \mathrm{~F} & 03 & 5.47 \mathrm{E} & 02 & 19.60 \\ 6.82 \mathrm{~F} & 03 & 1.72 \mathrm{E} & 03 & 5.13 \mathrm{E} & 02 & 20.00 \\ 3.92 \mathrm{E} & 03 & 9.86 \mathrm{~F} & 02 & 2.94 \mathrm{~F} & 02 & 24.49 \\ 4.42 \mathrm{~F} & 03 & 1.11 \mathrm{~F} & 03 & 3.32 \mathrm{~F} & 02 & 24.68 \\ 4.13 \mathrm{E} & 03 & 1.04 \mathrm{E} & 03 & 3.10 \mathrm{E} & 02 & 25.32 \\ 4.82 \mathrm{~F} & 03 & 1.21 \mathrm{E} & 03 & 3.62 \mathrm{~F} & 02 & 25.53 \\ 3.16 \mathrm{E} & 03 & 7.94 \mathrm{E} & 02 & 2.37 \mathrm{E} & 02 & 30.00 \\ 1.47 \mathrm{E} & 03 & 3.70 \mathrm{~F} & 02 & 1.11 \mathrm{E} & 02 & 40.00 \\ 8 . \mathrm{C} \mathrm{E} & 02 & 2.03 \mathrm{E} & 02 & 6.07 \mathrm{E} & 01 & 50.00 \\ 4.92 \mathrm{~F} & 02 & 1.24 \mathrm{E} & 02 & 3.70 \mathrm{E} & 01 & 60.00 \\ 2.24 \mathrm{E} & 02 & 5.64 \mathrm{E} & 01 & 1.69 \mathrm{~F} & 01 & 80.70 \\ 1.22 \mathrm{E} & 02 & 3.06 \mathrm{~F} & 01 & 9.15 \mathrm{E} & 00 & 100.00 \\ 5.66 \mathrm{E} & 01 & 1.42 \mathrm{~F} & 01 & 4.25 \mathrm{E} & 00 & 132.40\end{array}$

$\begin{array}{lllllll}7.19 \mathrm{E} & 03 & 1.82 \mathrm{E} & 03 & 5.49 \mathrm{E} & 02\end{array}$ $\begin{array}{llllll}7.19 E & 03 & 1.82 \mathrm{~F} & 33 & 5.49 \mathrm{~F} & 02\end{array}$ $3.81 \mathrm{~F} 03 \quad 9.63 \mathrm{E} 02$ 2.91F 02 $\begin{array}{llllll}4.30 \mathrm{E} & 03 & 1.09 \mathrm{E} & 03 & 3.29 \mathrm{~F} & 02\end{array}$ $\begin{array}{lllllll}4.02 \mathrm{E} & 03 & 1.02 \mathrm{~F} & 03 & 3.07 \mathrm{~F} & \mathrm{C} 2\end{array}$ $\begin{array}{lllllll}4.69 E & 03 & 1.19 P & 03 & 3.59 \mathrm{R} & 02\end{array}$ $\begin{array}{llllll}3.32 \mathrm{~F} & 03 & 8.40 \mathrm{~F} & 02 & 2.54 \mathrm{~F} & 02\end{array}$ $\begin{array}{llllll}1.55 \mathrm{~F} & 03 & 3.93 \mathrm{E} & 02 & 1.19 \mathrm{E} & 02\end{array}$ 8.53E $02 \quad 2.16 \mathrm{E} 02 \quad 6.52 \mathrm{E} 01$ $\begin{array}{lllllll}5.20 \mathrm{E} & 02 & 1.32 \mathrm{E} & 02 & 3.9 \mathrm{AE} & 01\end{array}$ $2.38 \mathrm{E} \quad 02 \quad 6.01 \mathrm{~F} 01 \quad 1.92 \mathrm{E} 01$

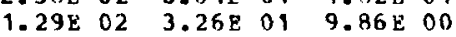
$\begin{array}{lllllllll}5.60 \mathrm{~F} & 01 & 1.42 \mathrm{E} & 01 & 4.28 \mathrm{~F} & 00\end{array}$
20.00 20.07 25.22 25.42 26.08 26.29 30.00 40.00 50.00 60.00 80.00 100.00 175.80

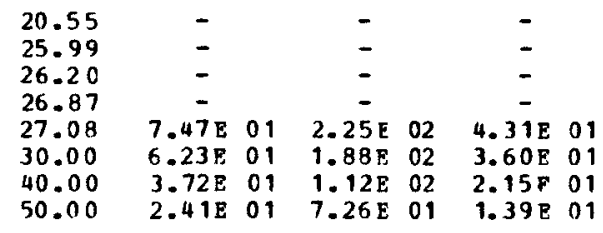

$$
\text { ES } \quad z=99
$$

$\begin{array}{cccc}\overline{-} & & \overline{-} \\ 2.81 \mathrm{E} & 03 & 7.07 \mathrm{E} & 02 \\ 2.65 \mathrm{E} & 03 & 6.66 \mathrm{E} & 02 \\ 2.66 \mathrm{E} & 03 & 6.68 \mathrm{E} & 02 \\ 2.05 \mathrm{E} & 03 & 5.15 \mathrm{E} & 02 \\ 1.01 \mathrm{~F} & 03 & 2.55 \mathrm{E} & 02 \\ 5.70 \mathrm{~F} & 02 & 1.47 \mathrm{E} & 02\end{array}$

$7.10 \mathrm{E} 03 \quad 1.01803-5.53 \mathrm{~B}^{2} 02$

20.55

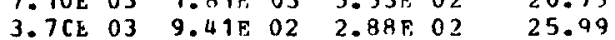

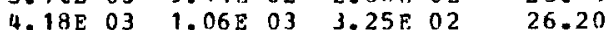

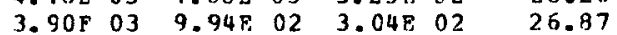
$\begin{array}{lllllll}4.57 \mathrm{E} & 03 & 1.16 \mathrm{E} & 03 & 3.56 \mathrm{E} & 02 & 27.08\end{array}$ $\begin{array}{lllllll}3.49 \mathrm{~F} & 03 & 8.89 \mathrm{E} & 02 & 2.72 \mathrm{E} & 02 & 30.00\end{array}$ $\begin{array}{lllllll}1.64 \mathrm{~F} & 03 & 4.17 \mathrm{~F} & 02 & 1.27 \mathrm{E} & 02 & 40.00\end{array}$

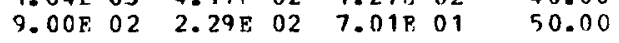


Table 8 (continued)

\begin{tabular}{|c|c|c|c|c|c|c|c|c|c|c|c|c|c|c|c|c|c|}
\hline $\begin{array}{l}\text { Photon } \\
\text { energy } \\
(\mathrm{keV})\end{array}$ & $L_{1} L_{2,3}$ & & $\begin{array}{c}L_{1} M_{3} \\
L \beta_{3}\end{array}$ & & $\begin{array}{c}L_{1} O_{2,3} \\
L \gamma_{4}\end{array}$ & & $\begin{array}{c}L_{2} M_{4} \\
L \beta_{1}\end{array}$ & & $\begin{array}{c}L_{2} N_{4} \\
L \gamma_{1}\end{array}$ & & $\begin{array}{c}L L_{3} M_{5} \\
L \alpha_{1}\end{array}$ & & $\begin{array}{l}L_{3} N_{4,5} \\
L \beta_{2,15}\end{array}$ & & $\begin{array}{c}L_{3} M_{1} \\
L \ell\end{array}$ & & $\begin{array}{l}\text { Photon } \\
\text { energy } \\
\text { (kev) }\end{array}$ \\
\hline $\begin{array}{r}60.00 \\
80.00 \\
100.00 \\
139.30\end{array}$ & $\begin{array}{l}1.66 \mathrm{E} \\
8.96 \mathrm{E} \\
5.46 \mathrm{~F} \\
2.55 \mathrm{E}\end{array}$ & $\begin{array}{l}01 \\
00 \\
00 \\
00\end{array}$ & $\begin{array}{l}5.00 \mathrm{E} \\
2.70 \mathrm{E} \\
1.65 \mathrm{~F} \\
7.68 \mathrm{~F}\end{array}$ & $\begin{array}{l}01 \\
01 \\
01 \\
00\end{array}$ & $\begin{array}{l}9.56 \mathrm{~F} \\
5.17 \mathrm{E} \\
3.15 \mathrm{~F} \\
1.47 \mathrm{~F}\end{array}$ & $\begin{array}{l}00 \\
00 \\
00 \\
00\end{array}$ & $\begin{array}{l}3.52 \mathrm{E} \\
1.62 \mathrm{~F} \\
8.81 \mathrm{~F} \\
3.54 \mathrm{E}\end{array}$ & $\begin{array}{l}02 \\
02 \\
01 \\
01\end{array}$ & $\begin{array}{l}8.84 \mathrm{~F} \\
4.07 \mathrm{~F} \\
2.21 \mathrm{~F} \\
8.88 \mathrm{E}\end{array}$ & $\begin{array}{ll}01 \\
01 \\
01 \\
0 & 0\end{array}$ & $\begin{array}{l}5.50 \mathrm{E} \\
2.51 \mathrm{~F} \\
1.37 \mathrm{E} \\
5.54 \mathrm{~F}\end{array}$ & $\begin{array}{l}02 \\
02 \\
02 \\
01\end{array}$ & $\begin{array}{l}1.40 \mathrm{P} \\
6.40 \mathrm{~F} \\
3.48 \mathrm{~F} \\
1.41 \mathrm{E}\end{array}$ & $\begin{array}{l}02 \\
01 \\
01 \\
01\end{array}$ & $\begin{array}{l}4.28 \mathrm{~F} \\
1.96 \mathrm{E} \\
1.06 \mathrm{~F} \\
4.31 \mathrm{E}\end{array}$ & $\begin{array}{l}01 \\
01 \\
01 \\
01\end{array}$ & $\begin{array}{r}60.00 \\
80.00 \\
100.00 \\
139.30\end{array}$ \\
\hline & & & & & & & $F M$ & $z$ & $=100$ & & & & & & & & \\
\hline $\begin{array}{r}21.04 \\
26.77 \\
26.99 \\
27.67 \\
27.89 \\
30.00 \\
40.00 \\
50.00 \\
60.00 \\
90.00 \\
100.00 \\
142.90\end{array}$ & $\begin{array}{c}- \\
- \\
- \\
7.92 \mathrm{E} \\
7.00 \mathrm{E} \\
4.18 \mathrm{~F} \\
2.70 \mathrm{E} \\
1.87 \mathrm{E} \\
1.01 \mathrm{E} \\
6.19 \mathrm{E} \\
2.73 \mathrm{E}\end{array}$ & $\begin{array}{l} \\
01 \\
01 \\
01 \\
01 \\
01 \\
01 \\
00 \\
00\end{array}$ & $\begin{array}{c}- \\
- \\
- \\
\overline{2} \\
2.18 \mathrm{E} \\
1.93 \mathrm{~F} \\
1.15 \mathrm{E} \\
7.44 \mathrm{E} \\
5.14 \mathrm{E} \\
2.79 \mathrm{~F} \\
1.70 \mathrm{E} \\
7.52 \mathrm{E}\end{array}$ & $\begin{array}{l}02 \\
02 \\
02 \\
01 \\
01 \\
01 \\
01 \\
00\end{array}$ & $\begin{array}{c}\overline{-} \\
\overline{-} \\
\overline{4} \\
4.28 \mathrm{~F} \\
3.79 \mathrm{~F} \\
2.26 \mathrm{~F} \\
1.46 \mathrm{~F} \\
1.01 \mathrm{~F} \\
5.49 \mathrm{~F} \\
3.35 \mathrm{~F} \\
1.48 \mathrm{~F}\end{array}$ & $\begin{array}{ll} & \\
0 & 1 \\
0 & 1 \\
01 & 1 \\
0 & 1 \\
01 \\
0 & 0 \\
0 & 0 \\
0 & 0\end{array}$ & $\begin{array}{c}\overline{-} \\
\overline{2} \\
2.76 \mathrm{~F} \\
2.61 \mathrm{E} \\
2.61 \mathrm{E} \\
2.16 \mathrm{~F} \\
1.08 \mathrm{~F} \\
6.08 \mathrm{~F} \\
3.77 \mathrm{~F} \\
1.74 \mathrm{~F} \\
9.50 \mathrm{~F} \\
3.57 \mathrm{E}\end{array}$ & $\begin{array}{l}03 \\
03 \\
03 \\
03 \\
03 \\
02 \\
02 \\
02 \\
01 \\
01\end{array}$ & $\begin{array}{c}- \\
- \\
6.99 \mathrm{E} \\
6.60 \mathrm{E} \\
6.61 \mathrm{E} \\
5.48 \mathrm{~F} \\
2.73 \mathrm{E} \\
1.54 \mathrm{~F} \\
9.53 \mathrm{~F} \\
4.41 \mathrm{~F} \\
2.41 \mathrm{E} \\
9.04 \mathrm{~F}\end{array}$ & $\begin{array}{l}02 \\
02 \\
02 \\
02 \\
02 \\
02 \\
01 \\
01 \\
01 \\
00\end{array}$ & $\begin{array}{l}7.00 \mathrm{E} \\
3.59 \mathrm{E} \\
4.05 \mathrm{E} \\
3.79 \mathrm{E} \\
4.42 \mathrm{~F} \\
3.66 \mathrm{E} \\
1.72 \mathrm{~F} \\
9.43 \mathrm{E} \\
5.76 \mathrm{E} \\
2.64 \mathrm{E} \\
1.44 \mathrm{E} \\
5.43 \mathrm{~F}\end{array}$ & $\begin{array}{l}03 \\
03 \\
03 \\
03 \\
03 \\
03 \\
03 \\
02 \\
02 \\
02 \\
02 \\
01\end{array}$ & $\begin{array}{l}1.79 \mathrm{~F} \\
9.18 \mathrm{~F} \\
1.04 \mathrm{E} \\
9.70 \mathrm{E} \\
1.13 \mathrm{E} \\
9.38 \mathrm{E} \\
4.39 \mathrm{E} \\
2.41 \mathrm{E} \\
1.47 \mathrm{E} \\
6.75 \mathrm{~F} \\
3.67 \mathrm{~F} \\
1.39 \mathrm{E}\end{array}$ & $\begin{array}{l}03 \\
02 \\
03 \\
02 \\
03 \\
02 \\
02 \\
02 \\
02 \\
01 \\
01 \\
01\end{array}$ & $\begin{array}{l}5.54 \mathrm{~F} \\
2.84 \mathrm{E} \\
3.21 \mathrm{E} \\
3.00 \mathrm{~F} \\
3.50 \mathrm{E} \\
2.90 \mathrm{~F} \\
1.36 \mathrm{E} \\
7.46 \mathrm{~F} \\
4.56 \mathrm{E} \\
2.09 \mathrm{E} \\
1.14 \mathrm{~F} \\
4.30 \mathrm{E}\end{array}$ & $\begin{array}{l}02 \\
02 \\
02 \\
02 \\
02 \\
02 \\
02 \\
01 \\
01 \\
01 \\
01 \\
00\end{array}$ & $\begin{array}{r}21.04 \\
26.77 \\
26.99 \\
27.67 \\
27.89 \\
30.00 \\
40.00 \\
50.00 \\
50.00 \\
90.00 \\
100.00 \\
142.90\end{array}$ \\
\hline & & & & & & & $M D$ & z & $=101$ & & & & & & & & \\
\hline $\begin{array}{r}21.53 \\
27.58 \\
27.80 \\
28.50 \\
28.73 \\
30.00 \\
40.00 \\
50.00 \\
60.00 \\
80.00 \\
100.00 \\
146.60\end{array}$ & $\begin{array}{c}- \\
- \\
- \\
- \\
8.32 \mathrm{E} \\
7.79 \mathrm{E} \\
4.66 \mathrm{~F} \\
3.02 \mathrm{E} \\
2.09 \mathrm{E} \\
1.14 \mathrm{E} \\
6.96 \mathrm{E} \\
2.91 \mathrm{E}\end{array}$ & $\begin{array}{l}01 \\
01 \\
01 \\
01 \\
01 \\
01 \\
00 \\
00\end{array}$ & $\begin{array}{c}- \\
- \\
- \\
- \\
2.09 \mathrm{E} \\
1.96 \mathrm{E} \\
1.17 \mathrm{E} \\
7.58 \mathrm{~F} \\
5.26 \mathrm{~F} \\
2.86 \mathrm{~F} \\
1.75 \mathrm{E} \\
7.31 \mathrm{E}\end{array}$ & $\begin{array}{l}02 \\
02 \\
02 \\
01 \\
01 \\
01 \\
01 \\
00\end{array}$ & $\begin{array}{c}- \\
- \\
- \\
- \\
4.22 \mathrm{E} \\
3.95 \mathrm{E} \\
2.36 \mathrm{E} \\
1.53 \mathrm{~F} \\
1.06 \mathrm{~F} \\
5.77 \mathrm{E} \\
3.53 \mathrm{~F} \\
1.48 \mathrm{~F}\end{array}$ & $\begin{array}{ll}01 \\
0 & 1 \\
0 & 1 \\
0 & 1 \\
0 & 1 \\
0 & 0 \\
0 & 0 \\
0 & 0\end{array}$ & $\begin{array}{c}- \\
-\overline{1} \\
2.71 \mathrm{~F} \\
2.56 \mathrm{E} \\
2.55 \mathrm{E} \\
2.29 \mathrm{~F} \\
1.14 \mathrm{~F} \\
6.44 \mathrm{~F} \\
4.00 \mathrm{~F} \\
1.86 \mathrm{~F} \\
1.01 \mathrm{E} \\
3.57 \mathrm{~F}\end{array}$ & $\begin{array}{l}03 \\
03 \\
03 \\
03 \\
03 \\
02 \\
02 \\
02 \\
02 \\
01\end{array}$ & $\begin{array}{c}- \\
- \\
6.92 \mathrm{E} \\
6.53 \mathrm{P} \\
6.51 \mathrm{E} \\
5.84 \mathrm{E} \\
2.92 \mathrm{P} \\
1.64 \mathrm{E} \\
1.02 \mathrm{E} \\
4.74 \mathrm{E} \\
2.59 \mathrm{E} \\
9.11 \mathrm{E}\end{array}$ & $\begin{array}{l}02 \\
02 \\
02 \\
02 \\
02 \\
02 \\
02 \\
01 \\
01 \\
01\end{array}$ & $\begin{array}{l}6.90 \mathrm{~F} \\
3.47 \mathrm{E} \\
3.92 \mathrm{E} \\
3.67 \mathrm{~F} \\
4.29 \mathrm{E} \\
3.84 \mathrm{~F} \\
1.80 \mathrm{E} \\
9.90 \mathrm{E} \\
6 . \mathrm{C} \mathrm{E} \\
2.78 \mathrm{E} \\
1.51 \mathrm{E} \\
5.35 \mathrm{E}\end{array}$ & $\begin{array}{l}03 \\
03 \\
03 \\
03 \\
03 \\
03 \\
03 \\
02 \\
02 \\
02 \\
02 \\
01\end{array}$ & $\begin{array}{l}1.78 \mathrm{E} \\
8.94 \mathrm{~F} \\
1.01 \mathrm{E} \\
9.44 \mathrm{E} \\
1.10 \mathrm{E} \\
9.88 \mathrm{E} \\
4.63 \mathrm{E} \\
2.55 \mathrm{E} \\
1.56 \mathrm{E} \\
7.15 \mathrm{~F} \\
3.89 \mathrm{~F} \\
1.38 \mathrm{E}\end{array}$ & $\begin{array}{l}03 \\
02 \\
03 \\
02 \\
03 \\
02 \\
02 \\
02 \\
02 \\
01 \\
01 \\
01\end{array}$ & $\begin{array}{l}5.55 \mathrm{~F} \\
2.79 \mathrm{~F} \\
3.15 \mathrm{E} \\
2.95 \mathrm{E} \\
3.45 \mathrm{E} \\
3.09 \mathrm{E} \\
1.45 \mathrm{~F} \\
7.96 \mathrm{E} \\
4.87 \mathrm{E} \\
2.23 \mathrm{~F} \\
1.22 \mathrm{E} \\
4.31 \mathrm{~F}\end{array}$ & $\begin{array}{l}02 \\
02 \\
02 \\
02 \\
02 \\
02 \\
02 \\
01 \\
01 \\
01 \\
01 \\
00\end{array}$ & $\begin{array}{r}21.53 \\
27.58 \\
27.80 \\
29.50 \\
28.73 \\
30.00 \\
40.00 \\
50.00 \\
60.00 \\
80.00 \\
100.00 \\
146.60\end{array}$ \\
\hline
\end{tabular}




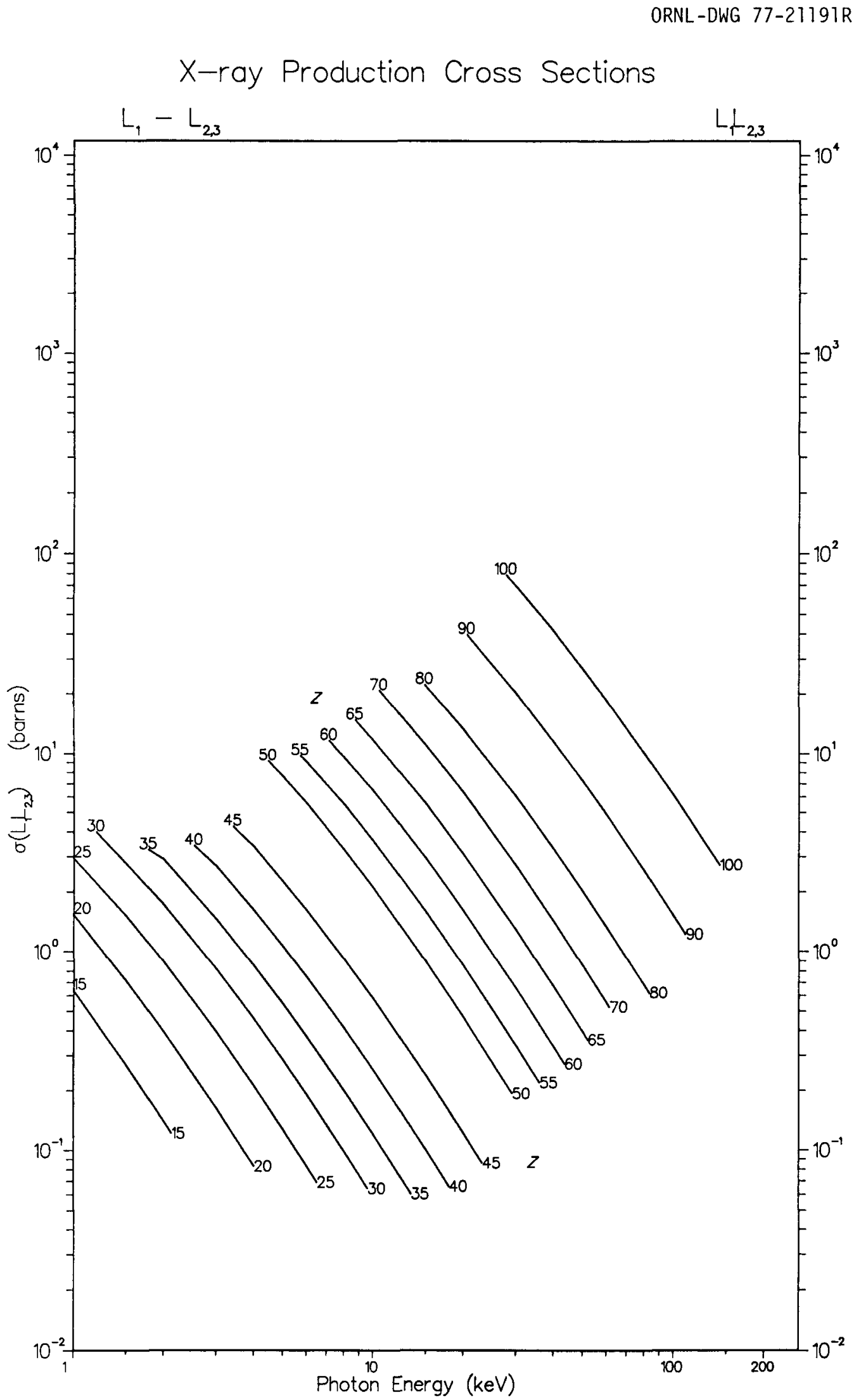

Fig. 6 
ORNL-DWG 77-21192R

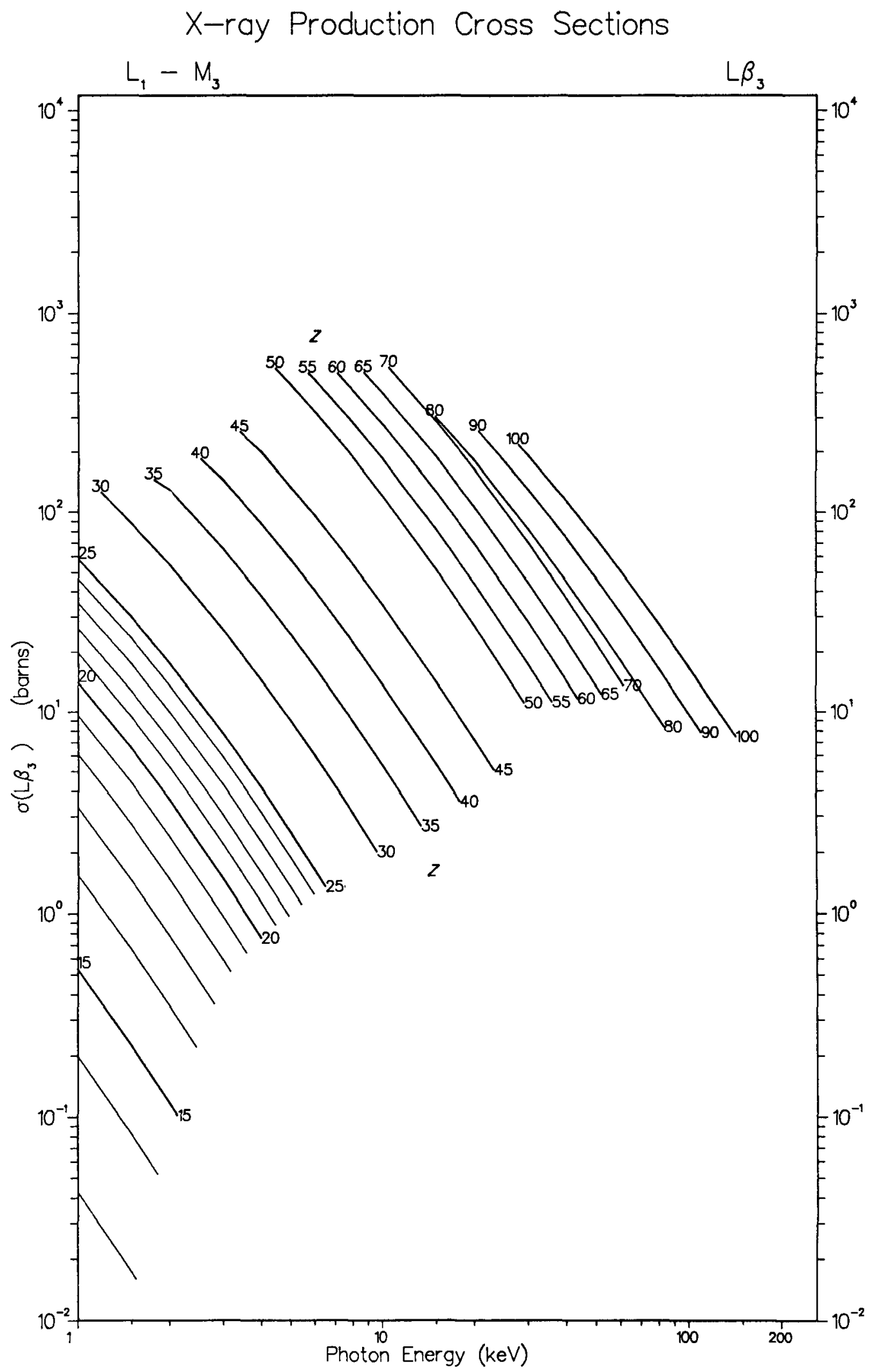

Fig. 7 
ORNL-DWG 77-21193R

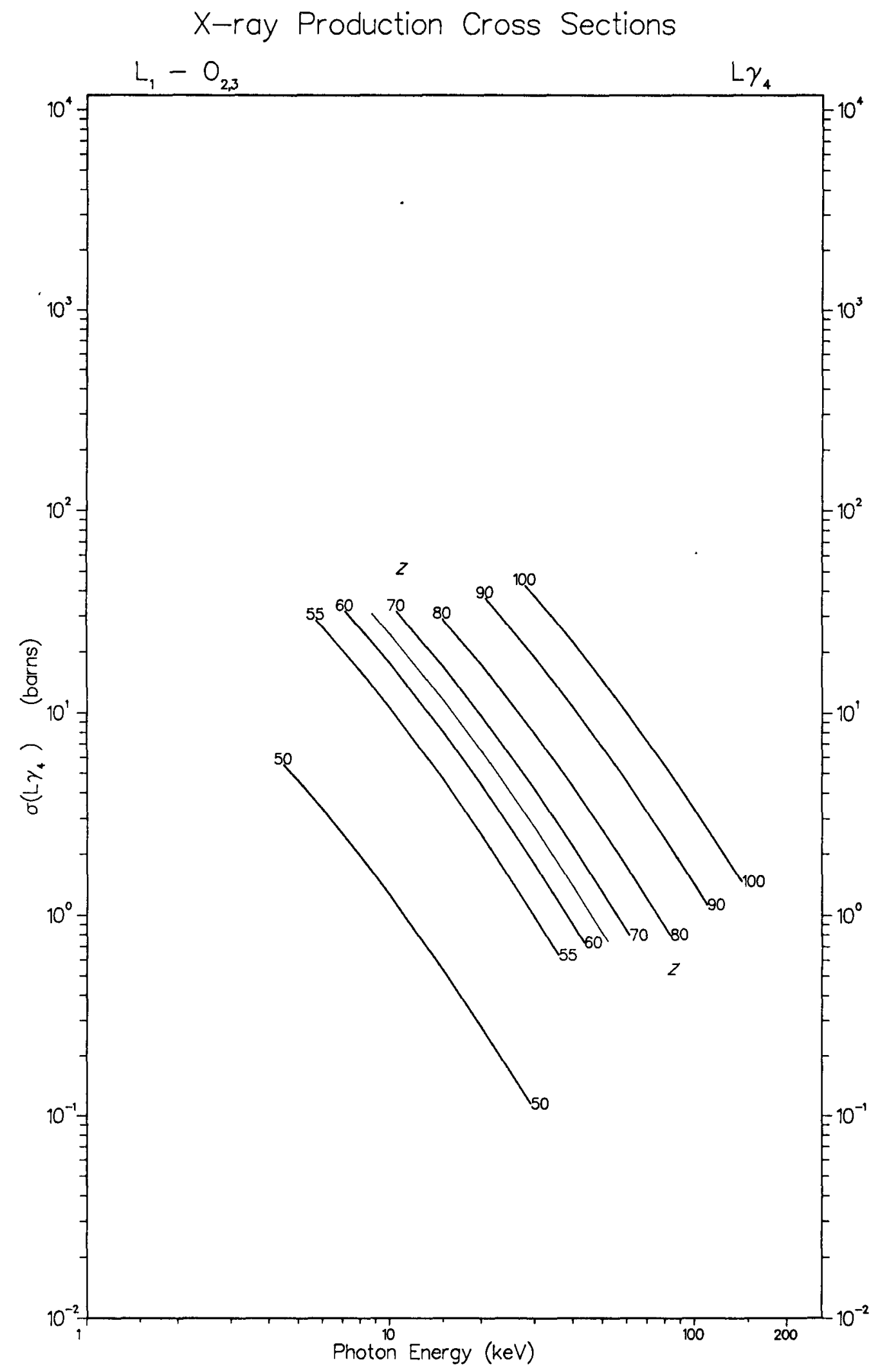

Fig. 8 


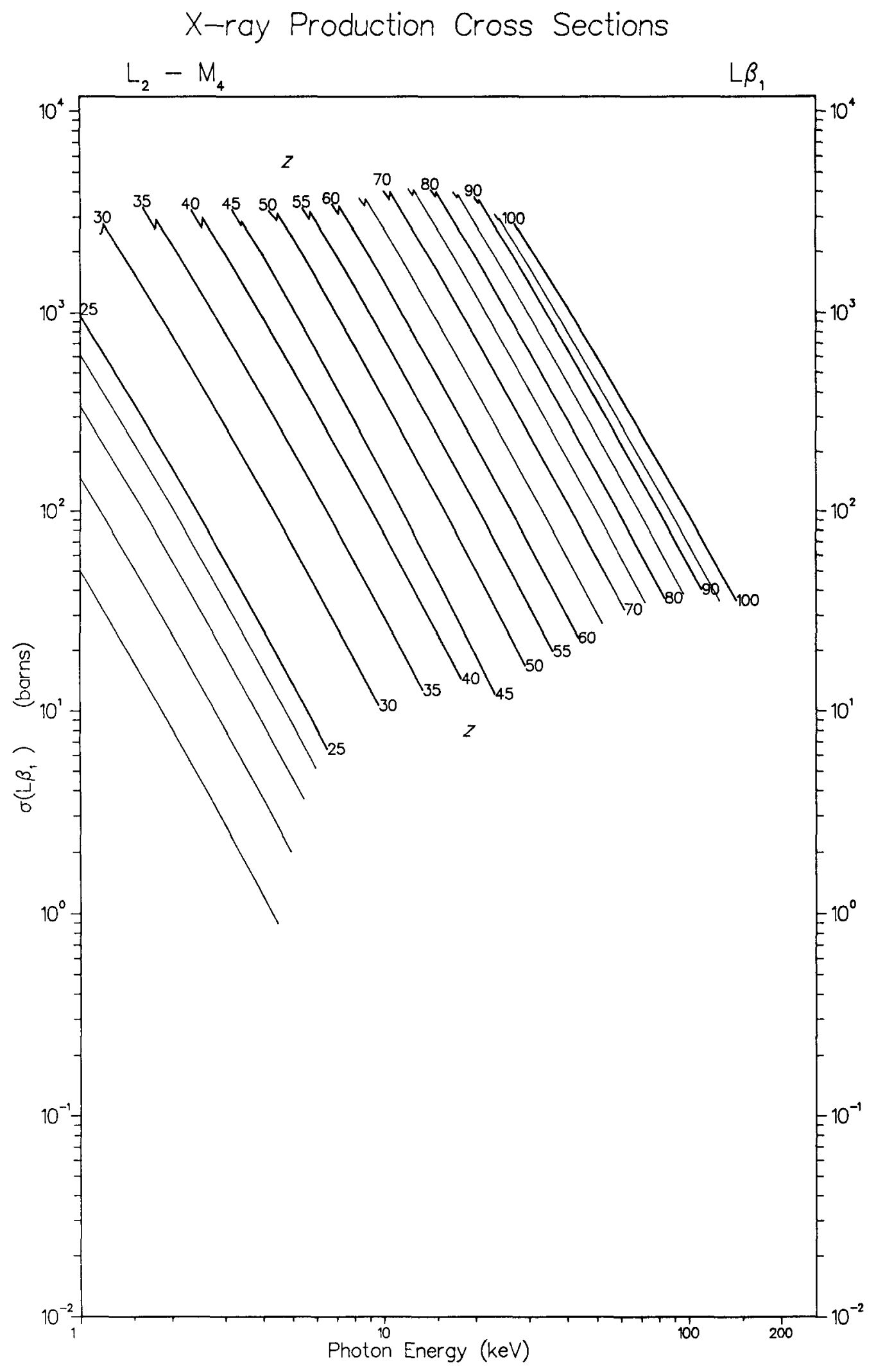

Fig. 9 
ORNL-DWG 77-21195R

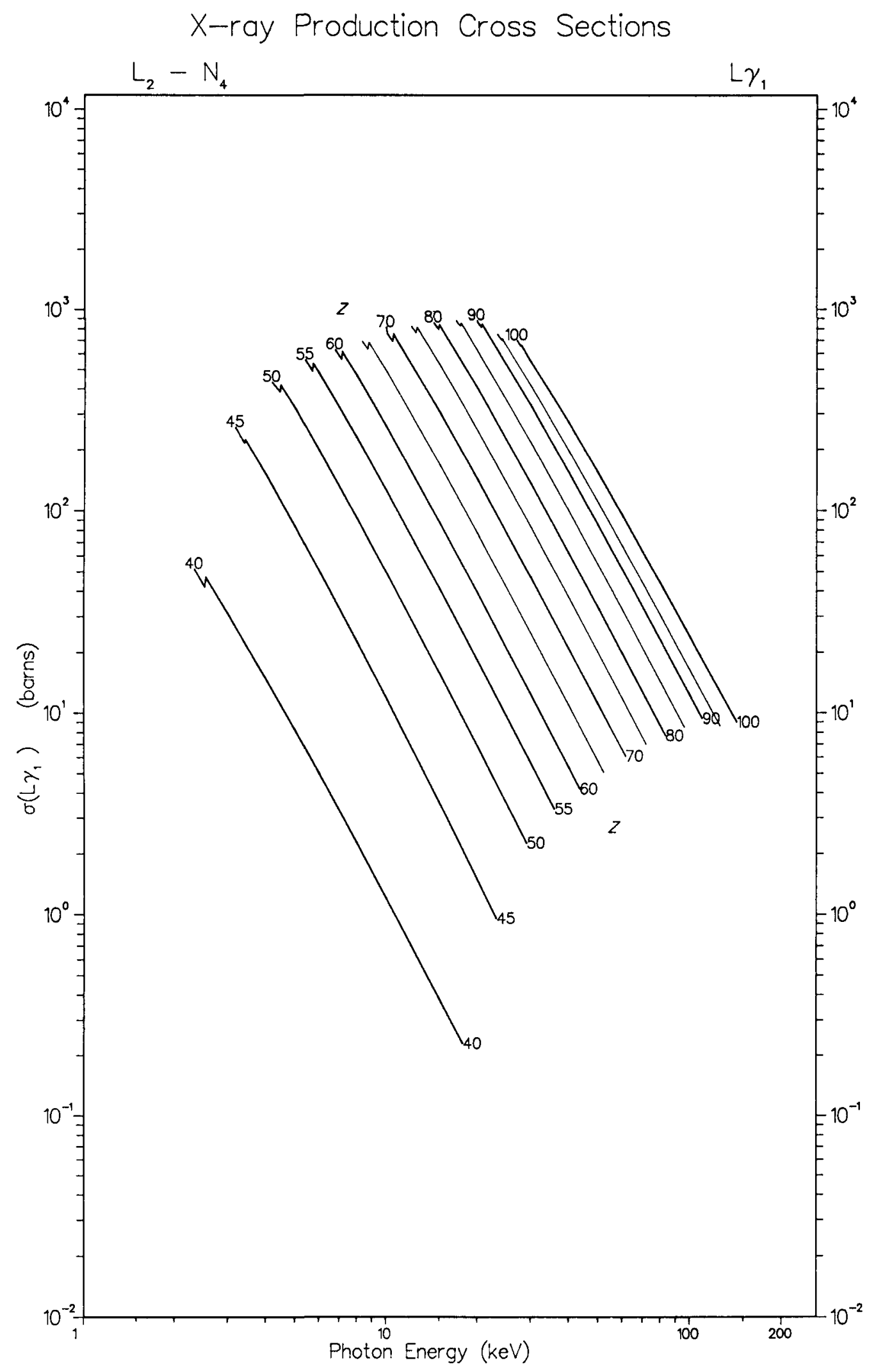

Fig. 10 
ORNL-DWG 77-21196R

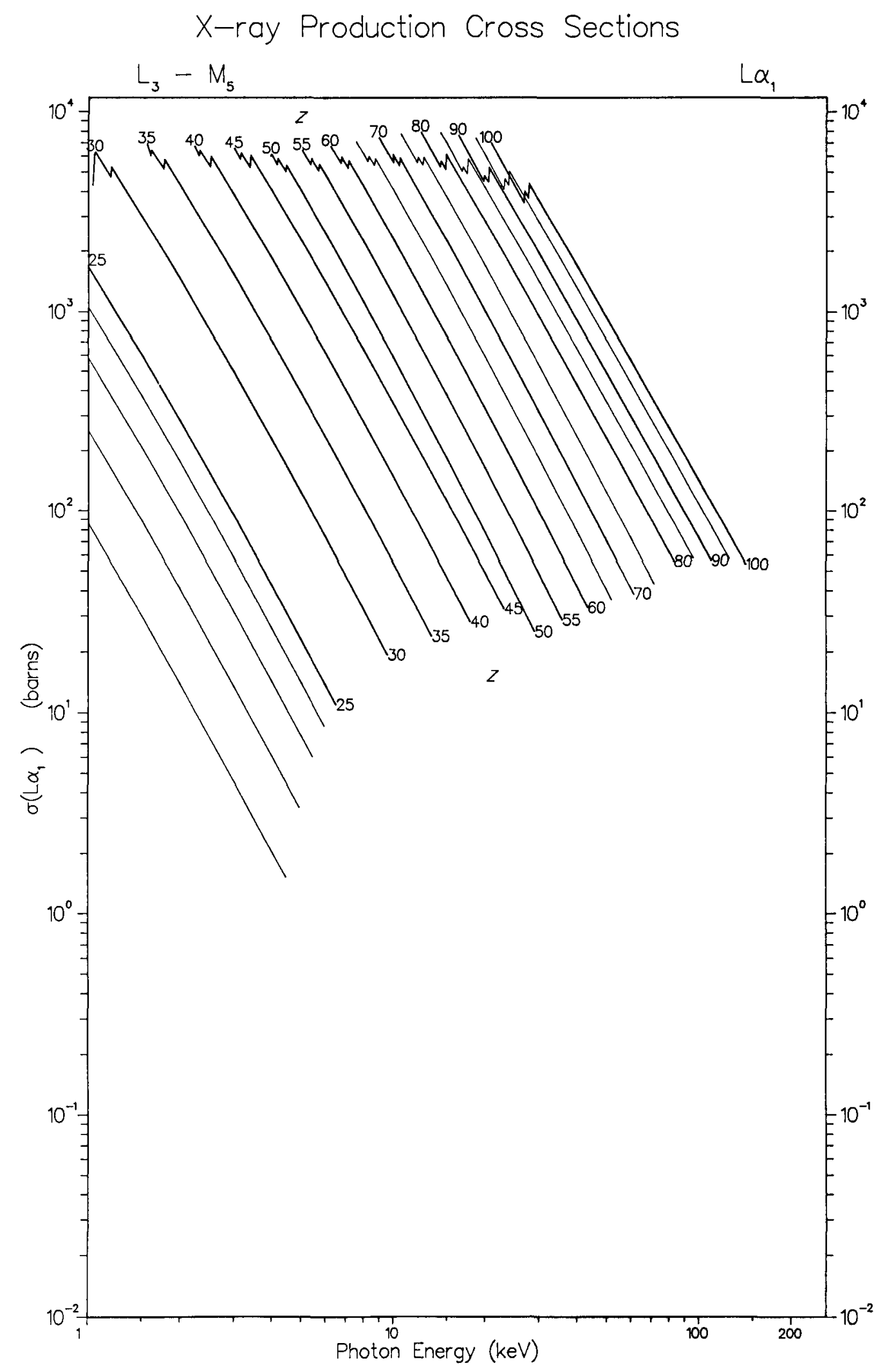

Fig. 11 


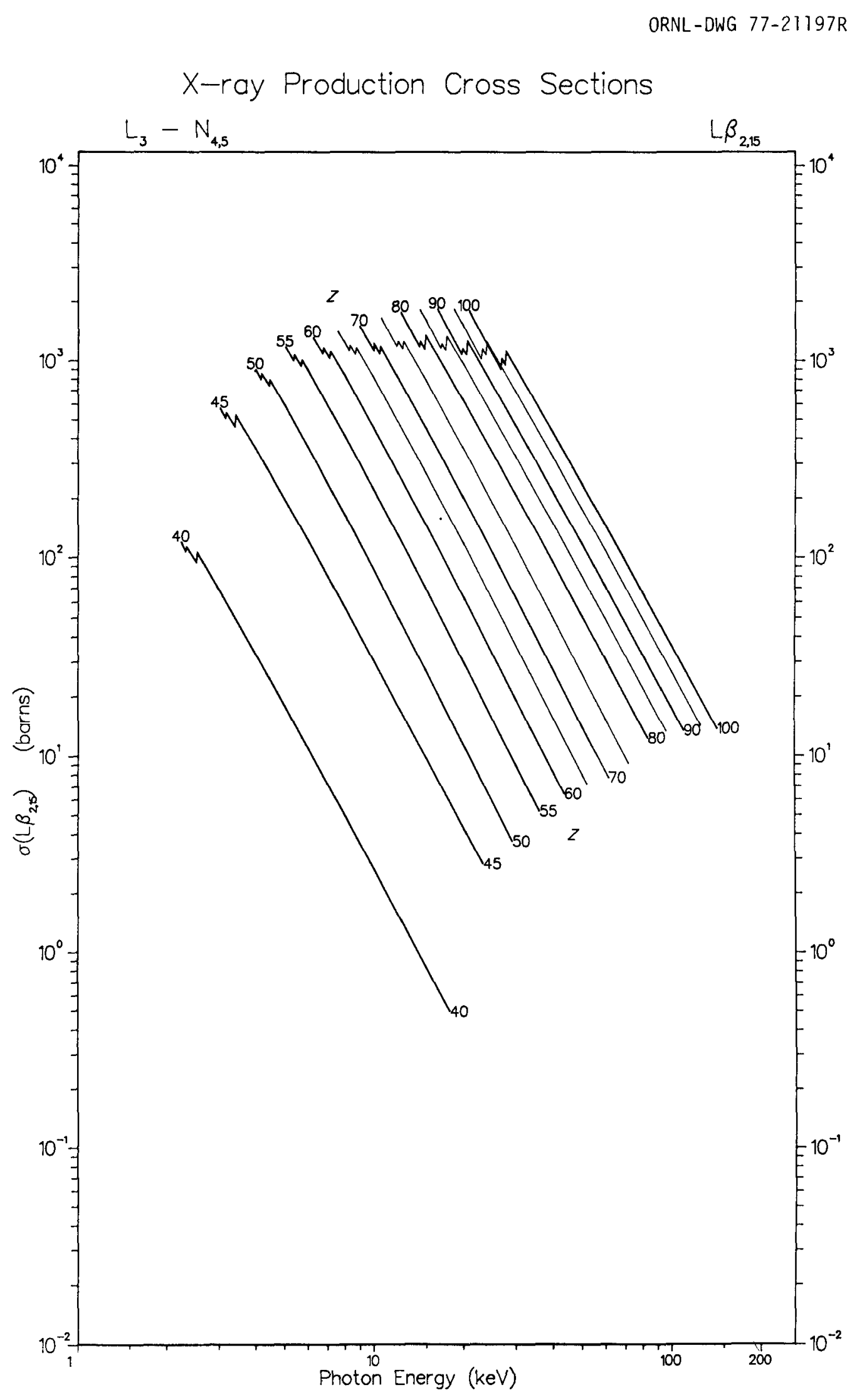

Fig. 12 
ORNL-DWG 77-21198R

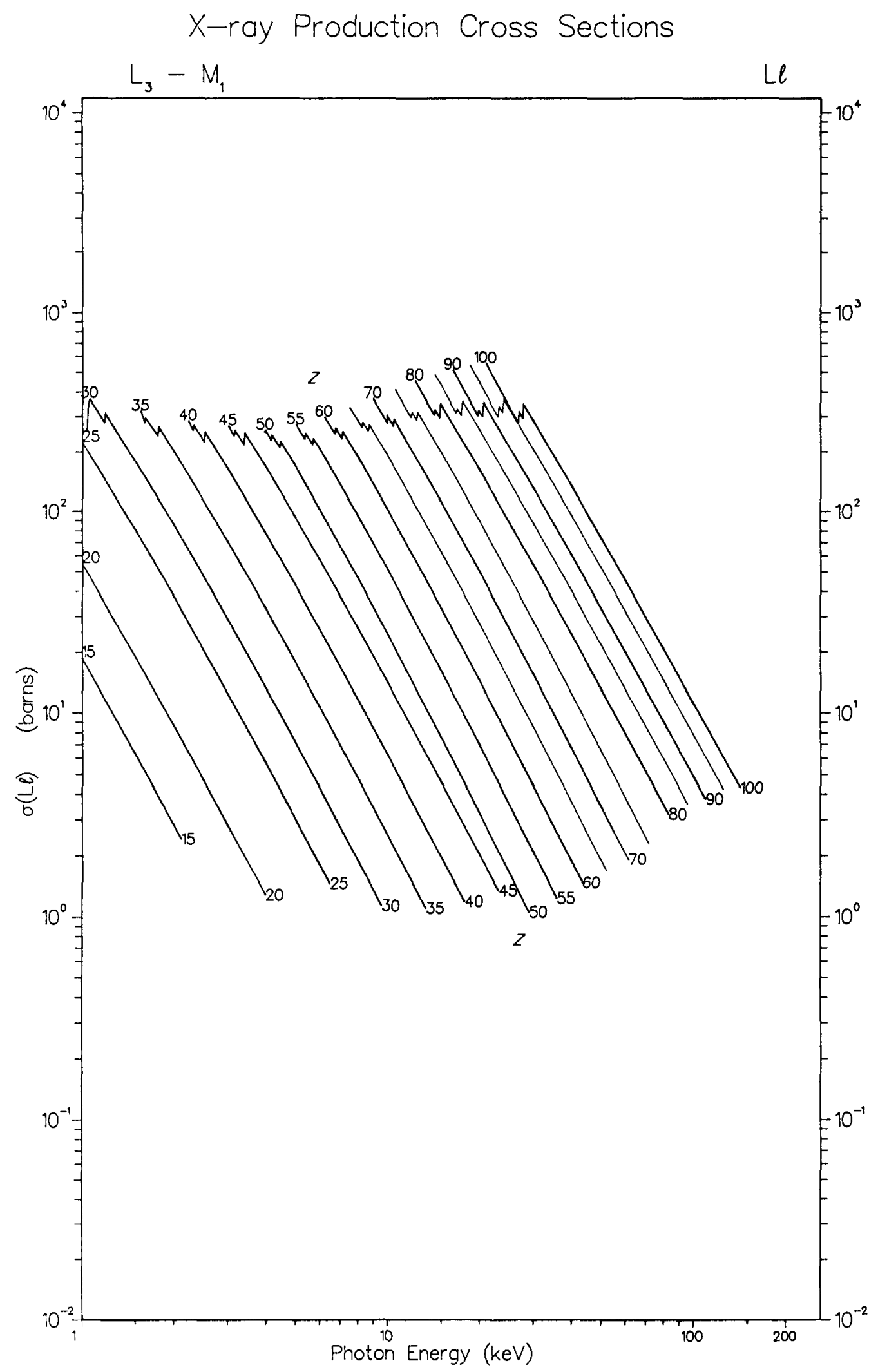

Fig. 13 
Table $9 K$-shell $\mathrm{x}$-ray fluorescence cross sections arranged in sequence by fluorescent $\mathrm{x}$ ray

Values are in units of barns/atom See Figs 14 to 17 for graphic representation

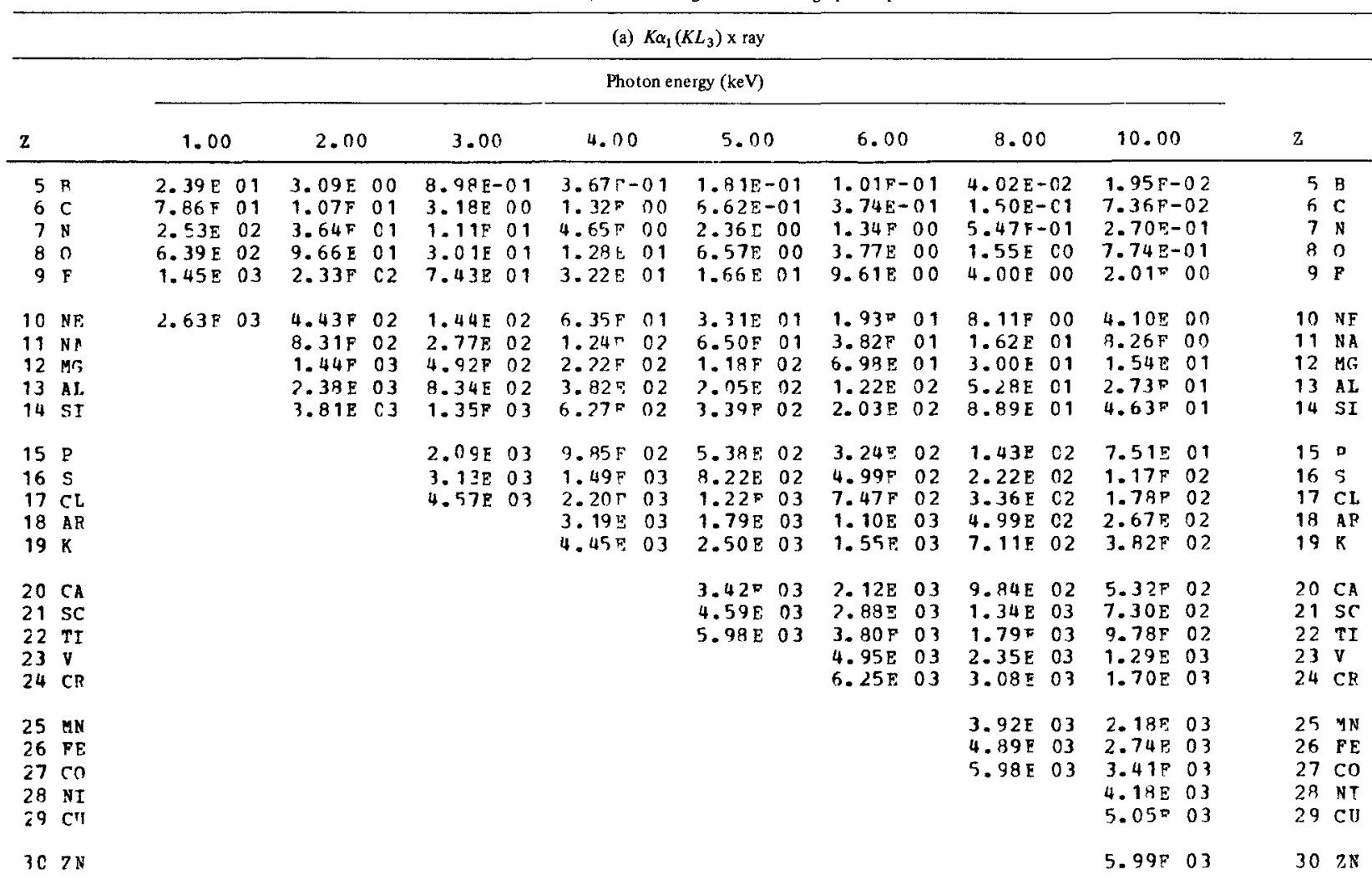


Table 9 (continued)

(a) $K \alpha_{1}\left(K L_{3}\right) \times$ ray

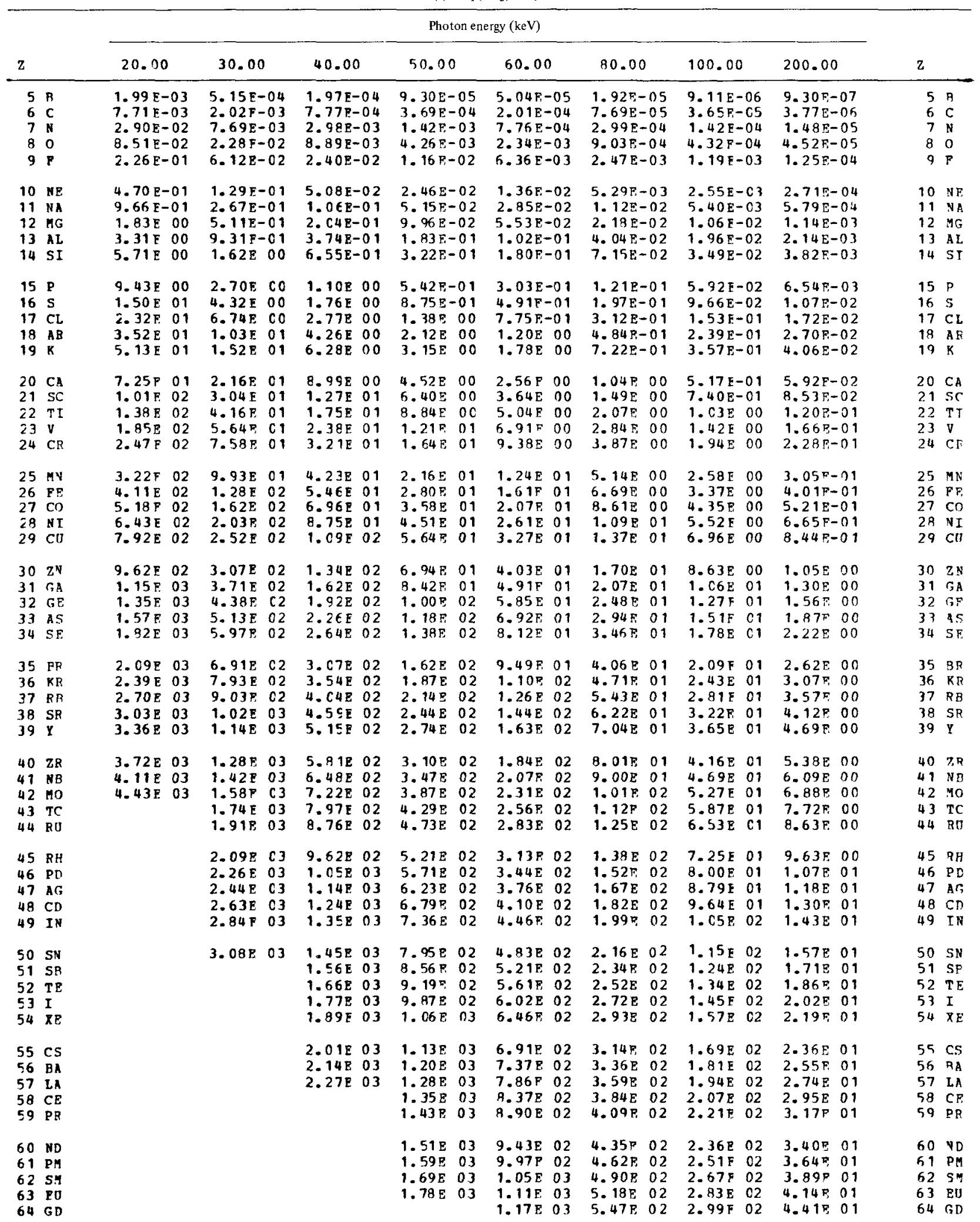


Table 9 (continued)

(a) $K \alpha_{1}\left(K L_{3}\right) \times$ ray

\begin{tabular}{|c|c|c|c|c|c|c|c|c|c|c|c|c|}
\hline \multirow{2}{*}{\multicolumn{2}{|c|}{$\mathbf{z}$}} & \multicolumn{9}{|c|}{ Photon energy (keV) } & \multirow[b]{2}{*}{$\mathbf{z}$} & \\
\hline & & 20.00 & 30.00 & 40.00 & 50.00 & 60.00 & 80.00 & 100.00 & & 200.00 & & \\
\hline $\begin{array}{l}65 \\
66 \\
67 \\
68 \\
69\end{array}$ & $\begin{array}{l}\text { TB } \\
\text { DY } \\
\text { HO } \\
\text { ER } \\
\text { TH }\end{array}$ & & & & & $\begin{array}{ll}1.228 & 03 \\
1.298 & 03 \\
1.368 & 03 \\
1.438 & 03 \\
1.49 E & 03\end{array}$ & $\begin{array}{ll}5.78 \mathrm{E} & 02 \\
6.10 \mathrm{E} & 02 \\
6.42 \mathrm{E} & 02 \\
6.75 \mathrm{E} & 02 \\
7.08 \mathrm{~B} & 02\end{array}$ & $\begin{array}{l}3.17 \mathrm{E} \\
3.35 \mathrm{E} \\
3.53 \mathrm{E} \\
3.72 \mathrm{E} \\
3.91 \mathrm{E}\end{array}$ & $\begin{array}{l}02 \\
02 \\
02 \\
02 \\
02\end{array}$ & 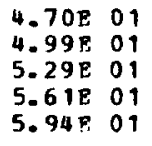 & $\begin{array}{l}65 \\
66 \\
67 \\
68 \\
69\end{array}$ & $\begin{array}{l}\text { TB } \\
\text { DY } \\
\text { HO } \\
\text { ER } \\
\text { TH }\end{array}$ \\
\hline $\begin{array}{l}70 \\
71 \\
72 \\
73 \\
74\end{array}$ & $\begin{array}{l}\text { YB } \\
\text { LU } \\
\text { HF } \\
\text { TA } \\
\text { V }\end{array}$ & & & & & & $\begin{array}{ll}7.42 \mathrm{E} & 02 \\
7.74 \mathrm{R} & 02 \\
\mathrm{B.09E} & 02 \\
8.44 \mathrm{E} & 02 \\
8.79 \mathrm{E} & 02\end{array}$ & $\begin{array}{l}4.11 E \\
4.31 E \\
4.52 E \\
4.74 E \\
4.95 E\end{array}$ & $\begin{array}{l}02 \\
02 \\
02 \\
02 \\
02\end{array}$ & 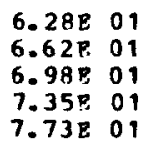 & $\begin{array}{l}70 \\
71 \\
72 \\
73 \\
74\end{array}$ & $\begin{array}{l}\text { YB } \\
\text { LU } \\
\text { HF } \\
T A \\
B\end{array}$ \\
\hline $\begin{array}{l}75 \\
76 \\
77 \\
78 \\
79\end{array}$ & $\begin{array}{l}\text { RE } \\
\text { OS } \\
\text { IR } \\
\text { PT } \\
\text { AU }\end{array}$ & & & & & & 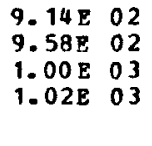 & $\begin{array}{l}5.16 \mathrm{E} \\
5.38 \mathrm{E} \\
5.60 \mathrm{E} \\
5.83 \mathrm{E} \\
6.05 \mathrm{E}\end{array}$ & $\begin{array}{l}02 \\
02 \\
02 \\
02 \\
02\end{array}$ & $\begin{array}{ll}8.10 E & 01 \\
8.51 E & 01 \\
8.938 & 01 \\
9.35 B & 01 \\
9.78 F & 01\end{array}$ & $\begin{array}{l}75 \\
76 \\
77 \\
78 \\
79\end{array}$ & $\begin{array}{l}\text { RF } \\
\text { OS } \\
\text { IP } \\
\text { PT } \\
\text { An }\end{array}$ \\
\hline $\begin{array}{l}80 \\
81 \\
82 \\
83 \\
84\end{array}$ & $\begin{array}{l}\text { HG } \\
\text { TL } \\
\text { PA } \\
\text { BI } \\
\text { PO }\end{array}$ & & & & & & & $\begin{array}{l}6.28 \mathrm{E} \\
6.51 \mathrm{E} \\
6.75 \mathrm{E} \\
7.01 \mathrm{E} \\
7.29 \mathrm{E}\end{array}$ & $\begin{array}{l}02 \\
02 \\
02 \\
02 \\
02\end{array}$ & $\begin{array}{ll}1.02 \mathrm{E} & 02 \\
1.07 \mathrm{E} & 02 \\
1.11 \mathrm{~F} & 02 \\
1.16 \mathrm{E} & 02 \\
1.21 \mathrm{E} & 02\end{array}$ & $\begin{array}{l}80 \\
81 \\
82 \\
83 \\
84\end{array}$ & $\begin{array}{l}\text { HG } \\
\text { TI } \\
\text { PB } \\
\text { BT } \\
\text { PO }\end{array}$ \\
\hline $\begin{array}{l}85 \\
86 \\
87 \\
88 \\
89\end{array}$ & $\begin{array}{l}A T \\
R N \\
F R \\
R A \\
A C\end{array}$ & & & & & & & $\begin{array}{l}7.49 \mathrm{E} \\
7.76 \mathrm{E}\end{array}$ & $\begin{array}{l}02 \\
02\end{array}$ & $\begin{array}{ll}1.26 \mathrm{P} & 02 \\
1.30 \mathrm{E} & 02 \\
1.35 \mathrm{E} & 02 \\
1.40 \mathrm{E} & 02 \\
1.46 \mathrm{E} & 02\end{array}$ & $\begin{array}{l}85 \\
86 \\
87 \\
88 \\
89\end{array}$ & $\begin{array}{l}A 7 \\
R N \\
P R \\
R A \\
\text { IC }\end{array}$ \\
\hline $\begin{array}{l}90 \\
91 \\
92 \\
93 \\
94\end{array}$ & $\begin{array}{l}\text { TH } \\
\text { PA } \\
\text { U } \\
\text { NP } \\
\text { PU }\end{array}$ & & & & & & & & & $\begin{array}{ll}1.51 \mathrm{P}, & 02 \\
1.56 \mathrm{P} & 02 \\
1.62 \mathrm{~B} & 02 \\
1.67 \mathrm{~B} & 02 \\
1.73 \mathrm{~F} & 02\end{array}$ & $\begin{array}{l}90 \\
91 \\
92 \\
93 \\
94\end{array}$ & $\begin{array}{l}\text { TH } \\
P A \\
\text { O } \\
\text { NP } \\
\text { P D }\end{array}$ \\
\hline $\begin{array}{l}95 \\
96 \\
97 \\
98 \\
99\end{array}$ & $\begin{array}{l}\text { AM } \\
\text { CM } \\
\text { BR } \\
\text { CP } \\
\text { ES }\end{array}$ & & & & & & & & & $\begin{array}{cc}1.78 F & 02 \\
1.83 \mathrm{P} & 02 \\
1.89 \mathrm{E} & 02 \\
1.95 \mathrm{P} & 02 \\
2.00 \mathrm{P} & 02\end{array}$ & $\begin{array}{l}95 \\
96 \\
97 \\
98 \\
99\end{array}$ & $\begin{array}{l}\text { AM } \\
\text { CH } \\
B K \\
\text { CF } \\
\text { FS }\end{array}$ \\
\hline $\begin{array}{l}100 \\
101\end{array}$ & $\begin{array}{l}\mathrm{FM} \\
\mathrm{HD}\end{array}$ & & & & & & & & & 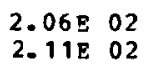 & $\begin{array}{l}100 \\
101\end{array}$ & FM \\
\hline
\end{tabular}


Table 9 (continued)

(b) $K \alpha_{1,2}\left(K L_{2,3}\right) \times$ ray

Photon energy (kev)

\begin{tabular}{|c|c|c|c|c|c|c|c|c|c|c|c|c|c|c|c|c|}
\hline \multicolumn{2}{|l|}{$z$} & \multicolumn{2}{|c|}{1.00} & \multicolumn{2}{|c|}{2.00} & \multicolumn{2}{|c|}{3.00} & \multicolumn{2}{|l|}{4.00} & 5.00 & 6.00 & \multicolumn{2}{|c|}{8.00} & 10.00 & \multicolumn{2}{|l|}{2} \\
\hline $\begin{array}{l}5 \\
6 \\
7 \\
8 \\
9\end{array}$ & $\begin{array}{l}B \\
C \\
N \\
0 \\
F\end{array}$ & $\begin{array}{l}3.59 \mathrm{E} \\
1.18 \mathrm{E} \\
3.81 \mathrm{E} \\
9.59 \mathrm{E} \\
2.18 \mathrm{~F}\end{array}$ & $\begin{array}{l}01 \\
02 \\
02 \\
02 \\
03\end{array}$ & $\begin{array}{l}4.64 \mathrm{E} \\
1.60 \mathrm{E} \\
5.47 \mathrm{E} \\
1.45 \mathrm{E} \\
3.50 \mathrm{E}\end{array}$ & $\begin{array}{ll}0 & 0 \\
01 & 1 \\
01 & 1 \\
02 \\
\mathrm{C} 2\end{array}$ & $\begin{array}{l}1.35 \mathrm{E} \\
4.77 \mathrm{E} \\
1.66 \mathrm{E} \\
4.52 \mathrm{E} \\
1.12 \mathrm{E}\end{array}$ & $\begin{array}{ll}0 & 0 \\
0 & 0 \\
0 & 1 \\
0 & 1 \\
0 & 2\end{array}$ & $\begin{array}{l}5.50 \mathrm{E}- \\
1.98 \mathrm{P} \\
7.00 \mathrm{E} \\
1.93 \mathrm{P} \\
4.84 \mathrm{E}\end{array}$ & $\begin{array}{ll}0 & 1 \\
0 & 0 \\
0 & 0 \\
0 & 1 \\
0 & 1\end{array}$ & $\begin{array}{l}2.72 \mathrm{E}-01 \\
9.91 \mathrm{E}-01 \\
3.54 \mathrm{E} 00 \\
9.96 \mathrm{E} 00 \\
2.50 \mathrm{~F} 01\end{array}$ & $\begin{array}{l}1.52 \mathrm{E}-01 \\
5.60 \mathrm{E}-01 \\
2.02 \mathrm{E}-00 \\
5.66 \mathrm{E} 00 \\
1.44 \mathrm{E} 01\end{array}$ & $\begin{array}{l}6.03 \mathrm{E}- \\
2.25 \mathrm{~F}- \\
8.23 \mathrm{~F}- \\
2.33 \mathrm{~F} \\
6.01 \mathrm{E}\end{array}$ & $\begin{array}{r}-02 \\
-01 \\
-01 \\
00 \\
00\end{array}$ & $\begin{array}{l}2.92 E-02 \\
1.10 F-01 \\
4.06 F-01 \\
1.16 E 00 \\
3.02 E 00\end{array}$ & $\begin{array}{l}5 \\
6 \\
7 \\
8 \\
9\end{array}$ & $\begin{array}{l}B \\
C \\
N \\
0 \\
P\end{array}$ \\
\hline $\begin{array}{l}10 \\
11 \\
12 \\
13 \\
14\end{array}$ & $\begin{array}{l}\text { NE } \\
\text { NA } \\
\text { MG } \\
\text { AL } \\
\text { SI }\end{array}$ & $3.95 \mathrm{E}$ & 03 & $\begin{array}{l}6.66 \mathrm{E} \\
1.25 \mathrm{E} \\
2.17 \mathrm{E} \\
3.58 \mathrm{E} \\
5.73 \mathrm{E}\end{array}$ & $\begin{array}{l}02 \\
03 \\
03 \\
03 \\
03\end{array}$ & $\begin{array}{l}2.17 \mathrm{E} \\
4.17 \mathrm{~F} \\
7.41 \mathrm{E} \\
1.25 \mathrm{E} \\
2.03 \mathrm{E}\end{array}$ & $\begin{array}{l}02 \\
02 \\
02 \\
03 \\
03\end{array}$ & $\begin{array}{l}9.54 \mathrm{E} \\
1.85 \mathrm{E} \\
3.34 \mathrm{E} \\
5.74 \mathrm{E} \\
9.43 \mathrm{E}\end{array}$ & $\begin{array}{l}01 \\
02 \\
02 \\
02 \\
02\end{array}$ & $\begin{array}{ll}4.97 \mathrm{E} & 01 \\
9.76 \mathrm{~F} & 01 \\
1.78 \mathrm{~F} & 02 \\
3.08 \mathrm{E} & 02 \\
5.10 \mathrm{E} & 02\end{array}$ & $\begin{array}{ll}2.90 \mathrm{E} & 01 \\
5.73 \mathrm{E} & 01 \\
1.05 \mathrm{E} & 02 \\
1.83 \mathrm{E} & 02 \\
3.05 \mathrm{E} & 02\end{array}$ & $\begin{array}{l}1.22 \mathrm{E} \\
2.44 \mathrm{E} \\
4.51 \mathrm{E} \\
7.94 \mathrm{E} \\
1.34 \mathrm{E}\end{array}$ & $\begin{array}{l}01 \\
01 \\
01 \\
01 \\
02\end{array}$ & $\begin{array}{ll}6.16 \mathrm{E} & 00 \\
1.24 \mathrm{E} & 01 \\
2.31 \mathrm{~F} & 01 \\
4.10 \mathrm{~F} & 01 \\
6.96 \mathrm{~F} & 01\end{array}$ & $\begin{array}{l}10 \\
11 \\
12 \\
13 \\
14\end{array}$ & $\begin{array}{l}\text { NE } \\
\text { NA } \\
\text { NG } \\
\text { AL } \\
\text { SI }\end{array}$ \\
\hline $\begin{array}{l}15 \\
16 \\
17 \\
18 \\
19\end{array}$ & $\begin{array}{l}\text { P } \\
\text { S } \\
\text { CL } \\
\text { A R } \\
\mathrm{K}\end{array}$ & & & & & $\begin{array}{l}3.15 \mathrm{E} \\
4.72 \mathrm{E} \\
6.88 \mathrm{E}\end{array}$ & $\begin{array}{l}03 \\
03 \\
03\end{array}$ & $\begin{array}{l}1.48 \mathrm{E} \\
2.24 \mathrm{E} \\
3.31 \mathrm{P} \\
4.80 \mathrm{E} \\
6.69 \mathrm{P}\end{array}$ & $\begin{array}{l}03 \\
03 \\
03 \\
03 \\
03\end{array}$ & $\begin{array}{ll}9.09 \mathrm{E} & 02 \\
1.24 \mathrm{E} & 03 \\
1.84 \mathrm{E} & 03 \\
2.69 \mathrm{E} & 03 \\
3.77 \mathrm{E} & 03\end{array}$ & $\begin{array}{ll}4.88 \mathrm{E} & 02 \\
7.51 \mathrm{E} & 02 \\
1.12 \mathrm{~F} & 0.3 \\
1.65 \mathrm{~F} & 03 \\
2.33 \mathrm{~F} & 03\end{array}$ & $\begin{array}{l}2.16 \mathrm{E} \\
3.35 \mathrm{E} \\
5.06 \mathrm{E} \\
7.52 \mathrm{E} \\
1.07 \mathrm{E}\end{array}$ & $\begin{array}{l}02 \\
02 \\
02 \\
02 \\
03\end{array}$ & $\begin{array}{ll}1.13 \mathrm{~F} & 02 \\
1.76 \mathrm{~F} & 02 \\
2.69 \mathrm{~F} & 02 \\
4.015 & 02 \\
5.75 \mathrm{~F} & 02\end{array}$ & $\begin{array}{l}15 \\
16 \\
17 \\
18 \\
19\end{array}$ & $\begin{array}{l}\text { P } \\
S \\
C L \\
\text { AP } \\
R\end{array}$ \\
\hline $\begin{array}{l}25 \\
26 \\
27 \\
28 \\
29\end{array}$ & $\begin{array}{l}\text { MN } \\
\text { PP } \\
\text { CO } \\
\text { NI } \\
\text { CU }\end{array}$ & & & & & & & & & & & $\begin{array}{l}5.92 E \\
7.39 E \\
9.04 E\end{array}$ & $\begin{array}{l}03 \\
03 \\
03\end{array}$ & $\begin{array}{ll}3.29 E & 03 \\
4.15 E & 03 \\
5.16 F & 03 \\
6.32 F & 03 \\
7.65 E & 03\end{array}$ & $\begin{array}{l}25 \\
26 \\
27 \\
28 \\
29\end{array}$ & $\begin{array}{l}M N \\
\text { FF } \\
C O \\
\text { NT } \\
C D\end{array}$ \\
\hline 30 & $\mathrm{ZN}$ & & & & & & & & & & & & & $9.08 \mathrm{~F}, 03$ & 30 & $\mathrm{ZN}$ \\
\hline
\end{tabular}


Table 9 (continued)

(b) $K \alpha_{1,2}\left(K L_{2,3}\right) \times$ ray

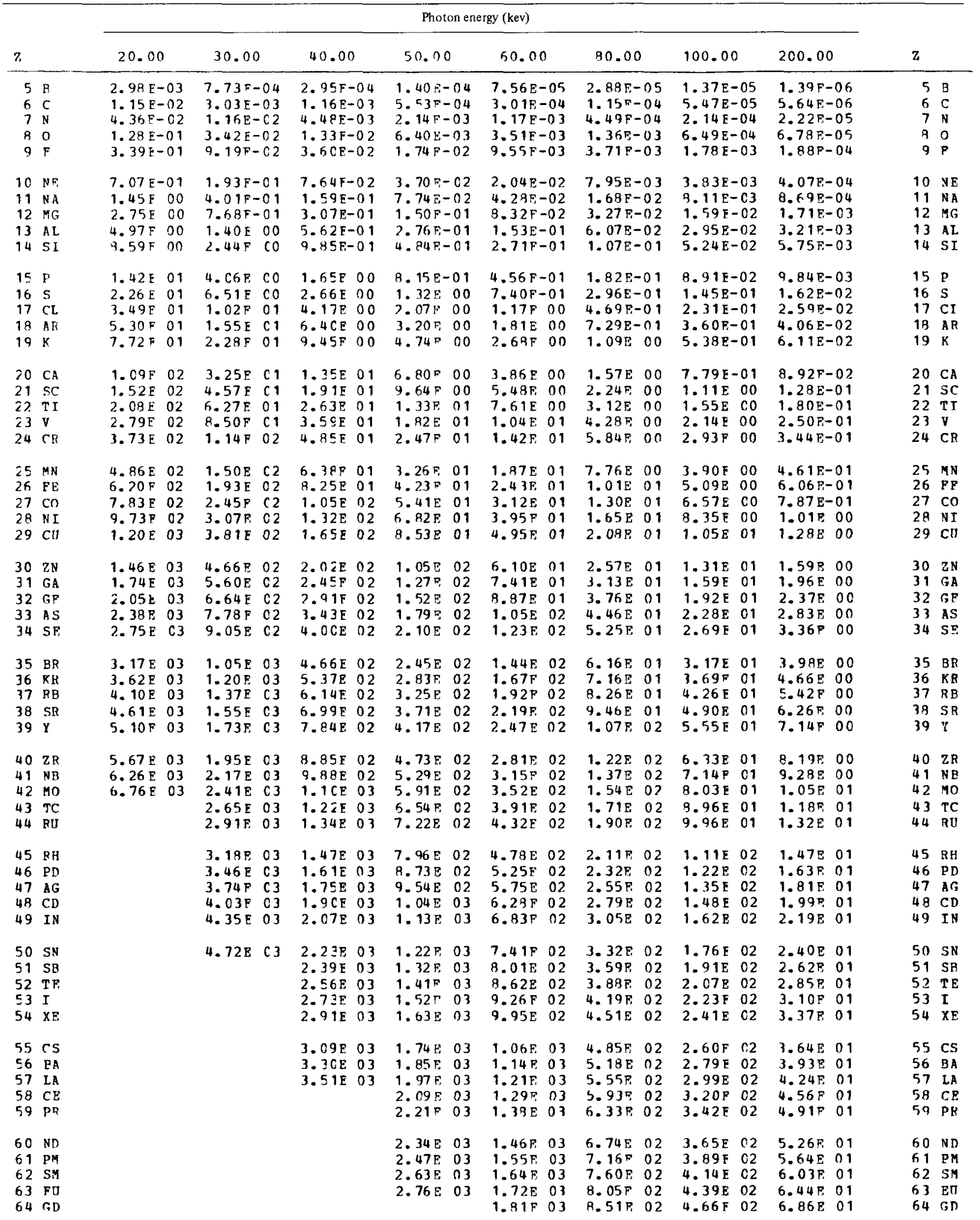


Table 9 (continued)

(b) $K \alpha_{1,2}\left(K L_{2,3}\right) \times$ ray

Photon energy (kev)

\begin{tabular}{|c|c|c|c|c|c|c|c|c|c|c|c|c|c|c|}
\hline \multirow[b]{2}{*}{$\mathrm{z}$} & & \multicolumn{11}{|c|}{ Photon energy (kev) } & \multirow{2}{*}{\multicolumn{2}{|c|}{$z$}} \\
\hline & & 20.00 & 30.00 & 40.00 & 50.00 & 60.00 & 80.00 & & 100.00 & & 200.00 & & & \\
\hline $\begin{array}{l}65 \\
66 \\
67 \\
68 \\
69\end{array}$ & $\begin{array}{l}T B \\
P Y \\
H O \\
F R \\
T M\end{array}$ & & & & & $\begin{array}{ll}1.91 \mathrm{E} & 03 \\
2.00 \mathrm{E} & 03 \\
2.12 \mathrm{E} & 03 \\
2.24 \mathrm{E} & 03 \\
2.33 \mathrm{E} & 03\end{array}$ & $\begin{array}{l}9.01 \mathrm{E} \\
9.51 \mathrm{E} \\
1.00 \mathrm{E} \\
1.06 \mathrm{E} \\
1.11 \mathrm{~F}\end{array}$ & $\begin{array}{l}02 \\
02 \\
03 \\
03 \\
03\end{array}$ & $\begin{array}{l}4.94 \mathrm{E} \\
5.22 \mathrm{E} \\
5.52 \mathrm{E} \\
5.82 \mathrm{E} \\
6.13 \mathrm{E}\end{array}$ & $\begin{array}{l}\mathrm{C} 2 \\
02 \\
\mathrm{C} 2 \\
\mathrm{C} 2 \\
\mathrm{O} 2\end{array}$ & $\begin{array}{l}7.32 \mathrm{~F} \\
7.78 \mathrm{E} \\
8.26 \mathrm{~F} \\
8.78 \mathrm{~F} \\
9.29 \mathrm{E}\end{array}$ & $\begin{array}{l}01 \\
01 \\
01 \\
01 \\
01 \\
01\end{array}$ & $\begin{array}{l}65 \\
66 \\
67 \\
68 \\
69\end{array}$ & $\begin{array}{l}T P \\
D Y \\
H O \\
\text { ER } \\
T M\end{array}$ \\
\hline $\begin{array}{l}70 \\
71 \\
72 \\
73 \\
74\end{array}$ & $\begin{array}{l}Y B \\
L U \\
H F \\
T A \\
W\end{array}$ & & & & & & $\begin{array}{l}1.16 \mathrm{E} \\
1.22 \mathrm{E} \\
1.27 \mathrm{~F} \\
1.33 \mathrm{~F} \\
1.38 \mathrm{E}\end{array}$ & $\begin{array}{l}03 \\
03 \\
03 \\
03 \\
03\end{array}$ & $\begin{array}{l}6.44 \mathrm{E} \\
6.76 \mathrm{E} \\
7.11 \mathrm{~F} \\
7.46 \mathrm{E} \\
7.80 \mathrm{E}\end{array}$ & $\begin{array}{l}02 \\
02 \\
02 \\
02 \\
02\end{array}$ & $\begin{array}{l}9.84 \mathrm{~F} \\
1.04 \mathrm{E} \\
1.10 \mathrm{E} \\
1.16 \mathrm{E} \\
1.22 \mathrm{~F}\end{array}$ & $\begin{array}{l}01 \\
02 \\
02 \\
02 \\
02\end{array}$ & $\begin{array}{l}70 \\
71 \\
72 \\
73 \\
74\end{array}$ & $\begin{array}{l}\text { YG } \\
\text { LU } \\
\text { HF } \\
\text { TA } \\
\text { W }\end{array}$ \\
\hline $\begin{array}{l}75 \\
76 \\
77 \\
78 \\
79\end{array}$ & $\begin{array}{l}\text { RE } \\
\text { OS } \\
\text { IR } \\
\text { PT } \\
\text { AU }\end{array}$ & & & & & & $\begin{array}{l}1.448 \\
1.51 \mathrm{E} \\
1.58 \mathrm{E} \\
1.62 \mathrm{E}\end{array}$ & $\begin{array}{l}03 \\
03 \\
03 \\
03\end{array}$ & $\begin{array}{l}8.13 \mathrm{~F} \\
8.50 \mathrm{~F} \\
8.86 \mathrm{~F} \\
9.23 \mathrm{E} \\
9.60 \mathrm{E}\end{array}$ & $\begin{array}{l}02 \\
\mathrm{C} 2 \\
02 \\
\mathrm{C2} \\
02\end{array}$ & $\begin{array}{l}1.28 \mathrm{~F} \\
1.34 \mathrm{~F} \\
1.41 \mathrm{~F} \\
1.48 \mathrm{~F} \\
1.55 \mathrm{~F}\end{array}$ & $\begin{array}{l}02 \\
02 \\
02 \\
02 \\
02\end{array}$ & $\begin{array}{l}75 \\
76 \\
77 \\
78 \\
79\end{array}$ & $\begin{array}{l}\text { PE } \\
\text { OS } \\
\text { IR } \\
\text { PT } \\
\text { AU }\end{array}$ \\
\hline $\begin{array}{l}80 \\
81 \\
82 \\
83 \\
84\end{array}$ & $\begin{array}{l}\text { HG } \\
\text { TL } \\
\text { PB } \\
\text { PI } \\
\text { PO }\end{array}$ & & & & & & & & $\begin{array}{l}9.98 \mathrm{E} \\
1.04 \mathrm{E} \\
1.08 \mathrm{E} \\
1.12 \mathrm{E} \\
1.17 \mathrm{E}\end{array}$ & $\begin{array}{l}02 \\
03 \\
03 \\
03 \\
03\end{array}$ & $\begin{array}{l}1.62 \mathrm{O} \\
1.70 \mathrm{E} \\
1.78 \mathrm{E} \\
1.85 \mathrm{E} \\
1.93 \mathrm{~F}\end{array}$ & $\begin{array}{l}02 \\
02 \\
02 \\
02 \\
02\end{array}$ & $\begin{array}{l}80 \\
81 \\
82 \\
83 \\
84\end{array}$ & $\begin{array}{l}\text { HG } \\
\text { TI } \\
\text { PB } \\
\text { BT } \\
\text { PO }\end{array}$ \\
\hline $\begin{array}{l}95 \\
86 \\
87 \\
88 \\
89\end{array}$ & $\begin{array}{l}A T \\
R N \\
F F \\
K A \\
A C\end{array}$ & & & & & & & & $\begin{array}{l}1.20 \mathrm{E} \\
1.25 \mathrm{E}\end{array}$ & $\begin{array}{l}03 \\
03\end{array}$ & $\begin{array}{l}2.01 \mathrm{~F} \\
2.09 \mathrm{E} \\
2.19 \mathrm{~F} \\
2.26 \mathrm{E} \\
2.35 \mathrm{~F}\end{array}$ & $\begin{array}{l}02 \\
02 \\
02 \\
02 \\
02\end{array}$ & $\begin{array}{l}85 \\
86 \\
87 \\
88 \\
89\end{array}$ & $\begin{array}{l}\text { AT } \\
\text { RN } \\
\text { PR } \\
\text { RA } \\
\text { AC }\end{array}$ \\
\hline $\begin{array}{l}90 \\
91 \\
92 \\
93 \\
94\end{array}$ & $\begin{array}{l}\text { IH } \\
\text { PA } \\
\text { U } \\
\text { NP } \\
\text { PU }\end{array}$ & & & & & & & & & & $\begin{array}{l}2.44 \mathrm{E} \\
2.54 \mathrm{~F} \\
2.63 \mathrm{E} \\
2.72 \mathrm{E} \\
2.82 \mathrm{~F}\end{array}$ & $\begin{array}{l}02 \\
02 \\
02 \\
02 \\
02\end{array}$ & $\begin{array}{l}90 \\
91 \\
92 \\
93 \\
94\end{array}$ & $\begin{array}{l}T H \\
P A \\
U \\
N P \\
P U\end{array}$ \\
\hline $\begin{array}{l}100 \\
101\end{array}$ & $\begin{array}{l}F M \\
M D\end{array}$ & & & & & & & & & & $\begin{array}{l}3.41 \mathrm{E} \\
3.50 \mathrm{E}\end{array}$ & $\begin{array}{l}02 \\
02\end{array}$ & $\begin{array}{l}100 \\
101\end{array}$ & $\begin{array}{l}\text { Fy } \\
\text { MD }\end{array}$ \\
\hline
\end{tabular}


Table 9 (continued)

(c) $K \beta_{1}\left(K M_{3}\right) \times$ ray

\begin{tabular}{|c|c|c|c|c|c|c|c|c|c|c|c|c|c|c|c|c|}
\hline \multirow[b]{3}{*}{$z$} & \multicolumn{16}{|c|}{ (c) $K \beta_{1}\left(K M_{3}\right) \times$ ray } \\
\hline & & \multicolumn{13}{|c|}{ Photon energy (keV) } & \multirow{2}{*}{\multicolumn{2}{|c|}{7}} \\
\hline & & 1.00 & 2.00 & & 3.00 & & 4.00 & & 5.00 & & 6.00 & & 8.00 & 10.00 & & \\
\hline $\begin{array}{l}13 \\
14 \\
15 \\
16 \\
17\end{array}$ & $\begin{array}{l}A L \\
S I \\
P \\
S \\
C L\end{array}$ & & $\begin{array}{ll}3.20 \mathrm{E} & 0 \\
1.12 \mathrm{~F} & 0\end{array}$ & $\begin{array}{l}01 \\
02\end{array}$ & $\begin{array}{l}1.12 \mathrm{E} \\
3.98 \mathrm{E} \\
9.87 \mathrm{E} \\
2.07 \mathrm{E} \\
3.95 \mathrm{E}\end{array}$ & $\begin{array}{ll}0 & 1 \\
0 & 1 \\
0 & 1 \\
0 & 2 \\
0 & 2\end{array}$ & $\begin{array}{l}5.12 \mathrm{~F} \\
1.34 \mathrm{E} \\
4.64 \mathrm{E} \\
9.83 \mathrm{E} \\
1.90 \mathrm{E}\end{array}$ & $\begin{array}{ll}0 & 0 \\
0 & 1 \\
0 & 1 \\
0 & 1 \\
0 & 2\end{array}$ & $\begin{array}{l}2.74 \mathrm{E} \\
9.97 \mathrm{E} \\
2.53 \mathrm{E} \\
5.42 \mathrm{E} \\
1.05 \mathrm{E}\end{array}$ & $\begin{array}{ll}0 & 0 \\
0 & 0 \\
0 & 1 \\
0 & 1 \\
0 & 2\end{array}$ & $\begin{array}{l}1.63 \mathrm{E} \\
5.97 \mathrm{E} \\
7.53 \mathrm{E} \\
3.29 \mathrm{E} \\
6.45 \mathrm{~F}\end{array}$ & $\begin{array}{ll}0 & 0 \\
0 & 0 \\
0 & 1 \\
0 & 1 \\
0 & 1\end{array}$ & $\begin{array}{ll}7.08 \mathrm{E} & -01 \\
2.62 \mathrm{E} & 00 \\
6.76 \mathrm{E} & 00 \\
1.47 \mathrm{E} & 01 \\
2.90 \mathrm{E} & 01\end{array}$ & 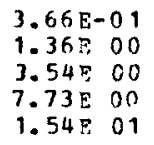 & $\begin{array}{l}13 \\
14 \\
15 \\
16 \\
17\end{array}$ & $\begin{array}{l}\text { AL } \\
\text { SI } \\
P \\
\text { S } \\
\text { CL. }\end{array}$ \\
\hline $\begin{array}{l}18 \\
19 \\
20 \\
21 \\
22\end{array}$ & $\begin{array}{l}A R \\
K \\
C A \\
S C \\
T I\end{array}$ & & & & & & $\begin{array}{l}3.47 \mathrm{E} \\
5.39 \mathrm{~F}\end{array}$ & $\begin{array}{l}02 \\
02\end{array}$ & $\begin{array}{l}1.95 E \\
3.03 F \\
4.50 E \\
6.14 E \\
8.12 E\end{array}$ & $\begin{array}{l}02 \\
02 \\
02 \\
02 \\
02\end{array}$ & $\begin{array}{l}1.20 \mathrm{E} \\
1.88 \mathrm{E} \\
2.79 \mathrm{E} \\
3.84 \mathrm{~F} \\
5.16 \mathrm{~F}\end{array}$ & $\begin{array}{l}02 \\
02 \\
02 \\
02 \\
02\end{array}$ & $\begin{array}{ll}5.44 \mathrm{E} & 01 \\
8.62 \mathrm{E} & 01 \\
1.30 \mathrm{~F} & 02 \\
1.79 \mathrm{~F} & 02 \\
2.43 \mathrm{~F} & 02\end{array}$ & $\begin{array}{ll}2.91 \mathrm{E} & 01 \\
4.63 \mathrm{E} & 01 \\
7.01 \mathrm{E} & 01 \\
9.75 \mathrm{~F} & 01 \\
1.33 \mathrm{E} & 02\end{array}$ & $\begin{array}{l}18 \\
19 \\
20 \\
21 \\
2 ?\end{array}$ & $\begin{array}{l}A R \\
R \\
C A \\
S C \\
T I\end{array}$ \\
\hline $\begin{array}{l}23 \\
24 \\
25 \\
26 \\
27\end{array}$ & $\begin{array}{l}V \\
\mathrm{CR} \\
\mathrm{MN} \\
\mathrm{EE} \\
\mathrm{CO}\end{array}$ & & & & & & & & & & $\begin{array}{l}6.77 \mathrm{E} \\
8.39 \mathrm{E}\end{array}$ & $\begin{array}{l}02 \\
02\end{array}$ & $\begin{array}{ll}3.22 \mathrm{E} & \mathrm{C2} \\
4.12 \mathrm{~F} & 02 \\
5.44 \mathrm{E} & 02 \\
6.32 \mathrm{~F} & 02 \\
9.37 \mathrm{E} & 02\end{array}$ & $\begin{array}{ll}1.77 \mathrm{E} & 02 \\
2.28 \mathrm{~F} & 02 \\
3.02 \mathrm{E} & 02 \\
3.83 \mathrm{~F} & 0 ? \\
4.77 \mathrm{~F}, & 02\end{array}$ & $\begin{array}{l}23 \\
24 \\
25 \\
2.6 \\
27\end{array}$ & $\begin{array}{l}V \\
C R \\
\text { IN } \\
\text { FE } \\
\text { CO }\end{array}$ \\
\hline $\begin{array}{l}28 \\
29 \\
30\end{array}$ & $\begin{array}{l}\mathrm{NI} \\
\mathrm{CU} \\
\mathrm{ZN}\end{array}$ & & & & & & & & & & & & & $\begin{array}{ll}5.87 F & 02 \\
6.98 \mathrm{E} & 02 \\
8.47 \mathrm{E} & 02\end{array}$ & $\begin{array}{l}28 \\
29 \\
30\end{array}$ & $\begin{array}{l}\mathrm{NI} \\
\mathrm{CUJ} \\
\mathrm{ZN}\end{array}$ \\
\hline
\end{tabular}


Table 9 (continued)

(c) $K \beta_{1}\left(K M_{3}\right) \times$ ray

\begin{tabular}{|c|c|c|c|c|c|c|c|c|c|c|c|c|}
\hline \multirow{2}{*}{\multicolumn{2}{|c|}{$\mathbf{z}$}} & \multicolumn{9}{|c|}{ Photon energy (keV) } & & \\
\hline & & 20.00 & 30.00 & 40.00 & 50.00 & & 60.00 & 80.00 & 100.00 & 200.00 & $\mathrm{z}$ & \\
\hline $\begin{array}{l}13 \\
14 \\
15 \\
16 \\
17\end{array}$ & $\begin{array}{l}A L \\
S I \\
P \\
S \\
C L\end{array}$ & $\begin{array}{l}4.44 \mathrm{E}-02 \\
1.68 \mathrm{~F}-01 \\
4.44 \mathrm{E}-01 \\
9.88 \mathrm{E}-01 \\
2.00 \mathrm{E} 00\end{array}$ & $\begin{array}{l}1.25 E-02 \\
4.77 E-02 \\
1.27 E-01 \\
2.85 E-01 \\
5.82 E-01\end{array}$ & $\begin{array}{l}5.02 \mathrm{~B}-03 \\
1.93 \mathrm{E}-02 \\
5.17 \mathrm{E}-02 \\
1.16 \mathrm{E}-01 \\
2.39 \mathrm{E}-01\end{array}$ & $\begin{array}{l}2.46 \mathrm{E} \\
9.48 \mathrm{E} \\
2.55 \mathrm{~F} \\
5.77 \mathrm{E} \\
1.19 \mathrm{E}\end{array}$ & $\begin{array}{l}-03 \\
-03 \\
-02 \\
-02 \\
-01\end{array}$ & $\begin{array}{l}1.37 E-03 \\
5.29 E-03 \\
1.43 E-02 \\
3.24 E-02 \\
6.69 E-02\end{array}$ & $\begin{array}{l}5.41 \mathrm{E}-04 \\
2.10 \mathrm{E}-03 \\
5.70 \mathrm{P}-03 \\
1.30 \mathrm{E}-02 \\
2.69 \mathrm{E}-02\end{array}$ & $\begin{array}{l}2.63 E-04 \\
1.03 E-03 \\
2.79 E-03 \\
6.37 E-03 \\
1.32 E-02\end{array}$ & $\begin{array}{l}2.86 R-05 \\
1.12 E-04 \\
3.08 R-04 \\
7.08 E-04 \\
1.48 R-03\end{array}$ & $\begin{array}{l}13 \\
14 \\
15 \\
16 \\
17\end{array}$ & $\begin{array}{l}\text { AL } \\
S I \\
p \\
S \\
C L\end{array}$ \\
\hline $\begin{array}{l}18 \\
19 \\
20 \\
21 \\
22\end{array}$ & $\begin{array}{l}A R \\
K \\
C A \\
S C \\
T I\end{array}$ & $\begin{array}{ll}3.84 \mathrm{E} & 00 \\
6.22 \mathrm{E} & 00 \\
9.55 \mathrm{E} & 00 \\
1.35 \mathrm{E} & 01 \\
1.87 \mathrm{E} & 01\end{array}$ & 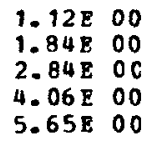 & $\begin{array}{l}4.64 \mathrm{E}-01 \\
7.61 \mathrm{E}-01 \\
1.18 \mathrm{E} 00 \\
1.7 \mathrm{CE} 00 \\
2.37 \mathrm{E} 00\end{array}$ & $\begin{array}{l}2.32 \mathrm{E} \\
3.81 \mathrm{E}- \\
5.95 \mathrm{E}- \\
8.56 \mathrm{E}- \\
1.20 \mathrm{E}\end{array}$ & $\begin{array}{rl}-0 & 1 \\
-0 & 1 \\
-0 & 1 \\
-0 & 1 \\
0 & 0\end{array}$ & $\begin{array}{l}1.31 E-01 \\
2.16 \mathrm{~F}-01 \\
3.38 \mathrm{E}-01 \\
4.87 \mathrm{E}-01 \\
6.85 \mathrm{E}-01\end{array}$ & $\begin{array}{l}\text { 5. } 28 \mathrm{E}-02 \\
8.75 \mathrm{E}-02 \\
1.37 \mathrm{E}-01 \\
1.99 \mathrm{E}-01 \\
2.81 \mathrm{E}-01\end{array}$ & $\begin{array}{l}2.60 \mathrm{E}-02 \\
4.33 \mathrm{E}-02 \\
6.82 \mathrm{E}-02 \\
9.89 \mathrm{E}-02 \\
1.40 \mathrm{E}-01\end{array}$ & $\begin{array}{l}2.948-03 \\
4.92 F-03 \\
7.80 E-03 \\
1.14 F-02 \\
1.62 E-02\end{array}$ & $\begin{array}{l}18 \\
19 \\
20 \\
21 \\
22\end{array}$ & $\begin{array}{l}A R \\
K \\
C A \\
S C \\
T I\end{array}$ \\
\hline $\begin{array}{l}23 \\
24 \\
25 \\
26 \\
27\end{array}$ & $\begin{array}{l}\nabla \\
C R \\
M N \\
P E \\
C O\end{array}$ & $\begin{array}{ll}2.54 \mathrm{E} & 01 \\
3.31 \mathrm{E} & 01 \\
4.46 \mathrm{E} & 01 \\
5.72 \mathrm{E} & 01 \\
7.24 \mathrm{E} & 01\end{array}$ & $\begin{array}{ll}7.72 \mathrm{E} & \mathrm{CO} \\
1.01 \mathrm{E} & 01 \\
1.38 \mathrm{E} & \mathrm{C1} \\
1.78 \mathrm{E} & 01 \\
2.27 \mathrm{E} & 01\end{array}$ & 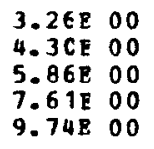 & $\begin{array}{l}1.65 \mathrm{E} \\
2.19 \mathrm{E} \\
3.00 \mathrm{E} \\
3.90 \mathrm{E} \\
5.00 \mathrm{E}\end{array}$ & $\begin{array}{ll}0 & 0 \\
0 & 0 \\
0 & 0 \\
0 & 0 \\
0 & 0\end{array}$ & $\begin{array}{rr}9.46 \mathrm{E}-01 \\
1.26 \mathrm{E} & 00 \\
1.72 \mathrm{E} & 00 \\
2.25 \mathrm{E} & 00 \\
2.89 \mathrm{E} & 00\end{array}$ & $\begin{array}{l}\text { 3. } 89 \mathrm{~F}-01 \\
5.18 \mathrm{E}-01 \\
7.13 \mathrm{E}-01 \\
9.33 \mathrm{E}-01 \\
1.20 \mathrm{E} 00\end{array}$ & $\begin{array}{l}\text { 1. } 95 \mathrm{E}-01 \\
2.60 \mathrm{E}-01 \\
3.58 \mathrm{E}-01 \\
4.70 \mathrm{E}-01 \\
6 . \mathrm{CBE}-01\end{array}$ & $\begin{array}{l}2.27 \mathrm{E}-02 \\
3.05 \mathrm{E}-02 \\
4.23 \mathrm{E}-02 \\
5.59 \mathrm{E}-02 \\
7.29 \mathrm{P}-02\end{array}$ & $\begin{array}{l}23 \\
24 \\
25 \\
26 \\
27\end{array}$ & $\begin{array}{l}V \\
C R \\
\text { HN } \\
F F \\
C O\end{array}$ \\
\hline $\begin{array}{l}28 \\
29 \\
30 \\
31 \\
32\end{array}$ & $\begin{array}{l}\text { NI } \\
\text { CO } \\
\mathrm{ZN} \\
G A \\
G P\end{array}$ & $\begin{array}{ll}9.03 \mathrm{E} & 01 \\
1.09 \mathrm{E} & 02 \\
1.36 \mathrm{E} & 02 \\
1.66 \mathrm{E} & 02 \\
1.99 \mathrm{E} & 02\end{array}$ & $\begin{array}{ll}2.85 \mathrm{E} & \mathrm{C1} \\
3.48 \mathrm{E} & 01 \\
4.34 \mathrm{E} & 01 \\
5.35 \mathrm{E} & \mathrm{C1} \\
6.47 \mathrm{E} & 01\end{array}$ & 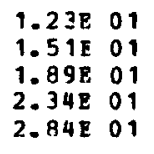 & $\begin{array}{l}6.33 \mathrm{E} \\
7.79 \mathrm{E} \\
9.80 \mathrm{E} \\
1.21 \mathrm{E} \\
1.48 \mathrm{E}\end{array}$ & $\begin{array}{ll}0 & 0 \\
0 & 0 \\
0 & 0 \\
0 & 1 \\
0 & 1\end{array}$ & $\begin{array}{ll}3.66 \mathrm{E} & 00 \\
4.52 \mathrm{E} & 00 \\
5.70 \mathrm{P} & 00 \\
7.08 \mathrm{P} & 00 \\
8.64 \mathrm{E} & 00\end{array}$ & $\begin{array}{ll}1.53 \mathrm{~F} & 00 \\
1.89 \mathrm{E} & 00 \\
2.40 \mathrm{E} & 00 \\
2.99 \mathrm{E} & 00 \\
3.66 \mathrm{E} & 00\end{array}$ & $\begin{array}{l}7.75 \mathrm{E}-01 \\
9.61 \mathrm{E}-01 \\
1.22 \mathrm{E} 00 \\
1.52 \mathrm{E} 00 \\
1.87 \mathrm{E} 00\end{array}$ & $\begin{array}{l}9.34 F-02 \\
1.178-01 \\
1.49 E-01 \\
1.87 F-01 \\
2.31 F-01\end{array}$ & $\begin{array}{l}28 \\
29 \\
30 \\
31 \\
32\end{array}$ & $\begin{array}{l}\mathrm{NI} \\
\mathrm{CU} \\
\mathrm{ZN} \\
\mathrm{GA} \\
\mathrm{GE}\end{array}$ \\
\hline $\begin{array}{l}33 \\
34 \\
35 \\
36 \\
37\end{array}$ & $\begin{array}{l}\text { AS } \\
\text { SE } \\
\text { BR } \\
\text { RR } \\
\text { RB }\end{array}$ & $\begin{array}{ll}2.37 \mathrm{E} & 02 \\
2.80 \mathrm{E} & 02 \\
3.26 \mathrm{E} & 02 \\
3.76 \mathrm{E} & 02 \\
4.33 \mathrm{E} & 02\end{array}$ & $\begin{array}{ll}7.73 \mathrm{~F} & 01 \\
9.20 \mathrm{~F} & 01 \\
1.08 \mathrm{E} & 02 \\
1.25 \mathrm{E} & 02 \\
1.45 \mathrm{~F} & 02\end{array}$ & 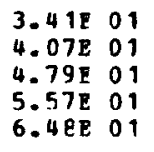 & $\begin{array}{l}1.78 \mathrm{E} \\
2.13 \mathrm{E} \\
2.52 \mathrm{E} \\
2.94 \mathrm{E} \\
3.43 \mathrm{E}\end{array}$ & $\begin{array}{ll}0 & 1 \\
0 & 1 \\
0 & 1 \\
0 & 1 \\
0 & 1\end{array}$ & $\begin{array}{ll}1.04 \mathrm{E} & 01 \\
1.25 \mathrm{P} & 01 \\
1.48 \mathrm{E} & 01 \\
1.73 \mathrm{~F} & 01 \\
2.02 \mathrm{E} & 01\end{array}$ & 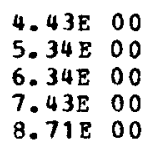 & $\begin{array}{ll}2.27 \mathrm{E} & 00 \\
2.74 \mathrm{E} & 00 \\
3.26 \mathrm{~B} & 00 \\
3.83 \mathrm{E} & 00 \\
4.50 \mathrm{E} & 00\end{array}$ & $\begin{array}{l}2.82 \mathrm{E}-01 \\
3.42 \mathrm{~F}-01 \\
4.09 \mathrm{E}-01 \\
4.84 \mathrm{~F}-01 \\
5.72 \mathrm{E}-01\end{array}$ & $\begin{array}{l}33 \\
34 \\
35 \\
36 \\
37\end{array}$ & $\begin{array}{l}A S \\
S E \\
B R \\
K R \\
R B\end{array}$ \\
\hline $\begin{array}{l}38 \\
39 \\
40 \\
41 \\
42\end{array}$ & $\begin{array}{l}S R \\
\text { IR } \\
\text { NB } \\
\text { MO }\end{array}$ & $\begin{array}{ll}4.94 \mathrm{E} & 02 \\
5.57 \mathrm{E} & 02 \\
6.26 \mathrm{E} & 02 \\
6.99 \mathrm{E} & 02 \\
7.66 \mathrm{E} & 02\end{array}$ & 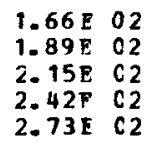 & $\begin{array}{ll}7.48 \mathrm{E} & 01 \\
8.56 \mathrm{E} & 01 \\
9.76 \mathrm{E} & 01 \\
1.1 \mathrm{CE} & 02 \\
1.25 \mathrm{E} & 02\end{array}$ & $\begin{array}{l}3.97 \mathrm{E} \\
4.56 \mathrm{E} \\
5.21 \mathrm{E} \\
5.91 \mathrm{E} \\
6.69 \mathrm{~F}\end{array}$ & $\begin{array}{ll}0 & 1 \\
0 & 1 \\
0 & 1 \\
0 & 1 \\
0 & 1\end{array}$ & $\begin{array}{ll}2.35 \mathrm{E} & 01 \\
2.70 \mathrm{E} & 01 \\
3.10 \mathrm{E} & 01 \\
3.52 \mathrm{P} & 01 \\
3.99 \mathrm{E} & 01\end{array}$ & $\begin{array}{ll}1.01 \mathrm{E} & 01 \\
1.17 \mathrm{~F} & 01 \\
1.34 \mathrm{~F} & 01 \\
1.53 \mathrm{E} & 01 \\
1.74 \mathrm{E} & 01\end{array}$ & $\begin{array}{ll}5.24 E & 00 \\
6.06 E & 00 \\
6.99 E & 00 \\
7.98 E & 00 \\
9.09 E & 00\end{array}$ & $\begin{array}{l}6.70 \mathrm{E}-01 \\
7.79 \mathrm{E}-01 \\
9.03 \mathrm{~F}-01 \\
1.04 \mathrm{~F} 00 \\
1.19 \mathrm{~F} 00\end{array}$ & $\begin{array}{l}38 \\
39 \\
40 \\
41 \\
42\end{array}$ & $\begin{array}{l}S R \\
Y \\
Z R \\
N B \\
M O\end{array}$ \\
\hline $\begin{array}{l}48 \\
49 \\
50 \\
51 \\
52\end{array}$ & $\begin{array}{l}\text { CD } \\
\text { IN } \\
\text { SN } \\
\text { SB } \\
\text { TE }\end{array}$ & & $\begin{array}{ll}4.85 \mathrm{E} & 02 \\
5.27 \mathrm{E} & \mathrm{C2} \\
5.78 \mathrm{E} & 02\end{array}$ & $\begin{array}{ll}2.29 \mathrm{E} & 02 \\
2.50 \mathrm{E} & 02 \\
2.72 \mathrm{E} & 02 \\
2.94 \mathrm{E} & 02 \\
3.18 \mathrm{E} & 02\end{array}$ & $\begin{array}{l}1.25 \mathrm{E} \\
1.37 \mathrm{~B} \\
1.49 \mathrm{E} \\
1.61 \mathrm{E} \\
1.76 \mathrm{~B}\end{array}$ & $\begin{array}{l}02 \\
02 \\
02 \\
02 \\
02\end{array}$ & $\begin{array}{ll}7.55 \mathrm{E} & 01 \\
8.28 \mathrm{E} & 01 \\
9.06 \mathrm{E} & 01 \\
9.82 \mathrm{E} & 01 \\
1.07 \mathrm{~F} & 02\end{array}$ & $\begin{array}{ll}3.36 \mathrm{E} & 01 \\
3.70 \mathrm{E} & 01 \\
4.06 \mathrm{~F} & 01 \\
4.41 \mathrm{E} & 01 \\
4.83 \mathrm{E} & 01\end{array}$ & $\begin{array}{ll}1.78 \mathrm{E} & 01 \\
1.96 \mathrm{E} & 01 \\
2.15 \mathrm{E} & 01 \\
2.34 \mathrm{E} & 01 \\
2.57 \mathrm{E} & 01\end{array}$ & $\begin{array}{ll}2.40 \mathrm{~F} & 00 \\
2.66 \mathrm{~F} & 00 \\
2.94 \mathrm{E} & 00 \\
3.22 \mathrm{E} & 00 \\
3.55 \mathrm{E} & 0 \mathrm{O}\end{array}$ & $\begin{array}{l}48 \\
49 \\
50 \\
51 \\
52\end{array}$ & $\begin{array}{l}C D \\
\text { IN } \\
S N \\
\text { SB } \\
\text { TP }\end{array}$ \\
\hline $\begin{array}{l}53 \\
54 \\
55 \\
56 \\
57\end{array}$ & $\begin{array}{l}\mathrm{I} \\
\mathrm{XE} \\
\mathrm{CS} \\
\mathrm{BA} \\
\mathrm{LA}\end{array}$ & & & $\begin{array}{ll}3.40 \mathrm{E} & 02 \\
3.67 \mathrm{E} & 02 \\
3.91 \mathrm{E} & 02 \\
4.19 \mathrm{~F} & 02 \\
4.50 \mathrm{E} & 02\end{array}$ & $\begin{array}{l}1.89 \mathrm{E} \\
2.06 \mathrm{R} \\
2.20 \mathrm{E} \\
2.35 \mathrm{E} \\
2.53 \mathrm{P}\end{array}$ & $\begin{array}{l}02 \\
02 \\
02 \\
02 \\
02\end{array}$ & $\begin{array}{ll}1.16 \mathrm{E} & 02 \\
1.25 \mathrm{E} & 02 \\
1.35 \mathrm{E} & 02 \\
1.44 \mathrm{E} & 02 \\
1.56 \mathrm{E} & 02\end{array}$ & $\begin{array}{ll}5.22 \mathrm{P} & 01 \\
5.69 \mathrm{E} & 01 \\
6.13 \mathrm{E} & 01 \\
6.58 \mathrm{E} & 01 \\
7.12 \mathrm{E} & 01\end{array}$ & $\begin{array}{ll}2.79 \mathrm{E} & 01 \\
3.05 \mathrm{E} & 01 \\
3.29 \mathrm{E} & 01 \\
3.53 \mathrm{E} & \mathrm{C1} \\
3.83 \mathrm{E} & 01\end{array}$ & $\begin{array}{ll}3.87 \mathrm{E} & 00 \\
4.25 \mathrm{~B} & 00 \\
4.61 \mathrm{E} & 00 \\
4.98 \mathrm{E} & 00 \\
5.44 \mathrm{E} & 00\end{array}$ & $\begin{array}{l}53 \\
54 \\
55 \\
56 \\
57\end{array}$ & $\begin{array}{l}I \\
X E \\
C S \\
\text { BA } \\
\text { LA }\end{array}$ \\
\hline $\begin{array}{l}58 \\
59 \\
60 \\
61 \\
62\end{array}$ & $\begin{array}{l}C P \\
\text { PR } \\
\text { HD } \\
\text { PM } \\
\text { SH }\end{array}$ & & & & $\begin{array}{l}2.68 \mathrm{E} \\
2.87 \mathrm{E} \\
3.04 \mathrm{~F} \\
3.25 \mathrm{E} \\
3.46 \mathrm{E}\end{array}$ & $\begin{array}{l}02 \\
02 \\
02 \\
02 \\
02\end{array}$ & $\begin{array}{ll}1.66 \mathrm{E} & 02 \\
1.79 \mathrm{E} & 02 \\
1.90 \mathrm{E} & 02 \\
2.03 \mathrm{E} & 02 \\
2.15 \mathrm{~F} & 02\end{array}$ & $\begin{array}{ll}7.62 \mathrm{~F} & 01 \\
8.22 \mathrm{P} & 01 \\
8.77 \mathrm{~F} & 01 \\
9.42 \mathrm{P} & 01 \\
1.00 \mathrm{E} & 02\end{array}$ & $\begin{array}{ll}4.11 E & 01 \\
4.45 E & 01 \\
4.75 F & 01 \\
5.12 E & 01 \\
5.45 E & 01\end{array}$ & $\begin{array}{ll}5.86 \mathrm{E} & 00 \\
6.37 \mathrm{E} & 00 \\
6.85 \mathrm{E} & 00 \\
7.41 \mathrm{~B} & 00 \\
7.94 \mathrm{~F} & 00\end{array}$ & $\begin{array}{l}58 \\
59 \\
60 \\
61 \\
62\end{array}$ & $\begin{array}{l}\text { CE } \\
\text { PR } \\
\text { ND } \\
\text { PM } \\
\text { SM }\end{array}$ \\
\hline $\begin{array}{l}63 \\
64 \\
65 \\
66 \\
67\end{array}$ & $\begin{array}{l}\text { EU } \\
\text { GD } \\
\text { TB } \\
\text { DY } \\
\text { HO }\end{array}$ & & & & $3.64 \mathrm{E}$ & 02 & $\begin{array}{ll}2.27 \mathrm{E} & 02 \\
2.42 \mathrm{E} & 02 \\
2.54 \mathrm{E} & 02 \\
2.68 \mathrm{E} & 02 \\
2.86 \mathrm{~B} & 02\end{array}$ & $\begin{array}{ll}1.06 \mathrm{E} & 02 \\
1.13 \mathrm{E} & 02 \\
1.20 \mathrm{E} & 02 \\
1.27 \mathrm{E} & 02 \\
1.35 \mathrm{E} & 02\end{array}$ & $\begin{array}{ll}5.798 & 01 \\
6.20 E & 01 \\
6.58 E & 01 \\
6.97 E & 01 \\
7.44 E & 01\end{array}$ & $\begin{array}{ll}8.49 \mathrm{~F} & 00 \\
9.15 \mathrm{E} & 00 \\
9.75 \mathrm{E} & 00 \\
1.04 \mathrm{~F} & 01 \\
1.11 \mathrm{E} & 01\end{array}$ & $\begin{array}{l}63 \\
64 \\
65 \\
66 \\
67\end{array}$ & $\begin{array}{l}\text { ET } \\
\text { FD } \\
\text { TR } \\
\text { DY } \\
\text { HO }\end{array}$ \\
\hline $\begin{array}{l}68 \\
69 \\
70 \\
71 \\
72\end{array}$ & $\begin{array}{l}\text { ER } \\
\text { TH } \\
\text { Y } \\
\text { L,U } \\
\text { HF }\end{array}$ & & & & & & $\begin{array}{ll}3.02 \mathrm{~F} & 02 \\
3.15 \mathrm{~F} & 02\end{array}$ & $\begin{array}{ll}1.42 \mathrm{~F} & 02 \\
1.50 \mathrm{E} & 02 \\
1.59 \mathrm{~F} & 02 \\
1.66 \mathrm{E} & 02 \\
1.74 \mathrm{~F} & 02\end{array}$ & 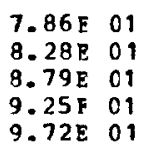 & 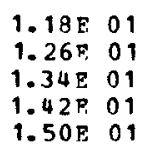 & $\begin{array}{l}68 \\
69 \\
70 \\
71 \\
72\end{array}$ & $\begin{array}{l}\text { ER } \\
T H \\
\text { YR } \\
\text { LU } \\
\text { HF }\end{array}$ \\
\hline
\end{tabular}


Table 9 (continued)

\begin{tabular}{|c|c|c|c|c|c|c|c|c|c|c|c|c|c|}
\hline \multirow[b]{3}{*}{$\mathrm{z}$} & \multicolumn{13}{|c|}{ (c) $K \beta_{1}\left(K M_{3}\right) \times$ ray } \\
\hline & & \multicolumn{10}{|c|}{ Photon energy (keV) } & \multirow[b]{2}{*}{$z$} & \\
\hline & & 20.00 & 30.00 & 40.00 & 50.00 & 60.00 & 80.00 & & 100.00 & & 200.00 & & \\
\hline $\begin{array}{l}73 \\
74 \\
75 \\
76 \\
77\end{array}$ & $\begin{array}{l}\text { TA } \\
\text { R } \\
\text { RF } \\
\text { OS } \\
\text { IR }\end{array}$ & & & & & & $\begin{array}{l}1.82 \mathrm{E} \\
1.90 \mathrm{P} \\
1.98 \mathrm{E} \\
2.10 \mathrm{~F} \\
2.20 \mathrm{P}\end{array}$ & $\begin{array}{l}02 \\
02 \\
02 \\
02 \\
02\end{array}$ & $\begin{array}{l}1.02 \mathrm{E} \\
1.07 \mathrm{E} \\
1.12 \mathrm{E} \\
1.18 \mathrm{E} \\
1.23 \mathrm{E}\end{array}$ & $\begin{array}{l}02 \\
02 \\
02 \\
02 \\
02\end{array}$ & $\begin{array}{ll}1.58 \mathrm{P} & 01 \\
1.67 \mathrm{E} & 01 \\
1.76 \mathrm{~F} & 01 \\
1.87 \mathrm{E} & 01 \\
1.96 \mathrm{P} & 01\end{array}$ & $\begin{array}{l}73 \\
74 \\
75 \\
76 \\
77\end{array}$ & $\begin{array}{l}T A \\
W \\
R E \\
O S \\
\text { IR }\end{array}$ \\
\hline $\begin{array}{l}78 \\
79 \\
80 \\
81 \\
82\end{array}$ & $\begin{array}{l}\text { PT } \\
\text { AU } \\
\text { BG } \\
\text { TL } \\
\text { PB }\end{array}$ & & & & & & $2.26 \mathrm{E}$ & 02 & $\begin{array}{l}1.28 \mathrm{E} \\
1.34 \mathrm{E} \\
1.39 \mathrm{E} \\
1.45 \mathrm{E} \\
1.52 \mathrm{E}\end{array}$ & $\begin{array}{l}02 \\
02 \\
02 \\
02 \\
02\end{array}$ & $\begin{array}{ll}2.06 \mathrm{E} & 01 \\
2.16 \mathrm{E} & 01 \\
2.26 \mathrm{E} & 01 \\
2.37 \mathrm{E} & 01 \\
2.50 \mathrm{E} & 01\end{array}$ & $\begin{array}{l}78 \\
79 \\
80 \\
81 \\
82\end{array}$ & $\begin{array}{l}\text { PT } \\
\text { AU } \\
\text { RG } \\
\text { TL } \\
\text { PB }\end{array}$ \\
\hline $\begin{array}{l}83 \\
84 \\
85 \\
86 \\
87\end{array}$ & $\begin{array}{l}\text { BI } \\
\text { PO } \\
\text { AT } \\
\text { RN } \\
\text { FR }\end{array}$ & & & & & & & & $\begin{array}{l}1.58 \mathrm{E} \\
1.65 \mathrm{E} \\
1.70 \mathrm{E} \\
1.77 \mathrm{E}\end{array}$ & $\begin{array}{l}02 \\
02 \\
02 \\
02\end{array}$ & $\begin{array}{ll}2.62 \mathrm{E} & 01 \\
2.73 \mathrm{E} & 01 \\
2.85 \mathrm{~F} & 01 \\
2.97 \mathrm{E} & 01 \\
3.10 \mathrm{E} & 01\end{array}$ & $\begin{array}{l}83 \\
84 \\
85 \\
86 \\
87\end{array}$ & $\begin{array}{l}\text { BI } \\
\text { PO } \\
\text { AT } \\
\text { RN } \\
\text { PR }\end{array}$ \\
\hline $\begin{array}{l}88 \\
89 \\
90 \\
91 \\
92\end{array}$ & $\begin{array}{l}\text { RA } \\
\text { AC } \\
\text { TH } \\
\text { PA } \\
\text { D }\end{array}$ & & & & & & & & & & $\begin{array}{ll}3.22 \mathrm{E} & 01 \\
3.35 \mathrm{E} & 01 \\
3.48 \mathrm{E} & 01 \\
3.65 \mathrm{E} & 01 \\
3.78 \mathrm{E} & 01\end{array}$ & $\begin{array}{l}88 \\
89 \\
90 \\
91 \\
92\end{array}$ & $\begin{array}{l}\text { RA } \\
\text { AC } \\
\text { TH } \\
\text { PA } \\
0\end{array}$ \\
\hline $\begin{array}{l}93 \\
94 \\
95 \\
96 \\
97\end{array}$ & $\begin{array}{l}\text { RP } \\
\text { PU } \\
\text { AM } \\
\text { CM } \\
\text { BK }\end{array}$ & & & & & & & & & & $\begin{array}{ll}3.92 \mathrm{E} & 01 \\
4.06 \mathrm{E} & 01 \\
4.20 \mathrm{E} & 01 \\
4.34 \mathrm{E} & 01 \\
4.49 \mathrm{E} & 01\end{array}$ & $\begin{array}{l}93 \\
94 \\
95 \\
96 \\
97\end{array}$ & $\begin{array}{l}\text { NP } \\
\text { PU } \\
A M \\
C H \\
B K\end{array}$ \\
\hline $\begin{array}{r}98 \\
99 \\
100 \\
101\end{array}$ & $\begin{array}{l}\text { CP } \\
\text { ES } \\
\text { PH } \\
\text { MD }\end{array}$ & & & & & & & & & & $\begin{array}{ll}4.64 \mathrm{E} & 01 \\
4.79 \mathrm{~F} & 01 \\
4.98 \mathrm{~F} & 01 \\
5.12 \mathrm{E} & 01\end{array}$ & $\begin{array}{r}99 \\
99 \\
100 \\
101\end{array}$ & $\begin{array}{l}C P \\
\text { RS } \\
P M \\
Y D\end{array}$ \\
\hline
\end{tabular}


Table 9 (continued)

(d) $K \beta_{1,3}\left(K M_{2,3}\right) \times$ ray

Photon energy $(\mathrm{keV})$

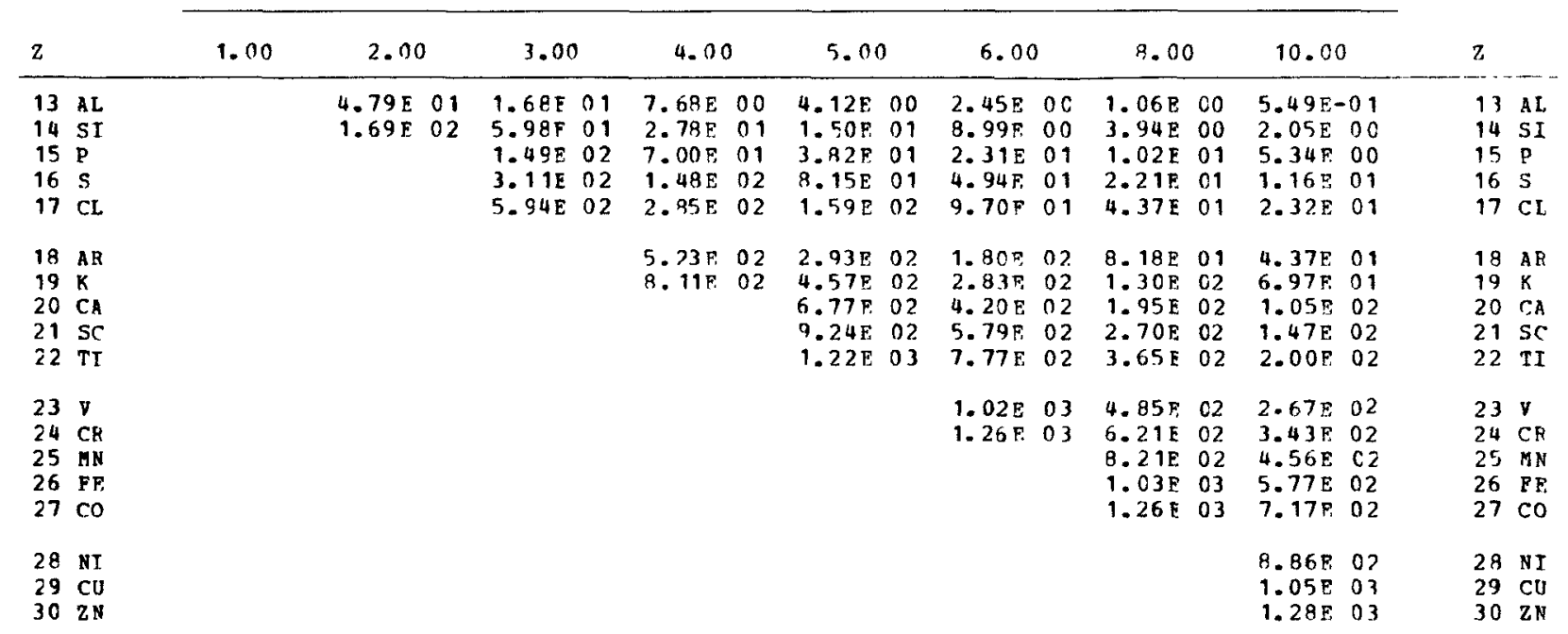


(d) $K \beta_{1,3}\left(K M_{2,3}\right) \times$ ray

Photon energy (keV)

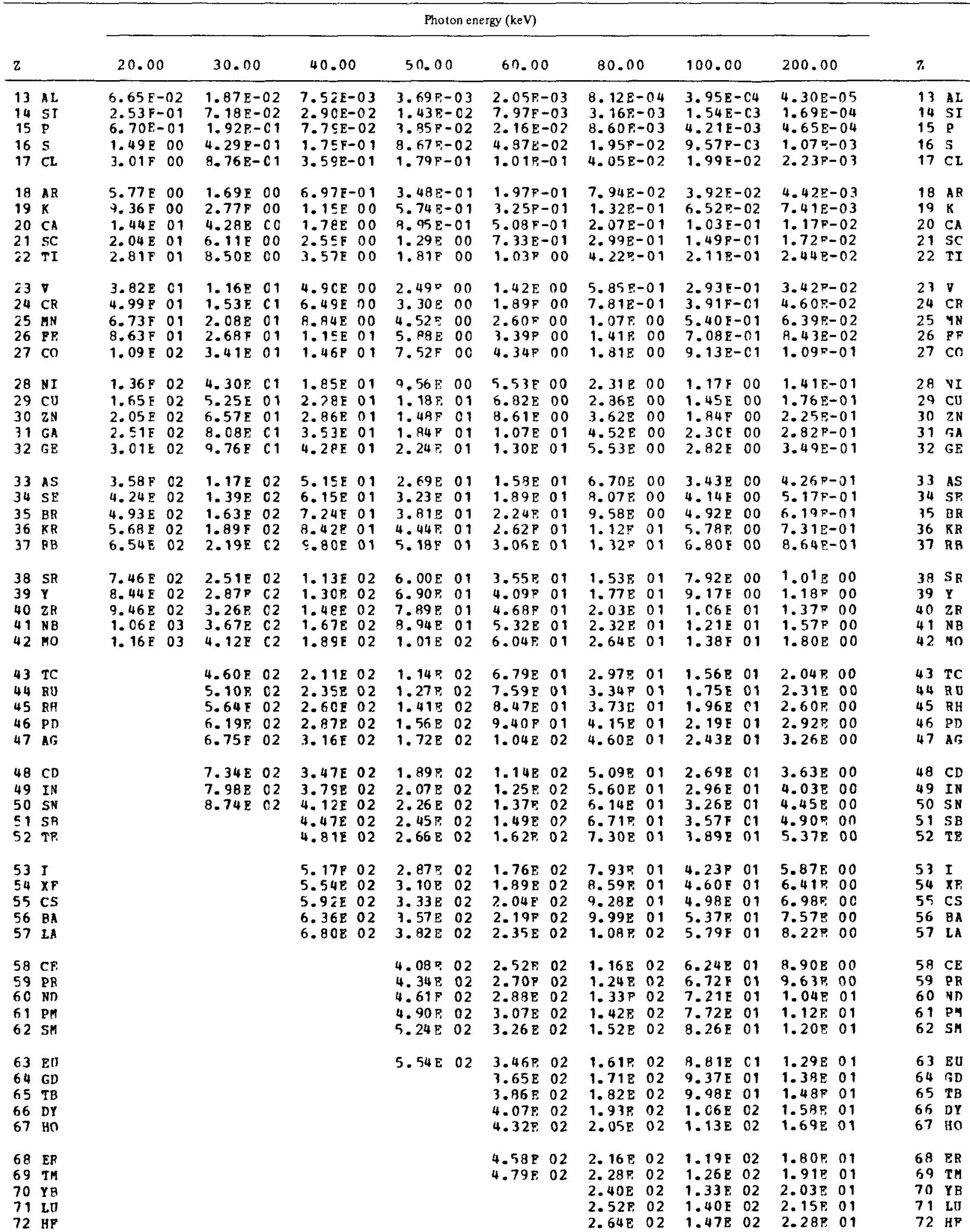


Table 9 (continued)

(d) $K \beta_{1,3}\left(K M_{2,3}\right) \times$ ray

\begin{tabular}{|c|c|c|c|c|c|c|c|c|c|c|c|c|c|c|}
\hline \multirow[b]{2}{*}{$\mathrm{z}$} & & \multicolumn{11}{|c|}{ Photon energy (keV) } & \multirow[b]{2}{*}{$\mathbf{z}$} & \\
\hline & & 20.00 & 30.00 & 40.00 & 50.00 & 60.00 & \multicolumn{2}{|c|}{80.00} & \multicolumn{2}{|c|}{100.00} & \multicolumn{2}{|c|}{200.00} & & \\
\hline 73 & TA & & & & & & $2.77 \mathrm{E}$ & 02 & $1.55 \mathrm{E}$ & $\mathrm{C} 2$ & $2.41 \mathrm{E}$ & 01 & 73 & $\mathbf{T A}_{\mathbf{A}}$ \\
\hline 74 & $\nabla$ & & & & & & $2.89 \mathrm{~F}$ & 02 & $1.63 \mathrm{E}$ & 02 & $2.54 \mathrm{~F}$ & 01 & 74 & H \\
\hline 75 & $\mathrm{RE}$ & & & & & & 3.035 & 02 & $1.71 \mathrm{E}$ & 02 & 2.685 & 01 & 75 & RE \\
\hline 76 & os & & & & & & $3.19 \mathrm{~F}$ & 02 & $1.79 \mathrm{E}$ & 02 & $2.83 \%$ & 01 & 76 & os \\
\hline 77 & IR & & & & & & 3. $33 \mathrm{~F}$ & 02 & $1.87 \mathrm{~F}$ & 02 & $2.97^{\mathrm{P}}$ & 01 & 77 & IR \\
\hline 78 & PT & & & & & & $3.42 \mathrm{E}$ & 02 & 1. $95 \mathrm{E}$ & 02 & $3.13 \mathrm{E}$ & 01 & 78 & PT \\
\hline 79 & $A U$ & & & & & & & & $2.03 \mathrm{~F}$ & 02 & $3.29 \mathrm{~F}$ & 01 & 79 & $\mathrm{AU}$ \\
\hline PO & $\mathrm{Hr}$ & & & & & & & & $2.12 \mathrm{~F}$ & 02 & $3.45 \mathrm{~F}$ & 01 & 8O & $\mathrm{HG}$ \\
\hline 81 & TL & & & & & & & & $2.20 \mathrm{E}$ & 02 & $3.61 \mathrm{~F}$ & 01 & 81 & TL \\
\hline 82 & $\mathrm{~PB}$ & & & & & & & & $2.29 \mathrm{~F}$ & 02 & $3.78 \mathrm{~F}$ & 01 & 82 & $P B$ \\
\hline 83 & AI & & & & & & & & $2.39 \mathrm{E}$ & 02 & $3.96 \mathrm{~F}$ & 01 & 83 & BI \\
\hline 84 & PO & & & & & & & & $2.49 \mathrm{~F}$ & 02 & $4.13 \mathrm{E}$ & 01 & 34 & PO \\
\hline ES & $A T$ & & & & & & & & $2.57 \mathrm{~F}$ & 02 & $4.31 \mathrm{~g}$ & 01 & 85 & $A T$ \\
\hline 86 & $\mathrm{RN}$ & & & & & & & & $2.67 \mathrm{E}$ & 02 & $4.49 \mathrm{E}$ & 01 & 86 & RN \\
\hline 87 & $\mathrm{FR}$ & & & & & & & & & & $4.68 \mathrm{~F}$ & 01 & 87 & F R \\
\hline 88 & $\mathrm{RA}$ & & & & & & & & & & $4.87 \mathrm{~F}$ & 01 & 88 & R A \\
\hline 89 & $A C$ & & & & & & & & & & $5.07 \mathrm{E}$ & 01 & 89 & $A C$ \\
\hline 90 & $T H$ & & & & & & & & & & $5.27 \mathrm{E}$ & 01 & 90 & TH \\
\hline 91 & PA & & & & & & & & & & $5.48 \mathrm{E}$ & 01 & 91 & PA \\
\hline 92 & 0 & & & & & & & & & & $5.70 \mathrm{E}$ & 01 & 92 & 'J \\
\hline 93 & NP & & & & & & & & & & $5.91 \mathrm{E}$ & 01 & 93 & NP \\
\hline 94 & PU & & & & & & & & & & $6.12 \mathrm{~F}$ & 01 & 94 & PI \\
\hline 95 & $A M$ & & & & & & & & & & $6.34 \mathrm{~F}$ & 01 & 95 & A H \\
\hline 96 & $\mathrm{CH}$ & & & & & & & & & & $6.55 \mathrm{~F}$ & 01 & 96 & $\mathrm{CM}$ \\
\hline 97 & $\mathrm{BK}$ & & & & & & & & & & $6.77 \mathrm{~F}$ & 01 & 97 & BK \\
\hline $\begin{array}{l}98 \\
99\end{array}$ & $\begin{array}{l}\text { CF } \\
\text { ES }\end{array}$ & & & & & & & & & & $\begin{array}{l}6.99 \mathrm{E} \\
7.22 \mathrm{E}\end{array}$ & $\begin{array}{l}01 \\
01\end{array}$ & $\begin{array}{l}98 \\
99\end{array}$ & $\begin{array}{l}\text { CF } \\
\text { FS }\end{array}$ \\
\hline 100 & $\mathrm{PM}$ & & & & & & & & & & $7.45 \mathrm{E}$ & 01 & 100 & $P M$ \\
\hline 101 & Mo & & & & & & & & & & $7.66 \mathrm{E}$ & 01 & 101 & MD \\
\hline
\end{tabular}


ORNL-DWG 77-18989R

X-ray Production Cross Sections

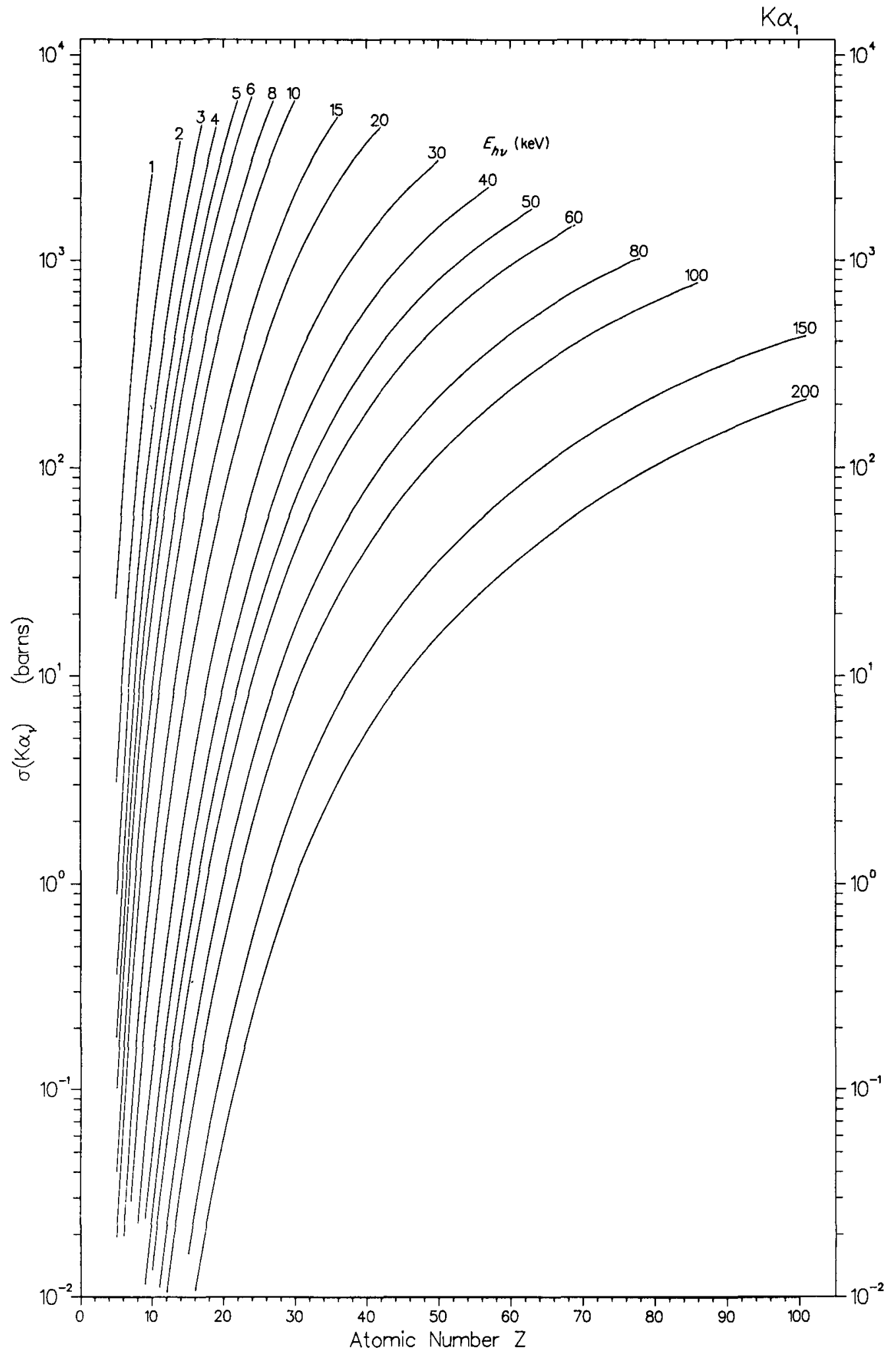

Fig. 14 
ORNL-DWG 77-18990R

\section{$X$-ray Production Cross Sections}

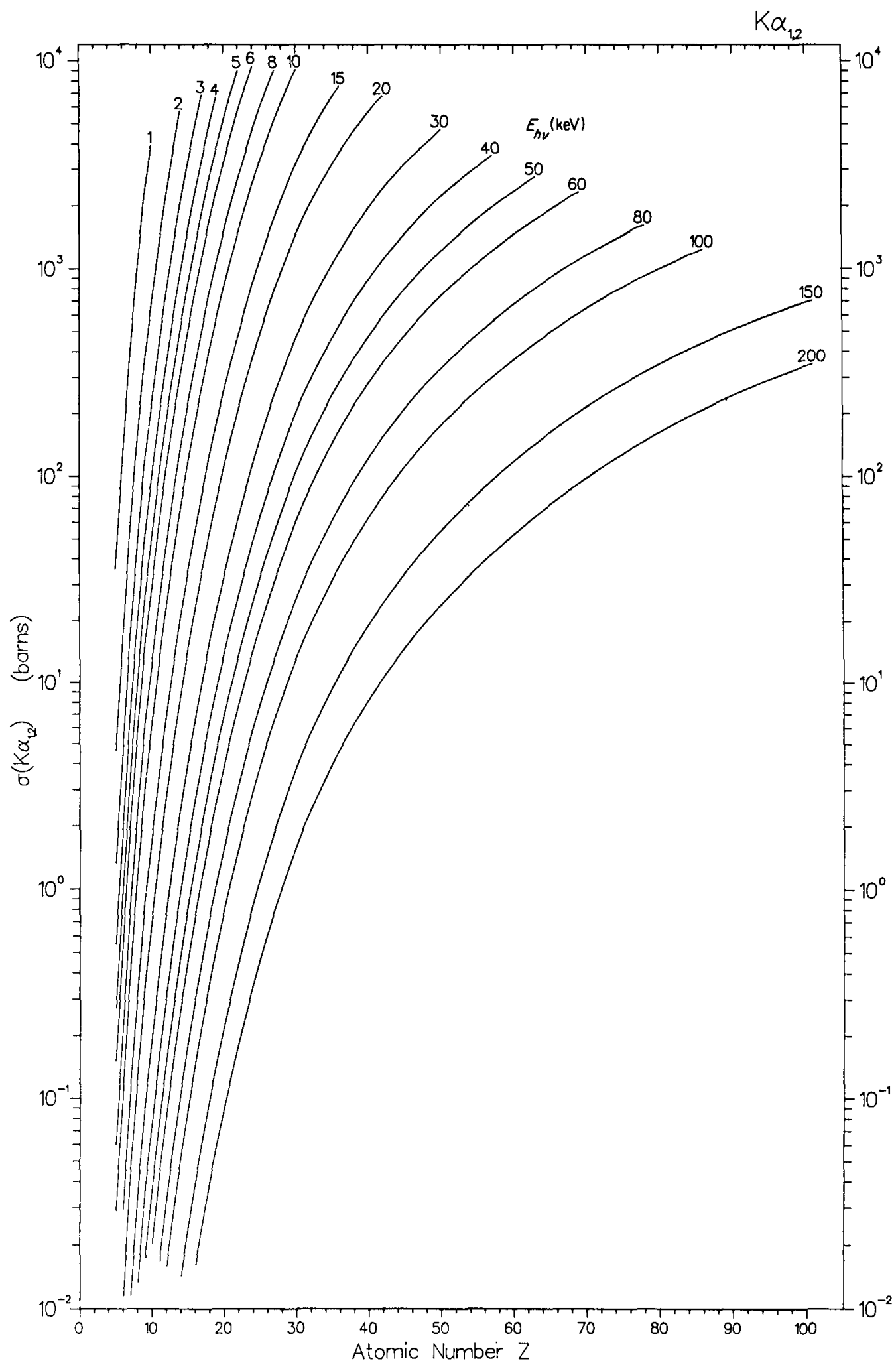

Fig. 15 
ORNL-DWG 77-18991R

X-ray Production Cross Sections

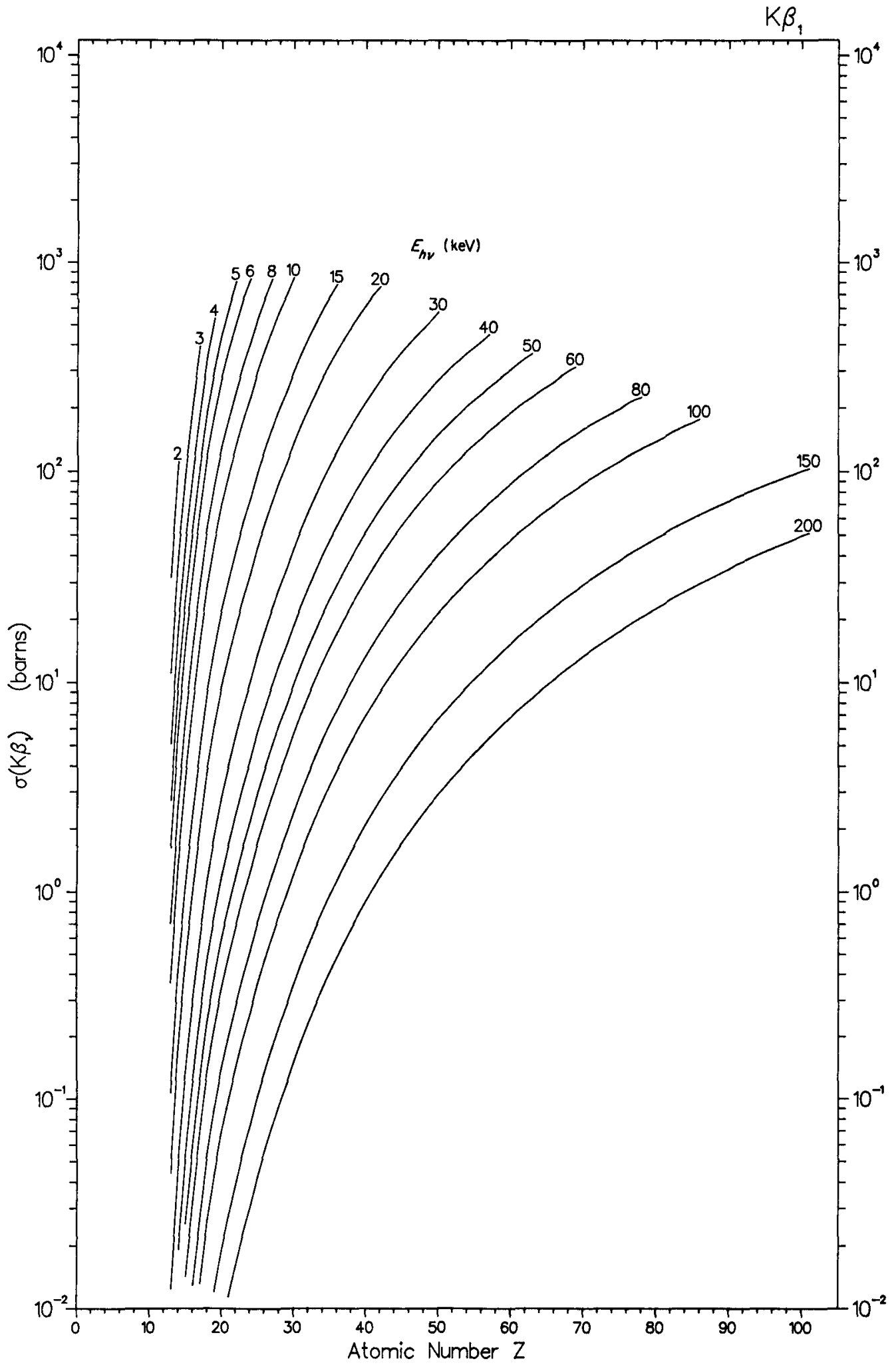

Fig. 16 
RNL-DWG 77-18992R

$X$-ray Production Cross Sections

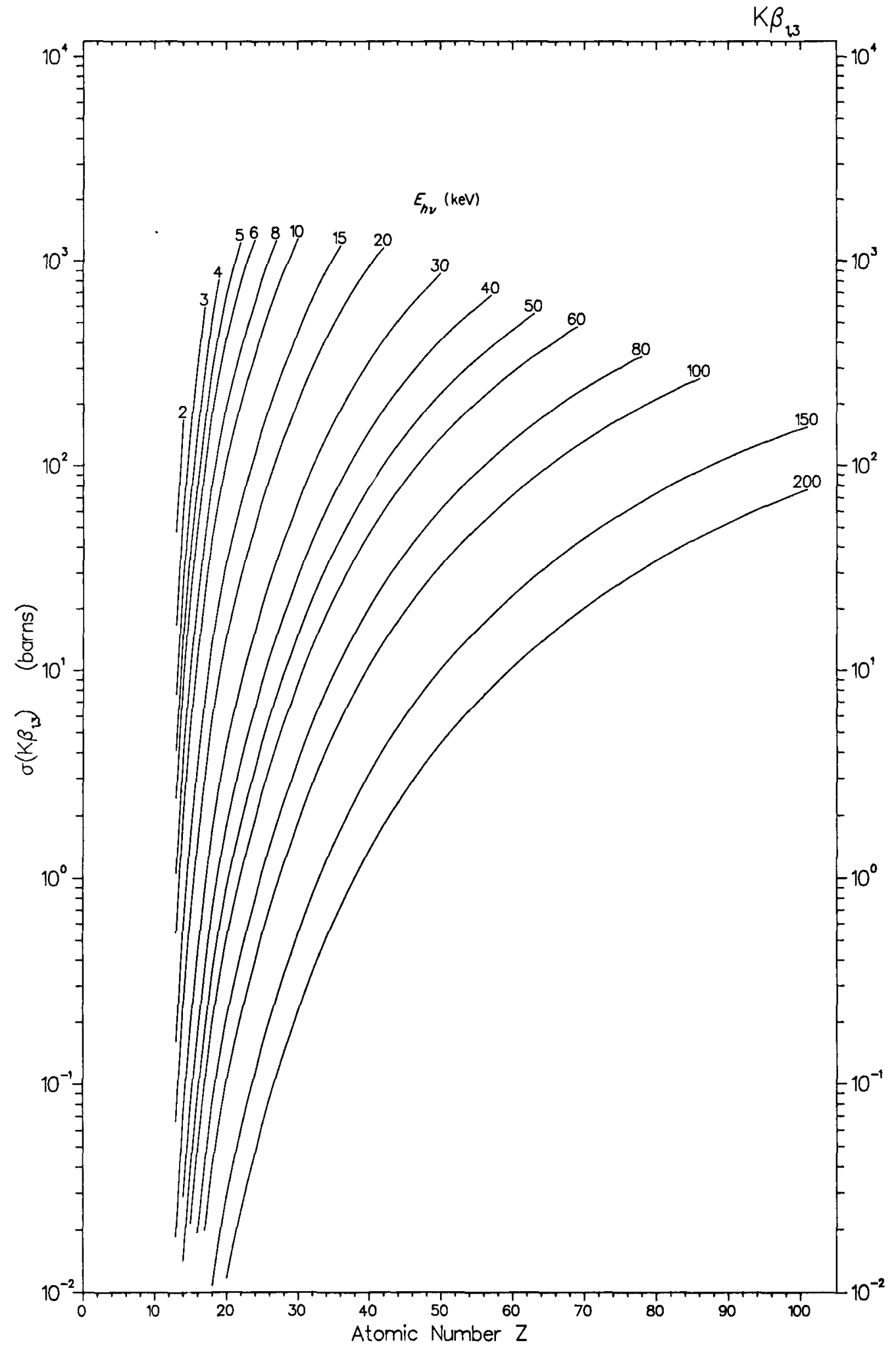

Fig. 17 
Table 10. $L$-shell $\mathrm{x}$-ray fluorescence cross sections arranged in sequence by fluorescent $\mathrm{x}$ ray Values are in units of barns/atom. See Figs. 18 to 25 for graphic representation.

\begin{tabular}{|c|c|c|c|c|c|c|c|c|c|c|c|}
\hline \multirow[b]{2}{*}{ z } & & \multicolumn{8}{|c|}{ Photon energy (keV) } & \multirow{2}{*}{\multicolumn{2}{|c|}{2}} \\
\hline & & 1.00 & 2.00 & 3.00 & 4.00 & 5.00 & 6.00 & 8.00 & 10.00 & & \\
\hline $\begin{array}{l}12 \\
13 \\
14\end{array}$ & $\begin{array}{l}M G \\
\text { AI } \\
\text { SI }\end{array}$ & $\begin{array}{l}5.65 \mathrm{E}-01 \\
5.81 \mathrm{E}-01 \\
6.21 \mathrm{E}-01\end{array}$ & & & & & & & & $\begin{array}{l}12 \\
13 \\
14\end{array}$ & $\begin{array}{l}M G \\
\text { AL } \\
\text { ST }\end{array}$ \\
\hline $\begin{array}{l}15 \\
16 \\
17 \\
18 \\
19\end{array}$ & $\begin{array}{l}P \\
S \\
C L \\
A R \\
K\end{array}$ & $\begin{array}{l}6.41 \mathrm{E}-01 \\
9.09 \mathrm{E}-01 \\
1.12 \mathrm{E} 00 \\
1.28 \mathrm{E} 00 \\
1.41 \mathrm{E} 00\end{array}$ & $\begin{array}{l}1.41 F-01 \\
2.07 E-01 \\
2.64 E-01 \\
3.12 E-01 \\
3.53 E-01\end{array}$ & $\begin{array}{l}1.24 P-01 \\
1.43 E-01\end{array}$ & . & & & & & $\begin{array}{l}15 \\
16 \\
17 \\
19 \\
19\end{array}$ & $\begin{array}{l}\mathrm{P} \\
\mathrm{S} \\
\mathrm{CI} \\
\mathrm{AP} \\
\mathrm{K}\end{array}$ \\
\hline $\begin{array}{l}20 \\
21 \\
22 \\
23 \\
24\end{array}$ & $\begin{array}{l}C A \\
S C \\
T I \\
Y \\
C R\end{array}$ & $\begin{array}{ll}1.55 E & C 0 \\
1.79 E & 00 \\
1.98 E & 00 \\
2.25 E & 00 \\
2.72 E & 00\end{array}$ & $\begin{array}{l}3.99 E-01 \\
4.78 E-01 \\
5.45 E-01 \\
6.39 E-01 \\
8.01 F-01\end{array}$ & $\begin{array}{l}1.64 E-01 \\
1.99 E-01 \\
2.31 F-01 \\
2.74 E-01 \\
3.49 E-01\end{array}$ & $\begin{array}{l}9.40 E-02 \\
1.03 F-01 \\
1.20 E-01 \\
1.45 E-01 \\
1.86 E-01\end{array}$ & $\begin{array}{l}3.61 E-02 \\
1.11 E-01\end{array}$ & & & & $\begin{array}{l}20 \\
21 \\
22 \\
23 \\
24\end{array}$ & $\begin{array}{l}\mathrm{Cl} \\
\mathrm{SC} \\
\mathrm{TI} \\
\mathrm{V} \\
\mathrm{CP}\end{array}$ \\
\hline $\begin{array}{l}25 \\
26 \\
27 \\
28 \\
29\end{array}$ & $\begin{array}{l}\text { HN } \\
\text { FE } \\
\text { CO } \\
\text { NI } \\
\text { CU }\end{array}$ & 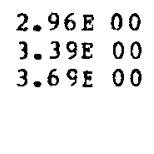 & 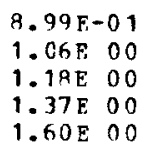 & $\begin{array}{l}3.97 E-01 \\
4.74 E-01 \\
5.39 E-01 \\
6.31 F-01 \\
7.49 E-01\end{array}$ & $\begin{array}{l}2.13 E-01 \\
2.57 E-01 \\
2.94 E-01 \\
3.48 E-01 \\
4.16 E-01\end{array}$ & $\begin{array}{l}1.29 \mathrm{~F}-01 \\
1.56 \mathrm{E}-01 \\
1.30 \mathrm{~F}-01 \\
2.14 \mathrm{E}-01 \\
2.59 \mathrm{~F}-01\end{array}$ & $\begin{array}{l}8.40 E-02 \\
1.02 E-01 \\
1.19 E-01 \\
1.42 E-01 \\
1.71 F-01\end{array}$ & $\begin{array}{l}7.22 \mathrm{E}-02 \\
8.80 \mathrm{E}-02\end{array}$ & & $\begin{array}{l}75 \\
25 \\
27 \\
29 \\
29\end{array}$ & $\begin{array}{l}\text { in } N \\
\mathrm{ED} \\
\mathrm{CO} \\
\mathrm{NI} \\
\mathrm{CI}\end{array}$ \\
\hline $\begin{array}{l}30 \\
31 \\
32 \\
33 \\
34\end{array}$ & $\begin{array}{l}\text { ZN } \\
\text { FA } \\
\text { GE } \\
\text { AS } \\
\text { SE }\end{array}$ & & $\begin{array}{ll}1.74 \mathrm{E} & 00 \\
2.04 \mathrm{E} & 100 \\
2.06 \mathrm{~F} & 00 \\
2.38 \mathrm{E} & 00 \\
2.67 \mathrm{~F} & 00\end{array}$ & $\begin{array}{l}8.26 \mathrm{E}-01 \\
9.83 \mathrm{E}-01 \\
1.01 \mathrm{E} 00 \\
1.18 \mathrm{~F} 00 \\
1.33 \mathrm{E} 00\end{array}$ & $\begin{array}{l}4.63 \mathrm{E}-01 \\
5.56 \mathrm{E}-01 \\
5.77 \mathrm{E}-01 \\
5.77 \mathrm{E}-01 \\
7.73 \mathrm{E}-01\end{array}$ & $\begin{array}{l}2.89 E-01 \\
3.48 E-01 \\
3.64 F-01 \\
4.30 E-01 \\
4.93 E-01\end{array}$ & $\begin{array}{l}1.93 E-01 \\
2.34 E-01 \\
2.46 F-01 \\
2.91 E-01 \\
3.36 E-01\end{array}$ & $\begin{array}{l}9.97 \mathrm{E}-02 \\
1.22 \mathrm{E}-01 \\
1.29 \mathrm{E}-01 \\
1.54 \mathrm{E}-01 \\
1.78 \mathrm{E}-01\end{array}$ & $\begin{array}{l}7.195-02 \\
7.535-02 \\
9.15 \pi-02 \\
1.075-01\end{array}$ & $\begin{array}{l}30 \\
37 \\
37 \\
33 \\
34\end{array}$ & $\begin{array}{l}\text { EN } \\
\text { TA } \\
\text { SE } \\
\text { AS } \\
\text { SP }\end{array}$ \\
\hline $\begin{array}{l}35 \\
36 \\
37 \\
38 \\
39\end{array}$ & $\begin{array}{l}\text { BR } \\
K R \\
\text { RB } \\
S R \\
Y\end{array}$ & & $\begin{array}{ll}2.93 E & 00 \\
3.16 E & 00\end{array}$ & 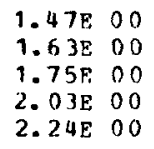 & $\begin{array}{l}8.61 \mathrm{E}-01 \\
9.6 \mathrm{CE}-01 \\
1.04 \mathrm{~F} 00 \\
1.22 \mathrm{~F} 00 \\
1.35 \mathrm{E} 00\end{array}$ & $\begin{array}{l}5.52 E-01 \\
6.20 E-01 \\
6.78 E-01 \\
7.98 E-01 \\
8.89 F-01\end{array}$ & $\begin{array}{l}\text { 3. } 78 E-01 \\
4.26 E-01 \\
4.60 F-01 \\
5.54 F-01 \\
6.19 E-01\end{array}$ & $\begin{array}{l}2.02 F-01 \\
2.29 F-01 \\
2.54 T-01 \\
3.02 E-01 \\
3.40 F-01\end{array}$ & $\begin{array}{l}1.22 F-01 \\
1.39 F-01 \\
1.54 E-01 \\
1.845-01 \\
2.98 E-01\end{array}$ & $\begin{array}{l}35 \\
35 \\
37 \\
30 \\
39\end{array}$ & $\begin{array}{l}3 \% \\
K ? \\
\text { RP } \\
\text { SR } \\
Y\end{array}$ \\
\hline $\begin{array}{l}40 \\
41 \\
42 \\
43 \\
44\end{array}$ & $\begin{array}{l}\text { ZR } \\
\text { NB } \\
\text { MO } \\
\text { TC } \\
\text { RU }\end{array}$ & & & $\begin{array}{ll}2.71 \mathrm{E} & 00 \\
3.73 \mathrm{~F} & 00 \\
3.93 \mathrm{E} & 00\end{array}$ & $\begin{array}{ll}1.64 \mathrm{E} & 00 \\
2.27 \mathrm{E} & 00 \\
2.44 \mathrm{E} & 00 \\
2.74 \mathrm{E} & 00 \\
3.09 \mathrm{E} & 00\end{array}$ & $\begin{array}{ll}1.08 \mathrm{E} & 00 \\
1.51 \mathrm{E} & 00 \\
1.63 \mathrm{E} & 00 \\
1.84 \mathrm{E} & 00 \\
2.078 & 00\end{array}$ & $\begin{array}{cc}7.55 \mathrm{~F} & -01 \\
1.06 \mathrm{E} & 00 \\
1.15 \mathrm{~F} & 00 \\
1.30 \mathrm{~F} & 00 \\
1.47 \mathrm{E} & 00\end{array}$ & $\begin{array}{l}4.17 \mathrm{E}-01 \\
5.89 \mathrm{E}-01 \\
6.44 \mathrm{E}-01 \\
7.34 \mathrm{E}-01 \\
8.35 \mathrm{~F}-01\end{array}$ & $\begin{array}{l}2.57 E-01 \\
3.64 E-01 \\
4.00 E-01 \\
4.53 E-01 \\
5.24 E-01\end{array}$ & $\begin{array}{l}40 \\
41 \\
42 \\
43 \\
44\end{array}$ & $\begin{array}{l}7, R \\
\text { iv B } \\
\text { iAn } \\
\text { TC } \\
\text { RII }\end{array}$ \\
\hline $\begin{array}{l}45 \\
46 \\
47 \\
48 \\
49\end{array}$ & $\begin{array}{l}\text { RH } \\
P D \\
\text { AG } \\
C D \\
\text { IN }\end{array}$ & & & & 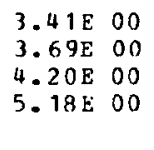 & $\begin{array}{ll}2.29 \mathrm{E} & 00 \\
2.50 \mathrm{E} & 00 \\
2.91 \mathrm{E} & 00 \\
3.45 \mathrm{E} & 00 \\
4.02 \mathrm{E} & 00\end{array}$ & $\begin{array}{ll}1.64 F & 00 \\
1.90 E & 00 \\
2.10 E & 00 \\
2.47 E & 00 \\
2.89 F & 00\end{array}$ & 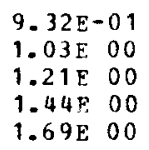 & $\begin{array}{l}5.87 E-01 \\
6.53 E-01 \\
7.68 F-01 \\
9.19 E-01 \\
1.09 E \quad 00\end{array}$ & $\begin{array}{l}45 \\
45 \\
47 \\
48 \\
49\end{array}$ & $\begin{array}{l}\text { RH } \\
\text { PD } \\
\text { AG } \\
\text { CN } \\
\text { IN }\end{array}$ \\
\hline $\begin{array}{l}50 \\
51 \\
52 \\
53 \\
54\end{array}$ & $\begin{array}{l}S N \\
S B \\
T E \\
I \\
X E\end{array}$ & & & & & 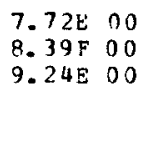 & $\begin{array}{ll}5.63 F & 00 \\
6.24 F & 00 \\
6.87 F & 00 \\
7.66 \mathrm{~F} & 00 \\
8.26 \mathrm{~F} & 00\end{array}$ & $\begin{array}{ll}3.30 \mathrm{E} & 00 \\
3.67 \mathrm{E} & 00 \\
4.05 \mathrm{~F} & 00 \\
4.56 \mathrm{E} & 00 \\
4.98 \mathrm{E} & 00\end{array}$ & $\begin{array}{ll}2.12 \mathrm{E} & 00 \\
2.37 \mathrm{~F} & 00 \\
2.63 \mathrm{~F} & 00 \\
2.97 \mathrm{E} & 00 \\
3.26 \mathrm{E} & 00\end{array}$ & $\begin{array}{l}50 \\
51 \\
52 \\
53 \\
54\end{array}$ & $\begin{array}{l}\mathrm{SN} \\
\mathrm{SB} \\
\mathrm{TE} \\
\mathrm{I} \\
\mathrm{XE}\end{array}$ \\
\hline $\begin{array}{l}55 \\
56 \\
57 \\
58 \\
59\end{array}$ & $\begin{array}{l}\text { CS } \\
\text { BA } \\
\text { LA } \\
\text { CE } \\
\text { PR }\end{array}$ & & & & & & $\begin{array}{ll}9.17 \mathrm{~F} & 00 \\
1.02 \mathrm{E} & 01\end{array}$ & $\begin{array}{ll}5.56 \mathrm{E} & 00 \\
6.25 \mathrm{E} & 00 \\
6.99 \mathrm{E} & 00 \\
7.76 \mathrm{E} & 00 \\
8.63 \mathrm{E} & 00\end{array}$ & $\begin{array}{ll}3.66 \mathrm{E} & 00 \\
4.12 \mathrm{~F} & 00 \\
4.61 \mathrm{E} & 00 \\
5.13 \mathrm{E} & 00 \\
5.74 \mathrm{E} & 00\end{array}$ & $\begin{array}{l}55 \\
56 \\
57 \\
59 \\
59\end{array}$ & $\begin{array}{l}\text { CS } \\
\text { SA } \\
L A \\
C E \\
\text { PR }\end{array}$ \\
\hline $\begin{array}{l}60 \\
61 \\
62 \\
63 \\
64\end{array}$ & $\begin{array}{l}\text { ND } \\
\text { PM } \\
\text { SN } \\
\text { RU } \\
\text { GD }\end{array}$ & . & & & & & & 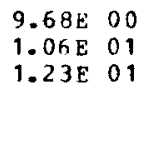 & $\begin{array}{ll}6.53 \mathrm{E} & 00 \\
7.23 \mathrm{~F} & 00 \\
8.33 \mathrm{~F} & 00 \\
9.45 \mathrm{~F} & 00 \\
1.06 \mathrm{~F} & 01\end{array}$ & $\begin{array}{l}60 \\
61 \\
62 \\
63 \\
64\end{array}$ & $\begin{array}{l}\text { ND } \\
\text { PM } \\
\text { SH } \\
\text { FU } \\
\text { FD }\end{array}$ \\
\hline $\begin{array}{l}65 \\
66 \\
67 \\
68\end{array}$ & $\begin{array}{l}\text { TB } \\
\text { DY } \\
\text { HO } \\
\text { ER }\end{array}$ & & & & & & & & $\begin{array}{ll}1.18 \mathrm{~F} & 01 \\
1.36 \mathrm{E} & 01 \\
1.55 \mathrm{E} & 01 \\
1.77 \mathrm{E} & 01\end{array}$ & $\begin{array}{l}65 \\
66 \\
67 \\
69\end{array}$ & $\begin{array}{l}\text { TR } \\
\text { DY } \\
\text { HO } \\
\text { ER }\end{array}$ \\
\hline
\end{tabular}


Table 10 (continued)

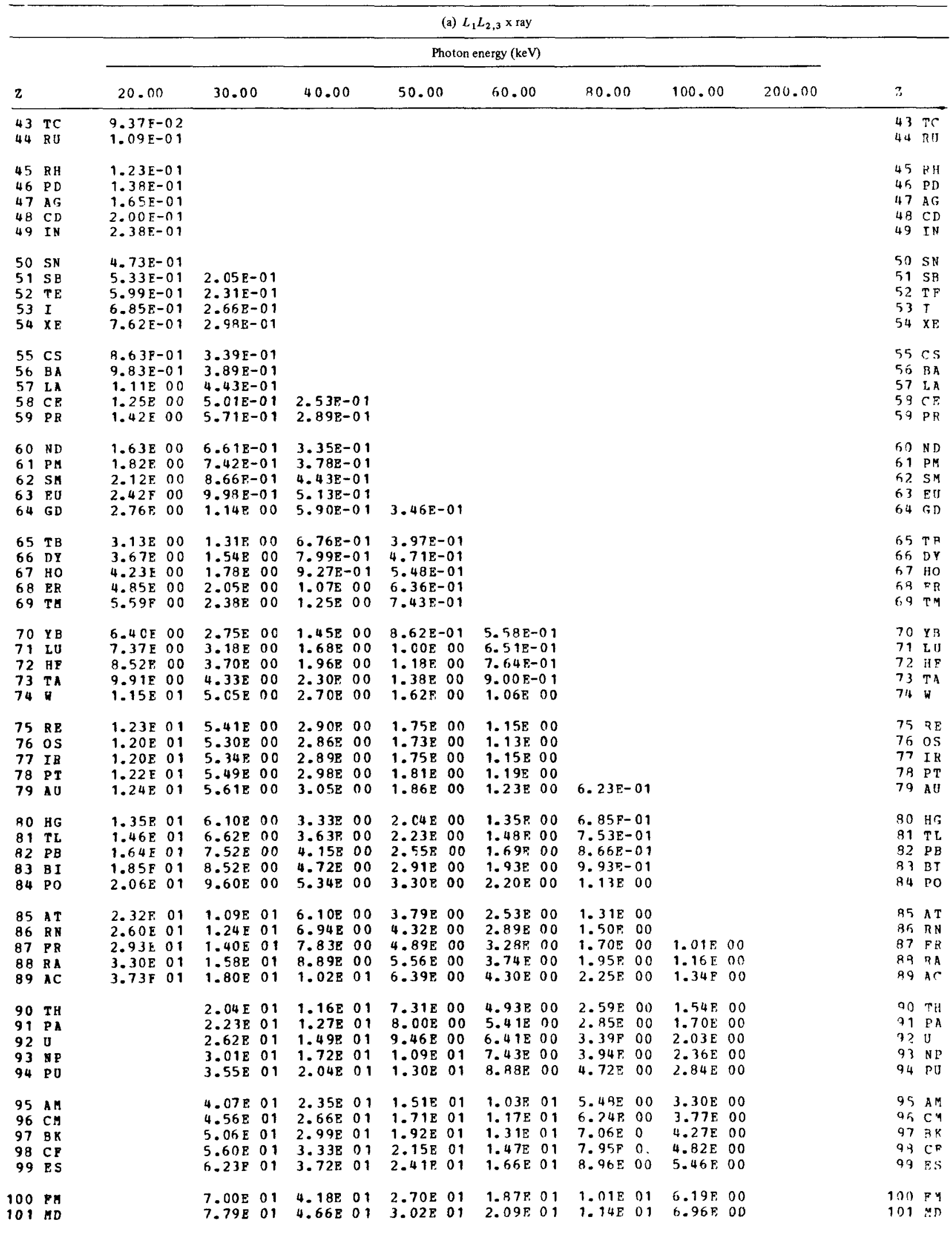


Table 10 (continued)

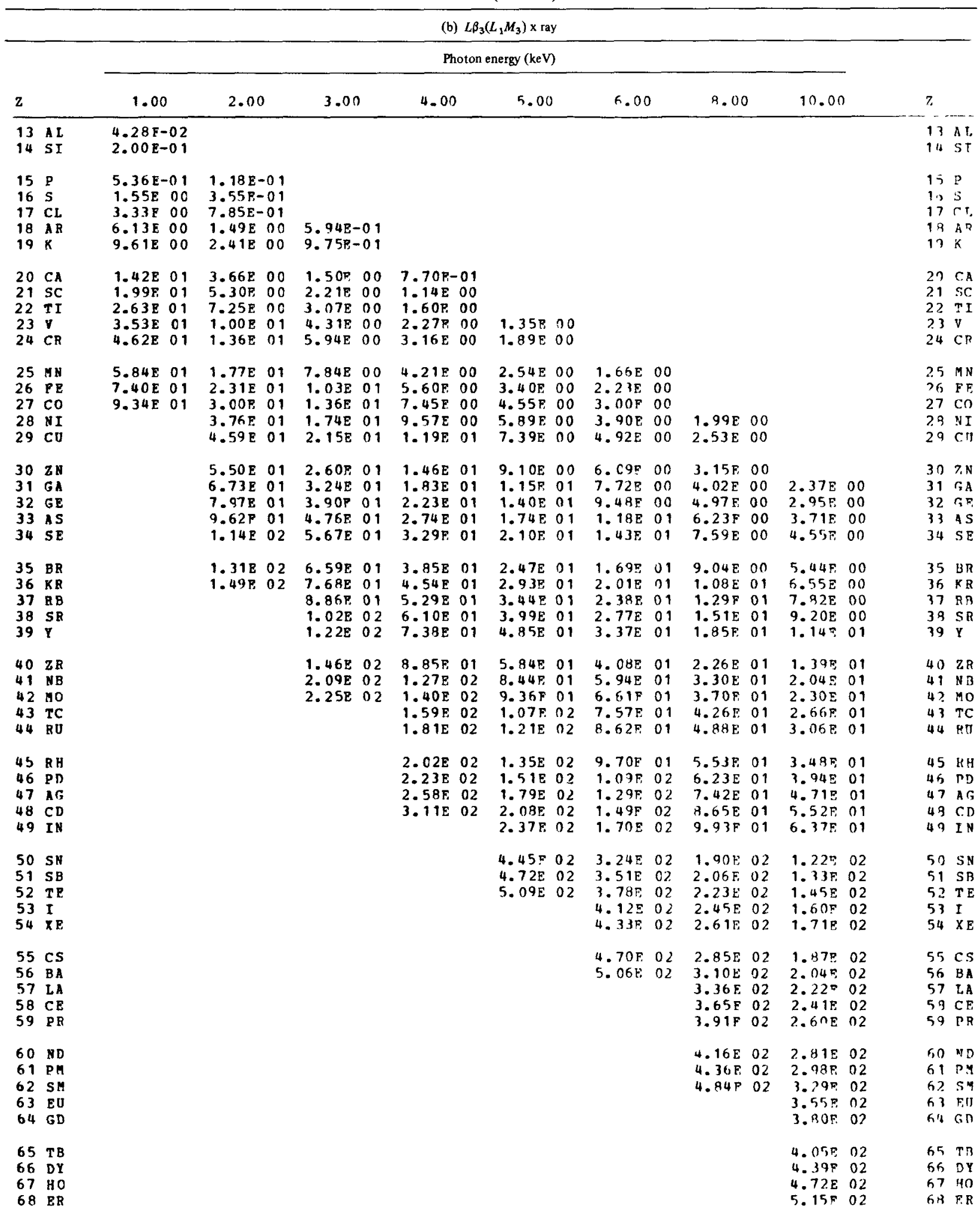


Table 10 (continued)

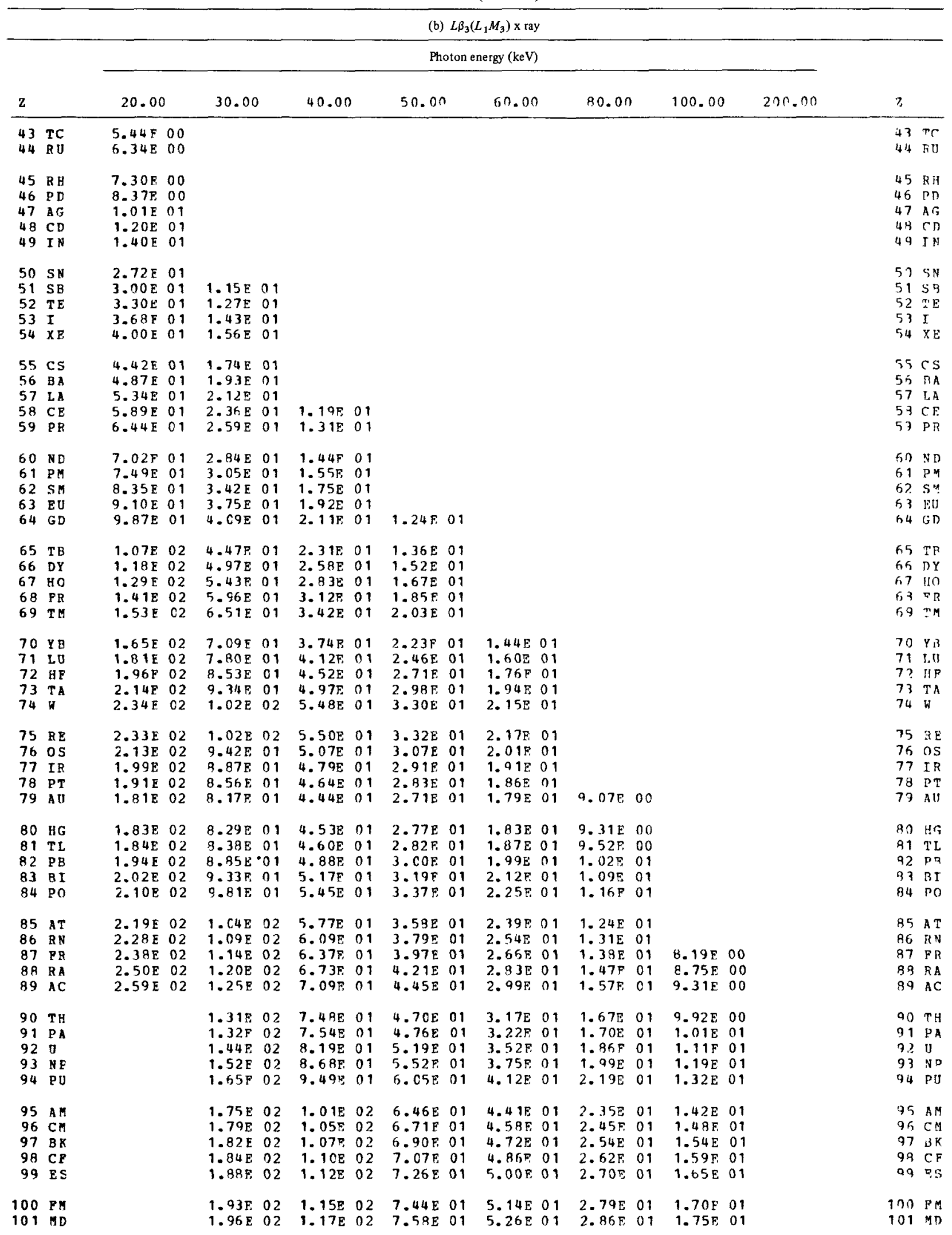


Table 10 (continued)

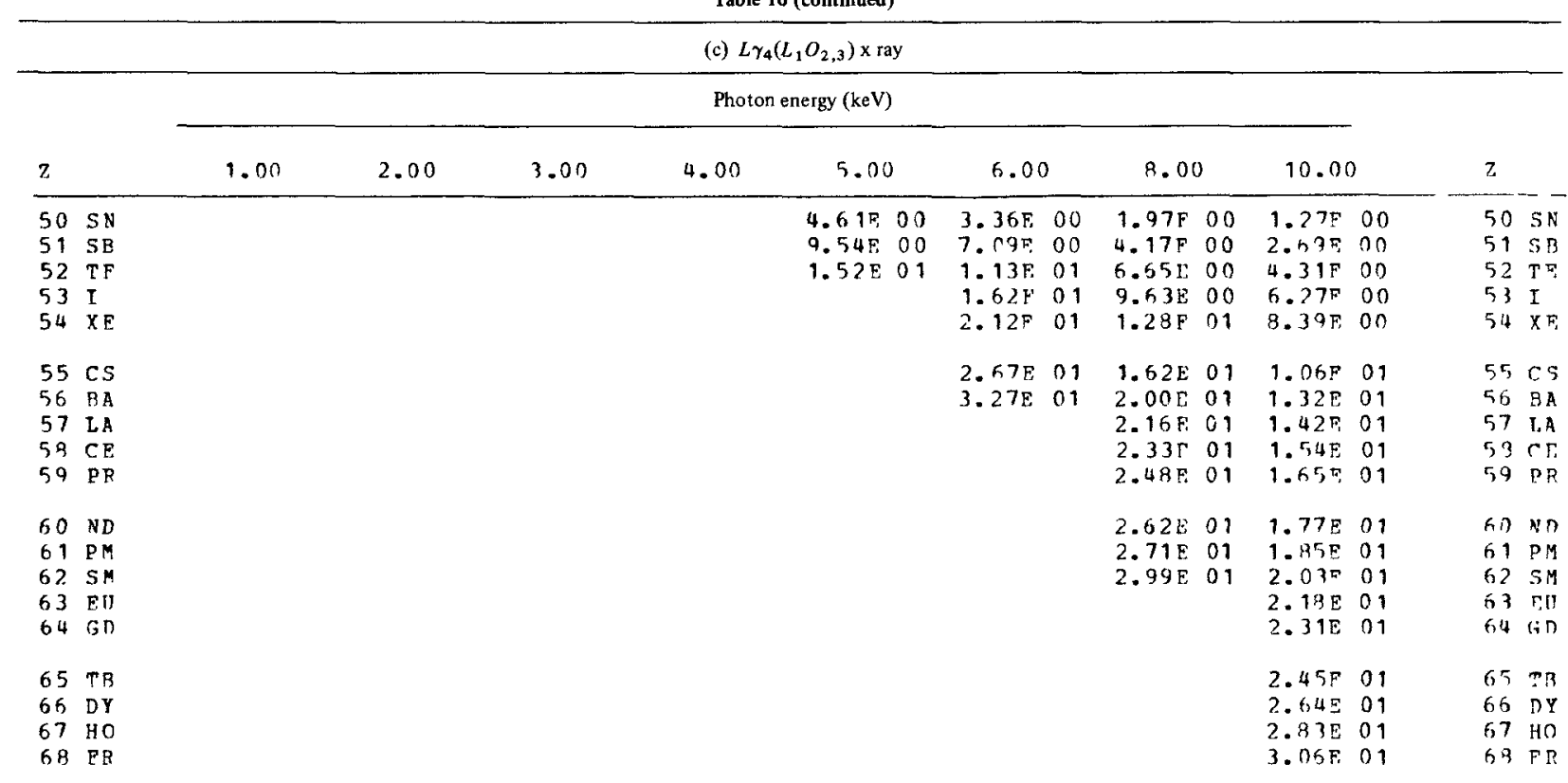


Table 10 (continued)

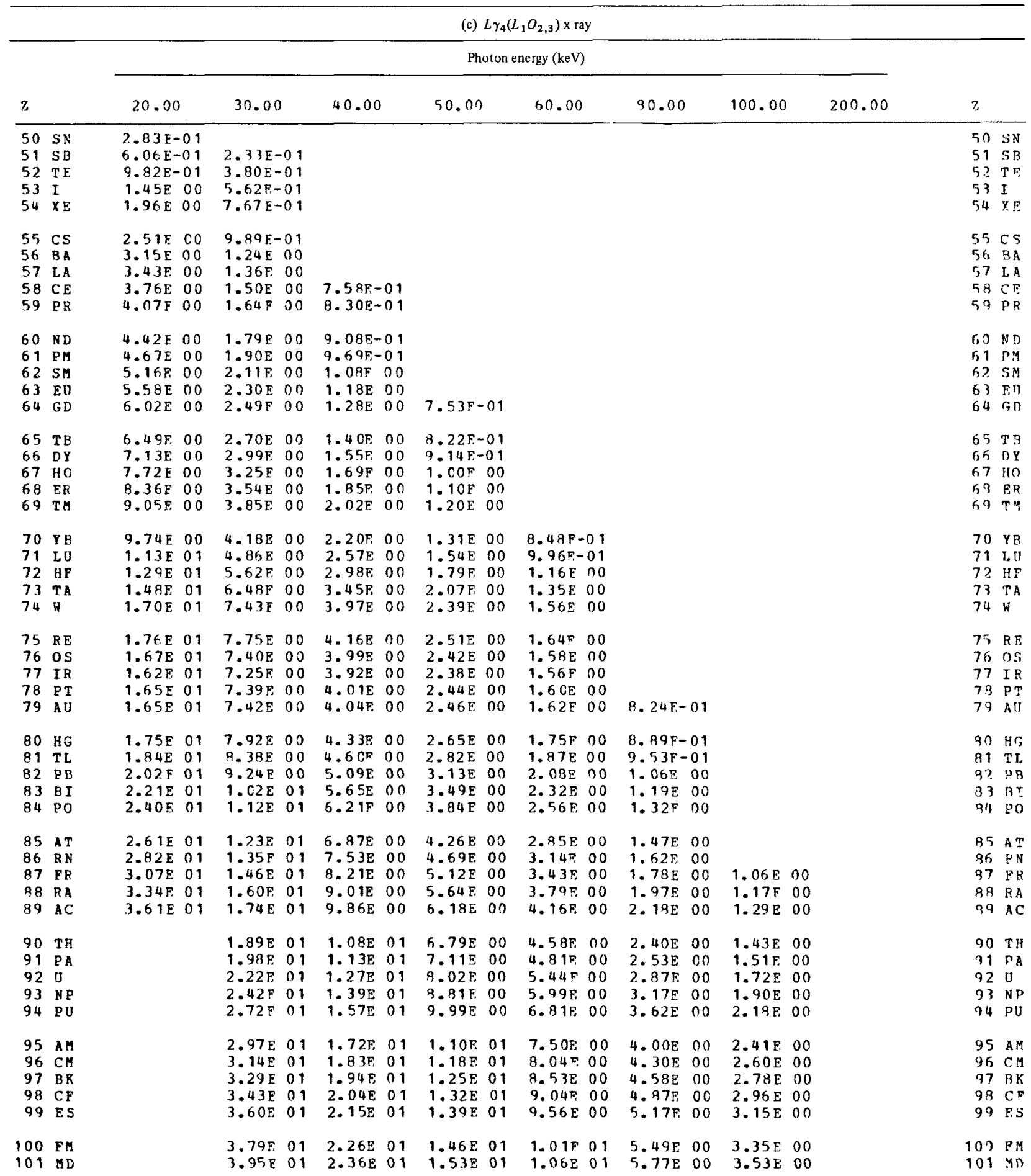


Table 10 (continued)

(d) $L \beta_{1}\left(L_{2} M_{4}\right) \times$ ray

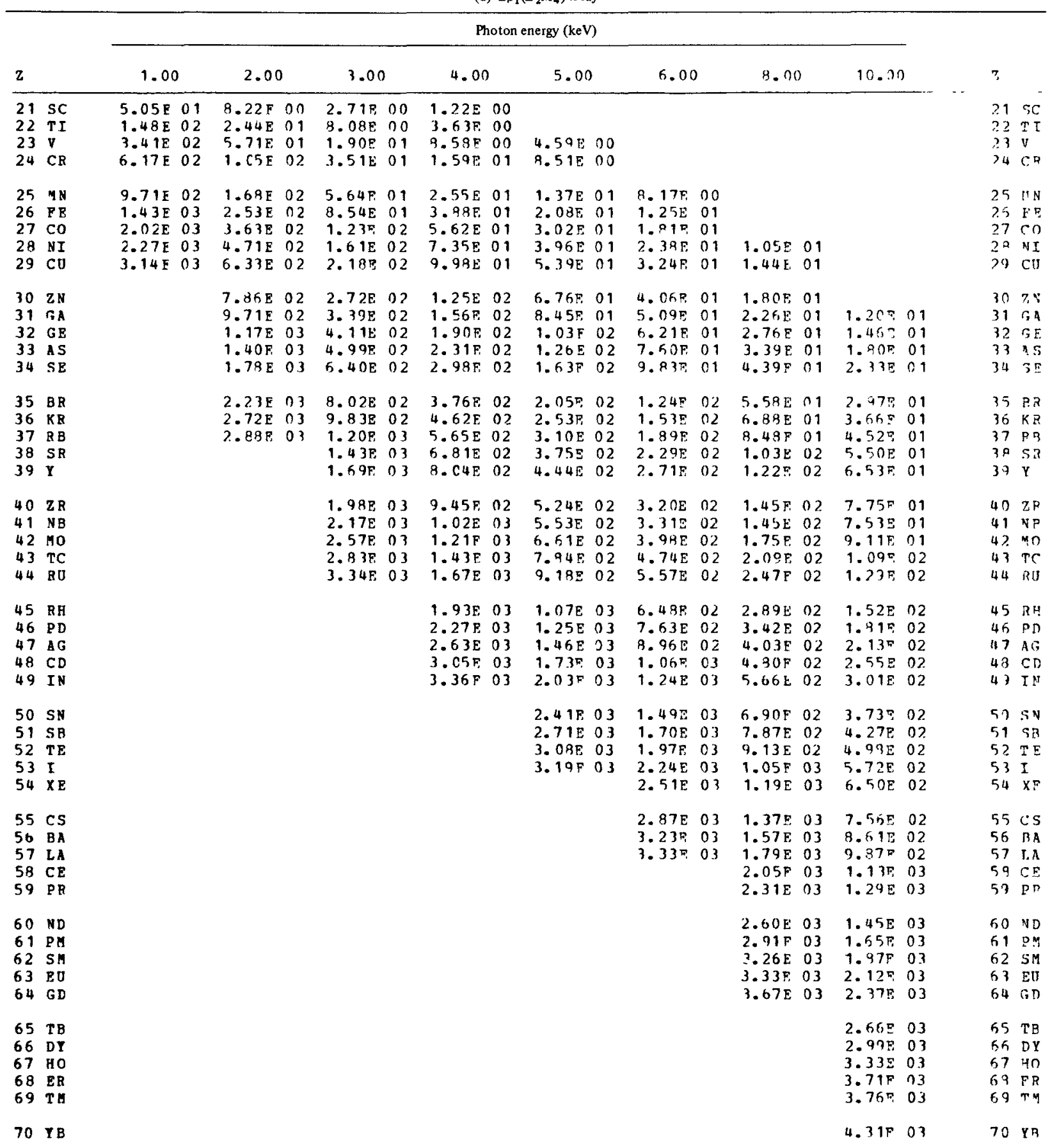


Table 10 (continued)

\begin{tabular}{|c|c|c|c|c|c|c|c|c|c|c|c|c|c|c|c|c|c|c|}
\hline \multicolumn{19}{|c|}{ (d) $L \beta_{1}\left(L_{2} M_{4}\right) \times$ ray } \\
\hline . & & \multicolumn{15}{|c|}{ Photon energy (keV) } & \multirow{2}{*}{\multicolumn{2}{|c|}{$?$}} \\
\hline $\mathbf{Z}$ & & 20.00 & & 30.00 & & 40.00 & & 50.00 & & 60.00 & & 80.00 & & 100.00 & & 200.00 & & \\
\hline $\begin{array}{l}43 \\
44\end{array}$ & $\begin{array}{l}\mathrm{TC} \\
\mathrm{RU}\end{array}$ & $\begin{array}{l}1.35 \mathrm{E} \\
1.62 \mathrm{E}\end{array}$ & $\begin{array}{l}01 \\
01\end{array}$ & & & & & & & & & & & & & & $\begin{array}{l}43 \\
44\end{array}$ & $\begin{array}{l}T C \\
d f 1\end{array}$ \\
\hline $\begin{array}{l}45 \\
46 \\
47 \\
48 \\
49\end{array}$ & $\begin{array}{l}R H \\
P D \\
A G \\
C D \\
I N\end{array}$ & $\begin{array}{l}1.91 \mathrm{E} \\
2.29 \mathrm{~F} \\
2.73 \mathrm{E} \\
3.29 \mathrm{E} \\
3.92 \mathrm{~F}\end{array}$ & $\begin{array}{ll}0 & 1 \\
0 & 1 \\
0 & 1 \\
0 & 1 \\
0 & 1\end{array}$ & & & & & & & & & & & & & & $\begin{array}{l}45 \\
46 \\
47 \\
49 \\
49\end{array}$ & $\begin{array}{l}\text { R1 } \\
\text { PD } \\
\text { AS } \\
\text { CD } \\
\text { IN }\end{array}$ \\
\hline $\begin{array}{l}50 \\
51 \\
52 \\
53 \\
54\end{array}$ & $\begin{array}{l}S N \\
S B \\
T E \\
I \\
X E\end{array}$ & $\begin{array}{l}5.13 \mathrm{E} \\
5.92 \mathrm{E} \\
6.99 \mathrm{E} \\
8.09 \mathrm{E} \\
9.32 \mathrm{E}\end{array}$ & $\begin{array}{ll}0 & 1 \\
0 & 1 \\
0 & 1 \\
0 & 1 \\
0 & 1\end{array}$ & $\begin{array}{l}1.79 \mathrm{E} \\
2.13 \mathrm{E} \\
2.48 \mathrm{~F} \\
2.87 \mathrm{E}\end{array}$ & $\begin{array}{ll}0 & 1 \\
0 & 1 \\
0 & 1 \\
0 & 1\end{array}$ & & & & & & & & & & & & $\begin{array}{l}50 \\
51 \\
52 \\
53 \\
54\end{array}$ & $\begin{array}{l}S N \\
S B \\
T E \\
I \\
X E\end{array}$ \\
\hline $\begin{array}{l}55 \\
56 \\
57 \\
58 \\
59\end{array}$ & $\begin{array}{l}\text { CS } \\
\text { BA } \\
\text { LA A } \\
\text { CE } \\
\text { PR }\end{array}$ & $\begin{array}{l}1.09 \mathrm{E} \\
1.26 \mathrm{E} \\
1.45 \mathrm{E} \\
1.68 \mathrm{E} \\
1.92 \mathrm{E}\end{array}$ & $\begin{array}{l}02 \\
02 \\
02 \\
02 \\
02\end{array}$ & $\begin{array}{l}3.38 E \\
3.90 E \\
4.52 E \\
5.24 E \\
6 . C 1 E\end{array}$ & $\begin{array}{ll}0 & 1 \\
0 & 1 \\
0 & 1 \\
0 & 1 \\
0 & 1\end{array}$ & $\begin{array}{l}2.26 \mathrm{E} \\
2.60 \mathrm{R}\end{array}$ & $\begin{array}{l}01 \\
01\end{array}$ & & & & & & & & & & $\begin{array}{l}55 \\
56 \\
57 \\
59 \\
59\end{array}$ & $\begin{array}{l}C S \\
B A \\
L A \\
C E \\
D Q\end{array}$ \\
\hline $\begin{array}{l}60 \\
61 \\
62 \\
63 \\
64\end{array}$ & $\begin{array}{l}\text { ND } \\
\text { PH } \\
\text { SI } \\
\text { EU } \\
\text { GD }\end{array}$ & $\begin{array}{l}2.19 \mathrm{E} \\
2.50 \mathrm{E} \\
2.85 \mathrm{E} \\
3.25 \mathrm{E} \\
3.68 \mathrm{E}\end{array}$ & $\begin{array}{l}02 \\
02 \\
02 \\
02 \\
02\end{array}$ & $\begin{array}{l}6.88 \mathrm{E} \\
7.89 \mathrm{E} \\
9.03 \mathrm{E} \\
1.03 \mathrm{E} \\
1.17 \mathrm{E}\end{array}$ & $\begin{array}{l}01 \\
01 \\
01 \\
02 \\
02\end{array}$ & $\begin{array}{l}2.98 \mathrm{E} \\
3.43 \mathrm{E} \\
3.94 \mathrm{E} \\
4.52 \mathrm{E} \\
5.14 \mathrm{E}\end{array}$ & $\begin{array}{ll}0 & 1 \\
0 & 1 \\
0 & 1 \\
0 & 1 \\
0 & 1\end{array}$ & $2.69 \mathrm{~F}$ & 01 & & & & & & & & $\begin{array}{l}60 \\
61 \\
62 \\
63 \\
64\end{array}$ & $\begin{array}{l}\text { VD } \\
\text { PM } \\
\text { SM } \\
\text { FU } \\
\text { GD }\end{array}$ \\
\hline $\begin{array}{l}65 \\
66 \\
67 \\
68 \\
69\end{array}$ & $\begin{array}{l}\text { TB } \\
\text { DY } \\
\text { HO } \\
\text { ER } \\
\text { TH }\end{array}$ & $\begin{array}{l}4.17 \mathrm{E} \\
4.76 \mathrm{E} \\
5.39 \mathrm{E} \\
6.08 \mathrm{E} \\
6.82 \mathrm{E}\end{array}$ & $\begin{array}{l}02 \\
02 \\
02 \\
02 \\
02\end{array}$ & $\begin{array}{l}1.34 \mathrm{E} \\
1.53 \mathrm{E} \\
1.74 \mathrm{E} \\
1.98 \mathrm{E} \\
2.23 \mathrm{E}\end{array}$ & $\begin{array}{l}02 \\
02 \\
02 \\
02 \\
02\end{array}$ & $\begin{array}{l}5.86 \mathrm{E} \\
6.75 \mathrm{E} \\
7.69 \mathrm{E} \\
8.73 \mathrm{E} \\
9.86 \mathrm{E}\end{array}$ & $\begin{array}{ll}01 \\
01 \\
01 \\
01 \\
01\end{array}$ & $\begin{array}{l}3.07 \mathrm{E} \\
3.54 \mathrm{E} \\
4.05 \mathrm{E} \\
4.60 \mathrm{~F} \\
5.21 \mathrm{E}\end{array}$ & $\begin{array}{l}01 \\
01 \\
01 \\
01 \\
01\end{array}$ & & & & & & & & $\begin{array}{l}65 \\
65 \\
57 \\
60 \\
69\end{array}$ & $\begin{array}{l}\text { TB } \\
\text { DY } \\
\text { HO } \\
\text { FR } \\
\text { TM }\end{array}$ \\
\hline $\begin{array}{l}70 \\
71 \\
72 \\
73 \\
74\end{array}$ & $\begin{array}{l}\text { YB } \\
\text { LU } \\
\text { HE } \\
\text { TA } \\
W\end{array}$ & $\begin{array}{l}7.60 \mathrm{E} \\
8.53 \mathrm{E} \\
9.33 \mathrm{E} \\
1.04 \mathrm{E} \\
1.13 \mathrm{E}\end{array}$ & $\begin{array}{l}02 \\
02 \\
02 \\
03 \\
03\end{array}$ & $\begin{array}{l}2.49 \mathrm{E} \\
2.80 \mathrm{E} \\
3.08 \mathrm{E} \\
3.43 \mathrm{E} \\
3.77 \mathrm{E}\end{array}$ & $\begin{array}{l}02 \\
02 \\
02 \\
02 \\
02\end{array}$ & $\begin{array}{l}1.11 F \\
1.25 F \\
1.37 \% \\
1.54 F \\
1.69 E\end{array}$ & $\begin{array}{l}02 \\
02 \\
02 \\
02 \\
02\end{array}$ & $\begin{array}{l}5.85 E \\
6.62 E \\
7.28 F \\
8.15 E \\
8.96 E\end{array}$ & $\begin{array}{l}01 \\
01 \\
01 \\
01 \\
01\end{array}$ & $\begin{array}{l}3.46 \mathrm{R} \\
3.92 \mathrm{E} \\
4.31 \mathrm{~F} \\
4.84 \mathrm{~F} \\
5.32 \mathrm{E}\end{array}$ & $\begin{array}{ll}0 & 1 \\
0 & 1 \\
01 & 1 \\
01 \\
01\end{array}$ & & & & & & $\begin{array}{l}70 \\
71 \\
72 \\
73 \\
714\end{array}$ & $\begin{array}{l}\text { Y } 0 \\
\text { LI } \\
H P \\
\text { TA } \\
\text { T }\end{array}$ \\
\hline $\begin{array}{l}75 \\
76 \\
77 \\
78 \\
79\end{array}$ & $\begin{array}{l}\text { RE } \\
\text { OS } \\
\text { IR } \\
\text { PT } \\
\text { AU }\end{array}$ & $\begin{array}{l}1.24 \mathrm{E} \\
1.37 \mathrm{E} \\
1.49 \mathrm{E} \\
1.62 \mathrm{E} \\
1.78 \mathrm{E}\end{array}$ & $\begin{array}{l}03 \\
03 \\
03 \\
03 \\
03\end{array}$ & $\begin{array}{l}4.15 \mathrm{E} \\
4.57 \mathrm{E} \\
4.99 \mathrm{E} \\
5.45 \mathrm{E} \\
5.98 \mathrm{E}\end{array}$ & $\begin{array}{l}02 \\
02 \\
02 \\
02 \\
02\end{array}$ & $\begin{array}{l}1.86 \mathrm{E} \\
2.05 \mathrm{E} \\
2.25 \mathrm{E} \\
2.46 \mathrm{E} \\
2.71 \mathrm{E}\end{array}$ & $\begin{array}{l}02 \\
02 \\
02 \\
02 \\
02\end{array}$ & $\begin{array}{l}9.87 \mathrm{E} \\
1.09 \mathrm{~F} \\
1.20 \mathrm{E} \\
1.31 \mathrm{E} \\
1.45 \mathrm{E}\end{array}$ & $\begin{array}{l}01 \\
02 \\
02 \\
02 \\
02\end{array}$ & $\begin{array}{l}5.86 \mathrm{E} \\
6.50 \mathrm{~F} \\
7.12 \mathrm{E} \\
7.80 \mathrm{E} \\
8.63 \mathrm{E}\end{array}$ & $\begin{array}{l}01 \\
01 \\
01 \\
01 \\
01\end{array}$ & $3.79 \mathrm{E}$ & 01 & & & & $\begin{array}{l}75 \\
76 \\
77 \\
78 \\
79\end{array}$ & $\begin{array}{l}\text { RE } \\
\text { OS } \\
\text { IR } \\
\text { Pm } \\
\text { AU }\end{array}$ \\
\hline $\begin{array}{l}80 \\
81 \\
82 \\
83 \\
84\end{array}$ & $\begin{array}{l}\text { HG } \\
\text { TL } \\
\text { PB } \\
\text { BI } \\
\text { PO }\end{array}$ & $\begin{array}{l}1.92 \mathrm{~B} \\
2.09 \mathrm{E} \\
2.25 \mathrm{E} \\
2.43 \mathrm{E} \\
2.61 \mathrm{E}\end{array}$ & $\begin{array}{l}03 \\
03 \\
03 \\
03 \\
03\end{array}$ & $\begin{array}{l}6.50 \mathrm{E} \\
7.12 \mathrm{~F} \\
7.70 \mathrm{E} \\
8.35 \mathrm{E} \\
9.09 \mathrm{E}\end{array}$ & $\begin{array}{l}02 \\
02 \\
02 \\
02 \\
02\end{array}$ & $\begin{array}{l}2.95 \mathrm{E} \\
3.24 \mathrm{P} \\
3.51 \mathrm{E} \\
3.81 \mathrm{E} \\
4.17 \mathrm{E}\end{array}$ & $\begin{array}{l}02 \\
02 \\
02 \\
02 \\
02\end{array}$ & $\begin{array}{l}1.58 \mathrm{E} \\
1.74 \mathrm{E} \\
1.88 \mathrm{E} \\
2.05 \mathrm{E} \\
2.25 \mathrm{E}\end{array}$ & $\begin{array}{l}02 \\
02 \\
02 \\
02 \\
02\end{array}$ & $\begin{array}{l}9.41 \mathrm{E} \\
1.04 \mathrm{~F} \\
1.13 \mathrm{E} \\
1.23 \mathrm{~F} \\
1.35 \mathrm{E}\end{array}$ & $\begin{array}{l}01 \\
02 \\
02 \\
02 \\
02\end{array}$ & $\begin{array}{l}4.13 \mathrm{E} \\
4.57 \mathrm{E} \\
4.96 \mathrm{E} \\
5.41 \mathrm{E} \\
5.96 \mathrm{E}\end{array}$ & $\begin{array}{l}01 \\
01 \\
01 \\
01 \\
01\end{array}$ & & & & $\begin{array}{l}80 \\
81 \\
32 \\
83 \\
84\end{array}$ & $\begin{array}{l}\text { IA } \\
\text { TL } \\
\text { PB } \\
\text { BI } \\
\text { PO }\end{array}$ \\
\hline $\begin{array}{l}85 \\
86 \\
87 \\
88 \\
89\end{array}$ & $\begin{array}{l}\text { AT } \\
\text { RN } \\
\text { PR } \\
\text { R A } \\
\text { AC }\end{array}$ & $\begin{array}{l}2.79 \mathrm{E} \\
3.00 \mathrm{E} \\
3.22 \mathrm{E} \\
3.45 \mathrm{E} \\
3.72 \mathrm{E}\end{array}$ & $\begin{array}{l}03 \\
03 \\
03 \\
03 \\
03\end{array}$ & $\begin{array}{l}9.80 \mathrm{E} \\
1.66 \mathrm{E} \\
1.15 \mathrm{E} \\
1.24 \mathrm{E} \\
1.33 \mathrm{E}\end{array}$ & $\begin{array}{l}02 \\
03 \\
03 \\
03 \\
03\end{array}$ & $\begin{array}{l}4.51 \mathrm{E} \\
4.91 \mathrm{E} \\
5.34 \mathrm{E} \\
5.74 \mathrm{E} \\
6.20 \mathrm{E}\end{array}$ & $\begin{array}{l}02 \\
02 \\
02 \\
02 \\
02\end{array}$ & $\begin{array}{l}2.43 \mathrm{E} \\
2.66 \mathrm{E} \\
2.90 \mathrm{E} \\
3.12 \mathrm{E} \\
3.38 \mathrm{E}\end{array}$ & $\begin{array}{l}02 \\
02 \\
02 \\
02 \\
02\end{array}$ & $\begin{array}{l}1.46 \mathrm{E} \\
1.60 \mathrm{E} \\
1.75 \mathrm{E} \\
1.89 \mathrm{E} \\
2.05 \mathrm{E}\end{array}$ & $\begin{array}{l}02 \\
02 \\
02 \\
02 \\
02\end{array}$ & $\begin{array}{l}6.47 \mathrm{E} \\
7.10 \mathrm{E} \\
7.80 \mathrm{~F} \\
8.41 \mathrm{E} \\
9.16 \mathrm{E}\end{array}$ & $\begin{array}{l}01 \\
01 \\
01 \\
01 \\
01\end{array}$ & $\begin{array}{l}4.14 \mathrm{E} \\
4.47 \mathrm{E} \\
4.98 \mathrm{~F}\end{array}$ & $\begin{array}{l}01 \\
01 \\
01\end{array}$ & & $\begin{array}{l}85 \\
95 \\
87 \\
89 \\
99\end{array}$ & $\begin{array}{l}A T \\
R N \\
F R \\
R A \\
A C\end{array}$ \\
\hline $\begin{array}{l}90 \\
91 \\
92 \\
93 \\
94\end{array}$ & $\begin{array}{l}\text { TH } \\
\mathbf{P A} \\
\mathbf{U} \\
\mathbf{H P} \\
\mathrm{PU}\end{array}$ & $3.72 \mathrm{E}$ & 03 & $\begin{array}{l}1.43 \mathrm{E} \\
1.46 \mathrm{E} \\
1.51 \mathrm{E} \\
1.57 \mathrm{E} \\
1.61 \mathrm{E}\end{array}$ & $\begin{array}{l}03 \\
03 \\
03 \\
03 \\
03\end{array}$ & $\begin{array}{l}6.67 \mathrm{E} \\
6.86 \mathrm{E} \\
7.13 \mathrm{E} \\
7.41 \mathrm{E} \\
7.62 \mathrm{E}\end{array}$ & $\begin{array}{l}02 \\
02 \\
02 \\
02 \\
02\end{array}$ & $\begin{array}{l}3.65 \mathrm{E} \\
3.76 \mathrm{E} \\
3.92 \mathrm{E} \\
4.09 \mathrm{E} \\
4.21 \mathrm{E}\end{array}$ & $\begin{array}{l}02 \\
02 \\
02 \\
02 \\
02\end{array}$ & $\begin{array}{l}2.21 \mathrm{~B} \\
2.28 \mathrm{E} \\
2.39 \mathrm{E} \\
2.49 \mathrm{E} \\
2.57 \mathrm{~B}\end{array}$ & $\begin{array}{l}02 \\
02 \\
02 \\
02 \\
02\end{array}$ & $\begin{array}{l}9.94 \mathrm{E} \\
1.03 \mathrm{E} \\
1.08 \mathrm{E} \\
1.13 \mathrm{~F} \\
1.16 \mathrm{E}\end{array}$ & $\begin{array}{l}01 \\
02 \\
02 \\
02 \\
02\end{array}$ & $\begin{array}{l}5.31 \mathrm{E} \\
5.50 \mathrm{E} \\
5.78 \mathrm{E} \\
6.05 \mathrm{E} \\
6.24 \mathrm{E}\end{array}$ & $\begin{array}{l}01 \\
01 \\
01 \\
01 \\
01\end{array}$ & & $\begin{array}{l}70 \\
91 \\
92 \\
33 \\
94\end{array}$ & $\begin{array}{l}\mathrm{TH} \\
\mathrm{PA} \\
\mathrm{U} \\
\mathrm{NP} \\
\mathrm{NU}\end{array}$ \\
\hline $\begin{array}{l}95 \\
96 \\
97 \\
98 \\
99\end{array}$ & $\begin{array}{l}\text { A H } \\
\text { CE } \\
B K \\
C F \\
\text { ES }\end{array}$ & & & $\begin{array}{l}1.70 \mathrm{E} \\
1.78 \mathrm{E} \\
1.88 \mathrm{E} \\
1.95 \mathrm{E} \\
2.05 \mathrm{E}\end{array}$ & $\begin{array}{l}03 \\
03 \\
03 \\
03 \\
03\end{array}$ & $\begin{array}{l}8.11 \mathrm{E} \\
8.57 \mathrm{E} \\
9.09 \mathrm{E} \\
9.56 \mathrm{E} \\
1.01 \mathrm{E}\end{array}$ & $\begin{array}{l}02 \\
02 \\
02 \\
02 \\
03\end{array}$ & $\begin{array}{l}4.50 \mathrm{E} \\
4.77 \mathrm{E} \\
5.07 \mathrm{E} \\
5.35 \mathrm{E} \\
5.70 \mathrm{E}\end{array}$ & $\begin{array}{l}02 \\
02 \\
02 \\
02 \\
02\end{array}$ & $\begin{array}{l}2.75 \mathrm{E} \\
2.92 \mathrm{E} \\
3.12 \mathrm{E} \\
3.30 \mathrm{E} \\
3.52 \mathrm{E}\end{array}$ & $\begin{array}{l}02 \\
02 \\
02 \\
02 \\
02\end{array}$ & $\begin{array}{l}1.25 \mathrm{E} \\
1.33 \mathrm{E} \\
1.43 \mathrm{E} \\
1.51 \mathrm{E} \\
1.62 \mathrm{E}\end{array}$ & $\begin{array}{l}02 \\
02 \\
02 \\
\mathrm{C} 2 \\
02\end{array}$ & $\begin{array}{l}6.73 \mathrm{E} \\
7.18 \mathrm{E} \\
7.72 \mathrm{~F} \\
8.19 \mathrm{E} \\
8.81 \mathrm{E}\end{array}$ & $\begin{array}{l}01 \\
01 \\
01 \\
01 \\
01\end{array}$ & & $\begin{array}{l}95 \\
96 \\
97 \\
93 \\
39\end{array}$ & $\begin{array}{l}A M \\
C M \\
\text { GK } \\
C P \\
\text { ES }\end{array}$ \\
\hline $\begin{array}{l}100 \\
101\end{array}$ & $\begin{array}{l}\text { PH } \\
\text { ID }\end{array}$ & & & $\begin{array}{l}2.16 \mathrm{E} \\
2.29 \mathrm{E}\end{array}$ & $\begin{array}{l}03 \\
03\end{array}$ & $\begin{array}{l}1.088 \\
1.14 \mathrm{E}\end{array}$ & $\begin{array}{l}03 \\
03\end{array}$ & $\begin{array}{l}6.088 \\
6.44 \mathrm{E}\end{array}$ & $\begin{array}{l}02 \\
02\end{array}$ & $\begin{array}{l}3.77 \mathrm{E} \\
4.00 \mathrm{E}\end{array}$ & $\begin{array}{l}02 \\
02\end{array}$ & $\begin{array}{l}1.74 E \\
1.86 \mathrm{E}\end{array}$ & $\begin{array}{l}02 \\
02\end{array}$ & $\begin{array}{l}9.50 F \\
1.01 \mathrm{~F}\end{array}$ & $\begin{array}{l}01 \\
02\end{array}$ & & $\begin{array}{l}10 ? \\
101\end{array}$ & $\begin{array}{l}P M \\
M n\end{array}$ \\
\hline
\end{tabular}


Table 10 (continued)

(e) $L \gamma_{1}\left(L_{2} N_{4}\right) \times$ ray

\begin{tabular}{|c|c|c|c|c|c|c|c|c|c|c|c|c|c|}
\hline \multirow{2}{*}{\multicolumn{2}{|c|}{$\mathbf{z}$}} & \multicolumn{10}{|c|}{ Photon energy (keV) } & \multirow{2}{*}{\multicolumn{2}{|c|}{7.}} \\
\hline & & 1.00 & 2.00 & 3.00 & 4.00 & 5.00 & 6.00 & & 8.00 & 10.00 & & & \\
\hline 39 & $\mathbf{Y}$ & & & 1.18801 & $5.64 \mathrm{E} 00$ & 3.11800 & $1.90 \mathrm{E}$ & 00 & $8.57 \mathrm{E}-0.2$ & $4.59 \mathrm{~F}-$ & 01 & 39 & $Y$ \\
\hline $\begin{array}{l}40 \\
41 \\
42 \\
43 \\
44\end{array}$ & $\begin{array}{l}\mathrm{ZR} \\
\mathrm{NB} \\
\mathrm{MO} \\
\mathrm{TC} \\
\mathrm{RO}\end{array}$ & & & $\begin{array}{ll}3.13 \mathrm{~F} & 01 \\
6.69 \mathrm{E} & 01 \\
1.07 \mathrm{~F} & 02 \\
1.51 \mathrm{E} & 02 \\
2.20 \mathrm{E} & 02\end{array}$ & $\begin{array}{ll}1.49 \mathrm{~F} & 01 \\
3.13 \mathrm{E} & 01 \\
5.04 \mathrm{E} & 01 \\
7.62 \mathrm{E} & 01 \\
1.10 \mathrm{E} & 02\end{array}$ & 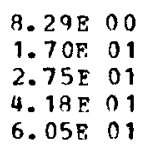 & $\begin{array}{l}5.07 \mathrm{E} \\
1.02 \mathrm{E} \\
1.66 \mathrm{~F} \\
2.53 \mathrm{~F} \\
3.67 \mathrm{E}\end{array}$ & $\begin{array}{ll}0 & 0 \\
01 \\
01 \\
01 \\
01\end{array}$ & 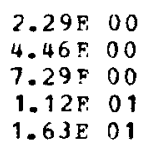 & $\begin{array}{l}1.23 \mathrm{~F} \\
2.31 \mathrm{~F} \\
3.80 \mathrm{~F} \\
5.83 \mathrm{~F} \\
8.51 \mathrm{~F}\end{array}$ & $\begin{array}{l}00 \\
00 \\
00 \\
00 \\
00\end{array}$ & $\begin{array}{l}40 \\
49 \\
47 \\
43 \\
44\end{array}$ & $\begin{array}{l}2 R \\
V R \\
40 \\
\text { TC } \\
\text { FII }\end{array}$ \\
\hline $\begin{array}{l}45 \\
46 \\
47 \\
48 \\
49\end{array}$ & $\begin{array}{l}\text { RH } \\
\text { PD } \\
\text { AG } \\
\text { CD } \\
\text { IN }\end{array}$ & & & & 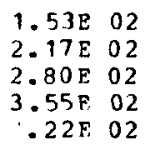 & $\begin{array}{ll}8.42 \mathrm{~F} & 01 \\
1.19 \mathrm{E} & 02 \\
1.56 \mathrm{E} & 02 \\
2.02 \mathrm{~F} & 02 \\
2.55 \mathrm{~F} & 02\end{array}$ & $\begin{array}{l}5.11 \mathrm{E} \\
7.30 \mathrm{E} \\
9.55 \mathrm{E} \\
1.24 \mathrm{E} \\
1.56 \mathrm{E}\end{array}$ & $\begin{array}{l}01 \\
01 \\
01 \\
02 \\
02\end{array}$ & $\begin{array}{ll}2.28 \mathrm{E} & 01 \\
3.27 \mathrm{E} & 01 \\
4.30 \mathrm{E} & 01 \\
5.59 \mathrm{E} & 01 \\
7.11 \mathrm{E} & 01\end{array}$ & $\begin{array}{l}1.20 \mathrm{E} \\
1.73 \mathrm{E} \\
2.27 \mathrm{~F} \\
2.96 \mathrm{~F} \\
3.79 \mathrm{E}\end{array}$ & $\begin{array}{l}01 \\
01 \\
01 \\
01 \\
01\end{array}$ & $\begin{array}{l}45 \\
45 \\
47 \\
44 \\
49\end{array}$ & $\begin{array}{l}\text { RH } \\
\text { PD } \\
\text { AG } \\
C D \\
\text { IN }\end{array}$ \\
\hline $\begin{array}{l}50 \\
51 \\
52 \\
53 \\
54\end{array}$ & $\begin{array}{l}S N \\
S B \\
T E \\
I \\
X E\end{array}$ & & & & & 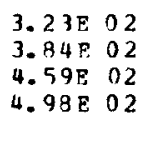 & $\begin{array}{l}2.00 \mathrm{E} \\
2.41 \mathrm{~F} \\
2.93 \mathrm{E} \\
3.49 \mathrm{~F} \\
4.07 \mathrm{E}\end{array}$ & $\begin{array}{l}02 \\
02 \\
02 \\
02 \\
02\end{array}$ & 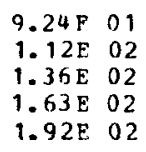 & $\begin{array}{l}5.00 \mathrm{~F} \\
6.06 \mathrm{E} \\
7.43 \mathrm{~B} \\
8.92 \mathrm{E} \\
1.05 \mathrm{~F}\end{array}$ & $\begin{array}{l}01 \\
01 \\
01 \\
01 \\
02\end{array}$ & $\begin{array}{l}50 \\
51 \\
5 ? \\
53 \\
54\end{array}$ & $\begin{array}{l}S N \\
S R \\
T E \\
I \\
X F\end{array}$ \\
\hline $\begin{array}{l}55 \\
56 \\
57 \\
58 \\
59\end{array}$ & $\begin{array}{l}\text { CS } \\
\text { BA } \\
\text { LA } \\
\text { CE } \\
\text { PR }\end{array}$ & & & & & & $\begin{array}{l}4.82 \mathrm{P} \\
5.63 \mathrm{E} \\
5.97 \mathrm{P}\end{array}$ & $\begin{array}{l}02 \\
02 \\
02\end{array}$ & $\begin{array}{ll}2.31 \mathrm{E} & 02 \\
2.73 \mathrm{E} & 02 \\
3.21 \mathrm{E} & 02 \\
3.64 \mathrm{E} & 02 \\
4.15 \mathrm{E} & 02\end{array}$ & $\begin{array}{l}1.27 \mathrm{E} \\
1.50 \mathrm{P} \\
1.77 \mathrm{E} \\
2.01 \mathrm{E} \\
2.31 \mathrm{E}\end{array}$ & $\begin{array}{l}02 \\
02 \\
02 \\
02 \\
02\end{array}$ & $\begin{array}{l}55 \\
56 \\
57 \\
59 \\
57\end{array}$ & $\begin{array}{l}\text { CS } \\
3 A \\
\text { LA } \\
\text { CF } \\
\text { PR }\end{array}$ \\
\hline $\begin{array}{l}60 \\
61 \\
62 \\
63 \\
64\end{array}$ & $\begin{array}{l}\text { ND } \\
\text { PA } \\
\text { SH } \\
\text { ED } \\
\text { GD }\end{array}$ & & & & & & & & $\begin{array}{ll}4.69 \mathrm{~F} & 02 \\
5.29 \mathrm{E} & 02 \\
5.97 \mathrm{~F} & 02 \\
6.11 \mathrm{~F} & 02 \\
6.84 \mathrm{~F} & 02\end{array}$ & $\begin{array}{l}2.63 \mathrm{E} \\
3.01 \mathrm{~F} \\
3.43 \mathrm{~F} \\
3.89 \mathrm{E} \\
4.43 \mathrm{~F}\end{array}$ & $\begin{array}{l}02 \\
02 \\
02 \\
02 \\
02\end{array}$ & $\begin{array}{l}60 \\
61 \\
62 \\
63 \\
64\end{array}$ & $\begin{array}{l}\text { VD } \\
\text { PM } \\
\text { SM } \\
\text { FU } \\
\text { GD }\end{array}$ \\
\hline $\begin{array}{l}65 \\
66 \\
67 \\
68 \\
69\end{array}$ & $\begin{array}{l}\text { TB } \\
\text { DY } \\
\text { HO } \\
\mathrm{ER} \\
\mathrm{TH}\end{array}$ & & & & & & & & & $\begin{array}{l}4.93 \mathrm{~F} \\
5.56 \mathrm{E} \\
6.23 \mathrm{E} \\
6.96 \mathrm{E} \\
7.07 \mathrm{E}\end{array}$ & $\begin{array}{l}02 \\
02 \\
02 \\
02 \\
02\end{array}$ & $\begin{array}{l}65 \\
55 \\
67 \\
69 \\
69\end{array}$ & $\begin{array}{l}T Q \\
D Y \\
H O \\
F R \\
T M\end{array}$ \\
\hline 70 & Y B & & & & & & & & & 8.145 & 02 & 70 & $Y B$ \\
\hline
\end{tabular}


Table 10 (continued)

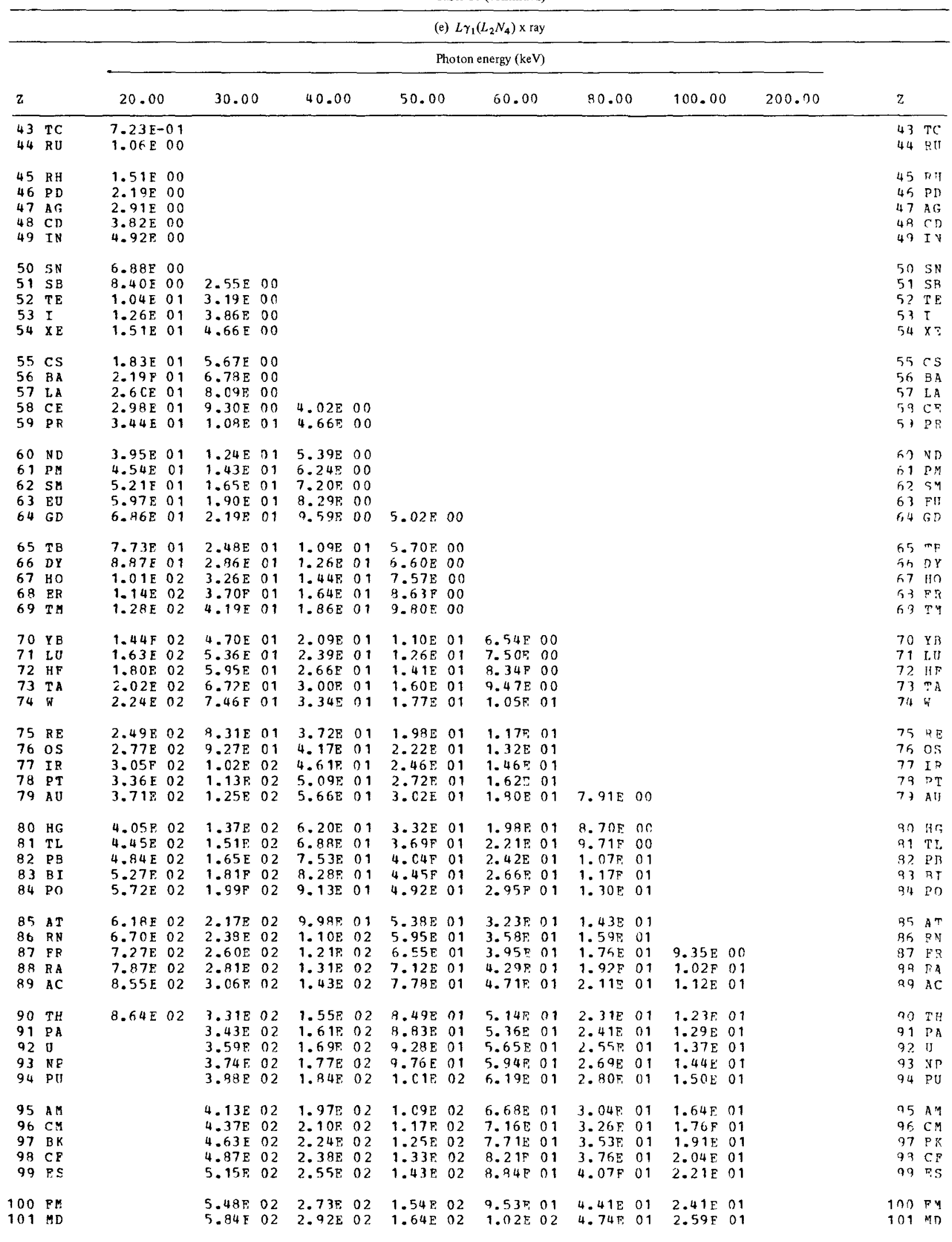


Table 10 (continued)

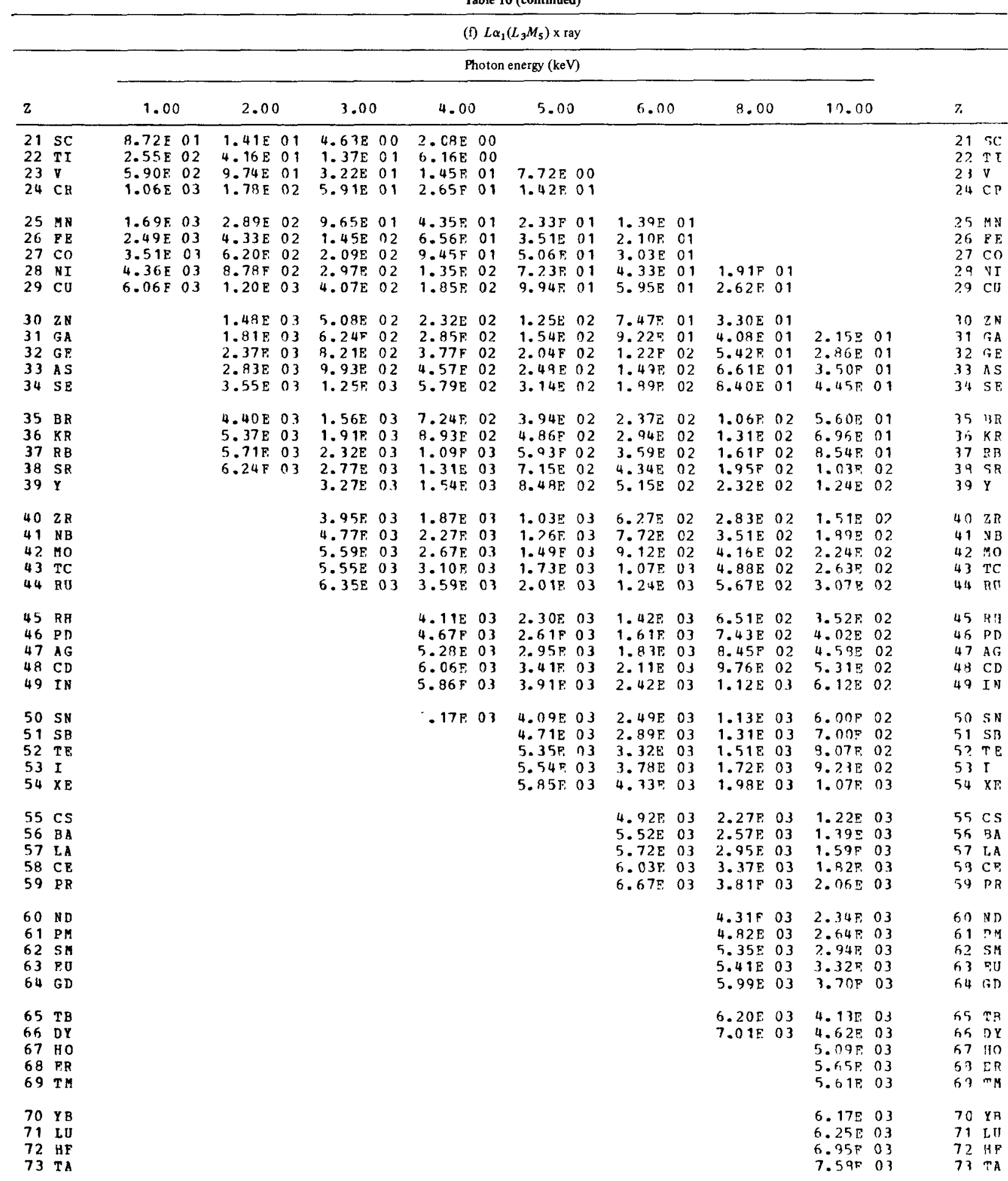


Table 10 (continued)

\begin{tabular}{|c|c|c|c|c|c|c|c|c|c|c|c|c|c|c|c|c|c|}
\hline \multicolumn{18}{|c|}{ (f) $L \alpha_{1}\left(L_{3} M_{5}\right) \times$ ray } \\
\hline \multirow[b]{2}{*}{2} & & \multicolumn{14}{|c|}{ Photon energy (keV) } & \multirow{2}{*}{\multicolumn{2}{|c|}{3}} \\
\hline & & \multicolumn{2}{|l|}{20.00} & \multicolumn{2}{|c|}{30.00} & \multicolumn{2}{|l|}{40.00} & \multicolumn{2}{|c|}{50.00} & \multicolumn{2}{|l|}{50.00} & \multicolumn{2}{|c|}{80.00} & 100.00 & \multirow[t]{2}{*}{200.00} & & \\
\hline $\begin{array}{l}43 \\
44\end{array}$ & $\begin{array}{l}\text { TC } \\
\text { RU }\end{array}$ & $\begin{array}{l}3.71 E \\
4.34 E\end{array}$ & $\begin{array}{l}01 \\
01\end{array}$ & & & & & & & & & & & & & $\begin{array}{l}43 \\
44\end{array}$ & $\begin{array}{l}\text { TC } \\
\text { RU }\end{array}$ \\
\hline $\begin{array}{l}50 \\
51 \\
52 \\
53 \\
54\end{array}$ & $\begin{array}{l}\text { SN } \\
S B \\
T E \\
T \\
X E\end{array}$ & $\begin{array}{l}7.88 \mathrm{E} \\
9.29 \mathrm{E} \\
1.08 \mathrm{E} \\
1.24 \mathrm{~F} \\
1.44 \mathrm{E}\end{array}$ & $\begin{array}{l}01 \\
01 \\
02 \\
02 \\
02\end{array}$ & $\begin{array}{l}2.75 \mathrm{E} \\
3.20 \mathrm{E} \\
3.70 \mathrm{E} \\
4.30 \mathrm{E}\end{array}$ & $\begin{array}{ll}01 \\
01 \\
01 \\
01\end{array}$ & & & & & & & & & & & $\begin{array}{l}50 \\
51 \\
5 ? \\
53 \\
54\end{array}$ & $\begin{array}{l}S N \\
S A \\
T E \\
I \\
X E\end{array}$ \\
\hline $\begin{array}{l}55 \\
56 \\
57 \\
58 \\
59\end{array}$ & $\begin{array}{l}\text { CS } \\
\text { BA } \\
\text { LA } \\
\text { CE } \\
\text { PR }\end{array}$ & $\begin{array}{l}1.66 \mathrm{~F} \\
1.90 \mathrm{~F} \\
2.20 \mathrm{E} \\
2.53 \mathrm{E} \\
2.88 \mathrm{E}\end{array}$ & $\begin{array}{l}02 \\
02 \\
02 \\
02 \\
02\end{array}$ & $\begin{array}{l}4.97 \mathrm{E} \\
5.71 \mathrm{E} \\
6.64 \mathrm{~F} \\
7.66 \mathrm{E} \\
8.75 \mathrm{E}\end{array}$ & $\begin{array}{l}01 \\
01 \\
01 \\
01 \\
01\end{array}$ & $\begin{array}{l}3.25 \mathrm{E} \\
3.71 \mathrm{E}\end{array}$ & $\begin{array}{l}01 \\
01\end{array}$ & & & & & & & & & $\begin{array}{l}55 \\
56 \\
57 \\
59 \\
59\end{array}$ & $\begin{array}{l}\mathrm{CS} \\
\mathrm{BA} \\
\mathrm{LA} \\
\mathrm{CE} \\
\mathrm{PR}\end{array}$ \\
\hline $\begin{array}{l}60 \\
61 \\
62 \\
63 \\
64\end{array}$ & $\begin{array}{l}\text { ND } \\
\text { PM } \\
\text { SM } \\
\text { EU } \\
\text { GD }\end{array}$ & $\begin{array}{l}3.30 \mathrm{E} \\
3.74 \mathrm{E} \\
4.19 \mathrm{~F} \\
4.74 \mathrm{E} \\
5.30 \mathrm{~F}\end{array}$ & $\begin{array}{l}02 \\
02 \\
02 \\
02 \\
02\end{array}$ & $\begin{array}{l}1.01 \mathrm{E} \\
1.14 \mathrm{E} \\
1.29 \mathrm{E} \\
1.46 \mathrm{E} \\
1.64 \mathrm{E}\end{array}$ & $\begin{array}{l}02 \\
02 \\
02 \\
02 \\
02\end{array}$ & $\begin{array}{l}4.29 \mathrm{~F} \\
4.87 \mathrm{E} \\
5.49 \mathrm{P} \\
6.23 \mathrm{E} \\
7.01 \mathrm{E}\end{array}$ & $\begin{array}{ll}01 \\
01 \\
01 \\
01 \\
01\end{array}$ & $3.61 \mathrm{E}$ & 01 & & & & & & & $\begin{array}{l}60 \\
61 \\
6 ? \\
63 \\
54\end{array}$ & $\begin{array}{l}\text { ND } \\
P A \\
S M \\
P A 1 \\
G D\end{array}$ \\
\hline $\begin{array}{l}65 \\
66 \\
67 \\
68 \\
69\end{array}$ & $\begin{array}{l}\text { TB } \\
\text { DY } \\
\text { HC } \\
\text { ER } \\
\text { TH }\end{array}$ & $\begin{array}{l}5.97 \mathrm{E} \\
6.73 \mathrm{E} \\
7.47 \mathrm{E} \\
8.36 \mathrm{E} \\
9.22 \mathrm{E}\end{array}$ & $\begin{array}{l}02 \\
02 \\
02 \\
02 \\
02\end{array}$ & $\begin{array}{l}1.85 \mathrm{~F} \\
2.09 \mathrm{E} \\
2.32 \mathrm{E} \\
2.61 \mathrm{E} \\
2.87 \mathrm{E}\end{array}$ & $\begin{array}{l}02 \\
02 \\
02 \\
02 \\
02\end{array}$ & $\begin{array}{l}7.92 \mathrm{E} \\
8.96 \mathrm{E} \\
1.00 \mathrm{E} \\
1.12 \mathrm{E} \\
1.24 \mathrm{E}\end{array}$ & $\begin{array}{ll}0 & 1 \\
0 & 1 \\
0 & 2 \\
02 & 2 \\
02\end{array}$ & $\begin{array}{l}4.09 \mathrm{E} \\
4.63 \mathrm{E} \\
5.17 \mathrm{E} \\
5.82 \mathrm{~F} \\
6.42 \mathrm{E}\end{array}$ & $\begin{array}{ll}01 \\
01 \\
01 \\
01 \\
01\end{array}$ & & & & & & & $\begin{array}{l}65 \\
65 \\
67 \\
68 \\
59\end{array}$ & $\begin{array}{l}m B \\
D Y \\
1 \mathrm{Y} O \\
F R \\
T H\end{array}$ \\
\hline $\begin{array}{l}75 \\
76 \\
77 \\
78 \\
79\end{array}$ & $\begin{array}{l}\text { RE } \\
\text { OS } \\
\text { IR } \\
\text { PT } \\
\text { A U }\end{array}$ & $\begin{array}{l}1.70 \mathrm{E} \\
1.91 \mathrm{E} \\
2.14 \mathrm{E} \\
2.37 \mathrm{E} \\
2.62 \mathrm{E}\end{array}$ & $\begin{array}{l}03 \\
03 \\
03 \\
03 \\
03\end{array}$ & $\begin{array}{l}5.43 \mathrm{E} \\
6.19 \mathrm{E} \\
7.01 \mathrm{~F} \\
7.85 \mathrm{E} \\
8.71 \mathrm{E}\end{array}$ & $\begin{array}{l}02 \\
02 \\
02 \\
02 \\
02\end{array}$ & $\begin{array}{l}2.38 \mathrm{E} \\
2.74 \mathrm{E} \\
3.13 \mathrm{E} \\
3.52 \mathrm{E} \\
3.93 \mathrm{E}\end{array}$ & $\begin{array}{l}02 \\
02 \\
02 \\
02 \\
02\end{array}$ & $\begin{array}{l}1.25 \mathrm{E} \\
1.45 \mathrm{E} \\
1.66 \mathrm{E} \\
1.88 \mathrm{E} \\
2.11 \mathrm{~F}\end{array}$ & $\begin{array}{l}02 \\
02 \\
02 \\
02 \\
02\end{array}$ & $\begin{array}{l}7.33 \mathrm{E} \\
9.55 \mathrm{~F} \\
9.89 \mathrm{E} \\
1.13 \mathrm{E} \\
1.26 \mathrm{E}\end{array}$ & $\begin{array}{l}01 \\
01 \\
01 \\
02 \\
02\end{array}$ & $5.62 \mathrm{E}$ & 01 & & & $\begin{array}{l}75 \\
76 \\
77 \\
78 \\
79\end{array}$ & $\begin{array}{l}R E \\
O S \\
\text { IR } \\
\text { PT } \\
\text { AU }\end{array}$ \\
\hline $\begin{array}{l}80 \\
81 \\
82 \\
83 \\
84\end{array}$ & $\begin{array}{l}\text { HG } \\
\text { TL } \\
\text { PB } \\
\text { BI } \\
\text { PO }\end{array}$ & $\begin{array}{l}2.87 \mathrm{~F} \\
3.13 \mathrm{E} \\
3.39 \mathrm{E} \\
3.64 \mathrm{E} \\
3.92 \mathrm{E}\end{array}$ & $\begin{array}{l}03 \\
03 \\
03 \\
03 \\
03\end{array}$ & $\begin{array}{l}9.60 \mathrm{E} \\
1 . \mathrm{C} \mathrm{E} \\
1.14 \mathrm{E} \\
1.23 \mathrm{E} \\
1.32 \mathrm{E}\end{array}$ & $\begin{array}{l}02 \\
03 \\
03 \\
03 \\
03\end{array}$ & $\begin{array}{l}4.35 \mathrm{~F} \\
4.76 \mathrm{~F} \\
5.19 \mathrm{~F} \\
5.50 \mathrm{E} \\
6.04 \mathrm{E}\end{array}$ & $\begin{array}{l}02 \\
02 \\
02 \\
02 \\
02\end{array}$ & $\begin{array}{l}2.34 \mathrm{~F} \\
2.57 \mathrm{~F} \\
2.80 \mathrm{~F} \\
3.03 \mathrm{~F} \\
3.27 \mathrm{~F}\end{array}$ & $\begin{array}{l}02 \\
02 \\
02 \\
02 \\
02\end{array}$ & $\begin{array}{l}1.41 \mathrm{E} \\
1.55 \mathrm{E} \\
1.69 \mathrm{~F} \\
1.83 \mathrm{~F} \\
1.97 \mathrm{E}\end{array}$ & $\begin{array}{l}02 \\
02 \\
02 \\
02 \\
02\end{array}$ & $\begin{array}{l}6.29 \mathrm{E} \\
6.93 \mathrm{E} \\
7.58 \mathrm{E} \\
8.21 \mathrm{~F} \\
8.89 \mathrm{E}\end{array}$ & $\begin{array}{l}01 \\
01 \\
01 \\
01 \\
01\end{array}$ & & & $\begin{array}{l}80 \\
81 \\
82 \\
93 \\
94\end{array}$ & $\begin{array}{l}\mathrm{HG} \\
\mathrm{TL} \\
\mathrm{PB} \\
\mathrm{BI} \\
\mathrm{PO}\end{array}$ \\
\hline $\begin{array}{l}85 \\
86 \\
87 \\
88 \\
89\end{array}$ & $\begin{array}{l}\text { AT } \\
\text { RN } \\
\text { PR } \\
\text { RA } \\
\text { AC }\end{array}$ & $\begin{array}{l}4.21 \mathrm{E} \\
4.48 \mathrm{E} \\
4.79 \mathrm{E} \\
5.11 \mathrm{E} \\
5.44 \mathrm{E}\end{array}$ & $\begin{array}{l}03 \\
03 \\
03 \\
03 \\
03\end{array}$ & $\begin{array}{l}1.43 \mathrm{E} \\
1.52 \mathrm{E} \\
1.63 \mathrm{~F} \\
1.75 \mathrm{~F} \\
1.87 \mathrm{~F}\end{array}$ & $\begin{array}{l}03 \\
03 \\
03 \\
03 \\
03\end{array}$ & $\begin{array}{l}6.54 F \\
6.97 \mathrm{E} \\
7.49 \mathrm{E} \\
8.03 \mathrm{~F} \\
8.60 \mathrm{P}\end{array}$ & $\begin{array}{l}02 \\
02 \\
02 \\
02 \\
02\end{array}$ & $\begin{array}{l}3.55 \mathrm{E} \\
3.79 \mathrm{E} \\
4.07 \mathrm{~F} \\
4.37 \mathrm{E} \\
4.68 \mathrm{E}\end{array}$ & $\begin{array}{l}02 \\
02 \\
02 \\
02 \\
02\end{array}$ & $\begin{array}{l}2.15 \mathrm{E} \\
2.29 \mathrm{~F} \\
2.47 \mathrm{E} \\
2.65 \mathrm{E} \\
2.84 \mathrm{~F}\end{array}$ & $\begin{array}{l}02 \\
02 \\
02 \\
02 \\
02\end{array}$ & $\begin{array}{l}9.68 \mathrm{E} \\
1.03 \mathrm{E} \\
1.11 \mathrm{E} \\
1.20 \mathrm{E} \\
1.29 \mathrm{E}\end{array}$ & $\begin{array}{l}01 \\
02 \\
02 \\
02 \\
02\end{array}$ & $\begin{array}{ll}6.00 F & 01 \\
6.46 \mathrm{E} & 01 \\
6.94 \mathrm{E} & 01\end{array}$ & & $\begin{array}{l}85 \\
86 \\
37 \\
89 \\
89\end{array}$ & $\begin{array}{l}\text { AT } \\
\text { RN } \\
\text { FR } \\
\text { RA } \\
\text { AC }\end{array}$ \\
\hline $\begin{array}{l}90 \\
91 \\
92 \\
93 \\
94\end{array}$ & $\begin{array}{l}\text { TH } \\
\text { PA } \\
\text { U } \\
\text { N F } \\
\text { PO }\end{array}$ & $\begin{array}{l}4.78 \mathrm{E} \\
4.73 \mathrm{~F} \\
5.04 \mathrm{~F} \\
5.38 \mathrm{E} \\
5.74 \mathrm{E}\end{array}$ & $\begin{array}{l}03 \\
03 \\
03 \\
03 \\
03\end{array}$ & $\begin{array}{l}1.99 \mathrm{E} \\
2.17 \mathrm{E} \\
2.34 \mathrm{E} \\
2.53 \mathrm{E} \\
2.68 \mathrm{E}\end{array}$ & $\begin{array}{l}03 \\
03 \\
03 \\
03 \\
03\end{array}$ & $\begin{array}{l}9.15 \mathrm{~F} \\
1.00 \mathrm{E} \\
1.08 \mathrm{E} \\
1.17 \mathrm{E} \\
1.24 \mathrm{E}\end{array}$ & $\begin{array}{l}02 \\
03 \\
03 \\
03 \\
03\end{array}$ & $\begin{array}{l}4.98 \mathrm{E} \\
5.46 \mathrm{E} \\
5.91 \mathrm{E} \\
6.40 \mathrm{E} \\
6.80 \mathrm{E}\end{array}$ & $\begin{array}{l}02 \\
02 \\
0 ? \\
02 \\
02\end{array}$ & $\begin{array}{l}3.02 \mathrm{E} \\
3.32 \mathrm{E} \\
3.59 \mathrm{E} \\
3.90 \mathrm{E} \\
4.14 \mathrm{E}\end{array}$ & $\begin{array}{l}02 \\
02 \\
02 \\
02 \\
02\end{array}$ & $\begin{array}{l}1.37 \mathrm{E} \\
1.51 \mathrm{E} \\
1.63 \mathrm{E} \\
1.77 \mathrm{E} \\
1.98 \mathrm{E}\end{array}$ & $\begin{array}{l}02 \\
02 \\
02 \\
02 \\
02\end{array}$ & $\begin{array}{ll}7.40 \mathrm{E} & 01 \\
8.15 \mathrm{E} & 01 \\
8.83 \mathrm{E} & 01 \\
9.60 \mathrm{E} & 01 \\
1.02 \mathrm{~F} & 02\end{array}$ & & $\begin{array}{l}90 \\
91 \\
92 \\
93 \\
94\end{array}$ & $\begin{array}{l}\mathrm{PI} \\
\mathrm{PA} \\
\mathrm{U} \\
\mathrm{NP} \\
\mathrm{PU}\end{array}$ \\
\hline $\begin{array}{l}95 \\
96 \\
97 \\
98 \\
99\end{array}$ & $\begin{array}{l}A M \\
C M \\
B K \\
C F \\
\text { ES }\end{array}$ & $\begin{array}{l}6.16 \mathrm{~F} \\
6.57 \mathrm{~F} \\
6.82 \mathrm{~F} \\
7.19 \mathrm{E}\end{array}$ & $\begin{array}{l}03 \\
03 \\
03 \\
03\end{array}$ & $\begin{array}{l}2.84 \mathrm{E} \\
3.00 \mathrm{E} \\
3.16 \mathrm{E} \\
3.32 \mathrm{~F} \\
3.49 \mathrm{E}\end{array}$ & $\begin{array}{l}03 \\
03 \\
03 \\
03 \\
03\end{array}$ & $\begin{array}{l}1.32 \mathrm{E} \\
1.40 \mathrm{E} \\
1.47 \mathrm{E} \\
1.55 \mathrm{E} \\
1.64 \mathrm{E}\end{array}$ & $\begin{array}{l}03 \\
03 \\
03 \\
03 \\
03\end{array}$ & $\begin{array}{l}7.22 \mathrm{E} \\
7.67 \mathrm{E} \\
8.07 \mathrm{E} \\
8.53 \mathrm{~F} \\
9.00 \mathrm{E}\end{array}$ & $\begin{array}{l}02 \\
02 \\
02 \\
02 \\
02\end{array}$ & $\begin{array}{l}4.40 \mathrm{E} \\
4.67 \mathrm{E} \\
4.92 \mathrm{E} \\
5.20 \mathrm{E} \\
5.50 \mathrm{~F}\end{array}$ & $\begin{array}{l}02 \\
02 \\
02 \\
02 \\
02\end{array}$ & $\begin{array}{l}2.00 \mathrm{E} \\
2.13 \mathrm{E} \\
2.24 \mathrm{E} \\
2.38 \mathrm{~F} \\
2.51 \mathrm{E}\end{array}$ & $\begin{array}{l}02 \\
02 \\
02 \\
02 \\
02\end{array}$ & $\begin{array}{ll}1.08 \mathrm{E} & 02 \\
1.16 \mathrm{E} & 02 \\
1.22 \mathrm{E} & 02 \\
1.29 \mathrm{~F} & 02 \\
1.37 \mathrm{E} & 02\end{array}$ & & $\begin{array}{l}95 \\
96 \\
97 \\
98 \\
99\end{array}$ & $\begin{array}{l}\text { AM } \\
\text { CM } \\
\text { BX } \\
\text { CF } \\
\text { ES }\end{array}$ \\
\hline $\begin{array}{l}100 \\
101\end{array}$ & $\begin{array}{l}\text { FM } \\
\text { MD }\end{array}$ & & & $\begin{array}{l}3.66 \mathrm{E} \\
3.84 \mathrm{E}\end{array}$ & $\begin{array}{l}0.3 \\
0.3\end{array}$ & $\begin{array}{l}1.72 \mathrm{E} \\
1.80 \mathrm{~F}\end{array}$ & $\begin{array}{l}03 \\
03\end{array}$ & $\begin{array}{l}9.43 \mathrm{E} \\
9.90 \mathrm{E}\end{array}$ & $\begin{array}{l}02 \\
02\end{array}$ & $\begin{array}{l}5.76 \mathrm{~F} \\
6.06 \mathrm{~F}\end{array}$ & $\begin{array}{l}02 \\
02\end{array}$ & $\begin{array}{l}2.64 \mathrm{E} \\
2.78 \mathrm{E}\end{array}$ & $\begin{array}{l}02 \\
02\end{array}$ & $\begin{array}{ll}1.44 \mathrm{E} & 02 \\
1.51 \mathrm{E} & 02\end{array}$ & & $\begin{array}{l}100 \\
101\end{array}$ & $\begin{array}{l}\text { Fi } \\
\text { MD }\end{array}$ \\
\hline
\end{tabular}


Table 10 (continued)

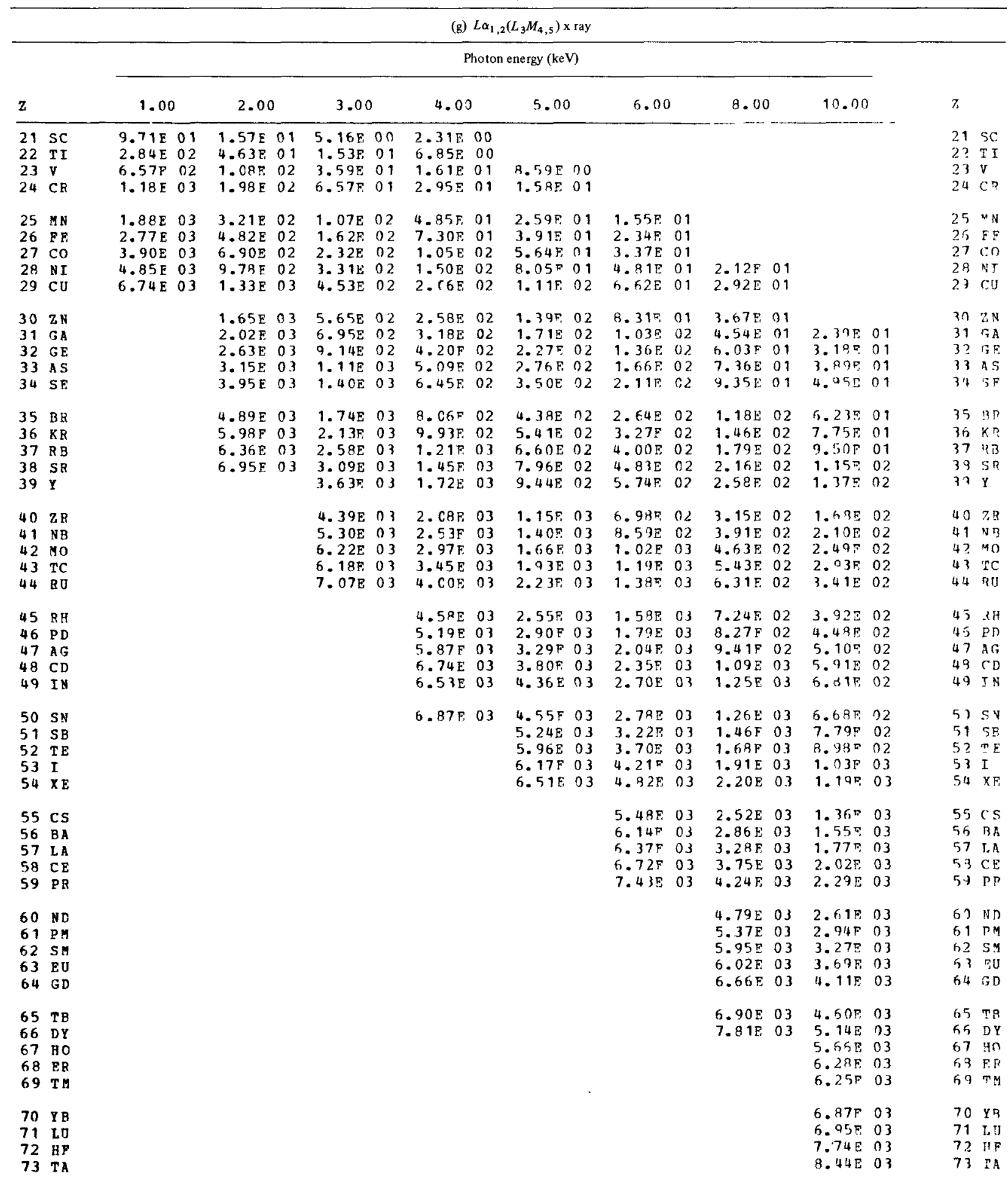


Table 10 (contınued)

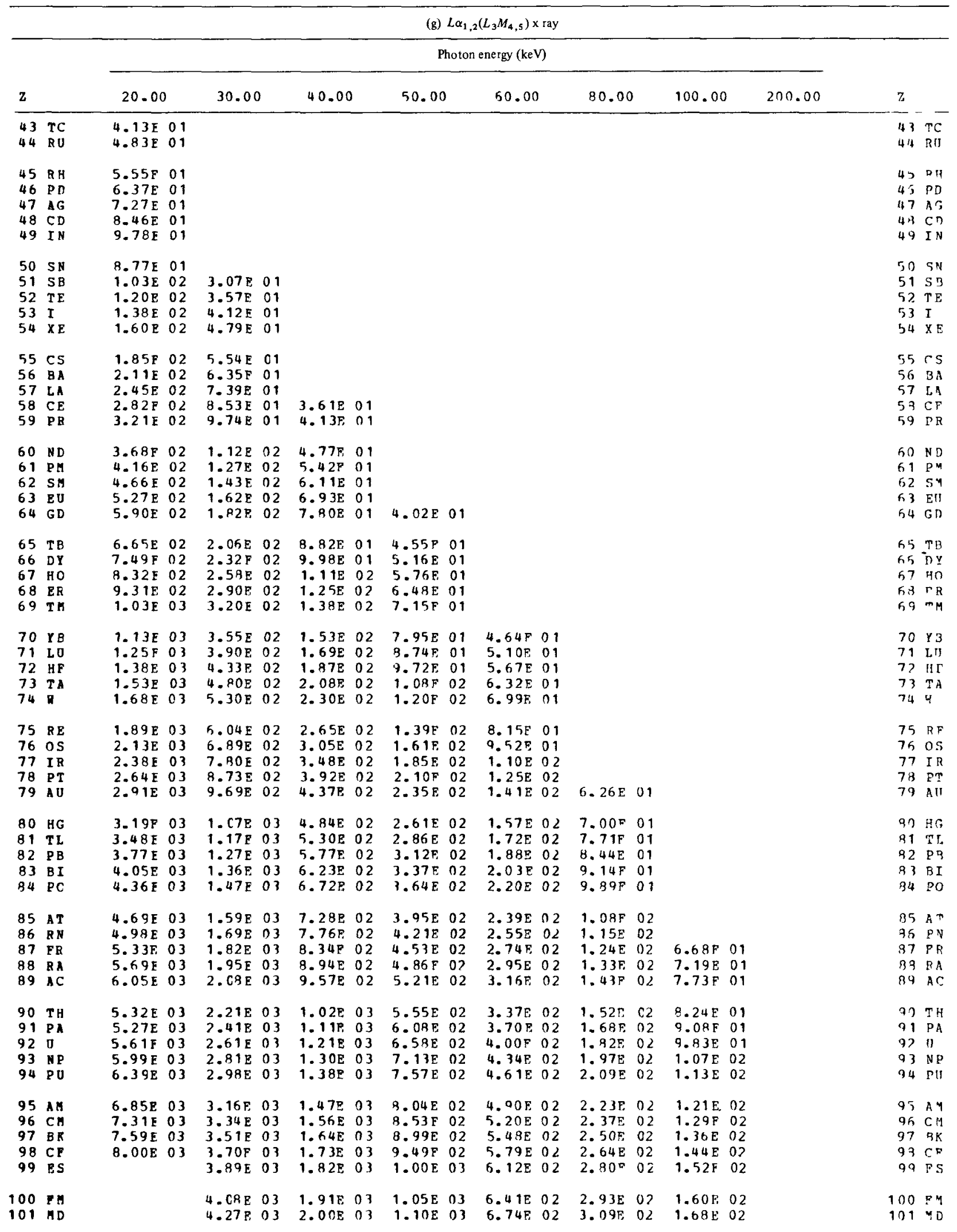


Table 10 (continued)

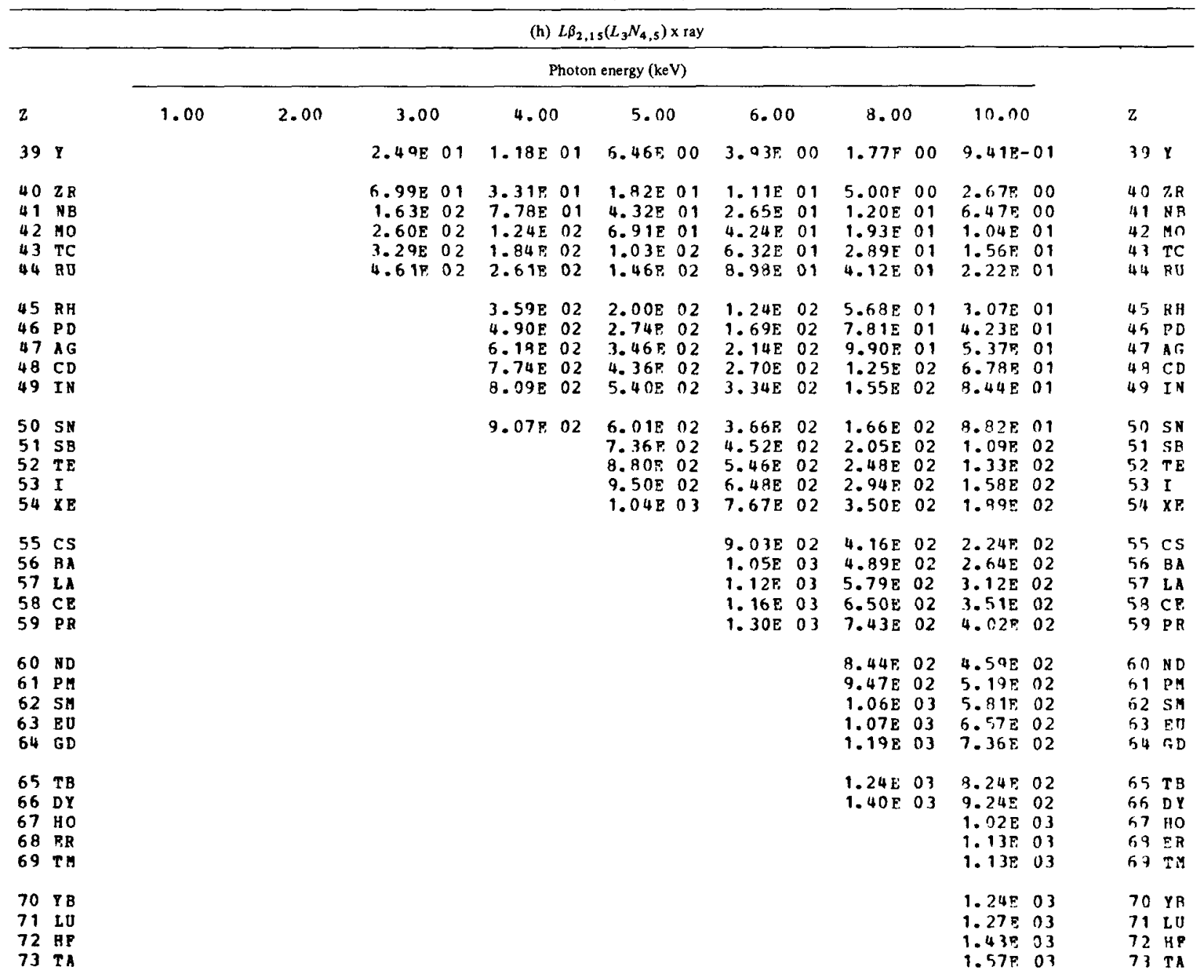


Table 10 (continued)

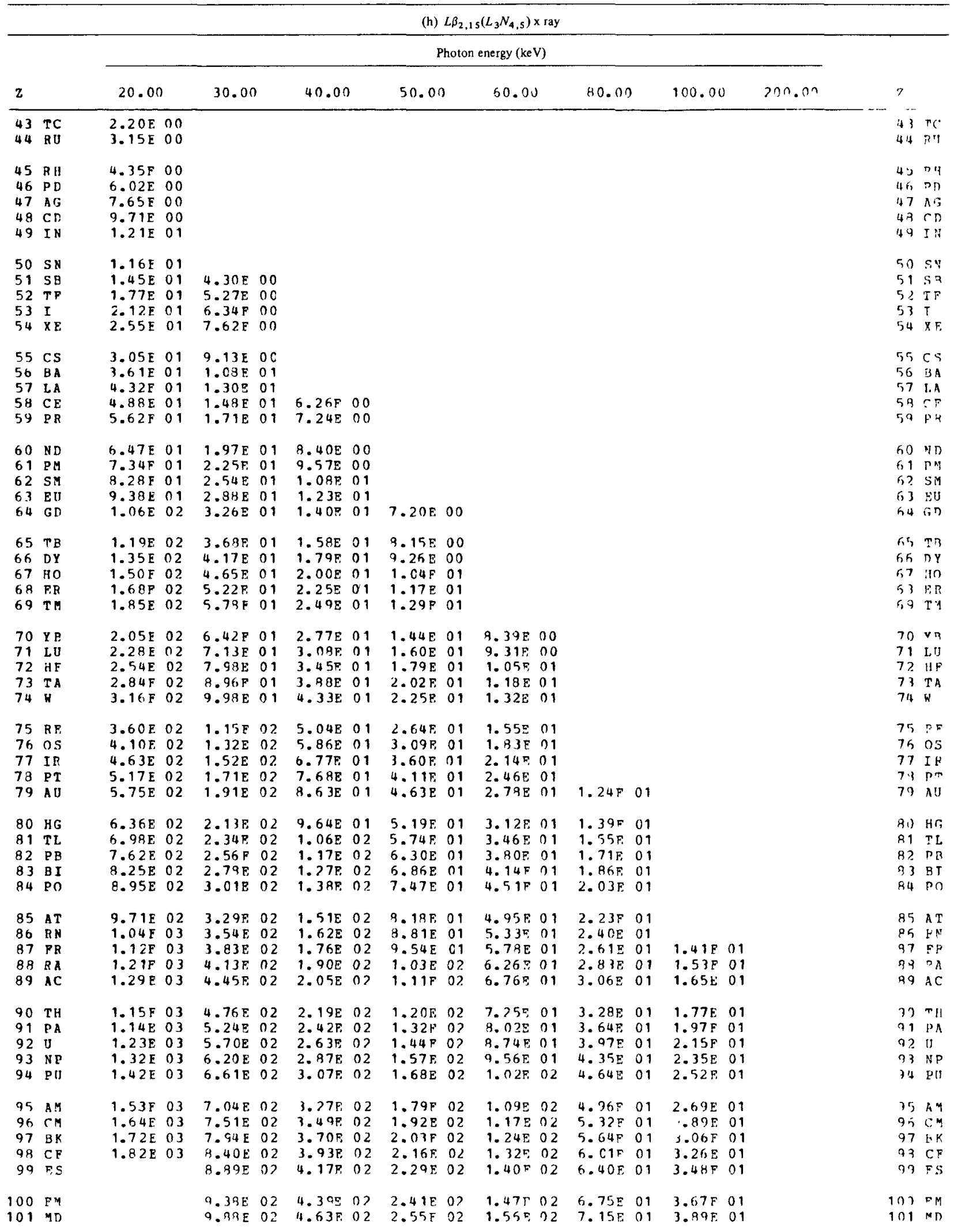


Table 10 (continued)

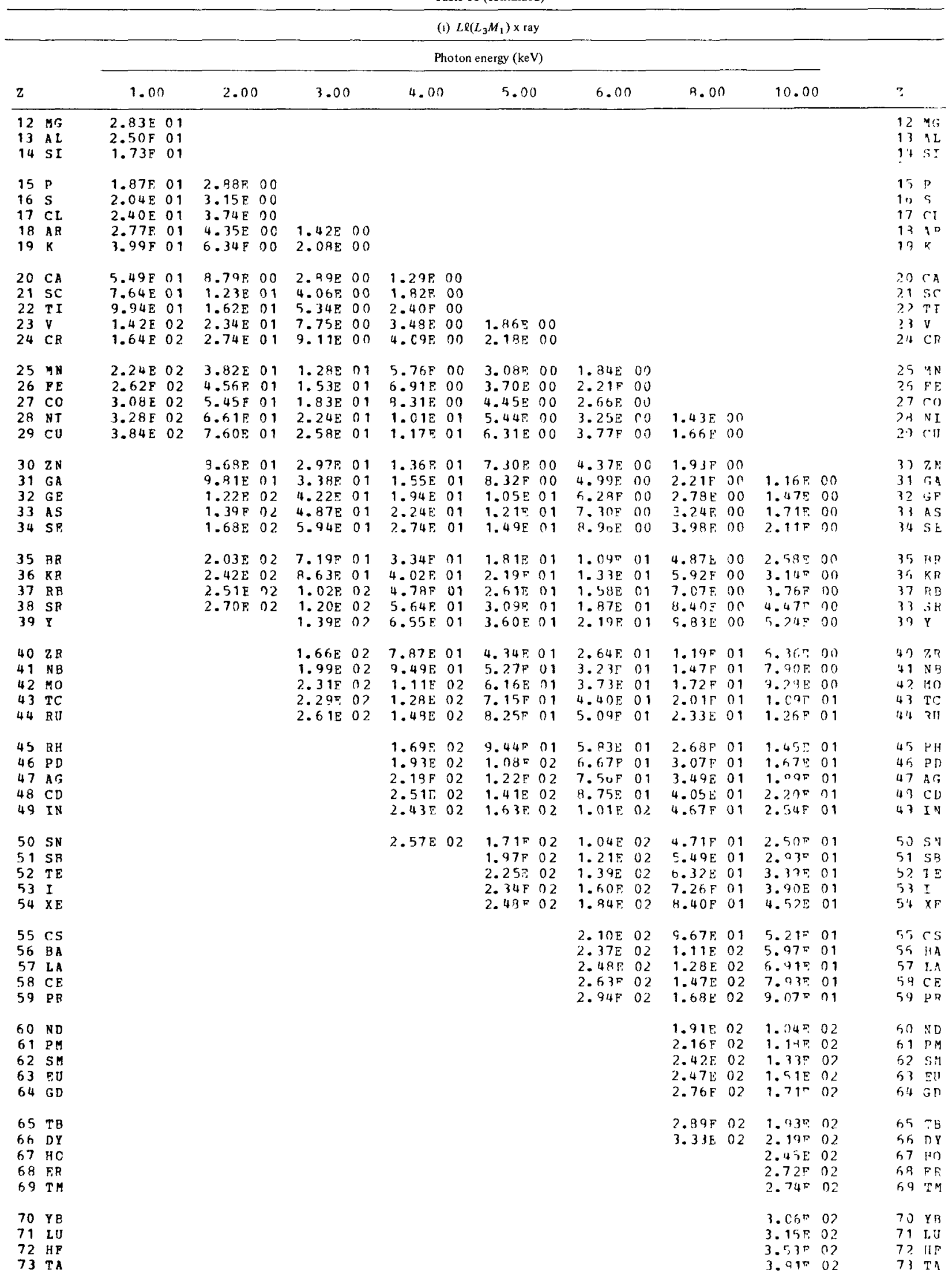


Table 10 (contmued)

\begin{tabular}{|c|c|c|c|c|c|c|c|c|c|c|c|c|c|c|c|c|c|}
\hline & & & & & & & & & (1) $\mathrm{Ler}$ & $\left.L_{3} M_{1}\right) \times$ ray & & & & & & & \\
\hline & & & & & & & & & hoton & energy $(\mathrm{keV})$ & & & & & & & \\
\hline 7 & & 20.00 & & 30.00 & & 40.00 & & 50.00 & & 60.00 & & 80.00 & & 100.00 & 200.00 & 7. & \\
\hline $\begin{array}{l}43 \\
44\end{array}$ & $\begin{array}{l}T C \\
R U\end{array}$ & $\begin{array}{l}1.53 \mathrm{E} \\
1.78 \mathrm{E}\end{array}$ & & & & & & & & & & & & & & $\begin{array}{l}43 \\
44\end{array}$ & $\begin{array}{l}{ }^{\pi} \mathrm{C} \\
\mathrm{RU}\end{array}$ \\
\hline $\begin{array}{l}45 \\
46 \\
47 \\
48 \\
49\end{array}$ & $\begin{array}{l}\text { RH } \\
\text { PD } \\
\text { AG } \\
\text { CD } \\
\text { I N }\end{array}$ & $\begin{array}{ll}2.05 \mathrm{~F} & 0 \\
2.37 \mathrm{~F} & 0 \\
2.70 \mathrm{~F} & 0 \\
3.15 \mathrm{~F} & 0 \\
3.6,5 \mathrm{~F} & 0\end{array}$ & $\begin{array}{lll}0 & 0 \\
0 & 0 \\
0 & 0 \\
0 & 0 \\
0 & 0\end{array}$ & & & & & & & & & & & & & $\begin{array}{l}45 \\
46 \\
47 \\
4-3 \\
49\end{array}$ & $\begin{array}{l}\text { PH } \\
\text { DD } \\
A C \\
\text { ID } \\
\text { IN }\end{array}$ \\
\hline $\begin{array}{l}50 \\
51 \\
52 \\
53 \\
54\end{array}$ & $\begin{array}{l}S N \\
S B \\
T E \\
I \\
X E\end{array}$ & $\begin{array}{ll}3.29 E & 0 \\
3.89 E & 0 \\
4.52 F & 0 \\
5.24 E & 0 \\
6.11 \mathrm{~F} & 0\end{array}$ & $\begin{array}{ll}0 & 0 \\
0 & 0 \\
0 & 0 \\
0 & 0 \\
0 & 0\end{array}$ & $\begin{array}{l}1.15 \mathrm{~F} \\
1.34 \mathrm{~F} \\
1.56 \mathrm{~F} \\
1.83 \mathrm{E}\end{array}$ & $\begin{array}{l}0 C \\
00 \\
00 \\
00\end{array}$ & & & & & & & & & & & $\begin{array}{l}59 \\
51 \\
57 \\
53 \\
54\end{array}$ & $\begin{array}{l}\text { SN } \\
\text { S } \\
\text { TF } \\
\text { I } \\
X F\end{array}$ \\
\hline $\begin{array}{l}55 \\
56 \\
57 \\
58 \\
59\end{array}$ & $\begin{array}{l}C S \\
B A \\
L A \\
C E \\
P R\end{array}$ & $\begin{array}{ll}7.08 \mathrm{E} & 0 \\
8.17 \mathrm{E} & 0 \\
9.54 \mathrm{~F} & 0 \\
1.10 \mathrm{E} & 0 \\
1.27 \mathrm{E} & 0\end{array}$ & $\begin{array}{ll}0 & 0 \\
0 & 0 \\
0 & 0 \\
0 & 1 \\
0 & 1\end{array}$ & $\begin{array}{l}2.12 \mathrm{~F} \\
2.46 \mathrm{E} \\
2.88 \mathrm{~F} \\
3.34 \mathrm{E} \\
3.85 \mathrm{E}\end{array}$ & $\begin{array}{l}00 \\
00 \\
00 \\
00 \\
00\end{array}$ & $\begin{array}{l}1.42 E \\
1.648\end{array}$ & $\begin{array}{l}00 \\
00\end{array}$ & & & & & & & & & $\begin{array}{l}55 \\
56 \\
57 \\
59 \\
59\end{array}$ & $\begin{array}{l}C S \\
R A \\
L A \\
C F \\
P R\end{array}$ \\
\hline $\begin{array}{l}60 \\
61 \\
62 \\
63 \\
64\end{array}$ & $\begin{array}{l}\text { ND } \\
\text { PM } \\
\text { SM } \\
\text { EU } \\
\text { GD }\end{array}$ & $\begin{array}{ll}1.47 \mathrm{E} & 0 \\
1.67 \mathrm{~F} & 0 \\
1.89 \mathrm{~F} & 0 \\
2.16 \mathrm{~F} & 0 \\
2.45 \mathrm{~F} & 0\end{array}$ & $\begin{array}{l}01 \\
01 \\
01 \\
01 \\
01\end{array}$ & $\begin{array}{l}4.47 \mathrm{E} \\
5.11 \mathrm{E} \\
5.81 \mathrm{E} \\
6.64 \mathrm{E} \\
7.55 \mathrm{~F}\end{array}$ & $\begin{array}{ll}0 & 0 \\
0 & 0 \\
0 & 0 \\
0 & 0 \\
0 & 0\end{array}$ & $\begin{array}{l}1.90 E \\
2.1 \mathrm{AE} \\
2.48 \mathrm{~F} \\
2.84 \mathrm{E} \\
3.73 \mathrm{E}\end{array}$ & $\begin{array}{ll}0 & 0 \\
0 & 0 \\
0 & 0 \\
0 & 0 \\
0 & 0\end{array}$ & $1.67 \mathrm{~F}$ & 00 & & & & & & & $\begin{array}{l}30 \\
61 \\
n ? \\
73 \\
64\end{array}$ & $\begin{array}{l}N D \\
P M \\
S M \\
-I I \\
G D\end{array}$ \\
\hline $\begin{array}{l}65 \\
66 \\
67 \\
68 \\
69\end{array}$ & $\begin{array}{l}\text { TR } \\
\text { DY } \\
\text { HO } \\
\text { ER } \\
\text { TM }\end{array}$ & $\begin{array}{ll}2.79 \mathrm{~F} & 0 \\
3.19 \mathrm{E} & 0 \\
3.59 \mathrm{~F} & 0 \\
4.03 \mathrm{~F} & 0 \\
4.50 \mathrm{~F} & 0\end{array}$ & $\begin{array}{l}01 \\
01 \\
01 \\
01 \\
01 \\
01\end{array}$ & $\begin{array}{l}8.63 \mathrm{E} \\
9.90 \mathrm{E} \\
1.12 \mathrm{~F} \\
1.26 \mathrm{E} \\
1.40 \mathrm{E}\end{array}$ & $\begin{array}{ll}0 & 0 \\
0 & 0 \\
0 & 1 \\
0 & 1 \\
0 & 1\end{array}$ & $\begin{array}{l}3.70 \mathrm{~F} \\
4.25 \mathrm{E} \\
4.80 \mathrm{E} \\
5.42 \mathrm{~F} \\
6.05 \mathrm{~F}\end{array}$ & $\begin{array}{ll}0 & 0 \\
0 & 0 \\
0 & 0 \\
0 & 0 \\
0 & 0\end{array}$ & $\begin{array}{l}1.91 \mathrm{E} \\
2.20 \mathrm{E} \\
2.49 \mathrm{~F} \\
2.80 \mathrm{~F} \\
3.13 \mathrm{~F}\end{array}$ & $\begin{array}{l}00 \\
00 \\
00 \\
00 \\
00\end{array}$ & & & & & & & $\begin{array}{l}65 \\
65 \\
67 \\
18 \\
57\end{array}$ & $\begin{array}{l}T B \\
r_{1} Y \\
4 O \\
F_{R} \\
m Y\end{array}$ \\
\hline $\begin{array}{l}70 \\
71 \\
72 \\
73 \\
74\end{array}$ & $\begin{array}{l}\text { YB } \\
\text { LU } \\
\text { HF } \\
\text { TA } \\
H\end{array}$ & $\begin{array}{ll}5.05 \mathrm{~F} & 0 \\
5.65 \mathrm{~F} & 0 \\
6.29 \mathrm{~F} & 0 \\
7.07 \mathrm{E} & 0 \\
7.89 \mathrm{E} & 0\end{array}$ & $\begin{array}{l}01 \\
01 \\
01 \\
01 \\
01 \\
01\end{array}$ & $\begin{array}{l}1.5 \mathrm{FE} \\
1.77 \mathrm{E} \\
1.98 \mathrm{~F} \\
2.23 \mathrm{E} \\
2.49 \mathrm{E}\end{array}$ & $\begin{array}{l}01 \\
01 \\
01 \\
01 \\
01\end{array}$ & $\begin{array}{l}6.82 F \\
7.65 \mathrm{E} \\
9.55 \mathrm{E} \\
9.64 \mathrm{E} \\
1.08 \mathrm{E}\end{array}$ & $\begin{array}{ll}0 & 0 \\
0 & 0 \\
0 & 0 \\
0 & 0 \\
0 & 1\end{array}$ & $\begin{array}{l}3.54 \mathrm{E} \\
3.97 \mathrm{~F} \\
4.44 \mathrm{~F} \\
5.01 \mathrm{E} \\
5.62 \mathrm{E}\end{array}$ & $\begin{array}{l}00 \\
00 \\
00 \\
00 \\
0\end{array}$ & $\begin{array}{l}2.06 \mathrm{E} \\
2.31 \mathrm{~F} \\
2.59 \mathrm{~F} \\
2.93 \mathrm{~F} \\
3.28 \mathrm{~F}\end{array}$ & $\begin{array}{ll}0 & 0 \\
0 & 0 \\
0 & 0 \\
0 & 0 \\
0 & 0\end{array}$ & & & & & $\begin{array}{l}70 \\
71 \\
73 \\
73 \\
74\end{array}$ & $\begin{array}{l}Y B \\
\text { Y II } \\
\text { HF } \\
T A \\
W\end{array}$ \\
\hline $\begin{array}{l}75 \\
76 \\
77 \\
78 \\
79\end{array}$ & $\begin{array}{l}\text { RF } \\
\text { OS } \\
\text { IR } \\
\text { PT } \\
\text { A U }\end{array}$ & 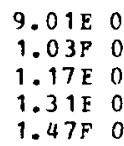 & $\begin{array}{l}01 \\
02 \\
02 \\
02 \\
02\end{array}$ & $\begin{array}{l}2.87 \mathrm{E} \\
3.32 \mathrm{~F} \\
3.82 \mathrm{~F} \\
4.34 \mathrm{~F} \\
4.98 \mathrm{E}\end{array}$ & $\begin{array}{ll}0 & 1 \\
0 & 1 \\
01 \\
01 \\
01 \\
01\end{array}$ & $\begin{array}{l}1.265 \\
1.47 E \\
1.70 E \\
1.95 E \\
2.20 E\end{array}$ & $\begin{array}{ll}0 & 1 \\
0 & 1 \\
01 \\
01 \\
01 \\
0 & 1\end{array}$ & $\begin{array}{l}6.6 \mathrm{CF} \\
7.75 \mathrm{E} \\
9.06 \mathrm{E} \\
1 . \mathrm{CHE} \\
1.19 \mathrm{~F}\end{array}$ & $\begin{array}{l}00 \\
00 \\
00 \\
01 \\
01\end{array}$ & $\begin{array}{l}3.89 \mathrm{~F} \\
4.59 \mathrm{E} \\
5.39 \mathrm{E} \\
6.24 \mathrm{E} \\
7.03 \mathrm{~F}\end{array}$ & $\begin{array}{ll}0 & 0 \\
00 & 0 \\
0 & 0 \\
00 & 0 \\
0 & 0\end{array}$ & $3.15=$ & 00 & & & $\begin{array}{l}75 \\
75 \\
77 \\
75 \\
73\end{array}$ & $\begin{array}{l}\text { RE } \\
\text { OS } \\
\text { IR } \\
\text { UT } \\
\text { AU }\end{array}$ \\
\hline $\begin{array}{l}80 \\
81 \\
82 \\
83 \\
84\end{array}$ & $\begin{array}{l}\text { HG } \\
\text { TL } \\
\text { PP } \\
\text { B I } \\
\text { PO }\end{array}$ & $\begin{array}{ll}1.64 \mathrm{E} & 0 \\
1.81 \mathrm{E} & 0 \\
1.99 \mathrm{E} & 0 \\
2.17 \mathrm{~F} & 0 \\
2.37 \mathrm{E} & 0\end{array}$ & $\begin{array}{l}02 \\
02 \\
02 \\
02 \\
02 \\
02\end{array}$ & $\begin{array}{l}5.47 \mathrm{E} \\
6.06 \mathrm{E} \\
6.69 \mathrm{E} \\
7.30 \mathrm{P} \\
7.99 \mathrm{E}\end{array}$ & $\begin{array}{l}01 \\
01 \\
01 \\
01 \\
01\end{array}$ & $\begin{array}{l}2.48 E \\
2.75 \mathrm{E} \\
3.05 \mathrm{~F} \\
3.34 \mathrm{E} \\
3.65 \mathrm{E}\end{array}$ & $\begin{array}{ll}0 & 1 \\
0 & 1 \\
0 & 1 \\
0 & 1 \\
01 & 1\end{array}$ & $\begin{array}{l}1.34 \mathrm{E} \\
1.49 \mathrm{~F} \\
1.65 \mathrm{E} \\
1.80 \mathrm{~F} \\
1.98 \mathrm{E}\end{array}$ & $\begin{array}{ll}0 & 1 \\
0 & 1 \\
0 & 1 \\
01 & 1 \\
01\end{array}$ & $\begin{array}{l}8.03 \mathrm{E} \\
8.95 \mathrm{E} \\
9.93 \mathrm{E} \\
1.09 \mathrm{~F} \\
1.20 \mathrm{E}\end{array}$ & $\begin{array}{ll}0 & 0 \\
0 & 0 \\
0 & 0 \\
0 & 1 \\
0 & 1\end{array}$ & $\begin{array}{l}3.59 \mathrm{~F} \\
4.01 \mathrm{E} \\
4.46 \mathrm{E} \\
4.89 \mathrm{E} \\
5.38 \mathrm{E}\end{array}$ & $\begin{array}{l}00 \\
00 \\
00 \\
00 \\
00\end{array}$ & & & $\begin{array}{l}87 \\
31 \\
37 \\
33 \\
94\end{array}$ & $\begin{array}{l}\angle I G \\
Z L \\
P B \\
11 T \\
D O\end{array}$ \\
\hline $\begin{array}{l}85 \\
86 \\
87 \\
88 \\
89\end{array}$ & $\begin{array}{l}\text { AT } \\
\text { RN } \\
\text { YR } \\
\text { RA } \\
\text { AC }\end{array}$ & $\begin{array}{ll}2.60 \mathrm{E} & 0 \\
2.80 \mathrm{~F} & 0 \\
3.05 \mathrm{E} & 0 \\
3.31 \mathrm{E} & 0 \\
3.57 \mathrm{E} & 0\end{array}$ & $\begin{array}{l}02 \\
02 \\
02 \\
02 \\
02\end{array}$ & $\begin{array}{l}3.79 \mathrm{E} \\
9.5 \mathrm{JE} \\
1.04 \mathrm{E} \\
1.13 \mathrm{E} \\
1.23 \mathrm{E}\end{array}$ & $\begin{array}{l}01 \\
01 \\
02 \\
02 \\
02\end{array}$ & $\begin{array}{l}4.03 \mathrm{E} \\
4.37 \mathrm{E} \\
4.76 \mathrm{E} \\
5.20 \mathrm{E} \\
5.65 \mathrm{E}\end{array}$ & $\begin{array}{ll}0 & 1 \\
0 & 1 \\
0 & 1 \\
0 & 1 \\
0 & 1\end{array}$ & $\begin{array}{l}2.19 \mathrm{I} \\
2.37 \mathrm{~F} \\
2.59 \mathrm{~F} \\
2.93 \mathrm{E} \\
3 . \mathrm{CBF}\end{array}$ & $\begin{array}{l}01 \\
01 \\
01 \\
01 \\
01\end{array}$ & $\begin{array}{ll}1.32 E & 0 \\
1.43 F & 0 \\
1.57 F & 0 \\
1.71 E & 0 \\
1.87 F & 0\end{array}$ & $\begin{array}{l}01 \\
01 \\
01 \\
01 \\
01 \\
01\end{array}$ & $\begin{array}{l}5.978 \\
6.47 \mathrm{~F} \\
7.08 \mathrm{~F} \\
7.75 \mathrm{E} \\
8.45 \mathrm{~F}\end{array}$ & $\begin{array}{l}00 \\
00 \\
00 \\
00 \\
00\end{array}$ & $\begin{array}{ll}3.82 \mathrm{~F} & 00 \\
4.18 \mathrm{~F} & 00 \\
4.57 \mathrm{E} & 00\end{array}$ & & $\begin{array}{l}35 \\
85 \\
97 \\
89 \\
89\end{array}$ & $\begin{array}{l}A T \\
4 N \\
F R \\
R A \\
A C\end{array}$ \\
\hline $\begin{array}{l}90 \\
91 \\
92 \\
93 \\
94\end{array}$ & $\begin{array}{l}\text { TH } \\
\text { PA } \\
\text { U } \\
N E \\
\text { PU }\end{array}$ & $\begin{array}{ll}3.20 \mathrm{~F} & 0 \\
3.22 \mathrm{E} & 0 \\
3.48 \mathrm{~F} & 0 \\
3.79 \mathrm{E} & 0 \\
4.10 \mathrm{E} & 0\end{array}$ & $\begin{array}{l}02 \\
02 \\
02 \\
02 \\
02 \\
02\end{array}$ & $\begin{array}{l}1.33 \mathrm{~F} \\
1.47 \mathrm{E} \\
1.62 \mathrm{E} \\
1.78 \mathrm{E} \\
1.91 \mathrm{E}\end{array}$ & $\begin{array}{l}02 \\
02 \\
02 \\
02 \\
02\end{array}$ & $\begin{array}{l}6.12 \mathrm{E} \\
6.81 \mathrm{~F} \\
7.48 \mathrm{~F} \\
8.24 \mathrm{E} \\
9.88 \mathrm{E}\end{array}$ & $\begin{array}{ll}0 & 1 \\
0 & 1 \\
0 & 1 \\
0 & 1 \\
01 & 1\end{array}$ & $\begin{array}{l}3.33 \mathrm{P} \\
3.72 \mathrm{~F} \\
4.09 \mathrm{E} \\
4.51 \mathrm{~F} \\
4.86 \mathrm{E}\end{array}$ & $\begin{array}{l}01 \\
01 \\
01 \\
01 \\
01\end{array}$ & $\begin{array}{ll}2.02 \mathrm{~F} & 0 \\
2.26 \mathrm{E} & 0 \\
2.49 \mathrm{E} & 0 \\
2.74 \mathrm{~F} & 0 \\
2.96 \mathrm{~B} & 0\end{array}$ & $\begin{array}{ll}0 & 1 \\
0 & 1 \\
0 & 1 \\
0 & 1 \\
0 & 1\end{array}$ & $\begin{array}{l}9.16 \mathrm{~F} \\
1.02 \mathrm{E} \\
1.13 \mathrm{E} \\
1.25 \mathrm{E} \\
1.34 \mathrm{E}\end{array}$ & $\begin{array}{l}0 \\
0 \\
01 \\
01 \\
01 \\
01\end{array}$ & 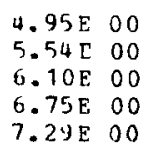 & & $\begin{array}{l}71 \\
71 \\
92 \\
93 \\
94\end{array}$ & $\begin{array}{l}\mathrm{TH} \\
\mathrm{PA} \\
\mathrm{U} \\
\mathrm{NP} \\
\mathrm{PU}\end{array}$ \\
\hline $\begin{array}{l}95 \\
96 \\
97 \\
98 \\
99\end{array}$ & $\begin{array}{l}\text { AM } \\
\text { CM } \\
\text { BK } \\
\text { CF } \\
\text { FS }\end{array}$ & $\begin{array}{ll}4.47 \mathrm{~F} & 0 \\
4.85 \mathrm{~F} & 0 \\
5.13 \mathrm{~F} & 0 \\
5.49 \mathrm{~F} & 0\end{array}$ & $\begin{array}{l}02 \\
02 \\
02 \\
02\end{array}$ & $\begin{array}{l}2.06 \mathrm{E} \\
2.22 \mathrm{~F} \\
2.37 \mathrm{E} \\
2.54 \mathrm{E} \\
2.72 \mathrm{E}\end{array}$ & $\begin{array}{l}02 \\
02 \\
02 \\
02 \\
02\end{array}$ & $\begin{array}{l}9.59 \mathrm{~F} \\
1.03 \mathrm{E} \\
1.11 \mathrm{E} \\
1.19 \mathrm{E} \\
1.27 \mathrm{E}\end{array}$ & $\begin{array}{l}01 \\
0 ? \\
02 \\
02 \\
02\end{array}$ & $\begin{array}{l}5.25 \mathrm{E} \\
5.66 \mathrm{E} \\
6.07 \mathrm{E} \\
6.52 \mathrm{E} \\
7.01 \mathrm{E}\end{array}$ & $\begin{array}{ll}0 & 1 \\
0 & 1 \\
0 & 1 \\
01 \\
01\end{array}$ & $\begin{array}{ll}3.20 \mathrm{E} & 0 \\
3.45 \mathrm{~F} & 0 \\
3.70 \mathrm{E} & 0 \\
3.98 \mathrm{E} & 0 \\
4.28 \mathrm{E} & 0\end{array}$ & $\begin{array}{l}01 \\
01 \\
01 \\
01 \\
01\end{array}$ & $\begin{array}{l}1.45 \mathrm{E} \\
1.57 \mathrm{E} \\
1.69 \mathrm{E} \\
1.82 \mathrm{~F} \\
1.76 \mathrm{~F}\end{array}$ & $\begin{array}{l}01 \\
01 \\
01 \\
01 \\
01\end{array}$ & $\begin{array}{ll}7.88 \mathrm{E} & 00 \\
3.53 \mathrm{~F} & 00 \\
9.15 \mathrm{E} & 00 \\
9.86 \mathrm{E} & 00 \\
1.06 \mathrm{E} & 01\end{array}$ & & $\begin{array}{l}95 \\
95 \\
37 \\
93 \\
93\end{array}$ & $\begin{array}{l}\text { AY } \\
\text { CM } \\
\text { BK } \\
\text { CF } \\
\text { ES }\end{array}$ \\
\hline $\begin{array}{l}100 \\
101\end{array}$ & $\begin{array}{l}\text { PM } \\
\text { MD }\end{array}$ & & & $\begin{array}{l}2.90 \mathrm{E} \\
3.09 \mathrm{E}\end{array}$ & $\begin{array}{l}02 \\
02\end{array}$ & $\begin{array}{l}1.36 \mathrm{E} \\
1.45 \mathrm{E}\end{array}$ & $\begin{array}{l}02 \\
02\end{array}$ & $\begin{array}{l}7.46 \mathrm{E} \\
7.96 \mathrm{~F}\end{array}$ & $\begin{array}{l}01 \\
01\end{array}$ & $\begin{array}{l}4.56 \mathrm{~F} \\
4.87 \mathrm{~F}\end{array}$ & $\begin{array}{l}01 \\
01\end{array}$ & $\begin{array}{l}2.09 \mathrm{~F} \\
2.23 \mathrm{~F}\end{array}$ & $\begin{array}{l}01 \\
01\end{array}$ & 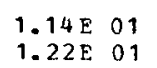 & & $\begin{array}{l}100 \\
101\end{array}$ & $\begin{array}{l}\text { PM } \\
M D\end{array}$ \\
\hline
\end{tabular}




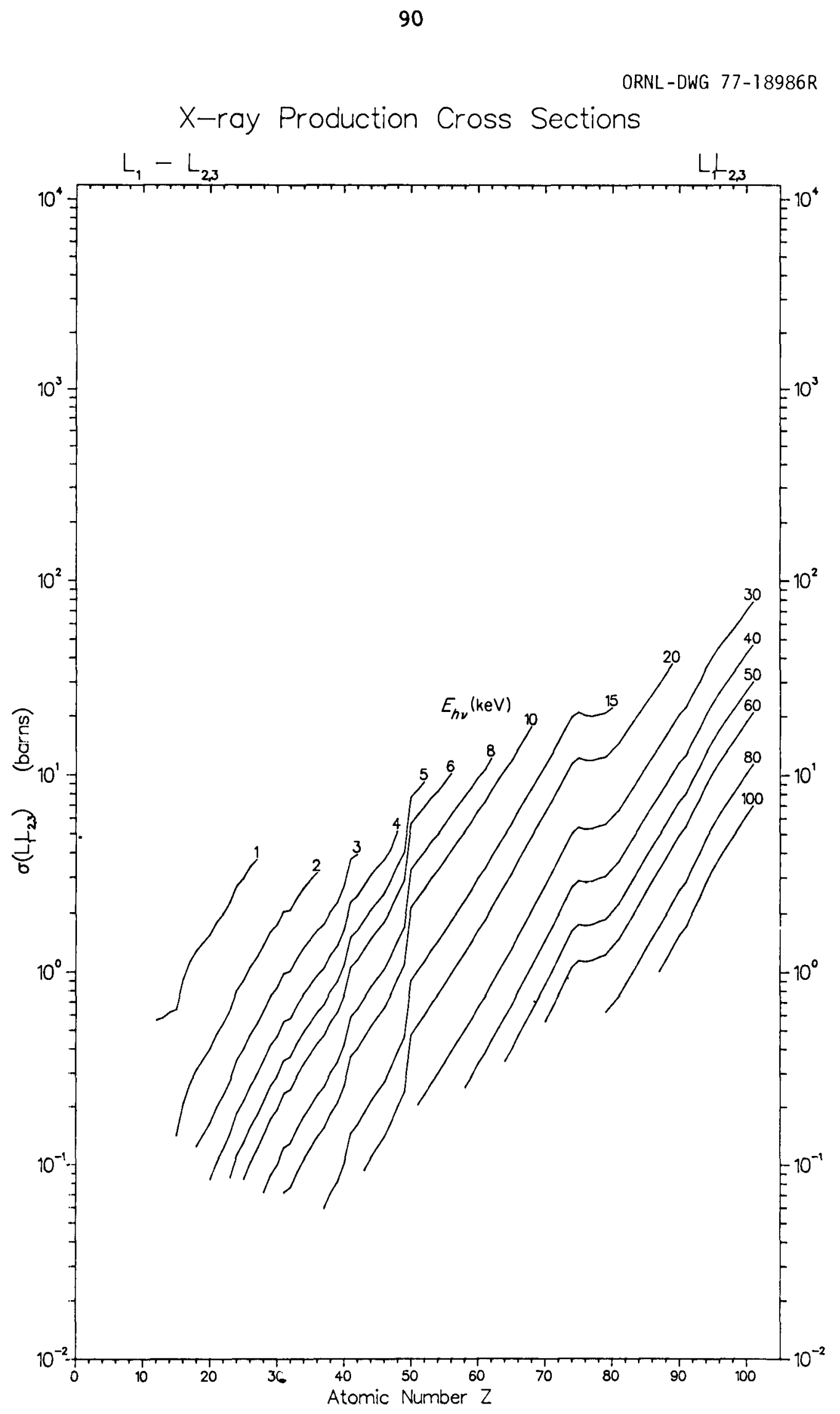

Fig. 18 
ORNL-DWG 77-21187R

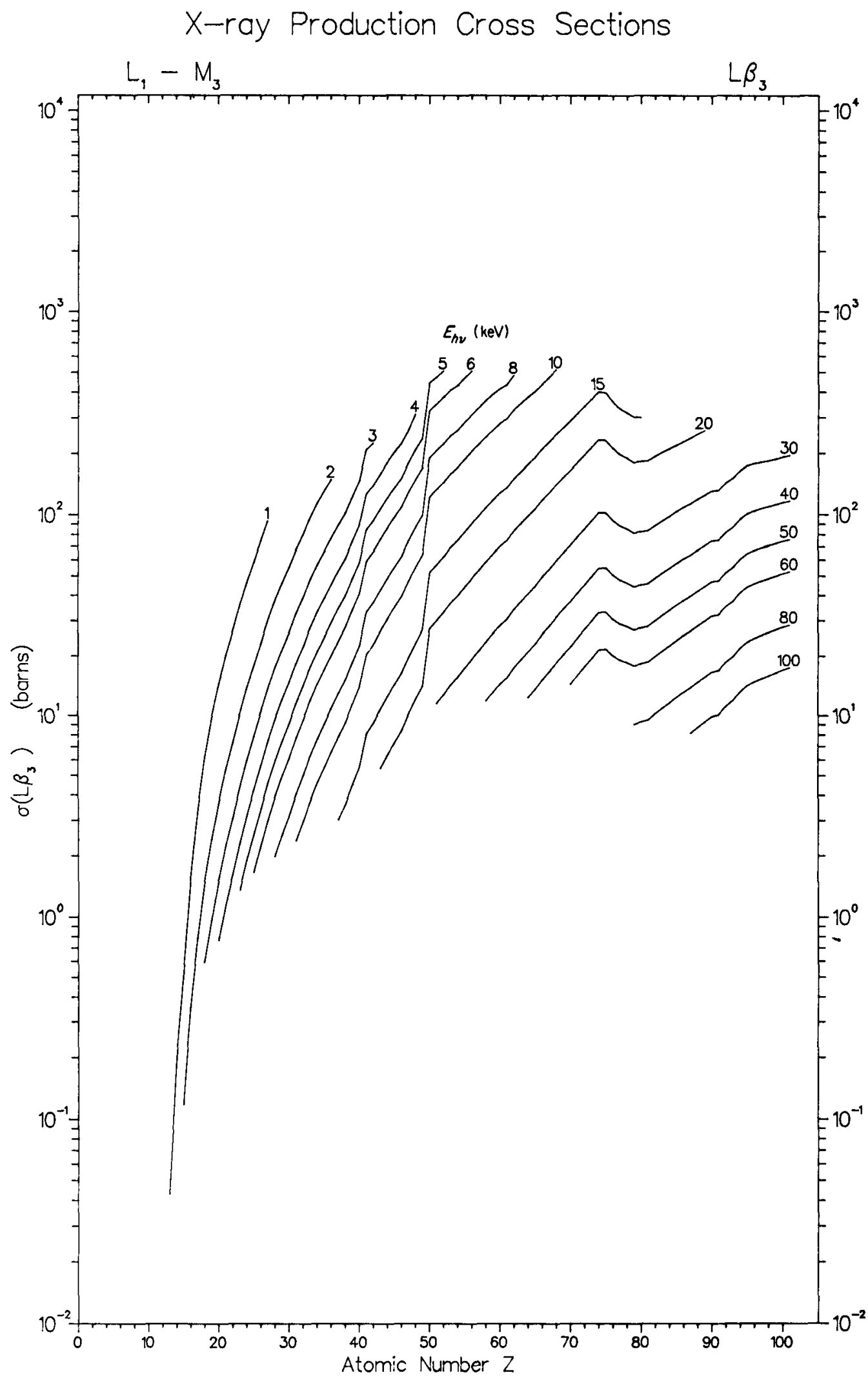

Fig. 19 
92

ORNL-DWG 77-21188R

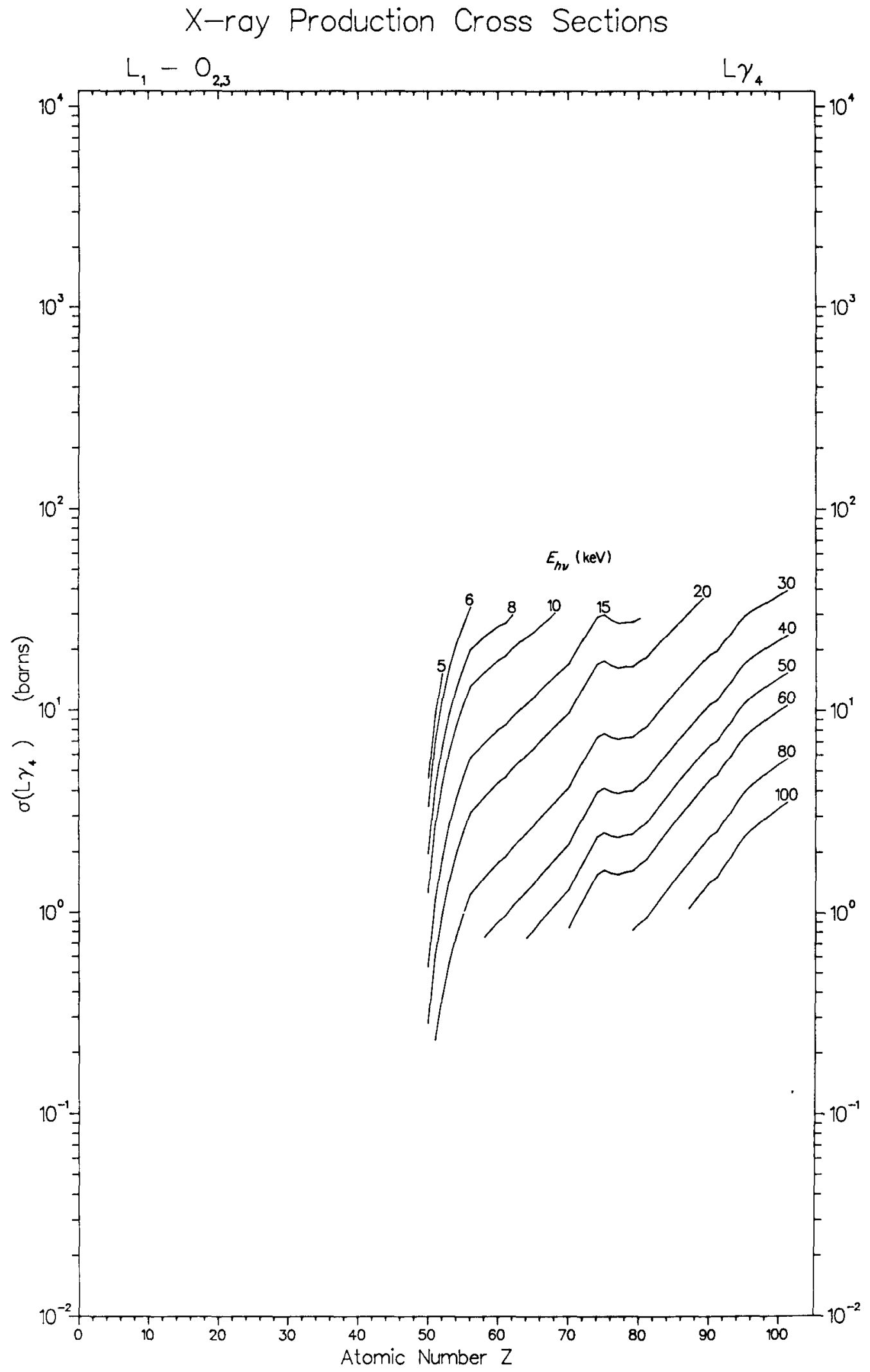

Fig. 20 
ORNL-DWG 77-21189R

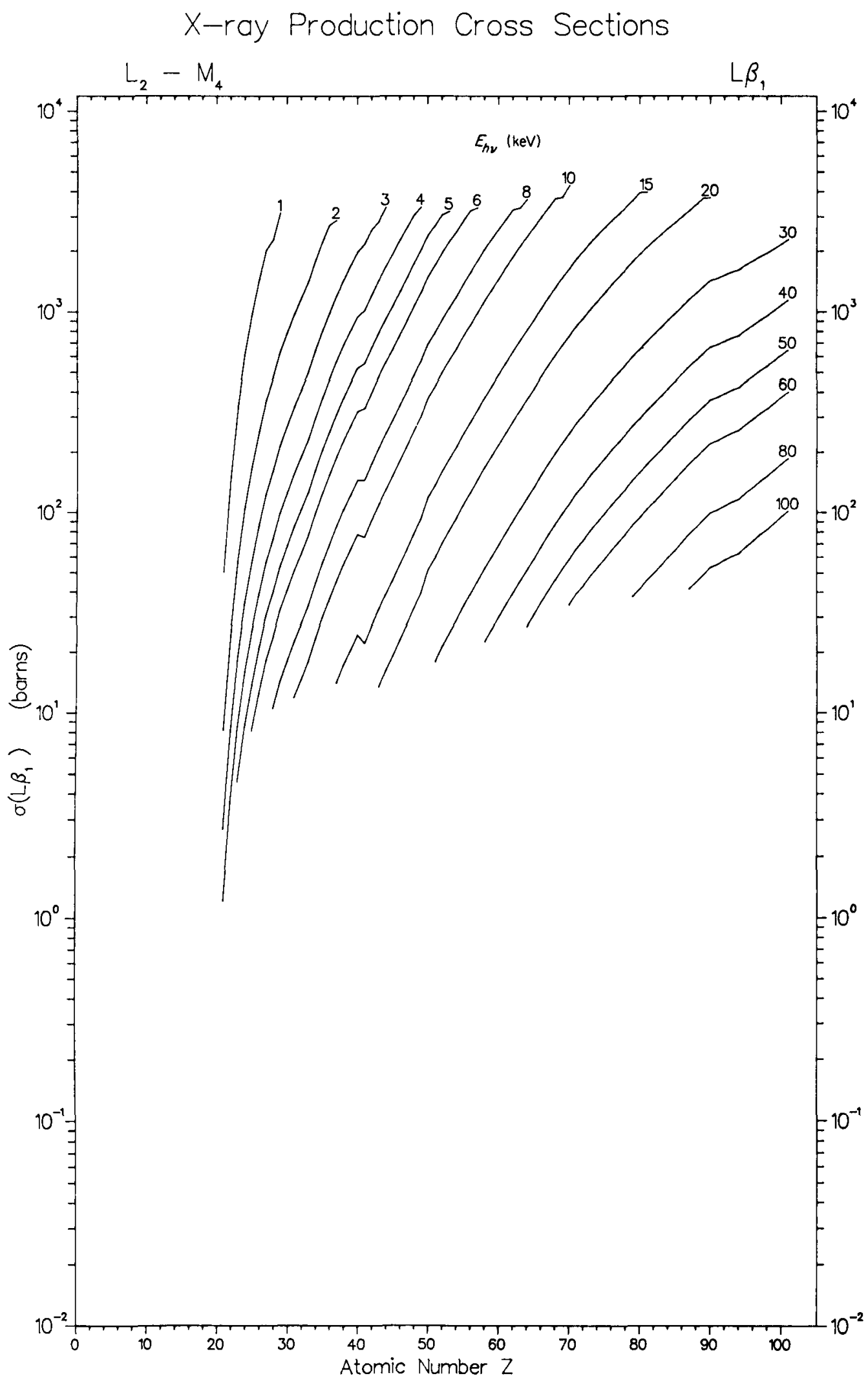

Fig. 21 


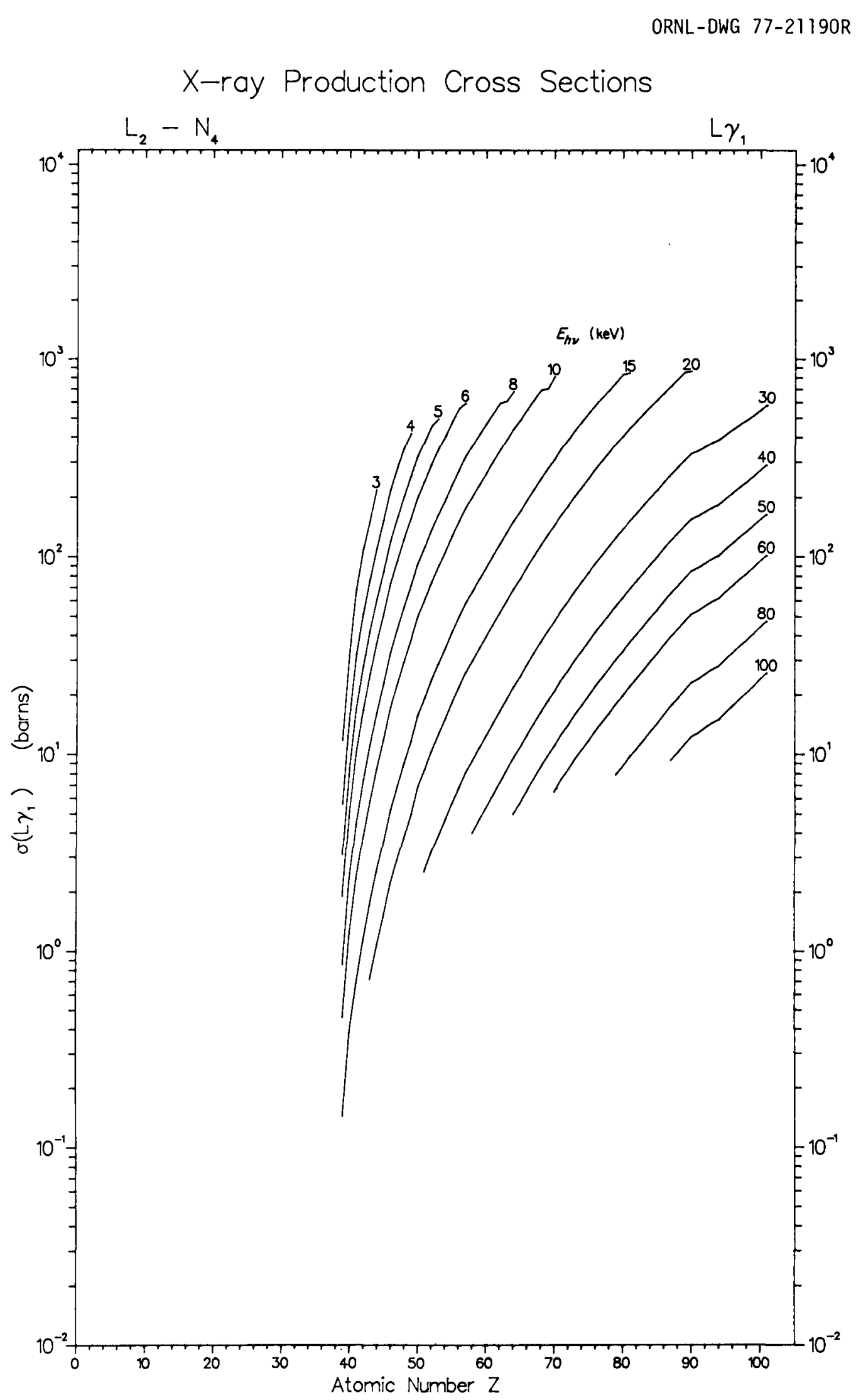

Fig. 22 
ORNL-DWG 77-21175R

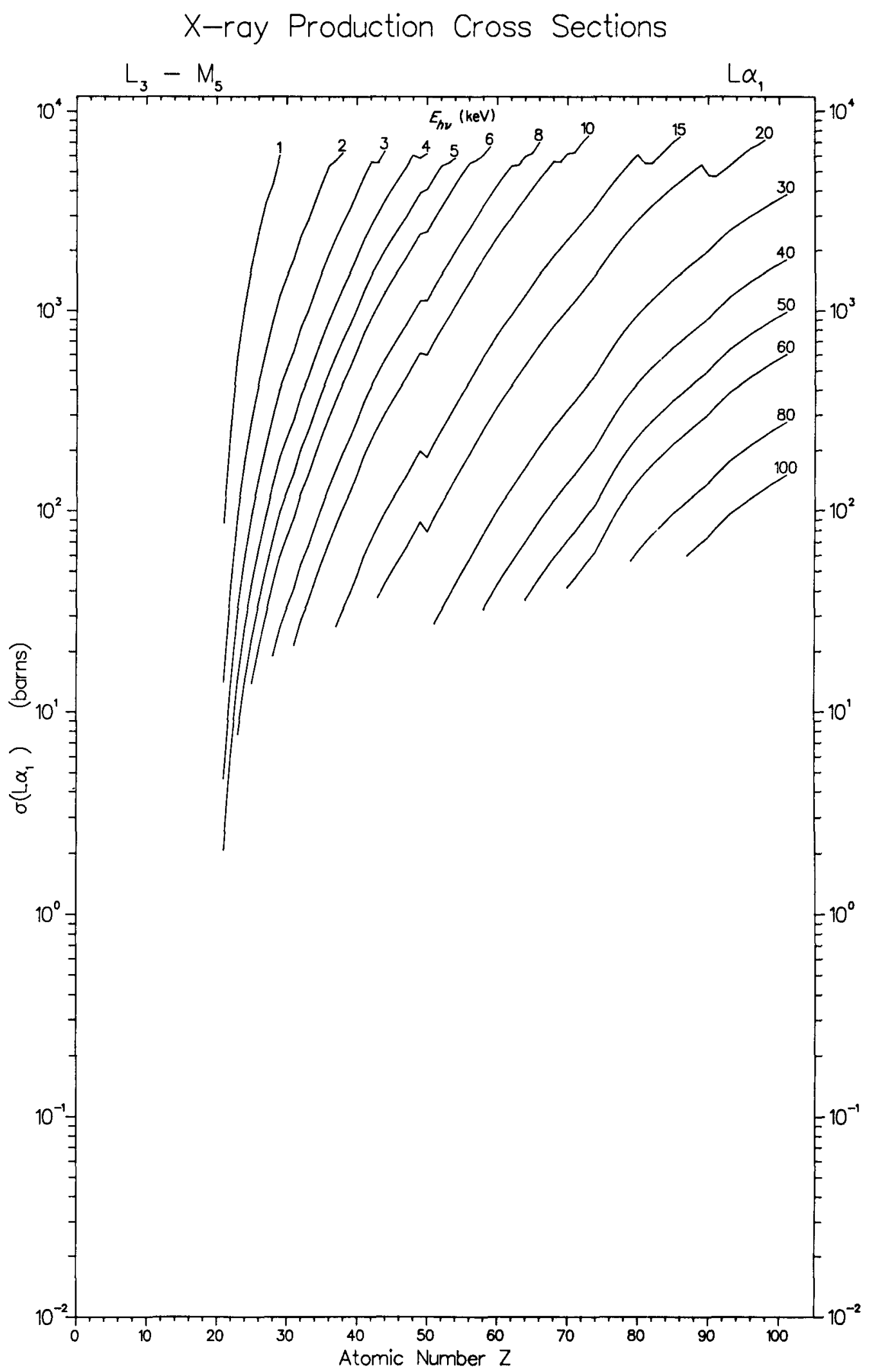

Fig. 23 
ORNL-DWG 77-21176R

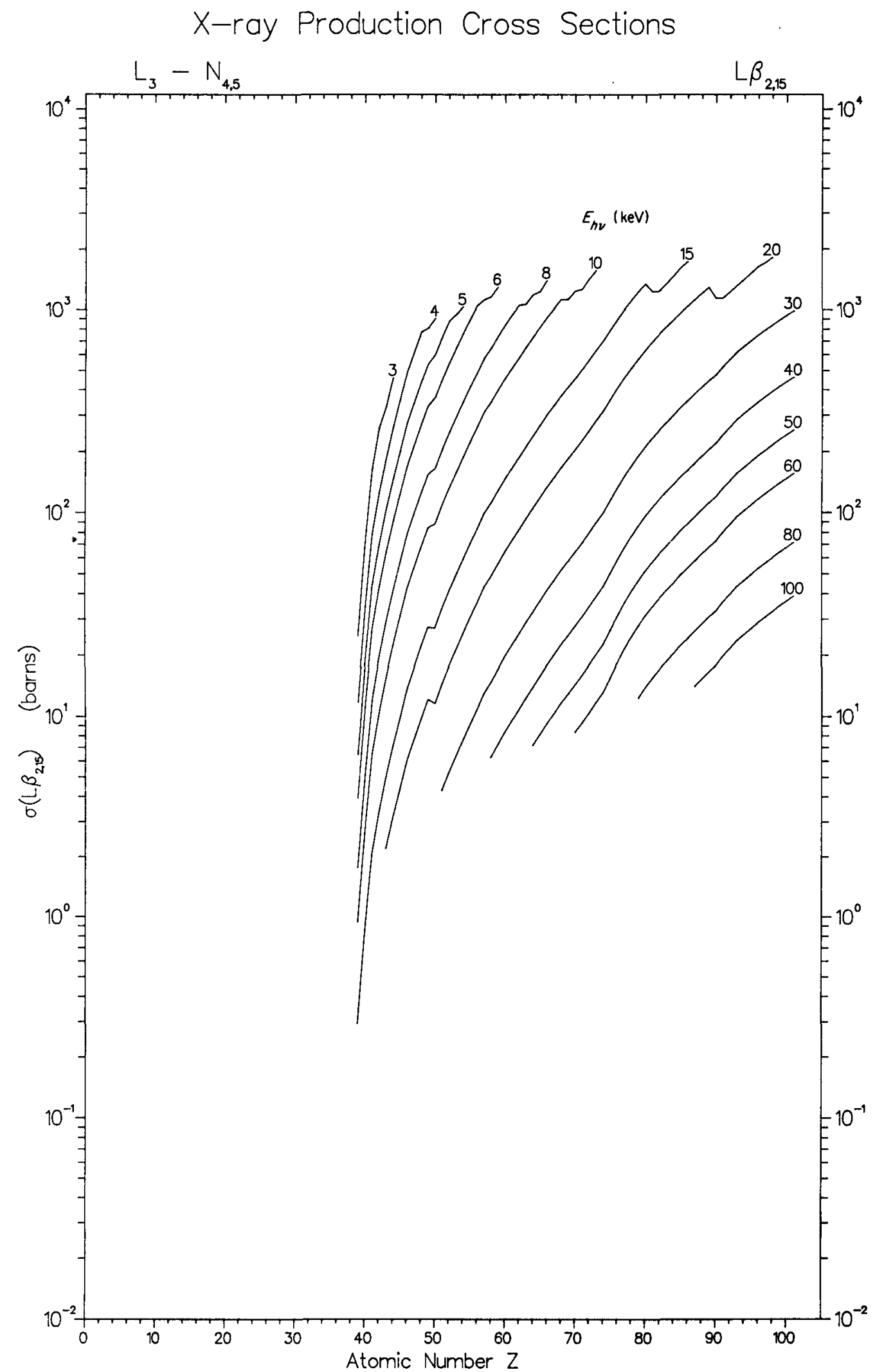

Fig. 24 


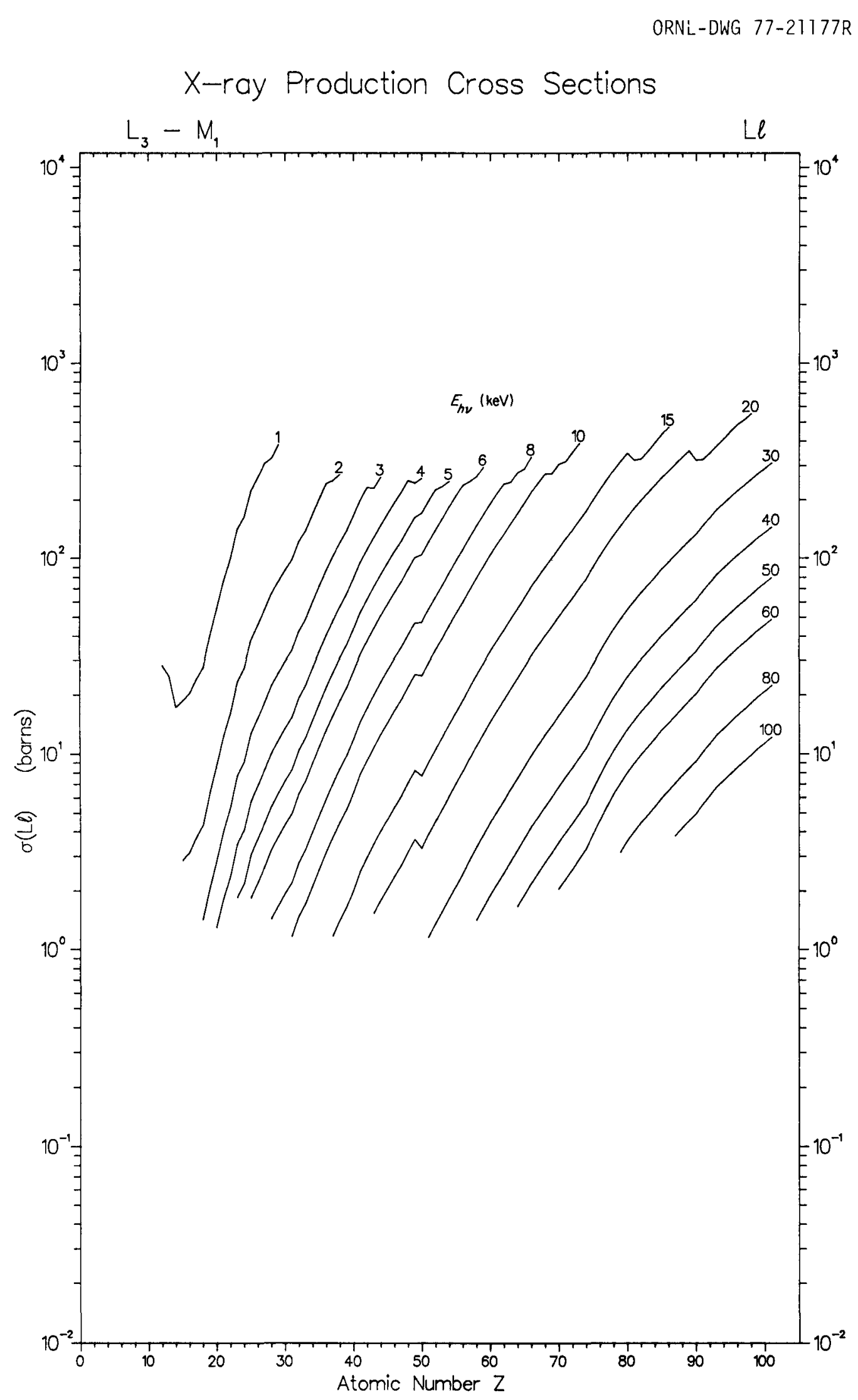

Fig. 25 
O

0

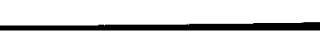


3. TABLES AND GRAPHS OF X-RAY FLUORESCENCE CROSS SECTIONS FOR SPECIFIC PHOTON SOURCES 
•

• 
In this section, fluorescence cross sections for the major $K$ and $L \mathrm{x}$ rays are given at the photon energies of eight excitation sources frequently used in $x$-ray fluorescence analysis. Tabulated cross sections are for $K \alpha_{1,2}$ and $L \alpha_{1,2}$ x-ray lines, both in units of $\mathrm{cm}^{2} / \mathrm{g}$ (Table 11) and in units of barns/atom (Table 12). Plotted cross sections (barns/atom) are for $K \alpha_{1}, K \alpha_{1,2}, K \beta_{1}, L \alpha_{1,2}, L \beta_{1}, L \beta_{2,15}$, and $L \gamma_{1}$ x-ray 1ines.

Energies of sources are the weighted means of all characteristic $K \times$ rays; for $M o K \alpha$, monochromatization was assumed, and source energy is the weighted mean of $K \alpha_{1}$ and $K \alpha_{2}$ x-ray energies. Underlying continuum (bremsstrahlung) was not considered. As a consequence, mean energies correspond most closely to sources used in fluorescent excitation.

Fluorescence cross section values at the energies of the specific sources were obtained by interpolation of the partial photoionization cross sections (cf. Eq. 1); using a three-point quadratic function,

$$
\begin{aligned}
\ln \sigma= & \frac{\left(\ln E-\ln E_{2}\right)\left(\ln E-\ln E_{3}\right)}{\left(\ln E_{1}-\ln E_{2}\right)\left(\ln E_{1}-\ln E_{3}\right)} \ln \sigma_{1} \\
& +\frac{\left(\ln E-\ln E_{3}\right)\left(\ln E-\ln E_{1}\right)}{\left(\ln E_{2}-\ln E_{3}\right)\left(\ln E_{2}-\ln E_{1}\right)} \ln \sigma_{2} \\
& +\frac{\left(\ln E-\ln E_{1}\right)\left(\ln E-\ln E_{2}\right)}{\left(\ln E_{3}-\ln E_{1}\right)\left(\ln E_{3}-\ln E_{2}\right)} \ln \sigma_{3},
\end{aligned}
$$

provided $\sigma_{1}>\sigma_{2}>\sigma_{3}$, that is, at energies greater than the $K$ or $L_{1}$ absorption edge, and, using a two-point linear function

$$
\ln \sigma=\frac{\ln E-\ln E_{1}}{\ln E_{2}-\ln E_{1}} \ln \sigma_{2}+\frac{\ln E_{2}-\ln E}{\ln E_{2}-\ln E_{1}} \ln \sigma_{1}
$$

for the regions between the absorption edges of the $L$ subshells. Indices 1 to 3 refer to coordinate points and not to subshells.

Note the following details:

1. Cross sections for $L \mathrm{x}$ rays at photon energies exceeding the $K$ binding energy refer only to initial ionization in the $L$ shell and do not include contributions from $K$-shell ionization with subsequent hole transfer by $K \alpha \times$ rays or $K L L$ Auger processes. 
2. The possibility that an $\mathrm{x}$ ray of the $K$ series from the excitation source may fall below an absorption edge of an element has not been considered. Thus the last entry of a column may be too large.

3. Theoretical binding energies used in ref, 2 and in this report are slightly greater than experimental binding energies.

4. In the case of $K \alpha \times$ rays if $Z \leqslant 15$, and $L \alpha \times$ rays if $Z \leqslant 30$, cross sections should be modified when investigating molecular or solidstate systems in accordance with the values in Table 16.

5. Read $9.13 \mathrm{E} 01$ as $9.13 \times 10^{1}$.

6. Cross section values 1 isted in Tables 11 and 12 are to be preferred over those given in ref. 1 because of the improved interpolation procedure used here. 
Table 11. $K \alpha$ and $L \alpha$ fluorescence cross sections for specific photon sources in units of $\mathrm{cm}^{2} / \mathrm{g}$

Excitation energles are weighted average energles of the $K$ x-ray series of the sources, except for MoKa and ${ }^{241} \mathrm{Am}$ Note $K \alpha=K \alpha_{1,2}$ and $L \alpha=L \alpha_{1,2}$

\begin{tabular}{|c|c|c|c|c|c|c|c|c|c|}
\hline \multirow{2}{*}{\multicolumn{2}{|c|}{$z$}} & \multicolumn{2}{|c|}{$\mathrm{T}_{\mathrm{I} K} \equiv 455 \mathrm{keV}$} & \multicolumn{2}{|c|}{$\mathrm{Cr}_{\mathrm{r} K} \equiv 5.46 \mathrm{keV}$} & \multicolumn{2}{|c|}{$\mathrm{CoK} \equiv 700 \mathrm{keV}$} & \multicolumn{2}{|c|}{$\mathrm{Cu} K \equiv 813 \mathrm{keV}$} \\
\hline & & $K \alpha$ & $L \alpha$ & $K \alpha$ & $L \alpha$ & $K \alpha$ & $L \alpha$ & $K a$ & $L \alpha$ \\
\hline 5 & & $2.04 E-02$ & & 1. $1 S E-02$ & & $5.17 E-03$ & & $3.19 \mathrm{E}-03$ & \\
\hline 6 & c & $6.66 \mathrm{E}-02$ & & $3.78 \mathrm{~F}-122$ & & $1.73 \mathrm{E}-02$ & & $1.07 \mathrm{E}-02$ & \\
\hline 7 & ง & $2.03 E-01$ & & $1.160-01$ & & $5.37 \mathrm{E}-02$ & & 3. $36 F_{1}-02$ & \\
\hline 8 & 0 & $4.93 \mathrm{E}-01$ & & $2.84 E-01$ & & $1.33 \mathrm{E}-01$ & & $3.35 E-02$ & \\
\hline 9 & $F$ & $1.05 \mathrm{E00}$ & & $6.085-01$ & & $2.87 F-01$ & & $1.81 \mathrm{~F}-01$ & \\
\hline 10 & $\mathrm{NE}$ & $1.96 \mathrm{E} \quad 00$ & & $1.14 E 00$ & & $5.45 \mathrm{E}-01$ & & $3.46 p-01$ & \\
\hline 11 & NA & $3.36 \mathrm{E} 00$ & & $1.98 E 00$ & & $9.50=-01$ & & $6.07 E-01$ & \\
\hline 12 & $M G$ & $5.76 \mathrm{~F} 00$ & & $3.42 \mathrm{E} 00$ & & $1.66 \mathrm{E} 00$ & & $1.06 \mathrm{E} 00$ & \\
\hline 13 & $A I$ & $8.96 \mathrm{E} 00$ & & 5.35800 & & $2.62 \mathrm{~F} 00$ & & $1.69 \mathrm{E} 00$ & \\
\hline 14 & SI & $1.42 \mathrm{E} 01$ & & 8.55500 & & $4.22 \mathrm{E} 00$ & & $2.74 \mathrm{E} 00$ & \\
\hline 15 & p & $2.04 E 01$ & & 1.23501 & & $5.14 E 00$ & & $4.01 E 00$ & \\
\hline 16 & $\mathrm{~s}$ & 2.99801 & & $1.93 \mathrm{E} 01$ & & $9.17 \mathrm{E} \quad 00$ & & $6.01 E 00$ & \\
\hline 17 & $\mathrm{CL}$ & $4.01 \mathrm{~F} 01$ & & $2.47 \mathrm{E} 01$ & & $1.25 \mathrm{E} 01$ & & $8.22 \mathrm{E} 00$ & \\
\hline 19 & $A R$ & 5.19501 & & $3.2+F 01$ & & $1.64 \mathrm{EO} 1$ & & $1.08 \mathrm{~F} 01$ & \\
\hline 19 & $\mathrm{k}$ & $7.41 \mathrm{E} 01$ & & 4.61801 & & $2.37 \mathrm{E} 01$ & & $1.58 \mathrm{~F} 01$ & \\
\hline 20 & $\mathrm{CA}$ & $9.78 E 01$ & & 6.15801 & & $3.19 \mathrm{E} 01$ & & $2.13 \mathrm{~F} 01$ & \\
\hline 21 & $\mathrm{sC}$ & $1.16 \mathrm{E} 02$ & $2.15 B-02$ & $7.40 \mathrm{~F} \quad 01$ & 1. $29 E-02$ & $3.86 \mathrm{E} 01$ & $6.41 F-03$ & $2.59 \mathrm{E} 01$ & $4.21 \mathrm{E}-03$ \\
\hline 22 & TI & & $5.99 p_{1}-02$ & $9.13 \mathrm{P} 01$ & $3.59 \mathrm{E}-02$ & $4.92 F 01$ & $1.78 E-02$ & $3.24 \mathrm{E} 01$ & $1.17 \mathrm{~F}-02$ \\
\hline 23 & $v$ & & $1.33 E-01$ & & $7.91 F-02$ & $5.94 F 01$ & $3.91 \mathrm{E}-02$ & $4.02 \mathrm{E} 01$ & $2.56 \mathrm{E}-02$ \\
\hline 24 & $\mathrm{CR}$ & & $2.390-01$ & & $1.42 \mathrm{E}-01$ & $7.53 \mathrm{E} 01$ & $7.038-02$ & $5.15 \mathrm{~F} 01$ & $4.608-02$ \\
\hline 25 & $M N$ & & $3.70 \Xi-01$ & & $2.22 E-01$ & 9.05501 & $1.09 \mathrm{E}-01$ & $6.23 \mathrm{~F} 01$ & $7.16 s-02$ \\
\hline 26 & $\mathrm{FE}$ & & $5.49 \mathrm{~F}-01$ & & $3.29 \mathrm{E}-01$ & (2) & $1.62 \mathrm{E}-01$ & $7.65 \mathrm{E} 01$ & $1.06 \mathrm{E}-01$ \\
\hline 27 & co & & $7.50 \mathrm{E}-01$ & & $4.49 \mathrm{E}-01$ & & $2.22 E-01$ & $8.98 \mathrm{E} 01$ & $1.45 F-01$ \\
\hline 28 & NT & & $1.03 \mathrm{E} 00$ & & $6.45 \mathrm{E}-01$ & & $3.19 \mathrm{E}-01$ & & $2.08 E-01$ \\
\hline 29 & $\mathrm{CU}$ & & $1.36 \mathrm{~F} 00$ & & $8.19 F-01$ & & $4.05 E-01$ & & $2.64 \mathrm{~F}-01$ \\
\hline 30 & $Z N$ & & $1.66 \mathrm{~F} \quad 00$ & & $9.98 \mathrm{~F}-01$ & & $4.95 E-01$ & & $3.23 E-01$ \\
\hline 31 & GA & & 1.92500 & & 1.16000 & & $5.73 \mathrm{E}-01$ & & $3.74 \mathrm{E}-01$ \\
\hline 32 & $\mathrm{rE}$ & & $2.44 E \quad 00$ & & $1.47 \mathrm{~F} 00$ & & $7.31 E-01$ & & $4.78 \mathrm{E}-01$ \\
\hline 33 & AS & & $2.83 F \quad 00$ & & 1.73500 & & $8.64 \mathrm{E}-01$ & & $5.65 F-01$ \\
\hline 34 & $S F$ & & $3.46 E 00$ & & $2.09 E 00$ & & $1.04 \mathrm{E} 00$ & & $6.81 E-01$ \\
\hline 35 & $B R$ & & $4.28 E 00$ & & $2.59 \mathrm{E} 00$ & & $1.29 \mathrm{E} 00$ & & $9.47 \mathrm{E}-01$ \\
\hline 36 & $K R$ & & $5.04 \mathrm{E} 00$ & & 3.05800 & & $1.53 \mathrm{E} 00$ & & $1.00 \mathrm{E} 00$ \\
\hline 37 & RB & & 6.02500 & & $3.66 \mathrm{~F} 00$ & & $1.83 \mathrm{E} 00$ & & $1.20 \mathrm{E} \quad 00$ \\
\hline 33 & $S R$ & & $7.07 \mathrm{~F} 00$ & & $4.30 E \quad 00$ & & 2.16800 & & $1.42 \mathrm{E} 00$ \\
\hline 39 & Y & & $8.25 \mathrm{E} \quad 00$ & & 5.03800 & & $2.54 \mathrm{~F} \quad 00$ & & $1.67 \mathrm{E} 00$ \\
\hline 40 & $2 R$ & & $9.74 \mathrm{E} \quad 00$ & & $5.96 E 00$ & & $3.01 \mathrm{E} 00$ & & $1.98 \% 00$ \\
\hline 41 & $\mathrm{NR}$ & & $1.17 \mathrm{E} 01$ & & $7.18 \mathrm{E} 00$ & & $3.66 \mathrm{~F}: 00$ & & $2.42 \mathrm{E} \quad 00$ \\
\hline 42 & MO & & $1.33 \mathrm{E} O 1$ & & $9.21 \mathrm{E} 00$ & & 4.19800 & & $2.78 \mathrm{~F} 00$ \\
\hline 43 & TC & & $1.50 \mathrm{~B} 01$ & & $9.29 \mathrm{E} 00$ & & $4.76 \mathrm{~F} 00$ & & $3.16 \mathrm{E} \quad 00$ \\
\hline 44 & $\mathrm{RO}$ & & $1.70 \mathrm{~F} 01$ & & $1.05,01$ & & 5.41800 & & $3.60 \mathrm{E} 00$ \\
\hline 45 & $\mathrm{RH}$ & & $1.91 \mathrm{E} \quad 01$ & & $1.19 \mathrm{E} 01$ & & $6.10 \mathrm{E} \quad 00$ & & $4.06 \mathrm{~F} 00$ \\
\hline 46 & PD & & $2.10 E \quad 01$ & & 1.30501 & & $6.72 \mathrm{P} 00$ & & $4.48 \mathrm{E} 00$ \\
\hline 47 & $9 G$ & & $2.35 \mathrm{E} 01$ & & $1.46 \mathrm{E} 01$ & & $7.53 \mathrm{E} 00$ & & 5.03800 \\
\hline 48 & $C D$ & & $2.59 \mathrm{E} 01$ & & $1.51 F$ Oi & & $8.35 E 00$ & & $5.57 \mathrm{E} \quad 00$ \\
\hline 49 & IN & & 2.90801 & & $1.81 \mathrm{E} \quad 01$ & & $9.39 \mathrm{E} 00$ & & $6.28 \mathrm{E} 00$ \\
\hline 50 & $\mathrm{SN}$ & & $2.94 \mathrm{E} \quad 01$ & & $1.82 \mathrm{E} \quad 01$ & & $9.24 \mathrm{E} \quad 00$ & & 6.09500 \\
\hline 51 & SB & & $3.02 \mathrm{E} 01$ & & $2.05 \mathrm{~F} 01$ & & $1.05 \mathrm{~F} 01$ & & $6.91 \mathrm{~F} 00$ \\
\hline 52 & $T E$ & & $2.98 E$ O1 & & $2.24 F 01$ & & $1.14 \mathrm{E} \quad 01$ & & $7.57 \mathrm{E} \quad 00$ \\
\hline 53 & I & & & & 2.55001 & & $1.31 \mathrm{E} 01$ & & $8.69 \mathrm{E} 00$ \\
\hline 54 & $X F$ & & & & 2.99501 & & $1.46 \mathrm{~F} 01$ & & 9.66800 \\
\hline 55 & $\operatorname{cs}$ & & & & $2.88 \mathrm{~F} 01$ & & $1.64 \mathrm{~F} \quad 01$ & & $1.09 \mathrm{E} \quad 01$ \\
\hline 56 & $\mathrm{BA}$ & & & & $2.99 F \quad 01$ & & $1.80 \mathrm{~F}$ o1 & & $1.20 \mathrm{E} 01$ \\
\hline 57 & LA & & & & & & $2.03 \mathrm{E} 01$ & & $1.36 \% 01$ \\
\hline 58 & $\mathrm{CE}$ & & & & & & $2.29 \mathrm{E} 01$ & & $1.54 \mathrm{E} \quad 01$ \\
\hline 59 & $P R$ & & & & & & $2.56 \mathrm{E} 01$ & & $1.73 \mathrm{~F} \quad 01$ \\
\hline 60 & ND & & & & & & $2.56 \mathrm{E} \quad 01$ & & $1.31 \% 01$ \\
\hline 61 & $P M$ & & & & & & 2.59801 & & $2.11 \mathrm{E} 01$ \\
\hline 62 & $5 M$ & & & & & & $2.80 \mathrm{E} 01$ & & $2.28 \mathrm{~F} \quad 01$ \\
\hline 63 & EU & & & & & & & & $2.52 \mathrm{P} 01$ \\
\hline 64 & GD & & & & & & & & 2.45801 \\
\hline 65 & $T R$ & & & & & & & & $2.50 \% 01$ \\
\hline 66 & $n Y$ & & & & & & & & $2.75 \%$ ol \\
\hline
\end{tabular}


Table 11 (continued)

\begin{tabular}{|c|c|c|c|c|c|c|c|c|c|}
\hline \multirow{2}{*}{\multicolumn{2}{|c|}{$z$}} & \multicolumn{2}{|c|}{$\mathrm{MoK} \alpha \equiv 17.44 \mathrm{keV}$} & \multicolumn{2}{|c|}{$\mathrm{Ag} K \equiv 22.5 \mathrm{keV}$} & \multicolumn{2}{|c|}{$\mathrm{Dy} K \equiv 47.0 \mathrm{keV}$} & \multicolumn{2}{|c|}{${ }^{241} \mathrm{Am} \equiv 5954 \mathrm{keV}$} \\
\hline & & $K \alpha$ & $L \alpha$ & $K \alpha$ & $L \alpha$ & $K \alpha$ & $L \alpha$ & $K \alpha$ & $L \alpha$ \\
\hline $\begin{array}{ll}5 & 8 \\
6 & 0 \\
7 & 1 \\
8 & 0 \\
9 & 8\end{array}$ & $\begin{array}{l}B \\
C \\
\text { N } \\
0 \\
P\end{array}$ & $\begin{array}{l}2.62 \mathrm{E}-04 \\
9.07 \mathrm{E}-04 \\
2.93 \mathrm{E}-03 \\
7.47 \mathrm{E}-03 \\
1.66 \mathrm{E}-02\end{array}$ & & $\begin{array}{l}1.12 E-04 \\
3.93 \mathrm{P}-04 \\
1.28 \mathrm{E}-03 \\
3.29 \mathrm{E}-03 \\
7.37 \mathrm{E}-03\end{array}$ & & $\begin{array}{l}9.57 \mathrm{E}-06 \\
3.41 \mathrm{E}-05 \\
1.13 \mathrm{E}-04 \\
2.95 \mathrm{E}-04 \\
6.74 \mathrm{E}-04\end{array}$ & & $\begin{array}{l}4.31 \mathrm{E}-06 \\
1.54 \mathrm{E}-05 \\
5.13 \mathrm{E}-05 \\
1.35 \mathrm{E}-04 \\
3.09 \mathrm{E}-04\end{array}$ & \\
\hline $\begin{array}{ll}10 & N \\
11 & 1 \\
12 & \\
13 & A \\
14 & 5\end{array}$ & $\begin{array}{l}N E \\
M A \\
M G \\
A L \\
\text { SI }\end{array}$ & $\begin{array}{l}3.25 \mathrm{~F}-02 \\
5.84 \mathrm{E}-02 \\
1.04 \mathrm{E}-01 \\
1.70 \mathrm{E}-01 \\
2.80 \mathrm{E}-01\end{array}$ & & $\begin{array}{l}1.45 E-02 \\
2.62 E-02 \\
4.72 E-02 \\
7.70 E-02 \\
1.28 F-01\end{array}$ & & $\begin{array}{l}1.35 \mathrm{E}-03 \\
2.48 \mathrm{E}-03 \\
4.53 \mathrm{E}-03 \\
7.50 \mathrm{E}-03 \\
1.27 \mathrm{E}-02\end{array}$ & & $\begin{array}{l}6.22 \mathrm{E}-04 \\
1.15 \mathrm{R}-03 \\
2.10 \mathrm{E}-03 \\
3.50 \mathrm{E}-03 \\
5.93 \mathrm{E}-03\end{array}$ & \\
\hline $\begin{array}{ll}15 & 8 \\
16 & 5 \\
17 & 0 \\
10 & \end{array}$ & $\begin{array}{l}\mathrm{P} \\
\mathrm{S} \\
\mathrm{CL}_{\mathrm{L}} \\
\mathrm{n}\end{array}$ & $\begin{array}{l}4.19 \mathrm{E}-01 \\
6.41 \mathrm{E}-01 \\
8.94 \mathrm{E}-01 \\
1.9 n \mathrm{NO}\end{array}$ & & $\begin{array}{l}1.92 E-01 \\
2.96 \mathrm{E}-01 \\
4.15 \mathrm{E}-01 \\
? .2 \mathrm{E}\end{array}$ & & $\begin{array}{l}1.93 \mathrm{~F}-02 \\
3.01 \mathrm{E}-02 \\
4.27 \mathrm{~F}-02\end{array}$ & & $\begin{array}{l}9.06 \mathrm{E}-03 \\
1.42 \mathrm{E}-02 \\
2.02 \mathrm{E}-02\end{array}$ & \\
\hline
\end{tabular}

$X$-ray Production Cross Sections

$\mathrm{Ag} \mathrm{Kh \nu}=22.5 \mathrm{keV}$

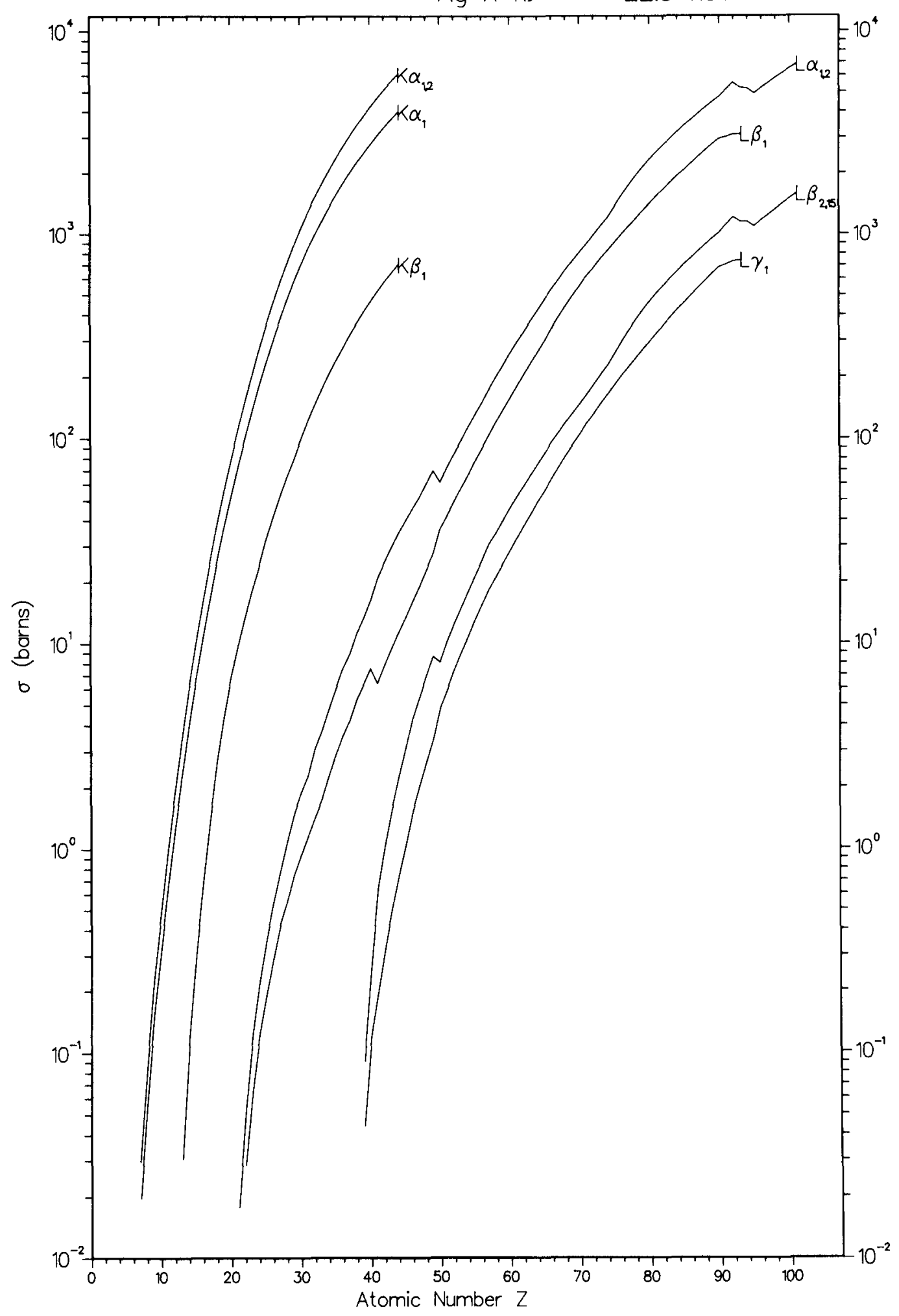

Fig. 31 
Table 11 (continued)

\begin{tabular}{|c|c|c|c|c|c|c|c|c|c|c|c|}
\hline & \multirow{2}{*}{$Z$} & \multicolumn{3}{|c|}{$M o K \alpha \equiv 17.44 \mathrm{keV}$} & \multicolumn{3}{|c|}{$\mathrm{AgK} \equiv 22.5 \mathrm{keV}$} & \multicolumn{2}{|c|}{$\mathrm{Dy} K \equiv 47.0 \mathrm{keV}$} & \multicolumn{2}{|c|}{${ }^{241} \mathrm{Am} \equiv 59.54 \mathrm{keV}$} \\
\hline & & $K \alpha$ & $L \alpha$ & & $K \alpha$ & $L \alpha$ & & $K \alpha$ & $L \alpha$ & $K \alpha$ & $L \alpha$ \\
\hline $\begin{array}{l}70 \\
71 \\
72 \\
73 \\
74\end{array}$ & $\begin{array}{l}\text { Y B } \\
\text { L U } \\
\text { H F } \\
\text { T A } \\
\mathbf{W}\end{array}$ & & $\begin{array}{ll}5.80 \mathrm{E} & 0 \\
6.30 \mathrm{E} & 0 \\
6.83 \mathrm{E} & 0 \\
7.45 \mathrm{E} & 0 \\
8.07 \mathrm{E} & 0\end{array}$ & $\begin{array}{l}00 \\
00 \\
00 \\
00 \\
00\end{array}$ & & $\begin{array}{l}2.82 \mathrm{E} \\
3.07 \mathrm{E} \\
3.33 \mathrm{E} \\
3.64 \mathrm{E} \\
3.94 \mathrm{E}\end{array}$ & $\begin{array}{lll}0 & 0 \\
0 & 0 \\
0 & 0 \\
0 & 0 \\
0 & 0\end{array}$ & & $\begin{array}{l}3.32 \mathrm{E}-01 \\
3.61 \mathrm{E}-01 \\
3.93 \mathrm{E}-01 \\
4.32 \mathrm{E}-01 \\
4.70 \mathrm{E}-01\end{array}$ & & $\begin{array}{l}1.65 \mathrm{E}-01 \\
1.79 \mathrm{E}-01 \\
1.95 \mathrm{E}-01 \\
2.14 \mathrm{E}-01 \\
2.34 \mathrm{E}-01\end{array}$ \\
\hline $\begin{array}{l}75 \\
76 \\
77 \\
78 \\
79\end{array}$ & $\begin{array}{l}\text { R F } \\
\text { OS } \\
\text { I R } \\
\text { PT } \\
\text { A U }\end{array}$ & & 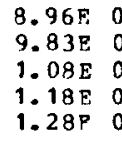 & $\begin{array}{l}00 \\
00 \\
01 \\
01 \\
01\end{array}$ & & $\begin{array}{l}4.41 \mathrm{E} \\
4.87 \mathrm{E} \\
5.41 \mathrm{E} \\
5.93 \mathrm{E} \\
6.48 \mathrm{E}\end{array}$ & $\begin{array}{ll}0 & 0 \\
0 & 0 \\
0 & 0 \\
0 & 0 \\
0 & 0\end{array}$ & & $\begin{array}{l}5.37 \mathrm{E}-01 \\
6.08 \mathrm{E}-01 \\
6.91 \mathrm{E}-01 \\
7.70 \mathrm{E}-01 \\
8.52 \mathrm{E}-01\end{array}$ & & $\begin{array}{l}2.69 E-01 \\
3.07 \mathrm{R}-01 \\
3.52 \mathrm{E}-01 \\
3.94 \mathrm{E}-01 \\
4.38 \mathrm{E}-01\end{array}$ \\
\hline $\begin{array}{l}80 \\
81 \\
82 \\
83 \\
84\end{array}$ & $\begin{array}{l}\text { HG } \\
\text { TL } \\
\text { PB } \\
\text { BI } \\
\text { PO }\end{array}$ & & $\begin{array}{l}1.38 \mathrm{E} \\
1.47 \mathrm{E} \\
1.57 \mathrm{E} \\
1.67 \mathrm{E} \\
1.79 \mathrm{E}\end{array}$ & $\begin{array}{l}01 \\
01 \\
01 \\
01 \\
01\end{array}$ & & $\begin{array}{l}6.99 \mathrm{~F} \\
7.48 \mathrm{E} \\
8.00 \mathrm{~S} \\
8.53 \mathrm{E} \\
9.17 \mathrm{~F}\end{array}$ & $\begin{array}{ll}0 & 0 \\
0 & 0 \\
0 & 0 \\
0 & 0 \\
0 & 0\end{array}$ & & 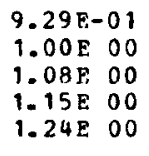 & & $\begin{array}{l}4.79 E-01 \\
5.17 E-01 \\
5.57 E-01 \\
5.97 E-01 \\
6.45 F-01\end{array}$ \\
\hline $\begin{array}{l}85 \\
86 \\
87 \\
88 \\
84\end{array}$ & $\begin{array}{l}A T \\
R N \\
P R \\
R A \\
A C\end{array}$ & & $\begin{array}{l}1.59 \mathrm{E} \\
1.54 \mathrm{~F} \\
1.59 \mathrm{~F} \\
1.69 \mathrm{~F} \\
1.79 \mathrm{E}\end{array}$ & $\begin{array}{l}01 \\
01 \\
01 \\
01 \\
01\end{array}$ & & $\begin{array}{l}9.16 \mathrm{E} \\
9.91 \mathrm{E} \\
1.06 \mathrm{E} \\
1.11 \mathrm{E} \\
1.18 \mathrm{E}\end{array}$ & $\begin{array}{ll}0 & 0 \\
0 & 0 \\
0 & 1 \\
0 & 1 \\
0 & 1\end{array}$ & & $\begin{array}{ll}1.35 \mathrm{E} & 00 \\
1.35 \mathrm{E} & 00 \\
1.45 \mathrm{E} & 00 \\
1.53 \mathrm{~F} & 00 \\
1.64 \mathrm{E} & 00\end{array}$ & & $\begin{array}{l}7.00 \mathrm{E}-01 \\
7.05 \mathrm{E}-01 \\
7.55 \mathrm{E}-01 \\
8.00 \mathrm{E}-01 \\
8.54 \mathrm{E}-01\end{array}$ \\
\hline $\begin{array}{l}90 \\
91 \\
92 \\
93 \\
94\end{array}$ & $\begin{array}{l}T H \\
P_{A} \\
U \\
N P \\
P U\end{array}$ & & $\begin{array}{l}1.89 \mathrm{E} \\
2.01 \mathrm{E} \\
2.09 \mathrm{E}\end{array}$ & $\begin{array}{l}01 \\
01 \\
01\end{array}$ & & $\begin{array}{l}1.23 \mathrm{E} \\
1.34 \mathrm{E} \\
1.40 \mathrm{~F} \\
1.33 \mathrm{E} \\
1.31 \mathrm{E}\end{array}$ & $\begin{array}{ll}0 & 1 \\
0 & 1 \\
0 & 1 \\
0 & 1 \\
0 & 1\end{array}$ & & $\begin{array}{ll}1.70 \mathrm{E} & 00 \\
1.88 \mathrm{E} & 00 \\
1.97 \mathrm{E} & 00 \\
2.14 \mathrm{E} & 00 \\
2.25 \mathrm{E} & 00\end{array}$ & & 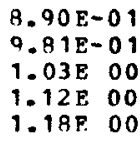 \\
\hline $\begin{array}{l}95 \\
96 \\
97 \\
98 \\
99\end{array}$ & $\begin{array}{l}A M \\
C M \\
B K \\
C F \\
E S\end{array}$ & & & & & $\begin{array}{l}1.22 \mathrm{E} \\
1.27 \mathrm{~F} \\
1.33 \mathrm{E} \\
1.41 \mathrm{~F} \\
1.46 \mathrm{E}\end{array}$ & $\begin{array}{ll}0 & 1 \\
0 & 1 \\
0 & 1 \\
0 & 1 \\
0 & 1\end{array}$ & & $\begin{array}{ll}2.36 \mathrm{E} & 00 \\
2.45 \mathrm{E} & 00 \\
2.57 \mathrm{E} & 00 \\
2.71 \mathrm{E} & 00 \\
2.82 \mathrm{E} & 00\end{array}$ & & $\begin{array}{ll}1.24 \mathrm{E} & 00 \\
1.29 \mathrm{E} & 00 \\
1.35 \mathrm{E} & 00 \\
1.43 \mathrm{E} & 00 \\
1.48 \mathrm{~F} & 00\end{array}$ \\
\hline $\begin{array}{l}100 \\
101\end{array}$ & $\begin{array}{l}F M \\
M D\end{array}$ & & & & & $\begin{array}{l}1.55 \mathrm{E} \\
1.59 \mathrm{E}\end{array}$ & $\begin{array}{l}01 \\
01\end{array}$ & & $\begin{array}{ll}2.96 \mathrm{E} & 00 \\
3.04 \mathrm{E} & 00\end{array}$ & & $\begin{array}{ll}1.56 \mathrm{~F} & 00 \\
1.60 \mathrm{~F} & 00\end{array}$ \\
\hline
\end{tabular}


Table $12 K \alpha$ and $L \alpha$ fluorescence cross sectrons for specific photon sources in units of barns/atom Exutation encrsics are weighted dverage encrgks of the $\alpha$ x raly serics ot the sources except for Moh $\alpha$ ind ${ }^{24}$ I Am $\mathrm{N} \| h \alpha-h \alpha_{12}$ and $l \alpha-l \alpha_{12}$

See Figs 26 to 33 for graphic representation

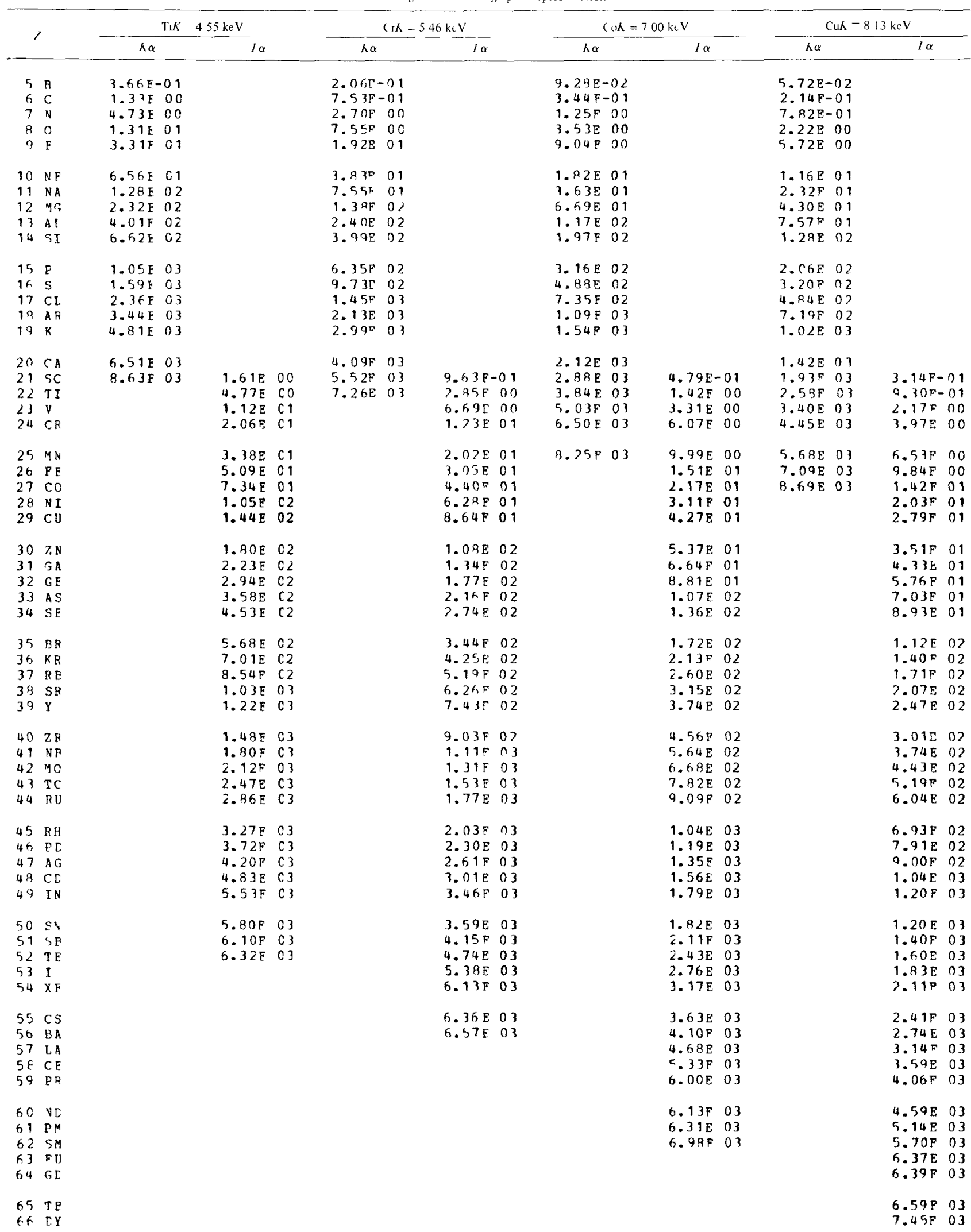


Table 12 (continued)

\begin{tabular}{|c|c|c|c|c|c|c|c|c|c|}
\hline \multirow{2}{*}{$Z$} & & \multicolumn{2}{|c|}{$\mathrm{Mo} K \alpha \equiv 17.44 \mathrm{keV}$} & \multicolumn{2}{|c|}{$\mathrm{Ag} K \equiv 22.5 \mathrm{keV}$} & \multicolumn{2}{|c|}{$\mathrm{Dy} K \equiv 47.0 \mathrm{keV}$} & \multicolumn{2}{|c|}{${ }^{241} \mathrm{Am}=5954 \mathrm{keV}$} \\
\hline & & $K \alpha$ & $L \alpha$ & $K a$ & $L \alpha$ & $K \alpha$ & $L \alpha$ & $K \alpha$ & $L \alpha$ \\
\hline $\begin{array}{l}5 \\
6 \\
7 \\
8 \\
9\end{array}$ & $\begin{array}{l}\mathrm{E} \\
\mathrm{C} \\
\mathrm{N} \\
\mathrm{O} \\
\mathrm{F}\end{array}$ & $\begin{array}{l}4.70 E-03 \\
1.81 F-02 \\
6.81 E-02 \\
1.99 E-01 \\
5.25 E-01\end{array}$ & & $\begin{array}{l}2.02 F-03 \\
7.83 F-03 \\
2.97 F-02 \\
8.73 F-02 \\
2.33 F-01\end{array}$ & & $\begin{array}{l}1.72 E-04 \\
6.79 E-04 \\
2.63 F-03 \\
7.85 E-03 \\
2.13 F-02\end{array}$ & & $\begin{array}{l}7.73 E-05 \\
3.08 E-04 \\
1.19 F-03 \\
3.59 E-03 \\
9.76 E-03\end{array}$ & \\
\hline $\begin{array}{l}10 \\
11 \\
12 \\
13 \\
14\end{array}$ & $\begin{array}{l}\text { NE } \\
\text { NA } \\
\text { Y } r \\
\text { AI } \\
S I\end{array}$ & $\begin{array}{ll}1.0 \mathrm{GF} & 00 \\
2.23 \mathrm{~F} & 00 \\
4.22 \mathrm{E} & 00 \\
7.5 \mathrm{GE} & \mathrm{CO} \\
1.31 \mathrm{E} & 01\end{array}$ & & $\begin{array}{rr}4.96 E-01 \\
1.00 \mathrm{E} & 00 \\
1.90 \mathrm{E} & 00 \\
3.45 \mathrm{E} & 00 \\
5.97 \mathrm{E} & 00\end{array}$ & & $\begin{array}{l}4.52 F-02 \\
9.45 F-02 \\
1.83 E-01 \\
3.36 F-01 \\
5.90 E-01\end{array}$ & & $\begin{array}{l}2.08 \mathrm{E}-02 \\
4.38 \mathrm{~F}-02 \\
8.50 \mathrm{E}-02 \\
1.57 \mathrm{E}-01 \\
2.76 \mathrm{E}-01\end{array}$ & \\
\hline $\begin{array}{l}15 \\
16 \\
17 \\
18 \\
19\end{array}$ & $\begin{array}{l}P \\
S \\
C L \\
A R \\
K\end{array}$ & $\begin{array}{ll}2.15 E & 01 \\
3.41 E & 01 \\
5.26 \mathrm{~F} & 01 \\
7.97 \mathrm{E} & 01 \\
1.16 \mathrm{~F} & 02\end{array}$ & & $\begin{array}{ll}9.89 E & 00 \\
1.59 F & 01 \\
2.44 F & 01 \\
3.72 F & 01 \\
5.44 E & 01\end{array}$ & & $\begin{array}{rr}9.91 \mathrm{E} & -01 \\
1.60 \mathrm{E} & 00 \\
2.52 \mathrm{~F} & 00 \\
3.88 \mathrm{~F} & 00 \\
5.74 \mathrm{~F} & 00\end{array}$ & & 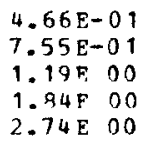 & \\
\hline $\begin{array}{l}20 \\
21 \\
22 \\
23 \\
24\end{array}$ & $\begin{array}{l}C A \\
S C \\
T I \\
V \\
C R\end{array}$ & $\begin{array}{ll}1.63 \mathrm{E} & 02 \\
2.27 \mathrm{E} & 02 \\
3.09 \mathrm{E} & 02 \\
4.14 \mathrm{E} & 02 \\
5.52 \mathrm{E} & 02\end{array}$ & $\begin{array}{l}3.67 E-C 2 \\
1.08 E-01 \\
2.49 E-01 \\
4.56 F-C 1\end{array}$ & $\begin{array}{ll}7.70 \mathrm{E} & 01 \\
1.09 \mathrm{GE} & 02 \\
1.47 \mathrm{E} & 02 \\
1.9 \mathrm{AE} & 02 \\
2.6 \mathrm{SE} & 02\end{array}$ & $\begin{array}{l}1.79 \mathrm{E}-02 \\
5.29 \mathrm{E}-02 \\
1.21 \mathrm{E}-01 \\
2.22 \mathrm{E}-01\end{array}$ & $\begin{array}{ll}8.245 & 00 \\
1.17 F & 01 \\
1.61 F & 01 \\
2.20 F & 01 \\
2.98 F & 01\end{array}$ & $\begin{array}{l}2.25 E-03 \\
6.65 E-03 \\
1.50 E-02 \\
2.74 E-02\end{array}$ & 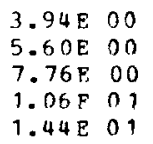 & $\begin{array}{l}1.15 E-03 \\
3.41 E-03 \\
7.66 E-03 \\
1.40 E-02\end{array}$ \\
\hline $\begin{array}{l}25 \\
26 \\
27 \\
28 \\
29\end{array}$ & $\begin{array}{l}\mathrm{MN} \\
\mathrm{FF} \\
\mathrm{CC} \\
\mathrm{NI} \\
\mathrm{CU}\end{array}$ & $\begin{array}{ll}7.16 \mathrm{E} & 02 \\
9.12 \mathrm{E} & 02 \\
1.15 \mathrm{E} & 03 \\
1.42 \mathrm{E} & 03 \\
1.75 \mathrm{~F} & 03\end{array}$ & 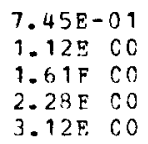 & $\begin{array}{ll}3.465 & 02 \\
4.43 F & 02 \\
5.6 C F & 02 \\
6.98 F & 02 \\
8.63 E & 02\end{array}$ & $\begin{array}{l}3.61 \mathrm{E}-01 \\
5.43 \mathrm{E}-01 \\
7.81 \mathrm{~F}-01 \\
1.10 \mathrm{~F} 00 \\
1.50 \mathrm{E} 00\end{array}$ & $\begin{array}{ll}3.93 \mathrm{~F} & 01 \\
5.09 \mathrm{E} & 01 \\
6.51 \mathrm{~F} & 01 \\
9.20 \mathrm{~F} & 01 \\
1.03 \mathrm{~F} & 02\end{array}$ & $\begin{array}{l}4.45 E-02 \\
6.68 E-02 \\
9.59 E-02 \\
1.34 E-01 \\
1.81 F-01\end{array}$ & 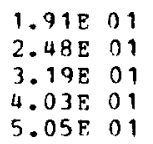 & $\begin{array}{l}2.26 \mathrm{E}-02 \\
3.40 \mathrm{E}-02 \\
4.87 \mathrm{E}-02 \\
6.78 \mathrm{E}-02 \\
9.17 \mathrm{E}-02\end{array}$ \\
\hline $\begin{array}{l}30 \\
31 \\
32 \\
33 \\
34\end{array}$ & $\begin{array}{l}Z N \\
G A \\
\text { GF } \\
A S \\
S E\end{array}$ & $\begin{array}{ll}2.12 \mathrm{~F} & 03 \\
2.53 \mathrm{~F} & \mathrm{C3} \\
2.97 \mathrm{E} & 03 \\
3.44 \mathrm{~F} & 03 \\
3.97 \mathrm{~F} & 03\end{array}$ & $\begin{array}{ll}3.93 \mathrm{~F} & \mathrm{CO} \\
4.80 \mathrm{E} & \mathrm{CO} \\
6.4 \mathrm{BF} & \mathrm{CO} \\
7.87 \mathrm{~F} & \mathrm{CO} \\
1.00 \mathrm{C} & \mathrm{C1}\end{array}$ & $\begin{array}{ll}1.05 \mathrm{E} & 03 \\
1.26 \mathrm{E} & 03 \\
1.48 \mathrm{~F} & 03 \\
1.73 \mathrm{~F} & 03 \\
2.00 \mathrm{E} & 03\end{array}$ & $\begin{array}{ll}1.89 \mathrm{E} & 00 \\
2.30 \mathrm{E} & 00 \\
3.11 \mathrm{E} & 00 \\
3.79 \mathrm{E} & 00 \\
4.30 \mathrm{E} & 00\end{array}$ & $\begin{array}{ll}1.26 \mathrm{E} & 02 \\
1.53 \mathrm{E} & 02 \\
1.82 \mathrm{~F} & 02 \\
2.15 \mathrm{E} & 02 \\
2.51 \mathrm{E} & 02\end{array}$ & $\begin{array}{l}2.29 \mathrm{~F}=01 \\
2.74 \mathrm{E}-01 \\
3.76 \mathrm{E}-01 \\
4.56 \mathrm{~F}-01 \\
5.78 \mathrm{~F}-01\end{array}$ & $\begin{array}{ll}6.23 \mathrm{E} & 01 \\
7.56 \mathrm{E} & 01 \\
9.05 \mathrm{E} & 01 \\
1.07 \mathrm{~F} & 02 \\
1.26 \mathrm{E} & 02\end{array}$ & $\begin{array}{l}1.16 \mathrm{E}-01 \\
1.38 \mathrm{E}-01 \\
1.90 \mathrm{E}-01 \\
2.31 \mathrm{E}-01 \\
2.92 \mathrm{E}-01\end{array}$ \\
\hline $\begin{array}{l}35 \\
36 \\
37 \\
38 \\
39\end{array}$ & $\begin{array}{l}\text { ER } \\
\text { KR } \\
R P \\
\text { SF } \\
Y\end{array}$ & $\begin{array}{ll}4.55 \mathrm{E} & 03 \\
5.17 \mathrm{~F} & 03 \\
5.83 \mathrm{~F} & 03 \\
6.53 \mathrm{~F} & 03 \\
7.20 \mathrm{~F} & 0.3\end{array}$ & $\begin{array}{ll}1.26 \mathrm{P} & 01 \\
1.57 \mathrm{E} & \mathrm{C1} \\
1.89 \mathrm{E} & 01 \\
2.33 \mathrm{E} & \mathrm{C1} \\
2.79 \mathrm{E} & \mathrm{C1}\end{array}$ & $\begin{array}{ll}2.31 F & 03 \\
2.64 F & 03 \\
3.00 E & 03 \\
3.38 \mathrm{E} & 03 \\
3.75 \mathrm{~F} & 03\end{array}$ & $\begin{array}{ll}6.04 \mathrm{E} & 00 \\
7.54 \mathrm{~F} & 00 \\
8.36 \mathrm{E} & 00 \\
1.12 \mathrm{E} & 01 \\
1.33 \mathrm{E} & 01\end{array}$ & $\begin{array}{ll}2.93 F & 02 \\
3.39 F & 02 \\
3.88 E & 02 \\
4.42 E & 02 \\
4.99 E & 02\end{array}$ & $\begin{array}{l}7.25 F-01 \\
9.09 E-01 \\
9.86 E-01 \\
1.32 E-00 \\
1.58 E \quad 00\end{array}$ & 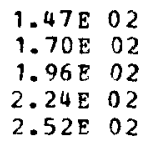 & $\begin{array}{l}3.66 \mathrm{E}-01 \\
4.60 \mathrm{E}-01 \\
4.86 \mathrm{E}-01 \\
6.66 \mathrm{E}-01 \\
7.96 \mathrm{E}-01\end{array}$ \\
\hline $\begin{array}{l}40 \\
41 \\
42 \\
43 \\
44\end{array}$ & $\begin{array}{l}7 \mathrm{~F} \\
\mathrm{NB} \\
\mathrm{MC} \\
\mathrm{TC} \\
\mathrm{RU}\end{array}$ & & $\begin{array}{ll}3.41 \mathrm{~F} & \mathrm{C1} \\
4.35 \mathrm{E} & 01 \\
5.19 \mathrm{~F} & 01 \\
6.11 \mathrm{~F} & 01 \\
7.13 \mathrm{E} & 01\end{array}$ & $\begin{array}{ll}4.1 \mathrm{AE} & 03 \\
4.63 \mathrm{~F} & 03 \\
5.10 \mathrm{~F} & 03 \\
5.59 \mathrm{E} & 03 \\
6.07 \mathrm{E} & 03\end{array}$ & 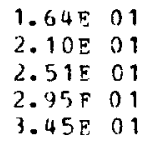 & $\begin{array}{ll}5.63 \mathrm{~F} & 02 \\
6.29 \mathrm{E} & 02 \\
7.03 \mathrm{~F} & 02 \\
7.78 \mathrm{~F} & 02 \\
8.58 \mathrm{E} & 02\end{array}$ & $\begin{array}{ll}1.95 \mathrm{E} & 00 \\
2.57 \mathrm{E} & 00 \\
3.07 \mathrm{E} & 00 \\
3.56 \mathrm{E} & 00 \\
4.20 \mathrm{E} & 00\end{array}$ & $\begin{array}{ll}2.86 \mathrm{E} & 02 \\
3.21 \mathrm{E} & 02 \\
3.59 \mathrm{E} & 02 \\
3.99 \mathrm{E} & 02 \\
4.41 \mathrm{E} & 02\end{array}$ & 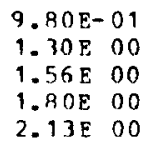 \\
\hline $\begin{array}{l}45 \\
46 \\
47 \\
48 \\
49\end{array}$ & $\begin{array}{l}\mathrm{RH} \\
\mathrm{PL} \\
\mathrm{AG} \\
\mathrm{CL} \\
\mathrm{IN}\end{array}$ & & $\begin{array}{ll}8.20 E & C 1 \\
9.41 E & 01 \\
1.07 E & C 2 \\
1.25 E & C 2 \\
1.44 E & 02\end{array}$ & & 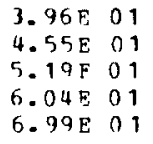 & $\begin{array}{ll}9.45 \mathrm{~F} & 02 \\
1.04 \mathrm{E} & 03 \\
1.13 \mathrm{E} & 03 \\
1.23 \mathrm{E} & 03 \\
1.34 \mathrm{~F} & 03\end{array}$ & $\begin{array}{ll}4.80 \mathrm{E} & 00 \\
5.53 \mathrm{~F} & 00 \\
6.29 \mathrm{E} & 00 \\
7.33 \mathrm{E} & 00 \\
8.50 \mathrm{E} & 00\end{array}$ & $\begin{array}{ll}4.87 \mathrm{E} & 02 \\
5.35 \mathrm{~F} & 02 \\
5.86 \mathrm{~F} & 02 \\
6.40 \mathrm{~F} & 02 \\
6.96 \mathrm{E} & 02\end{array}$ & $\begin{array}{ll}2.43 \mathrm{E} & 00 \\
2.80 \mathrm{E} & 00 \\
3.18 \mathrm{~F} & 00 \\
3.71 \mathrm{E} & 00 \\
4.31 \mathrm{E} & 00\end{array}$ \\
\hline $\begin{array}{l}50 \\
51 \\
52 \\
53 \\
54\end{array}$ & $\begin{array}{l}S N \\
S F \\
T E \\
I \\
X F\end{array}$ & & $\begin{array}{ll}1.32 \mathrm{E} & 02 \\
1.55 \mathrm{~F} & \mathrm{C} 2 \\
1.80 \mathrm{E} & \mathrm{C} 2 \\
2.07 \mathrm{E} & \mathrm{C} 2 \\
2.40 \mathrm{~F} & \mathrm{C} 2\end{array}$ & & 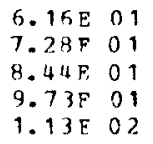 & $\begin{array}{ll}1.44 \mathrm{E} & 03 \\
1.55 F & 03 \\
1.67 F & 03 \\
1.79 \mathrm{E} & 03 \\
1.92 F & 03\end{array}$ & $\begin{array}{ll}6.72 E & 00 \\
7.90 E & 00 \\
9.24 E & 00 \\
1.06 E & 01 \\
1.24 E & 01\end{array}$ & $\begin{array}{ll}7.55 \mathrm{E} & 02 \\
8.16 \mathrm{E} & 02 \\
8.78 \mathrm{E} & 02 \\
9.44 \mathrm{E} & 02 \\
1.01 \mathrm{E} & 03\end{array}$ & $\begin{array}{ll}3.29 \mathrm{E} & 00 \\
3.96 \mathrm{E} & 00 \\
4.52 \mathrm{E} & 00 \\
5.20 \mathrm{E} & 00 \\
6.07 \mathrm{~F} & 00\end{array}$ \\
\hline $\begin{array}{l}55 \\
56 \\
57 \\
58 \\
59\end{array}$ & $\begin{array}{l}\text { CS } \\
\text { BA } \\
\text { LA } \\
\text { CE } \\
\text { PH }\end{array}$ & & $\begin{array}{ll}2.76 \mathrm{~F} & \mathrm{C2} \\
3.15 \mathrm{~F} & \mathrm{C} 2 \\
3.64 \mathrm{E} & \mathrm{C2} \\
4.19 \mathrm{~F} & \mathrm{C} 2 \\
4.77 \mathrm{~F} & \mathrm{C} 2\end{array}$ & & $\begin{array}{ll}1.30 \mathrm{E} & 02 \\
1.49 \mathrm{E} & 02 \\
1.73 \mathrm{E} & 0 ? \\
1.99 \mathrm{~F} & 02 \\
2.27 \mathrm{E} & 02\end{array}$ & $\begin{array}{ll}2.05 \mathrm{~F} & 03 \\
2.19 \mathrm{~F} & 03 \\
2.31 \mathrm{~F} & 03 \\
2.45 \mathrm{E} & 03 \\
2.60 \mathrm{E} & 03\end{array}$ & $\begin{array}{ll}1.44 \mathrm{E} & 01 \\
1.65 \mathrm{E} & 01 \\
1.93 \mathrm{~F} & 01 \\
2.23 \mathrm{E} & 01 \\
2.55 \mathrm{E} & 01\end{array}$ & 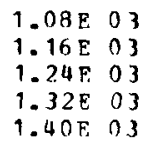 & $\begin{array}{ll}7.04 \mathrm{E} & 00 \\
9.09 \mathrm{E} & 00 \\
9.50 \mathrm{~F} & 00 \\
1.09 \mathrm{E} & 01 \\
1.25 \mathrm{E} & 01\end{array}$ \\
\hline $\begin{array}{l}60 \\
61 \\
62 \\
63 \\
64\end{array}$ & $\begin{array}{l}N E \\
\text { PM } \\
S M \\
F U \\
G E\end{array}$ & & $\begin{array}{ll}5.46 \mathrm{E} & 02 \\
6.16 \mathrm{E} & \mathrm{C} 2 \\
6.91 \mathrm{E} & \mathrm{C2} \\
7.80 \mathrm{E} & \mathrm{C} 2 \\
8.72 \mathrm{E} & \mathrm{C} 2\end{array}$ & & $\begin{array}{ll}2.61 \mathrm{~F} & 02 \\
2.95 \mathrm{~F} & 02 \\
3.32 \mathrm{~F} & 02 \\
3.75 \mathrm{E} & 02 \\
4.20 \mathrm{~F} & 02\end{array}$ & $\begin{array}{ll}2.75 \mathrm{E} & 03 \\
2.91 \mathrm{~F} & 03\end{array}$ & $\begin{array}{ll}2.95 \mathrm{E} & 01 \\
3.35 \mathrm{E} & 01 \\
3.79 \mathrm{~F} & 01 \\
4.29 \mathrm{E} & 01 \\
4.84 \mathrm{~F} & 01\end{array}$ & $\begin{array}{ll}1.49 \mathrm{E} & 03 \\
1.57 \mathrm{E} & 03 \\
1.66 \mathrm{~F} & 03 \\
1.75 \mathrm{~F} & 03 \\
1.84 \mathrm{E} & 03\end{array}$ & $\begin{array}{ll}1.45 \mathrm{E} & 01 \\
1.65 \mathrm{E} & 01 \\
1.87 \mathrm{E} & 01 \\
2.12 \mathrm{E} & 01 \\
2.38 \mathrm{E} & 01\end{array}$ \\
\hline $\begin{array}{l}65 \\
66 \\
67 \\
69 \\
69\end{array}$ & $\begin{array}{l}\text { TH } \\
\text { DY } \\
\text { HO } \\
\text { EN } \\
\text { TM }\end{array}$ & & $\begin{array}{ll}9.92 F & C 2 \\
1.11 E & C 3 \\
1.23 F & 03 \\
1.37 E & 03 \\
1.51 F & C 3\end{array}$ & & 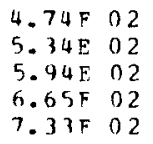 & & $\begin{array}{ll}5.47 \mathrm{~F} & 01 \\
6.19 \mathrm{E} & 01 \\
6.91 \mathrm{E} & 01 \\
7.78 \mathrm{E} & 01 \\
8.58 \mathrm{E} & 01\end{array}$ & $\begin{array}{ll}1.948 & 03 \\
2.04 \mathrm{E} & 03 \\
2.15 \mathrm{E} & 03 \\
2.27 \mathrm{E} & 03\end{array}$ & $\begin{array}{cc}2.70 \mathrm{E} & 01 \\
3.06 \mathrm{E} & 01 \\
3.42 \mathrm{E} & 01 \\
3.85 \mathrm{E} & 01 \\
4.25 \mathrm{E} & 01\end{array}$ \\
\hline
\end{tabular}


Table 12 (continued)

\begin{tabular}{|c|c|c|c|c|c|c|c|c|c|c|}
\hline \multirow{2}{*}{\multicolumn{2}{|c|}{$z$}} & \multicolumn{2}{|c|}{$\operatorname{Mo} K \alpha \equiv 17.44 \mathrm{keV}$} & \multicolumn{2}{|c|}{$\mathrm{Ag} K \equiv 22.5 \mathrm{keV}$} & \multicolumn{2}{|c|}{ Dy $K \equiv 47.0 \mathrm{keV}$} & \multicolumn{3}{|c|}{${ }^{241} \mathrm{Am} \cong 59.54 \mathrm{keV}$} \\
\hline & & $K \alpha$ & $L \alpha$ & $K \alpha$ & $L \alpha$ & $K \alpha$ & $L \alpha$ & $K \alpha$ & $L \alpha$ & \\
\hline $\begin{array}{l}70 \\
71 \\
72 \\
73 \\
74\end{array}$ & $\begin{array}{l}\text { Y E } \\
\text { LU } \\
\text { HF } \\
\text { TA } \\
\text { W }\end{array}$ & & $\begin{array}{ll}1.67 \mathrm{E} & 03 \\
1.83 \mathrm{E} & 03 \\
2.02 \mathrm{E} & \mathrm{C3} \\
2.24 \mathrm{E} & 03 \\
2.46 \mathrm{E} & 03\end{array}$ & & 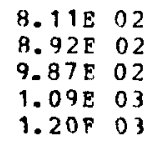 & & $\begin{array}{ll}9.54 \mathrm{E} & 01 \\
1.05 \mathrm{E} & 02 \\
1.17 \mathrm{E} & 02 \\
1.30 \mathrm{E} & 02 \\
1.43 \mathrm{E} & 02\end{array}$ & & $\begin{array}{l}4.73 \mathrm{E} \\
5.20 \mathrm{E} \\
5.78 \mathrm{E} \\
6.44 \mathrm{E} \\
7.13 \mathrm{E}\end{array}$ & $\begin{array}{l}01 \\
01 \\
01 \\
01 \\
01\end{array}$ \\
\hline $\begin{array}{l}75 \\
76 \\
77 \\
78 \\
79\end{array}$ & $\begin{array}{l}\text { RF } \\
\text { OS } \\
\text { IR } \\
\text { PT } \\
\text { AU }\end{array}$ & & 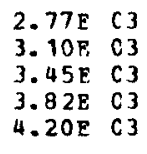 & & 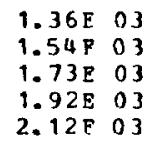 & & $\begin{array}{ll}1.66 E & 02 \\
1.92 E & 02 \\
2.21 E & 02 \\
2.50 E & 02 \\
2.79 E & 02\end{array}$ & & $\begin{array}{l}8.32 \mathrm{E} \\
9.71 \mathrm{E} \\
1.12 \mathrm{E} \\
1.28 \mathrm{E} \\
1.43 \mathrm{P}\end{array}$ & $\begin{array}{l}01 \\
01 \\
02 \\
02 \\
02\end{array}$ \\
\hline $\begin{array}{l}80 \\
81 \\
82 \\
83 \\
84\end{array}$ & $\begin{array}{l}\text { HG } \\
\text { TL } \\
\text { PE } \\
\text { BI } \\
\text { PO }\end{array}$ & & 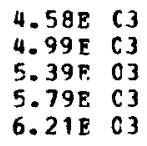 & & 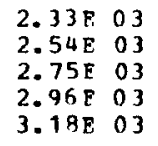 & & $\begin{array}{ll}3.09 \mathrm{~F} & 02 \\
3.39 \mathrm{E} & 02 \\
3.70 \mathrm{E} & 02 \\
4.00 \mathrm{~F} & 02 \\
4.31 \mathrm{E} & 02\end{array}$ & & $\begin{array}{l}1.60 \mathrm{E} \\
1.75 \mathrm{E} \\
1.92 \mathrm{E} \\
2.07 \mathrm{E} \\
2.24 \mathrm{E}\end{array}$ & $\begin{array}{l}02 \\
02 \\
02 \\
02 \\
02\end{array}$ \\
\hline $\begin{array}{l}85 \\
86 \\
87 \\
88 \\
89\end{array}$ & $\begin{array}{l}A T \\
R N \\
F R \\
R A \\
A C\end{array}$ & & $\begin{array}{ll}5.51 E & C 3 \\
5.66 \mathrm{~F} & 03 \\
5.87 E & 03 \\
6.30 \mathrm{E} & 03 \\
6.75 E & C 3\end{array}$ & & 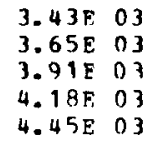 & & $\begin{array}{ll}4.68 \mathrm{E} & 02 \\
4.99 \mathrm{E} & 02 \\
5.37 \mathrm{E} & 02 \\
5.76 \mathrm{E} & 02 \\
6.17 \mathrm{E} & 02\end{array}$ & & $\begin{array}{l}2.43 \mathrm{E} \\
2.60 \mathrm{E} \\
2.79 \mathrm{E} \\
3.00 \mathrm{E} \\
3.22 \mathrm{E}\end{array}$ & $\begin{array}{l}02 \\
02 \\
02 \\
02 \\
02\end{array}$ \\
\hline $\begin{array}{l}90 \\
91 \\
92 \\
93 \\
94\end{array}$ & $\begin{array}{l}T H \\
P A \\
U \\
N P \\
P U\end{array}$ & & 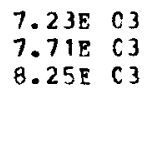 & & 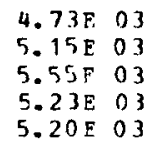 & & $\begin{array}{ll}6.57 \mathrm{E} & 02 \\
7.20 \mathrm{E} & 02 \\
7.79 \mathrm{E} & 02 \\
8.43 \mathrm{E} & 02 \\
8.95 \mathrm{E} & 02\end{array}$ & & $\begin{array}{l}3.438 \\
3.76 \mathrm{E} \\
4.07 \mathrm{E} \\
4.42 \mathrm{E} \\
4.69 \mathrm{E}\end{array}$ & $\begin{array}{l}02 \\
02 \\
02 \\
02 \\
02\end{array}$ \\
\hline $\begin{array}{l}95 \\
96 \\
97 \\
98 \\
99\end{array}$ & $\begin{array}{l}A M \\
C M \\
B K \\
C F \\
E S\end{array}$ & & & & 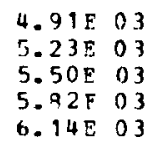 & & $\begin{array}{ll}9.51 \mathrm{E} & 02 \\
1.01 \mathrm{E} & 03 \\
1.06 \mathrm{E} & 03 \\
1.12 \mathrm{E} & 03 \\
1.18 \mathrm{E} & 03\end{array}$ & & $\begin{array}{l}4.99 \mathrm{E} \\
5.30 \mathrm{E} \\
5.58 \mathrm{~F} \\
5.90 \mathrm{E} \\
6.23 \mathrm{E}\end{array}$ & $\begin{array}{l}02 \\
02 \\
02 \\
02 \\
02\end{array}$ \\
\hline $\begin{array}{l}100 \\
101\end{array}$ & $\begin{array}{l}P M \\
M L\end{array}$ & & & & 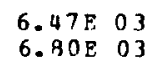 & & $\begin{array}{ll}1.24 \mathrm{E} & 03 \\
1.30 \mathrm{E} & 03\end{array}$ & & $\begin{array}{l}6.53 \mathrm{E} \\
6.86 \mathrm{~F}\end{array}$ & $\begin{array}{l}02 \\
02\end{array}$ \\
\hline
\end{tabular}


ORNL-DWG 77-21185

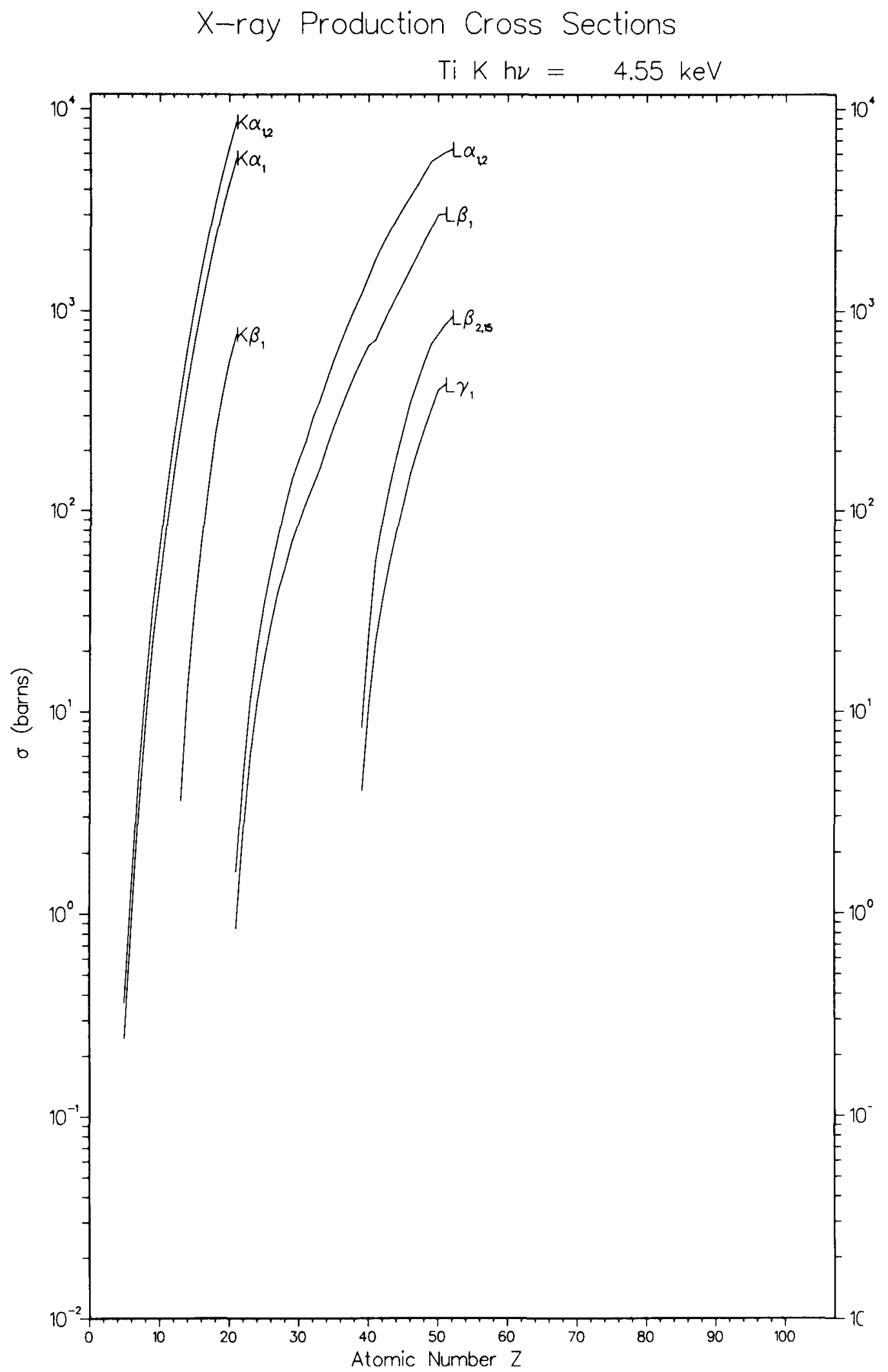

Fig. 26 


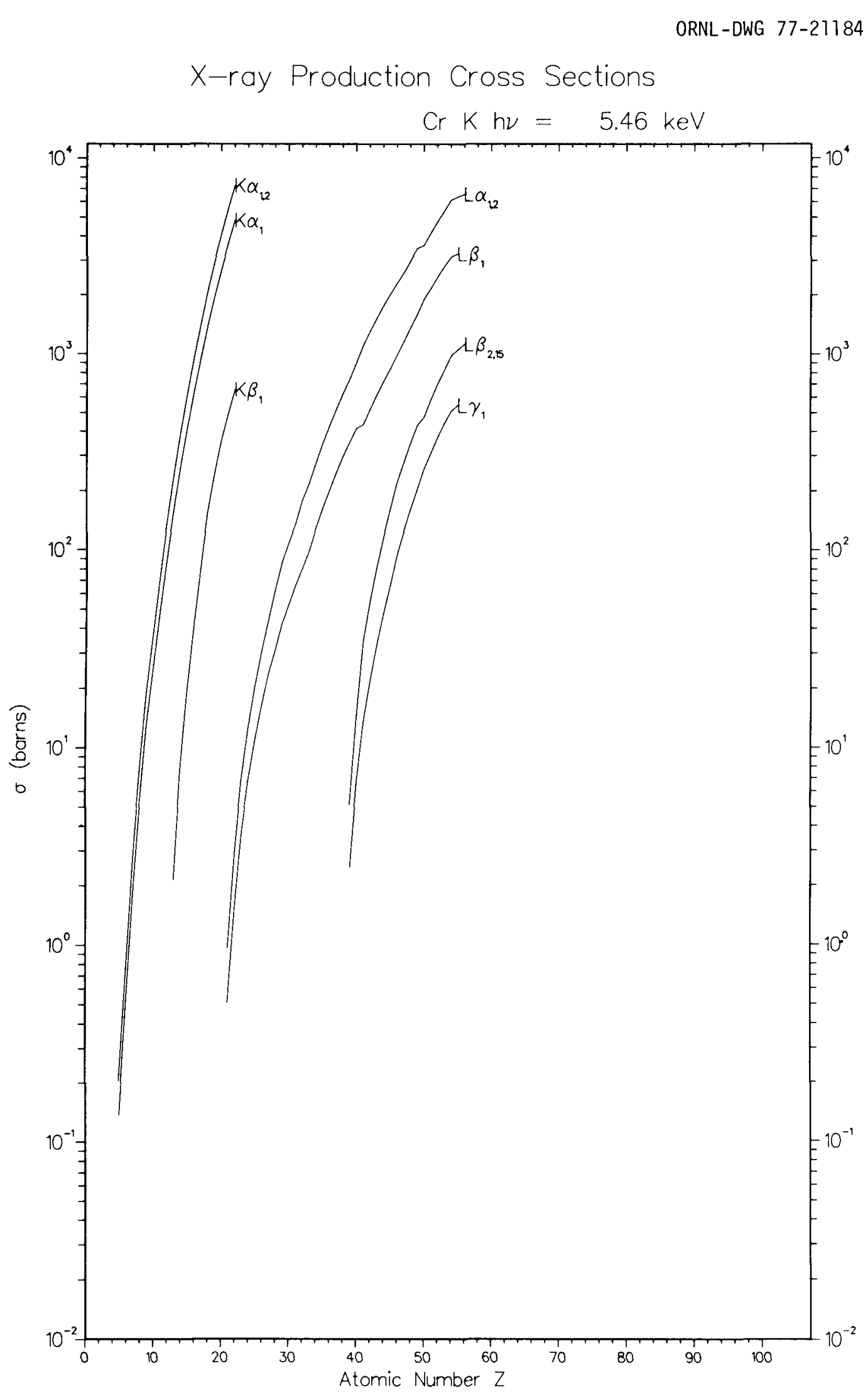

Fig. 27 


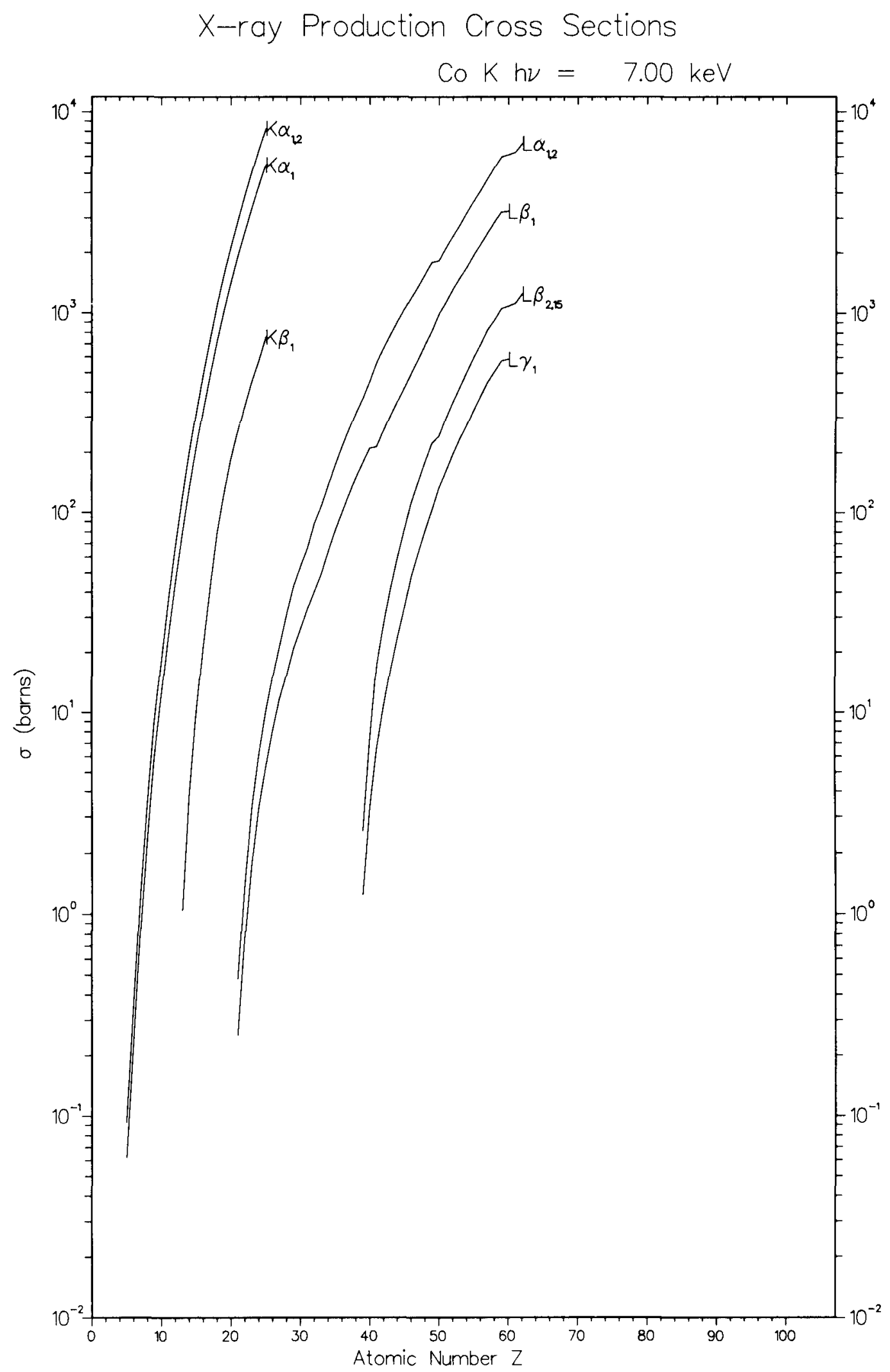

Fig. 28 
ORNL-DWG 77-21182

$X$-ray Production Cross Sections

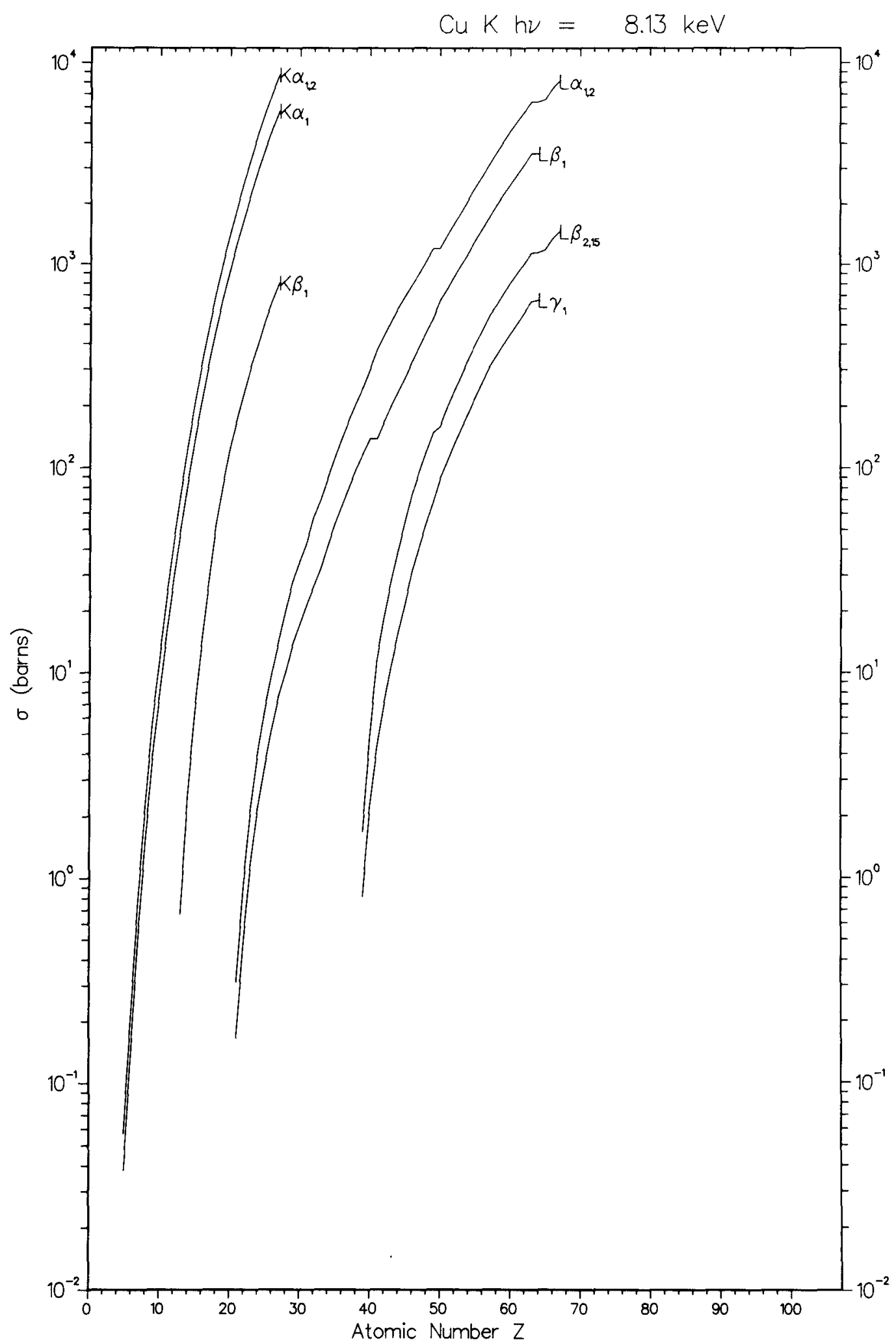

Fig. 29 
ORNL-DWG 77-21181

$X$-ray Production Cross Sections

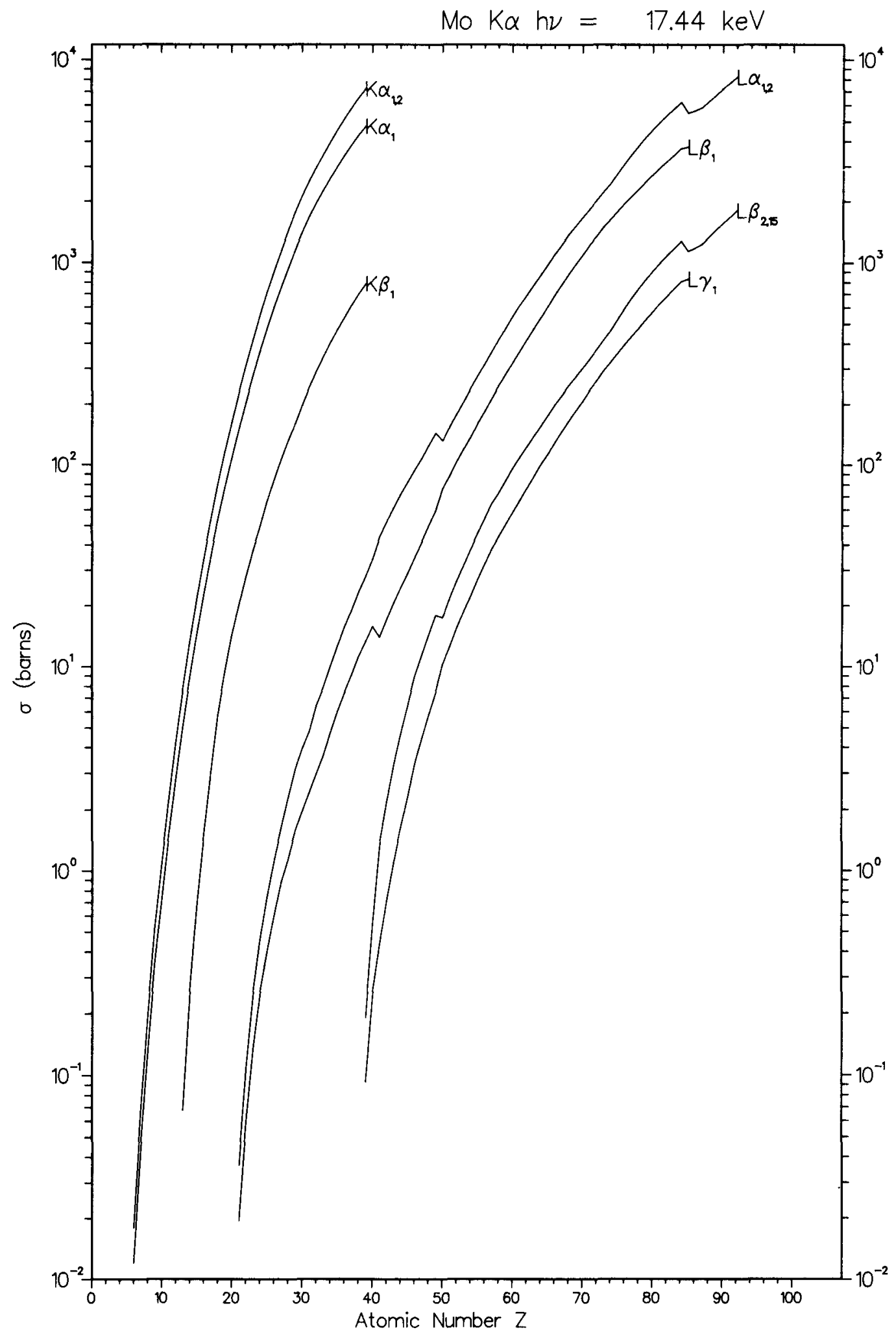

Fig. 30 
ORNL-DWG 77-21180

$X$-ray Production Cross Sections

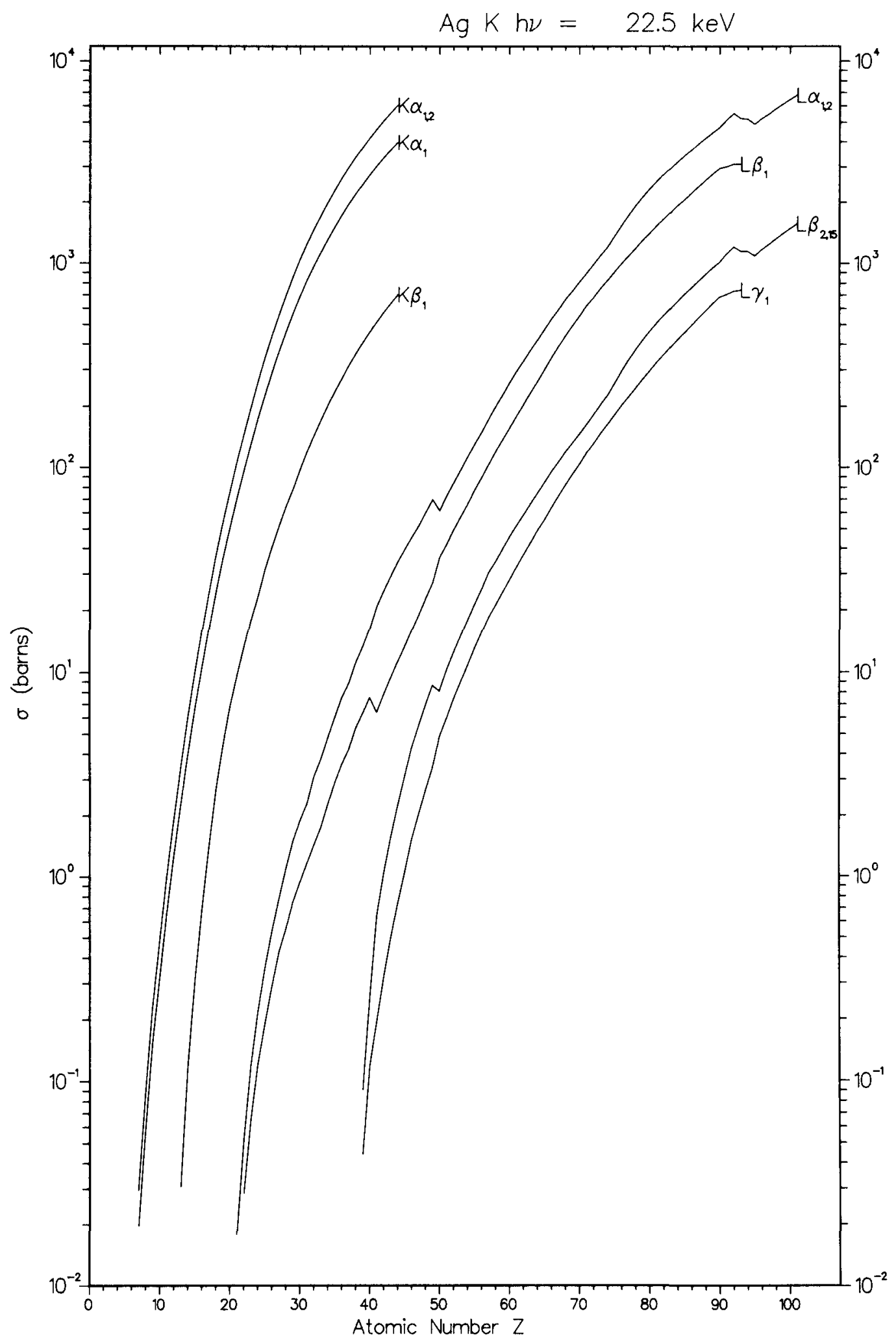

Fig. 31 
115

ORNL-DWG 77-21179

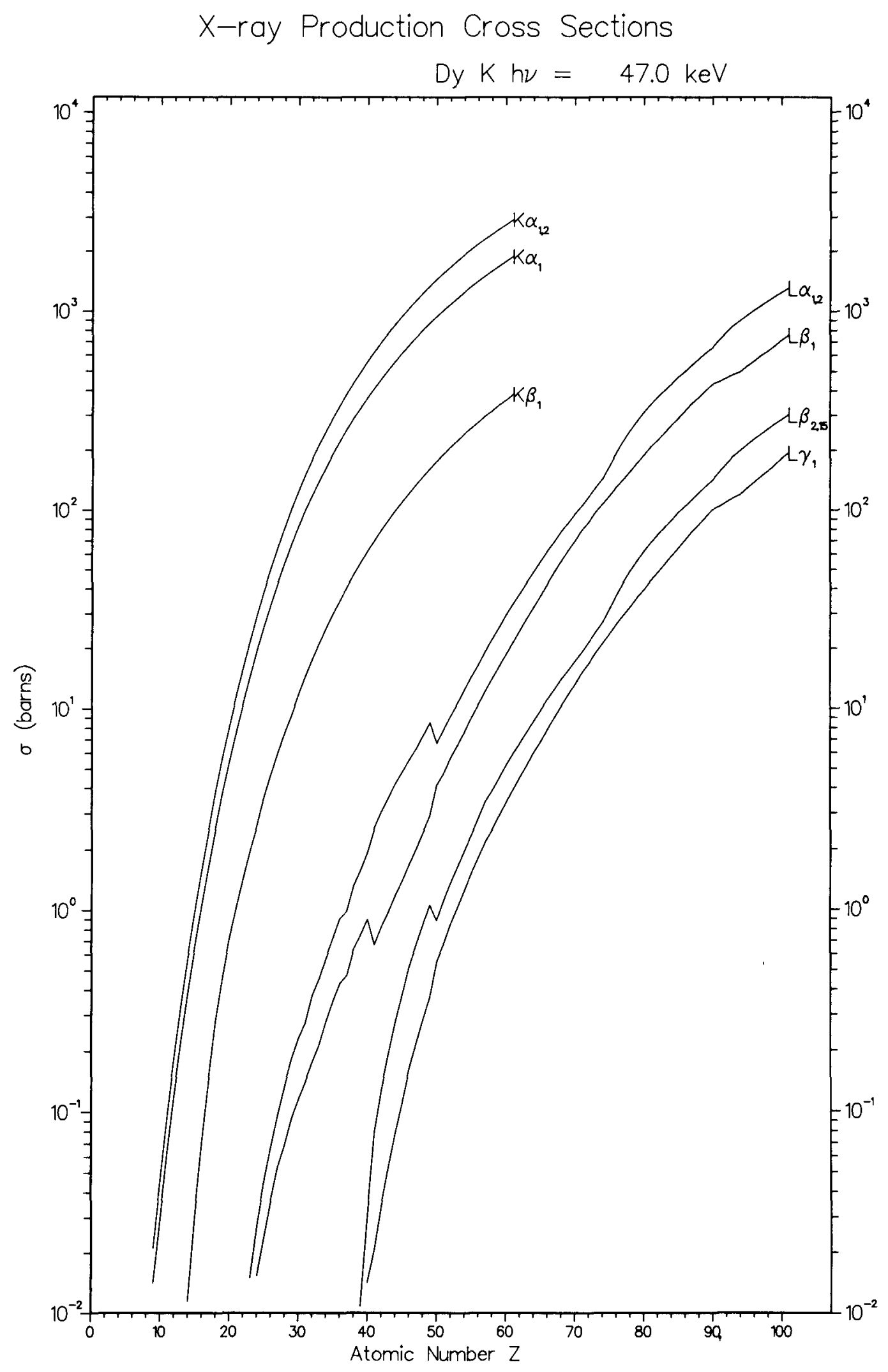

Fig. 32 
ORNL-DWG 77-21178

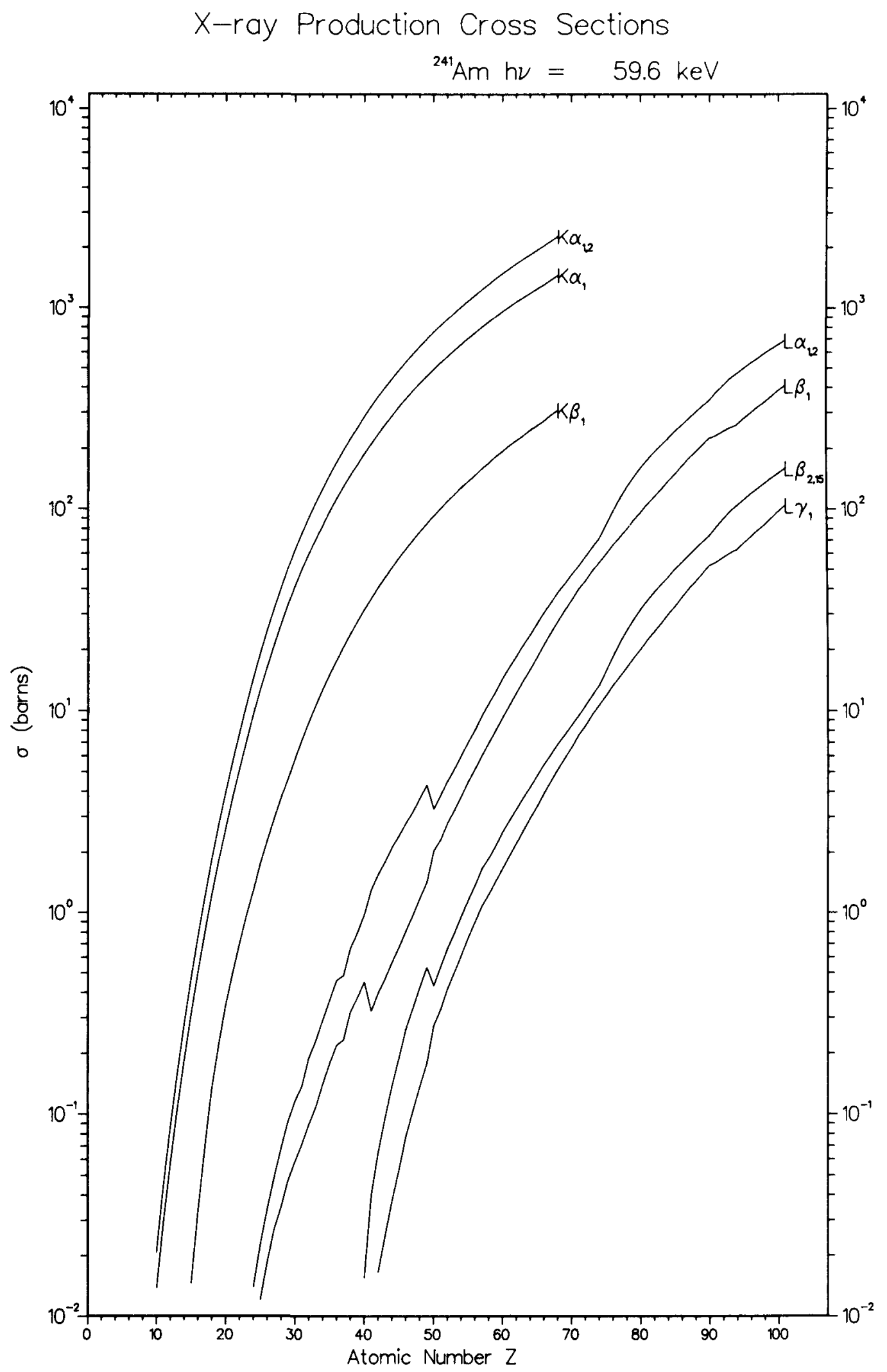

F1g. 33 
4. SUPPLEMENT 
•

○ 
For the benefit of readers who are interested in background and auxiliary information, the following supplementary material is presented (Tables 13 through 22):

- Fractional $K$ - and $L$-shell radiative rates,

- Fluorescence and Coster-Kronig yields,

- Fluorescence and Coster-Kronig yields in condensed matter,

- Fractional fluorescence and hole-transfer yields,

- Effective fluorescence yields,

- Average L-shell fluorescence yield,

- Total L-shell fluorescence cross sections,

- L-subshell photoionization cross section ratios,

- Conversion factors.

Although tables are self-explanatory, the following comments may be usefu1.

1. The total L-shell fluorescence cross section (Table 20) and average $L$-shell fluorescence yield (Table 19) are insensitive to the partition of the $L$ ionization cross section into its subshell components because the effective fluorescence yields $\nu_{1}, \nu_{2}$, and $\omega_{3}$ (Tables 15 and 18) are nearly the same numerically.

2. Because photoionization cross section ratios for the $L$ subshells (Table 21) vary rapidly with photon energy, jump ratios can be applied only in a very narrow energy range above an absorption edge.

3. Fractional fluorescence and hole-transfer yields (Table 17) allow ready calculation of the $x$-ray production cross sections for ionization by electrons, protons, and other particles or modes. For example, the $L \alpha_{1,2} x$-ray production cross section under proton bombardment is given by

$$
\sigma_{L \alpha_{1,2}}^{P}=\omega_{3} F_{L \alpha_{1,2}}\left(\sigma_{L_{3}}^{P}+\sigma_{L_{2}}^{P} f_{2,3}+\sigma_{L_{1}}^{P} F_{1,3}\right) \text {, }
$$

where $\sigma_{L_{i}}^{P}$ designates the partial cross sections for proton ionization. 
Table 13 Fractional radiative rates $F_{y}$ for $K$ shell

Calculated from Eq 2 with radiative rates $S$ from refs $4-8$ for example $F_{K \alpha_{1}}=S_{K \alpha_{1}} / S_{K R}$

\begin{tabular}{|c|c|c|c|c|c|c|c|c|c|c|c|}
\hline$Z$ & & $\begin{array}{l}K L_{3} \\
K \alpha_{1}\end{array}$ & $\begin{array}{l}K L_{2} 3 \\
K \alpha_{1}\end{array}$ & $\begin{array}{c}K M_{3} \\
K \beta_{1}\end{array}$ & $\begin{array}{c}K M_{23} \\
K \beta_{1}\end{array}$ & $Z$ & & $\begin{array}{l}K L_{3} \\
K \alpha_{1}\end{array}$ & $\begin{array}{l}K L_{23} \\
K \alpha_{1}\end{array}$ & $\begin{array}{c}K M_{3} \\
K \beta_{1}\end{array}$ & $\begin{array}{c}K M_{2} 3 \\
K \beta_{13}\end{array}$ \\
\hline $\begin{array}{l}5 \\
6 \\
7 \\
8 \\
9\end{array}$ & $\begin{array}{l}\mathrm{B} \\
\mathrm{C} \\
\mathrm{N} \\
\mathrm{C} \\
\mathrm{F}\end{array}$ & $\begin{array}{l}0.6667 \\
0.6580 \\
0.6650 \\
0.6659 \\
0.6659\end{array}$ & $\begin{array}{l}1.0000 \\
1.0000 \\
1.0000 \\
1.0000 \\
1.0000\end{array}$ & & & $\begin{array}{l}55 \\
56 \\
57 \\
58 \\
59\end{array}$ & $\begin{array}{l}\text { CS } \\
\text { BA } \\
\text { LA } \\
\text { CE } \\
\text { PR }\end{array}$ & $\begin{array}{l}0.5235 \\
0.5213 \\
0.5200 \\
0.5187 \\
0.5173\end{array}$ & $\begin{array}{l}0.8067 \\
0.8043 \\
0.8030 \\
0.8018 \\
0.8006\end{array}$ & $\begin{array}{l}0.102 \\
0.102 \\
0.103 \\
0.103 \\
0.104\end{array}$ & $\begin{array}{l}0.1545 \\
0.1530 \\
0.1557 \\
0.15+, 4 \\
0.1572\end{array}$ \\
\hline $\begin{array}{l}10 \\
11 \\
12 \\
13 \\
14\end{array}$ & $\begin{array}{l}N E \\
N A \\
M G \\
A L \\
S I\end{array}$ & $\begin{array}{l}0.6654 \\
0.6661 \\
0.6645 \\
0.6564 \\
0.6460\end{array}$ & $\begin{array}{l}1.0000 \\
1.0000 \\
1.0000 \\
0.9868 \\
0.9714\end{array}$ & $\begin{array}{l}0.0088 \\
0.0190\end{array}$ & $\begin{array}{l}0.0132 \\
0.0286\end{array}$ & $\begin{array}{l}60 \\
61 \\
62 \\
63 \\
64\end{array}$ & $\begin{array}{l}\text { ND } \\
\text { PM } \\
\text { SM } \\
\text { En } \\
\text { SD }\end{array}$ & $\begin{array}{l}0.5163 \\
0.5149 \\
0.5138 \\
0.5127 \\
0.5113\end{array}$ & $\begin{array}{l}0.7997 \\
0.7994 \\
0.7977 \\
0.7959 \\
0.7955\end{array}$ & $\begin{array}{l}0.104 \\
0.105 \\
0.105 \\
0.105 \\
0.176\end{array}$ & $\begin{array}{l}0.1579 \\
0.1535 \\
0.1571 \\
0.1597 \\
0.1501\end{array}$ \\
\hline $\begin{array}{l}15 \\
16 \\
17 \\
19 \\
19\end{array}$ & $\begin{array}{l}\mathrm{P} \\
S \\
\mathrm{CL} \\
\mathrm{AR} \\
\mathrm{K}\end{array}$ & $\begin{array}{l}0.6346 \\
0.6232 \\
0.6115 \\
0.5993 \\
0.5925\end{array}$ & $\begin{array}{l}0.9549 \\
0.9387 \\
0.9706 \\
0.9019 \\
0.9920\end{array}$ & $\begin{array}{l}0.0799 \\
0.0411 \\
0.0528 \\
0.0653 \\
0.0719\end{array}$ & $\begin{array}{l}0.0451 \\
0.0618 \\
0.0794 \\
0.0992 \\
0.1099\end{array}$ & $\begin{array}{l}65 \\
66 \\
67 \\
68 \\
69\end{array}$ & $\begin{array}{l}\text { TB } \\
D Y \\
\text { FO } \\
P R \\
T M\end{array}$ & $\begin{array}{l}0.5105 \\
0.5092 \\
0.5081 \\
0.5072 \\
0.5059\end{array}$ & $\begin{array}{l}0.7952 \\
0.7939 \\
0.7932 \\
0.7979 \\
0.7970\end{array}$ & $\begin{array}{l}0.106 \\
0.106 \\
0.107 \\
0.107 \\
0.107\end{array}$ & $\begin{array}{l}0.1623 \\
0.1613 \\
0.1619 \\
0.16>4 \\
0.16>8\end{array}$ \\
\hline $\begin{array}{l}20 \\
21 \\
22 \\
23 \\
24\end{array}$ & $\begin{array}{l}C A \\
S C \\
T I \\
V \\
C A\end{array}$ & $\begin{array}{l}0.5868 \\
0.5850 \\
0.5842 \\
0.5833 \\
0.5845\end{array}$ & $\begin{array}{l}0.3838 \\
0.8919 \\
0.8907 \\
0.8797 \\
0.8921\end{array}$ & $\begin{array}{l}0.0773 \\
0.0783 \\
0.0793 \\
0.0799 \\
0.0783\end{array}$ & $\begin{array}{l}0.1163 \\
0.1179 \\
0.1194 \\
0.1202 \\
0.1190\end{array}$ & $\begin{array}{l}70 \\
71 \\
72 \\
73 \\
74\end{array}$ & $\begin{array}{l}Y B \\
\text { LU } \\
H F \\
T A \\
W\end{array}$ & $\begin{array}{l}0.5050 \\
0.5032 \\
0.5024 \\
0.5011 \\
0.4998\end{array}$ & $\begin{array}{l}0.7915 \\
0.7999 \\
0.7895 \\
0.7995 \\
0.7875\end{array}$ & $\begin{array}{l}0.109 \\
2.128 \\
0.108 \\
0.109 \\
1.109\end{array}$ & $\begin{array}{l}0.1632 \\
0.1635 \\
0.1639 \\
0.1642 \\
0.1545\end{array}$ \\
\hline $\begin{array}{l}25 \\
26 \\
27 \\
29 \\
29\end{array}$ & $\begin{array}{l}M N \\
F E \\
C O \\
N I \\
C U\end{array}$ & $\begin{array}{l}0.5317 \\
0.5811 \\
0.5805 \\
0.5709 \\
0.5807\end{array}$ & $\begin{array}{l}0.8783 \\
0.8779 \\
0.8775 \\
0.8771 \\
0.8798\end{array}$ & $\begin{array}{l}0.0807 \\
0.0810 \\
0.0812 \\
0.0914 \\
0.0802\end{array}$ & $\begin{array}{l}0.1217 \\
0.1221 \\
0.1220 \\
0.1229 \\
0.1212\end{array}$ & $\begin{array}{l}75 \\
76 \\
77 \\
78 \\
79\end{array}$ & $\begin{array}{l}\mathrm{PF} \\
\mathrm{OS} \\
\mathrm{TH} \\
\mathrm{PT} \\
\mathrm{AII}\end{array}$ & $\begin{array}{l}0.4970 \\
0.4967 \\
0.4957 \\
0.4945 \\
0.4932\end{array}$ & $\begin{array}{l}0.7849 \\
0.7943 \\
0.7340 \\
0.7839 \\
0.7830\end{array}$ & $\begin{array}{l}0.108 \\
0.109 \\
0.109 \\
0.109 \\
0.109\end{array}$ & $\begin{array}{l}0.1647 \\
0.1549 \\
0.1650 \\
0.1653 \\
0.1657\end{array}$ \\
\hline $\begin{array}{l}30 \\
31 \\
32 \\
33 \\
34\end{array}$ & $\begin{array}{l}2 N \\
G A \\
G F \\
A S \\
S E\end{array}$ & $\begin{array}{l}0.5788 \\
0.5767 \\
0.5738 \\
0.5709 \\
0.5675\end{array}$ & $\begin{array}{l}0.8764 \\
0.8712 \\
0.8693 \\
0.8651 \\
0.8603\end{array}$ & $\begin{array}{l}0.0813 \\
0.0832 \\
0.0847 \\
0.0860 \\
0.0875\end{array}$ & $\begin{array}{l}0.1236 \\
0.1257 \\
0.1279 \\
0.1300 \\
0.1323\end{array}$ & $\begin{array}{l}80 \\
81 \\
82 \\
83 \\
84\end{array}$ & $\begin{array}{l}\mathrm{HG} \\
\mathrm{TL} \\
\mathrm{PB} \\
\mathrm{BI} \\
\text { PO }\end{array}$ & $\begin{array}{l}0.4918 \\
0.4965 \\
0.4890 \\
0.4877 \\
0.4865\end{array}$ & $\begin{array}{l}0.7370 \\
0.7917 \\
0.7800 \\
0.7791 \\
0.7786\end{array}$ & $\begin{array}{l}0.109 \\
3.109 \\
0.110 \\
0.110 \\
0.110\end{array}$ & $\begin{array}{l}0.1638 \\
0.1551 \\
0.1021 \\
0.1622 \\
0.1662\end{array}$ \\
\hline $\begin{array}{l}35 \\
36 \\
37 \\
39 \\
39\end{array}$ & $\begin{array}{l}E R \\
K R \\
\text { EB } \\
S R \\
Y\end{array}$ & $\begin{array}{l}0.5638 \\
0.5615 \\
0.5587 \\
0.5559 \\
0.5510\end{array}$ & $\begin{array}{l}0.8550 \\
0.8527 \\
0.8489 \\
0.8452 \\
0.8381\end{array}$ & $\begin{array}{l}0.0980 \\
0.0985 \\
0.0895 \\
0.0905 \\
0.0915\end{array}$ & $\begin{array}{l}0.1330 \\
0.1337 \\
0.1354 \\
0.1369 \\
0.1385\end{array}$ & $\begin{array}{l}95 \\
86 \\
87 \\
88 \\
39\end{array}$ & $\begin{array}{l}A T \\
R N \\
F P \\
R A \\
A C\end{array}$ & $\begin{array}{l}0.4845 \\
0.4829 \\
0.4814 \\
0.4801 \\
0.4787\end{array}$ & $\begin{array}{l}0.7769 \\
0.7756 \\
0.7745 \\
0.7779 \\
0.7731\end{array}$ & $\begin{array}{l}0.110 \\
0.110 \\
0.110 \\
0.110 \\
3.110\end{array}$ & $\begin{array}{l}0.1653 \\
0.1603 \\
0.1654 \\
0.1655 \\
0.1653\end{array}$ \\
\hline $\begin{array}{l}40 \\
49 \\
42 \\
43 \\
44\end{array}$ & $\begin{array}{l}Z R \\
N R \\
M C \\
T C \\
\text { Til }\end{array}$ & $\begin{array}{l}0.5513 \\
0.5495 \\
0.5474 \\
0.5451 \\
0.5430\end{array}$ & $\begin{array}{l}0.8394 \\
0.4369 \\
0.8347 \\
0.8313 \\
0.8287\end{array}$ & $\begin{array}{l}0.0920 \\
0.0935 \\
0.0945 \\
0.0353 \\
0.0961\end{array}$ & $\begin{array}{l}0.1401 \\
2.1415 \\
2.1430 \\
0.1443 \\
0.1455\end{array}$ & $\begin{array}{l}90 \\
91 \\
92 \\
93 \\
94\end{array}$ & $\begin{array}{l}\mathrm{TH} \\
\mathrm{PA} \\
\text { If } \\
\mathrm{NP} \\
\mathrm{PU}\end{array}$ & $\begin{array}{l}0.4771 \\
0.476 .0 \\
0.4744 \\
0.4730 \\
0.4716\end{array}$ & $\begin{array}{l}0.7721 \\
0.7717 \\
0.7707 \\
0.7707 \\
0.7695\end{array}$ & $\begin{array}{r}0.110 \\
0.111 \\
0.111 \\
0.111 \\
0.111\end{array}$ & $\begin{array}{l}1.1656 \\
0.1659 \\
0.1073 \\
0.1673 \\
0.16 .73\end{array}$ \\
\hline $\begin{array}{l}45 \\
46 \\
47 \\
48 \\
49\end{array}$ & $\begin{array}{l}\text { FH } \\
\text { ED } \\
A G \\
C D \\
\text { IN }\end{array}$ & $\begin{array}{l}0.5422 \\
0.5403 \\
0.5396 \\
0.5362 \\
0.5348\end{array}$ & $\begin{array}{l}0.8277 \\
0.9261 \\
0.9244 \\
0.8214 \\
0.9194\end{array}$ & $\begin{array}{l}0.0969 \\
0.0973 \\
0.0997 \\
0.0989 \\
0.0994\end{array}$ & $\begin{array}{l}0.1466 \\
0.1477 \\
0.1487 \\
0.1496 \\
0.1505\end{array}$ & $\begin{array}{l}95 \\
95 \\
77 \\
99 \\
79\end{array}$ & $\begin{array}{l}A M \\
C Y \\
B K \\
C F \\
L S\end{array}$ & $\begin{array}{l}0.4596 \\
0.4697 \\
0.4679 \\
0.4660 \\
0.4544\end{array}$ & $\begin{array}{l}0.7685 \\
0.7691 \\
6.7679 \\
0.7670 \\
0.7653\end{array}$ & $\begin{array}{l}1.111 \\
0.111 \\
0.111 \\
0.111 \\
0.111\end{array}$ & $\begin{array}{l}0.1673 \\
0.1673 \\
0.1174 \\
0.1674 \\
0.1674\end{array}$ \\
\hline $\begin{array}{l}50 \\
51 \\
52 \\
53 \\
54\end{array}$ & $\begin{array}{l}S N \\
S E \\
T F \\
I \\
X=\end{array}$ & $\begin{array}{l}0.5329 \\
0.5169 \\
0.5282 \\
0.5245 \\
0.5 \angle 51\end{array}$ & $\begin{array}{l}0.8177 \\
0.3153 \\
0.8119 \\
0.8091 \\
0.8304\end{array}$ & $\begin{array}{l}0.100 ? \\
0.1707 \\
3.101 \\
0.101 \\
0.10 \%\end{array}$ & $\begin{array}{l}0.1513 \\
0.1521 \\
0.1524 \\
0.1234 \\
0.1540\end{array}$ & $\begin{array}{l}100 \\
101 \\
102 \\
103 \\
104\end{array}$ & $\begin{array}{l}\text { Fi } \\
\times 5 \\
17 \\
\text { 1 }\end{array}$ & $\begin{array}{l}0.4679 \\
0.4615 \\
0.4598 \\
0.4567 \\
0.4569\end{array}$ & $\begin{array}{l}0.7,57 \\
0.7,53 \\
0.7645 \\
6.76,44 \\
0.7639\end{array}$ & $\begin{array}{l}0.112 \\
0.112 \\
0.112 \\
0.112 \\
0.112\end{array}$ & $\begin{array}{l}0.1674 \\
0.1675 \\
0.1675 \\
0.1675 \\
0.1675\end{array}$ \\
\hline
\end{tabular}


Table 14 Fractıonal radiative rates $F_{y}$ for $L$ subshells

Calculated from Eq 2 with radiative rates $S$ from refs 48 for example $F_{L \alpha_{1}}=S_{I \alpha_{1}} / S_{L_{3}} R$ Note $F_{L \alpha_{12}}=1113 F_{L \alpha_{1}}$ independent of atomic number $Z$

\begin{tabular}{|c|c|c|c|c|c|c|c|c|c|c|c|}
\hline$Z$ & 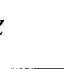 & $I_{1} I_{23}$ & $\begin{array}{c}I_{1} M_{3} \\
I \beta_{3}\end{array}$ & $\begin{array}{c}L_{1} O_{23} \\
L \gamma_{4}\end{array}$ & $\begin{array}{c}I_{2} M_{4} \\
L \beta_{1}\end{array}$ & $\begin{array}{c}L_{2} N_{4} \\
L \gamma_{1}\end{array}$ & $\begin{array}{c}I_{3} M_{5} \\
L \alpha_{1}\end{array}$ & $\begin{array}{l}L_{3} N_{4} 5 \\
L \beta_{2} \quad 15\end{array}$ & $\begin{array}{c}I_{3} M_{1} \\
I \ell\end{array}$ & $Z$ & \\
\hline $\begin{array}{l}10 \\
11 \\
12 \\
13 \\
14\end{array}$ & $\begin{array}{l}\text { NF } \\
\text { NA } \\
\text { MG } \\
\text { A L } \\
\text { SI }\end{array}$ & $\begin{array}{l}1.000 \\
1.000 \\
1.000 \\
0.899 \\
0.672\end{array}$ & $\begin{array}{l}0.0663 \\
0.2160\end{array}$ & & & & & & $\begin{array}{l}1.000 \\
1.000 \\
1.000 \\
1.000\end{array}$ & $\begin{array}{l}10 \\
11 \\
12 \\
13 \\
14\end{array}$ & $\begin{array}{l}N E \\
N A \\
M G \\
A L \\
S I\end{array}$ \\
\hline $\begin{array}{l}15 \\
16 \\
17 \\
18 \\
19\end{array}$ & $\begin{array}{l}P \\
S \\
C L \\
A . F \\
K\end{array}$ & $\begin{array}{l}0.441 \\
0.278 \\
0.182 \\
0.121 \\
0.088\end{array}$ & $\begin{array}{l}0.3688 \\
0.4755 \\
0.540 C \\
0.5787 \\
0.6013\end{array}$ & & & & & & $\begin{array}{l}1.000 \\
1.007 \\
1.000 \\
1.000 \\
0.95\end{array}$ & $\begin{array}{l}15 \\
16 \\
17 \\
18 \\
19\end{array}$ & $\begin{array}{l}\mathrm{P} \\
\mathrm{S} \\
\mathrm{CL} \\
\mathrm{A} \mathrm{B} \\
\mathrm{K}\end{array}$ \\
\hline $\begin{array}{l}20 \\
21 \\
22 \\
23 \\
24\end{array}$ & $\begin{array}{l}C A \\
S C \\
T I \\
V \\
C B\end{array}$ & $\begin{array}{l}C .067 \\
0.056 \\
c .047 \\
C .040 \\
0.037\end{array}$ & $\begin{array}{l}0.6145 \\
0.5210 \\
0.6248 \\
0.6285 \\
0.6300\end{array}$ & & $\begin{array}{l}0.5150 \\
0.7078 \\
0.8002 \\
0.8719\end{array}$ & & $\begin{array}{l}0.4565 \\
0.5291 \\
0.7147 \\
0.7783\end{array}$ & & $\begin{array}{l}0.88 \\
0.40 \\
0.245 \\
0.172 \\
0.120\end{array}$ & $\begin{array}{l}20 \\
21 \\
22 \\
23 \\
24\end{array}$ & $\begin{array}{l}C A \\
S C \\
T I \\
V \\
C R\end{array}$ \\
\hline $\begin{array}{l}75 \\
26 \\
27 \\
28 \\
29\end{array}$ & $\begin{array}{l}\mathrm{MN} \\
\mathrm{PE} \\
\mathrm{CO} \\
\mathrm{NI} \\
\mathrm{CU}\end{array}$ & $\begin{array}{l}0.032 \\
0.029 \\
0.025 \\
0.023 \\
0.022\end{array}$ & $\begin{array}{l}0.6315 \\
0.6322 \\
0.6328 \\
0.6334 \\
0.6315\end{array}$ & & $\begin{array}{l}0.8878 \\
0.9104 \\
0.9253 \\
0.9348 \\
0.9460\end{array}$ & & $\begin{array}{l}0.7931 \\
0.8138 \\
0.8284 \\
0.8378 \\
0.8492\end{array}$ & & $\begin{array}{l}0.105 \\
0.0857 \\
0.0729 \\
0.0630 \\
0.0539\end{array}$ & $\begin{array}{l}25 \\
26 \\
27 \\
28 \\
29\end{array}$ & $\begin{array}{l}\mathrm{MN} \\
\mathrm{FE} \\
\mathrm{CO} \\
\mathrm{NI} \\
\mathrm{CU}\end{array}$ \\
\hline $\begin{array}{l}30 \\
31 \\
32 \\
33 \\
34\end{array}$ & $\begin{array}{l}Z N \\
\text { GA } \\
\text { GE } \\
\text { AS } \\
\text { SF }\end{array}$ & $\begin{array}{l}0.020 \\
0.019 \\
0.016 \\
0.015 \\
0.014\end{array}$ & $\begin{array}{l}0.6310 \\
0.6260 \\
0.6175 \\
0.6072 \\
0.5956\end{array}$ & & $\begin{array}{l}0.9507 \\
0.9542 \\
0.9555 \\
0.9570 \\
0.9591\end{array}$ & & $\begin{array}{l}0.8524 \\
0.8535 \\
0.8546 \\
0.8567 \\
0.8572\end{array}$ & & $\begin{array}{l}0.0499 \\
0.0462 \\
0.0439 \\
0.0420 \\
0.0406\end{array}$ & $\begin{array}{l}30 \\
31 \\
32 \\
33 \\
34\end{array}$ & $\begin{array}{l}\text { ZN } \\
\text { rA } \\
\text { GF } \\
\text { AS } \\
\text { SE }\end{array}$ \\
\hline $\begin{array}{l}35 \\
36 \\
37 \\
38 \\
39\end{array}$ & $\begin{array}{l}B R \\
K F \\
R R \\
S R \\
Y\end{array}$ & $\begin{array}{l}0.013 \\
0.012 \\
0.011 \\
0.011 \\
0.010\end{array}$ & $\begin{array}{l}0.5818 \\
0.5666 \\
0.3575 \\
0.5474 \\
0.5452\end{array}$ & & $\begin{array}{l}0.9598 \\
0.9609 \\
0.9611 \\
0.9594 \\
0.9556\end{array}$ & 0.0067 & $\begin{array}{l}0.8576 \\
0.8584 \\
0.8589 \\
0.8588 \\
0.8533\end{array}$ & 0.0065 & $\begin{array}{l}0.0395 \\
0.0387 \\
0.0378 \\
0.0371 \\
0.0362\end{array}$ & $\begin{array}{l}75 \\
36 \\
37 \\
38 \\
39\end{array}$ & $\begin{array}{l}B R \\
K P \\
R B \\
S R \\
Y\end{array}$ \\
\hline $\begin{array}{l}40 \\
41 \\
42 \\
43 \\
44\end{array}$ & $\begin{array}{l}\mathrm{ZR} \\
\mathrm{NB} \\
\mathrm{MO} \\
\mathrm{TC} \\
\mathrm{RU}\end{array}$ & $\begin{array}{l}0.010 \\
9.6 E-3 \\
9.3 E-3 \\
9.1 E-3 \\
9.0 E-3\end{array}$ & $\begin{array}{l}0.54 C 6 \\
0.5376 \\
0.5335 \\
0.5288 \\
0.5262\end{array}$ & & $\begin{array}{l}0.9479 \\
0.9363 \\
0.9270 \\
0.9194 \\
0.9068\end{array}$ & $\begin{array}{l}0.0150 \\
0.0288 \\
0.0386 \\
0.0490 \\
0.0597\end{array}$ & $\begin{array}{l}0.8474 \\
0.8372 \\
0.8286 \\
0.9193 \\
C .8102\end{array}$ & $\begin{array}{l}0.0150 \\
0.0297 \\
0.0385 \\
0.0496 \\
0.0583\end{array}$ & $\begin{array}{l}0.0357 \\
0.0350 \\
0.0343 \\
0.0338 \\
0.0333\end{array}$ & $\begin{array}{l}40 \\
41 \\
42 \\
43 \\
44\end{array}$ & $\begin{array}{l}7 \mathrm{P} \\
\text { NB } \\
\text { MO } \\
\text { TC } \\
\text { RI }\end{array}$ \\
\hline $\begin{array}{l}45 \\
46 \\
47 \\
48 \\
49\end{array}$ & $\begin{array}{l}\text { RH } \\
\text { PD } \\
A G \\
C D \\
I N\end{array}$ & $\begin{array}{l}8.8 E-3 \\
8.6 E-3 \\
8.4 E-3 \\
8.5 E-3 \\
8.6 E-3\end{array}$ & $\begin{array}{l}0.5219 \\
0.5197 \\
0.5157 \\
0.5114 \\
0.5059\end{array}$ & & $\begin{array}{l}0.8958 \\
0.9943 \\
0.8762 \\
0.8678 \\
0.8611\end{array}$ & $\begin{array}{l}0.0707 \\
0.0845 \\
0.0933 \\
0.1010 \\
0.1082\end{array}$ & $\begin{array}{l}0.8001 \\
0.7879 \\
0.7815 \\
0.7739 \\
0.7682\end{array}$ & $\begin{array}{l}0.0698 \\
0.0828 \\
0.0915 \\
0.0989 \\
0.1060\end{array}$ & $\begin{array}{l}0.0329 \\
0.0326 \\
0.0323 \\
0.0321 \\
0.0319\end{array}$ & $\begin{array}{l}45 \\
46 \\
47 \\
48 \\
49\end{array}$ & $\begin{array}{l}\text { RH } \\
P D \\
A G \\
C D \\
I N\end{array}$ \\
\hline $\begin{array}{l}.0 \\
51 \\
52 \\
53 \\
54\end{array}$ & $\begin{array}{l}S N \\
S B \\
T E \\
I \\
X E\end{array}$ & $\begin{array}{l}8.7 E-3 \\
8.8 E-3 \\
8.9 E-3 \\
9.0 E-3 \\
9.1 E-3\end{array}$ & $\begin{array}{l}0.5094 \\
0.4951 \\
0.4899 \\
0.4834 \\
0.4773\end{array}$ & $\begin{array}{l}0.0052 \\
0.0100 \\
0.0146 \\
0.0190 \\
0.0234\end{array}$ & $\begin{array}{l}0.8543 \\
0.8498 \\
0.8436 \\
0.8389 \\
7.9344\end{array}$ & $\begin{array}{l}0.1145 \\
0.1204 \\
0.1259 \\
0.1308 \\
0.1353\end{array}$ & $\begin{array}{l}0.7622 \\
0.7578 \\
0.7529 \\
0.7484 \\
0.7440\end{array}$ & $\begin{array}{l}0.1120 \\
0.1194 \\
0.1238 \\
0.1292 \\
0.1319\end{array}$ & $\begin{array}{l}0.0318 \\
0.0317 \\
0.0316 \\
0.0316 \\
0.0316\end{array}$ & $\begin{array}{l}50 \\
51 \\
52 \\
53 \\
54\end{array}$ & $\begin{array}{l}S N \\
S B \\
T E \\
I \\
X E\end{array}$ \\
\hline $\begin{array}{l}55 \\
56 \\
57 \\
58 \\
59\end{array}$ & $\begin{array}{l}\text { CS } \\
\text { BA } \\
\text { LA } \\
\text { CF } \\
\text { PA }\end{array}$ & $\begin{array}{l}9.2 \mathrm{E}-3 \\
9.4 \mathrm{E}-3 \\
9.6 \mathrm{~F}-3 \\
9.8 \mathrm{E}-3 \\
0.0101\end{array}$ & $\begin{array}{l}0.4716 \\
0.4659 \\
0.4608 \\
0.4604 \\
0.458 \mathrm{C}\end{array}$ & $\begin{array}{l}0.0268 \\
0.0301 \\
0.0296 \\
0.0294 \\
0.0290\end{array}$ & $\begin{array}{l}0.8309 \\
0.8271 \\
0.8232 \\
0.9250 \\
0.8213\end{array}$ & $\begin{array}{l}0.1396 \\
0.1440 \\
0.1475 \\
0.1465 \\
0.1473\end{array}$ & $\begin{array}{l}0.7407 \\
0.7366 \\
0.7323 \\
0.7336 \\
0.7314\end{array}$ & $\begin{array}{l}0.1360 \\
0.1400 \\
0.1438 \\
0.1414 \\
0.1476\end{array}$ & $\begin{array}{l}0.0316 \\
0.0317 \\
0.0318 \\
0.0320 \\
0.0322\end{array}$ & $\begin{array}{l}55 \\
56 \\
57 \\
58 \\
59\end{array}$ & $\begin{array}{l}\text { CS } \\
\text { BA } \\
\text { LA } \\
\text { CF } \\
\text { PB }\end{array}$ \\
\hline $\begin{array}{l}60 \\
61 \\
62 \\
63 \\
64\end{array}$ & $\begin{array}{l}\text { ND } \\
P M \\
S Y \\
\text { FU } \\
\text { GD }\end{array}$ & $\begin{array}{l}0.0106 \\
0.0110 \\
0.0114 \\
0.0119 \\
0.0124\end{array}$ & $\begin{array}{l}0.4556 \\
0.4525 \\
0.4499 \\
0.4467 \\
0.4432\end{array}$ & $\begin{array}{l}0.0297 \\
0.0282 \\
0.0278 \\
0.0274 \\
0.0270\end{array}$ & $\begin{array}{l}0.8211 \\
0.8196 \\
0.3210 \\
0.8199 \\
0.8171\end{array}$ & $\begin{array}{l}0.1483 \\
0.1490 \\
0.1502 \\
0.1505 \\
0.1524\end{array}$ & $\begin{array}{l}0.7325 \\
0.7327 \\
0.7300 \\
0.7307 \\
0.7281\end{array}$ & $\begin{array}{l}0.1435 \\
0.1440 \\
0.1443 \\
0.1447 \\
0.1450\end{array}$ & $\begin{array}{l}0.0325 \\
0.0328 \\
0.0330 \\
0.0333 \\
0.0336\end{array}$ & $\begin{array}{l}60 \\
61 \\
62 \\
63 \\
54\end{array}$ & $\begin{array}{l}\text { ND } \\
\text { PM } \\
\text { SM } \\
\text { EI } \\
\text { GD }\end{array}$ \\
\hline $\begin{array}{l}65 \\
06 \\
67 \\
68 \\
63\end{array}$ & $\begin{array}{l}\text { TE } \\
D Y \\
H C \\
E R \\
T Y\end{array}$ & $\begin{array}{l}0.0129 \\
0.0136 \\
0.0143 \\
0.0149 \\
0.0157\end{array}$ & $\begin{array}{l}0.4413 \\
0.4396 \\
0.4357 \\
0.4326 \\
0.4295\end{array}$ & $\begin{array}{l}0.0267 \\
0.0264 \\
0.0261 \\
0.0257 \\
0.0254\end{array}$ & $\begin{array}{l}0.8187 \\
0.8220 \\
0.8215 \\
0.8210 \\
0.8200\end{array}$ & $\begin{array}{l}0.1518 \\
0.1530 \\
0.1536 \\
0.1540 \\
0.1544\end{array}$ & $\begin{array}{l}0.7278 \\
0.7268 \\
0.7260 \\
0.7263 \\
0.7252\end{array}$ & $\begin{array}{l}0.1451 \\
0.1453 \\
0.1455 \\
0.1450 \\
0.1458\end{array}$ & $\begin{array}{l}0.0347 \\
0.0345 \\
0.0347 \\
0.0357 \\
0.0354\end{array}$ & $\begin{array}{l}65 \\
66 \\
67 \\
68 \\
69\end{array}$ & $\begin{array}{l}\text { TE } \\
\text { DY } \\
\text { HO } \\
\text { FR } \\
T M\end{array}$ \\
\hline
\end{tabular}


Table 14 (contmued)

\begin{tabular}{|c|c|c|c|c|c|c|c|c|c|c|c|}
\hline 2 & $Z$ & $L_{1} L_{23}$ & $\begin{array}{c}L_{1} M_{3} \\
L \beta_{3}\end{array}$ & $\begin{array}{c}L_{1} O_{23} \\
L \gamma_{4}\end{array}$ & $\begin{array}{c}L_{2} M_{4} \\
L \beta_{1}\end{array}$ & $\begin{array}{c}L L_{2} N_{4} \\
L \gamma_{1}\end{array}$ & $\begin{array}{c}L_{3} M_{5} \\
L a_{1}\end{array}$ & $\begin{array}{l}L_{3} N_{45} \\
L \beta_{2} 15\end{array}$ & $\begin{array}{c}L_{3} M_{1} \\
L \ell\end{array}$ & $Z$ & $z$ \\
\hline $\begin{array}{l}70 \\
71 \\
72 \\
73 \\
74\end{array}$ & $\begin{array}{l}\text { Y B } \\
\text { LU } \\
\text { HE } \\
\text { TA } \\
W\end{array}$ & $\begin{array}{l}0.0165 \\
0.0172 \\
0.0181 \\
0.0191 \\
0.0201\end{array}$ & $\begin{array}{l}0.4262 \\
0.4216 \\
0.4174 \\
0.4123 \\
0.4078\end{array}$ & $\begin{array}{l}0.0251 \\
0.0263 \\
0.0275 \\
0.0286 \\
0.0296\end{array}$ & $\begin{array}{l}0.8161 \\
0.8190 \\
0.8098 \\
0.8087 \\
0.8048\end{array}$ & $\begin{array}{l}0.1542 \\
0.1563 \\
0.1566 \\
0.1582 \\
0.1594\end{array}$ & $\begin{array}{l}0.7246 \\
0.7227 \\
0.7204 \\
0.7175 \\
0.7139\end{array}$ & $\begin{array}{l}0.1459 \\
0.1469 \\
0.1479 \\
0.1489 \\
0.1497\end{array}$ & $\begin{array}{l}0.0759 \\
0.0365 \\
0.0366 \\
0.0370 \\
0.0373\end{array}$ & $\begin{array}{l}70 \\
71 \\
72 \\
73 \\
74\end{array}$ & $\begin{array}{l}\text { YB } \\
\text { LU } \\
\text { HE } \\
\text { TA } \\
W\end{array}$ \\
\hline $\begin{array}{l}75 \\
76 \\
77 \\
78 \\
79\end{array}$ & $\begin{array}{l}\text { RE } \\
O S \\
\text { I R } \\
\text { PT } \\
\text { A U }\end{array}$ & $\begin{array}{l}c .0213 \\
0.0224 \\
0.0237 \\
0.0243 \\
0.0263\end{array}$ & $\begin{array}{l}0.4033 \\
0.3990 \\
0.3936 \\
0.3883 \\
0.3831\end{array}$ & $\begin{array}{l}0.0305 \\
0.0313 \\
0.0322 \\
0.0335 \\
0.0348\end{array}$ & $\begin{array}{l}0.8019 \\
0.7983 \\
0.7938 \\
0.7924 \\
0.7889\end{array}$ & $\begin{array}{l}0.1608 \\
0.1620 \\
0.1629 \\
0.1641 \\
0.1647\end{array}$ & $\begin{array}{l}0.7120 \\
0.7096 \\
0.7047 \\
0.7025 \\
0.6993\end{array}$ & $\begin{array}{l}0.1508 \\
0.1517 \\
0.1525 \\
0.1532 \\
0.1537\end{array}$ & $\begin{array}{l}0.0377 \\
0.0381 \\
0.0384 \\
0.0389 \\
0.0392\end{array}$ & $\begin{array}{l}75 \\
76 \\
77 \\
78 \\
79\end{array}$ & $\begin{array}{l}\text { RE } \\
\text { OS } \\
\text { IR } \\
\text { PT } \\
\text { AU }\end{array}$ \\
\hline $\begin{array}{l}80 \\
81 \\
82 \\
93 \\
84\end{array}$ & $\begin{array}{l}\text { HG } \\
\text { TI } \\
\text { P R } \\
\text { B I } \\
\text { PC }\end{array}$ & $\begin{array}{l}0.0278 \\
0.0294 \\
0.0311 \\
0.0329 \\
0.0347\end{array}$ & $\begin{array}{l}0.3779 \\
0.3719 \\
0.366 \mathrm{C} \\
0.3603 \\
0.3545\end{array}$ & $\begin{array}{l}0.0361 \\
0.0372 \\
0.0382 \\
0.0394 \\
0.0404\end{array}$ & $\begin{array}{l}0.7859 \\
0.7830 \\
0.7796 \\
0.7772 \\
0.7741\end{array}$ & $\begin{array}{l}0.1654 \\
0.1665 \\
0.1674 \\
0.1688 \\
0.1695\end{array}$ & $\begin{array}{l}0.6960 \\
0.6933 \\
0.6909 \\
0.6881 \\
0.6855\end{array}$ & $\begin{array}{l}0.1543 \\
0.1549 \\
0.1554 \\
0.1560 \\
0.1566\end{array}$ & $\begin{array}{l}0.0397 \\
0.0401 \\
0.0406 \\
0.0410 \\
0.0415\end{array}$ & $\begin{array}{l}80 \\
81 \\
82 \\
83 \\
84\end{array}$ & $\begin{array}{l}\text { HG } \\
\text { TL } \\
\text { PB } \\
\text { BI } \\
\text { PO }\end{array}$ \\
\hline $\begin{array}{l}85 \\
96 \\
87 \\
38 \\
89\end{array}$ & $\begin{array}{l}A T \\
R N \\
\text { FR } \\
\text { RA } \\
\text { AC }\end{array}$ & $\begin{array}{l}0.0368 \\
C .0389 \\
0.0412 \\
0.0434 \\
0.0463\end{array}$ & $\begin{array}{l}0.3481 \\
0.3413 \\
0.3351 \\
0.3287 \\
0.3222\end{array}$ & $\begin{array}{l}0.0414 \\
0.0422 \\
0.0432 \\
0.0440 \\
0.0448\end{array}$ & $\begin{array}{l}0.7712 \\
0.7684 \\
0.7659 \\
0.7633 \\
0.7602\end{array}$ & $\begin{array}{l}0.1706 \\
0.1718 \\
0.1728 \\
0.1739 \\
0.1750\end{array}$ & $\begin{array}{l}0.6830 \\
0.6801 \\
0.6776 \\
0.6751 \\
0.6722\end{array}$ & $\begin{array}{l}0.1574 \\
0.1582 \\
0.1588 \\
0.1596 \\
0.1601\end{array}$ & $\begin{array}{l}0.0421 \\
0.0426 \\
0.0431 \\
0.0437 \\
0.0442\end{array}$ & $\begin{array}{l}85 \\
86 \\
87 \\
89 \\
89\end{array}$ & $\begin{array}{l}A T \\
R N \\
P R \\
R A \\
A C\end{array}$ \\
\hline $\begin{array}{l}90 \\
91 \\
92 \\
93 \\
94\end{array}$ & $\begin{array}{l}T H \\
P A \\
U \\
N P \\
\text { PU }\end{array}$ & $\begin{array}{l}0.0490 \\
0.0520 \\
0.0552 \\
0.0586 \\
0.0622\end{array}$ & $\begin{array}{l}0.3154 \\
0.3092 \\
0.3027 \\
0.2956 \\
0.2899\end{array}$ & $\begin{array}{l}0.0455 \\
0.0462 \\
0.0468 \\
0.0472 \\
0.0477\end{array}$ & $\begin{array}{l}0.7575 \\
0.7557 \\
0.7534 \\
0.7512 \\
0.7490\end{array}$ & $\begin{array}{l}0.1761 \\
0.1773 \\
0.1783 \\
0.1794 \\
0.1806\end{array}$ & $\begin{array}{l}0.6698 \\
0.6678 \\
0.6658 \\
0.6637 \\
0.6620\end{array}$ & $\begin{array}{l}0.1606 \\
0.1614 \\
0.1620 \\
0.1628 \\
0.1633\end{array}$ & $\begin{array}{l}0.0448 \\
0.0454 \\
0.0460 \\
0.0467 \\
0.0473\end{array}$ & $\begin{array}{l}90 \\
91 \\
92 \\
93 \\
94\end{array}$ & $\begin{array}{l}\text { TH } \\
\text { PA } \\
\text { I } \\
\text { NP } \\
\text { PU }\end{array}$ \\
\hline $\begin{array}{l}95 \\
96 \\
97 \\
98 \\
69\end{array}$ & $\begin{array}{l}A M \\
C M \\
\text { AK } \\
C F \\
F S\end{array}$ & $\begin{array}{l}0.0660 \\
c .0700 \\
0.0744 \\
0.0789 \\
0.0837\end{array}$ & $\begin{array}{l}0.2834 \\
0.2749 \\
0.2674 \\
0.2600 \\
0.2525\end{array}$ & $\begin{array}{l}0.0482 \\
0.0482 \\
0.0483 \\
0.0484 \\
0.0483\end{array}$ & $\begin{array}{l}0.7474 \\
0.7451 \\
0.7431 \\
0.7415 \\
0.7396\end{array}$ & $\begin{array}{l}0.1815 \\
0.1826 \\
0.1836 \\
0.1848 \\
0.1858\end{array}$ & $\begin{array}{l}0.6603 \\
0.6580 \\
0.6560 \\
0.6544 \\
0.6526\end{array}$ & $\begin{array}{l}0.1638 \\
0.1644 \\
0.1650 \\
0.1655 \\
0.1661\end{array}$ & $\begin{array}{l}0.0490 \\
0.0496 \\
0.0493 \\
0.0500 \\
0.0508\end{array}$ & $\begin{array}{l}95 \\
96 \\
97 \\
98 \\
99\end{array}$ & $\begin{array}{l}A M \\
C M \\
B K \\
C F \\
E S\end{array}$ \\
\hline $\begin{array}{l}100 \\
101 \\
102 \\
103 \\
104\end{array}$ & $\begin{array}{l}\text { FM } \\
\text { ML } \\
\text { NO } \\
\text { LR }\end{array}$ & $\begin{array}{l}0.0899 \\
0.0942 \\
0.1001 \\
0.1060 \\
0.1125\end{array}$ & $\begin{array}{l}0.2447 \\
0.2368 \\
0.2287 \\
0.2205 \\
0.2120\end{array}$ & $\begin{array}{l}0.0481 \\
0.0478 \\
0.0473 \\
0.0469 \\
0.0461\end{array}$ & $\begin{array}{l}0.7375 \\
0.7357 \\
0.7337 \\
0.7314 \\
0.7293\end{array}$ & $\begin{array}{l}0.1867 \\
0.1878 \\
0.1888 \\
0.1896 \\
0.1906\end{array}$ & $\begin{array}{l}0.6509 \\
0.6490 \\
0.6472 \\
0.6452 \\
0.6431\end{array}$ & $\begin{array}{l}0.1666 \\
0.1671 \\
0.1676 \\
0.1680 \\
0.1683\end{array}$ & $\begin{array}{l}0.0515 \\
0.0522 \\
0.0530 \\
0.0533 \\
0.0545\end{array}$ & $\begin{array}{l}100 \\
101 \\
102 \\
103 \\
104\end{array}$ & $\begin{array}{l}\text { PM } \\
\text { MD } \\
\text { NO } \\
\text { LR }\end{array}$ \\
\hline $\begin{array}{l}105 \\
106 \\
107 \\
108 \\
109\end{array}$ & & & & & $\begin{array}{l}0.7275 \\
0.7253 \\
0.7230 \\
0.7205 \\
0.7180\end{array}$ & $\begin{array}{l}0.1916 \\
0.1924 \\
0.1932 \\
0.1939 \\
0.1946\end{array}$ & $\begin{array}{l}0.641 \\
0.639 \\
0.637 \\
0.634 \\
0.632\end{array}$ & $\begin{array}{l}0.1697 \\
0.1690 \\
0.1693 \\
0.1696 \\
0.1699\end{array}$ & $\begin{array}{l}0.0553 \\
0.0561 \\
0.0569 \\
0.0577 \\
0.0585\end{array}$ & $\begin{array}{l}105 \\
106 \\
107 \\
108 \\
109\end{array}$ & \\
\hline 110 & & & & & 0.7155 & 0.1952 & 0.629 & 0.1700 & 0.0593 & 110 & \\
\hline
\end{tabular}


Table 15 Fluorescence and Coster-Kronig yeelds for $K$ and $L$ shells

See ref 3 for detats For low $Z$, consult Table 16 Note $f_{1}=f_{1,2}+f_{13}, f^{\prime}{ }_{1,3} \equiv L_{1} \rightarrow L_{3}$ intrashell radiative yreld

\begin{tabular}{|c|c|c|c|c|c|c|c|c|c|c|c|c|}
\hline & $Z$ & $\omega_{K}$ & $\omega_{1}$ & $\omega_{2}$ & $\omega_{3}$ & $f_{1}$ & $f_{12}$ & $f_{1,3}$ & $f_{1,3}^{\prime}$ & $f_{2,3}$ & & $Z$ \\
\hline $\begin{array}{l}5 \\
6 \\
7 \\
9 \\
9\end{array}$ & $\begin{array}{l}\mathrm{P} \\
\mathrm{C} \\
\mathrm{N} \\
\mathrm{C} \\
\mathrm{F}\end{array}$ & $\begin{array}{r}1.7 E-03 \\
2.8 E-03 \\
5.2 E-C 3 \\
8.3 E-03 \\
0.013\end{array}$ & & & & & & & & & $\begin{array}{l}5 \\
6 \\
7 \\
8 \\
9\end{array}$ & $\begin{array}{l}B \\
C \\
N \\
0 \\
5\end{array}$ \\
\hline $\begin{array}{l}10 \\
11 \\
12 \\
13 \\
14\end{array}$ & $\begin{array}{l}\text { NE } \\
\text { NA } \\
M F \\
\text { AL } \\
\text { SI }\end{array}$ & $\begin{array}{l}0.018 \\
0.023 \\
0.030 \\
0.039 \\
c .05 \mathrm{C}\end{array}$ & $\begin{array}{l}2.9 \mathrm{E}-05 \\
2.6 \mathrm{E}-05 \\
3.0 \mathrm{E}-05\end{array}$ & $\begin{array}{l}1.2 E-03 \\
7.5 E-04 \\
3.7 F-04\end{array}$ & $\begin{array}{l}1.2 E-03 \\
7.5 F-04 \\
3.8 E-04\end{array}$ & $\begin{array}{l}0.962 \\
0.965 \\
0.959\end{array}$ & $\begin{array}{l}0.32 \\
0.32 \\
0.32\end{array}$ & $\begin{array}{l}0.64 \\
0.64 \\
0.64\end{array}$ & $\begin{array}{l}2.0 \mathrm{E}-05 \\
1.6 \mathrm{E}-05 \\
1.4 \mathrm{E}-05\end{array}$ & & $\begin{array}{l}10 \\
11 \\
12 \\
13 \\
14\end{array}$ & $\begin{array}{l}\text { NE } \\
\text { HA } \\
\text { HG } \\
\text { A L } \\
\text { S I }\end{array}$ \\
\hline $\begin{array}{l}15 \\
16 \\
17 \\
18 \\
19\end{array}$ & $\begin{array}{l}P \\
S \\
C L \\
A R \\
K\end{array}$ & $\begin{array}{l}0.063 \\
0.078 \\
0.097 \\
0.118 \\
c .140\end{array}$ & $\begin{array}{l}3.9 E-05 \\
7.4 E-05 \\
1.2 E-04 \\
1.8 E-04 \\
2.4 E-04\end{array}$ & $\begin{array}{l}3.1 E-04 \\
2.6 F-04 \\
2.4 F-04 \\
2.2 E-04 \\
2.7 E-04\end{array}$ & $\begin{array}{l}3.1 F-04 \\
2.6 F-04 \\
2.4 F-04 \\
2.2 F-04 \\
2.7 E-04\end{array}$ & $\begin{array}{l}0.951 \\
0.944 \\
0.939 \\
0.934 \\
0.929\end{array}$ & $\begin{array}{l}0.32 \\
0.32 \\
0.32 \\
0.31 \\
0.31\end{array}$ & $\begin{array}{l}0.63 \\
0.62 \\
0.62 \\
0.62 \\
0.62\end{array}$ & $\begin{array}{l}1.2 F-05 \\
1.4 E-05 \\
1.4 E-05 \\
1.3 E-05 \\
1.2 E-05\end{array}$ & & $\begin{array}{l}15 \\
16 \\
17 \\
18 \\
19\end{array}$ & $\begin{array}{l}\text { P } \\
S \\
C L \\
A R \\
K\end{array}$ \\
\hline $\begin{array}{l}20 \\
21 \\
22 \\
23 \\
24\end{array}$ & $\begin{array}{l}\text { C A } \\
\text { SC } \\
\text { T I } \\
\text { V } \\
\text { C E }\end{array}$ & $\begin{array}{l}0.163 \\
0.188 \\
0.214 \\
0.243 \\
0.275\end{array}$ & $\begin{array}{l}3.1 E-04 \\
3.9 E-04 \\
4.7 E-04 \\
5.8 E-04 \\
7.1 E-04\end{array}$ & $\begin{array}{l}3.3 E-04 \\
8.4 E-04 \\
1.5 F-03 \\
2.6 F-03 \\
3.7 E-03\end{array}$ & $\begin{array}{l}3.3 E-04 \\
8.4 E-04 \\
1.5 E-03 \\
2.6 F-03 \\
3.7 F-03\end{array}$ & $\begin{array}{l}0.920 \\
0.912 \\
0.902 \\
0.894 \\
0.895\end{array}$ & $\begin{array}{l}0.31 \\
0.31 \\
0.31 \\
0.31 \\
0.31\end{array}$ & $\begin{array}{l}0.61 \\
0.60 \\
0.59 \\
0.58 \\
0.57\end{array}$ & $\begin{array}{l}1.4 \mathrm{E}-05 \\
1.4 \mathrm{E}-05 \\
1.5 \mathrm{E}-05 \\
1.6 \mathrm{E}-05 \\
1.8 \mathrm{E}-05\end{array}$ & & $\begin{array}{ll}2 & 0 \\
2 & 1 \\
2 & 2 \\
2 & 3 \\
2 & 4\end{array}$ & $\begin{array}{l}C A \\
S C \\
T I \\
\mathbf{Y} \\
C R\end{array}$ \\
\hline $\begin{array}{l}25 \\
26 \\
27 \\
28 \\
29\end{array}$ & $\begin{array}{l}M N \\
F E \\
C O \\
N I \\
C U\end{array}$ & $\begin{array}{l}0.308 \\
0.340 \\
0.373 \\
0.406 \\
0.440\end{array}$ & $\begin{array}{l}8.4 E-04 \\
1.0 E-03 \\
1.2 F-03 \\
1.4 E-03 \\
1.6 E-03\end{array}$ & $\begin{array}{r}5.0 E-03 \\
6.3 E-03 \\
7.7 E-03 \\
8.6 E-03 \\
0.010\end{array}$ & $\begin{array}{r}5.0 F-03 \\
6.3 E-03 \\
7.7 E-03 \\
9.3 E-03 \\
0.011\end{array}$ & $\begin{array}{l}0.877 \\
0.868 \\
0.856 \\
0.847 \\
0.839\end{array}$ & $\begin{array}{l}0.30 \\
0.30 \\
0.30 \\
0.30 \\
0.30\end{array}$ & $\begin{array}{l}0.58 \\
c .57 \\
C .56 \\
C .55 \\
C .54\end{array}$ & $\begin{array}{l}1.9 E-05 \\
2.1 E-05 \\
2.3 E-05 \\
2.4 F-05 \\
2.6 E-05\end{array}$ & $\begin{array}{l}0.028 \\
0.028\end{array}$ & $\begin{array}{l}25 \\
26 \\
27 \\
28 \\
29\end{array}$ & $\begin{array}{l}H N \\
F E \\
C O \\
N I \\
C O\end{array}$ \\
\hline $\begin{array}{l}30 \\
31 \\
32 \\
33 \\
34\end{array}$ & $\begin{array}{l}\text { ZN } \\
\text { GA } \\
\text { GE } \\
\text { AS } \\
\text { SE }\end{array}$ & $\begin{array}{l}0.474 \\
0.507 \\
0.535 \\
0.562 \\
0.589\end{array}$ & $\begin{array}{l}1.8 E-03 \\
2.1 E-03 \\
2.4 E-03 \\
2.8 E-03 \\
3.2 E-03\end{array}$ & $\begin{array}{l}0.0111 \\
0.012 \\
0.013 \\
0.014 \\
0.016\end{array}$ & $\begin{array}{l}0.012 \\
0.013 \\
0.015 \\
0.016 \\
0.018\end{array}$ & $\begin{array}{l}0.831 \\
0.822 \\
0.815 \\
0.809 \\
0.804\end{array}$ & $\begin{array}{l}0.29 \\
0.29 \\
0.28 \\
0.29 \\
0.28\end{array}$ & $\begin{array}{l}0.54 \\
0.53 \\
0.53 \\
0.53 \\
0.52\end{array}$ & $\begin{array}{l}2.8 E-05 \\
3.0 E-05 \\
3.2 E-05 \\
3.4 E-05 \\
3.6 E-05\end{array}$ & $\begin{array}{l}0.026 \\
0.032 \\
0.050 \\
0.063 \\
0.076\end{array}$ & $\begin{array}{l}30 \\
31 \\
32 \\
33 \\
34\end{array}$ & $\begin{array}{l}\text { ZN } \\
\text { GA } \\
\text { GE } \\
\text { AS } \\
\text { SE }\end{array}$ \\
\hline $\begin{array}{l}35 \\
36 \\
37 \\
38 \\
39\end{array}$ & $\begin{array}{l}\text { BR } \\
K R \\
\text { RE } \\
\text { SB } \\
Y\end{array}$ & $\begin{array}{l}0.618 \\
0.643 \\
0.667 \\
0.690 \\
0.710\end{array}$ & $\begin{array}{l}3.6 E-03 \\
4.1 E-03 \\
4.6 E-03 \\
5.1 E-03 \\
5.9 E-03\end{array}$ & $\begin{array}{l}0.018 \\
0.020 \\
0.022 \\
0.024 \\
0.026\end{array}$ & $\begin{array}{l}0.020 \\
0.022 \\
0.024 \\
0.026 \\
0.028\end{array}$ & $\begin{array}{l}0.800 \\
0.797 \\
0.794 \\
0.790 \\
0.785\end{array}$ & $\begin{array}{l}0.28 \\
0.27 \\
0.27 \\
0.27 \\
0.26\end{array}$ & $\begin{array}{l}0.52 \\
0.52 \\
0.52 \\
0.52 \\
0.52\end{array}$ & $\begin{array}{l}3.8 \mathrm{~F}-05 \\
4.1 \mathrm{E}-05 \\
4.4 \mathrm{E}-05 \\
4.7 \mathrm{E}-05 \\
5.2 \mathrm{E}-05\end{array}$ & $\begin{array}{l}0.088 \\
0.100 \\
0.109 \\
0.117 \\
0.126\end{array}$ & $\begin{array}{l}35 \\
76 \\
37 \\
39 \\
39\end{array}$ & $\begin{array}{l}\text { BR } \\
\text { R R } \\
\text { R B } \\
\text { SR } \\
Y\end{array}$ \\
\hline $\begin{array}{l}40 \\
41 \\
42 \\
43 \\
44\end{array}$ & $\begin{array}{l}\text { ZR } \\
\text { NE } \\
\text { MO } \\
\text { TC } \\
\text { RU }\end{array}$ & $\begin{array}{l}0.730 \\
0.747 \\
0.765 \\
0.780 \\
0.794\end{array}$ & $\begin{array}{r}6.8 E-03 \\
9.4 E-03 \\
0.010 \\
0.011 \\
0.012\end{array}$ & $\begin{array}{l}0.028 \\
0.031 \\
0.034 \\
0.037 \\
0.040\end{array}$ & $\begin{array}{l}0.031 \\
0.034 \\
0.037 \\
0.040 \\
0.043\end{array}$ & $\begin{array}{l}0.779 \\
0.713 \\
0.712 \\
0.711 \\
0.709\end{array}$ & $\begin{array}{l}0.26 \\
0.10 \\
0.10 \\
0.10 \\
0.10\end{array}$ & $\begin{array}{l}0.52 \\
0.61 \\
0.61 \\
0.61 \\
0.61\end{array}$ & $\begin{array}{l}5.8 E-05 \\
7.8 E-05 \\
8.1 E-05 \\
8.8 E-05 \\
9.6 E-05\end{array}$ & $\begin{array}{l}0.132 \\
0.137 \\
0.141 \\
0.144 \\
0.148\end{array}$ & $\begin{array}{l}40 \\
41 \\
42 \\
43 \\
44\end{array}$ & $\begin{array}{l}\text { ZR } \\
\text { NB } \\
\text { HO } \\
\text { TC } \\
\text { R O }\end{array}$ \\
\hline $\begin{array}{l}45 \\
46 \\
47 \\
48 \\
49\end{array}$ & $\begin{array}{l}R H \\
P E \\
A G \\
C D \\
I N\end{array}$ & $\begin{array}{l}0.868 \\
0.820 \\
0.831 \\
0.843 \\
0.853\end{array}$ & $\begin{array}{l}0.013 \\
0.014 \\
0.016 \\
0.018 \\
0.020\end{array}$ & $\begin{array}{l}0.043 \\
0.047 \\
0.051 \\
0.056 \\
0.061\end{array}$ & $\begin{array}{l}0.046 \\
0.049 \\
0.052 \\
0.056 \\
0.050\end{array}$ & $\begin{array}{l}0.765 \\
0.700 \\
0.694 \\
0.688 \\
0.681\end{array}$ & $\begin{array}{l}0.10 \\
0.10 \\
0.10 \\
0.10 \\
0.10\end{array}$ & $\begin{array}{l}0.60 \\
0.60 \\
0.59 \\
0.59 \\
0.59\end{array}$ & $\begin{array}{l}1.0 \mathrm{E}-04 \\
1.1 \mathrm{E}-04 \\
1.2 \mathrm{E}-04 \\
1.4 \mathrm{E}-04 \\
1.6 \mathrm{~F}-04\end{array}$ & $\begin{array}{l}0.150 \\
0.151 \\
0.153 \\
0.155 \\
0.157\end{array}$ & $\begin{array}{l}45 \\
46 \\
47 \\
48 \\
49\end{array}$ & $\begin{array}{l}\text { R H } \\
\text { P D } \\
\text { A G } \\
\text { CD } \\
\text { I N }\end{array}$ \\
\hline $\begin{array}{l}50 \\
51 \\
52 \\
53 \\
54\end{array}$ & $\begin{array}{l}\text { SN } \\
\text { SB } \\
T E \\
I \\
X E\end{array}$ & $\begin{array}{l}0.862 \\
0.870 \\
0.877 \\
0.894 \\
0.891\end{array}$ & $\begin{array}{l}0.037 \\
0.039 \\
0.041 \\
0.044 \\
0.046\end{array}$ & $\begin{array}{l}0.065 \\
0.069 \\
0.074 \\
0.079 \\
0.083\end{array}$ & $\begin{array}{l}0.064 \\
0.069 \\
0.074 \\
0.079 \\
0.085\end{array}$ & $\begin{array}{l}0.439 \\
0.448 \\
0.455 \\
0.461 \\
0.466\end{array}$ & $\begin{array}{l}0.17 \\
0.17 \\
0.18 \\
0.18 \\
0.19\end{array}$ & $\begin{array}{l}0.27 \\
0.28 \\
0.28 \\
0.28 \\
0.28\end{array}$ & $\begin{array}{l}3.0 F-04 \\
3.2 \mathrm{E}-04 \\
3.4 \mathrm{E}-04 \\
3.7 F-04 \\
4.0 \mathrm{E}-04\end{array}$ & $\begin{array}{l}0.157 \\
0.156 \\
0.155 \\
0.154 \\
0.154\end{array}$ & $\begin{array}{l}50 \\
51 \\
52 \\
53 \\
54\end{array}$ & $\begin{array}{l}\text { S N } \\
\text { SB } \\
\text { TE } \\
\text { I } \\
X E\end{array}$ \\
\hline $\begin{array}{l}55 \\
56 \\
57 \\
59 \\
59\end{array}$ & $\begin{array}{l}\text { CS } \\
\text { PA } \\
\text { IA } \\
\text { CE } \\
\text { FP }\end{array}$ & $\begin{array}{l}0.897 \\
0.902 \\
0.907 \\
0.912 \\
0.917\end{array}$ & $\begin{array}{l}0.049 \\
0.052 \\
0.055 \\
0.058 \\
0.061\end{array}$ & $\begin{array}{l}0.090 \\
0.096 \\
0.103 \\
0.110 \\
0.117\end{array}$ & $\begin{array}{l}0.091 \\
0.097 \\
0.104 \\
0.111 \\
0.118\end{array}$ & $\begin{array}{l}0.470 \\
0.474 \\
0.478 \\
0.492 \\
0.485\end{array}$ & $\begin{array}{l}0.19 \\
0.19 \\
0.19 \\
0.19 \\
0.19\end{array}$ & $\begin{array}{l}0.28 \\
0.28 \\
0.29 \\
0.29 \\
0.29\end{array}$ & $\begin{array}{l}4.3 F-04 \\
4.7 E-04 \\
5.1 F-04 \\
5.5 E-04 \\
6.0 E-04\end{array}$ & $\begin{array}{l}0.154 \\
0.153 \\
0.153 \\
0.153 \\
0.153\end{array}$ & $\begin{array}{l}55 \\
56 \\
57 \\
58 \\
59\end{array}$ & $\begin{array}{l}\text { CS } \\
B A \\
L A \\
C P \\
\text { PR }\end{array}$ \\
\hline $\begin{array}{l}6 C \\
61 \\
62 \\
63 \\
64\end{array}$ & $\begin{array}{l}N C \\
\text { PM } \\
\text { SM } \\
\text { EU } \\
\text { GL }\end{array}$ & $\begin{array}{l}0.921 \\
0.925 \\
0.929 \\
0.932 \\
0.935\end{array}$ & $\begin{array}{l}0.064 \\
0.066 \\
0.071 \\
0.075 \\
0.079\end{array}$ & $\begin{array}{l}0.124 \\
0.132 \\
0.140 \\
0.149 \\
0.158\end{array}$ & $\begin{array}{l}0.125 \\
0.132 \\
0.139 \\
0.147 \\
0.155\end{array}$ & $\begin{array}{l}0.489 \\
0.490 \\
0.492 \\
0.493 \\
0.493\end{array}$ & $\begin{array}{l}0.19 \\
0.19 \\
0.19 \\
0.19 \\
0.19\end{array}$ & $\begin{array}{l}0.30 \\
0.30 \\
0.30 \\
0.30 \\
0.30\end{array}$ & $\begin{array}{l}6.6 F-04 \\
7.2 E-04 \\
7.9 E-04 \\
8.7 F-04 \\
9.6 F-04\end{array}$ & $\begin{array}{l}0.152 \\
0.151 \\
0.150 \\
0.149 \\
0.147\end{array}$ & $\begin{array}{l}60 \\
61 \\
62 \\
61 \\
64\end{array}$ & $\begin{array}{l}\text { ND } \\
\text { PA } \\
\text { SH } \\
\text { EU } \\
\text { GD }\end{array}$ \\
\hline
\end{tabular}


Table 15 (cont inued)

\begin{tabular}{|c|c|c|c|c|c|c|c|c|c|c|c|c|}
\hline 7 & & $\omega_{K}$ & $\omega_{1}$ & $\omega_{2}$ & $\omega_{3}$ & $f_{1}$ & $f_{12}$ & $f_{13}$ & $f_{13}$ & $f_{23}$ & $Z$ & \\
\hline $\begin{array}{l}65 \\
66 \\
67 \\
68 \\
69\end{array}$ & $\begin{array}{l}\text { T B } \\
\text { DY } \\
\text { HS } \\
\text { F R } \\
\text { TM }\end{array}$ & $\begin{array}{l}0.938 \\
0.941 \\
0.944 \\
0.947 \\
0.949\end{array}$ & $\begin{array}{l}0.083 \\
0.089 \\
0.094 \\
0.100 \\
c .106\end{array}$ & $\begin{array}{l}0.167 \\
0.178 \\
0.189 \\
0.200 \\
0.211\end{array}$ & $\begin{array}{l}0.164 \\
0.174 \\
0.192 \\
0.192 \\
0.201\end{array}$ & $\begin{array}{l}0.493 \\
0.492 \\
0.490 \\
0.487 \\
0.483\end{array}$ & $\begin{array}{l}0.19 \\
0.19 \\
0.19 \\
0.19 \\
0.19\end{array}$ & $\begin{array}{l}0.30 \\
0.30 \\
0.30 \\
0.30 \\
0.29\end{array}$ & $\begin{array}{l}1.9 F-03 \\
1.2 E-03 \\
1.3 E-03 \\
1.4 F-03 \\
1.6 F-03\end{array}$ & $\begin{array}{l}0.145 \\
0.143 \\
0.142 \\
0.140 \\
0.139\end{array}$ & $\begin{array}{l}65 \\
65 \\
67 \\
68 \\
69\end{array}$ & $\begin{array}{l}\text { TB } \\
\text { DY } \\
\text { HO } \\
\text { E R } \\
\text { TM }\end{array}$ \\
\hline $\begin{array}{l}70 \\
71 \\
72 \\
73 \\
74\end{array}$ & $\begin{array}{l}\text { YE } \\
\text { LU } \\
\text { HF } \\
\text { TA } \\
W\end{array}$ & $\begin{array}{l}0.951 \\
0.953 \\
0.954 \\
0.957 \\
0.958\end{array}$ & $\begin{array}{l}0.112 \\
0.120 \\
0.128 \\
C .137 \\
0.147\end{array}$ & $\begin{array}{l}0.227 \\
0.234 \\
0.246 \\
0.258 \\
0.270\end{array}$ & $\begin{array}{l}0.210 \\
0.220 \\
0.231 \\
0.243 \\
0.255\end{array}$ & $\begin{array}{l}0.478 \\
0.472 \\
0.465 \\
0.457 \\
0.447\end{array}$ & $\begin{array}{l}0.19 \\
0.19 \\
0.19 \\
0.19 \\
0.17\end{array}$ & $\begin{array}{l}0.29 \\
0.28 \\
0.29 \\
0.29 \\
0.29\end{array}$ & $\begin{array}{l}1.9 F-03 \\
2.0 F-03 \\
2.3 F-03 \\
2.6 E-03 \\
2.8 F-03\end{array}$ & $\begin{array}{l}0.138 \\
0.136 \\
0.135 \\
0.134 \\
0.133\end{array}$ & $\begin{array}{ll}7 & 0 \\
71 \\
72 \\
73 \\
74\end{array}$ & $\begin{array}{l}\text { YR } \\
\text { LU } \\
\text { HE } \\
\text { TA } \\
\text { W }\end{array}$ \\
\hline $\begin{array}{l}75 \\
76 \\
77 \\
78 \\
79\end{array}$ & $\begin{array}{l}\text { PE } \\
\text { OS } \\
\text { I } \\
\text { FI } \\
\text { A U }\end{array}$ & $\begin{array}{l}0.959 \\
0.961 \\
0.962 \\
0.963 \\
0.964\end{array}$ & $\begin{array}{l}0.144 \\
0.130 \\
0.120 \\
0.114 \\
0.107\end{array}$ & $\begin{array}{l}0.283 \\
0.295 \\
0.308 \\
0.321 \\
0.334\end{array}$ & $\begin{array}{l}0.268 \\
0.241 \\
0.294 \\
0.356 \\
0.320\end{array}$ & $\begin{array}{l}0.495 \\
0.552 \\
0.603 \\
0.640 \\
0.672\end{array}$ & $\begin{array}{l}0.16 \\
0.16 \\
0.15 \\
0.14 \\
0.14\end{array}$ & $\begin{array}{l}0.33 \\
0.39 \\
0.45 \\
0.50 \\
0.53\end{array}$ & $\begin{array}{l}3.0 E-03 \\
2.9 E-03 \\
2.8 F-03 \\
2.9 E-03 \\
2.8 F-03\end{array}$ & $\begin{array}{l}0.130 \\
0.128 \\
0.126 \\
0.124 \\
0.127\end{array}$ & $\begin{array}{l}75 \\
76 \\
77 \\
78 \\
79\end{array}$ & $\begin{array}{l}\text { R F } \\
\text { OS } \\
\text { I R } \\
\text { PT } \\
\text { AO }\end{array}$ \\
\hline $\begin{array}{l}90 \\
81 \\
82 \\
93 \\
94\end{array}$ & $\begin{array}{l}\text { HG } \\
T L \\
E B \\
\text { EI } \\
\text { PO }\end{array}$ & $\begin{array}{l}\text { C. .965 } \\
0.966 \\
C .967 \\
0.968 \\
0.968\end{array}$ & $\begin{array}{l}c=107 \\
0.107 \\
0.112 \\
0.117 \\
0.122\end{array}$ & $\begin{array}{l}0.347 \\
0.360 \\
0.373 \\
0.387 \\
0.401\end{array}$ & $\begin{array}{l}0.333 \\
0.347 \\
0.260 \\
0.373 \\
0.396\end{array}$ & $\begin{array}{l}0.690 \\
0.696 \\
0.696 \\
0.694 \\
0.689\end{array}$ & $\begin{array}{l}0.13 \\
0.13 \\
0.12 \\
0.11 \\
0.11\end{array}$ & $\begin{array}{l}0.56 \\
0.57 \\
0.58 \\
0.58 \\
0.58\end{array}$ & $\begin{array}{l}3.0 F-03 \\
3.2 F-03 \\
3.5 F-13 \\
3.8 F-03 \\
4.2 E-03\end{array}$ & $\begin{array}{l}0.120 \\
0.118 \\
0.116 \\
0.113 \\
0.111\end{array}$ & $\begin{array}{l}80 \\
81 \\
82 \\
83 \\
84\end{array}$ & $\begin{array}{l}\mathrm{HI} \\
\mathrm{TL} \\
\mathrm{PB} \\
\mathrm{BI} \\
\mathrm{PO}\end{array}$ \\
\hline $\begin{array}{l}85 \\
86 \\
17 \\
89 \\
89\end{array}$ & $\begin{array}{l}\text { AT } \\
\text { RN } \\
\text { F ? } \\
\text { RA } \\
\text { AC }\end{array}$ & $\begin{array}{l}0.969 \\
0.969 \\
0.970 \\
0.970 \\
0.971\end{array}$ & $\begin{array}{l}0.128 \\
0.134 \\
0.139 \\
0.146 \\
0.153\end{array}$ & $\begin{array}{l}0.415 \\
0.429 \\
0.443 \\
0.456 \\
0.468\end{array}$ & $\begin{array}{l}0.399 \\
0.411 \\
0.424 \\
0.437 \\
0.450\end{array}$ & $\begin{array}{l}0.685 \\
0.682 \\
0.677 \\
0.672 \\
0.664\end{array}$ & $\begin{array}{l}0.10 \\
0.10 \\
0.10 \\
0.09 \\
0.09\end{array}$ & $\begin{array}{l}0.59 \\
0.58 \\
0.58 \\
0.58 \\
0.58\end{array}$ & $\begin{array}{l}4.7 F-03 \\
5.2 F-03 \\
5.8 F-03 \\
6.4 F-03 \\
7.15-03\end{array}$ & $\begin{array}{l}0.111 \\
0.110 \\
0.109 \\
0.108 \\
0.108\end{array}$ & $\begin{array}{l}85 \\
96 \\
87 \\
99 \\
99\end{array}$ & $\begin{array}{l}A T \\
R N \\
\text { FP } \\
\text { RA } \\
\text { AC }\end{array}$ \\
\hline $\begin{array}{l}96 \\
91 \\
92 \\
93 \\
94\end{array}$ & $\begin{array}{l}\text { I H } \\
\text { PA } \\
\text { U } \\
\text { P I }\end{array}$ & $\begin{array}{l}0.971 \\
0.972 \\
0.972 \\
0.973 \\
0.973\end{array}$ & $\begin{array}{l}0.167 \\
C .162 \\
0.176 \\
0.187 \\
0.205\end{array}$ & $\begin{array}{l}0.479 \\
0.472 \\
0.457 \\
0.446 \\
0.464\end{array}$ & $\begin{array}{l}0.453 \\
0.476 \\
0.479 \\
0.502 \\
0.514\end{array}$ & $\begin{array}{l}0.660 \\
0.664 \\
0.652 \\
0.642 \\
0.605\end{array}$ & $\begin{array}{l}0.09 \\
0.09 \\
0.08 \\
0.07 \\
0.05\end{array}$ & $\begin{array}{l}0.57 \\
0.58 \\
0.57 \\
0.57 \\
0.56\end{array}$ & $\begin{array}{r}7.3 E-03 \\
8.4 E-03 \\
9.7 E-03 \\
0.011 \\
0.013\end{array}$ & $\begin{array}{l}0.108 \\
0.139 \\
0.167 \\
0.192 \\
0.198\end{array}$ & $\begin{array}{l}90 \\
91 \\
92 \\
93 \\
94\end{array}$ & $\begin{array}{l}\mathrm{TH} \\
\mathrm{PA} \\
\mathrm{U} \\
\mathrm{NP} \\
\mathrm{PU}\end{array}$ \\
\hline $\begin{array}{l}95 \\
45 \\
97 \\
98 \\
79\end{array}$ & $\begin{array}{l}A N \\
C N \\
P K \\
C E \\
F C\end{array}$ & $\begin{array}{l}0.974 \\
0.974 \\
0.975 \\
0.975 \\
0.975\end{array}$ & $\begin{array}{l}0.218 \\
0.228 \\
0.236 \\
0.244 \\
0.253\end{array}$ & $\begin{array}{l}0.471 \\
0.479 \\
0.495 \\
0.490 \\
0.497\end{array}$ & $\begin{array}{l}0.576 \\
1.539 \\
0.550 \\
0.550 \\
0.570\end{array}$ & $\begin{array}{l}0.595 \\
0.597 \\
0.599 \\
0.573 \\
0.565\end{array}$ & $\begin{array}{l}0.05 \\
0.04 \\
0.04 \\
0.03 \\
0.03\end{array}$ & $\begin{array}{l}0.55 \\
0.55 \\
0.54 \\
0.54 \\
0.54\end{array}$ & $\begin{array}{l}0.014 \\
0.016 \\
0.017 \\
0.019 \\
0.021\end{array}$ & $\begin{array}{l}0.203 \\
0.200 \\
0.199 \\
0.197 \\
0.196\end{array}$ & $\begin{array}{l}95 \\
96 \\
97 \\
99 \\
99\end{array}$ & $\begin{array}{l}A M \\
C M \\
B K \\
C F \\
\text { PS }\end{array}$ \\
\hline $\begin{array}{l}100 \\
101 \\
102 \\
103 \\
1>4\end{array}$ & $\begin{array}{l}\text { EY } \\
M C \\
\text { MC } \\
\text { L? }\end{array}$ & $\begin{array}{l}0.976 \\
0.976 \\
0.976 \\
0.977 \\
0.977\end{array}$ & $\begin{array}{l}0.263 \\
0.272 \\
0.280 \\
0.242 \\
C .291\end{array}$ & $\begin{array}{l}0.506 \\
0.515 \\
0.524 \\
0.533 \\
0.544\end{array}$ & $\begin{array}{l}0.579 \\
0.598 \\
0.596 \\
0.604 \\
0.611\end{array}$ & $\begin{array}{l}0.556 \\
0.548 \\
0.540 \\
0.539 \\
0.531\end{array}$ & $\begin{array}{l}0.03 \\
0.02 \\
0.02 \\
0.01 \\
0.01\end{array}$ & $\begin{array}{l}0.53 \\
0.53 \\
0.52 \\
0.53 \\
0.52\end{array}$ & $\begin{array}{l}0.023 \\
0.026 \\
0.028 \\
0.030 \\
0.033\end{array}$ & $\begin{array}{l}0.194 \\
0.191 \\
0.199 \\
0.185 \\
0.181\end{array}$ & $\begin{array}{l}100 \\
104 \\
102 \\
103 \\
104\end{array}$ & $\begin{array}{l}\text { FH } \\
\text { MD } \\
\text { NO } \\
\text { LR }\end{array}$ \\
\hline $\begin{array}{l}105 \\
106 \\
107 \\
109 \\
1119\end{array}$ & & $\begin{array}{l}0.977 \\
0.978 \\
0.97 F \\
0.978 \\
0.978\end{array}$ & $\begin{array}{l}0.300 \\
0.310 \\
0.370 \\
0.331 \\
0.343\end{array}$ & $\begin{array}{l}0.553 \\
0.562 \\
0.573 \\
0.504 \\
0.500\end{array}$ & $\begin{array}{l}0.618 \\
0.624 \\
0.630 \\
0.635 \\
0.640\end{array}$ & $\begin{array}{l}0.522 \\
0.513 \\
0.505 \\
0.497 \\
0.488\end{array}$ & 0.01 & $\begin{array}{l}0.51 \\
0.51 \\
0.50 \\
0.50 \\
0.49\end{array}$ & $\begin{array}{l}0.035 \\
0.038 \\
0.042 \\
0.046 \\
0.050\end{array}$ & $\begin{array}{l}0.178 \\
0.174 \\
0.171 \\
0.165 \\
0.163\end{array}$ & $\begin{array}{l}105 \\
106 \\
107 \\
109 \\
109\end{array}$ & \\
\hline 116 & & 0.974 & $0.3^{2} 4$ & 0.598 & 0.6164 & 0.477 & & 0.48 & 0.054 & 0.158 & 110 & \\
\hline
\end{tabular}


Table 16. Fluorescence, Auger, and Coster-Kronig yields for light atoms in condensed matter ${ }^{a}$

$$
\left(9.0 \mathrm{E}-5 \equiv 9.0 \times 10^{-5}\right)
$$

\begin{tabular}{|c|c|c|c|c|c|c|}
\hline \multicolumn{3}{|c|}{$K$ shell } & \multicolumn{4}{|c|}{$L_{2}$ subshel1 } \\
\hline$Z$ & $\omega_{K}$ & $a_{K}$ & 2 & $\omega_{2}$ & $a_{2}$ & $f_{2,3}$ \\
\hline 3 & $9.0 \mathrm{E}-5$ & 1.000 & 22 & $8.3 \mathrm{E}-4^{b}$ & $0.54^{b}$ & $0.46^{b}$ \\
\hline 4 & $3.3 \mathrm{E}-4$ & 1.000 & 23 & $1.2 \mathrm{E}-3^{b}$ & $0.49^{b}$ & $0.51^{b}$ \\
\hline 5 & $7.0 \mathrm{E}-4$ & 1.000 & 24 & $1.6 \mathrm{E}-3^{b}$ & $0.44^{b}$ & $0.56^{b}$ \\
\hline 6 & $1.4 E-3$ & 0.999 & 25 & $2.1 \mathrm{E}-3$ & 0.42 & 0.58 \\
\hline 7 & $3.1 \mathrm{E}-3$ & 0.997 & 26 & $2.4 \mathrm{E}-3$ & 0.39 & 0.61 \\
\hline 8 & $5.8 \mathrm{E}-3$ & 0.994 & 27 & $3.0 \mathrm{E}-3$ & 0.39 & 0.61 \\
\hline 9 & $9.2 \mathrm{E}-3$ & 0.991 & 28 & $3.5 \mathrm{E}-3$ & 0.38 & 0.62 \\
\hline 10 & $(0.016)$ & $(0.984)$ & 29 & $4.1 \mathrm{E}-3$ & 0.38 & 0.62 \\
\hline 11 & 0.021 & 0.979 & 30 & $9.0 \mathrm{E}-3$ & 0.71 & 0.28 \\
\hline 12 & 0.028 & 0.972 & & & & \\
\hline 13 & 0.036 & 0.964 & & & & \\
\hline 14 & 0.048 & 0.952 & & & & \\
\hline 15 & 0.061 & 0.939 & & & & \\
\hline \multicolumn{7}{|c|}{ 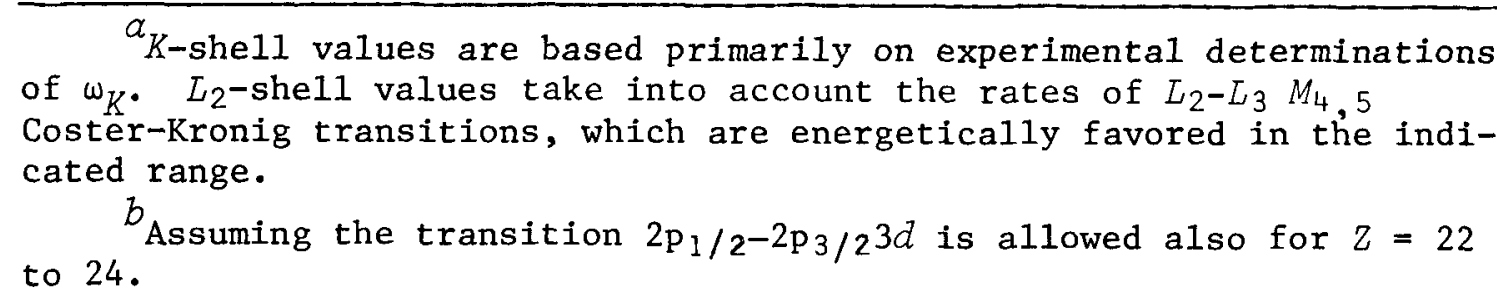 } \\
\hline
\end{tabular}


Table 17. Fractional fluorescence yields $\omega_{i} F_{i j}$ for major $K$ and $L \mathrm{x}$ rays and hole transfer yield $F_{1,3}$

From Tables 13 10 15. Definition: $F_{1,3}=f_{1,3}+f_{1,3}^{\prime}+f_{1,2} f_{2,3}$.

Table columns arranged for ready use in Eqs. 3 to 6.

\begin{tabular}{|c|c|c|c|c|c|c|c|c|c|c|}
\hline & $Z$ & $\omega_{K} F_{K \alpha_{1}}$ & $\omega_{K} F_{K \alpha_{1,2}}$ & $\omega_{1} F_{L \beta_{3}}$ & $\omega_{2} F_{L \beta_{1}}$ & $f_{2,3}$ & $\omega_{3} F_{L \alpha_{1}}$ & $F_{1,3}$ & $z$ & $z$ \\
\hline $\begin{array}{l}5 \\
6 \\
7 \\
8 \\
9\end{array}$ & $\begin{array}{l}B \\
C \\
N \\
O \\
F\end{array}$ & $\begin{array}{l}0.001 \\
0.002 \\
0.003 \\
0.006 \\
0.009\end{array}$ & $\begin{array}{l}0.002 \\
0.003 \\
0.005 \\
0.008 \\
0.013\end{array}$ & & & & & & $\begin{array}{l}5 \\
6 \\
7 \\
8 \\
9\end{array}$ & $\begin{array}{l}B \\
C \\
N \\
0 \\
F\end{array}$ \\
\hline $\begin{array}{l}10 \\
11 \\
12 \\
13 \\
14\end{array}$ & $\begin{array}{l}\text { NE } \\
\text { NA } \\
\text { MG } \\
\text { AL. } \\
\text { SI }\end{array}$ & $\begin{array}{l}0.012 \\
0.015 \\
0.020 \\
0.026 \\
0.032\end{array}$ & $\begin{array}{l}0.018 \\
0.023 \\
0.030 \\
0.038 \\
0.049\end{array}$ & & & & & $\begin{array}{l}0.640 \\
0.640 \\
0.640\end{array}$ & $\begin{array}{l}10 \\
11 \\
12 \\
13 \\
14\end{array}$ & $\begin{array}{l}\text { NE } \\
\text { NA } \\
\text { MI } \\
\text { AL } \\
\text { SI }\end{array}$ \\
\hline $\begin{array}{l}15 \\
16 \\
17 \\
18 \\
19\end{array}$ & $\begin{array}{l}\mathbf{P} \\
\mathbf{S} \\
\mathrm{CI} \\
\mathbf{A R} \\
\mathrm{K}\end{array}$ & $\begin{array}{l}0.040 \\
0.049 \\
0.059 \\
0.071 \\
0.083\end{array}$ & $\begin{array}{l}0.060 \\
0.073 \\
0.089 \\
0.106 \\
0.125\end{array}$ & & & & & $\begin{array}{l}0.630 \\
0.620 \\
0.620 \\
0.620 \\
0.620\end{array}$ & $\begin{array}{l}15 \\
16 \\
17 \\
18 \\
19\end{array}$ & $\begin{array}{l}p \\
S \\
C L \\
A R \\
K\end{array}$ \\
\hline $\begin{array}{l}20 \\
21 \\
22 \\
23 \\
24\end{array}$ & $\begin{array}{l}\mathrm{CA} \\
\mathrm{SC} \\
\mathrm{TI} \\
\mathrm{V} \\
\mathrm{CR}\end{array}$ & $\begin{array}{l}0.096 \\
0.110 \\
0.125 \\
0.142 \\
0.161\end{array}$ & $\begin{array}{l}0.144 \\
0.166 \\
0.188 \\
0.214 \\
0.243\end{array}$ & & $\begin{array}{l}0.001 \\
0.002 \\
0.003\end{array}$ & & $\begin{array}{l}0.001 \\
0.002 \\
0.003\end{array}$ & $\begin{array}{l}0.610 \\
0.600 \\
0.590 \\
0.580 \\
0.570\end{array}$ & $\begin{array}{l}20 \\
21 \\
22 \\
23 \\
24\end{array}$ & $\begin{array}{l}C A \\
S C \\
T I \\
V \\
C R\end{array}$ \\
\hline $\begin{array}{l}25 \\
26 \\
27 \\
28 \\
29\end{array}$ & $\begin{array}{l}\mathrm{MN} \\
\mathrm{PE} \\
\mathrm{CO} \\
\mathrm{MI} \\
\mathrm{CII}\end{array}$ & $\begin{array}{l}0.179 \\
0.198 \\
0.217 \\
0.235 \\
0.256\end{array}$ & $\begin{array}{l}0.271 \\
0.298 \\
0.327 \\
0.356 \\
0.387\end{array}$ & $\begin{array}{l}0.001 \\
0.001 \\
0.051 \\
0.001 \\
0.001\end{array}$ & $\begin{array}{l}0.004 \\
0.006 \\
0.007 \\
0.008 \\
0.009\end{array}$ & $\begin{array}{l}0.028 \\
0.028\end{array}$ & $\begin{array}{l}0.004 \\
0.005 \\
0.006 \\
0.008 \\
0.009\end{array}$ & $\begin{array}{l}0.580 \\
0.570 \\
0.560 \\
0.558 \\
0.548\end{array}$ & $\begin{array}{l}25 \\
26 \\
27 \\
28 \\
29\end{array}$ & $\begin{array}{l}\text { MN } \\
\text { PE } \\
\text { CO } \\
\text { NI } \\
\text { CU }\end{array}$ \\
\hline $\begin{array}{l}30 \\
31 \\
32 \\
33 \\
34\end{array}$ & $\begin{array}{l}\text { ZN } \\
G A \\
G E \\
A S \\
S E\end{array}$ & $\begin{array}{l}0.274 \\
0.292 \\
0.307 \\
0.321 \\
0.334\end{array}$ & $\begin{array}{l}0.415 \\
0.442 \\
0.465 \\
0.486 \\
0.507\end{array}$ & $\begin{array}{l}0.001 \\
0.001 \\
0.001 \\
0.002 \\
0.002\end{array}$ & $\begin{array}{l}0.0110 \\
0.011 \\
0.012 \\
0.013 \\
0.015\end{array}$ & $\begin{array}{l}0.026 \\
0.032 \\
0.050 \\
0.053 \\
0.076\end{array}$ & $\begin{array}{l}0.010 \\
0.011 \\
0.013 \\
0.0114 \\
0.015\end{array}$ & $\begin{array}{l}0.548 \\
0.539 \\
0.544 \\
0.548 \\
0.541\end{array}$ & $\begin{array}{l}30 \\
31 \\
32 \\
33 \\
34\end{array}$ & $\begin{array}{l}\text { ZN } \\
\text { GA } \\
\text { GE } \\
\text { AS } \\
\text { SE }\end{array}$ \\
\hline $\begin{array}{l}35 \\
36 \\
37 \\
38 \\
39\end{array}$ & $\begin{array}{l}B R \\
K R \\
R B \\
S R \\
Y\end{array}$ & $\begin{array}{l}0.348 \\
0.361 \\
0.373 \\
0.384 \\
0.391\end{array}$ & $\begin{array}{l}0.529 \\
0.548 \\
0.566 \\
0.583 \\
0.595\end{array}$ & $\begin{array}{l}0.002 \\
0.002 \\
0.003 \\
3.003 \\
0.003\end{array}$ & $\begin{array}{l}0.017 \\
0.019 \\
0.021 \\
0.023 \\
0.025\end{array}$ & $\begin{array}{l}0.088 \\
0.100 \\
0.109 \\
0.117 \\
0.126\end{array}$ & $\begin{array}{l}0.017 \\
0.019 \\
0.021 \\
0.022 \\
0.024\end{array}$ & $\begin{array}{l}0.545 \\
0.547 \\
0.549 \\
0.552 \\
0.553\end{array}$ & $\begin{array}{l}35 \\
36 \\
37 \\
38 \\
39\end{array}$ & $\begin{array}{l}\text { BR } \\
K R \\
R B \\
\text { SR } \\
Y\end{array}$ \\
\hline $\begin{array}{l}40 \\
41 \\
42 \\
43 \\
44\end{array}$ & $\begin{array}{l}\text { ZR } \\
\text { NB } \\
\text { MO } \\
\text { TC } \\
\text { RU }\end{array}$ & $\begin{array}{l}0.402 \\
0.410 \\
0.419 \\
0.425 \\
0.431\end{array}$ & $\begin{array}{l}0.613 \\
0.625 \\
0.639 \\
0.648 \\
0.658\end{array}$ & $\begin{array}{l}0.004 \\
0.005 \\
0.035 \\
0.035 \\
0.006\end{array}$ & $\begin{array}{l}0.027 \\
0.029 \\
0.032 \\
0.034 \\
0.036\end{array}$ & $\begin{array}{l}0.132 \\
0.137 \\
0.141 \\
0.144 \\
0.148\end{array}$ & $\begin{array}{l}0.026 \\
0.028 \\
0.031 \\
0.033 \\
0.035\end{array}$ & $\begin{array}{l}0.554 \\
0.624 \\
0.624 \\
0.624 \\
0.625\end{array}$ & $\begin{array}{l}40 \\
41 \\
42 \\
43 \\
44\end{array}$ & $\begin{array}{l}\text { ZR } \\
\text { NB } \\
\text { MO } \\
\text { TC } \\
\text { RU }\end{array}$ \\
\hline $\begin{array}{l}45 \\
46 \\
47 \\
48 \\
49\end{array}$ & $\begin{array}{l}\text { RH } \\
P D \\
A G \\
C D \\
\text { IN }\end{array}$ & $\begin{array}{l}0.438 \\
0.443 \\
0.449 \\
0.452 \\
0.456\end{array}$ & $\begin{array}{l}0.669 \\
0.677 \\
0.695 \\
0.692 \\
0.699\end{array}$ & $\begin{array}{l}0.007 \\
0.007 \\
0.008 \\
0.009 \\
0.010\end{array}$ & $\begin{array}{l}0.039 \\
0.042 \\
0.045 \\
0.049 \\
0.053\end{array}$ & $\begin{array}{l}0.150 \\
0.151 \\
0.153 \\
0.155 \\
0.157\end{array}$ & $\begin{array}{l}0.037 \\
0.039 \\
0.041 \\
0.043 \\
0.046\end{array}$ & $\begin{array}{l}0.615 \\
0.615 \\
0.605 \\
0.606 \\
0.606\end{array}$ & $\begin{array}{l}45 \\
46 \\
47 \\
48 \\
49\end{array}$ & $\begin{array}{l}\text { RH } \\
\text { PD } \\
\text { AG } \\
\text { CD } \\
\text { IN }\end{array}$ \\
\hline $\begin{array}{l}50 \\
51 \\
52 \\
53 \\
54\end{array}$ & $\begin{array}{l}\text { SN } \\
\text { SB } \\
T E \\
I \\
X E\end{array}$ & $\begin{array}{l}0.459 \\
0.462 \\
0.463 \\
0.465 \\
0.468\end{array}$ & $\begin{array}{l}0.705 \\
0.709 \\
0.712 \\
0.716 \\
0.720\end{array}$ & $\begin{array}{l}0.019 \\
0.019 \\
0.020 \\
0.021 \\
0.022\end{array}$ & $\begin{array}{l}0.056 \\
0.059 \\
0.062 \\
0.066 \\
0.069\end{array}$ & $\begin{array}{l}0.157 \\
0.156 \\
0.155 \\
0.154 \\
0.154\end{array}$ & $\begin{array}{l}0.049 \\
0.052 \\
0.056 \\
0.059 \\
0.063\end{array}$ & $\begin{array}{l}0.297 \\
0.307 \\
0.308 \\
0.308 \\
0.310\end{array}$ & $\begin{array}{l}50 \\
51 \\
52 \\
53 \\
54\end{array}$ & $\begin{array}{l}S N \\
S B \\
T E \\
I \\
X E\end{array}$ \\
\hline $\begin{array}{l}55 \\
56 \\
57 \\
58 \\
59\end{array}$ & $\begin{array}{l}\text { CS } \\
\text { BA } \\
L A \\
\text { CE } \\
\text { PR }\end{array}$ & $\begin{array}{l}0.470 \\
0.470 \\
0.472 \\
0.473 \\
0.474\end{array}$ & $\begin{array}{l}0.724 \\
0.725 \\
0.728 \\
0.731 \\
0.734\end{array}$ & $\begin{array}{l}0.023 \\
0.024 \\
0.025 \\
0.027 \\
0.029\end{array}$ & $\begin{array}{l}0.075 \\
0.079 \\
0.085 \\
0.091 \\
0.096\end{array}$ & $\begin{array}{l}0.154 \\
0.153 \\
0.153 \\
0.153 \\
0.153\end{array}$ & $\begin{array}{l}0.067 \\
0.071 \\
0.076 \\
0.081 \\
0.086\end{array}$ & $\begin{array}{l}0.310 \\
0.310 \\
0.320 \\
0.320 \\
0.320\end{array}$ & $\begin{array}{l}55 \\
56 \\
57 \\
58 \\
59\end{array}$ & $\begin{array}{l}\mathrm{CS} \\
\mathrm{BA} \\
\mathrm{LA} \\
\mathrm{CE} \\
\mathrm{PR}\end{array}$ \\
\hline
\end{tabular}


Table 17 (continued)

\begin{tabular}{|c|c|c|c|c|c|c|c|c|c|c|}
\hline$Z$ & & $\omega_{K} F_{K \alpha_{1}}$ & $\omega_{K} F_{K \alpha_{1,2}}$ & $\omega_{1} F_{I \beta_{3}}$ & $\omega_{2} F_{I \beta_{1}}$ & $f_{2,3}$ & $\omega_{3} F_{L \alpha_{1}}$ & $F_{13}$ & $Z$ & \\
\hline $\begin{array}{l}60 \\
61 \\
62 \\
63 \\
64\end{array}$ & $\begin{array}{l}\text { ND } \\
\text { PM } \\
\text { SM } \\
\text { EV } \\
\text { GD }\end{array}$ & $\begin{array}{l}0.476 \\
0.476 \\
0.477 \\
0.478 \\
0.478\end{array}$ & $\begin{array}{l}0.737 \\
0.739 \\
0.741 \\
0.743 \\
0.744\end{array}$ & $\begin{array}{l}0.029 \\
0.030 \\
0.032 \\
0.034 \\
0.035\end{array}$ & $\begin{array}{l}0.102 \\
0.108 \\
0.115 \\
0.122 \\
0.129\end{array}$ & $\begin{array}{l}0.152 \\
0.151 \\
0.150 \\
0.149 \\
0.147\end{array}$ & $\begin{array}{l}0.092 \\
0.097 \\
0.101 \\
0.107 \\
0.113\end{array}$ & $\begin{array}{l}0.330 \\
0.329 \\
0.329 \\
0.329 \\
0.329\end{array}$ & $\begin{array}{l}60 \\
61 \\
62 \\
63 \\
64\end{array}$ & $\begin{array}{l}\text { ND } \\
\text { PM } \\
\text { SM } \\
\text { EU } \\
\text { GD }\end{array}$ \\
\hline $\begin{array}{l}65 \\
66 \\
67 \\
68 \\
69\end{array}$ & $\begin{array}{l}\text { TB } \\
\text { DY } \\
\text { HO } \\
\text { ER } \\
\text { TM }\end{array}$ & $\begin{array}{l}0.479 \\
0.479 \\
0.480 \\
0.480 \\
0.480\end{array}$ & $\begin{array}{l}0.746 \\
0.747 \\
0.749 \\
0.751 \\
0.752\end{array}$ & $\begin{array}{l}0.037 \\
0.039 \\
0.041 \\
0.043 \\
0.046\end{array}$ & $\begin{array}{l}0.137 \\
0.146 \\
0.155 \\
0.164 \\
0.173\end{array}$ & $\begin{array}{l}0.145 \\
0.143 \\
0.142 \\
0.140 \\
0.133\end{array}$ & $\begin{array}{l}0.119 \\
0.126 \\
0.132 \\
0.139 \\
0.146\end{array}$ & $\begin{array}{l}0.329 \\
0.329 \\
0.329 \\
0.328 \\
0.318\end{array}$ & $\begin{array}{l}65 \\
66 \\
67 \\
68 \\
69\end{array}$ & $\begin{array}{l}\text { TA } \\
\text { DY } \\
\text { HO } \\
\text { ER } \\
\text { TM }\end{array}$ \\
\hline $\begin{array}{l}70 \\
71 \\
72 \\
73 \\
74\end{array}$ & $\begin{array}{l}\text { YB } \\
\text { LU } \\
\text { HE } \\
\text { TA } \\
\text { W }\end{array}$ & $\begin{array}{l}0.480 \\
0.480 \\
0.480 \\
0.480 \\
0.479\end{array}$ & $\begin{array}{l}0.753 \\
0.753 \\
0.754 \\
0.755 \\
0.754\end{array}$ & $\begin{array}{l}0.048 \\
0.051 \\
0.053 \\
0.056 \\
0.060\end{array}$ & $\begin{array}{l}0.181 \\
0.191 \\
0.199 \\
0.209 \\
0.217\end{array}$ & $\begin{array}{l}0.138 \\
0.136 \\
0.135 \\
0.134 \\
0.133\end{array}$ & $\begin{array}{l}0.152 \\
0.159 \\
0.166 \\
0.174 \\
0.182\end{array}$ & $\begin{array}{l}0.318 \\
0.308 \\
0.307 \\
0.307 \\
0.305\end{array}$ & $\begin{array}{l}70 \\
71 \\
72 \\
73 \\
74\end{array}$ & $\begin{array}{l}Y B \\
L V \\
\text { HP } \\
\text { TA } \\
W\end{array}$ \\
\hline $\begin{array}{l}75 \\
76 \\
77 \\
78 \\
79\end{array}$ & $\begin{array}{l}\text { RE } \\
\text { OS } \\
\text { IR } \\
\text { PT } \\
\text { AU }\end{array}$ & $\begin{array}{l}0.477 \\
0.477 \\
0.477 \\
0.476 \\
0.475\end{array}$ & $\begin{array}{l}0.753 \\
0.754 \\
0.754 \\
0.755 \\
0.755\end{array}$ & $\begin{array}{l}0.058 \\
0.052 \\
0.047 \\
0.044 \\
0.041\end{array}$ & $\begin{array}{l}0.227 \\
0.235 \\
0.244 \\
0.254 \\
0.267\end{array}$ & $\begin{array}{l}0.130 \\
0.128 \\
0.126 \\
0.124 \\
0.122\end{array}$ & $\begin{array}{l}0.191 \\
0.199 \\
0.207 \\
0.215 \\
0.224\end{array}$ & $\begin{array}{l}0.354 \\
0.413 \\
0.472 \\
0.529 \\
0.550\end{array}$ & $\begin{array}{l}75 \\
76 \\
77 \\
78 \\
79\end{array}$ & $\begin{array}{l}\mathrm{RE} \\
\mathrm{OS} \\
\mathrm{IR} \\
\mathrm{PT} \\
\mathrm{A} \text { IJ }\end{array}$ \\
\hline $\begin{array}{l}80 \\
81 \\
82 \\
83 \\
84\end{array}$ & $\begin{array}{l}\mathrm{HG} \\
\mathrm{TL} \\
\mathrm{PB} \\
\mathrm{BI} \\
\mathrm{PO}\end{array}$ & $\begin{array}{l}0.475 \\
0.474 \\
0.473 \\
0.472 \\
0.471\end{array}$ & $\begin{array}{l}0.755 \\
0.754 \\
0.754 \\
0.754 \\
0.754\end{array}$ & $\begin{array}{l}0.040 \\
0.040 \\
0.041 \\
0.042 \\
0.043\end{array}$ & $\begin{array}{l}0.273 \\
0.292 \\
0.291 \\
0.301 \\
0.310\end{array}$ & $\begin{array}{l}0.120 \\
0.119 \\
0.116 \\
0.113 \\
0.111\end{array}$ & $\begin{array}{l}0.232 \\
0.241 \\
0.249 \\
0.257 \\
0.265\end{array}$ & $\begin{array}{l}0.579 \\
0.589 \\
0.597 \\
0.596 \\
0.596\end{array}$ & $\begin{array}{l}80 \\
81 \\
82 \\
83 \\
94\end{array}$ & $\begin{array}{l}H G \\
T I \\
\text { PB } \\
\text { BI } \\
\text { PO }\end{array}$ \\
\hline $\begin{array}{l}85 \\
86 \\
87 \\
88 \\
89\end{array}$ & $\begin{array}{l}A T \\
R N \\
F R \\
R A \\
A C\end{array}$ & $\begin{array}{l}0.469 \\
0.468 \\
0.467 \\
0.466 \\
0.465\end{array}$ & $\begin{array}{l}0.753 \\
0.752 \\
0.751 \\
0.751 \\
0.751\end{array}$ & $\begin{array}{l}0.045 \\
0.046 \\
0.047 \\
0.048 \\
0.049\end{array}$ & $\begin{array}{l}0.320 \\
0.330 \\
0.339 \\
0.348 \\
0.356\end{array}$ & $\begin{array}{l}0.111 \\
0.110 \\
0.109 \\
0.108 \\
0.108\end{array}$ & $\begin{array}{l}0.273 \\
0.280 \\
0.287 \\
0.295 \\
0.302\end{array}$ & $\begin{array}{l}0.506 \\
0.595 \\
0.597 \\
0.595 \\
0.597\end{array}$ & $\begin{array}{l}85 \\
86 \\
87 \\
88 \\
89\end{array}$ & $\begin{array}{l}\text { AT } \\
\text { BN } \\
\text { FR } \\
\text { BA } \\
\text { AC }\end{array}$ \\
\hline $\begin{array}{l}90 \\
91 \\
92 \\
93 \\
94\end{array}$ & $\begin{array}{l}\text { TH } \\
\text { PA } \\
\text { U } \\
\text { NP } \\
\text { PU }\end{array}$ & $\begin{array}{l}0.463 \\
0.463 \\
0.461 \\
0.460 \\
0.459\end{array}$ & $\begin{array}{l}0.750 \\
0.750 \\
0.749 \\
0.749 \\
0.749\end{array}$ & $\begin{array}{l}0.051 \\
0.050 \\
0.053 \\
0.055 \\
0.059\end{array}$ & $\begin{array}{l}0.363 \\
0.357 \\
0.357 \\
0.350 \\
0.148\end{array}$ & $\begin{array}{l}0.109 \\
0.139 \\
0.157 \\
0.192 \\
0.198\end{array}$ & $\begin{array}{l}0.310 \\
0.318 \\
0.326 \\
0.333 \\
0.340\end{array}$ & $\begin{array}{l}0.588 \\
0.600 \\
0.593 \\
0.594 \\
0.583\end{array}$ & $\begin{array}{l}90 \\
91 \\
92 \\
93 \\
94\end{array}$ & $\begin{array}{l}\text { Tम } \\
\mathrm{PA} \\
\text { U } \\
\text { NP } \\
\mathrm{PU}\end{array}$ \\
\hline $\begin{array}{l}95 \\
96 \\
97 \\
98 \\
99\end{array}$ & $\begin{array}{l}A M \\
C A \\
B K \\
C F \\
E S\end{array}$ & $\begin{array}{l}0.458 \\
0.457 \\
0.455 \\
0.454 \\
0.453\end{array}$ & $\begin{array}{l}0.749 \\
0.748 \\
0.748 \\
0.748 \\
0.747\end{array}$ & $\begin{array}{l}0.052 \\
0.053 \\
0.063 \\
0.063 \\
0.054\end{array}$ & $\begin{array}{l}0.352 \\
0.357 \\
0.360 \\
0.363 \\
0.368\end{array}$ & $\begin{array}{l}0.203 \\
0.200 \\
0.198 \\
0.197 \\
0.196\end{array}$ & $\begin{array}{l}0.347 \\
0.355 \\
0.361 \\
0.366 \\
0.372\end{array}$ & $\begin{array}{l}0.574 \\
0.574 \\
0.565 \\
0.565 \\
0.567\end{array}$ & $\begin{array}{l}95 \\
96 \\
97 \\
98 \\
99\end{array}$ & $\begin{array}{l}A M \\
C M \\
B K \\
C F \\
E S\end{array}$ \\
\hline $\begin{array}{l}100 \\
101 \\
102 \\
103 \\
104\end{array}$ & $\begin{array}{l}\text { FM } \\
\text { MD } \\
\text { NO } \\
\text { LR }\end{array}$ & $\begin{array}{l}0.452 \\
0.450 \\
0.449 \\
0.448 \\
0.446\end{array}$ & $\begin{array}{l}0.747 \\
0.747 \\
0.746 \\
0.747 \\
0.746\end{array}$ & $\begin{array}{l}0.054 \\
0.064 \\
0.054 \\
0.052 \\
0.062\end{array}$ & $\begin{array}{l}0.373 \\
0.379 \\
0.384 \\
0.390 \\
0.397\end{array}$ & $\begin{array}{l}0.194 \\
0.191 \\
0.199 \\
0.195 \\
0.181\end{array}$ & $\begin{array}{l}0.377 \\
0.382 \\
0.386 \\
0.390 \\
0.393\end{array}$ & $\begin{array}{l}0.559 \\
0.560 \\
0.552 \\
0.562 \\
0.555\end{array}$ & $\begin{array}{l}100 \\
101 \\
102 \\
103 \\
104\end{array}$ & $\begin{array}{l}\text { FM } \\
\text { KD } \\
\text { NO } \\
\text { LR }\end{array}$ \\
\hline $\begin{array}{l}105 \\
106 \\
107 \\
108 \\
109\end{array}$ & & & & & $\begin{array}{l}0.402 \\
0.408 \\
0.414 \\
0.421 \\
0.424\end{array}$ & $\begin{array}{l}0.178 \\
0.174 \\
0.171 \\
0.165 \\
0.163\end{array}$ & $\begin{array}{l}0.396 \\
0.399 \\
0.401 \\
0.403 \\
0.404\end{array}$ & $\begin{array}{l}0.547 \\
0.548 \\
0.542 \\
0.546 \\
0.540\end{array}$ & $\begin{array}{l}105 \\
106 \\
107 \\
108 \\
109\end{array}$ & \\
\hline 110 & & & & & 0.428 & 0.158 & 0.405 & 0.534 & 110 & \\
\hline
\end{tabular}


Table 18. Effective fluorescence yields for $L_{1}$ and $L_{2}$ subshells

Definittons: $v_{1}=\omega_{1}+\omega_{2} f_{1,2}+\omega_{3}\left(f_{1,3}+f^{\prime}{ }_{1,3}+f_{1,2} f_{2,3}\right)$, and $\nu_{2}=\omega_{2}+\omega_{3} f_{2,3}$. See also ref. 3 .

\begin{tabular}{|c|c|c|c|c|c|c|c|c|c|c|c|}
\hline \multicolumn{2}{|c|}{$Z$} & $\nu_{1}$ & $\nu_{2}$ & \multicolumn{2}{|c|}{$Z$} & $\frac{\nu_{1}}{0.067}$ & $\frac{\nu_{2}}{0.075}$ & \multicolumn{2}{|c|}{$Z$} & $\begin{array}{c}v_{1} \\
0.476\end{array}$ & $\frac{\nu_{2}}{0.525}$ \\
\hline $\begin{array}{l}10 \\
11 \\
12 \\
13 \\
14\end{array}$ & $\begin{array}{l}N E \\
N A \\
M G \\
A L \\
S I\end{array}$ & $\begin{array}{l}1.2 E-03 \\
7.5 E-04 \\
3.9 E-04\end{array}$ & $\begin{array}{l}1.2 E-03 \\
7.5 E-04 \\
3.7 E-04\end{array}$ & $\begin{array}{l}50 \\
51 \\
52 \\
53 \\
54\end{array}$ & $\begin{array}{l}\text { SN } \\
S B \\
T E \\
I \\
X E\end{array}$ & $\begin{array}{l}0.067 \\
0.072 \\
0.077 \\
0.083 \\
0.088\end{array}$ & $\begin{array}{l}0.075 \\
0.080 \\
0.085 \\
0.091 \\
0.096\end{array}$ & $\begin{array}{l}90 \\
91 \\
92 \\
93 \\
94\end{array}$ & $\begin{array}{l}\text { TH } \\
\text { PA } \\
\text { D } \\
\text { N P } \\
\text { PU }\end{array}$ & $\begin{array}{l}0.476 \\
0.485 \\
0.503 \\
0.518 \\
0.528\end{array}$ & $\begin{array}{l}0.529 \\
0.538 \\
0.549 \\
0.562 \\
0.566\end{array}$ \\
\hline $\begin{array}{l}15 \\
16 \\
17 \\
18 \\
19\end{array}$ & $\begin{array}{l}\text { F } \\
S \\
C L \\
A F \\
K\end{array}$ & $\begin{array}{l}3.3 E-04 \\
3.2 E-04 \\
3.5 E-04 \\
3.8 E-04 \\
4.9 F-04\end{array}$ & $\begin{array}{l}3.1 E-04 \\
2.6 F-04 \\
2.4 E-04 \\
2.2 E-04 \\
2.7 E-04\end{array}$ & $\begin{array}{l}55 \\
56 \\
57 \\
58 \\
59\end{array}$ & $\begin{array}{l}\text { CS } \\
\text { BA } \\
\text { LA } \\
\text { CF } \\
\text { PR }\end{array}$ & $\begin{array}{l}0.094 \\
0.100 \\
0.108 \\
0.114 \\
0.121\end{array}$ & $\begin{array}{l}0.104 \\
0.111 \\
0.119 \\
0.127 \\
0.135\end{array}$ & $\begin{array}{l}95 \\
96 \\
97 \\
98 \\
99\end{array}$ & $\begin{array}{l}\text { A H } \\
\text { CM } \\
\text { BR } \\
\text { CF } \\
\text { ES }\end{array}$ & $\begin{array}{l}0.544 \\
0.557 \\
0.566 \\
0.575 \\
0.591\end{array}$ & $\begin{array}{l}0.578 \\
0.587 \\
0.594 \\
0.600 \\
0.609\end{array}$ \\
\hline $\begin{array}{l}20 \\
21 \\
22 \\
23 \\
24\end{array}$ & $\begin{array}{l}C A \\
S C \\
T I \\
V \\
C R\end{array}$ & $\begin{array}{l}6.1 E-04 \\
1.2 E-03 \\
1.9 E-03 \\
2.9 E-03 \\
4.0 E-C Z\end{array}$ & $\begin{array}{l}3.3 E-04 \\
8.4 E-04 \\
1.5 E-03 \\
2.6 E-03 \\
3.7 E-03\end{array}$ & $\begin{array}{l}60 \\
61 \\
62 \\
63 \\
64\end{array}$ & $\begin{array}{l}\text { ND } \\
\text { PM } \\
\text { SM } \\
\text { EU } \\
\text { GD }\end{array}$ & $\begin{array}{l}0.129 \\
0.135 \\
0.143 \\
0.152 \\
0.160\end{array}$ & $\begin{array}{c}0.143 \\
0.152 \\
0.161 \\
0.171 \\
0.181\end{array}$ & $\begin{array}{l}100 \\
101 \\
102 \\
103 \\
104\end{array}$ & $\begin{array}{l}\text { FN } \\
\text { MD } \\
\text { NO } \\
\text { LR }\end{array}$ & $\begin{array}{l}0.602 \\
0.611 \\
0.619 \\
0.627 \\
0.635\end{array}$ & $\begin{array}{l}0.618 \\
0.627 \\
0.637 \\
0.645 \\
0.655\end{array}$ \\
\hline $\begin{array}{l}25 \\
26 \\
27 \\
28 \\
29\end{array}$ & $\begin{array}{l}M N \\
F E \\
C O \\
N I \\
C D\end{array}$ & $\begin{array}{r}5.2 E-C 3 \\
6.5 E-03 \\
7.8 E-03 \\
9.2 E-03 \\
0.011\end{array}$ & $\begin{array}{r}5.0 \mathrm{E}-03 \\
6.3 \mathrm{E}-03 \\
7.7 \mathrm{E}-03 \\
8.9 \mathrm{E}-03 \\
0.010\end{array}$ & $\begin{array}{l}65 \\
66 \\
67 \\
68 \\
69\end{array}$ & $\begin{array}{l}\text { PB } \\
\text { DY } \\
\text { HO } \\
\text { ER } \\
\text { TM }\end{array}$ & $\begin{array}{l}0.169 \\
0.180 \\
0.190 \\
0.201 \\
0.210\end{array}$ & $\begin{array}{l}0.191 \\
0.203 \\
0.215 \\
0.227 \\
0.239\end{array}$ & $\begin{array}{l}105 \\
106 \\
107 \\
108 \\
109\end{array}$ & & $\begin{array}{l}0.643 \\
0.652 \\
0.661 \\
0.678 \\
0.689\end{array}$ & $\begin{array}{l}0.663 \\
0.671 \\
0.681 \\
0.689 \\
0.694\end{array}$ \\
\hline $\begin{array}{l}30 \\
31 \\
32 \\
33 \\
34\end{array}$ & $\begin{array}{l}\text { ZN } \\
\text { GA } \\
\text { GE } \\
\text { AS } \\
\text { SE }\end{array}$ & $\begin{array}{l}0.012 \\
0.013 \\
0.014 \\
0.015 \\
0.017\end{array}$ & $\begin{array}{l}0.011 \\
0.012 \\
0.014 \\
0.015 \\
0.017\end{array}$ & $\begin{array}{l}70 \\
79 \\
72 \\
73 \\
74\end{array}$ & $\begin{array}{l}\text { YB } \\
\text { L I } \\
\text { HP } \\
\text { TA } \\
\text { W }\end{array}$ & $\begin{array}{l}0.221 \\
0.232 \\
0.243 \\
0.258 \\
0.271\end{array}$ & $\begin{array}{l}0.251 \\
0.264 \\
0.277 \\
0.291 \\
0.304\end{array}$ & 110 & & 0.698 & 0.700 \\
\hline $\begin{array}{l}35 \\
36 \\
37 \\
38 \\
39\end{array}$ & $\begin{array}{l}E R \\
K B \\
R B \\
\text { S R } \\
Y\end{array}$ & $\begin{array}{l}0.020 \\
0.022 \\
0.024 \\
0.026 \\
0.028\end{array}$ & $\begin{array}{l}0.020 \\
0.022 \\
0.025 \\
0.027 \\
0.030\end{array}$ & $\begin{array}{l}75 \\
76 \\
77 \\
78 \\
79\end{array}$ & $\begin{array}{l}\text { RE } \\
\text { OS } \\
\text { I R } \\
\text { PT } \\
\text { AU }\end{array}$ & $\begin{array}{l}0.234 \\
0.293 \\
0.305 \\
0.318 \\
0.330\end{array}$ & $\begin{array}{l}0.318 \\
0.331 \\
0.345 \\
0.359 \\
0.373\end{array}$ & & & & \\
\hline $\begin{array}{l}40 \\
41 \\
42 \\
43 \\
44\end{array}$ & $\begin{array}{l}2 R \\
\text { NE } \\
\text { MO } \\
\text { TC } \\
\text { RU }\end{array}$ & $\begin{array}{l}0.031 \\
0.034 \\
0.036 \\
0.040 \\
0.043\end{array}$ & $\begin{array}{l}0.032 \\
0.036 \\
0.039 \\
0.043 \\
0.046\end{array}$ & $\begin{array}{l}80 \\
81 \\
82 \\
83 \\
84\end{array}$ & $\begin{array}{l}\mathrm{HS} \\
\text { TL } \\
\text { PB } \\
\text { BI } \\
\text { FO }\end{array}$ & $\begin{array}{l}0.345 \\
0.358 \\
0.372 \\
0.382 \\
0.396\end{array}$ & $\begin{array}{l}0.387 \\
0.401 \\
0.415 \\
0.429 \\
0.444\end{array}$ & & & & \\
\hline $\begin{array}{l}45 \\
46 \\
47 \\
48 \\
49\end{array}$ & $\begin{array}{l}\text { RH } \\
P D \\
A G \\
C D \\
\text { IN }\end{array}$ & $\begin{array}{l}0.046 \\
0.049 \\
0.053 \\
0.058 \\
0.062\end{array}$ & $\begin{array}{l}0.050 \\
0.054 \\
0.059 \\
0.065 \\
0.070\end{array}$ & $\begin{array}{l}85 \\
86 \\
87 \\
88 \\
89\end{array}$ & $\begin{array}{l}\text { AT } \\
\text { RN } \\
\text { FR } \\
\text { RA } \\
\text { AC }\end{array}$ & $\begin{array}{l}0.411 \\
0.422 \\
0.436 \\
0.448 \\
0.464\end{array}$ & $\begin{array}{l}0.459 \\
0.474 \\
0.489 \\
0.503 \\
0.517\end{array}$ & & & & \\
\hline
\end{tabular}


Table 19. Average fluorescence yield for $L$ shell of the elements $Z=28(4) 100$

Definition $\bar{\omega}_{L}=\left(\sigma_{L_{1}} \nu_{1}+\sigma_{L_{2}} \nu_{2}+\sigma_{L_{3}} \omega_{3}\right) / \sigma_{L}$

Values are calculated for photon energies just above the $L_{1}$ edge and just below the $K$ edge

Effective fluorescence yields $\nu_{1}, \nu_{2}$, and $\omega_{3}$ are given for comparison See also Tables 15 and 18

\begin{tabular}{|c|c|c|c|c|c|c|c|c|}
\hline 2 & $z$ & $E_{L_{1}}$ & $\bar{\omega}_{L}$ & $E_{K}$ & $\bar{\omega}_{L}$ & $v_{1}$ & $\nu_{2}$ & $\omega_{3}$ \\
\hline $\begin{array}{l}28 \\
32 \\
36 \\
40\end{array}$ & $\begin{array}{l}\text { NI } \\
\text { GE } \\
\text { K R } \\
\text { Z R }\end{array}$ & $\begin{array}{l}1.01 \\
1.41 \\
1.92 \\
2.53\end{array}$ & $\begin{array}{l}9.15 \mathrm{E}-03 \\
1.45 \mathrm{E}-02 \\
2.20 \mathrm{E}-02 \\
3.14 \mathrm{E}-02\end{array}$ & $\begin{array}{r}3.36 \\
11.14 \\
14.38 \\
19.09\end{array}$ & $\begin{array}{l}\text { 9. } 16 E-03 \\
\text { 1. } 44 E-02 \\
2.18 E-02 \\
\text { 3. } 13 E-02\end{array}$ & $\begin{array}{l}9.17 E-03 \\
1.42 F-02 \\
2.15 E-02 \\
3.13 E-02\end{array}$ & $\begin{array}{l}8.86 \mathrm{E}-03 \\
1.37 \mathrm{E}-02 \\
2.22 \mathrm{E}-02 \\
3.21 \mathrm{E}-02\end{array}$ & $\begin{array}{l}9.30 E-03 \\
1.50 E-02 \\
2.20 E-02 \\
3.10 E-02\end{array}$ \\
\hline $\begin{array}{l}44 \\
48 \\
52 \\
56 \\
60\end{array}$ & $\begin{array}{l}R \mathrm{BU} \\
\mathrm{CD} \\
\mathrm{TE} \\
\mathrm{BA} \\
\mathrm{ND}\end{array}$ & $\begin{array}{l}3.23 \\
4.00 \\
4.95 \\
6.00 \\
7.14\end{array}$ & $\begin{array}{l}4.40 \mathrm{E}-02 \\
5.88 \mathrm{E}-02 \\
7.79 \mathrm{E}-02 \\
1.02 \mathrm{E}-01 \\
1.31 \mathrm{E}-01\end{array}$ & $\begin{array}{l}22.24 \\
26.88 \\
32.03 \\
37.71 \\
43.91\end{array}$ & $\begin{array}{l}\text { 4. } 35 \mathrm{E}-02 \\
5.34 \mathrm{E}-02 \\
\text { 7. } 78 \mathrm{E}-02 \\
\text { 1. } 01 \mathrm{E}-01 \\
1.31 \mathrm{E}-01\end{array}$ & $\begin{array}{l}4.29 \mathrm{E}-02 \\
5.75 \mathrm{E}-02 \\
7.71 \mathrm{E}-02 \\
1.00 \mathrm{E}-01 \\
1.29 \mathrm{E}-01\end{array}$ & $\begin{array}{l}4.64 \mathrm{E}-02 \\
6.47 \mathrm{E}-02 \\
8.55 \mathrm{E}-02 \\
1.11 \mathrm{E}-01 \\
1.43 \mathrm{E}-01\end{array}$ & $\begin{array}{l}4.30 E-02 \\
5.60 E-02 \\
7.40 F-02 \\
9.70 E-02 \\
1.25 E-01\end{array}$ \\
\hline $\begin{array}{l}64 \\
68 \\
72 \\
76 \\
80\end{array}$ & $\begin{array}{l}\text { GD } \\
\text { ER } \\
\text { HF } \\
\text { OS } \\
\text { HG }\end{array}$ & $\begin{array}{r}8.41 \\
9.79 \\
11.33 \\
13.04 \\
14.94\end{array}$ & $\begin{array}{l}1.64 \mathrm{E}-01 \\
2.04 \mathrm{E}-01 \\
2.48 \mathrm{E}-01 \\
2.99 \mathrm{E}-01 \\
3.53 \mathrm{E}-01\end{array}$ & $\begin{array}{l}53.66 \\
57.99 \\
65.96 \\
74.62 \\
83.99\end{array}$ & $\begin{array}{l}1.63 \mathrm{E}-01 \\
2.04 \mathrm{E}-01 \\
2.48 \mathrm{E}-01 \\
2.99 \mathrm{E}-01 \\
3.52 \mathrm{E}-01\end{array}$ & $\begin{array}{l}1.60 \mathrm{E}-01 \\
2.01 \mathrm{E}-01 \\
2.43 \mathrm{E}-01 \\
2.93 \mathrm{E}-01 \\
3.45 \mathrm{E}-01\end{array}$ & $\begin{array}{l}1.81 \mathrm{E}-01 \\
2.27 \mathrm{E}-01 \\
2.77 \mathrm{E}-01 \\
3.31 \mathrm{E}-01 \\
3.87 \mathrm{E}-01\end{array}$ & $\begin{array}{l}1.55 E-01 \\
1.92 E-01 \\
2.31 F-01 \\
2.81 F-01 \\
3.33 E-01\end{array}$ \\
\hline $\begin{array}{r}84 \\
88 \\
92 \\
96 \\
100\end{array}$ & $\begin{array}{l}\text { PO } \\
\text { RA } \\
\text { U } \\
\text { C I } \\
\text { P }\end{array}$ & $\begin{array}{l}17.05 \\
19.39 \\
21.95 \\
24.77 \\
27.89\end{array}$ & $\begin{array}{l}4.07 E-01 \\
4.62 E-01 \\
5.12 E-01 \\
5.59 E-01 \\
5.99 E-01\end{array}$ & $\begin{array}{r}94.15 \\
105.17 \\
117.10 \\
130.03 \\
144.09\end{array}$ & $\begin{array}{l}4.06 \mathrm{E}-01 \\
4.61 \mathrm{E}-01 \\
5.14 \mathrm{E}-01 \\
5.62 \mathrm{E}-01 \\
6.02 \mathrm{E}-01\end{array}$ & $\begin{array}{l}3.96 \mathrm{E}-01 \\
4.48 \mathrm{E}-01 \\
5.03 \mathrm{E}-01 \\
5.57 \mathrm{E}-01 \\
6.02 \mathrm{E}-01\end{array}$ & $\begin{array}{l}4.44 E-01 \\
5.03 E-01 \\
5.49 E-01 \\
5.87 E-01 \\
6.18 E-01\end{array}$ & $\begin{array}{l}3.86 F-01 \\
4.37 F-01 \\
4.89 E-01 \\
5.39 E-01 \\
5.79 F-01\end{array}$ \\
\hline
\end{tabular}

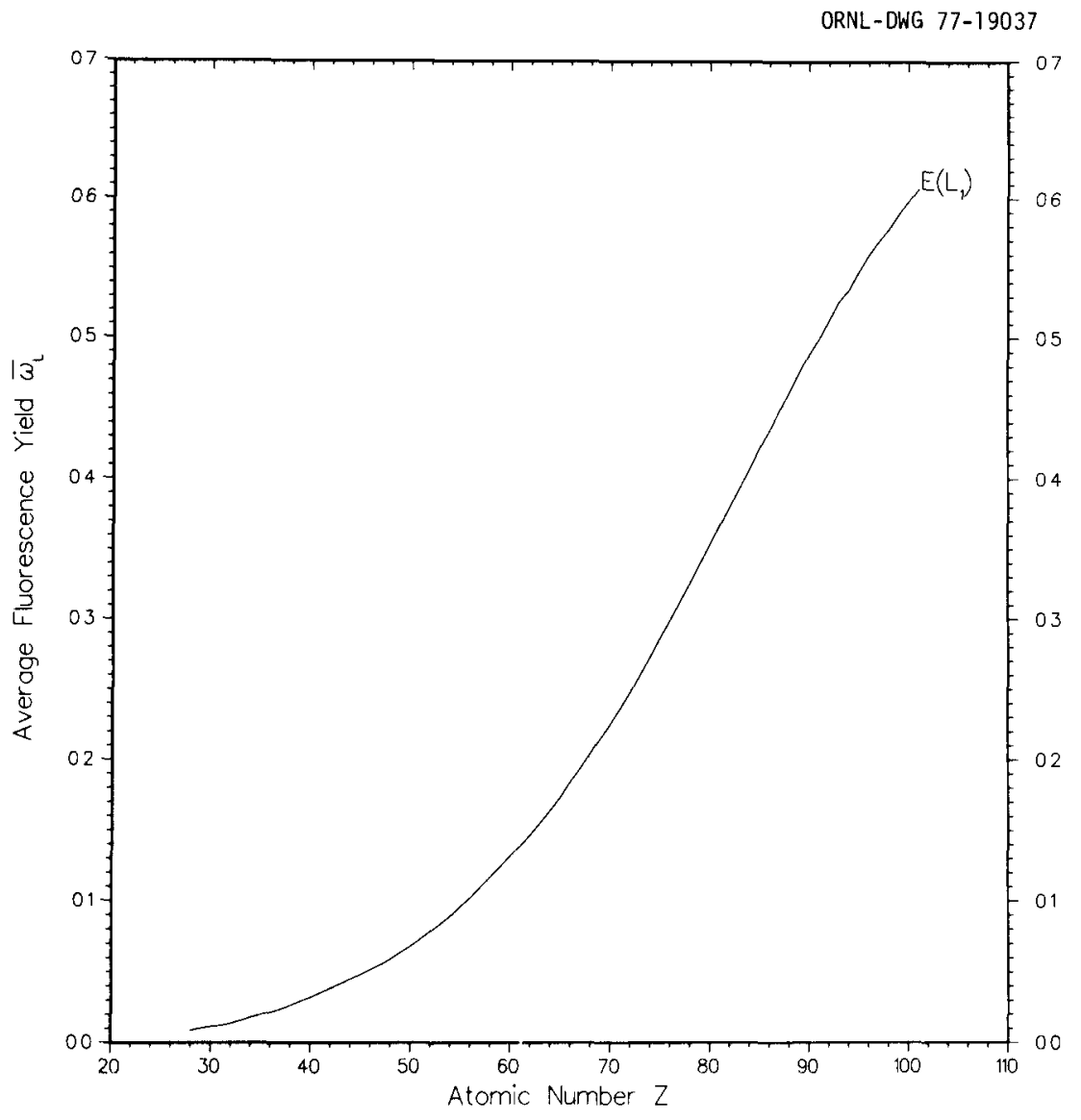

Fig. 34. Average fluorescence yield for $L$ shell calculated for photolonization just above the $L_{1}$ absorption edge. Note, however, that the average fluorescence yield is insensitive to the initial hole distribution as shown in Table 19. 
Table 20. Total $L$-shell $\mathrm{x}$-ray fluorescence cross sections

Values are in barns/atom; photon energy is in $\mathrm{keV}$. Energy range between $L_{3}$ and $K$ absorption edges is covered. From refs. 2 and 3 . This cross section is defined as $\sigma_{T, R}=\sigma_{L_{1}} \nu_{1}+\sigma_{L_{2}} \nu_{2}+\sigma_{L_{3}} \omega_{3}$

\begin{tabular}{|c|c|c|c|c|c|c|c|c|c|c|c|c|c|c|}
\hline$h \nu$ & ${ }^{\sigma} T_{T, R}$ & & $h \nu$ & ${ }^{o} T, R$ & & $h \nu$ & $\sigma_{T, R}$ & & $h \nu$ & $o_{T, R}$ & & $h v$ & $o_{T, R}$ & \\
\hline$M G \quad z$ & $=12$ & & SC 2 & $=21$ & & N I Z & $=28$ & & GE Z & $=32$ & & RR Z & $=36$ & \\
\hline 1.00 & $4.30 \mathrm{E}$ & 01 & 4.00 & 8. $76 \mathrm{E}$ & 00 & 1.00 & $7.63 \mathrm{E}$ & 03 & 2.00 & $4.12 \mathrm{E}$ & 03 & 3.00 & $3.39 \mathrm{E}$ & 03 \\
\hline 1.29 & $2.15 E$ & 09 & 4.46 & $6.51 \mathrm{~F}$ & 00 & $\begin{array}{l}1.01 \\
1.01\end{array}$ & $\begin{array}{l}7.53 \mathrm{E} \\
8.56 \mathrm{E}\end{array}$ & $\begin{array}{l}03 \\
03\end{array}$ & $\begin{array}{l}3.00 \\
4.00\end{array}$ & $\begin{array}{l}1.46 \mathrm{E} \\
6.76 \mathrm{~F}\end{array}$ & $\begin{array}{l}03 \\
02\end{array}$ & $\begin{array}{l}4.00 \\
5.00\end{array}$ & $\begin{array}{l}1.60 \mathrm{E} \\
8.82 \mathrm{E}\end{array}$ & $\begin{array}{l}03 \\
02\end{array}$ \\
\hline$A L \quad Z$ & $=13$ & & T I 2 & $=22$ & & $\begin{array}{l}1.50 \\
2.00\end{array}$ & $\begin{array}{l}3.34 \mathrm{E} \\
1.61 \mathrm{E}\end{array}$ & $\begin{array}{l}03 \\
03\end{array}$ & & $\begin{array}{l}3.69 \mathrm{~F} \\
2.23 \mathrm{E}\end{array}$ & $\begin{array}{l}02 \\
02\end{array}$ & $\begin{array}{l}6.00 \\
8.00\end{array}$ & $\begin{array}{l}5.398 \\
2.44 E\end{array}$ & $\begin{array}{l}02 \\
02\end{array}$ \\
\hline $\begin{array}{l}1.00 \\
1.50\end{array}$ & $3 . \varepsilon 2 F$ & 01 & 1.00 & $6.57 \mathrm{E}$ & 02 & 3.00 & $5.55 \mathrm{P}$ & 02 & 8.00 & $1.00 \mathrm{E}$ & 02 & 10.00 & 1. $31 \mathrm{~F}$ & 02 \\
\hline $\begin{array}{l}1.50 \\
1.55\end{array}$ & $\begin{array}{l}1.28 \mathrm{E} \\
1.18 \mathrm{E}\end{array}$ & $\begin{array}{l}01 \\
01\end{array}$ & $\begin{array}{l}1.50 \\
2.00\end{array}$ & $\begin{array}{l}2.37 \mathrm{~F} \\
1.12 \mathrm{~F}\end{array}$ & $\begin{array}{l}02 \\
02\end{array}$ & $\begin{array}{l}4.00 \\
5.00\end{array}$ & $\begin{array}{l}2.55 \mathrm{E} \\
1.38 \mathrm{E}\end{array}$ & $\begin{array}{l}02 \\
02\end{array}$ & $\begin{array}{l}10.00 \\
11.06\end{array}$ & $\begin{array}{l}5.35 \mathrm{E} \\
4.03 \mathrm{~F}\end{array}$ & $\begin{array}{l}01 \\
01\end{array}$ & 14.27 & $4.79 E$ & 01 \\
\hline & & & 3.00 & $3.81 \mathrm{E}$ & 01 & 6.00 & $8.32 \mathrm{E}$ & 01 & & & & RB Z & $=37$ & \\
\hline SI Z & $=14$ & & $\begin{array}{l}4.00 \\
4.94\end{array}$ & $\begin{array}{l}1.75 \mathrm{~F} \\
9.82 \mathrm{~F}\end{array}$ & $\begin{array}{l}01 \\
00\end{array}$ & $\begin{array}{l}8.00 \\
8.29\end{array}$ & $\begin{array}{l}3.72 \mathrm{E} \\
3.36 \mathrm{E}\end{array}$ & $\begin{array}{l}01 \\
01\end{array}$ & AS Z & $=33$ & & 1.82 & $7.73 \mathrm{E}$ & 03 \\
\hline $\begin{array}{l}1.00 \\
1.50\end{array}$ & $2.67 \mathrm{~F}$ & 01 & & & & & & & 1. 34 & $6.79 \mathrm{E}$ & 03 & & $\begin{array}{l}7.29 \mathrm{E} \\
1.10 \mathrm{E}\end{array}$ & $\begin{array}{l}03 \\
04\end{array}$ \\
\hline $\begin{array}{l}1.50 \\
1.83\end{array}$ & $\begin{array}{l}9.08 E \\
5.32 E\end{array}$ & $\begin{array}{ll}0 & 0 \\
0 & 0\end{array}$ & 2 & $=2.3$ & & $\mathrm{CU} \mathrm{Z}$ & $=29$ & & $\begin{array}{l}1.36 \\
1.38\end{array}$ & $\begin{array}{l}6.84 \mathrm{E} \\
9.99 \mathrm{E}\end{array}$ & $\begin{array}{l}03 \\
03\end{array}$ & $\begin{array}{l}1.88 \\
2.00\end{array}$ & $\begin{array}{l}1.10 \mathrm{E} \\
9.64 \mathrm{E}\end{array}$ & $\begin{array}{l}04 \\
03\end{array}$ \\
\hline $\mathbf{z}$ & $=15$ & & $\begin{array}{l}1.00 \\
1.50 \\
2.00\end{array}$ & $\begin{array}{l}1.31 E \\
4.73 E \\
2.24 E\end{array}$ & $\begin{array}{l}03 \\
02 \\
02\end{array}$ & $\begin{array}{l}1.00 \\
1.08 \\
1.09\end{array}$ & $\begin{array}{l}1.05 E \\
9.335 \\
9.40 \mathrm{~F}\end{array}$ & $\begin{array}{l}04 \\
03 \\
03\end{array}$ & $\begin{array}{l}1 . \\
1 .\end{array}$ & $\begin{array}{l}8.61 \mathrm{E} \\
8.36 \mathrm{E} \\
9.44 \mathrm{E}\end{array}$ & $\begin{array}{l}03 \\
03 \\
03\end{array}$ & & $\begin{array}{l}9.13 \mathrm{E} \\
1.05 \mathrm{E} \\
4.10 \mathrm{E}\end{array}$ & $\begin{array}{l}03 \\
04 \\
03\end{array}$ \\
\hline 1.00 & 2. $97 \mathrm{E}$ & 01 & 3.00 & $7.57 \mathrm{~F}$ & 01 & 1.50 & $4.42 \mathrm{~F}$ & 03 & 2.00 & $4.93 F$ & 03 & 4.00 & $1.95 \mathrm{E}$ & 03 \\
\hline 1.50 & $1.02 \mathrm{~F}$ & 01 & 4.00 & 3.465 & 01 & 2.00 & $2.15 \mathrm{~F}$ & 03 & 3. & $1.76 \mathrm{E}$ & 63 & 5.00 & 1.0 & 03 \\
\hline .00 & $4.67 \mathrm{E}$ & 00 & 5.00 & 1. $\mathrm{A7F}$ & 01 & 3.00 & $7.44 \mathrm{~F}$ & 02 & 4.00 & $8.21 \mathrm{E}$ & 02 & 6.00 & 6.5 & 02 \\
\hline 2.13 & $3.54 \mathrm{E}$ & 00 & 5.43 & $1.48 F$ & 01 & $\begin{array}{l}4.00 \\
5.00\end{array}$ & $\begin{array}{l}3.42 \% \\
1.86 \%\end{array}$ & $\begin{array}{l}02 \\
02\end{array}$ & $\begin{array}{l}5.00 \\
6.00\end{array}$ & $\begin{array}{l}4.49 E \\
2.73 E\end{array}$ & $\begin{array}{l}02 \\
\mathrm{C2}\end{array}$ & $\begin{array}{r}8.00 \\
10.00\end{array}$ & $\begin{array}{l}2.94 \mathrm{E} \\
1.60 \mathrm{E}\end{array}$ & $\begin{array}{l}02 \\
02\end{array}$ \\
\hline S Z & $=16$ & & $C R \quad Z$ & $=24$ & & $\begin{array}{l}6.00 \\
8.00\end{array}$ & $\begin{array}{l}1.12 \mathrm{E} \\
5.01 \mathrm{~F}\end{array}$ & $\begin{array}{l}02 \\
01\end{array}$ & $\begin{array}{r}3.00 \\
10.00\end{array}$ & $\begin{array}{l}1.23 \mathrm{E} \\
6.57 \mathrm{E}\end{array}$ & $\begin{array}{l}02 \\
01\end{array}$ & $\begin{array}{l}15.00 \\
15.14\end{array}$ & $\begin{array}{l}5.12 \mathrm{E} \\
4.98 \mathrm{~B}\end{array}$ & $\begin{array}{l}01 \\
01\end{array}$ \\
\hline 1.00 & $3.41 \mathrm{E}$ & 01 & 1.00 & $2.15 \mathrm{~F}$ & 03 & 9.93 & $3.67 \mathrm{E}$ & 01 & 11.82 & $4.10 \mathrm{~F}$ & 01 & & & \\
\hline 1.50 & $1.18 \mathrm{E}$ & 01 & 1.50 & $7.82 F$ & 0 & & & & & & & SR $\mathrm{Z}$ & $=38$ & \\
\hline 2.00 & $5.51 \mathrm{~F}$ & 00 & 2.00 & $3.71 \mathrm{~F}$ & 02 & $\mathrm{ZN}$ & $=30$ & & $5: 2$ & $=34$ & & & & \\
\hline 2.45 & $3.18 \mathrm{E}$ & 00 & 3.00 & $1.26 \mathrm{~F}$ & 0 & & & & & & & 1.96 & $7.80 \mathrm{E}$ & 03 \\
\hline & & & 4.00 & $5.73 \mathrm{~F}$ & 01 & 1.03 & $5.09 \mathrm{E}$ & 03 & 1.45 & $7.49 \mathrm{E}$ & 03 & 2.00 & $7.27 \mathrm{E}$ & 03 \\
\hline$C L \quad z$ & $=17$ & & $\begin{array}{l}5.00 \\
5.95\end{array}$ & $\begin{array}{l}3.10 \mathrm{E} \\
1.91 \mathrm{~F}\end{array}$ & $\begin{array}{l}01 \\
01\end{array}$ & $\begin{array}{l}1.05 \\
1.06\end{array}$ & $\begin{array}{l}7.29 F \\
9.68 F\end{array}$ & $\begin{array}{l}0 \\
0\end{array}$ & $\begin{array}{l}1.48 \\
1.49\end{array}$ & $\begin{array}{l}7.05 \mathrm{E} \\
1.06 \mathrm{E}\end{array}$ & $\begin{array}{l}03 \\
04\end{array}$ & $\begin{array}{l}2.01 \\
2.03\end{array}$ & $\begin{array}{l}7.20 \mathrm{E} \\
1.10 \mathrm{~F}\end{array}$ & $\begin{array}{l}03 \\
04\end{array}$ \\
\hline 1.00 & $4.25 F$ & 01 & & & & 1.18 & $8.30 \mathrm{~F}$ & 03 & 1.50 & $1.04 \mathrm{~F}$ & 04 & 2.20 & $9.10 \mathrm{E}$ & 03 \\
\hline 1.50 & $1.51 \mathrm{E}$ & 01 & $M N Z$ & $=25$ & & 1.19 & $9.41 \mathrm{E}$ & 03 & 1.64 & $8.69 \mathrm{E}$ & 03 & 2.22 & 1. C $4 F$ & 04 \\
\hline 2.00 & $7.13 \mathrm{~F}$ & 00 & & & & 1.50 & $5.40 \mathrm{E}$ & 03 & 1.65 & $9.82 \mathrm{E}$ & C3 & 3.00 & $4.90 \mathrm{E}$ & 03 \\
\hline $2.8 \mathrm{C}$ & $2 . c 1 F$ & 00 & $\begin{array}{l}1.00 \\
1.50\end{array}$ & $\begin{array}{l}3.32 F \\
1.22 F\end{array}$ & $\begin{array}{l}03 \\
0.3\end{array}$ & $\begin{array}{l}2.00 \\
3.00\end{array}$ & $\begin{array}{l}2.65 \mathrm{~F} \\
9.24 \mathrm{E}\end{array}$ & $\begin{array}{l}03 \\
02\end{array}$ & $\begin{array}{l}2.00 \\
3.00\end{array}$ & $\begin{array}{l}6.19 \mathrm{E} \\
2.23 \mathrm{~F}\end{array}$ & $\begin{array}{l}03 \\
03\end{array}$ & $\begin{array}{l}4.00 \\
5.00\end{array}$ & $\begin{array}{l}2.34 \mathrm{P} \\
1.30 \mathrm{E}\end{array}$ & $\begin{array}{l}0.3 \\
03\end{array}$ \\
\hline AR $Z$ & $=18$ & & $\begin{array}{l}2.00 \\
3.00\end{array}$ & $\begin{array}{l}5.81 \mathrm{~F} \\
1.98 \mathrm{~F}\end{array}$ & $\begin{array}{l}02 \\
02\end{array}$ & $\begin{array}{l}4.00 \\
5.00\end{array}$ & $\begin{array}{l}4.26 \mathrm{E} \\
2.37 \mathrm{E}\end{array}$ & $\begin{array}{l}02 \\
02\end{array}$ & $\begin{array}{l}4.00 \\
5.00\end{array}$ & $\begin{array}{l}1.04 \mathrm{~F} \\
5.71 \mathrm{~F}\end{array}$ & $\begin{array}{l}03 \\
02\end{array}$ & $\begin{array}{l}6.00 \\
8.00\end{array}$ & $\begin{array}{l}7.948 \\
3.618\end{array}$ & $\begin{array}{l}02 \\
02\end{array}$ \\
\hline $\begin{array}{l}1.00 \\
1.50\end{array}$ & $\begin{array}{l}5.23 \mathrm{~F} \\
1.91 \mathrm{E}\end{array}$ & $\begin{array}{l}01 \\
01\end{array}$ & $\begin{array}{l}4.00 \\
5.00\end{array}$ & $\begin{array}{l}9.01 \mathrm{E} \\
4.88 \mathrm{E}\end{array}$ & $\begin{array}{l}01 \\
01\end{array}$ & $\begin{array}{l}6.00 \\
8.00\end{array}$ & $\begin{array}{l}1.40 \mathrm{E} \\
6.27 \mathrm{~F}\end{array}$ & $\begin{array}{l}02 \\
01\end{array}$ & $\begin{array}{l}6.00 \\
8.00\end{array}$ & $\begin{array}{l}3.47 \mathrm{E} \\
1.57 \mathrm{E}\end{array}$ & $\begin{array}{l}02 \\
02\end{array}$ & $\begin{array}{l}10.00 \\
15.00\end{array}$ & $\begin{array}{l}1.95 \mathrm{E} \\
6.22 \mathrm{E}\end{array}$ & $\begin{array}{l}02 \\
01\end{array}$ \\
\hline 2.00 & $9.14 \mathrm{E}$ & 00 & 6.00 & $2.94 \mathrm{~F}$ & 01 & 9.61 & $3.73 \mathrm{E}$ & 01 & 10.00 & B. $38 \mathrm{~F}$ & 01 & 96.05 & $5.13 \mathrm{~F}$ & 01 \\
\hline $\begin{array}{l}3.00 \\
7.17\end{array}$ & $3.17 \mathrm{E}$ & $0 C$ & 6.50 & $2.34 \mathrm{~F}$ & 01 & & & & 2.61 & $4.35 \mathrm{E}$ & 61 & & & \\
\hline 3.17 & $2.73 \mathrm{E}$ & & FE $Z$ & $=26$ & & GA Z & $=31$ & & BR Z & $=35$ & & $Y$ & $=39$ & \\
\hline k z & $=19$ & & 1.00 & $4.74 \mathrm{E}$ & 0 & $\begin{array}{l}1.13 \\
1.15\end{array}$ & $\begin{array}{l}5.54 \mathrm{E} \\
6.94 \mathrm{E}\end{array}$ & $\begin{array}{l}03 \\
03\end{array}$ & 1. 56 & 8.1 & & $\begin{array}{l}2.10 \\
2.16\end{array}$ & & $\begin{array}{l}03 \\
03\end{array}$ \\
\hline 1.00 & $7.54 \mathrm{E}$ & 01 & 1.50 & $1.77 \mathrm{~F}$ & 0 & 1.16 & $9.47 \mathrm{E}$ & 03 & 1.60 & 7.21 & & 2.17 & $1.09 \mathrm{E}$ & 04 \\
\hline 1.50 & $2.92 \mathrm{E}$ & 01 & 2.00 & $8.46 \mathrm{E}$ & 0 & 1.29 & $8.20 \mathrm{E}$ & 0 & 1.61 & 1.1 & C & 2.35 & $9.67 \%$ & 03 \\
\hline 2.00 & $1.41 \mathrm{~F}$ & 01 & 3.00 & $2.89 \mathrm{~F}$ & 0 & 1.30 & $9.27 \mathrm{~F}$ & 03 & 1.77 & 8.9 & & & 1. C4E & 04 \\
\hline 3.00 & 4. $\mathrm{C} 2 \mathrm{E}$ & $O C$ & 4.00 & $1.32 \mathrm{~F}$ & 0 & 1.50 & $6.57 \mathrm{E}$ & 03 & 1.78 & $1.01 \mathrm{E}$ & 04 & & $5.92 \mathrm{E}$ & 03 \\
\hline 3.58 & 3. $C 9 E$ & 00 & 5.00 & $7.14 \mathrm{~F}$ & 0 & 2.00 & $3.25 \mathrm{E}$ & 03 & 2.00 & $7.67 \mathrm{E}$ & 03 & & $2.79 \mathrm{E}$ & 03 \\
\hline & & & 6.00 & $4.30 \mathrm{~F}$ & 0 & 3.00 & $1.14 \mathrm{E}$ & 03 & 3.00 & $2.77 \mathrm{E}$ & 03 & 5.00 & $1.55 \mathrm{E}$ & 03 \\
\hline CA $\mathrm{Z}$ & $=20$ & & 7.08 & $2.71 \mathrm{~F}$ & 01 & $\begin{array}{l}4.00 \\
5.00\end{array}$ & $\begin{array}{l}5.27 \mathrm{E} \\
2.97 \mathrm{~F}\end{array}$ & $\begin{array}{l}02 \\
02\end{array}$ & $\begin{array}{l}4.00 \\
5.00\end{array}$ & $\begin{array}{l}1.30 \mathrm{~F} \\
7.16 \mathrm{E}\end{array}$ & $\begin{array}{l}03 \\
\mathrm{C} 2\end{array}$ & $\begin{array}{l}6.00 \\
8.00\end{array}$ & $\begin{array}{l}9.49 \mathrm{E} \\
4.34 \mathrm{E}\end{array}$ & $\begin{array}{l}02 \\
02\end{array}$ \\
\hline $\begin{array}{l}1.00 \\
1.50\end{array}$ & $\begin{array}{l}1.17 \mathrm{E} \\
4.35 \mathrm{E}\end{array}$ & $\begin{array}{l}02 \\
01\end{array}$ & $\mathrm{CO} 2$ & $=27$ & & $\begin{array}{l}6.00 \\
8.00\end{array}$ & $\begin{array}{l}1.74 \mathrm{E} \\
7.79 \mathrm{E}\end{array}$ & $\begin{array}{l}02 \\
01\end{array}$ & $\begin{array}{l}6.00 \\
8.00\end{array}$ & $\begin{array}{l}4.36 \mathrm{E} \\
1.97 \mathrm{E}\end{array}$ & $\begin{array}{l}02 \\
02\end{array}$ & $\begin{array}{l}10.00 \\
15.00\end{array}$ & $\begin{array}{l}2.34 \mathrm{E} \\
7.50 \mathrm{E}\end{array}$ & $\begin{array}{l}02 \\
01\end{array}$ \\
\hline 2.00 & $2.11 \mathrm{E}$ & 01 & 1.00 & $6.56 \mathrm{~F}$ & 03 & 10.00 & $4.15 \mathrm{~F}$ & 01 & 10.00 & 1.06 & 02 & 16.98 & $5.27 \mathrm{~F}$ & 01 \\
\hline 3.00 & $7.42 \mathrm{E}$ & 00 & 1.50 & $2.48 \mathrm{~F}$ & 03 & 10.32 & $3.80 \%$ & 01 & 13.42 & $4.60 \mathrm{E}$ & 01 & & & \\
\hline 4.00 & $3.48 E$ & on & 2.00 & $1.19 \mathrm{~F}$ & 03 & & & & & & & & & \\
\hline 4.01 & $3.45 \mathrm{E}$ & $O C$ & $\begin{array}{l}3.00 \\
4.00\end{array}$ & $\begin{array}{l}4.07 \mathrm{~F} \\
1.87 \mathrm{~F}\end{array}$ & $\begin{array}{l}02 \\
02\end{array}$ & $G F \quad Z$ & $=32$ & & $\mathrm{~K} \mathrm{R}$ & $=36$ & & & & \\
\hline SC $z$ & $=21$ & & 5.00 & $1.01 \mathrm{~F}$ & 02 & 1.23 & 5.44 & 0 & & 8.7 & & & & \\
\hline & & & & $6.09 \mathrm{~F}$ & 01 & 1.25 & $7.08 \mathrm{E}$ & 03 & 1.73 & $7.32 \mathrm{E}$ & & & & \\
\hline 1.00 & $3.21 \mathrm{E}$ & 02 & 7.67 & $3.05 \mathrm{~F}$ & 01 & 1.26 & $9.99 \mathrm{E}$ & 03 & 1.74 & $1.15 \mathrm{E}$ & 04 & & & \\
\hline 1.50 & $1.16 \mathrm{E}$ & 02 & & & & 1.40 & $8.51 \mathrm{E}$ & 0 & & $9.11 \mathrm{~F}$ & 03 & & & \\
\hline 2.00 & $5.53 \mathrm{~F}$ & 01 & & & & 1.41 & 9.597 & 0 & 1.92 & $1.03 \mathrm{E}$ & C4 & & & \\
\hline 3.00 & $1.90 \mathrm{~F}$ & 01 & & & & 1.50 & $8.33 \mathrm{~F}$ & 03 & 2.00 & $9.36 \mathrm{~F}$ & 03 & & & \\
\hline
\end{tabular}


Table 20 (contınued)

\begin{tabular}{|c|c|c|c|c|c|c|c|c|c|c|c|c|c|c|}
\hline$h v$ & $\sigma_{T R}$ & & $h v$ & $\sigma_{T R}$ & & $h v$ & $\sigma_{T R}$ & & $h \nu$ & $\sigma_{T R}$ & & $h \nu$ & $\sigma_{T R}$ & \\
\hline $2 R \quad Z$ & $=40$ & & RU 7 & $=44$ & & $C D \quad Z$ & $=48$ & & $\mathrm{TE} \mathrm{Z}$ & $=52$ & & BA 7 & $=56$ & \\
\hline 2.24 & R. $\mathrm{Cl}^{1 \mathrm{E}}$ & 03 & 2.99 & $1.15 \mathrm{~F}$ & 04 & 4.00 & $1.20 \mathrm{~F}$ & 04 & 3.00 & $3.54 \mathrm{E}$ & 03 & 37.41 & $8.90 \mathrm{~F}$ & 01 \\
\hline 2.31 & $7.25 \mathrm{~F}$ & 01 & 3.00 & $1.15 E$ & 04 & $4 . C 2$ & $1.17 \mathrm{E}$ & 04 & 10.00 & $1.96 \mathrm{~F}$ & C 3 & & & \\
\hline 2.33 & $1.11=$ & 04 & 3.20 & $9.78 \mathrm{~F}$ & 03 & 5.00 & $6.91 \mathrm{~F}$ & 03 & 15.00 & $6.50 \mathrm{~F}$ & $\mathrm{C} 2$ & LA Z & $=57$ & \\
\hline 2.51 & $9.23 \mathrm{~F}$ & 03 & 3.23 & $1.13 \mathrm{~F}$ & 04 & 6.00 & $4.24 \mathrm{E}$ & 03 & 20.00 & $2.93 E$ & $\mathrm{C} 2$ & & & \\
\hline 2.53 & 1. $C 6 E$ & 04 & 4.00 & 6.625 & 03 & 9.00 & $1.98 \mathrm{E}$ & 03 & 30.00 & $9.39 \mathrm{E}$ & 01 & 5.52 & R. $81 \mathrm{E}$ & 03 \\
\hline 3.00 & $7.02 \mathrm{E}$ & 03 & 5.00 & $3.72 \mathrm{~F}$ & 03 & 10.00 & $1.07 \mathrm{E}$ & 03 & 31.79 & $7.97 \mathrm{~F}$ & C 1 & & $7.53 \mathrm{~F}$ & 03 \\
\hline 4.00 & $3.36 \mathrm{~F}$ & 03 & 6.00 & $2.31 \mathrm{~F}$ & 03 & 15.00 & $3.57 \mathrm{~g}$ & 02 & & & & & $1.21 \mathrm{R}$ & 04 \\
\hline 5.00 & $1 . E R T$ & 03 & 2.00 & $1.07 \mathrm{~F}$ & 03 & 20.00 & 1.607 & 02 & $I$ & $=53$ & & 6.00 & $1.19 \mathrm{E}$ & 04 \\
\hline 0.00 & $1.15 \mathrm{E}$ & 03 & 10.00 & $5.79 F$ & 02 & 26.66 & $7.07 \mathrm{E}$ & 01 & & & & 24 & $1.07 \mathrm{~F}$ & 04 \\
\hline 8.00 & $5.24 E$ & 02 & 15.00 & $1.88 \mathrm{~F}$ & 02 & & & & 4.59 & $8.20 \mathrm{E}$ & C 3 & 6.29 & $1.27 \mathrm{~F}$ & 04 \\
\hline 10.00 & 2. EfF & 07 & 20.00 & $8.34 \mathrm{~F}$ & 01 & I N 7 & $=49$ & & 4. 95 & $7.37 \mathrm{~F}$ & 03 & 8.00 & $6.97 F$ & 03 \\
\hline 15.00 & $9.19 \mathrm{~F}$ & 01 & 22.07 & $6.31 E$ & 01 & & & & 4.89 & $1.17 \mathrm{~F}$ & 04 & 10. & 3. $85 F$ & 03 \\
\hline 17.94 & $5.53 \mathrm{~F}$ & 01 & & & & 3.75 & $8.05 \mathrm{~F}$ & 03 & 3.00 & $1.12 \mathrm{E}$ & C4 & 15.00 & $1.30 \mathrm{~F}$ & 03 \\
\hline & & & RH 2 & $=45$ & & 3.94 & $7.31 \mathrm{E}$ & 03 & 5. 16 & 1.03 & 04 & 00 & $5.92 \mathrm{~F}$ & 02 \\
\hline NB 2 & $=41$ & & & & & 3.97 & $1.16 \mathrm{~F}$ & 04 & 5.20 & $1.22 \mathrm{E}$ & $\mathrm{CH}$ & .00 & $1.92 \mathrm{E}$ & 02 \\
\hline & & & 3.02 & $8.24 \mathrm{E}$ & 03 & 4.00 & $1.15 \mathrm{E}$ & 04 & 6.00 & $9.58 \mathrm{E}$ & C3 & 38.91 & $9.21 \mathrm{~F}$ & 01 \\
\hline 2.39 & B. 16E & 03 & 3.15 & $7.40 \mathrm{~F}$ & 03 & 4.21 & $1.02 \mathrm{~F}$ & 04 & 9.00 & $4.75 \mathrm{~F}$ & 03 & & & \\
\hline 2.46 & $7.37 \mathrm{E}$ & 03 & 3.17 & $1.15 \mathrm{~F}$ & 04 & 4.24 & 1. 1 $\mathrm{AE}$ & 04 & 10.00 & $2.25 \mathrm{E}$ & $C 3$ & CE 2 & 58 & \\
\hline 2.48 & $1.13 \mathrm{E}$ & 04 & 3.39 & $9.81 \mathrm{~F}$ & 03 & 5.00 & $7.92 \mathrm{E}$ & 03 & 15.00 & $7.49 \mathrm{E}$ & $\mathrm{C2}$ & & & \\
\hline 2.67 & $9.47 \mathrm{~F}$ & 03 & 3.49 & $1.17 \mathrm{E}$ & 04 & 6.00 & $4.93 \mathrm{~F}$ & 03 & 20.00 & $3.38 \mathrm{~F}$ & 02 & 5.75 & 9. $99 \mathrm{E}$ & 03 \\
\hline 2.69 & 1. C9E & 04 & 4.00 & $7.69 \mathrm{~F}$ & 03 & 3.00 & $2.32=$ & 03 & 30.00 & $1.09 \mathrm{~F}$ & 02 & 6.00 & $8.23 \mathrm{E}$ & 01 \\
\hline 3.00 & $8.40 \mathrm{~F}$ & 03 & 5.00 & $4.32^{5}$ & 03 & $10 . \mathrm{co}$ & $1.27 \mathrm{E}$ & 03 & 33.13 & B. $19 \mathrm{~F}$ & $C^{\prime}$ & 6. & 7.6 & 03 \\
\hline 4.00 & $4 . \mathrm{C} 3 \mathrm{~F}$ & 03 & 5.00 & $2.68 \mathrm{~F}$ & 03 & 15.00 & $4.19 \mathrm{E}$ & 02 & & & & 6.21 & $1.22 \mathrm{~F}$ & 04 \\
\hline 5.00 & $2.25 \mathrm{~F}$ & 03 & 9.00 & $1.24 \mathrm{~F}$ & 03 & 20.00 & $1.8 A E$ & 02 & $X E Z$ & $=54$ & & 6.51 & $1 . C^{\circ}$ & 04 \\
\hline 6.00 & $1.39 \mathrm{~F}$ & 03 & 10.00 & $6.76 \mathrm{~F}$ & 02 & 27.89 & $7.33 \mathrm{E}$ & 01 & & & & & 1.29 & 04 \\
\hline 9.00 & $6.3+E$ & 02 & 15.00 & $2.20 \mathrm{~F}$ & 02 & & & & 4.91 & $8.37 \mathrm{~F}$ & 03 & & 7.87 & 03 \\
\hline 10.00 & $3.44 \mathrm{~F}$ & 02 & 20.00 & $9.77 \mathrm{E}$ & 01 & S॥ 7 & $=50$ & & 5.00 & $7.96 E$ & C 3 & 10. & 4.35 & 03 \\
\hline 15.00 & $1.11 \mathrm{~F}$ & 02 & 21.17 & $6.43 \mathrm{E}$ & 01 & & & & 5.10 & $7.44 \mathrm{~F}$ & C 3 & & $1.48 \mathrm{E}$ & 03 \\
\hline 18.93 & $5.73 \mathrm{E}$ & 01 & & & & 1.95 & $7.93 \mathrm{E}$ & 03 & 5. 14 & 1. $17 \mathrm{~F}$ & C4 & 20 & $6.76 \mathrm{~F}$ & 02 \\
\hline & & & F L $\quad 7$ & $=46$ & & 4.00 & $B .10 E$ & 03 & 5.41 & $1.04 \mathrm{~F}$ & 04 & 30 & $2.19 F$ & 02 \\
\hline M Z Z & $=42$ & & & & & 4.16 & $7.27 \mathrm{E}$ & 03 & 3.45 & $1.23 \mathrm{~F}$ & $\mathrm{C} 4$ & 40.00 & $9.75 \mathrm{~F}$ & 01 \\
\hline & & & 3.19 & $8.08 \mathrm{E}$ & 03 & 4.19 & $1.15 \mathrm{~F}$ & 04 & 6.00 & $9.74 \mathrm{~F}$ & C3 & 40.42 & $9.46 \mathrm{~F}$ & 01 \\
\hline 2.54 & $9.24 \mathrm{~F}$ & 03 & 3.33 & $7.38 \mathrm{~F}$ & 03 & 4.44 & $1.01 \mathrm{E}$ & 04 & 3.00 & $4.63 \mathrm{E}$ & 03 & & & \\
\hline 2.63 & $7.42 \mathrm{~F}$ & 03 & 3.36 & $1.15 \mathrm{~F}$ & 04 & 4.47 & $1.19 \mathrm{~F}$ & 04 & 10.00 & $2.57 \mathrm{~F}$ & $C 3$ & PR Z & $=57$ & \\
\hline 2.65 & $1.14 \mathrm{~F}$ & 04 & 3.58 & $9.91 \mathrm{~F}$ & 03 & 5.00 & $9.07 \mathrm{~F}$ & 03 & 15.00 & $8.60 \mathrm{E}$ & $C 2$ & & & \\
\hline 2.94 & a. $f 1 E$ & 03 & 3.60 & $1.15 E$ & 04 & 6.00 & $5.66 \mathrm{~F}$ & 03 & 20.00 & $3.99 \mathrm{~F}$ & $C 2$ & 6.00 & $9.13 \mathrm{E}$ & 03 \\
\hline 2.87 & $1.11 \mathrm{E}$ & 04 & 4.00 & $8.92 \mathrm{E}$ & 03 & 3.00 & $2.67 \mathrm{~F}$ & 03 & 30.00 & $1.25 \mathrm{E}$ & $C 2$ & 00 & 9.1 & 03 \\
\hline 3.00 & $9.94 F$ & 03 & 5.00 & $5.02 \pi$ & 03 & 10.00 & $1.47 \mathrm{E}$ & 03 & 34.52 & $8.41 \mathrm{~F}$ & $C 1$ & 44 & $7.67 \mathrm{E}$ & 03 \\
\hline 4.05 & $4.79 \mathrm{E}$ & 03 & 6.00 & $3.12 \mathrm{~F}$ & 03 & 15.00 & $4.85 \mathrm{E}$ & 02 & & & & 6.49 & $1.23 \mathrm{~F}$ & 04 \\
\hline 3.00 & $2.68 \mathrm{E}$ & 03 & 8.00 & $1.45 \mathrm{~F}$ & 03 & 20.00 & $2.19 \mathrm{~F}$ & 02 & $\operatorname{cs} 7$ & $=55$ & & 6.79 & $1.10 \mathrm{E}$ & 04 \\
\hline 6.00 & $1.65 \mathrm{~F}$ & 03 & 10.00 & $7.91 \mathrm{~F}$ & 02 & 29.16 & $7.53 \mathrm{~F}$ & 01 & & & & 6.85 & $1.30 \mathrm{E}$ & 04 \\
\hline 2.00 & $7.60 F$ & 02 & 15.00 & $2.59 \mathrm{~F}$ & 02 & & & & 5.04 & R. $69 \mathrm{E}$ & 03 & 8.00 & $8.89 \mathrm{~F}$ & 03 \\
\hline 10.00 & $4.12 F$ & 02 & 20.00 & $1.15 \mathrm{~F}$ & 02 & $\triangle B$ & $=51$ & & 5.36 & $7.47 \mathrm{~F}$ & $\mathrm{C} 3$ & 10.00 & $4.95 \mathrm{E}$ & 03 \\
\hline 15.00 & $1.33 \mathrm{E}$ & 02 & 24.30 & $6.50 E$ & $n 1$ & & & & 5.40 & $1.19 \mathrm{E}$ & C4 & 15.00 & $1.68 \mathrm{E}$ & 03 \\
\hline .95 & $5 . \subseteq 2 E$ & 01 & & & & 4.16 & $8.71 \mathrm{~F}$ & 03 & 5.68 & $1.05 \mathrm{~F}$ & 04 & 20.00 & $7.69 \mathrm{~F}$ & 02 \\
\hline & & & $A S_{2} 7$ & $=47$ & & 4.38 & $7.33 \mathrm{E}$ & 03 & 5.72 & $1.24 \mathrm{~F}$ & 04 & 30.00 & $? .49 \%$ & 02 \\
\hline IC 7 & $=43$ & & & & & 4.42 & $1.15 \mathrm{E}$ & 04 & 6.70 & $1.11 \mathrm{~F}$ & 04 & 40.00 & $1.11 \mathrm{~F}$ & 02 \\
\hline & & & 3.37 & $8.12 F$ & 03 & 4.67 & $1.02 \mathrm{~F}$ & 04 & 8.00 & $5.32 \mathrm{~F}$ & 03 & 41.97 & $9.69 \mathrm{E}$ & 01 \\
\hline $2.7 \mathrm{C}$ & p. $27 k$ & 03 & 3.52 & $7.29 \mathrm{~F}$ & 03 & 4.71 & $1.20 \mathrm{E}$ & 04 & 10.00 & $2.96 \mathrm{~F}$ & $\mathrm{C3}$ & & & \\
\hline 2.79 & $7.44 \mathrm{~F}$ & 03 & 3.55 & $1.15 \mathrm{~F}$ & 34 & 5.00 & $1.04 \mathrm{~F}$ & 04 & .00 & $9.91 \mathrm{~F}$ & $\mathrm{C} 2$ & ND Z & $=60$ & \\
\hline 2.82 & $1.15 \mathrm{~F}$ & 04 & 3.78 & $9.91 \mathrm{~F}$ & 0 & 6.00 & $6.53 \mathrm{~F}$ & 03 & .00 & $4.49 \mathrm{E}$ & $\mathrm{C}$ & & & \\
\hline $3.0 \mathrm{C}$ & 9. EbF & 03 & 3.91 & $1.15 \mathrm{~F}$ & 04 & 3.00 & $3.09 F$ & 03 & 30.00 & $1.45 \mathrm{E}$ & 02 & 6.24 & $9.24 \mathrm{~F}$ & 03 \\
\hline 3.02 & $9.71 \mathrm{E}$ & 03 & 4.00 & $1.03 \mathrm{~F}$ & 04 & 10.00 & $1.7 \mathrm{CF}$ & 03 & 35.95 & B. $68 \mathrm{E}$ & 01 & 6.72 & $7.7 \cap \mathrm{F}$ & 03 \\
\hline 3.04 & $1.12 \mathrm{~F}$ & 04 & 5.00 & $5.30 \mathrm{~F}$ & 03 & 15.00 & $5.62 \mathrm{~F}$ & 02 & & & & 6.77 & $1.74 \mathrm{E}$ & ou \\
\hline 4.00 & $5.64 \mathrm{~F}$ & 03 & 6.00 & $3.61 \mathrm{~F}$ & 03 & 20.00 & $2.53 \mathrm{E}$ & 02 & $B 12$ & $=56$ & & 7.09 & 1.1 & 04 \\
\hline 5.00 & $3.17 F$ & 03 & 8.00 & $1.69 \mathrm{~F}$ & 03 & 30.00 & $8.08 \mathrm{~F}$ & 01 & & & & 7.14 & $1.31 \mathrm{~F}$ & 04 \\
\hline 6.00 & $1.96 \mathrm{~F}$ & 03 & 10.00 & $9.22 \mathrm{~F}$ & 02 & 30.45 & $7.74 \mathrm{E}$ & 01 & 5.28 & $8.66 \mathrm{E}$ & 03 & 8.00 & $9.96 \mathrm{E}$ & 03 \\
\hline 9.00 & $9 . C 4 E$ & 02 & 15.00 & $3.01 \mathrm{E}$ & 02 & & & & & $7.47 \mathrm{~F}$ & 03 & & 5.5 & 03 \\
\hline 10.00 & $4.90 \mathrm{E}$ & 02 & 20.00 & $1.34 \mathrm{~F}$ & $n 2$ & $T F \quad z$ & $=52$ & & 5.67 & $1.19 \mathrm{E}$ & $\mathrm{C} 4$ & 15.00 & $1.90 \mathrm{E}$ & 03 \\
\hline $15.0 n$ & $1.59 \mathrm{~F}$ & 02 & 25.46 & $6.78 \mathrm{~F}$ & 09 & & & & 5.95 & $1.06 \mathrm{E}$ & $\mathrm{CH}$ & 20.00 & $8.71 \mathrm{~F}$ & 02 \\
\hline 20.00 & $7 .(3 F$ & 01 & & & & 4.37 & $8.11 \mathrm{E}$ & 03 & 6.00 & $1.25 \mathrm{~F}$ & 04 & 30.00 & $2.84 \mathrm{E}$ & 02 \\
\hline 20.99 & $6.13 E$ & 01 & CD ? & $=48$ & & 4.61 & $7.36 \mathrm{E}$ & 03 & 6.00 & $1.25 \mathrm{~F}$ & 04 & 40.00 & $1.27 \mathrm{~F}$ & 02 \\
\hline & & & & & & 4.65 & $1.16 \mathrm{E}$ & 04 & 8. 00 & $6.05 E$ & C 3 & 43.56 & $9.95 \mathrm{E}$ & 01 \\
\hline RU 2 & $=44$ & & 3.56 & E. 18F & 03 & 4.91 & $1.03 \mathrm{~F}$ & 04 & 10.00 & $3.36 \mathrm{E}$ & 03 & & & \\
\hline & & & 3.73 & $7.32 \mathrm{~F}$ & 03 & 4.95 & $1.21 \mathrm{E}$ & 04 & $1 \mathrm{s.00}$ & $1.13 \mathrm{t}$ & 03 & & & \\
\hline 2.86 & $8.27 \mathrm{E}$ & 03 & 3.76 & $1.16 \mathrm{~F}$ & 04 & 5.00 & 1. $18 \mathrm{~F}$ & 04 & 20.00 & $5.14 \mathrm{E}$ & 02 & & & \\
\hline$\therefore 97$ & $7.43 \mathrm{~F}$ & 03 & 3.99 & $1.01 \mathrm{~F}$ & 04 & 6.00 & $7.51 \mathrm{~F}$ & 03 & 30.00 & $1.66 \mathrm{~F}$ & $\mathrm{C} 2$ & & & \\
\hline
\end{tabular}


Table 20 (continued)

\begin{tabular}{|c|c|c|c|c|c|c|c|c|c|c|c|c|c|c|}
\hline$h v$ & \multicolumn{2}{|c|}{$\sigma_{T R}$} & $h \nu$ & \multicolumn{2}{|c|}{$\sigma_{T R}$} & $h \nu$ & \multicolumn{2}{|l|}{${ }^{0} T R$} & $h \nu$ & \multicolumn{2}{|l|}{$\sigma_{T R}$} & $h \nu$ & \multicolumn{2}{|l|}{$o_{T R}$} \\
\hline PM Z & $=61$ & & $\mathrm{~TB} \quad \mathrm{Z}$ & $=65$ & & $T * Z$ & $=6.9$ & & $H$ & $=74$ & & ET $z$ & $=79$ & \\
\hline 6.50 & $9.3 .3 \mathrm{~L}$ & 03 & 10.00 & 9.945 & 03 & 57.44 & $1.23 \mathrm{~F}$ & 02 & $10 \cdot 28$ & $1.08 \mathrm{~F}$ & 04 & 30.00 & $2.03 E$ & 03 \\
\hline 7.01 & $7.70 \mathrm{E}$ & 03 & 15.00 & $3.40 \mathrm{~F}$ & 03 & & & & 11.56 & $7.87 \mathrm{~F}$ & 03 & 40.00 & $9.32 \mathrm{E}$ & 02 \\
\hline 7.07 & $1.25 \mathrm{~F}$ & 04 & 20.00 & $1.57 \mathrm{~F}$ & 03 & $Y P Z$ & $=70$ & & 11.65 & $1.34 \mathrm{~F}$ & 04 & 50.00 & $5.07 \mathrm{r}$ & 02 \\
\hline 7.39 & $1.12 E$ & 04 & 30.00 & $5.19 \mathrm{E}$ & 02 & & & & 12.07 & $1.24 \mathrm{E}$ & 04 & 60.00 & 3.075 & 02 \\
\hline 7.45 & $1.32 \mathrm{~F}$ & 04 & 40.00 & $2.33 \mathrm{~F}$ & 02 & 9.00 & $1.03 \mathrm{E}$ & 04 & 12.17 & $1.47 \mathrm{E}$ & 04 & 78.58 & $1.45 \mathrm{~F}$ & 02 \\
\hline 8.00 & $1.11 \mathrm{E}$ & 04 & 50.00 & $1.25 \mathrm{~F}$ & 02 & 9.98 & $7.96 \mathrm{E}$ & 03 & 75.00 & $8.71 \mathrm{~F}$ & 03 & & & \\
\hline 10.00 & $6.28 E$ & 03 & 52.02 & $1.11 \mathrm{E}$ & 02 & 10.00 & 1. $3 \mathrm{AF}$ & 04 & 20.00 & $4.09 \mathrm{E}$ & $\mathrm{C} \overline{3}$ & AO Z & 79 & \\
\hline 15.00 & $2.14 \mathrm{E}$ & 03 & & & & 10.06 & $1.33 \mathrm{E}$ & 04 & 30.00 & $1.39 \mathrm{~F}$ & $C 3$ & & & \\
\hline $20.0 \mathrm{C}$ & $9.80 E$ & 02 & DY $Z$ & $=65$ & & 10.45 & $1.21 \mathrm{E}$ & 04 & 40.00 & $6.33 \mathrm{~F}$ & 02 & 12.00 & 1. $14 F$ & 04 \\
\hline 30.00 & $3.20 \mathrm{E}$ & 02 & & & & 10.53 & $1.44 \mathrm{~F}$ & 04 & 50.00 & $3.43 \mathrm{E}$ & $C 2$ & 13.76 & $7.84 \mathrm{E}$ & 03 \\
\hline 40.00 & $1.43 \mathrm{~F}$ & 02 & 7.83 & $9.97 \mathrm{~F}$ & 03 & 15.00 & $5.82 \mathrm{E}$ & 03 & 60.00 & $2.07 \mathrm{E}$ & 02 & 13.87 & $1.35 \mathrm{E}$ & 04 \\
\hline 45.19 & 1. C $1 \mathrm{~F}$ & 02 & $\begin{array}{l}8.00 \\
8.58\end{array}$ & $\begin{array}{l}9.05 \mathrm{E} \\
7.88 \mathrm{E}\end{array}$ & $\begin{array}{l}03 \\
03\end{array}$ & $\begin{array}{l}20.00 \\
30.00\end{array}$ & $\begin{array}{l}2.73 \mathrm{E} \\
9.11 \mathrm{~F}\end{array}$ & $\begin{array}{l}03 \\
02\end{array}$ & 69.65 & $1.37 \mathrm{~F}$ & 62 & $\begin{array}{l}14.33 \\
14.44\end{array}$ & $\begin{array}{l}1.25 \mathrm{E} \\
1.49 \mathrm{E}\end{array}$ & $\begin{array}{l}04 \\
04\end{array}$ \\
\hline SM Z & $=62$ & & 8.65 & $1.30 \mathrm{~F}$ & 04 & 40.00 & $4.13 E$ & 02 & $R E \quad z$ & $=75$ & & 15.00 & $1.34 \mathrm{E}$ & 04 \\
\hline & & & 9.00 & $1.18 \mathrm{E}$ & 04 & 50.00 & $2.22 E$ & 02 & & & & 20.00 & $6.47 \mathrm{E}$ & 03 \\
\hline 6.75 & $9.40 \mathrm{~F}$ & 03 & 9.08 & $1.40 \mathrm{~F}$ & 04 & 60.00 & $1.34 \mathrm{E}$ & 02 & 10.61 & $1.10 \mathrm{~F}$ & 04 & 30.00 & $2.22 \mathrm{E}$ & 03 \\
\hline 7.31 & $7.70 \mathrm{~F}$ & 03 & 10.00 & $1.10 \mathrm{E}$ & 04 & 61.40 & $1.25=$ & 02 & 11.98 & 7. $89 \mathrm{E}$ & C3 & 40.00 & $1.02 \mathrm{~F}$ & 03 \\
\hline 7.37 & $1.25 \mathrm{~F}$ & 04 & 15.00 & $3.93 \mathrm{~F}$ & 03 & & & & 12.09 & $1.35 \mathrm{E}$ & 04 & 50.00 & $5.5 n E$ & 02 \\
\hline $7.7 \mathrm{C}$ & $1.13 \mathrm{~F}$ & 04 & 20.00 & $1.78 \mathrm{~F}$ & 03 & $\mathrm{LU} 2$ & $=71$ & & 12.50 & $1.24 \mathrm{~F}$ & 04 & 60.00 & 3.378 & 02 \\
\hline 7.76 & $1.33 \mathrm{~F}$ & 04 & 30.00 & $5.87 \mathrm{~F}$ & 02 & & & & 12.60 & $1.48 \mathrm{E}$ & 04 & 80.00 & 1. $52 \mathrm{~F}$ & 02 \\
\hline 8.00 & $1.24 \mathrm{~F}$ & 04 & 40.00 & $2.64 \mathrm{~F}$ & 02 & 9.31 & $1.04 \mathrm{E}$ & 04 & 15.00 & $9.57 \mathrm{E}$ & $C 3$ & 80.92 & $1.47 \mathrm{E}$ & 02 \\
\hline $10.0 \mathrm{C}$ & 7. $C 4 \mathrm{E}$ & 03 & 50.00 & $1.41 \mathrm{~F}$ & 02 & 10.00 & $8.64 \mathrm{E}$ & 03 & 20.00 & $4.52 \mathrm{E}$ & $\mathrm{C} 3$ & & & \\
\hline 15.00 & $2.41 \mathrm{~F}$ & 03 & 53.82 & $1.15 \mathrm{~F}$ & 02 & 10.36 & $7.85=$ & 03 & 30.00 & $1.53 \mathrm{E}$ & $C 3$ & $\mathrm{HC} \mathrm{Z}$ & $=80$ & \\
\hline 20.00 & $1.11 E$ & 03 & & & & 10.45 & $1.33 \mathrm{E}$ & 04 & 40.00 & $7.02 \mathrm{E}$ & $C 2$ & & & \\
\hline 30.00 & $3.62 E$ & 02 & $\mathrm{HO} \quad 2$ & $=67$ & & 10.84 & $1.22 \mathrm{E}$ & 04 & 50.00 & $3.90 \mathrm{~F}$ & 02 & 12.37 & $1.14 \mathrm{E}$ & 04 \\
\hline 40.00 & $1.62 \mathrm{E}$ & 02 & & & & 10.92 & $1.44 \mathrm{E}$ & 04 & 60.00 & $2.30 \mathrm{E}$ & $C 2$ & 14.24 & $7.79 \mathrm{~F}$ & 03 \\
\hline 46.84 & $1.04 \mathrm{~F}$ & 02 & 9.12 & $1.00 \mathrm{~F}$ & 04 & $15 . \mathrm{co}$ & $6.46 \mathrm{E}$ & 03 & 71.91 & $1.39 \mathrm{~F}$ & $C 2$ & 14.35 & $1.34 \mathrm{~F}$ & 04 \\
\hline Fif 7 & 61 & & 8.92 & 7. $85 F$ & $\begin{array}{l}03 \\
04\end{array}$ & $\begin{array}{l}20.00 \\
30.00\end{array}$ & $\begin{array}{l}3.02 \mathrm{E} \\
1.01 \mathrm{E}\end{array}$ & $\begin{array}{l}03 \\
03\end{array}$ & 057 & & & $\begin{array}{r}14.82 \\
14.94\end{array}$ & $\begin{array}{l}1.24 \mathrm{E} \\
1.48 \mathrm{E}\end{array}$ & $\begin{array}{l}04 \\
04\end{array}$ \\
\hline & 63 & & $\begin{array}{l}0.74 \\
9.35\end{array}$ & $1.19 \mathrm{E}$ & 04 & 40.00 & $4.60 \mathrm{E}$ & 02 & & & & 15.00 & $1.46 \mathrm{E}$ & 04 \\
\hline 7.02 & $9.53 \mathrm{E}$ & 03 & 9.43 & $1.41 \mathrm{E}$ & 04 & 50.00 & $2.49 \mathrm{E}$ & 02 & 10.95 & 1. $11 \mathrm{~F}$ & 04 & 20.00 & $7.05 \mathrm{E}$ & 03 \\
\hline 7.02 & 7.745 & 03 & 10.00 & $1.21 \mathrm{~F}$ & 04 & 60.00 & $1.49 \mathrm{~F}$ & 02 & 12.41 & 7. 900 & C 3 & 30.00 & $2.43 F$ & 03 \\
\hline $\begin{array}{l}7.02 \\
7.68\end{array}$ & $1.27 \mathrm{~F}$ & 04 & 15.00 & $4.27 \mathrm{~F}$ & 03 & 53.40 & $1.29 \mathrm{E}$ & 02 & 12.51 & $1.35 \%$ & 04 & 40.00 & $1.12 \mathrm{E}$ & 03 \\
\hline 9.00 & $1.15 \mathrm{E}$ & 04 & 20.00 & $1.98 \mathrm{~F}$ & 03 & & & & 12.94 & 1. $25 \mathrm{~F}$ & 04 & 50.00 & $6.10 \mathrm{E}$ & 02 \\
\hline 8.01 & $1.14 \mathrm{E}$ & 04 & 30.00 & $6.57 \mathrm{~F}$ & 02 & HF Z & $=72$ & & 13.05 & $1.48 \mathrm{~F}$ & $\mathrm{C} 4$ & $6 C .00$ & $3.70 \mathrm{E}$ & 02 \\
\hline 8.08 & $1.35 \mathrm{E}$ & 04 & 40.00 & $2.96 \mathrm{~F}$ & 02 & & & & 15.00 & $1.04 \mathrm{E}$ & 04 & 80.00 & $1.69 \mathrm{E}$ & 02 \\
\hline 10.00 & 7. $\subseteq 2 \mathrm{~F}$ & 03 & 50.00 & $1.59 \mathrm{~F}$ & 02 & 9.63 & $1.05 \mathrm{E}$ & 04 & 20.00 & $4.95 \mathrm{~F}$ & 03 & 83.32 & $1.50 \mathrm{~F}$ & 02 \\
\hline 15.00 & $2.71 \mathrm{E}$ & 03 & 55.66 & $1.18 \mathrm{~F}$ & 02 & 10.00 & $9.65 \mathrm{E}$ & 03 & 30.70 & $1.68 \mathrm{~F}$ & 03 & & & \\
\hline 20.00 & $1.25 \mathrm{E}$ & 03 & & & & 10.75 & $7.85 \mathrm{E}$ & 03 & 40.00 & $7.71 \mathrm{E}$ & $c 2$ & TL 7 & $=81$ & \\
\hline 30.00 & $4.09 \mathrm{E}$ & 02 & F R Z & $=68$ & & 10.84 & $1.33 \mathrm{E}$ & 04 & 50.00 & 4. $18 \mathrm{E}$ & $C 2$ & & & \\
\hline 40.00 & $1.83 E$ & 02 & & & & 11.24 & $1.22 \mathrm{~F}$ & 04 & 60.00 & $2.53 \mathrm{~F}$ & $c 2$ & 12.75 & $1.15 \mathrm{E}$ & 04 \\
\hline 48.53 & 1. $C 7 \mathrm{~F}$ & 02 & 8.41 & $1.02 \mathrm{~F}$ & 04 & 11.33 & $1.45^{\circ}$ & 04 & 74.02 & $1.41 \mathrm{E}$ & 02 & 14.73 & $7.74 \mathrm{E}$ & 03 \\
\hline & & & 9.27 & $7.90 \mathrm{E}$ & 03 & 15.00 & $7.14 \mathrm{E}$ & 03 & & & & 14.95 & $1.34 \mathrm{~F}$ & 04 \\
\hline GD 2 & $=64$ & & 9.34 & $1.32 \mathrm{E}$ & 04 & 20.00 & $3.34 \mathrm{E}$ & 03 & IR $\quad Z$ & $=77$ & & 15.00 & $1.30 \mathrm{E}$ & 04 \\
\hline & & & 9.71 & $1.20 \mathrm{E}$ & 04 & 30.00 & 1.125 & 03 & & & & 15.32 & $1.24 \mathrm{E}$ & 04 \\
\hline 7.29 & $9.61 \mathrm{~F}$ & 03 & 9.79 & $1.42 F$ & 04 & 40.00 & $5.11 \mathrm{P}$ & 02 & 11.29 & $1.13 \mathrm{~F}$ & 04 & 15.45 & $1.47 \mathrm{E}$ & 04 \\
\hline 7.94 & $7.73 E$ & 03 & 10.00 & $1.35 \mathrm{~F}$ & 04 & 50.00 & $2.76 F$ & 02 & 12.85 & $7.90 \mathrm{~F}$ & 03 & 20.00 & 7.698 & 01 \\
\hline 8.00 & $1.27 \mathrm{~F}$ & 04 & 15.00 & $4.76 \mathrm{~F}$ & 03 & 60.00 & $1.66 \mathrm{E}$ & 02 & 12.95 & $1.35 \mathrm{E}$ & 04 & 30.00 & $2.65 \mathrm{E}$ & 03 \\
\hline 9.00 & $1.27 \mathrm{~F}$ & 04 & 20.00 & $2.22 \mathrm{~F}$ & 03 & 55.44 & $1.30 F$ & 02 & 13.39 & $1.25 \mathrm{~F}$ & 04 & 40.00 & $1.22 \mathrm{E}$ & 03 \\
\hline 9.34 & $1.15 \mathrm{~F}$ & 04 & 30.00 & $7.37 \mathrm{~F}$ & 02 & & & & 13.50 & $1.48 \mathrm{~F}$ & $\mathrm{CH}$ & 50.00 & $6.69 \mathrm{E}$ & 02 \\
\hline 9.41 & $1.36 \mathrm{E}$ & 04 & 40.00 & 3. $33 \mathrm{~F}$ & 02 & $T A$ & $=73$ & & 15.00 & $1.14 \mathrm{E}$ & 04 & 60.00 & $4.06 \mathrm{E}$ & 02 \\
\hline 10.00 & $8.84 \mathrm{~F}$ & 03 & 50.00 & $1.79 \mathrm{~F}$ & 02 & & & & 20.00 & $5.42 \mathrm{E}$ & 03 & 80.00 & $1.84 \mathrm{E}$ & 02 \\
\hline 15.00 & 3. $\mathrm{CHE}$ & 03 & 57.53 & $1.21 \mathrm{~F}$ & 02 & 3.95 & $1.07 \mathrm{~F}$ & 04 & 30.00 & $1.85 \mathrm{~F}$ & 03 & 85.77 & $1.52 \mathrm{P}$ & 02 \\
\hline 20.00 & $1.4 C E$ & 03 & & & & 10.00 & $1.06 \mathrm{E}$ & 04 & 40.00 & $8.48 \mathrm{E}$ & 02 & & & \\
\hline 30.00 & $4.61 \mathrm{E}$ & 02 & TM 2 & $=69$ & & 11.15 & $7.87 \mathrm{E}$ & 03 & 50.00 & $4.61 \mathrm{E}$ & $\mathrm{C} 2$ & & & \\
\hline 40.00 & $2.07 \mathrm{~F}$ & 02 & & & & 11.24 & $1.34 \mathrm{E}$ & 04 & 60.00 & $2.79 \mathrm{E}$ & $C 2$ & & & \\
\hline $50.0 \mathrm{C}$ & 1. $10 \mathrm{E}$ & 02 & 8.70 & $1.03 E$ & 04 & 11.65 & $1.23 \mathrm{~F}$ & 04 & 76.27 & $1.43 \mathrm{~F}$ & 02 & & & \\
\hline 50.26 & 1. C9E & 02 & 9.62 & $7.89 \mathrm{E}$ & 03 & 11.74 & $1.46 \mathrm{E}$ & 04 & & & & & & \\
\hline & & & 9.70 & $1.32 \mathrm{~F}$ & 04 & 15.00 & $7.90 \mathrm{E}$ & 03 & PT $Z$ & $=78$ & & & & \\
\hline TB $Z$ & $=65$ & & 10.00 & $1.23 \mathrm{~F}$ & 04 & 20.00 & $3.71 \mathrm{~F}$ & 03 & & & & & & \\
\hline & & & & $1.21 \mathrm{E}$ & 04 & 10.00 & $1.25 \mathrm{~F}$ & 03 & 11.65 & 1. $13 \mathrm{E}$ & 0,4 & & & \\
\hline 7.56 & $9.78 \mathrm{~F}$ & 03 & 10.15 & $1.43 \mathrm{~F}$ & 34 & 40.00 & $5.71 \mathrm{~F}$ & 02 & 13.30 & 7.845 & 03 & & & \\
\hline 3.00 & $8.51 \mathrm{E}$ & 03 & 15.00 & c. $27 \mathrm{~F}$ & 03 & $50 . \mathrm{CC}$ & $3.09 \mathrm{~F}$ & 02 & 13.41 & $1.35 \mathrm{~B}$ & $\mathrm{CH}$ & & & \\
\hline 8.25 & 7. EOF & 03 & 20.00 & $2.46 \mathrm{~F}$ & 03 & 60.00 & $1.86 \mathrm{P}$ & 02 & 13. 95 & $1.25 \mathrm{E}$ & 04 & & & \\
\hline 8.32 & 1. $28 \mathrm{E}$ & 04 & 30.00 & $8.19 \mathrm{~F}$ & 02 & 67.52 & 1. $34 \mathrm{E}$ & 02 & 13.77 & $1.48 \mathrm{E}$ & $\mathrm{CH}_{4}$ & & & \\
\hline 8.67 & $1.16 F$ & 04 & 40.00 & $3.71 \mathrm{~F}$ & 02 & & & & 15.00 & $1.23 \mathrm{~F}$ & 04 & & & \\
\hline 9.73 & 1. $37 \mathrm{E}$ & 04 & 50.00 & $1.99 \mathrm{E}$ & 02 & & & & 20.00 & $5.92 \mathrm{E}$ & C 3 & & & \\
\hline
\end{tabular}


Table 20 (contınued)

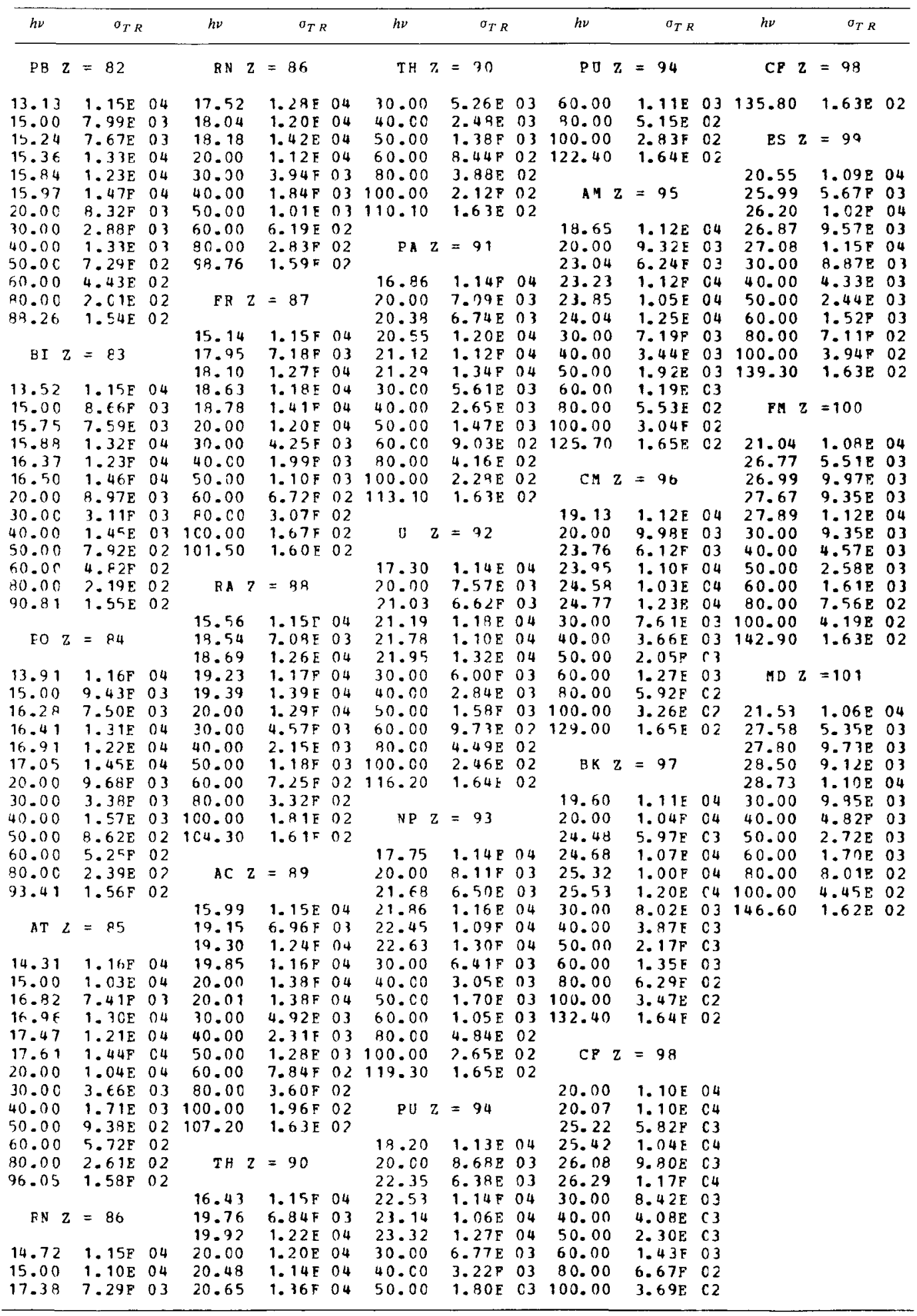


Table 21. $L$-subshell photoionization cross section ratios for $Z=12(8) 100$ (ref. 2)

\begin{tabular}{|c|c|c|c|c|c|c|c|c|c|}
\hline $\begin{array}{l}\text { Photon } \\
\text { energy } \\
(\mathrm{keV})\end{array}$ & $\frac{\sigma_{L_{1}}}{\sigma_{L}}$ & $\frac{\sigma_{L_{2}}}{\sigma_{L}}$ & $\frac{\sigma_{L_{3}}}{\sigma_{L}}$ & $\frac{\sigma_{L_{1}}}{\sigma_{L_{2,3}}}$ & $\frac{\sigma_{L_{2}}}{\sigma_{L_{3}}}$ & $\frac{\sigma_{L_{1}}}{\sigma_{\text {total }}}$ & $\frac{\sigma_{L_{2}}}{\sigma_{\text {total }}}$ & $\frac{\sigma_{L_{3}}}{\sigma_{\text {total }}}$ & $\begin{array}{l}\text { Photon } \\
\text { energy } \\
\text { (keV) }\end{array}$ \\
\hline \multicolumn{10}{|c|}{$M G Z=12$} \\
\hline $\begin{array}{l}1.00 \\
1.29\end{array}$ & $\begin{array}{l}0.538 \\
0.600\end{array}$ & $\begin{array}{l}0.155 \\
0.135\end{array}$ & $\begin{array}{l}0.306 \\
0.265\end{array}$ & $\begin{array}{l}1.165 \\
1.503\end{array}$ & $\begin{array}{l}0.507 \\
0.508\end{array}$ & $\begin{array}{l}0.524 \\
0.583\end{array}$ & $\begin{array}{l}0.151 \\
0.131\end{array}$ & $\begin{array}{l}0.298 \\
0.257\end{array}$ & $\begin{array}{l}1.00 \\
1.29\end{array}$ \\
\hline \multicolumn{10}{|c|}{$C A Z=20$} \\
\hline $\begin{array}{l}1.00 \\
1.50 \\
2.00 \\
3.00 \\
4.00 \\
4.01\end{array}$ & $\begin{array}{l}0.255 \\
0.340 \\
0.406 \\
0.504 \\
0.572 \\
0.573\end{array}$ & $\begin{array}{l}0.252 \\
0.224 \\
0.202 \\
0.170 \\
0.147 \\
0.146\end{array}$ & $\begin{array}{l}0.493 \\
0.436 \\
0.392 \\
0.327 \\
0.281 \\
0.281\end{array}$ & $\begin{array}{l}0.342 \\
0.514 \\
0.683 \\
1.014 \\
1.338 \\
1.341\end{array}$ & $\begin{array}{l}0.512 \\
0.515 \\
0.517 \\
0.520 \\
0.522 \\
0.522\end{array}$ & $\begin{array}{l}0.230 \\
0.304 \\
0.362 \\
0.448 \\
0.508 \\
0.508\end{array}$ & $\begin{array}{l}0.227 \\
0.201 \\
0.181 \\
0.151 \\
0.130 \\
0.130\end{array}$ & $\begin{array}{l}0.444 \\
0.391 \\
0.350 \\
C .290 \\
0.249 \\
C .249\end{array}$ & $\begin{array}{l}1.00 \\
1.50 \\
2.00 \\
3.00 \\
4.00 \\
4.01\end{array}$ \\
\hline \multicolumn{10}{|c|}{ NI $Z=28$} \\
\hline $\begin{array}{l}1.00 \\
1.01 \\
1.01 \\
1.50 \\
2.00 \\
3.00 \\
4.00 \\
5.00 \\
6.00 \\
8.00 \\
8.29\end{array}$ & $\begin{array}{l}0.0 \\
0.0 \\
0.138 \\
0.190 \\
0.241 \\
0.323 \\
0.388 \\
0.441 \\
0.484 \\
0.552 \\
0.561\end{array}$ & $\begin{array}{l}0.339 \\
0.339 \\
0.292 \\
0.276 \\
0.260 \\
0.234 \\
0.212 \\
0.195 \\
0.180 \\
0.157 \\
0.155\end{array}$ & $\begin{array}{l}0.661 \\
0.661 \\
0.570 \\
0.533 \\
0.498 \\
0.443 \\
0.399 \\
0.364 \\
0.335 \\
0.290 \\
0.285\end{array}$ & $\begin{array}{l}0.0 \\
0.0 \\
0.160 \\
0.235 \\
0.318 \\
0.478 \\
0.634 \\
0.788 \\
0.939 \\
1.234 \\
1.277\end{array}$ & $\begin{array}{l}0.513 \\
0.513 \\
0.513 \\
0.518 \\
0.522 \\
0.528 \\
0.532 \\
0.535 \\
0.538 \\
0.543 \\
0.543\end{array}$ & $\begin{array}{l}0.0 \\
0.0 \\
0.122 \\
0.168 \\
0.213 \\
0.285 \\
0.341 \\
0.386 \\
0.424 \\
0.483 \\
0.490\end{array}$ & $\begin{array}{l}0.295 \\
0.295 \\
0.259 \\
0.245 \\
0.230 \\
0.206 \\
0.187 \\
0.171 \\
0.158 \\
0.138 \\
0.135\end{array}$ & $\begin{array}{l}0.574 \\
0.574 \\
0.504 \\
0.472 \\
0.440 \\
0.390 \\
0.351 \\
0.320 \\
0.294 \\
0.253 \\
0.249\end{array}$ & $\begin{array}{l}1.00 \\
1.01 \\
1.01 \\
1.50 \\
2.00 \\
3.00 \\
4.00 \\
5.00 \\
6.00 \\
8.00 \\
8.29\end{array}$ \\
\hline \multicolumn{10}{|c|}{$K R z=36$} \\
\hline $\begin{array}{r}1.68 \\
1.73 \\
1.74 \\
1.90 \\
1.92 \\
2.00 \\
3.00 \\
4.00 \\
5.00 \\
6.00 \\
8.00 \\
10.00 \\
14.27\end{array}$ & $\begin{array}{l}0.0 \\
0.0 \\
0.0 \\
0.0 \\
0.138 \\
0.151 \\
0.214 \\
0.267 \\
0.313 \\
0.352 \\
0.418 \\
0.470 \\
0.553\end{array}$ & $\begin{array}{l}0.0 \\
0.0 \\
0.363 \\
0.343 \\
C .296 \\
0.292 \\
0.274 \\
0.257 \\
0.243 \\
0.230 \\
C .208 \\
0.191 \\
0.163\end{array}$ & $\begin{array}{l}1.000 \\
1.000 \\
0.637 \\
0.657 \\
0.566 \\
0.557 \\
0.512 \\
0.475 \\
0.444 \\
0.418 \\
0.374 \\
0.339 \\
0.284\end{array}$ & $\begin{array}{l}0.0 \\
0.0 \\
0.0 \\
0.0 \\
0.160 \\
0.178 \\
0.273 \\
0.365 \\
0.455 \\
0.544 \\
0.719 \\
0.889 \\
1.238\end{array}$ & $\begin{array}{l}0.0 \\
0.0 \\
0.570 \\
0.521 \\
0.522 \\
0.525 \\
0.534 \\
0.541 \\
0.546 \\
0.550 \\
0.557 \\
0.563 \\
0.573\end{array}$ & $\begin{array}{l}0.0 \\
0.0 \\
0.0 \\
0.0 \\
0.117 \\
0.129 \\
0.183 \\
0.228 \\
0.267 \\
0.300 \\
0.356 \\
0.400 \\
0.470\end{array}$ & $\begin{array}{l}0.0 \\
0.0 \\
0.302 \\
0.284 \\
0.251 \\
0.249 \\
0.233 \\
0.219 \\
0.207 \\
0.196 \\
0.177 \\
0.162 \\
0.138\end{array}$ & $\begin{array}{l}0.777 \\
0.756 \\
0.530 \\
0.545 \\
0.481 \\
0.474 \\
0.437 \\
0.406 \\
0.379 \\
0.356 \\
0.318 \\
0.288 \\
0.241\end{array}$ & $\begin{array}{r}1.68 \\
1.73 \\
1.74 \\
1.90 \\
1.92 \\
2.00 \\
3.00 \\
4.00 \\
5.00 \\
6.00 \\
8.00 \\
10.00 \\
14.27\end{array}$ \\
\hline \multicolumn{10}{|c|}{$\operatorname{RO} Z=44$} \\
\hline $\begin{array}{r}2.86 \\
2.97 \\
2.99 \\
3.00 \\
3.20 \\
3.23 \\
4.00 \\
5.00 \\
6.00 \\
8.00 \\
10.00 \\
15.00 \\
20.00 \\
22.07\end{array}$ & $\begin{array}{l}0.0 \\
0.0 \\
0.0 \\
0.0 \\
0.0 \\
0.154 \\
0.190 \\
0.226 \\
0.260 \\
0.318 \\
0.366 \\
0.459 \\
0.524 \\
0.546\end{array}$ & $\begin{array}{l}0.0 \\
0.0 \\
C .350 \\
0.353 \\
0.347 \\
0.294 \\
0.286 \\
0.276 \\
C .266 \\
0.248 \\
0.233 \\
0.202 \\
C .180 \\
0.172\end{array}$ & $\begin{array}{l}1.000 \\
1.000 \\
0.650 \\
0.647 \\
0.653 \\
0.552 \\
0.524 \\
0.497 \\
0.474 \\
0.434 \\
0.401 \\
0.339 \\
0.296 \\
0.281\end{array}$ & $\begin{array}{l}0.0 \\
0.0 \\
0.0 \\
0.0 \\
0.0 \\
0.183 \\
0.235 \\
0.293 \\
0.351 \\
0.466 \\
0.578 \\
0.847 \\
1.103 \\
1.205\end{array}$ & $\begin{array}{l}0.0 \\
0.0 \\
0.539 \\
0.547 \\
0.531 \\
0.532 \\
0.547 \\
0.555 \\
0.561 \\
0.572 \\
0.580 \\
0.596 \\
0.609 \\
0.614\end{array}$ & $\begin{array}{l}0.0 \\
0.0 \\
0.0 \\
0.0 \\
0.0 \\
0.127 \\
0.156 \\
0.187 \\
0.214 \\
0.262 \\
0.302 \\
0.378 \\
0.432 \\
0.450\end{array}$ & $\begin{array}{l}0.0 \\
0.0 \\
0.278 \\
0.281 \\
0.275 \\
0.241 \\
0.236 \\
0.228 \\
0.220 \\
0.205 \\
0.192 \\
0.167 \\
0.148 \\
0.142\end{array}$ & $\begin{array}{l}0.715 \\
0.713 \\
0.515 \\
0.513 \\
0.519 \\
0.453 \\
0.431 \\
0.410 \\
0.392 \\
C .358 \\
0.331 \\
0.280 \\
0.244 \\
0.232\end{array}$ & $\begin{array}{r}2.86 \\
2.97 \\
2.99 \\
3.00 \\
3.20 \\
3.23 \\
4.00 \\
5.00 \\
6.00 \\
8.00 \\
10.00 \\
15.00 \\
20.00 \\
22.07\end{array}$ \\
\hline
\end{tabular}


Table 21 (continued)

\begin{tabular}{cccccccccc}
\hline $\begin{array}{c}\text { Photon } \\
\text { energy } \\
(\mathrm{keV})\end{array}$ & $\frac{\sigma_{L_{1}}}{\sigma_{L}}$ & $\frac{\sigma_{L_{2}}}{\sigma_{L}}$ & $\frac{\sigma_{L_{3}}}{\sigma_{L}}$ & $\frac{\sigma_{L_{1}}}{\sigma_{L_{2,3}}}$ & $\frac{\sigma_{L_{2}}}{\sigma_{L_{3}}}$ & $\frac{\sigma_{L_{1}}}{\sigma_{\text {total }}}$ & $\frac{\sigma_{L_{2}}}{\sigma_{\text {total }}}$ & $\frac{\sigma_{L_{3}}}{\sigma_{\text {total }}}$ & $\begin{array}{l}\text { Photon } \\
\text { energy } \\
(\mathrm{keV})\end{array}$ \\
\hline
\end{tabular}

$\begin{array}{lll}4.37 & 0.0 & 0.0 \\ 4.61 & 0.0 & 0.0 \\ 4.65 & 0.0 & 0.346 \\ 4.91 & 0.0 & 0.355 \\ 4.95 & 0.167 & 0.295 \\ 5.00 & 0.167 & 0.295 \\ 6.00 & 0.195 & C .292 \\ 8.00 & 0.245 & 0.278 \\ 10.00 & 0.287 & 0.266 \\ 15.00 & 0.372 & 0.240 \\ 20.00 & 0.436 & 0.219 \\ 30.00 & 0.526 & 0.189 \\ 31.78 & 0.538 & C .185\end{array}$

1.000
1.000
0.654
0.645
0.538
0.537
0.513
0.477
0.447
0.388
0.345
0.285
0.277

TE $z=52$

$\begin{array}{rll}6.24 & 0.0 & 0.0 \\ 6.72 & 0.0 & 0.0 \\ 6.77 & 0.0 & 0.362 \\ 7.09 & 0.0 & 0.366 \\ 7.14 & 0.173 & 0.303 \\ 8.00 & 0.188 & 0.300 \\ 10.00 & 0.226 & 0.292 \\ 15.00 & 0.302 & 0.272 \\ 20.00 & 0.362 & 0.254 \\ 30.00 & 0.449 & 0.226 \\ 40.00 & 0.510 & 0.206 \\ 43.56 & 0.528 & 0.200\end{array}$

1.000
1.000
0.638
0.634
0.525
0.512
0.482
0.426
0.384
0.325
0.284
0.272

0.0

0.0

$0.0 \quad 0.0$

0.0

0.0

0.667

4.37

0.0

0.529

0.0

0.0

0.201

0.550

0.0

0.263

0.677

4.61

0.2010 .550

0.133

0.243

0.133

0.271

0.498

4.65

0.494

0.428

4.91

$0.324 \quad 0.583$

0.235

0.428

0.403

0.156

0.234

0.411

.196

0.223

0.383

$0.592 \quad 0.618$

0.231
0.299

0.214

0.772

0.635

1. 109

0.662

0.351

0.193

0.359

0.313

0.423

0.152

0.278

0.230

4.95

5.00

6.00

8.00

10.00

15.00

20.00

1.165

ND $Z=60$

$\begin{array}{llllll}0.0 & 0.0 & 0.0 & 0.0 & 0.646 & 6.24 \\ 0.0 & 0.0 & 0.0 & 0.0 & 0.648 & 6.72 \\ 0.0 & 0.567 & 0.0 & 0.269 & 0.474 & 6.77 \\ 0.0 & 0.578 & 0.0 & 0.273 & 0.472 & 7.09 \\ 0.209 & 0.577 & 0.134 & 0.236 & 0.408 & 7.14 \\ 0.232 & 0.587 & 0.147 & 0.235 & 0.400 & 8.00 \\ 0.292 & 0.607 & 0.177 & 0.229 & 0.378 & 10.00 \\ 0.434 & 0.637 & 0.238 & 0.214 & 0.336 & 15.00 \\ 0.567 & 0.661 & 0.285 & 0.200 & 0.303 & 20.00 \\ 0.815 & 0.696 & 0.354 & 0.178 & 0.256 & 30.00 \\ 1.043 & 0.724 & 0.403 & 0.162 & 0.224 & 40.00 \\ 1.119 & 0.733 & 0.417 & 0.157 & 0.215 & 43.56\end{array}$

$\begin{array}{rl}8.41 & 0.0 \\ 9.27 & 0.0 \\ 9.34 & 0.0 \\ 9.71 & 0.0 \\ 9.79 & 0.176 \\ 10.00 & 0.180 \\ 15.00 & 0.246 \\ 20.00 & 0.300 \\ 30.00 & 0.382 \\ 40.00 & 0.442 \\ 50.00 & 0.488 \\ 57.53 & 0.515\end{array}$

$\begin{array}{ll}0.0 & 1.000 \\ 0.0 & 1.000 \\ 0.373 & 0.627 \\ 0.377 & 0.623 \\ 0.310 & 0.513 \\ 0.308 & 0.511 \\ 0.299 & 0.455 \\ 0.285 & 0.415 \\ 0.261 & 0.357 \\ 0.242 & 0.316 \\ C .227 & 0.285 \\ 0.218 & 0.267\end{array}$

ER $z=68$

0.00 .0

$\begin{array}{ll}0.0 & 0.0 \\ 0.0 & 0.0 \\ 0.0 & 0.596 \\ 0.0 & 0.606 \\ 0.214 & 0.605 \\ 0.220 & 0.603 \\ 0.327 & 0.657 \\ 0.429 & 0.686 \\ 0.619 & 0.732 \\ 0.792 & 0.768 \\ 0.952 & 0.798 \\ 1.064 & 0.818\end{array}$

0.0

0.0

0.0

0.0

0.135

0.139

0.191

0.234

0.298

0.345

0.390

0.0

0.273

0.629

8.41

0.276

0.629

9.27

9.34

0.238

0.456

9.71

9.79

0.237

10.00

0.232

0.353

15.00

0.221

0.323

20.00

0.278

30.00

$0.189 \quad 0.246$

40.00

0.402

0.170

0.222

50.00

OS $z=76$

$\begin{array}{llll}10.95 & 0.0 & 0.0 & 1.000 \\ 12.41 & 0.0 & 0.0 & 1.000 \\ 12.51 & 0.0 & 0.388 & 0.612 \\ 12.94 & 0.0 & 0.392 & 0.608 \\ 13.05 & 0.179 & 0.321 & 0.500 \\ 15.00 & 0.199 & 0.319 & 0.482 \\ 20.00 & 0.249 & 0.311 & 0.440 \\ 30.00 & 0.324 & 0.294 & 0.382 \\ 40.00 & 0.381 & 0.278 & 0.341 \\ 50.00 & 0.425 & 0.264 & 0.310 \\ 60.00 & 0.461 & 0.253 & 0.286 \\ 74.02 & 0.500 & 0.240 & 0.259\end{array}$

$\begin{array}{ll}0.0 & 0.0 \\ 0.0 & 0.0 \\ 0.0 & 0.633 \\ 0.0 & 0.645 \\ 0.218 & 0.643 \\ 0.249 & 0.661 \\ 0.331 & 0.708 \\ 0.480 & 0.769 \\ 0.616 & 0.814 \\ 0.740 & 0.853 \\ 0.854 & 0.886 \\ 1.001 & 0.927\end{array}$

0.0
0.0
0.0
0.0
0.136
0.152
0.191
0.249
0.294
0.328
0.355
0.386

0.0

0.613

10.95

0.0

0.279

0.611

12.41

0.283

0.441

12.51

0.500

0.482

0.244

0.438
0.379

12.94

13.05

$0.368 \quad 15.00$

$\begin{array}{lll}0.239 & 0.337 & 20.00\end{array}$

$\begin{array}{lll}0.226 & 0.294 & 30.00\end{array}$

$\begin{array}{lll}0.226 & 0.294 & 30.00 \\ 0.214 & 0.263 & 40.00\end{array}$

0.310

0.259

$\begin{array}{ll}0.854 & 0.886 \\ 1.001 & 0.927\end{array}$

0.204

0.239

50.00

0.195

0.221

60.00

0.185

0.200

74.02 
Table 21 (continued)

\begin{tabular}{|c|c|c|c|c|c|c|c|c|c|}
\hline $\begin{array}{l}\text { Photon } \\
\text { energy } \\
(\mathrm{keV})\end{array}$ & $\frac{\sigma_{L_{1}}}{\sigma_{L}}$ & $\frac{\sigma_{L_{2}}}{\sigma_{L}}$ & $\frac{\sigma_{L_{3}}}{\sigma_{L}}$ & $\frac{\sigma_{L_{1}}}{\sigma_{L_{2,3}}}$ & $\frac{\sigma_{L_{2}}}{\sigma_{L_{3}}}$ & $\frac{\sigma_{L_{1}}}{\sigma_{\text {total }}}$ & $\frac{\sigma_{L_{2}}}{\sigma_{\text {total }}}$ & $\frac{\sigma_{L_{3}}}{\sigma_{\text {total }}}$ & $\begin{array}{l}\text { Photon } \\
\text { energy } \\
\text { (keV) }\end{array}$ \\
\hline \multicolumn{10}{|c|}{ PO $z=84$} \\
\hline $\begin{array}{l}13.91 \\
15.00 \\
16.28 \\
16.41 \\
16.91 \\
17.05 \\
20.00 \\
30.00 \\
40.00 \\
50.00 \\
60.00 \\
80.00 \\
93.41\end{array}$ & $\begin{array}{l}0.0 \\
0.0 \\
0.0 \\
0.0 \\
0.0 \\
0.181 \\
0.205 \\
0.274 \\
0.326 \\
0.368 \\
0.403 \\
0.455 \\
0.482\end{array}$ & $\begin{array}{l}0.0 \\
0.0 \\
0.0 \\
0.404 \\
0.409 \\
0.335 \\
C .332 \\
0.323 \\
0.312 \\
C .301 \\
C .292 \\
C .276 \\
C .268\end{array}$ & $\begin{array}{l}1.000 \\
1.000 \\
1.000 \\
0.596 \\
0.591 \\
0.484 \\
0.464 \\
0.403 \\
0.362 \\
0.330 \\
0.306 \\
0.269 \\
0.250\end{array}$ & $\begin{array}{l}0.0 \\
0.0 \\
0.0 \\
0.0 \\
0.0 \\
0.221 \\
0.257 \\
0.377 \\
0.485 \\
0.583 \\
0.674 \\
0.835 \\
0.930\end{array}$ & $\begin{array}{l}0.0 \\
0.0 \\
0.0 \\
0.677 \\
0.692 \\
0.692 \\
0.715 \\
0.803 \\
0.863 \\
0.912 \\
0.955 \\
1.027 \\
1.069\end{array}$ & $\begin{array}{l}0.0 \\
0.0 \\
0.0 \\
0.0 \\
0.0 \\
0.136 \\
0.154 \\
0.207 \\
0.248 \\
0.280 \\
0.307 \\
0.347 \\
0.367\end{array}$ & $\begin{array}{l}0.0 \\
0.0 \\
0.0 \\
0.286 \\
0.290 \\
0.251 \\
0.250 \\
0.245 \\
0.237 \\
0.229 \\
0.222 \\
0.211 \\
0.204\end{array}$ & $\begin{array}{l}0.596 \\
0.595 \\
0.591 \\
0.422 \\
0.420 \\
0.362 \\
0.349 \\
0.305 \\
0.275 \\
0.251 \\
0.233 \\
0.205 \\
0.191\end{array}$ & $\begin{array}{l}13.91 \\
15.00 \\
16.28 \\
16.41 \\
16.91 \\
17.05 \\
20.00 \\
30.00 \\
40.00 \\
50.00 \\
60.00 \\
80.00 \\
93.41\end{array}$ \\
\hline \multicolumn{10}{|c|}{ v $2=92$} \\
\hline $\begin{array}{r}17.30 \\
20.00 \\
21.03 \\
21.19 \\
21.78 \\
21.95 \\
30.00 \\
40.00 \\
50.00 \\
60.00 \\
80.00 \\
100.00 \\
116.20\end{array}$ & $\begin{array}{l}0.0 \\
0.0 \\
0.0 \\
0.0 \\
0.0 \\
0.182 \\
0.230 \\
0.278 \\
0.317 \\
0.349 \\
0.399 \\
0.436 \\
0.460\end{array}$ & $\begin{array}{l}0.0 \\
0.0 \\
0.0 \\
0.423 \\
0.426 \\
0.350 \\
C .35 C \\
0.344 \\
C .337 \\
C .330 \\
C .318 \\
0.308 \\
0.301\end{array}$ & $\begin{array}{l}1.000 \\
1.000 \\
1.000 \\
0.577 \\
0.574 \\
0.469 \\
0.420 \\
0.378 \\
0.346 \\
0.321 \\
0.283 \\
0.256 \\
0.239\end{array}$ & $\begin{array}{l}0.0 \\
0.0 \\
0.0 \\
0.0 \\
0.0 \\
0.222 \\
0.299 \\
0.384 \\
0.463 \\
0.536 \\
0.663 \\
0.773 \\
0.851\end{array}$ & $\begin{array}{l}0.0 \\
0.0 \\
0.0 \\
0.734 \\
0.742 \\
0.747 \\
0.833 \\
0.909 \\
0.973 \\
1.029 \\
1.123 \\
1.202 \\
1.258\end{array}$ & $\begin{array}{l}0.0 \\
0.0 \\
0.0 \\
0.0 \\
0.0 \\
0.134 \\
0.172 \\
0.208 \\
0.238 \\
0.263 \\
0.300 \\
0.329 \\
0.347\end{array}$ & $\begin{array}{l}0.0 \\
0.0 \\
0.0 \\
0.296 \\
0.298 \\
0.259 \\
0.261 \\
0.258 \\
0.253 \\
0.249 \\
0.240 \\
0.232 \\
0.227\end{array}$ & $\begin{array}{l}0.581 \\
0.574 \\
0.573 \\
0.403 \\
0.402 \\
0.347 \\
0.314 \\
0.284 \\
0.260 \\
0.242 \\
0.213 \\
0.193 \\
0.180\end{array}$ & $\begin{array}{r}17.30 \\
20.00 \\
29.03 \\
21.19 \\
21.78 \\
21.95 \\
30.00 \\
40.00 \\
50.00 \\
60.00 \\
80.00 \\
100.00 \\
116.20\end{array}$ \\
\hline \multicolumn{10}{|c|}{ PE $z=100$} \\
\hline $\begin{array}{r}21.04 \\
26.77 \\
26.99 \\
27.67 \\
27.89 \\
30.00 \\
40.00 \\
50.00 \\
60.00 \\
80.00 \\
100.00 \\
142.90\end{array}$ & $\begin{array}{l}0.0 \\
0.0 \\
0.0 \\
0.0 \\
0.180 \\
0.191 \\
0.234 \\
0.269 \\
0.299 \\
0.345 \\
0.380 \\
0.433\end{array}$ & $\begin{array}{l}0.0 \\
0.0 \\
0.443 \\
0.445 \\
C .367 \\
0.365 \\
0.372 \\
0.371 \\
0.368 \\
0.361 \\
0.354 \\
0.342\end{array}$ & $\begin{array}{l}1.000 \\
1.000 \\
0.557 \\
0.555 \\
0.453 \\
0.444 \\
0.393 \\
0.360 \\
0.333 \\
0.294 \\
0.266 \\
0.226\end{array}$ & $\begin{array}{l}0.0 \\
0.0 \\
0.0 \\
0.0 \\
0.220 \\
0.237 \\
0.306 \\
0.368 \\
0.426 \\
0.527 \\
0.613 \\
0.763\end{array}$ & $\begin{array}{l}0.0 \\
0.0 \\
0.795 \\
0.803 \\
0.811 \\
0.823 \\
0.946 \\
1.032 \\
1.106 \\
1.230 \\
1.333 \\
1.514\end{array}$ & $\begin{array}{l}0.0 \\
0.0 \\
0.0 \\
0.0 \\
0.132 \\
0.141 \\
0.174 \\
0.200 \\
0.223 \\
0.258 \\
0.284 \\
0.324\end{array}$ & $\begin{array}{l}0.0 \\
0.0 \\
0.307 \\
0.308 \\
0.269 \\
0.268 \\
0.276 \\
0.276 \\
0.275 \\
0.270 \\
0.265 \\
0.256\end{array}$ & $\begin{array}{l}0.568 \\
0.557 \\
0.386 \\
0.384 \\
0.332 \\
0.326 \\
0.292 \\
0.268 \\
0.248 \\
0.219 \\
0.199 \\
0.169\end{array}$ & $\begin{array}{r}21.04 \\
26.77 \\
26.99 \\
27.67 \\
27.89 \\
30.00 \\
40.00 \\
50.00 \\
60.00 \\
80.00 \\
100.00 \\
142.90\end{array}$ \\
\hline
\end{tabular}


Table 22. Conversion factor $C=\frac{\text { Atomic weight }(\mathrm{g} / \mathrm{mole})}{\text { Avogadro number }} \times 10^{24}$

Divide values in Tables 7 to 14 by $C$ to obtain cross section values in units of $\mathrm{cm}^{2} / \mathrm{g}, \mu / \rho\left(\mathrm{cm}^{2} / \mathrm{g}\right)=\sigma / C$ (barns/atom). Note that for unstable elements, $C$ was calculated for the most common isotope that is either the longest lived or the most often used one.

\begin{tabular}{|c|c|c|c|c|c|}
\hline $\begin{array}{l}1 \\
2 \\
3 \\
4 \\
5\end{array}$ & $\begin{array}{l}\text { H } \\
\text { HF } \\
\text { LI } \\
\text { BE } \\
\text { B }\end{array}$ & $\begin{array}{l}1.674 \\
6.647 \\
11.52 \\
14.96 \\
17.95\end{array}$ & $\begin{array}{l}51 \\
52 \\
53 \\
54 \\
55\end{array}$ & $\begin{array}{l}S B \\
T E \\
I \\
X E \\
C S\end{array}$ & $\begin{array}{l}202.2 \\
211.9 \\
210.7 \\
218.0 \\
220.7\end{array}$ \\
\hline $\begin{array}{r}6 \\
7 \\
8 \\
9 \\
10\end{array}$ & $\begin{array}{l}\text { C } \\
N \\
O \\
F \\
N E\end{array}$ & $\begin{array}{l}19.94 \\
23.26 \\
26.57 \\
31.55 \\
33.51\end{array}$ & $\begin{array}{l}56 \\
57 \\
58 \\
59 \\
60\end{array}$ & $\begin{array}{l}\text { BA } \\
\text { LA } \\
\text { CE } \\
\text { PR } \\
\text { ND }\end{array}$ & $\begin{array}{l}228.1 \\
230.7 \\
232.7 \\
234.0 \\
239.6\end{array}$ \\
\hline $\begin{array}{l}11 \\
12 \\
13 \\
14 \\
15\end{array}$ & $\begin{array}{l}\text { NA } \\
\text { HG } \\
\text { AL } \\
\text { SI } \\
\text { P }\end{array}$ & $\begin{array}{l}38.19 \\
40.38 \\
44.78 \\
46.63 \\
51.43\end{array}$ & $\begin{array}{l}61 \\
62 \\
63 \\
64 \\
65\end{array}$ & $\begin{array}{l}\text { PM } \\
\text { SM } \\
\text { EO } \\
\text { GD } \\
\text { TB }\end{array}$ & $\begin{array}{l}244.1 \\
249.6 \\
252.4 \\
261.1 \\
263.9\end{array}$ \\
\hline $\begin{array}{l}16 \\
17 \\
18 \\
19 \\
20\end{array}$ & $\begin{array}{l}S \\
C L \\
A R \\
k \\
C A\end{array}$ & $\begin{array}{l}53.24 \\
58.87 \\
66.32 \\
64.93 \\
66.55\end{array}$ & $\begin{array}{l}66 \\
67 \\
68 \\
69 \\
70\end{array}$ & $\begin{array}{l}\text { DY } \\
\text { HO } \\
\text { ER } \\
\text { TH } \\
\text { YB }\end{array}$ & $\begin{array}{l}269.8 \\
273.9 \\
277.7 \\
280.5 \\
287.3\end{array}$ \\
\hline $\begin{array}{l}21 \\
22 \\
23 \\
24 \\
25\end{array}$ & $\begin{array}{l}\mathrm{SC} \\
\mathrm{TI} \\
\mathrm{V} \\
\mathrm{CH} \\
\mathrm{MN}\end{array}$ & $\begin{array}{l}74.65 \\
79.53 \\
84.59 \\
86.34 \\
91.22\end{array}$ & $\begin{array}{l}71 \\
72 \\
73 \\
74 \\
75\end{array}$ & $\begin{array}{l}\text { LU } \\
\text { HF } \\
\text { TA } \\
W \\
\text { RE }\end{array}$ & $\begin{array}{l}290.6 \\
296.4 \\
300.5 \\
305.4 \\
309.2\end{array}$ \\
\hline $\begin{array}{l}26 \\
27 \\
28 \\
29 \\
30\end{array}$ & $\begin{array}{l}P E \\
C D \\
N I \\
C U \\
Z N\end{array}$ & $\begin{array}{l}92.74 \\
97.85 \\
97.45 \\
105.5 \\
108.6\end{array}$ & $\begin{array}{l}76 \\
77 \\
78 \\
79 \\
80\end{array}$ & $\begin{array}{l}\text { OS } \\
\text { IR } \\
\text { PT } \\
\text { AU } \\
\text { HG }\end{array}$ & $\begin{array}{l}315.8 \\
319.1 \\
323.9 \\
327.4 \\
333.1\end{array}$ \\
\hline $\begin{array}{l}31 \\
32 \\
33 \\
34 \\
35\end{array}$ & $\begin{array}{l}\text { GA } \\
\text { GE } \\
\text { AS } \\
\text { SE } \\
\text { BR }\end{array}$ & $\begin{array}{l}115.8 \\
120.5 \\
124.4 \\
131.1 \\
132.7\end{array}$ & $\begin{array}{l}81 \\
82 \\
83 \\
84 \\
85\end{array}$ & $\begin{array}{l}\text { TL } \\
\text { PB } \\
\text { BI } \\
\text { PO } \\
\text { A T }\end{array}$ & $\begin{array}{l}339.4 \\
344.1 \\
347.0 \\
347.1 \\
347.8\end{array}$ \\
\hline $\begin{array}{l}36 \\
37 \\
38 \\
39 \\
40\end{array}$ & $\begin{array}{l}K R \\
R B \\
S Q \\
Y \\
Z R\end{array}$ & $\begin{array}{l}139.1 \\
141.9 \\
145.5 \\
147.6 \\
151.5\end{array}$ & $\begin{array}{l}86 \\
87 \\
88 \\
89 \\
90\end{array}$ & $\begin{array}{l}\text { RN } \\
\text { FR } \\
\text { RA } \\
\text { AC } \\
\text { TH }\end{array}$ & $\begin{array}{l}368.6 \\
370.3 \\
375.3 \\
377.0 \\
385.3\end{array}$ \\
\hline $\begin{array}{l}41 \\
42 \\
43 \\
44 \\
45\end{array}$ & $\begin{array}{l}\text { NR } \\
\text { MO } \\
\text { TC } \\
\text { RU } \\
\text { RH }\end{array}$ & $\begin{array}{l}154.3 \\
159.3 \\
164.4 \\
167.8 \\
170.9\end{array}$ & $\begin{array}{l}91 \\
92 \\
93 \\
94 \\
95\end{array}$ & $\begin{array}{l}\text { PA } \\
\text { U } \\
\text { NP } \\
\text { PU } \\
\text { A }\end{array}$ & $\begin{array}{l}383.6 \\
395.3 \\
393.6 \\
397.0 \\
403.6\end{array}$ \\
\hline $\begin{array}{l}46 \\
47 \\
48 \\
49 \\
50\end{array}$ & $\begin{array}{l}\text { PN } \\
\text { A3 } \\
\text { CD } \\
\text { IN } \\
\text { SN }\end{array}$ & $\begin{array}{l}176.7 \\
179.1 \\
186.6 \\
190.7 \\
197.1\end{array}$ & $\begin{array}{r}96 \\
97 \\
98 \\
99 \\
100\end{array}$ & $\begin{array}{l}\text { CH } \\
B K \\
C P \\
\text { ES } \\
\text { FM }\end{array}$ & $\begin{array}{l}411.9 \\
413.6 \\
413.6 \\
420.2 \\
418.6\end{array}$ \\
\hline & & & 101 & MD & 428.4 \\
\hline
\end{tabular}


0

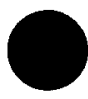




\section{REFERENCES}

1. M. 0. Krause, E. Ricci, C. J. Sparks, Jr., and C. W. Nestor, Jr., Adv. X-Ray AnaZ. 21: 119 (1978).

2. J. H. Scofield, Report UCRL-51326, Lawrence Livermore Laboratory, 1973 (unpublished).

3. M. 0. Krause, J. Phys. Chem. Ref. Data (to be published).

4. J. H. Scofield, Phys. Rev. A 9: 1041 (1974).

5. J. H. Scofield, Phys. Rev. A 10: 1507 (1974).

6. J. H. Scofield, Phys. Rev. A 12: 345 (1975).

7. J. H. Scofield, in Atomic Innershezl Processes, B. Crasemann, Ed., vo1. I, Academic Press, 1975, chap. 6.

8. Values for elements not listed in refs. 4-7 were interpolated with the aid of Scofield's relativistic Hartree-Slater predictions [At. Data Nucl. Data Tables 14: 121 (1974)].

9. E. F. Plechaty, D. E. Cullen, and R. J. Howerton, Report UCRL-50400, Lawrence Livermore Laboratory, 1975 (unpublished).

10. Values given in ref. 9 are identical with those of W. H. McMaster et al., Report UCRL-50174, Lawrence Livermore Laboratory, 1969, for most elements in the present energy range.

11. Wm. J. Veigele, At. Data Nucl. Data Tables 5: 51 (1973).

12. Predictions by E. Storm and H. I. Israel, At. Data Nucl. Data Tables A7: 565 (1970) are up to $2 \%$ smaller than Scofield's.

13. F. Wuilleumier and M. 0. Krause, Phys. Rev. A 10: 242 (1974).

14. J. H. Scofield, J. Electron Spectrosc. Relat. Phenom. 8: 129 (1976).

15. R. H. Pratt, A. Ron, and H. K. Tseng, Rev. Mod. Phys. 45: 273 (1973), specifically Fig. 5.

16. S. I. Salem, S. L. Panossian, and R. A. Krause, At. Data Nucl. Data Tables 14: 91 (1974).

17. C. S. Fad1ey, Chem. Phys. Lett. 25: 225 (1974).

18. M. 0. Krause, F. Wuilleumier, and C. W. Nestor, Jr., Phys. Rev. A 6: 871 (1972). 
19. R. L. Watson, M. W. Michael, J. Hernandez, A. K. Leeper, and C. D. Wendt, Adv. X-Ray Anal. (to be published in vol. 21).

20. R. D. Giauque and J. M. Jaklevic, Adv. X-Ray AnaZ. 15: 164 (1971).

21. For work earlier than refs. 19 and 20, see K. I. Narbutt, BuZZ. Acad. Sci. USSR, Phys. Ser. 28: 765 (1964).

22. R. J. Grader, A. J. Oliver, and P. J. Ebert, Phys. Rev. A 16: 2388 (1977).

23. J. H. Scofield, Phys. Rev. A 14: 1418 (1976).

24. J. H. Scofield, private communication. 


\section{ORNL-5399 \\ Distribution Category UC-4}

\section{INTERNAL DISTRIBUTION}

1-3. Central Research Library

4. ORNL - Y-12 Technical Library Document Reference Section

5-18. Laboratory Records Department

19. Laboratory Records, ORNL R.C.

20. ORNL Patent office

21. C. F. Barnett

22. C. E. Bemis

23. R. L. Hahn

24. R. F. Hibbs

25. L. D. Hulett

26. L. H. Jenkins

27. 0. L. Keller

\author{
28-58. M. 0. Krause \\ 59. C. J. McHargue \\ 60. P. D. Miller \\ 61-74. C. W. Nestor, Jr. \\ 75. H. Postma \\ 76-85. E. Ricci \\ 86. R. W. Roussin \\ 87. W. D. Shults \\ 88. A. H. Smith \\ 89-104. C. J. Sparks \\ 105. J. R. Stokely, Jr. \\ 106. A. Zucker
}

\section{EXTERNAL DISTRIBUTION}

107. Dr. Don Bilderback, Department of Materials Science and Engineering, Cornel1 University, Bard Ha11, Ithaca, NY 14850

108. K. Maack Bisgard, Physics Laboratory, Royal Veterinary- and Agricultural University, Thorvaldsensvej 40, DK 1871, Copenhagen V, Denmark

109. P. G. Burkhalter, Naval Research Laboratory, Code 6680, Washington, DC 20390

110. Dr. W. J. Campbe11, U.S. Department of the Interior, Bureau of Mines, College Park, MD 20740

111. James L. Cate, L-520, University of California, Lawrence Livermore Laboratory, P.0. Box 808, Livermore, CA 94550

112. Dr. L. F. De Filippis, Department of Botany, La Trobe University, Bundoora, Melbourne, Victoria, Australia 3083

113. David W. Dugan, United States Department of the Interior, Geological Survey, Water Resources Division, 5110 College Avenue, College Park, MD 20740

114. Robert Etherton, Box 2109, University Station, Murray, KY 42071

115. Dr. R. W. Fink, School of Chemistry, Georgia Institute of Technology, Atlanta, GA 30332

116. Dr. Dale Gedcke, ORTEC, Inc., 100 Midland Road, Oak Ridge, TN 37830

117. Dr. Robert D. Giauque, Lawrence Berkeley Laboratory, University of California, Berkeley, CA 94720

118. Gene Golovchenko, Bell Laboratories, 600 Mountain Avenue, Murray Hill, $\mathrm{NJ}$

119. Dr. L. Gonzalez, Junta de Energia Nuclear, Dirision de Fisica-Reactor Avda. Complutense, 22, Madrid-3, Spain

120. Dr. Barry Gordon, Department of Applied Scieiıce, Brookhaven National Laboratory, Upton, NY 11973 
121. Prof. R. W. Gould, Department of Materials Science and Engineering, College of Engineering, University of Florida, Gainesville, FL 32611

122. P. Halpin, Environmental Protection Agency, OAWP, OAOPS, APTIC, Research Triangle Park, NC 27711

123. Dr. K. F. J. Heinrich, Section Chief, 310.02 Div., B1dg Chem. A121, U.S. Department of Commerce, National Bureau of Standards, Washington, DC 20234

124. Dr. L. Husain, Division of Laboratories and Research, New York State Department of Health, New Scotland Avenue, Albany, NY 12201

125. Louis C. Iannie11o, Division of Materials Science, Office of Basic Energy Science, Department of Energy, Washington, DC 20545

126. Gerald Kaplan, Johnson \& Johnson, Central Analytical Labs, New Brunswick, NJ 08903

127. Virginia Kobelt, Parma Tech Center, Union Carbide, P.o. Box 6116 Cleveland, $\mathrm{OH} 44101$

128. Dr. Daniel B. Lister, Mgr., Industrial Systems, Princeton GammaTech Inc., Box 641, Princeton, NJ 08540

129. Kirk Melson, Battelle-Northwest, P.0. Box 999, Richland, WA 99352

130. Donald Mentzer, Union Carbide Corporation, Parma Technical Center, 12900 Snow Road, Cleveland, $\mathrm{OH}$

131. S. G. Metcalf, Atlantic Richfield Handford Company, Building 2225, Area 200 West, Richland, WA 99352

132. Dr. Lee Middleman, Nuclear Semiconductor, 161 Constitution Drive, Menlo Park, CA 94025

133. Prof. Nolan F. Mongelson, Chemistry Department 255ESC, Brigham Young University, Provo, UT 84601

134. Jim Mulvaney, Nuclear Physical Laboratory, University of Colorado, Boulder, CO 80302

135. Dr. Peter A. Pella, Division 310.02, Building Chemistry A121 U.S. Department of Commerce, National Bureau of Standards, Washington, DC 20234

136. Sta. Miriam Pino, Instituto de Higiene del Trabajo Y Contaminacion Atmosferica, S.N.S., Santo Domingo 2398, Casilla 3979, Santiago, Chile

137. Alan P. Quinn, Corning Glass Works, Corning, NY 14830

138. Rodney R. Ruch, Chemist, Analytical Chemistry Section, Illinois State Geological Survey, Natural Resources Building, Urbana, IL 61801

139. Richard W. Ryon, L-404, Lawrence Livermore Laboratory, University of California, P.0. Box 808, Livermore, CA 94550

140. R. A. Semmler, Chemistry Research Division, IIT Research Institute, 10 West 35 Street, Chicago, IL 60616

141. Dr. Saiyid Shan, Physics Department, The University of Texas System Cancer Center, M. D. Anderson Hospital and Tumor Institute, Texas Medical Center, Houston, TX 77025

142. R. W. Shaw, Jr., Department of Chemistry, Duke University, Durham, NC 27706

143. Dr. Yair Talmi, Princeton Applied Research Corporation, P.0. Box 2565, Princeton, NJ 08540 
144. Dr. Robert Towns, Department of Chemistry, Cleveland State University, Cleveland, $\mathrm{OH} 44115$

145. Nancy Ulmer, USEPA, 4676 Columbia Parkway, Cincinnati, OH 45268

146. Dr. E. Unger, Staatliches Forschungsinstitut fur Geochemie, 86 Bamberg, Concordiastraße 28, Postfach 4041, BRD, West Germany

147. Dr. N. E. Whitehead, The Institute of Nuclear Sciences, DSIR, Lower Hutt, Wellington, New Zealand

148. Director, Research and Technical Support Division, DOE-ORO, Oak Ridge, TN 37830

149-343. Given distribution as shown in TID-4500 under distribution category UC-4 (25 copies - NTIS) 